
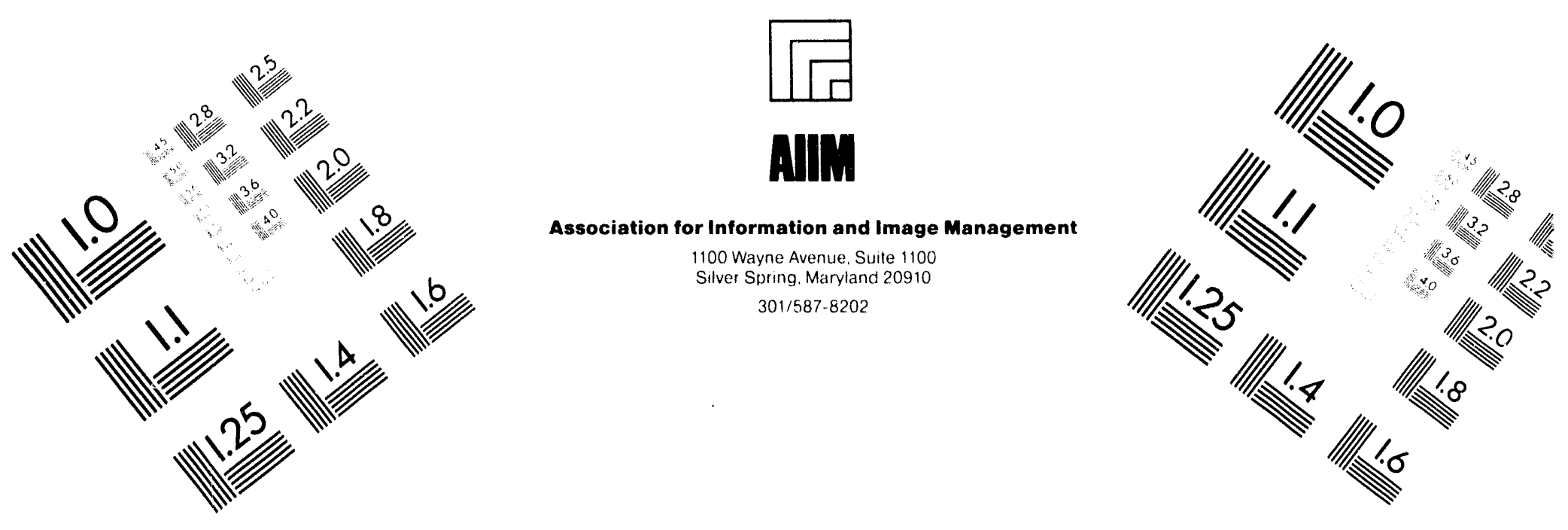

\title{
Centimeter
}

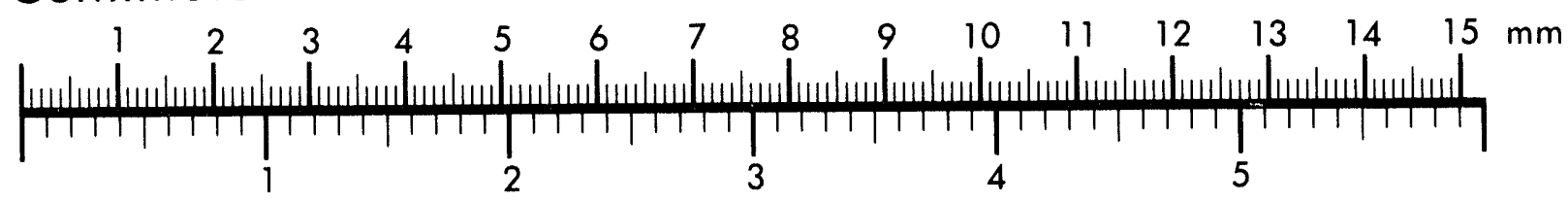
Inches
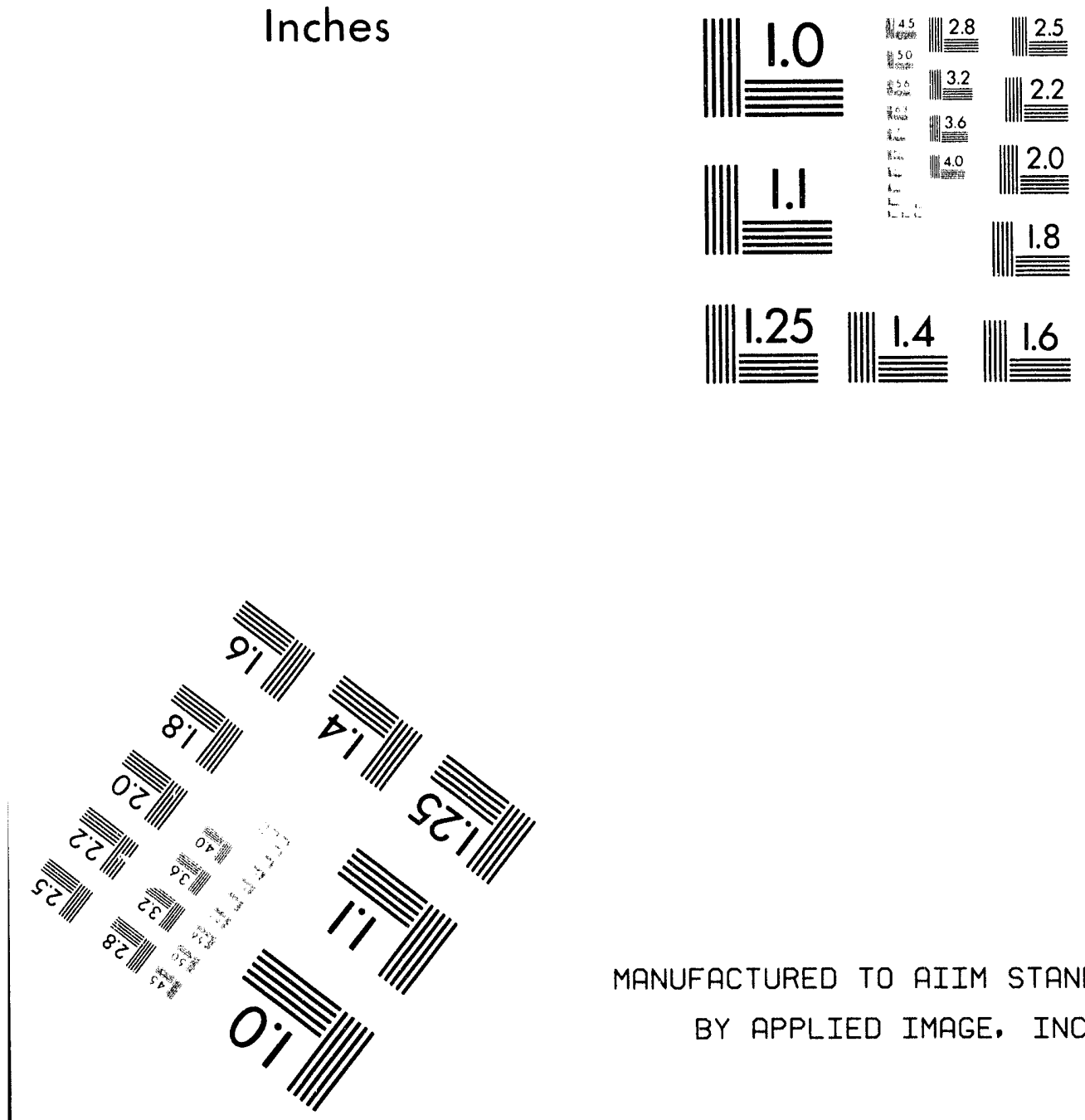

MANUFACTURED TO AIIM STANDARDS BY APPLIED IMAGE. INC.

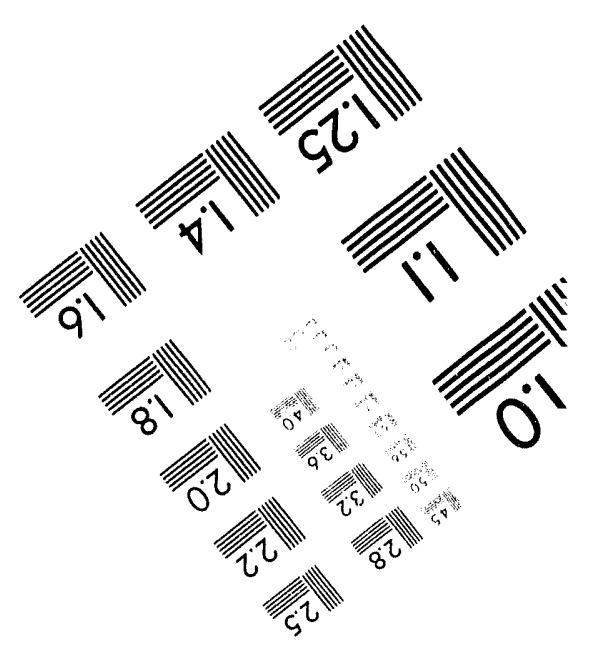



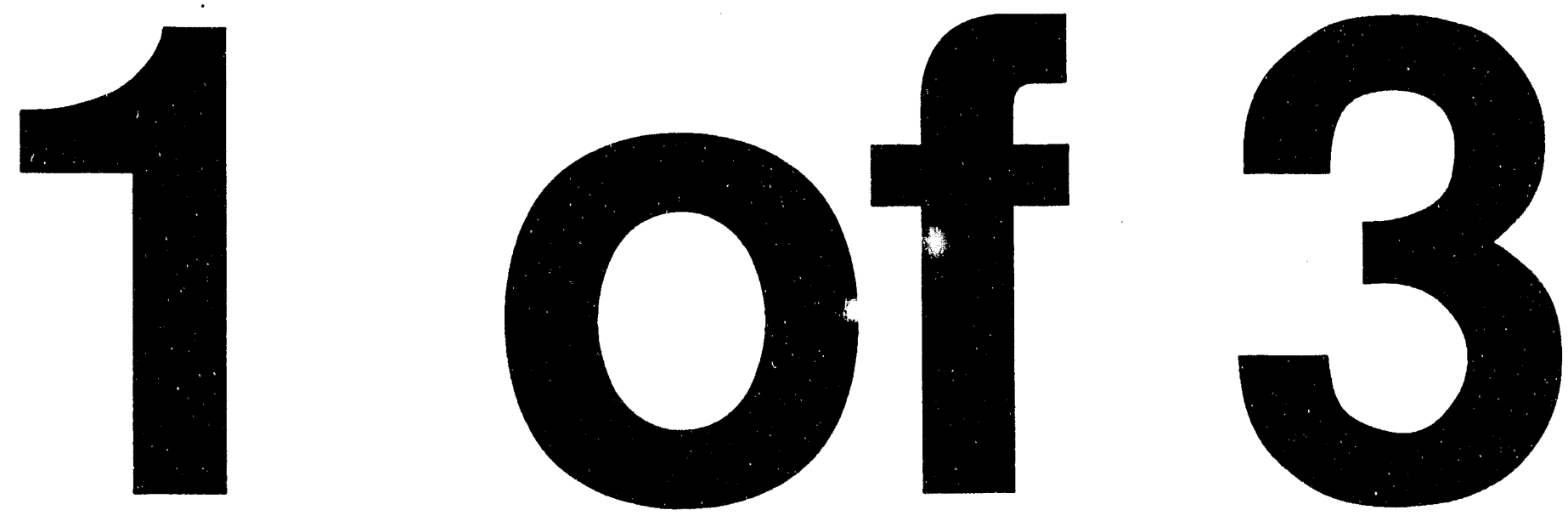


\title{
UNITED STATES DEPARTMENT OF THE INTERIOR GEOLOGICAL SURVEY
}

\author{
Denver, Colorado
}

\section{PRELIMINARY SEISMICITY AND FOCAL MECHANISMS FOR THE SOUTHERN GREAT BASIN OF NEVADA AND CALIFORNIA: JANUARY 1992 THROUGH SEPTEMBER 1992}

by

\author{
S.C. Harmsen
}

\author{
DISCLAIMER
}

This report was prepared as an account of work sponsored by an agency of the United States This report was prepared as an account of work sponsored by an agency thereof, nor any of their employees, makes any warranty, express or implied, or assumes any legal liability or responsibility for the accuracy, completeness, or usefulness of any information, apparatus, product, or prucess disclosed, or represents that its use would not infringe privately owned rights. Reference herein to any specific commercial product, process, or service by trade name, trademark, manufacturer, or otherwise does not necessarily constitute or imply its endorsement, recommendation, or favoring by the United States Government or any agency thereof. The views and opinions of authors expressed herein do not necessarily state or reflect those of the United States Government or any agency thereof.

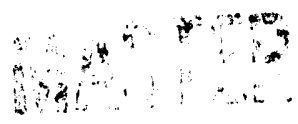




\section{U.S. DEPARTMENT OF THE INTERIOR \\ BRUCE BABBITT, Secretary}

U.S. GEOLOGICAL SURVEY

Gordon P. Eaton, Director

The use of trade, product, industry, or firm names is for descriptive purposes only and does not imply endorsement by the U.S. Government.

For additional information write to:

Chief, Geologic Studies Program Yucca Mountain Project Branch

U.S. Geological Survey

Box 25046, MS 421

Denver Federal Center

Denver, CO 80225
Copies of this report can be purchased from:

U.S. Geological Survey

Earth Science Information Center

Open-File Reports Section

Box 25286, MS 517

Denver Federal Center

Denver, CO 80225 


\section{CONTENTS}

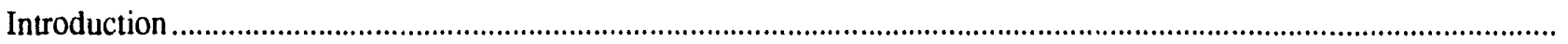

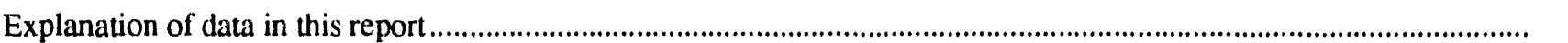

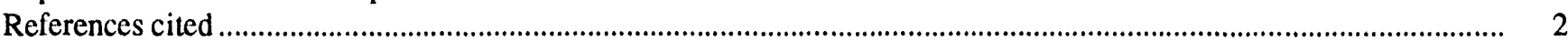

Appendix A. Preliminary southern Great Basin earthquake locations for January to September 1992 ........................... 5

Appendix B. Preliminary SGB chemical and nuclear explosion location data, January 1 to September 30, 1992............ 93

Appendix C. Southern Great Basin preliminary earthquake focal mechanisms, January to September, 1992 .................. 99

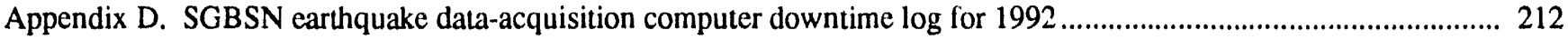

\section{FIGURES}

1. Map of southern Great Basin showing SGBSN station locations, major faults that are known or suspected to have moved in the last 2-3 million years, epicenters for the period January 1 through September 30, 1992, cities and towns.

2. Map of vicinity of Little Skull Mountain with preliminary SGBSN-derived epicenters for the period January 1, 1992 through September 30, 1992

B1. Map of epicenters for chemical explosions and nuclear device tests in the southern Great Basin of Nevada and California for the period January 1, 1992 to September 30, 1992

C1. Map of earthquake epicenters for 110 earthquakes for which focal mechanisms were computed for the period January 1, 1992 to September 30, 1992. Lower-hemisphere projections of the focal mechanisms for the six largest of these earthquakes with magnitude $\mathrm{M}_{\mathrm{L}} \geq 4.0$ are shown...

Remaining figures of Appendix C. - Focal mechanism solutions for SGB earthquakes, January through September, 1992 .

\section{TABLES}

B1. Announced NTS nuclear device tests at Nevada Test Site in first 9 months of 1992 .............................................. 93

C1. USGS/Little Skull Mountain, temporary seismographic station coordinates ......................................................... 100

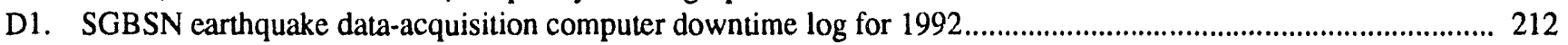




\title{
Preliminary Seismicity and Focal Mechanisms for the Southern Great Basin of Nevada and California: January 1992 through September 1992
}

\author{
by S.C. Harmsen
}

\section{INTRODUCTION}

The telemetered southern Great Basin seismic network (SGBSN) is operated for the Department of Energy's Yucca Mountain Project (YMP). The U.S. Geological Survey, Branch of Earthquake and Landslide Hazards, maintained this network until September 30,1992, at which time all operational and analysis responsibilities were transferred to the University of Nevada at Reno Seismological Laboratory (UNRSL). This report contains preliminary earthquake and chemical explosion hypocenter listings and preliminary earthquake focal mechanism solutions for USGS/ SGBSN data for the period January 1, 1992 through September 30, 1992, 15:00 UTC.

\section{EXPLANATION OF DATA IN THIS REPORT}

The local earthquake and blast hypocenter summaries of Appendices A and B, respectively, follow the same format as previous SGBSN seismicity open-file reports, such as that by Harmsen (1993), which contain definitions, lists of station coordinates, and velocity models used. Most data in this report were obtained from computer-digitized seismograms, except for periods when the Golden, Colorado, seismic monitoring computer was not operating. The seismic backup data consists of 16 millimeter develocorder films, which were scaled for local earthquake data through May 31, 1992, and for the Little Skull Mountain mainshock of June 29, 1992, 10:14:22 UTC. The develocorder films are currently archived by the YMP/USGS Local Records Center, Denver, Co. A list of computer downtime for which USGS/SGBSN hypocenter data are not available is given in Appendix D.

Preliminary hypocenter parameters were derived primarily on the basis of SGBSN data. Supplemental $\mathrm{P}$-wave polarity and arrival time data were supplied by neighboring regional seismic networks for some of the largest southern Great Basin (SGB) earthquakes of 1992. Some hypocenters in the southwestern part of the SGB were obtained from the USGS Southern Califormia seismic network (L. Wald, USGS, Pasadena,
CA, written commun., 1992), and are noted in the listing. In a few instances, magnitude data from other organizations or other branches of the USGS has been included in this listing. For example, the Little Skull Mountain mainshock of June 29, 1992, is listed with magnitude $M_{L}=5.6$, an estimate supplied by the USGS National Earthquake Information Center, Golden, Co. Many focal mechanism solutions presented in Appendix C incorporate data from a temporary seismic network that the USGS deployed around Little Skull Mountain from June 30, 1992 to July 14, 1992. Details of the deployment are contained in a report by Meremonte and others (1993). Figure 1 is a map of January through September earthquake epicenters in the SGB plotted from the USGS/SGBSN hypocenter data given in Appendix A. Figure 2 is a map of earthquake epicenters for the same period, for the area within the central rectangle of figure 1. Many of the faults shown in figure 2 seem to define a left lateral strike slip fault domain (Carr, 1984), but the Little Skull Mountain mainshock and the majority of aftershock focal mechanisms shown in Appendix $\mathrm{C}$ are predominantly normal slip.

The earthquake hypocenter listing of Appendix A excludes all low-coda-frequency seismicity associated with Nevada Test Site (NTS) nuclear device tests. Such phenomena include aftershocks at ultra-shallow hypocentral depths and cavity collapses. If these events are primarily induced by nuclear testing, then their inclusion in an earthquake catalog would bias any effort to characterize natural seismicity rates in the northern NTS from this catalog. Continued research on low-frequency phenomena at northern NTS recorded by SGBSN may have value relative to monitoring compliance with nuclear test-ban treatics (P. Richards, Lamont Doherty Obs., Columbia Univ., NJ, written commun., 1993).

A variety of studies have been completed and others are underway discussing the seismicity of the SGB in 1992. This report is being presented without analysis of the seismicity data in order to fulfill require. ments of a Memorandum of Agreement, and to provide 
many previously unpublished focal mechanism solutions. A comprehensive catalog of SGB earthquakes for the calendar year 1992 is presently being compiled for publication by the UNRSL (D. von Seggern, oral commun., 1993), and will supercede this interim report's data.

\section{REFERENCES CITED}

Brocher, T.M., Hart, P.E., and Carle, S.F., 1990, Feasibility study of the seismic reflection method in Amargosa Desert, Nye County, Nevada: U.S. Geological Survey Open File Report 89-133, 150 pp. (NNA.901106.0099)

Carr, W.J., 1984, Regional structural setting of Yucca Mountain, southwestern Nevada, and late Cenozoic rates of tectonic activity in part of the southwestern Great Basin, Nevada and California: U.S. Geological Survey Open-File Report 84-854, 109 p. (NNA.870325.0475)

Harmsen, S.C., and Bufe, C.G., 1992, Seismicity and focal mechanisms in the southern Great Basin of Nevada and California: 1987 through 1989: U.S. Geological Survey Open-File Report 91-572, 216 p. (NNA.920408.0001)

Harmsen, S.C., 1993, Seismicity and focal mechanisms for the southern Great Basin of Nevada and California in 1991: U.S. Geological Survey Open-File Report 92-340, 100 p. (NNA.920629.0129)
Lee, W.H.K., and Lahr, J.C., 1975, HYPO7l (revised): A computer program for determining hypocenter, magnitude, and first-motion pattern of local earthquakes: U.S. Geological Survey Open-File Report 75-311, 116 pp. (NNA.920211.0038)

Maldonado, F., 1985, Geologic map of the Jackass Flats area, Nye County, Nevada, USGS Misc. Investigations Map 1519. (HQS.880517.1925)

Meremonte, M., Cranswick. E., Gomberg, J., Worley, D., Carver, D., Brooks, J., Banfill, R., Overturf, D., and Bice, T., 1993, Report on seismologic field investigations of the 29 June 1992 Little Skull Mountain earthquake: U.S. Geological Survey Open-File Report 93-555, 95 pp. (NNA.931117.0008)

Snoke, J.A., Munsey, J.W., Teague, A.G., and Bollinger, G.A., 1984, A program for focal mechanism determination by combined use of polarity and amplitude ratio data: Earthquake Notes, v. 55, p. 15. (NNA.920211.0046)

Wald, L.A., Watts, K., Mori, J., and Douglass, K., 1993, The Southern California Network Bulletin, JanuaryDecember 1992: U.S. Geological Survey Open-File Report 93-227, 50 pp. (NNA.931117.0009)

NOTE: Parenthesized numbers following each cited reference are for U.S. Department of Energy OCRWM Records Management purposes only and should not be used when ordering the publication. 


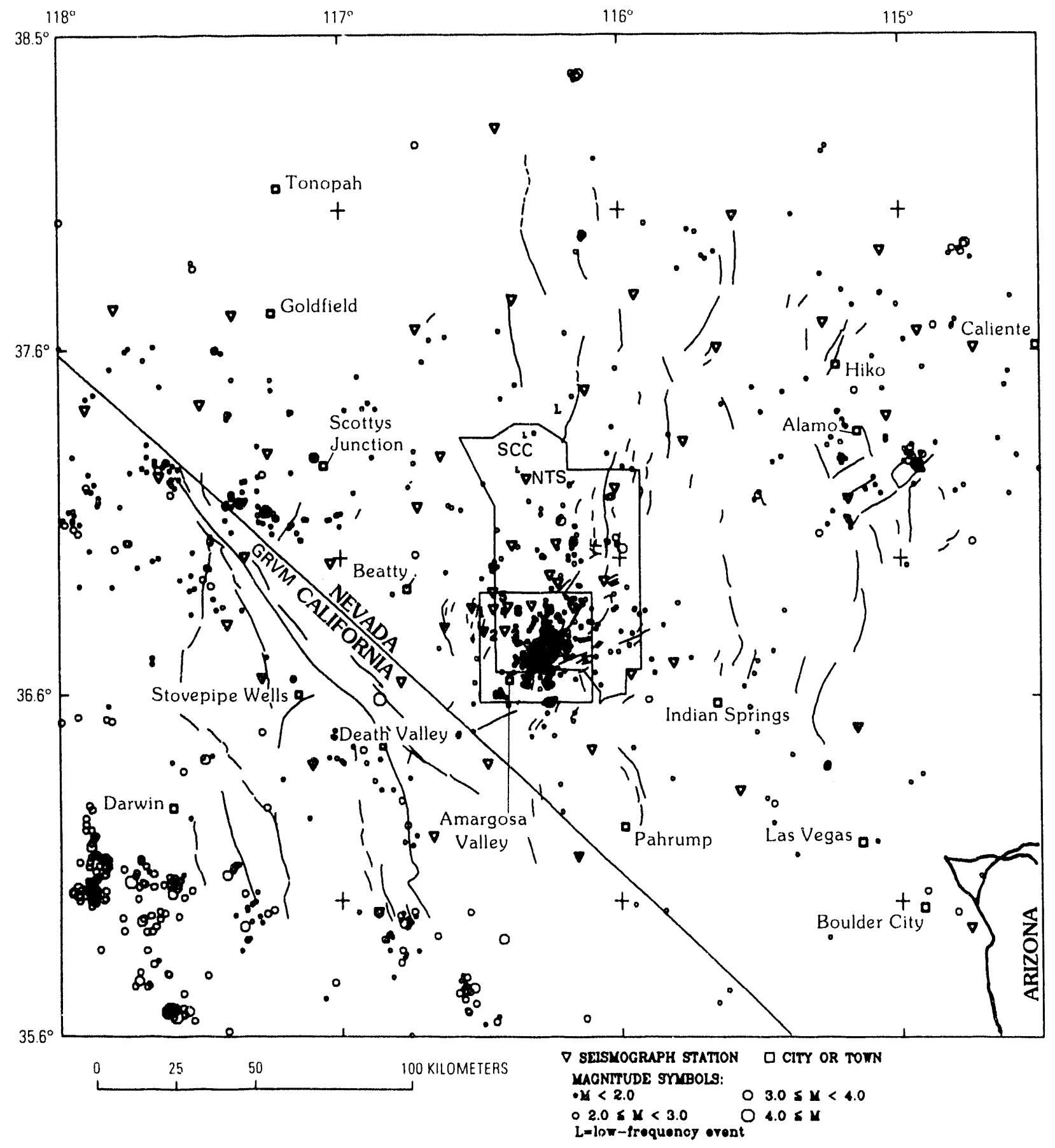

Figure 1. - Map of southern Great Basin showing southern Great Basin seismic network station locations, major faults that are known or suspected to have moved in the last 2-3 million years, epicenters for the period January 1 through September 30,1992, cities and towns. GRVM=Grapevine Mountains, NTS= Nevada Test Site, $S C C=$ Silent Canyon Caldera, YF=Yucca Flat. Figure 2, below, shows data for the box over the southwestern NTS, containing the study area for the USCS Little Skull Mountain aftershock investigation (Meremonte and others, 1993). 


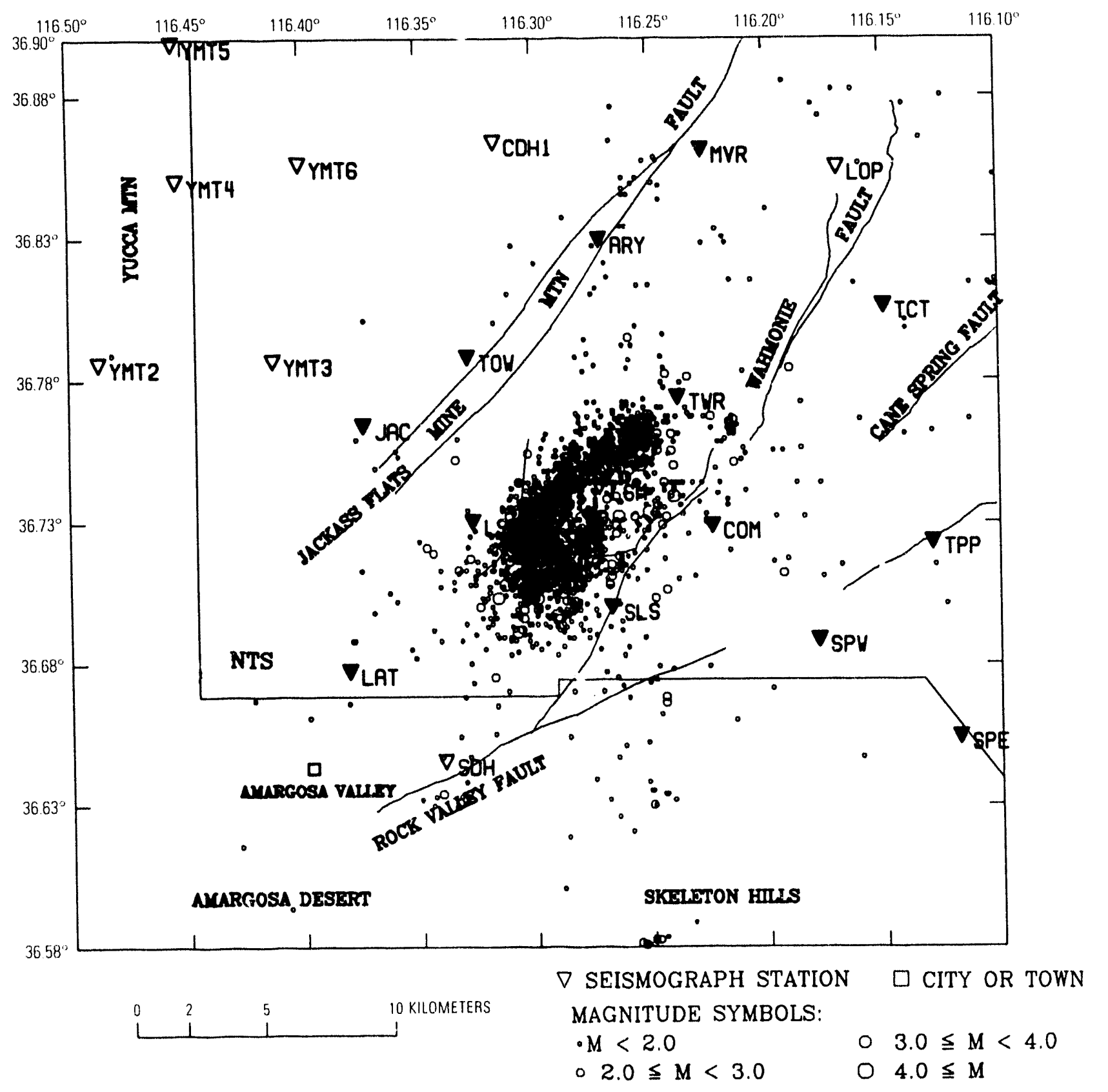

Figure 2. - Map of vicinity of Little Skull Mountain with preliminary southern Great Basin seismic network-derived epicenters for the period January 1, 1992 through September 30, 1992. Relatively major faults are shown, digitized from Maldonado (1985). Southwestern NTS boundary is shown. Permanent SGBSN stations are shown as open triangles, temporary network stations as darkened triangles. 


\section{Appendix A}

\section{Preliminary SGB earthquake locations for January to September 1992}

Approximately 3800 distinct earthquake hypocenters are reported here. All SGBSN preliminary hypocenters in this appendix were computed using the Fortran program, HYPO71 (Lee and Lahr, 1975). Hypocenters for the Little Skull Mountain foreshocks, mainshock, and aftershocks incorporate P-arrival delays determined in a hypocenter relocation study (J. Gomberg, written commun., 1992). S-arrival delays are defined by the formula, $S_{\text {delay }}=$ $P_{\text {delay }} \times 1.71$. The local hypocenter summary column headings are for the most part selfexplanatory. UTC is Universal Coordinated Time. Horizontal error equals $\sqrt{s d x^{2}+s d y^{2}}$, where $s d x$ and $s d y$ refer to the HYPO71 standard errors in longitude and latitude, respectively. Vertical error is the HYPO71 standard error in depth $(s d z)$. "AZI GAP" is the azimuthal gap, that is, the largest angle subtended by the epicenter and any two circularly adjacent stations with positive phase weight. "Q1" and "Q2" represent two HYPO71 hypocenter quality estimates as defined by Lee and Lahr (1975). "DS" is a code for data source: A for analog seismograms, (data scaled from Develocorder films, starting depth, $z_{0}$, at $7 \mathrm{~km}$ for iterations), I for data scaled from digital seismograms. In Appendix A, preliminary hypocenter data in the SGB computed by the Southern California Seismic Network (SCSN) for the period January 1, 1992, to July 21,1992 , have been included as originally reported (L. Wald, written commun., 1992). SCSN hypocenters for $M_{L} \geq 3$ earthquakes were subsequently reviewed and published (Wald and others, 1993) and may differ from the preliminary data given here. Hypocenter data from SCSN may be of value in assessments of possible triggering of the Little Skull Mountain, Nv., earthquake of June 29, 1992 by the Landers, Ca., mainshock of June 28, 1992. All SCSN hypocenters are identified by the letter "P" in the DS column (Pasadena, Ca., data source). The SCSN hypocenters reported here contain no standard horizontal error, AZI GAP, vertical error, or DELMIN (see below) information. These parameter estimates have been set to zero, 999, - , and blank, respectively, in this appendix. A few hypocenters are listed twice, as determined by SGBSN and by SCSN, respectively, with an equal sign tying them (example, July 17, 1992, 03:04:56).

Various magnitude estimates are computed for the earthquake hypocenters. Mca is the coda-average magnitude, $\mathrm{Md}$ is the duration magnitude estimate, MLh is local magnitude from horizontal-component instruments, MLv is local magnitude from vertical-component instruments, MLc is the maximum of station magnitudes scaled from overdriven (clipped) records. Wavelet amplitudes scaled from vertical-component seismograms are multiplied by 1.75 to approximate the horizontal-component amplitude (see Harmsen and Bufe, 1992). The depth estimates may be followed by one or two stars. One star means that the depth-of-focus standard error estimate was very large ( $\geq$ half crustal thickness). For SGBSN data, two stars imply that the depth was fixed by HYPO71 during the last several iterations for hypocenter, because the data lacked resolving power for that parameter. For SCSN data, two stars merely mean that no information was supplied on depth-of-focus resolution. DELMIN is the minimum source to station distance in $\mathrm{km}$, and RMS RES. is the root-mean-square traveltime residual in sec. \#N PH. is the number of $(\mathrm{P}+\mathrm{S})$ phases having positive weight in the solution. Finally, U.S.G.S. quadrangle is the name of $7 \frac{1}{2}$-minute or 15-minute tcpographic quadrangle in which the 'picenter lies. See Harmsen (1993) for maps of topographi: quadrangle names appearing in this report. 
1992 LOCAL HYPOCENTER SUROARY - SGB EARTHQUAKES

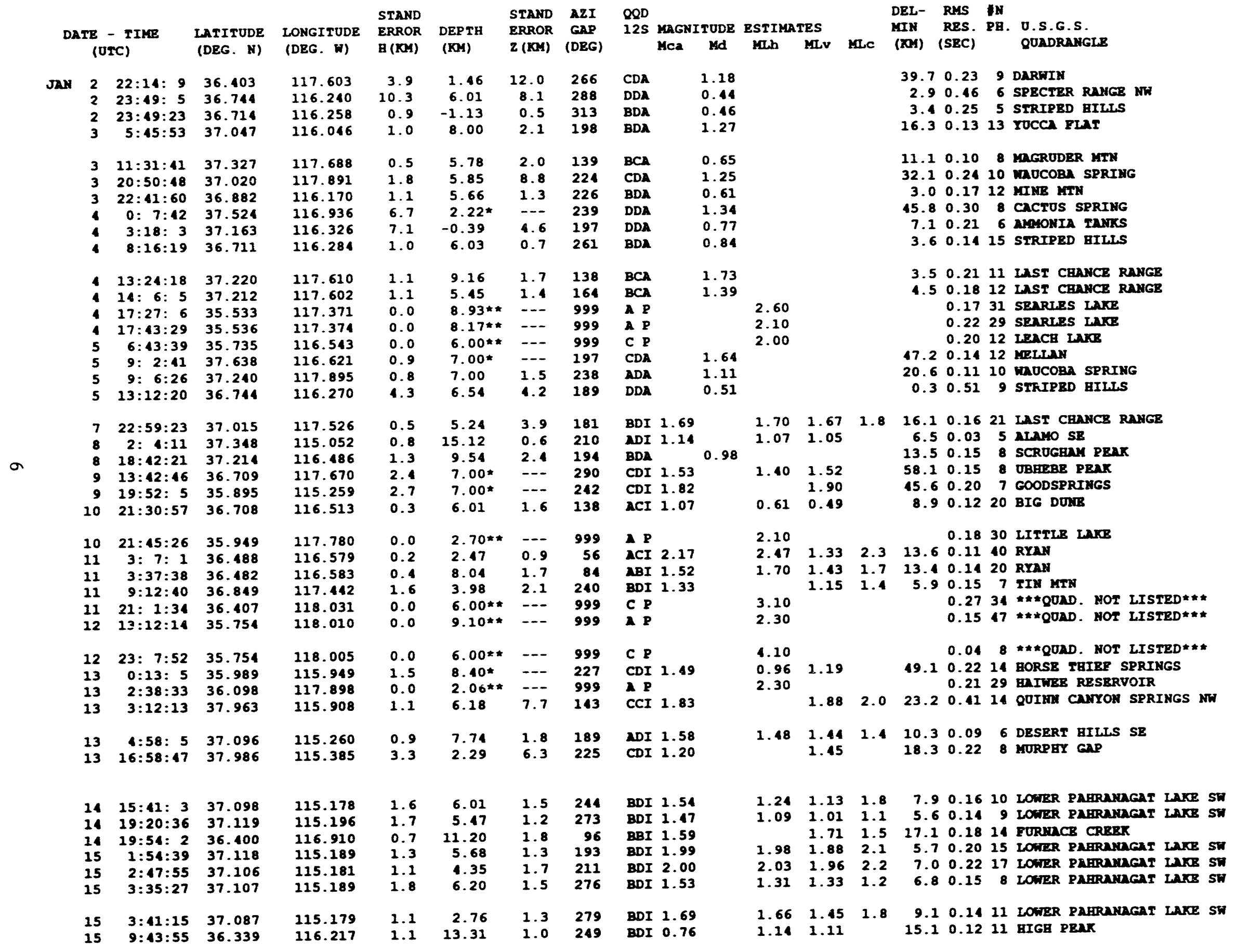


1992 LOCAL HYPOCENTER SULMARY - SGB EARTHQUAKES

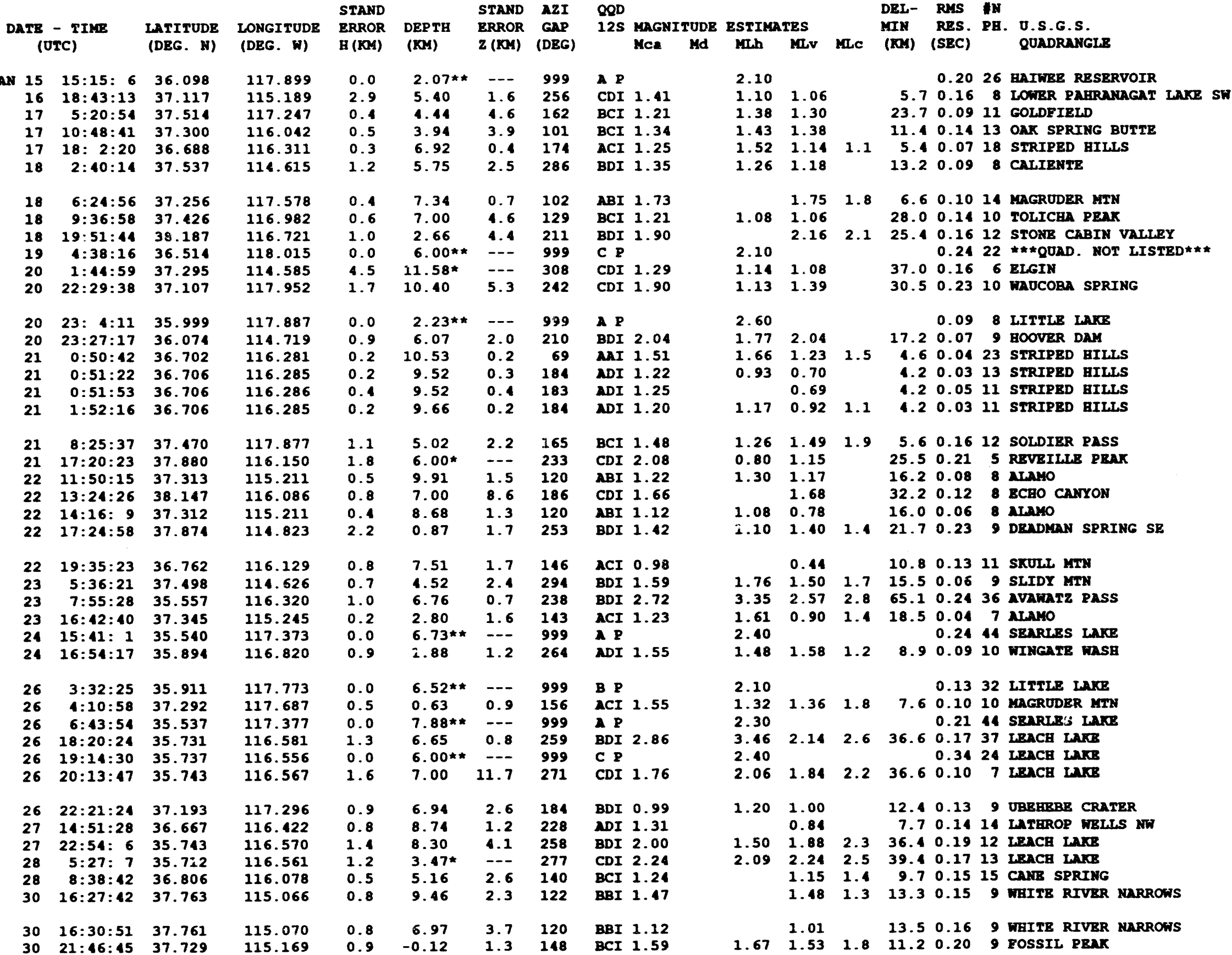


1992 LOCAL HYPOCENTER SULMARY - SGB EARTHQUAKES

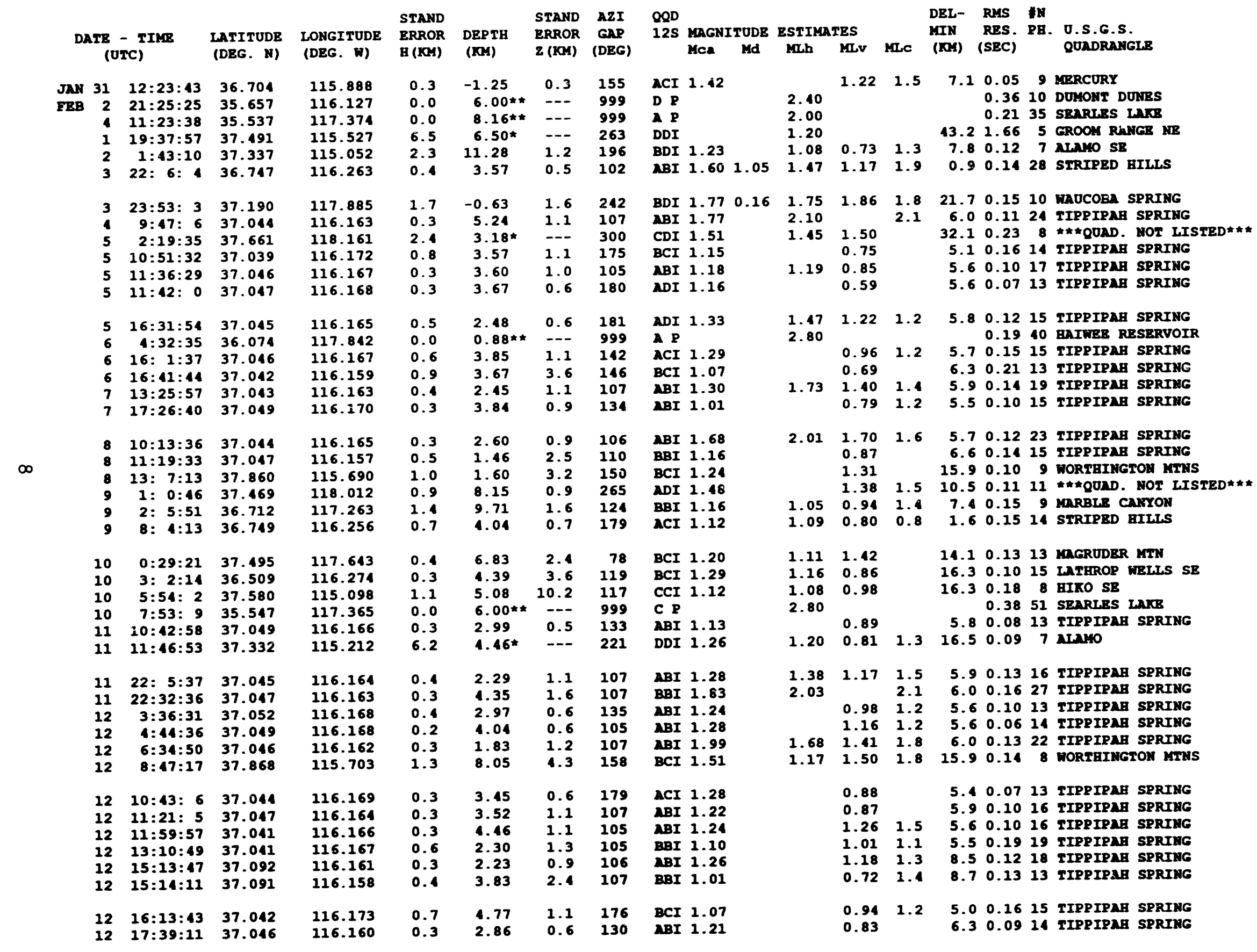




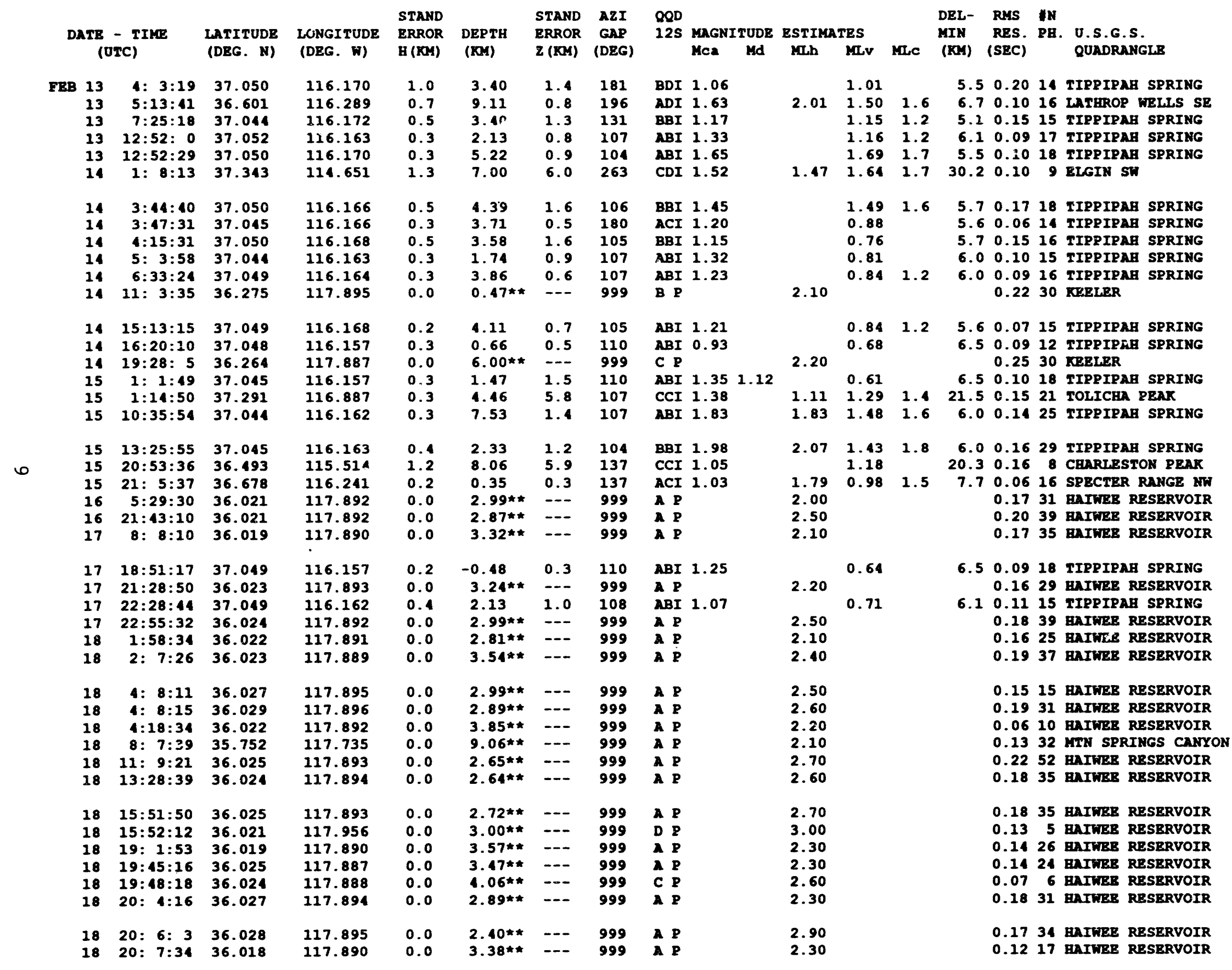


1992 LOCAL HYPOCENTER SUMEARY - SGB EARTBQUAKRS

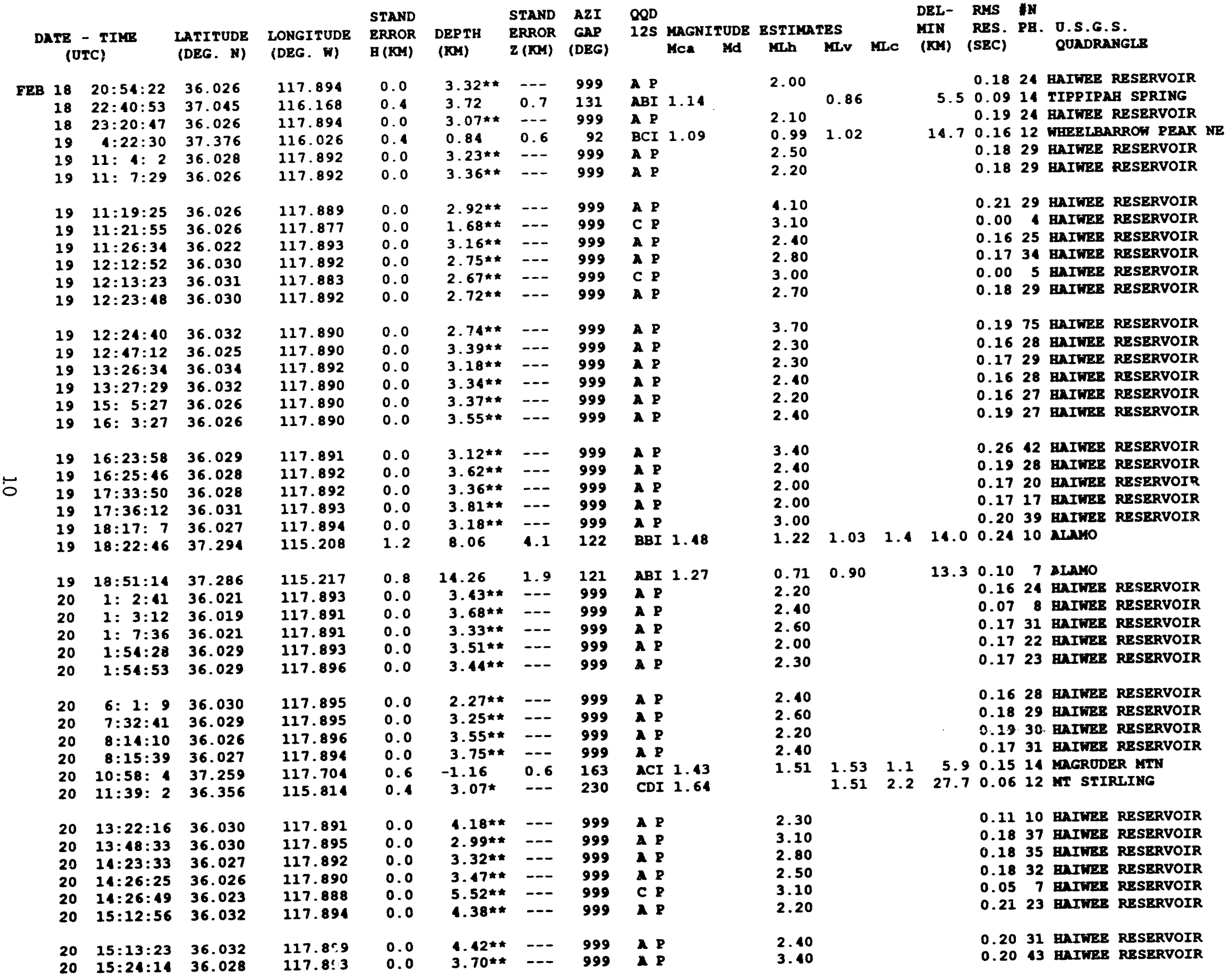


1992 LOCAL HYPOCENTER SURMARY - SGB EARTHQUAKES

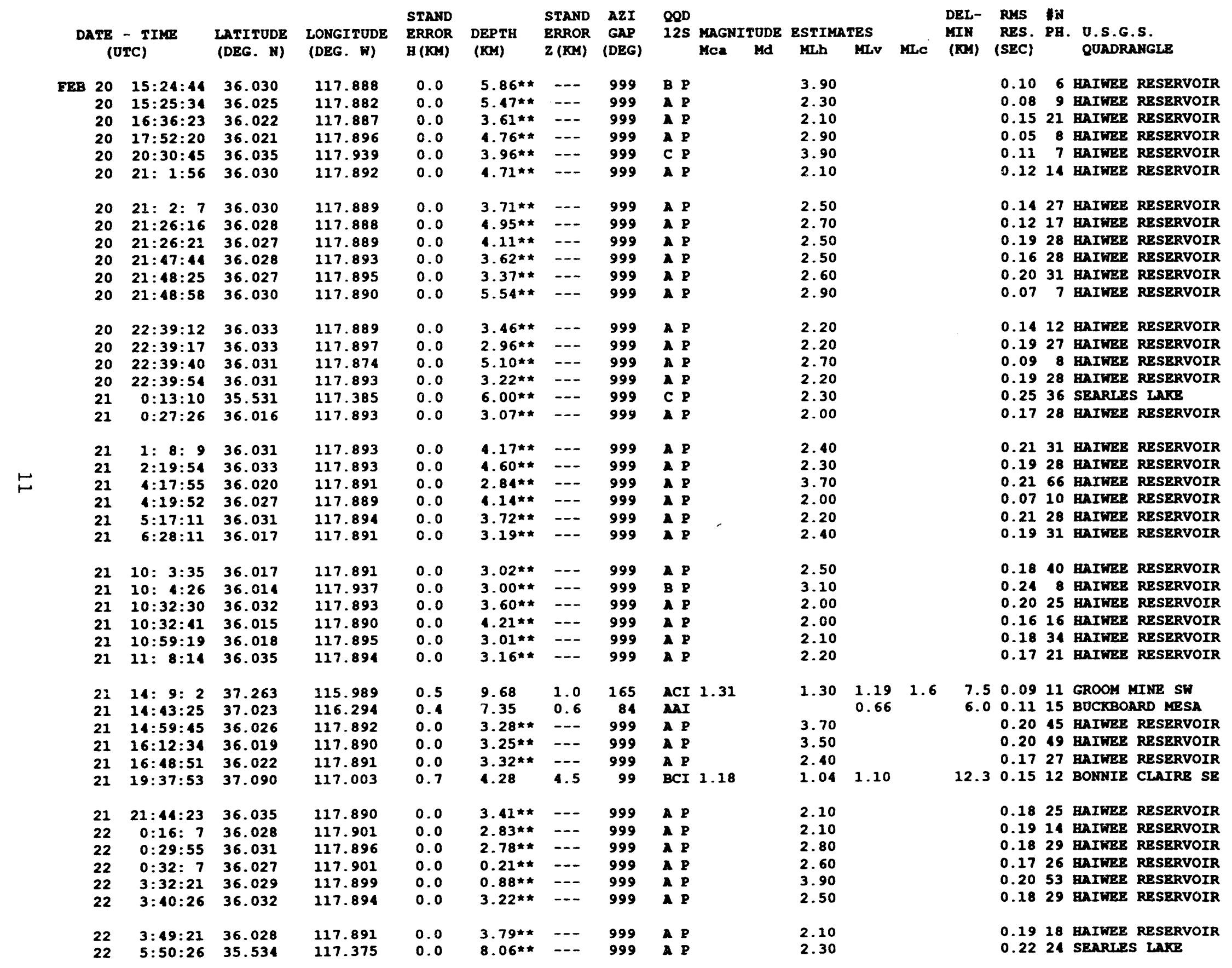


1992 LOCAL HTPOCENTER SUMMARY - SGB RARTHQUAKES

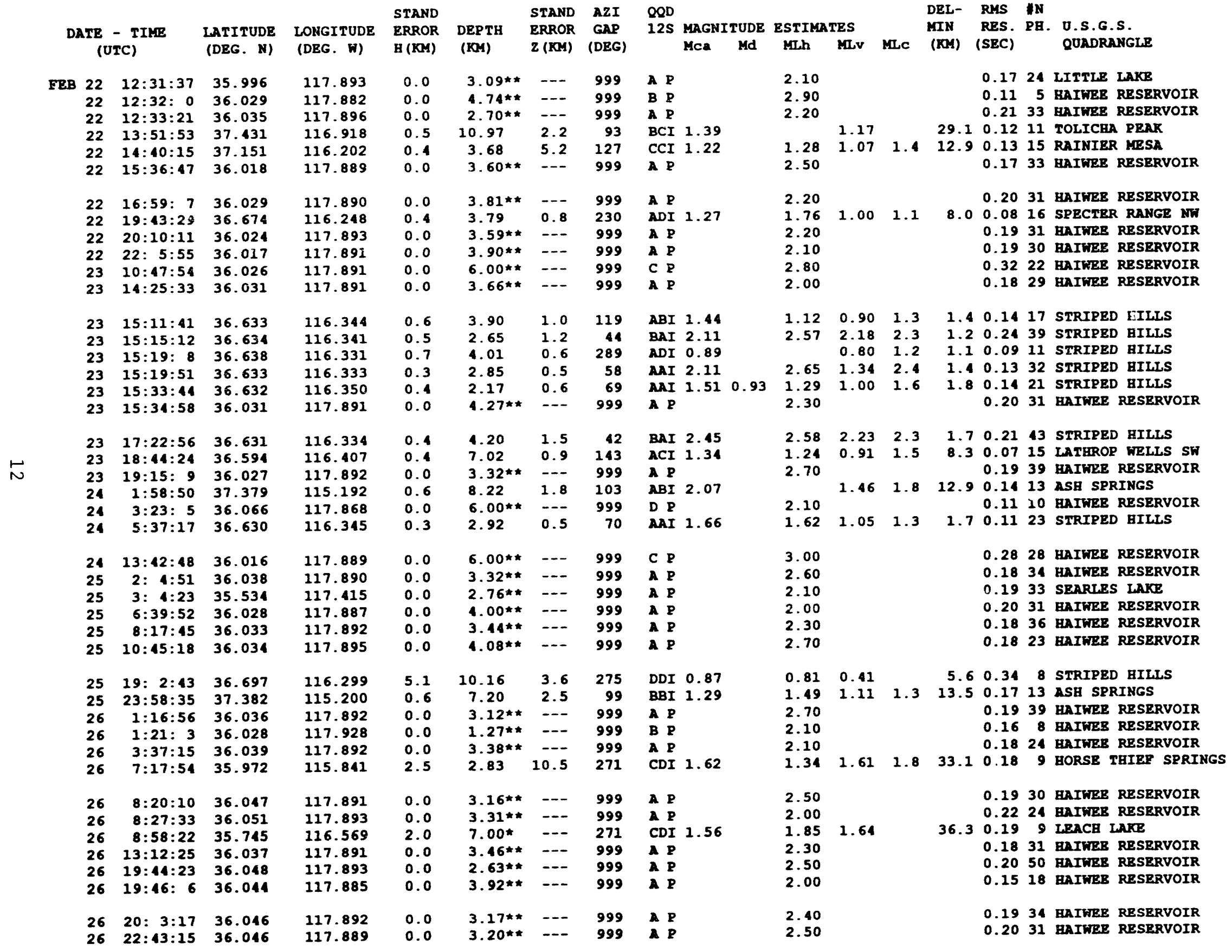


1992 LOCAL HYPOCENTER SUMMARY - SGB EARTHQUAKES

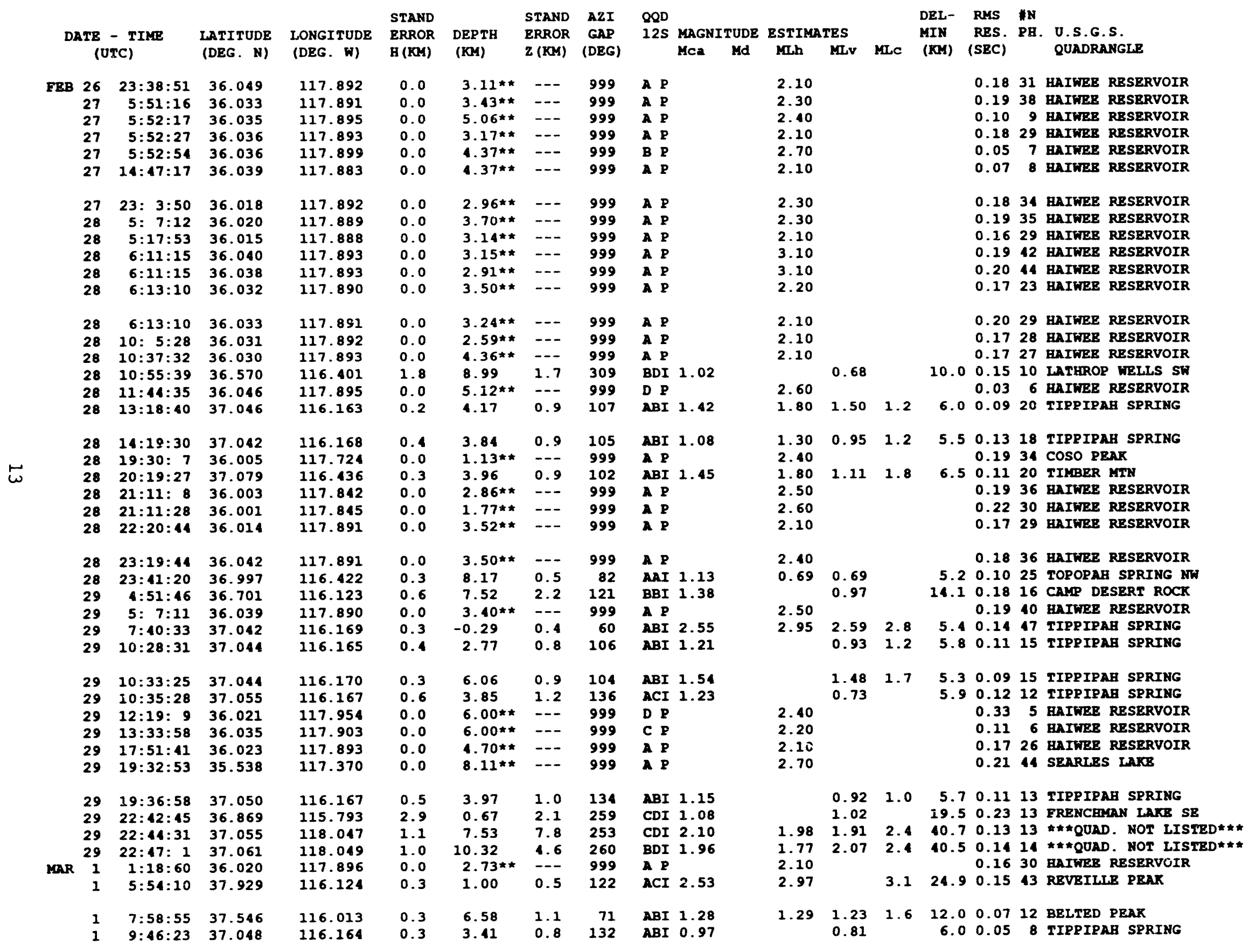


1992 LOCAL HYPOCENTER SUROAAY - SGB EARTHQUAKRS

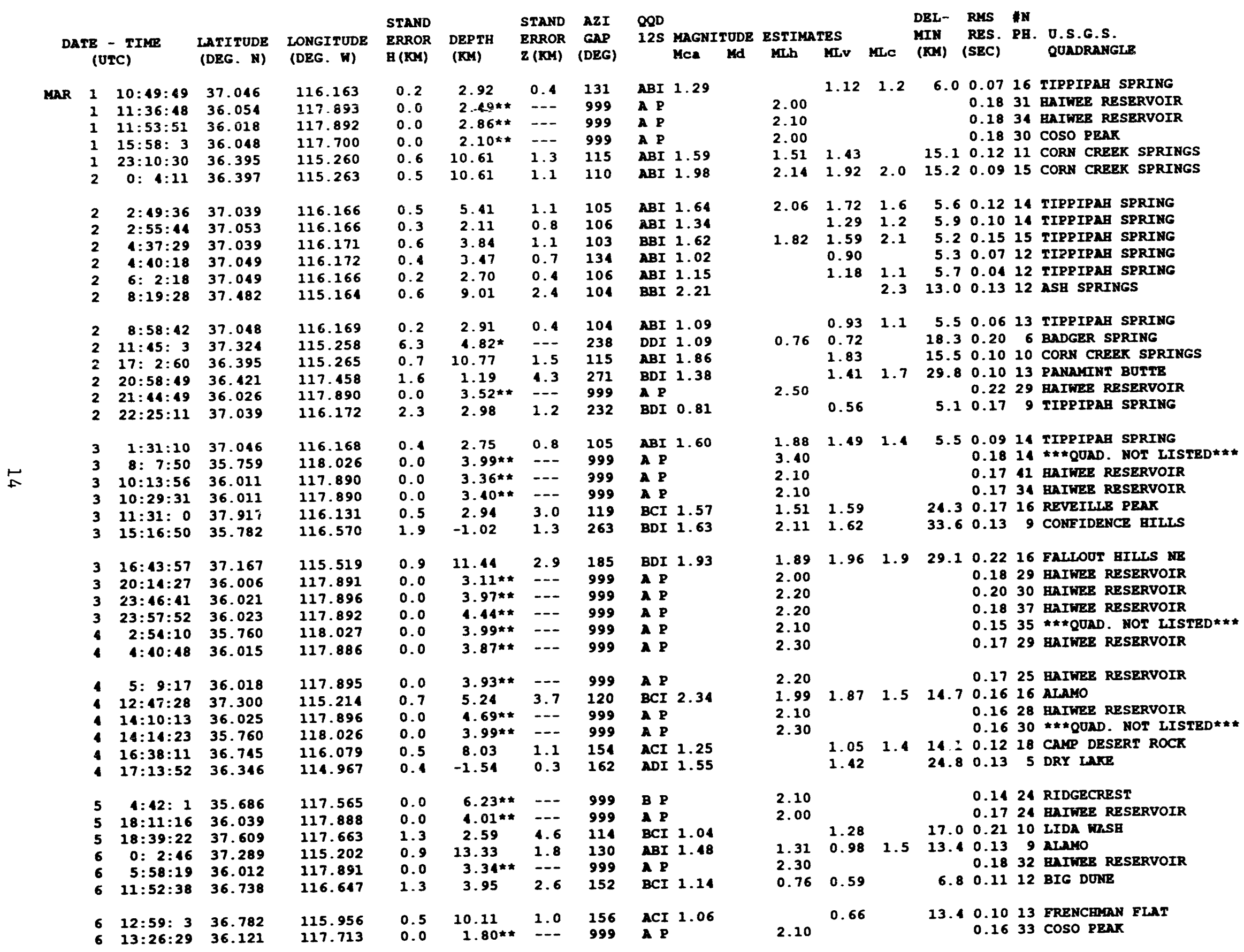


1992 LOCAL HYPOCENTER SUMARAY - SGB EARTHQUAKES

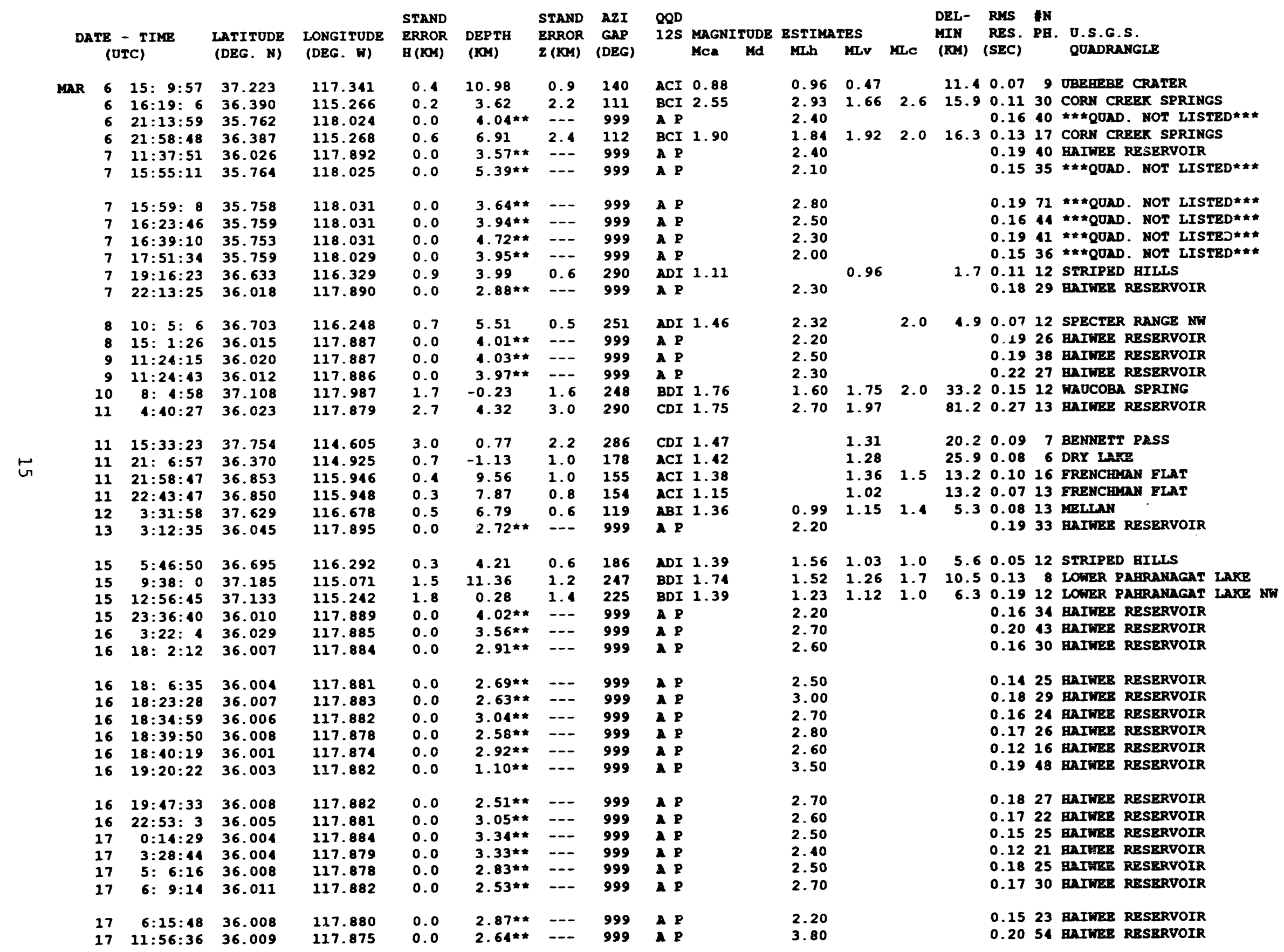


1992 LOCAL HYPOCENTER SUMAARY - SGB EARTHQUAKES

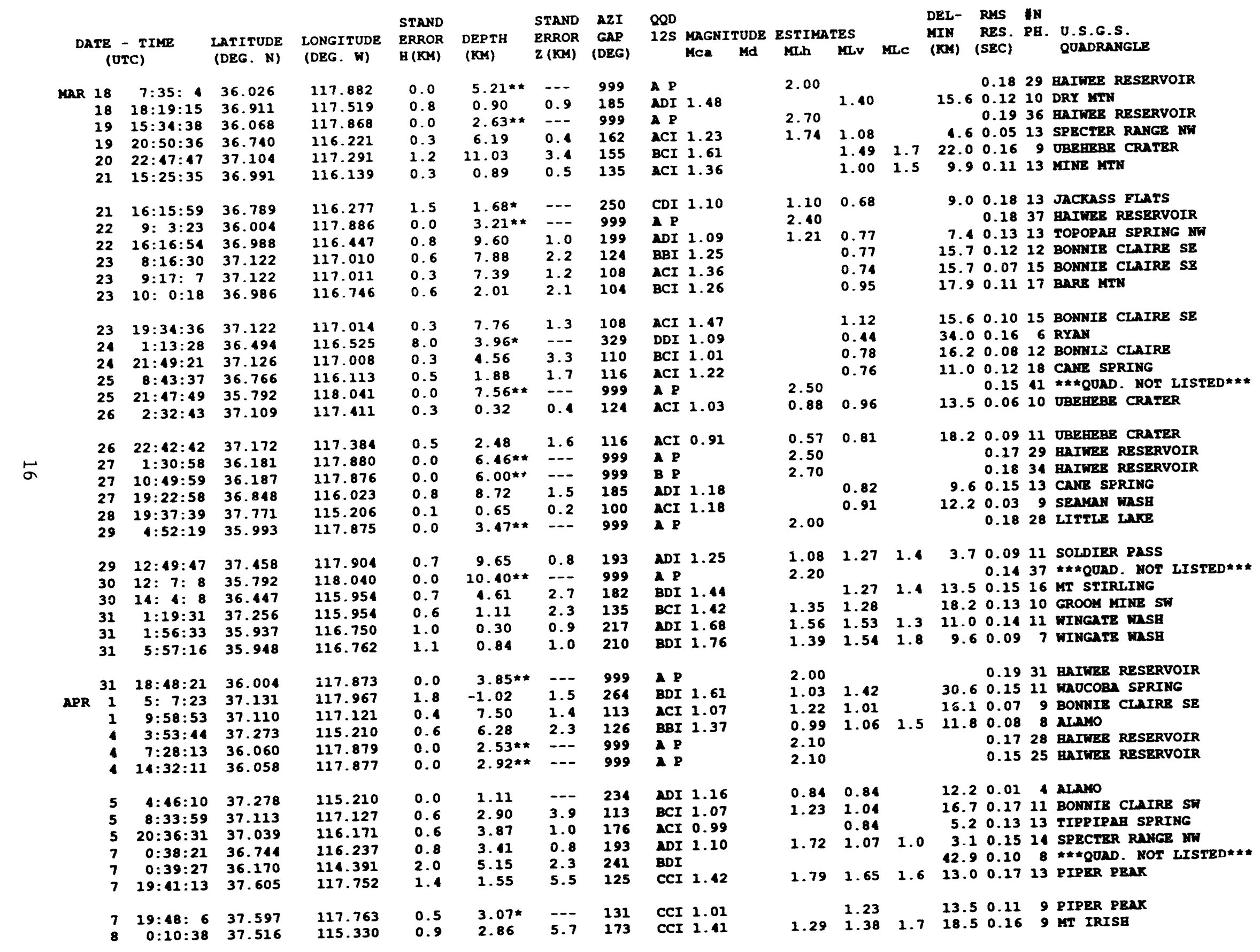




\begin{tabular}{|c|c|c|c|c|c|c|c|c|c|c|c|c|c|c|c|c|c|c|}
\hline \multicolumn{3}{|c|}{$\begin{array}{l}\text { DATE - TIKE } \\
\text { (UTC) }\end{array}$} & $\begin{array}{l}\text { LATITUDB } \\
\text { (DEG. N) }\end{array}$ & $\begin{array}{l}\text { LONGITUDE } \\
\text { (DEG. W) }\end{array}$ & $\begin{array}{l}\text { STAND } \\
\text { ERROR } \\
\text { E (KA) }\end{array}$ & $\begin{array}{l}\text { DEPTH } \\
\text { (IOY) }\end{array}$ & $\begin{array}{l}\text { STAND } \\
\text { ERROR } \\
2 \text { (KM) }\end{array}$ & $\begin{array}{c}\text { AZI } \\
\text { GAP } \\
\text { (DEG) }\end{array}$ & $\begin{array}{l}Q Q D \\
125\end{array}$ & \multicolumn{2}{|c|}{ MAGNITUDE } & \multicolumn{2}{|c|}{ EsTIMATES } & MLC & $\begin{array}{l}\text { DEL- } \\
\begin{array}{l}\text { MIN } \\
\text { (ROY) }\end{array} \\
10.6\end{array}$ & $\begin{array}{l}\text { RMS } \\
\text { RES. } \\
\text { (SEC) }\end{array}$ & $\begin{array}{l}\mathbf{H} \\
\mathbf{P H} .\end{array}$ & $\begin{array}{l}\text { U.S.G.S. } \\
\text { QUADRANGLE }\end{array}$ \\
\hline APR & $\begin{array}{l}8 \\
8 \\
8 \\
8 \\
9 \\
9\end{array}$ & $\begin{array}{c}0: 40: 1 \\
11: 39: 12 \\
16: 0: 18 \\
18: 40: 20 \\
10: 16: 35 \\
16: 19: 20\end{array}$ & $\begin{array}{l}37.142 \\
37.256 \\
36.548 \\
37.331 \\
36.575 \\
36.885\end{array}$ & $\begin{array}{l}116.279 \\
114.505 \\
116.261 \\
114.874 \\
116.526 \\
116.191\end{array}$ & $\begin{array}{l}0.4 \\
1.6 \\
1.1 \\
1.3 \\
0.4 \\
0.9\end{array}$ & $\begin{array}{c}3.68 \\
3.63^{\star} \\
1.16 \\
18.78 \\
5.20 \\
5.19\end{array}$ & $\begin{array}{l}2.0 \\
-2 . \\
1.4 \\
1.9 \\
1.2 \\
1.0\end{array}$ & $\begin{array}{l}168 \\
289 \\
192 \\
216 \\
120 \\
180\end{array}$ & $\begin{array}{l}\mathrm{ACI} \\
\mathrm{CDI} \\
\mathrm{BDI} \\
\mathrm{BDI} \\
\mathrm{BCI} \\
\mathrm{BCI}\end{array}$ & $\begin{array}{l}1.03 \\
1.84 \\
1.26 \\
1.20 \\
1.38 \\
1.26\end{array}$ & & $\begin{array}{l}1.49 \\
1.96 \\
0.83 \\
1.08 \\
1.19\end{array}$ & $\begin{array}{l}0.79 \\
1.87 \\
i .05 \\
1.03 \\
1.25 \\
1.07\end{array}$ & $\begin{array}{l}1.2 \\
1.9 \\
1.4 \\
1.5 \\
1.6 \\
1.1\end{array}$ & $\begin{array}{r}10.6 \\
14.0 \\
12.9 \\
17.8 \\
18.4 \\
3.9\end{array}$ & $\begin{array}{l}0.06 \\
0.17 \\
0.13 \\
0.06 \\
0.16 \\
0.15\end{array}$ & $\begin{array}{r}10 \\
10 \\
14 \\
7 \\
20 \\
13\end{array}$ & $\begin{array}{l}\text { AMAONIA TANKS } \\
\text { BLGIN } \\
\text { LATEROR WELLS SE } \\
\text { GREGBRSON BASIN } \\
\text { BIG DONE } \\
\text { MINE MTN }\end{array}$ \\
\hline & $\begin{array}{r}9 \\
10 \\
12 \\
13 \\
13 \\
13\end{array}$ & $\begin{array}{r}23: 40: 58 \\
7: 45: 55 \\
23: 19: 29 \\
0: 10: 55 \\
17: 16: 13 \\
17: 51: 16\end{array}$ & $\begin{array}{l}35.923 \\
37.110 \\
36.010 \\
36.510 \\
35.752 \\
36.478\end{array}$ & $\begin{array}{l}118.033 \\
117.130 \\
117.873 \\
118.128 \\
118.019 \\
117.029\end{array}$ & $\begin{array}{l}0.0 \\
0.1 \\
0.0 \\
0.0 \\
0.0 \\
0.7\end{array}$ & $\begin{array}{l}11.91^{\star \star} \\
7.00 \\
2.78^{\star \star} \\
6.00^{\star \star} \\
2.83^{\star \star} \\
14.45\end{array}$ & $\begin{array}{l}--. \\
1.6 \\
--- \\
--. \\
--- \\
0.8\end{array}$ & $\begin{array}{r}999 \\
112 \\
999 \\
999 \\
999 \\
96\end{array}$ & $\begin{array}{ll}C & P \\
\text { ACI } \\
\text { A } & \text { P } \\
\text { C } & P \\
\text { A } & P \\
\text { BBI }\end{array}$ & 1.37 & 0.29 & $\begin{array}{l}2.10 \\
1.41 \\
2.30 \\
2.30 \\
2.00\end{array}$ & 1.53 & 1.4 & 16.5 & $\begin{array}{l}0.12 \\
0.08 \\
0.19 \\
0.2 \\
0.18 \\
0.18\end{array}$ & $\begin{array}{l}8 \\
9 \\
31 \\
20 \\
30 \\
16\end{array}$ & 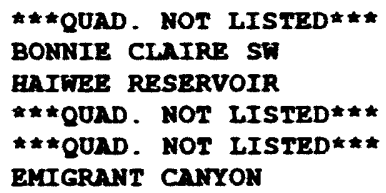 \\
\hline & $\begin{array}{l}13 \\
13 \\
13 \\
13 \\
13 \\
14\end{array}$ & $\begin{array}{l}19: 3: 40 \\
19: 8: 28 \\
19: 23: 4 \\
21: 27: 1 \\
21: 27: 28 \\
9: 2: 19\end{array}$ & $\begin{array}{l}36.949 \\
36.876 \\
36.476 \\
36.666 \\
36.227 \\
37.221\end{array}$ & $\begin{array}{l}117.532 \\
116.265 \\
117.023 \\
116.088 \\
115.455 \\
117.339\end{array}$ & $\begin{array}{l}1.0 \\
0.6 \\
0.7 \\
0.3 \\
1.2 \\
0.3\end{array}$ & $\begin{array}{r}6.70 \\
-0.24 \\
11.44 \\
5.82 \\
11.75 \\
8.80\end{array}$ & $\begin{array}{l}3.2 \\
0.5 \\
1.3 \\
1.4 \\
1.0 \\
0.6\end{array}$ & $\begin{array}{r}196 \\
117 \\
92 \\
102 \\
292 \\
95\end{array}$ & $\begin{array}{l}\mathrm{EDI} \\
\mathrm{ABI} \\
\mathrm{BBI} \\
\mathrm{ABI} \\
\mathrm{BDI} \\
\mathrm{ABI}\end{array}$ & $\begin{array}{l}1.37 \\
0.82 \\
1.80 \\
1.18 \\
1.64 \\
0.87\end{array}$ & & $\begin{array}{l}1.00 \\
1.36 \\
0.84\end{array}$ & $\begin{array}{l}1.37 \\
0.34 \\
1.93 \\
1.08 \\
1.38 \\
0.73\end{array}$ & $\begin{array}{l}1.4 \\
2.1 \\
1.4 \\
1.4\end{array}$ & $\begin{array}{r}17.4 \\
4.8 \\
11.6 \\
11.4 \\
15.8 \\
11.4\end{array}$ & $\begin{array}{l}0.15 \\
0.13 \\
0.22 \\
0.08 \\
0.14 \\
0.05\end{array}$ & $\begin{array}{r}10 \\
10 \\
21 \\
13 \\
11 \\
9\end{array}$ & $\begin{array}{l}\text { DRY MTN } \\
\text { TOPOPAH SPRING } \\
\text { EMIGRANT CANYON } \\
\text { CAYP DESERT RDCK } \\
\text { LA MADRE MTN } \\
\text { UBEREBB CRATER }\end{array}$ \\
\hline$\varpi$ & $\begin{array}{l}14 \\
15 \\
15 \\
17 \\
17 \\
17\end{array}$ & $\begin{array}{r}23: 30: 1 \\
0: 2: 18 \\
2: 26: 28 \\
1: 46: 38 \\
16: 43: 8 \\
18: 27: 22\end{array}$ & $\begin{array}{l}35.669 \\
35.666 \\
36.683 \\
36.834 \\
37.923 \\
36.181\end{array}$ & $\begin{array}{l}117.560 \\
117.562 \\
116.262 \\
116.260 \\
116.127 \\
118.071\end{array}$ & $\begin{array}{l}0.0 \\
0.0 \\
0.6 \\
0.4 \\
0.3 \\
0.0\end{array}$ & $\begin{array}{l}2.33^{\star \star} \\
3.33^{\star \star} \\
0.87 \\
5.43 \\
5.19 \\
6.00 \star \star\end{array}$ & $\begin{array}{l}--- \\
--- \\
0.5 \\
1.1 \\
4.8 \\
---\end{array}$ & $\begin{array}{l}999 \\
999 \\
216 \\
112 \\
120 \\
999\end{array}$ & $\begin{array}{l}A P \\
A P \\
A D I \\
A B I \\
B C I \\
C P\end{array}$ & $\begin{array}{l}1.24 \\
0.95 \\
1.66\end{array}$ & & $\begin{array}{l}2.10 \\
2.20 \\
2.00\end{array}$ & $\begin{array}{l}1.00 \\
0.57 \\
1.64\end{array}$ & $\begin{array}{l}1.4 \\
2.2\end{array}$ & $\begin{array}{r}6.7 \\
6.0 \\
24.6\end{array}$ & $\begin{array}{l}0.20 \\
0.21 \\
0.11 \\
0.10 \\
0.15 \\
0.13\end{array}$ & $\begin{array}{l}39 \\
31 \\
14 \\
13 \\
15 \\
27\end{array}$ & 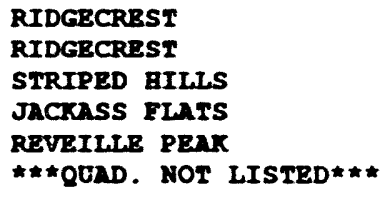 \\
\hline & $\begin{array}{l}17 \\
20 \\
21 \\
21 \\
21 \\
22\end{array}$ & $\begin{array}{r}23: 42: 37 \\
20: 55: 1 \\
9: 13: 20 \\
16: 18: 16 \\
20: 22: 14 \\
19: 56: 17\end{array}$ & $\begin{array}{l}37.929 \\
37.203 \\
36.905 \\
36.949 \\
36.710 \\
36.114\end{array}$ & $\begin{array}{l}116.124 \\
116.754 \\
117.536 \\
116.799 \\
115.746 \\
117.714\end{array}$ & $\begin{array}{l}0.4 \\
0.4 \\
0.8 \\
0.3 \\
0.6 \\
0.0\end{array}$ & $\begin{array}{r}0.39 \\
-0.39 \\
1.99 \\
9.01 \\
-0.32 \\
1.57 \star \star\end{array}$ & $\begin{array}{l}0.7 \\
0.4 \\
1.5 \\
1.5 \\
0.4 \\
-.-\end{array}$ & $\begin{array}{l}122 \\
180 \\
199 \\
102 \\
211 \\
999\end{array}$ & $\begin{array}{l}\text { BCI } \\
A C I \\
\text { ADI } \\
\text { ACI } \\
\text { ADI } \\
\text { A P }\end{array}$ & $\begin{array}{l}2.26 \\
1.58 \\
1.19 \\
1.74 \\
1.51\end{array}$ & & $\begin{array}{l}2.02 \\
1.02 \\
2.70\end{array}$ & $\begin{array}{l}1.29 \\
1.11 \\
2.33\end{array}$ & $\begin{array}{l}2.3 \\
1.3\end{array}$ & $\begin{array}{r}25.0 \\
6.7 \\
16.1 \\
21.3 \\
6.0\end{array}$ & $\begin{array}{l}0.16 \\
0.13 \\
0.12 \\
0.10 \\
0.12 \\
0.19\end{array}$ & $\begin{array}{l}22 \\
16 \\
10 \\
18 \\
14 \\
32\end{array}$ & $\begin{array}{l}\text { REVEILLE PEAX } \\
\text { SPRINGDALE } \\
\text { DRY MTN } \\
\text { BULLFROG } \\
\text { INDIAN SPRINGS NW } \\
\text { COSO PEAK }\end{array}$ \\
\hline & $\begin{array}{l}23 \\
24 \\
25 \\
25 \\
25 \\
26\end{array}$ & $\begin{array}{r}23: 21: 5 \\
16: 1: 23 \\
4: 12: 3 \\
9: 14: 55 \\
14: 21: 38 \\
17: 13: 27\end{array}$ & $\begin{array}{l}36.835 \\
37.219 \\
36.973 \\
37.185 \\
36.712 \\
37.150\end{array}$ & $\begin{array}{l}117.531 \\
117.351 \\
117.720 \\
114.612 \\
116.086 \\
117.386\end{array}$ & $\begin{array}{l}1.1 \\
0.4 \\
2.4 \\
3.7 \\
0.4 \\
0.3\end{array}$ & $\begin{array}{c}9.18 \\
8.68 \\
16.19 \\
7.00 \\
5.13 \\
8.99\end{array}$ & $\begin{array}{l}1.6 \\
0.8 \\
8.2 \\
-. . \\
1.6 \\
0.7\end{array}$ & $\begin{array}{l}205 \\
109 \\
249 \\
281 \\
114 \\
109\end{array}$ & $\begin{array}{l}\text { BDI } \\
A B I \\
C D I \\
C D I \\
\text { ACI } \\
\text { ABI }\end{array}$ & $\begin{array}{l}1.32 \\
1.50 \\
1.20 \\
1.92 \\
1.32 \\
1.28\end{array}$ & & $\begin{array}{l}1.09 \\
1.57\end{array}$ & $\begin{array}{l}1.15 \\
1.40 \\
1.14 \\
1.64 \\
1.10 \\
1.27\end{array}$ & $\begin{array}{l}1.6 \\
1.6\end{array}$ & $\begin{array}{l}11.7 \\
12.3 \\
29.5 \\
16.1 \\
12.5 \\
17.1\end{array}$ & $\begin{array}{l}0.18 \\
0.13 \\
0.20 \\
0.27 \\
0.10 \\
0.09\end{array}$ & $\begin{array}{r}13 \\
23 \\
6 \\
11 \\
18 \\
17\end{array}$ & $\begin{array}{l}\text { DRY MTN } \\
\text { UBEBRBB CRATER } \\
\text { DRY MTN } \\
\text { VIGO NB } \\
\text { CANP DESERT ROCK } \\
\text { UBEBEBE CRATER }\end{array}$ \\
\hline & $\begin{array}{l}26 \\
26 \\
27 \\
28 \\
28 \\
29\end{array}$ & $\begin{array}{l}18: 58: 29 \\
21: 24: 59 \\
13: 22: 39 \\
2: 36: 58 \\
20: 36: 14 \\
10: 36: 15\end{array}$ & $\begin{array}{l}37.565 \\
37.275 \\
37.066 \\
36.793 \\
37.037 \\
36.714\end{array}$ & $\begin{array}{l}116.705 \\
115.454 \\
116.153 \\
116.084 \\
116.009 \\
118.109\end{array}$ & $\begin{array}{l}0.6 \\
0.5 \\
0.7 \\
0.3 \\
0.1 \\
0.0\end{array}$ & $\begin{array}{r}1.46 \\
2.27 \\
-0.31 \\
5.50 \\
0.73 \\
6.00 \star\end{array}$ & $\begin{array}{l}2.1 \\
2.7 \\
0.6 \\
1.5 \\
0.6 \\
-.-\end{array}$ & $\begin{array}{l}167 \\
144 \\
139 \\
135 \\
123 \\
999\end{array}$ & $\begin{array}{l}\text { BCI } \\
\text { BCI } \\
\text { BCI } \\
\text { ABI } \\
\text { BCI } \\
\text { D P }\end{array}$ & $\begin{array}{l}1.58 \\
1.34\end{array}$ & & $\begin{array}{l}0.67 \\
2.20\end{array}$ & $\begin{array}{l}1.46 \\
1.16 \\
0.61 \\
0.72 \\
1.01\end{array}$ & $\begin{array}{l}1.9 \\
1.7\end{array}$ & $\begin{array}{r}10.3 \\
26.5 \\
7.5 \\
10.2 \\
12.8\end{array}$ & $\begin{array}{l}0.07 \\
0.17 \\
0.18 \\
0.10 \\
0.15 \\
0.20\end{array}$ & $\begin{array}{l}12 \\
16 \\
11 \\
17 \\
20 \\
12\end{array}$ & 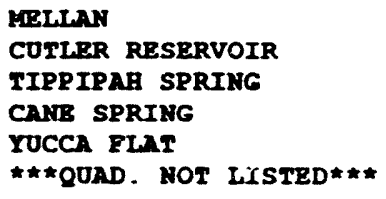 \\
\hline & $\begin{array}{l}29 \\
30\end{array}$ & $\begin{array}{l}11: 1: 24 \\
11: 43: 25\end{array}$ & $\begin{array}{l}35.792 \\
36.837\end{array}$ & $\begin{array}{l}117.716 \\
115.930\end{array}$ & $\begin{array}{l}0.0 \\
0.3\end{array}$ & $\begin{array}{l}10.18 * \pi \\
-0.59\end{array}$ & $\overline{0.6}$ & $\begin{array}{r}999 \\
85\end{array}$ & $\begin{array}{l}\text { A P } \\
\text { ACI }\end{array}$ & 1.64 & & .40 & 1.42 & 1.6 & 15.4 & $\begin{array}{l}0.13 \\
0.13\end{array}$ & $\begin{array}{l}30 \\
24\end{array}$ & $\begin{array}{l}\text { MTN SPRINGS CANXON } \\
\text { FRENCHYAN FLAT }\end{array}$ \\
\hline
\end{tabular}


1992 LOCAL GYPOCENTER SUMRARY - SGB EARTHQUAKES DATE - TIME
(UTC)
(DEG. N)

APR $30 \quad 14: 11: 48 \quad 36.847$ $20: 51: 8$ 8:27: 37.485 $6: 11: 4937.925$

$7: 18: 21 \quad 37.147$ $2: 22: 29 \quad 37.832$ $2: 24: 59 \quad 37.849$ 3. 2.1435 .936 $7: 23: 51 \quad 36.856$ 4: $5: 29 \quad 37.005$

6 9: $2: 56 \quad 35.790$ $15: 46: 18 \quad 36.806$ $7: 45: 34 \quad 35.790$ $0: 32: 736.970$ $0: 56: 36 \quad 36.968$

8 $18: 28: 13 \quad 35.994$

\subsection{0} 115.910 115.187 117.292 117.292 116.130

117.784 117.519 117.526

118.040 115.844 118.042 117.473 117.464 117.879 116.305 116.264 114.920 116.936 114.952 115.281

117.405 117.874 117.690 117.890 117.880 117.841

116.429 117.885 116.940 117.535 116.829
117.460 115.274
115.261 116.761 116.761 114.804 114.804 116.270 115.558
STAND $\begin{array}{llll}\text { (IN) } & \text { (KM) } & 2 \text { (KO) } & \text { (DEG) }\end{array}$

$\begin{array}{rrrr}0.6 & 6.76 & 1.7 & 206\end{array}$

$\begin{array}{llll}2.1 & 12.97 & 1.0 & 210\end{array}$

$2.5 \quad 3.01 * \quad--273$

4.9156

5.80

3.9120

$\begin{array}{llll}1.0 & 8.30 & 1.9 & 202 \\ 0.9 & 7.13 & 2.8 & 223\end{array}$

$\begin{array}{llll}1.7 & 5.12 & 6.7 & 228\end{array}$

$\begin{array}{llll}0.8 & 9.37 & 1.3 & 162\end{array}$

$0.7 \quad 3.63 \quad 1.4 \quad 175$

$0.0 \quad 8.29 *$ - $\quad 999$

$\begin{array}{llll}0.5 & 13.10 & 0.7 & 221\end{array}$

$\begin{array}{llll}0.0 & 8.46 * \star & --\overline{7} & 999 \\ 0.4 & 5.47 & 1.7 & 161\end{array}$

$\begin{array}{llll}0.6 & 1.05 & 3.8 & 167\end{array}$

$0.0 \quad 1.51 * \star \ldots 99$

$\begin{array}{llllll}0.6 & 7.18 & 1.0 & 247 & \text { ADI } 1.36 \\ 0.3 & 5.08 & 0.2 & 190 & \text { ADI } & 1.43\end{array}$

$0.47 .62 \quad 1.0 \quad 147$ ACI 1.26

$\begin{array}{llllll}7.4 & 7.86 & 8.0 & 216 & \text { CDI } 1.42\end{array}$

$\begin{array}{llllll}2.0 & 10.43 & 3.1 & 208 & \text { BDI } 1.77\end{array}$

$\begin{array}{lrrrrr}1.1 & 1.10 & 3.4 & 177 & \text { BCI } & 1.33\end{array}$

$0.0 \quad 8.30 \pm \ldots \quad--\quad 999 \quad$ A P

$\begin{array}{lllll}0.0 & 4.73 * * & -- & 999 & \text { A P } \\ 0.0 & 8.50 * * & -- & 999 & \text { A }\end{array}$

$\begin{array}{lllll}0.0 & 8.500 & 2.1 & 291 & \text { BDI } \\ 1.9 & 7.00 & 2.1 & 291\end{array}$

$\begin{array}{lllll}2.4 & 7.00 & 5.9 & 276 & \text { CDI }\end{array}$

$\begin{array}{lllll}1.4 & 7.00 & 2.6 & 273 & \end{array}$

$\begin{array}{llllll}0.7 & 6.28 & 2.1 & 112 & \text { BBI } 1.45\end{array}$

$\begin{array}{lllll}5.2 & 5.00 * & -- & 291 & \text { DDI }\end{array}$

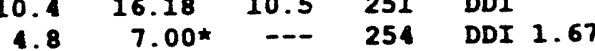

$\begin{array}{lllll}1.2 & -1.39 & 0.8 & 274 & \text { BDI } 1.34\end{array}$

$\begin{array}{lllll}0.3 & 2.89 & 1.3 & 176 & \text { ACI } 2.21\end{array}$

$3.27 .48 * \quad--2269$ CDI 1.60

$2.0 \quad 1.38 \quad 4.8227$ BDI 1.41

1.4181 BDI 1.37

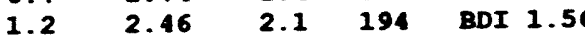

$\begin{array}{lllll}0.5 & 2.49 & 0.9 & 177 & \text { ACI } 1.06\end{array}$

$\begin{array}{lllll}0.5 & 8.61 & 0.4 & 216 & \text { NDI } 1.26 \\ 2.0 & 2.67 & 8.0 & 238 & \text { CDI } 1.54\end{array}$
DEL- RMS N

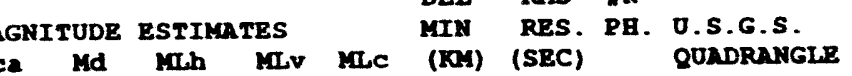

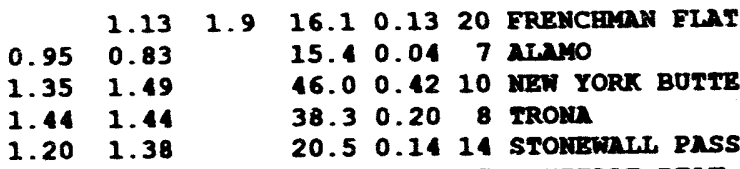

24. 0.1217 PEVBILLB PEAX

$\begin{array}{lllllll}1.13 & 1.31 & 1.7 & 15.5 & 0.16 & 12 & \text { EAST.OF JOSHOA FLATS }\end{array}$

$\begin{array}{lllllll}2.5 & 18.8 & 0.17 & 15 & \text { GOAT ISLAND }\end{array}$

$\begin{array}{llll}.12 & 1.50 & 20.6 & 0.2210 \mathrm{GOAT} \text { ISLAND }\end{array}$

$\begin{array}{llllllll}2.05 & 1.88 & 1.8 & 37.6 & 0.10 & 8 & \text { TRON }\end{array}$

$0.74 \quad 9.0 \quad 0.1413$ CANB SPRING

$0.83 \quad 5.30 .1111$ IIPPIPAB SPRING

$2.1000 .1227 * *$ QOAD. HOT LISTED**

$1.32 \quad 12.80 .0511$ FRENCEMAN LAKE SE

作

$241.911 .70 .1420 \mathrm{IIN}$ MOONTAIN

$1.13 \quad 1.431 .2 \quad 11.10 .1311$ IIN MOUNTaIs

$\begin{array}{llll}2.50 & 0.19 & 28 \text { LITTLB LAKE }\end{array}$

$\begin{array}{lllllll}1.52 & 1.33 & 1.3 & 15.1 & 0.07 & 12 & \text { DRAD HORSE FLAT }\end{array}$

$\begin{array}{lllllll}2.42 & 1.87 & 1.5 & 2.8 & 0.04 & 10 & \text { STRIPED BILLS }\end{array}$

$\begin{array}{lllll}0.87 & 1.10 & 11.5 & 0.08 & 9 \\ & \text { PAKROC SURAT PASS }\end{array}$

$\begin{array}{llllllll}1.85 & 1.35 & 1.5 & 17.5 & 0.13 & 8 & \text { DELAMaR LAKB }\end{array}$

$\begin{array}{llllll}1.58 & 2.0 & 15.4 & 0.17 & 10 & \text { DELANAR IAKE }\end{array}$

$0.72 \quad 1.6 \quad 15.40 .12 \quad 8$ YURPEY GAP SE

$2.10 \quad 0.1720$ SEARLES LAKE

$2.60 \quad 0.2235$ EATWER RESERVOIR

2.10

$\begin{array}{lllllll}3.10 & 2.47 & 2.8 & 82.5 & 0.18 & 12 & \text { EATWEE RESERVOIR }\end{array}$

$\begin{array}{llllll}2.60 & 2.12 & 82.3 & 0.39 & 12 & \text { EMIKEB RESERVOIR }\end{array}$

$\begin{array}{lllllll}2.60 & 2.29 & 2.4 & 78.0 & 0.16 & 13 & \text { EAIMEE RESERVOIR }\end{array}$

$\begin{array}{lllllll}1.44 & 1.4 & 10.7 & 0.11 & 10 & \text { WILD BORSE RANCB }\end{array}$

$\begin{array}{lllll}2.12 & 1.78 & 82.4 & 0.5910 & \text { GRIWEB RBSERVOIR }\end{array}$

$\begin{array}{llrrrr}128 & 1.04 & 6.6 & 0.68 & 7 \text { wrReATE wh } \\ 1.47 & 42.6 & 0.64 & 7 \text { coso PEAR }\end{array}$

$\begin{array}{lllllll}1.34 & 1.23 & 1.3 & 12.6 & 0.07 & 8 & \text { GIMGATE WASB }\end{array}$

$\begin{array}{llllll}1.71 & 2.2 & 13.5 & 0.13 & 32 & \text { IIN MOONTRIN }\end{array}$

$\begin{array}{lllllll}1.57 & 1.46 & 34.8 & 0.3411 \text { WATER GAP NE }\end{array}$

$\begin{array}{llllllll}2.01 & 1.66 & 2.2 & 36.7 & 0.11 & 9 \text { WATER GNP } \mathrm{MB}\end{array}$

$\begin{array}{llllll}1.33 & 1.2 & 10.6 & 0.16 & 8 & \text { WINGATR WASH }\end{array}$

$0.871 .33 \quad 9.90 .107$ PARROC SPRING $\mathrm{NE}$

$\begin{array}{lllllll}1.19 & 1.47 & 1.5 & 10.8 & 0.21 & 10 & \text { PARROC SPRIMG MB }\end{array}$

0.810 .95

9.40 .078 PABROC SPRING NE

$1.16 \quad 0.84$

7.10 .0814 STRIPED BILLS

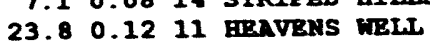


1992 LOCAL HYPOCENTER SUTMARY - SGB EARTHQUAKES

IAITTUDE LONGITUDE STA (UTC)

(DEG H) (DEG TU)

$\operatorname{MAY} 18 \quad 18: 52: 50 \quad 36.663$

1820 :

$\begin{array}{ccc}18 & 20: 7: 22 & 37.203 \\ 19 & 3: 55: 25 & 36.702\end{array}$

19 6: $0: 45 \quad 37.518$

$196: 21: 29 \quad 37.069$

$1: 34: 29 \quad 37.151$

$20 \quad 1: 36: 49 \quad 37.160$

20 19:0:55 35.905

20 19:0:55 35.905

$21 \quad 22: 56: 21 \quad 37302$

$22 \quad 6: 10: 39 \quad 37.648$

$22 \quad 6: 35: 50 \quad 37.118$

$2210: 45: 35 \quad 37.921$

$22 \quad 17: 14: 34 \quad 35.784$

$22 \quad 17: 51: 25 \quad 35.887$

$23 \quad 5: 50: 39 \quad 36.621$

$23 \quad 17: 7: 53 \quad 37.499$

$23 \quad 18: 17: 39 \quad 37.117$

$\sqrt{6}$

116.304

116.203

117.242

117.864

117.859

116.828

116.169

116.193

115.185

116.136

118.056

116.846

116.259

116.373

116.207

116.554

117.661 $\begin{array}{llllll}115.522 & 1.7 & 4.68 * & \ldots & 247 & \text { CDI } 1.43\end{array}$

\begin{tabular}{llllll}
115.126 & 1.2 & 6.81 & 1.6 & 247 & CDI 1.43 \\
\hline & 0.7 & 3.45 & 1.5 & 175 & BDI 1.39
\end{tabular}

118.033

116.998

117.296

116.551

116.299

116.840

116.840

114.928

116.833
117.352

$22: 45: 45 \quad 35.900$

$6: 41: 936.849$

$26 \quad 17: 9: 46 \quad 36.651$

26 23: 5:48 36.090

$27 \quad 22: 32: 53 \quad 36.126$

$3: 25: 58 \quad 37.429$

9: $4: 42 \quad 37.932$

$28 \quad 23: 12: 1 \quad 35.998$

$29 \quad 3: 26: 29 \quad 37.459$ $8: 29: 9 \quad 36.881$ $22: 10: 56 \quad 35.284$

$\begin{array}{lrr}31 & 5: 50: 33 & 36.282 \\ 31 & 14: 29: 26 & 36.983\end{array}$

JU木 1 5:50:37 35.944

$1 \quad 10: 18: 14 \quad 37.189$

1 12:26:56 36.566
116.262

117.834

117.889

116.874

116.128

117.932

116.423

117.452

117.608
115.452

115.452
116.161

116.790

115.502

115.538 $\begin{array}{llll}3.45 & 1.5 & 175 & \text { ACI } 0.68\end{array}$

$\begin{array}{llll}-1.10 & 0.3 & 91 & \text { ABI } 1.14\end{array}$

BBI 1.14

BDI 2.11

$\begin{array}{rrrr}5.03 & 0.8 & 296 & \text { ADI } 1.10 \\ 4.74 & 4.2 & 59 & \text { BCI } 1.42\end{array}$

$\begin{array}{rrrr}5.03 & 0.8 & 296 & \text { ADI } 1.10 \\ 4.74 & 4.2 & 59 & \text { BCI } 1.42\end{array}$

ABI 1.61

DCI 1.

$2.24 * 0.9269$ DDI

$\begin{array}{llllll}0.2 & 4.48 & 0.4 & 178 & \text { ACI } 1.07\end{array}$

CDI

ADI 1.56

CDI 1.32

BDI

ACI 1.10

ACI 2.30

DDI

BDI 2.1

ACI 0.93

$7.00 \quad 4.7 \quad 312$

$\begin{array}{lll}7.00 & 2.9 & 239\end{array}$

$\begin{array}{lll}7.00 & 5.4 & 217\end{array}$

CDI

$\begin{array}{lllll}1.1 & 1.23 & 3.4 & 139 & \text { BCI } 1.46\end{array}$
STAND AZI QOD

SS Magitude RSTIMATES

MIN RES. PH. D.S.G.S

(SEC) QUADRANGLE

$26.0 \quad 0.187$ BEAVENS WELT

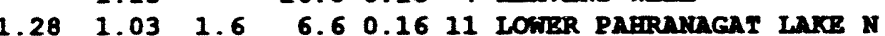

$\begin{array}{lllll}0.12 & 1.0 & 5.3 & 0.07 & 9\end{array}$

$\begin{array}{llllll}1.44 & 1.6 & 12.1 & 0.17 & 12 & \text { BONRIE CINIRE S }\end{array}$

1.001 .4421 .40 .1111 EAst. OF JOSBOA FLATS

$\begin{array}{lllllll}1.39 & 1.58 & 1.7 & 20.6 & 0.09 & 11 & \text { EAST. OF JOSHOA FLATS }\end{array}$

$1.297 .50 .21 \quad 8$ mINGATE masE

$\begin{array}{llllllll}0.44 & 0.79 & 1.3 & 1.5 & 0.20 & 10 & \text { SUNDONN RESERVOIR }\end{array}$

$\begin{array}{llllll}1.02 & 0.93 & 13.2 & 0.06 & 8 & \text { DELAMaR LAKE }\end{array}$

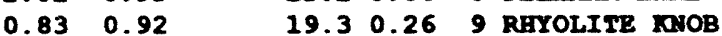

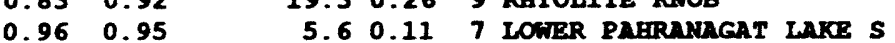

$\begin{array}{lllllll}1.68 & 1.65 & 1.9 & 24.9 & 0.18 & 15 & \text { REVEILLE PEAK NW }\end{array}$

2.20

$0.1533 * \pi 20 \mathrm{DD}$. NOT LIS

$2.07 \quad 2.1$

80.159 mINGATB mas

$\begin{array}{lll}7.7 & 0.0711 & 11 \\ \text { SKRLETON BILLS }\end{array}$

$\begin{array}{lllll}1.82 & 1.41 & 22.2 & 0.12 & 20 \\ \text { APACHE TEAR CANYON }\end{array}$

$\begin{array}{llllll}1.81 & 1.47 & 9.1 & 0.14 & 22 & \text { IIPPIPAH SPRIYG }\end{array}$

$\begin{array}{llllll}2.32 & 1.8 & 35.1 & 0.09 & 10 & \text { CONFIDENCE BILLS }\end{array}$

1.24 5.4 0.1215 LAST CHANCB MTM

$1.6187 .00 .177 * *$ OQOAD. NOT LISTED***

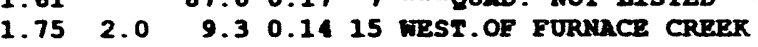

$1.40 \quad 1.0 \quad 4.20 .1412$ GOLD POINT

1.3637 .00 .277 CONFIDENCE aILLS

$\begin{array}{lllllll}1.18 & 0.83 & 1.0 & 5.5 & 0.05 & 15 & \text { STRIPED HILLS }\end{array}$

$\begin{array}{lllllll}1.56 & 1.63 & 1.2 & 9.3 & 0.07 & 9 & \text { wINGATE WASB }\end{array}$

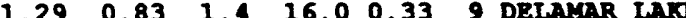

1.371 .27 .80 .2211 TIVGate

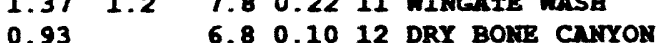

$\begin{array}{lllllll}0.82 & 1.2 & 7.0 & 0.14 & 17 & \text { STRIPED BILLS }\end{array}$

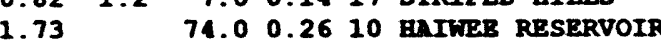

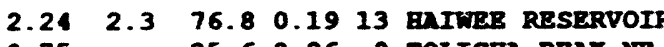

\begin{tabular}{llll}
0.75 & 25.6 & 0.26 & 9 \\
\hline & TOLICBA PBAK NE
\end{tabular}

$\begin{array}{lllllll}3.08 & 1.76 & 2.4 & 25.4 & 0.12 & 31 & \text { REVEILLE PEAK } \mathrm{MH}\end{array}$

$2.02 \quad 2.486 .60 .297$ LITTLE LAKB

$\begin{array}{lllllll}1.17 & 0.93 & 1.5 & 26.6 & 0.13 & 19 & \text { GOLD FLAT EAST }\end{array}$

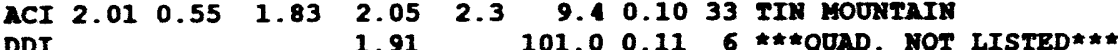

0.3

2.155 .90 .1216 GRAPEVIKE SPRING

$\begin{array}{llll}8.3 & 0.09 & 13 & \text { MTNE MTN }\end{array}$

$0.71 \quad 1.28$

$7.3 \quad 0.35 \quad 8$ wIMGaTE wask

$\begin{array}{rr}1.02 & 1.13 \\ 1.34 & 1.29\end{array}$

$\begin{array}{llll}28.0 & 0.19 & 13 & \text { FALLOUT HILLS NR } \\ 28.3 & 0.29 & 11 & \end{array}$ 
1992 LOCAL HYPOCENTER SURAARY - SGB EARTHQUAKES

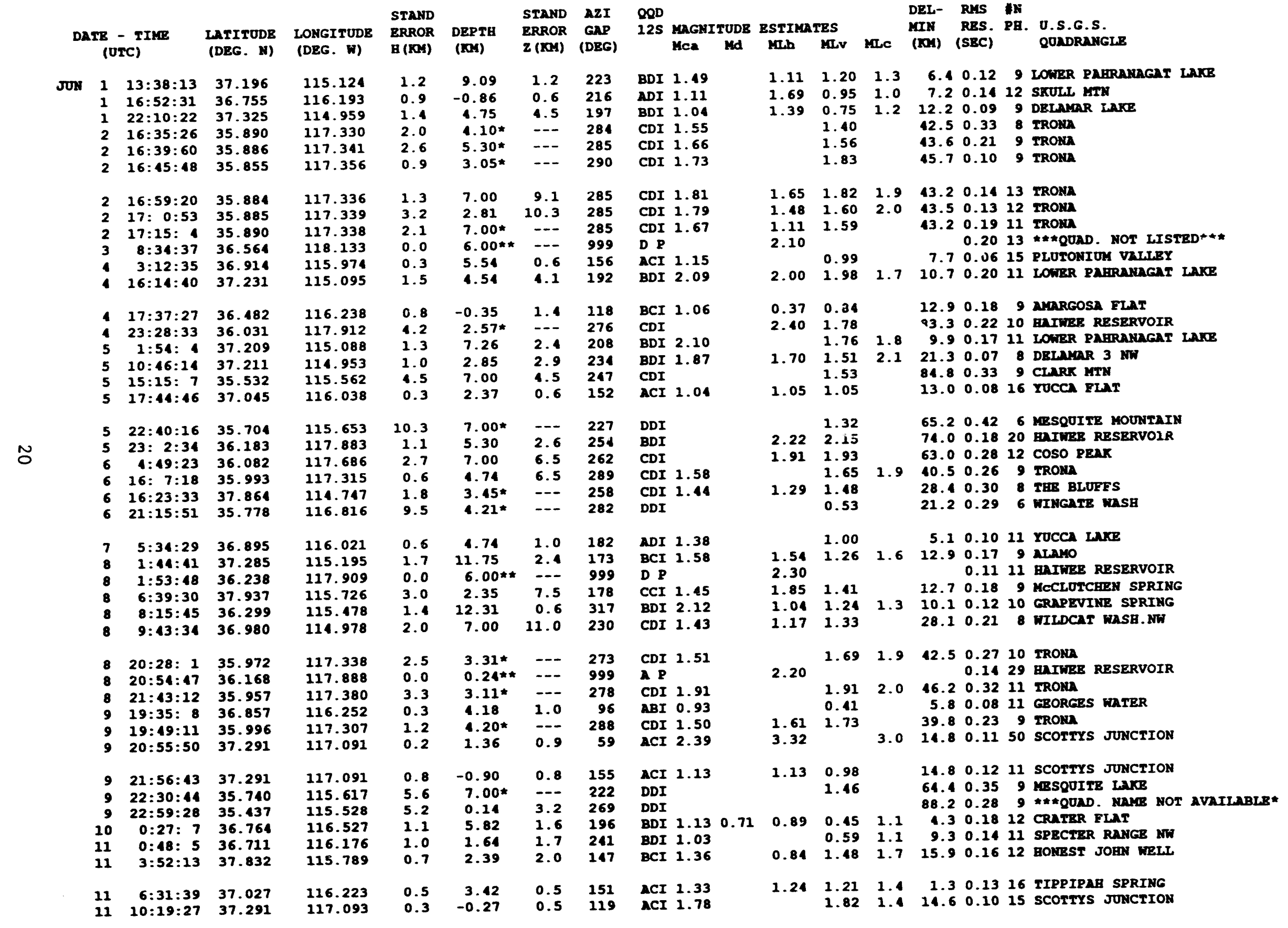


1992 LOCAL HYPOCENTER SUMEARY - SGB EARTHQUAKRS

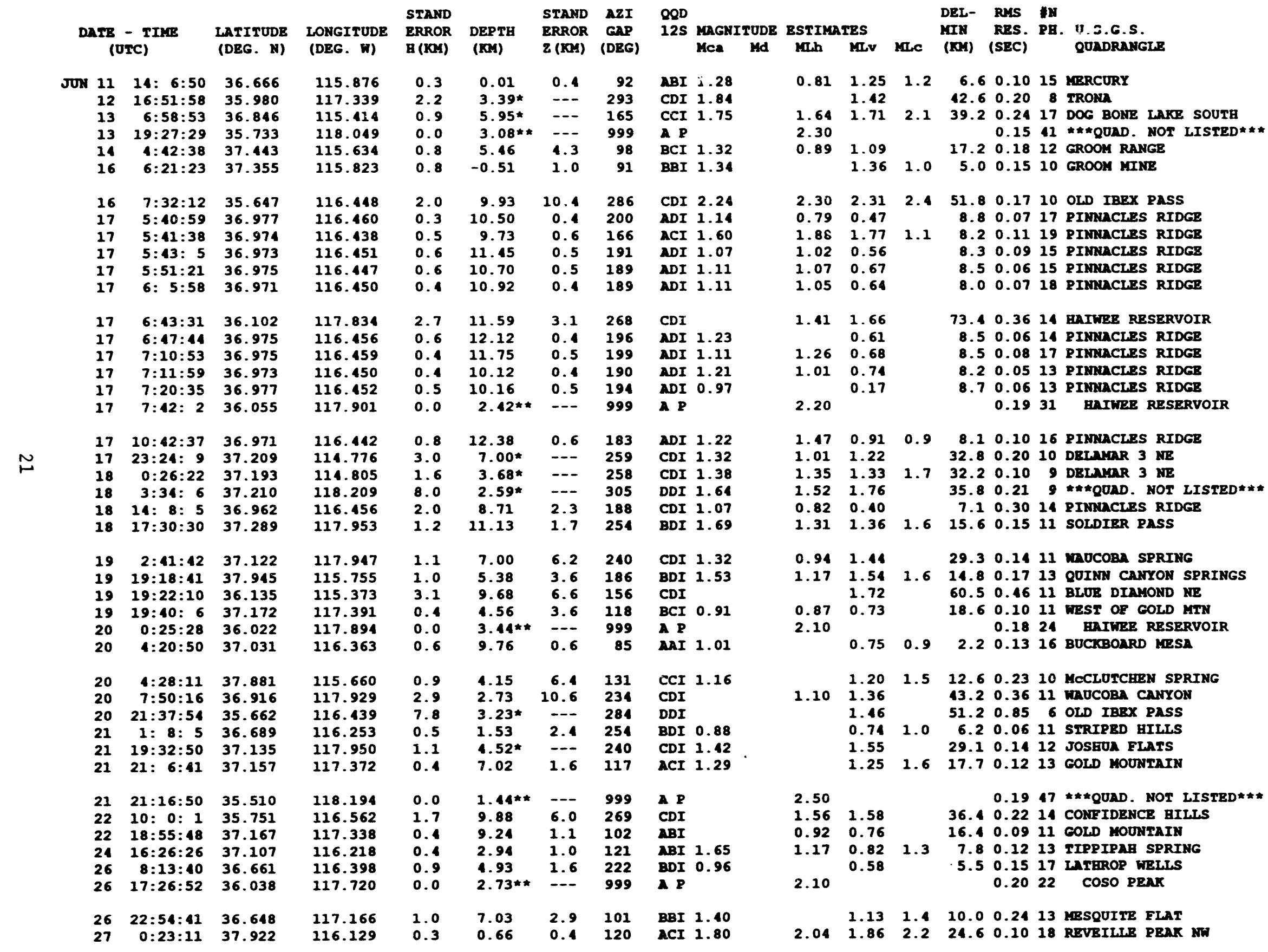


1992 LOCAL HYPOCENTER SURQRARY - SGB RARTBQOAKES

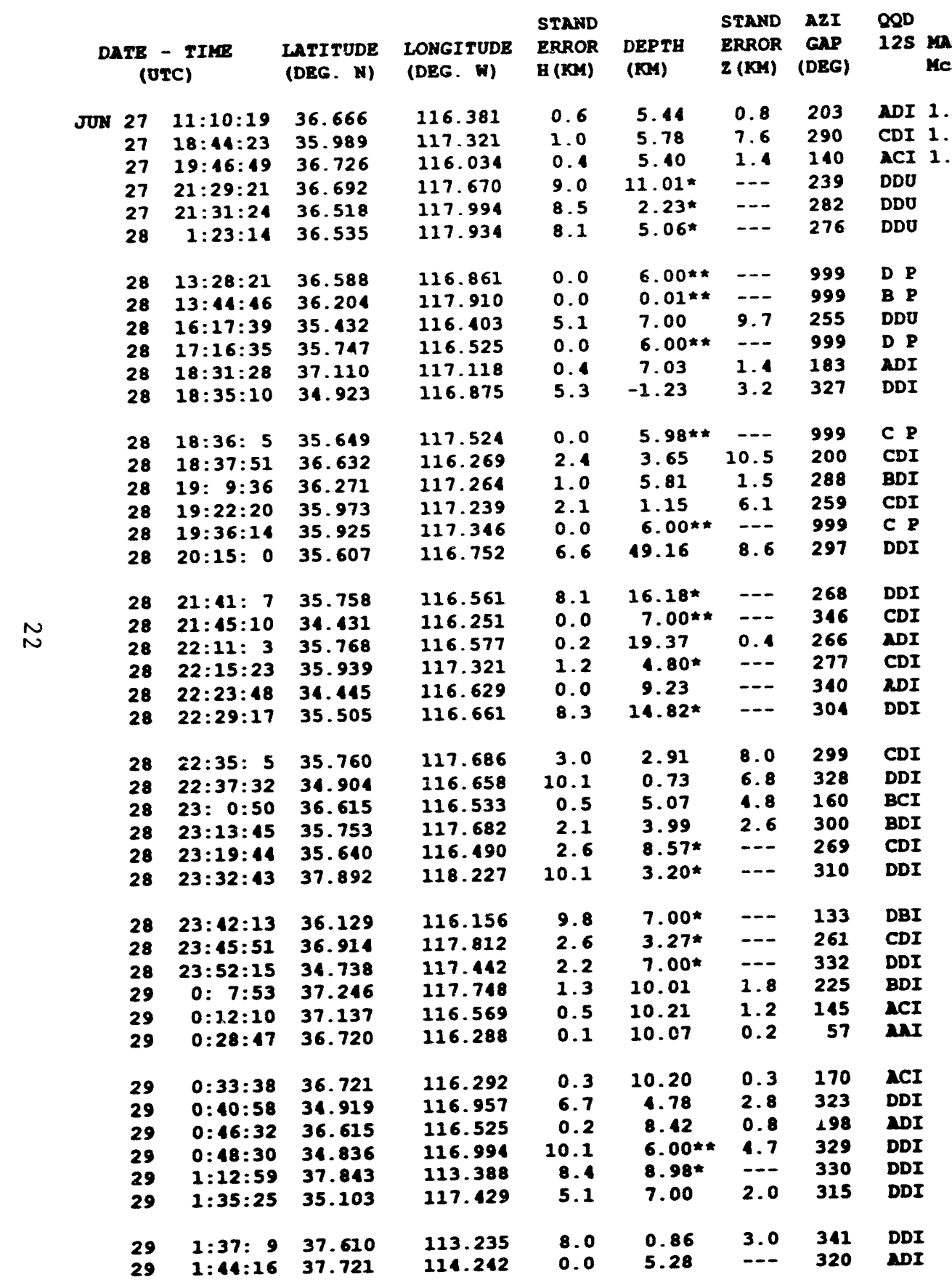

DRL- RMS HN

MII RES. PB. O.S.G.S.

MAGITUDE ESTIMATES KLC (KA) (SEC) OOADRANGLE

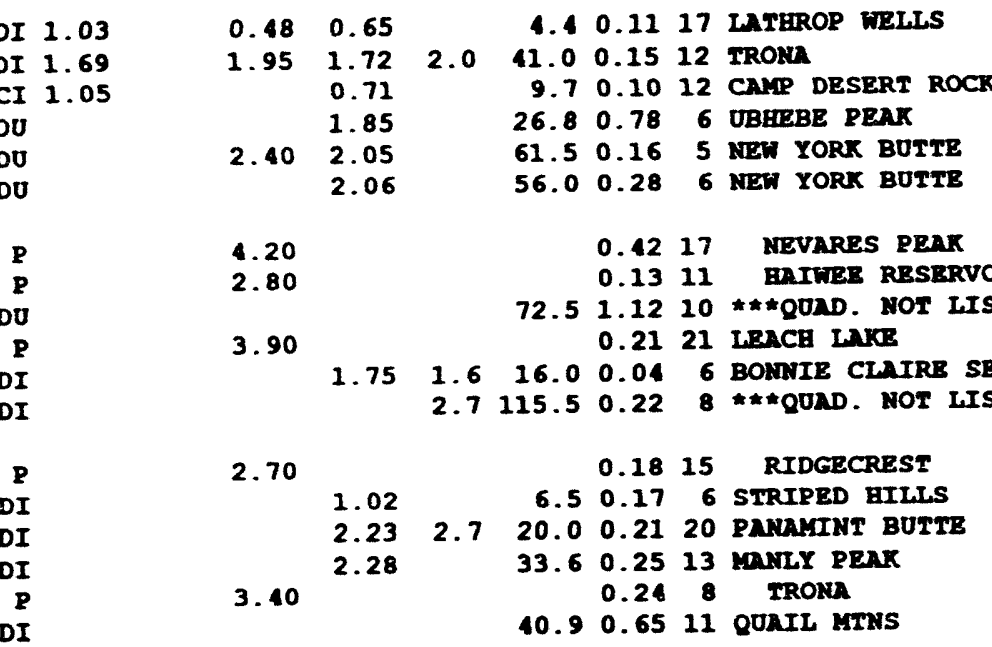

35.80 .829 CONFIDENCE gILLS

$188.0 .40,3 * \pi *$ OOAD. NOT LISTED***

18.1 0.016 CONFIDENCE BILLS

34.10 .01 C CONTID

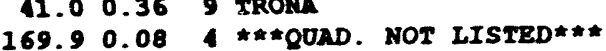

$\begin{array}{rr}169.9 & 0.08 \\ 54.3 & 0.527 \text { LEACH LAKR }\end{array}$

77.30 .3414 MTN SPRINGS CANYON

$119.20 .296 * *$ QOAD. NOT LISTBD***

17.60 .1316 LEERTAND

77.10 .2916 MTM SPRINGS CANYON

49.60 .239 OLD IBEX PASS

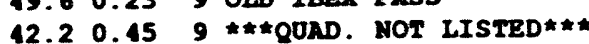

0.28 .179 STEWHRT VALIEY

$\begin{array}{rrrr}0.2 & 8.17 & 9 & \text { STEWART VALLEY } \\ 38.2 & 0.22 & 8 & \text { EAST OF muCOBA CANYON }\end{array}$ 145.71 .696 * 140 ON. NOT LISTED:4"

$1.33 \quad 9.10 .127$ maNGING ROCK CANYON

$14.0 \quad 0.05 \quad 8$ TaIRSTY CarYoN

$\begin{array}{lllllll}2.28 & 1.96 & 1.8 & 2.8 & 0.05 & 27 & \text { SIRIPED BILLS }\end{array}$

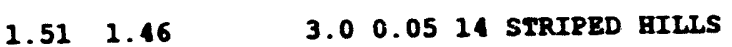

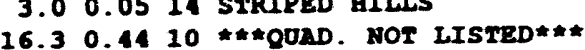

$1.19 \quad \begin{array}{ll}16.9 & 0.0513 \text { LEELND }\end{array}$

$2.73 \quad 125.70 .548 *$ \&OND. NOT LISTED*** $121.90 .128 * *$ QOND. NOT LISTED*** $108.30 .3010 * *$ QUND. NOT IISTED***

$2.74 \begin{array}{rrrr}2.7 & 132.8 & 0.40 & 9 * * \text { *OND. NOT LISTED*** } \\ 45.6 & 0.03 & 4 * * \text { QOND. NOT LISTED*** }\end{array}$ 
1992 LOCAL HYPOCENTER SUEMARY - SGB EARTHQUAKES

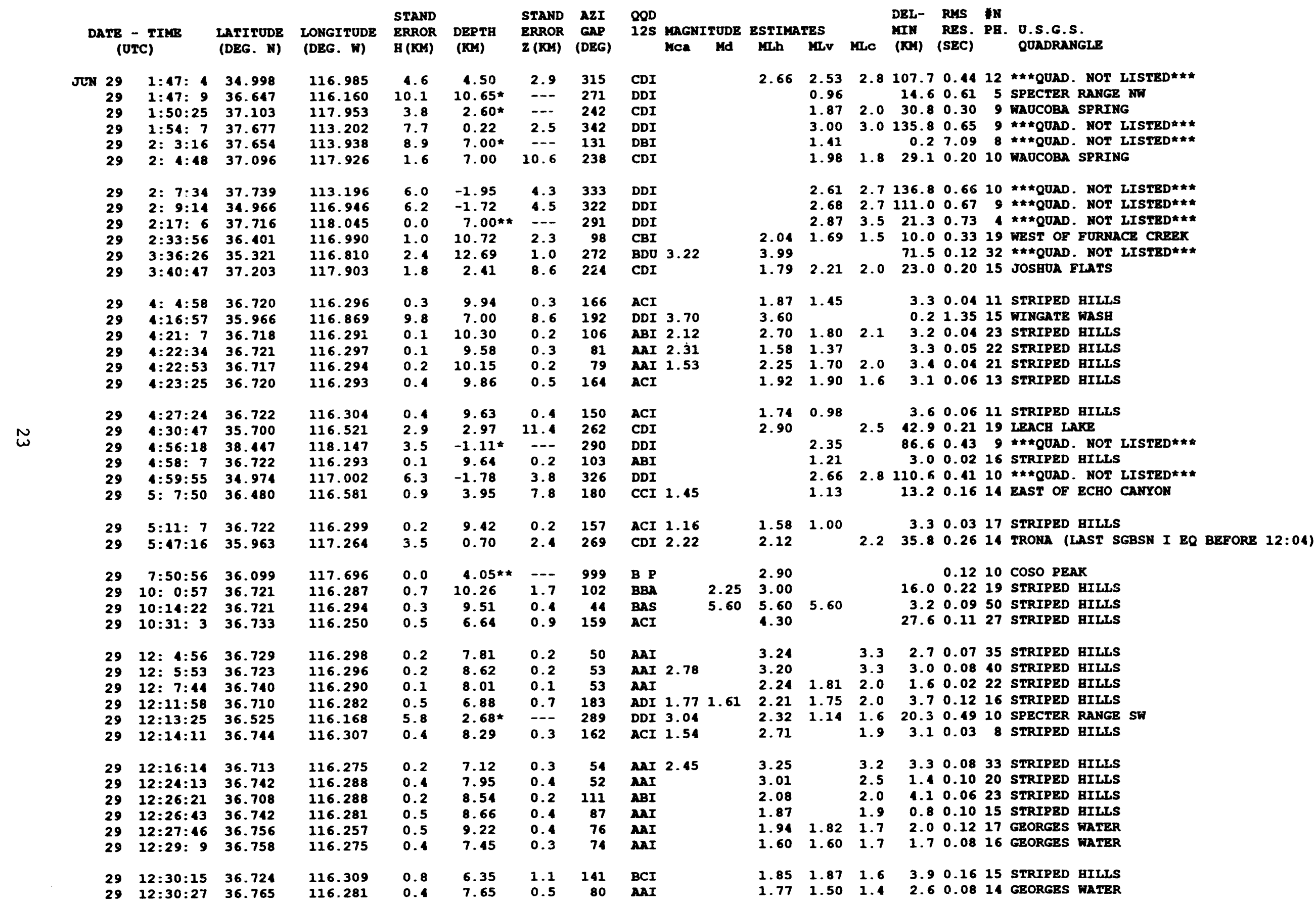




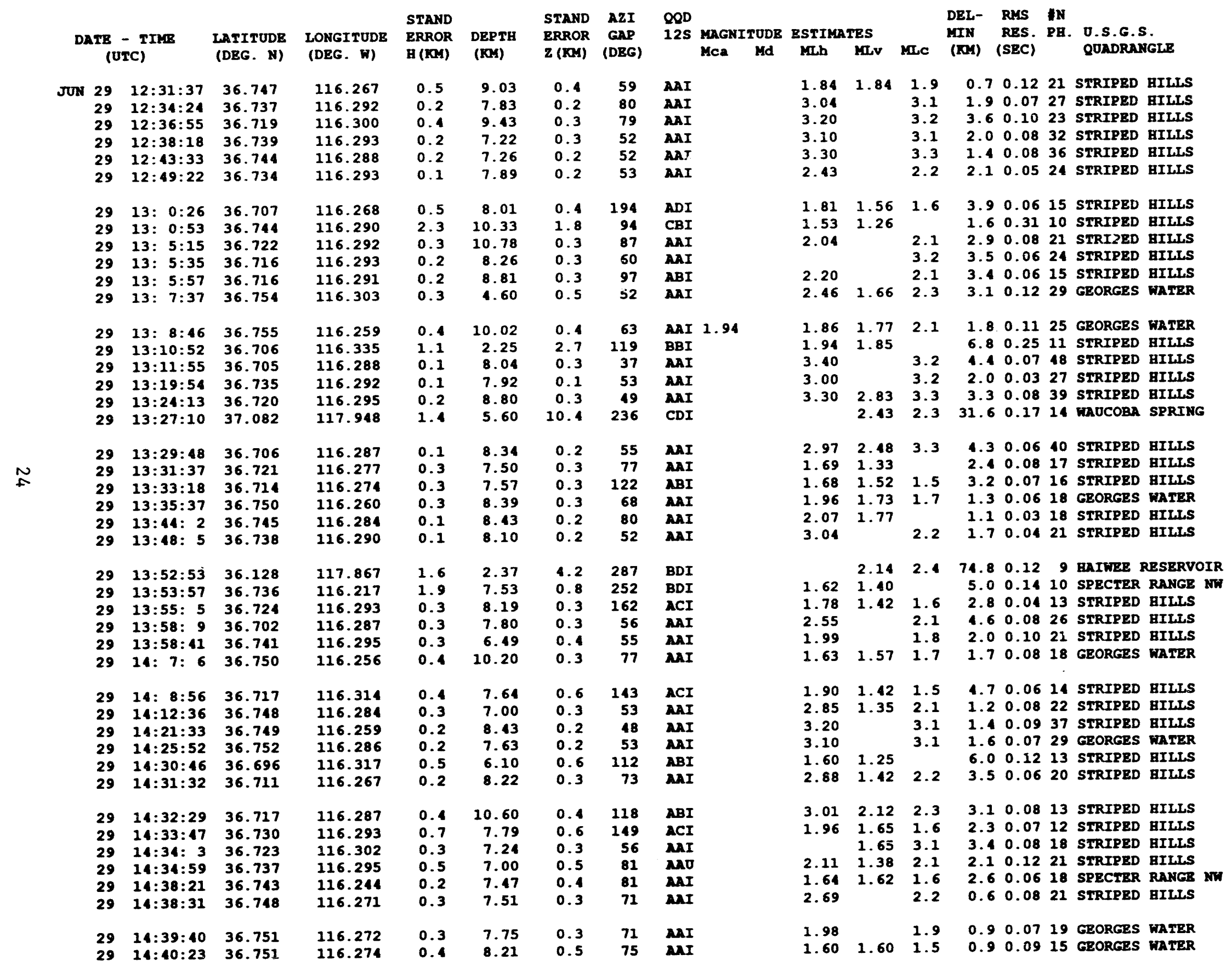


$\begin{array}{lllllll} & & \text { STAND } & \text { STAND } & \text { A2I } & \text { QQD } \\ \text { DATE - TIKE LATITUDE LONGITUDE } & \text { ERROR DEPTH } & \text { ERROR } & \text { GAP } & \text { 12S }\end{array}$ (UTC)

JUN $29 \quad 14: 41: 58 \quad 36.723$ $14: 50: 32 \quad 36.737$ 29 14:57:14 36.744

$\begin{array}{llll}\text { ERROR } & \text { DEPTH } & \text { ERROR } & \text { GAP } \\ \text { H(KM) } & \text { (KA) } & \text { Z (KOY) } & \text { (DEG) }\end{array}$

\section{$\begin{array}{lllll}0.6 & 9.21 & 0.5 & 103 & \mathrm{ABI}\end{array}$}

$\begin{array}{lllrr}0.2 & 9.92 & 0.2 & 61 & \text { AAI } \\ 0.3 & 8.78 & 0.3 & 105 & \text { ABI }\end{array}$

$\begin{array}{lllll}1.5 & 13.65 & 1.4 & 104 & \text { BBI }\end{array}$

$\begin{array}{lllll}0.3 & 9.09 & 0.3 & 184 & \text { ADI }\end{array}$

$\begin{array}{lllll}0.3 & 7.36 & 0.5 & 96 & \mathrm{ABI}\end{array}$

$0.3 \quad 8.78$

$0.5 \quad 9.26$

$\begin{array}{ll}0.5 & 7.00 \\ 0.2 & 7.18\end{array}$

7.23
8.07

0.491

$0.4102 \quad A B I$

0.483 AAU

$0.3 \quad 56$ AAI

$0.2 \quad 101 \quad A B I$

$\begin{array}{lllll}0.2 & 7.94 & 0.2 & 116 & \text { ABI }\end{array}$

0.29 .00

.00

7.61

7.21

0.26 .50

$0.3 \quad 55$

0.233

$0.5 \quad 143$ ACI

$\begin{array}{lllll}1.4 & 6.52 & 1.2 & 190 & \text { BDI }\end{array}$

$\begin{array}{lllll}0.2 & 8.27 & 0.2 & 71 & \text { AAI }\end{array}$ $29 \quad 14: 46: 31 \quad 36.696$ $29 \quad 14: 48: 55 \quad 36.723$ 14:50:37 36.696 $14: 52: 37,36.696$

$29 \quad 14: 53: 41 \quad 36.713$ $29 \quad 14: 56: 19 \quad 36.750$ 29 15: $3: 44 \quad 36.727$ $29 \quad 15: 4: 57 \quad 36.732$ 29 15: 8: $6 \quad 36.724$

$29 \quad 15: 14: 10 \quad 36.707$ $29 \quad 15: 15: 17 \quad 36.715$ $29 \quad 15: 17: 38 \quad 36.741$ $29 \quad 15: 20: 52 \quad 36.723$ 29 15:32:0 36.726 $29 \quad 15: 32: 21 \quad 36.723$

$29 \quad 15: 33: 34 \quad 36.751$

116.293 116.305 116.298 116.284 116.300 116.258 116.291 116.302 116.243 116.297

116.283 116.304 116.389 116.301 16.275 116.308

116.214 116.269 116.287 116.360 116.293 116.270

29 15:36:43 36.

$29 \quad 15: 37: 1 \quad 36.735$

$29 \quad 15: 38: 42 \quad 36.744$

$29 \quad 15: 38: 57 \quad 36.728$ $29 \quad 15: 39: 34 \quad 36.744$ $29 \quad 15: 41: 17 \quad 36.727$ $29 \quad 15: 44: 44 \quad 36.318$ $29 \quad 15: 45: 14 \quad 36.746$ $29 \quad 15: 52: 43 \quad 36.708$

$29 \quad 15: 55: 1 \quad 36.750$ $29 \quad 15: 55: 23 \quad 36.717$ $29 \quad 15: 56: 23 \quad 36.697$ 29 15:58: 136.706 $29 \quad 15: 58: 18 \quad 36.727$ 29 15:59:43 36.720

$29 \quad 15: 59: 43 \quad 36.716$ 29 16: $1: 5$ 36.721 29 16:1: 5 36.721 29 16: 2:14 36.706 29 16: 8:17 36.762 29 16: 9:58 36.711

$29 \quad 16: 12: 0 \quad 36.717$ $29 \quad 16: 12: 46 \quad 36.752$

116.268

116.295

116.274

116.303

116.260

116.289

116.273

116.296

116.310

116.290

116.300

116.274

116.295

116.302

116.303

116.295

116.255

116.302

116.277

116.252
S MAGITUDE ESTIMATES

$\begin{array}{lllll}1.0 & 5.85 & 1.1 & 182 & \text { ADI } \\ 1.6 & 7.00 & 2.3 & 115 & \text { BBD }\end{array}$

$0.253 \quad 19$
0.65 .77

$0.9 \quad 7.69$

$0.3 \quad 6.57$

8.21
8.61

8.40$$
0.3
$$

$0.3 \quad 76$ AAI

$0.3 \quad 45$ AAI 2.60

0.28 .19

0.375 AAI

0.3111

$0.3 \quad 83$

$\begin{array}{rr}0.2 & 56 \\ 0.9 & 183\end{array}$

ABA

AAI

AAI

0.27 .40

10.66

$0.7 \quad 173$

$0.8 \quad 9.62$

$0.7 \quad 6.20$

$\begin{array}{rrr}0.7 & 173 & \text { ACI } \\ 0.9 & 56 & \text { BAI }\end{array}$

$\begin{array}{lll}1.0 & 56 & \mathrm{BAI}\end{array}$

$\begin{array}{rrrrr}0.2 & 8.10 & 0.3 & 57 & \text { MAI } \\ 0.5 & 8.23 & 0.6 & 168 & \text { ACI }\end{array}$

$0.3 \quad 7.79$

$0.3 \quad 114$

$\begin{array}{lrr}0.2 & 58 & \text { ABI }\end{array}$
$0.3105 \quad A B I$

0.9169 BCI

$\begin{array}{lllll}.6 & 11.45 & 0.5 & 183 & \mathrm{ADI}\end{array}$ $\begin{array}{llllllll}1.88 & 1.43 & 1.6 & 2.9 & 0.09 & 12 & \text { STRIPED }\end{array}$ $\begin{array}{lllllll}2.39 & 1.80 & 2.2 & 6.0 & 0.06 & 23 & \text { STRIPED EILIS }\end{array}$ $\begin{array}{lllllll}1.94 & 1.49 & 1.5 & 3.2 & 0.06 & 15 & \text { STRIPED } \\ 1.6 I L L S\end{array}$ $\begin{array}{lllllll}1.63 & 1.34 & 1.7 & 1.2 & 0.25 & 12 & \text { STRIPED HILLS }\end{array}$ $\begin{array}{llllll}1.52 & 1.27 & 5.5 & 0.04 & 15 & \text { STRIPED HILLS }\end{array}$ $\begin{array}{lllll}2.49 & 2.1 & 6.3 & 0.09 & 20 \\ & \text { STRIPED HILLS }\end{array}$

$\begin{array}{lllllll}2.08 & 1.47 & 2.1 & 1.1 & 0.07 & 17 & \text { STRIPED HILLS }\end{array}$ $\begin{array}{lllllll}1.80 & 1.76 & 1.7 & 1.5 & 0.11 & 19 & \text { GEORGES WATER }\end{array}$ $\begin{array}{lllllll}1.93 & 1.41 & 1.9 & 1.7 & 0.10 & 16 & \text { STRIPED HILLS }\end{array}$ $2.95 \quad 3.1 \quad 3.20 .0526$ STRIPED BILLS $\begin{array}{lllllll}2.91 & 1.85 & 3.0 & 2.8 & 0.04 & 15 & \text { SPECTER RANGE }\end{array}$ $\begin{array}{llllll}3.03 & 1.99 & 2.2 & 3.0 & 0.05 & 23 \\ \text { STRIFED HILLS }\end{array}$

$\begin{array}{lllllll}1.63 & 1.28 & 1.5 & 4.0 & 0.04 & 16 & \text { STRIPED HILLS }\end{array}$

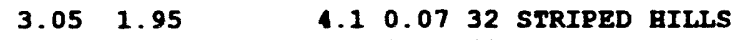
$\begin{array}{lllllll}3.10 & 2.20 & 3.1 & 1.5 & 0.05 & 40 & \text { STRIPED HILLS }\end{array}$

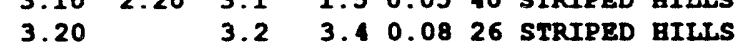
$\begin{array}{lllllll}3.20 & & 3.2 & 3.4 & 0.08 & 26 & \text { STRIPED BILLS } \\ 1.61 & 1.45 & & 1.9 & 0.04 & 13 & \text { STRIPED BILLS }\end{array}$ $\begin{array}{lllllll}1.78 & 1.45 & 1.4 & 3.8 & 0.05 & 12 & \text { STRIPED HILLS }\end{array}$

$\begin{array}{lllll}2.03 & 2.2 & 5.3 & 0.1612 & \text { SKOLL MTN }\end{array}$

$\begin{array}{lllllll}1.97 & 1.62 & 2.0 & 1.2 & 0.05 & 20 & \text { GEORGES WATER }\end{array}$

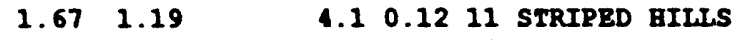
$\begin{array}{lllll}1.76 & 1.30 & 5.90 .3012 \text { GEORGES WATER }\end{array}$ $\begin{array}{lllllll}1.82 & 1.67 & 1.6 & 2.0 & 0.06 & 23 & \text { STRIPED GILLS }\end{array}$ $\begin{array}{lllll}1.66 & 1.42 & 0.3 & 0.0913 & \text { STRIPED HILLS }\end{array}$

$\begin{array}{llllll}1.92 & 1.54 & 1.6 & 0.06 & 9 & \text { STRIPED BILLS }\end{array}$

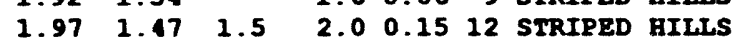
$\begin{array}{lllllll}1.53 & 1.39 & 1.5 & 1.7 & 0.06 & 17 & \text { STRIPED HILLS }\end{array}$ $\begin{array}{llllllll}2.16 & 1.53 & 2.1 & 3.9 & 0.06 & 19 & \text { STRIPED HILLS }\end{array}$ $\begin{array}{lllllll}1.56 & 1.47 & 1.7 & 1.2 & 0.07 & 18 & \text { STRIPED HILLS }\end{array}$ $3.40 \quad 1.10 .0950$ STRIPED BILLS

$2.03 \quad 2.2 \quad 0.90 .0826$ GEORGES WATER $\begin{array}{lllllll}2.07 & 1.35 & 2.1 & 3.6 & 0.15 & 11 & \text { STRIPED BILLS }\end{array}$ $\begin{array}{llllll}1.63 & 1.44 & 6.1 & 0.06 & 15 & \text { STRIPED EILLS }\end{array}$ $1.54136 \quad 430.0617$ STRTPED BILLS $\begin{array}{lllllll}1.81 & 1.34 & 1.9 & 3.1 & 0.06 & 18 & \text { STRIPED BILLS }\end{array}$ $\begin{array}{lllllll}2.27 & 1.53 & 1.7 & 2.5 & 0.15 & 13 & \text { STRIPED BILLS }\end{array}$

$\begin{array}{lllllll}2.25 & 1.53 & 1.6 & 3.6 & 0.10 & 14 & \text { STRIPED BILLS }\end{array}$ $\begin{array}{llllll}2.10 & 2.1 & 3.6 & 0.21 & 24 & \text { STRIPED BILLS }\end{array}$

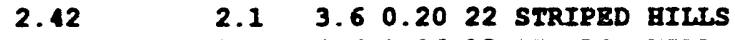
$\begin{array}{lllllll}2.07 & 1.37 & 1.6 & 4.6 & 0.06 & 12 & \text { STRIPED HILLS }\end{array}$ $\begin{array}{llllll}3.30 & 3.2 & 2.7 & 0.09 & 37 & \text { GEORGES WATER }\end{array}$ $\begin{array}{lllll}2.27 & 2.0 & 4.4 & 0.08 & 15\end{array}$

$\begin{array}{lllll}2.36 & 2.8 & 0.07 & 18 & \text { STRIPED BILLS }\end{array}$ $\begin{array}{lllll}2.46 & 2.3 & 2.1 & 0.05 & 22 \\ & & \text { GEORGES WATER }\end{array}$ 


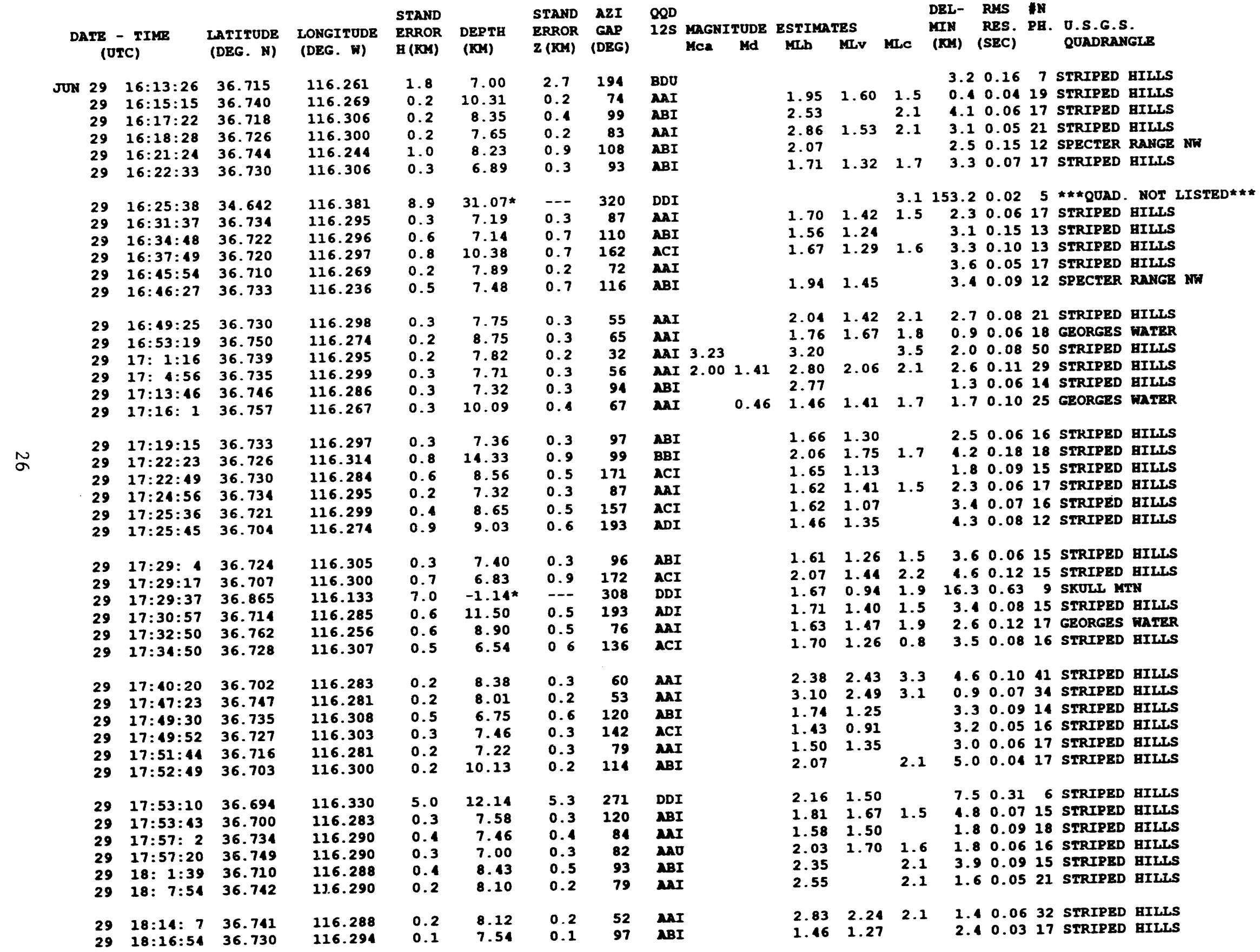


1992 LOCAL HYPOCENTER SURAARY - SGB EARTHQUAKES

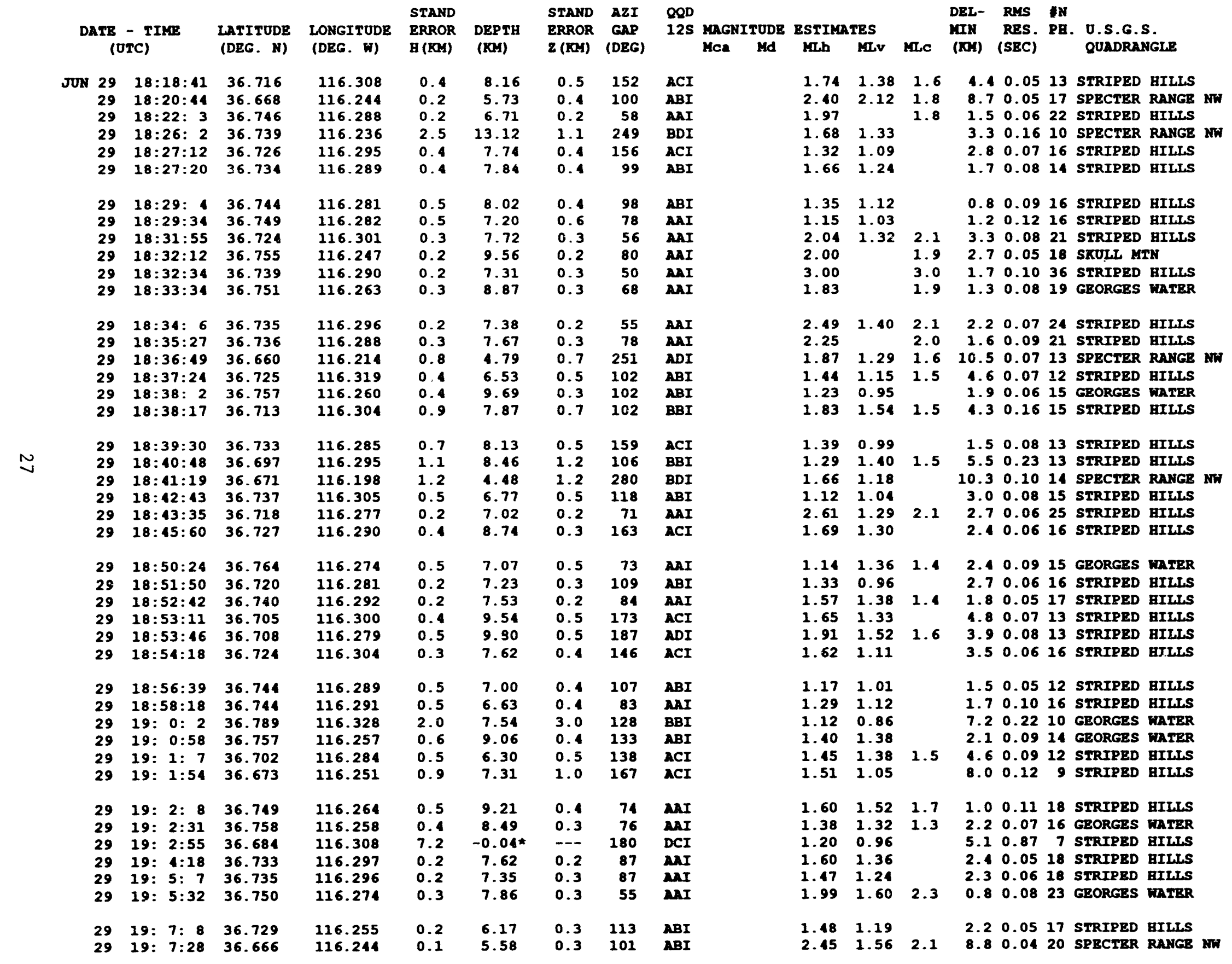


1992 LOCAL HYPOCENTER SURAARY - SGB EARTHQUAKES

STAND STAND AZI QQD

DATE - TIME LATITUDE LONGITUDE ERROR DEPTH ERAND GAP 12S MAGNITUDE ESTIMATES

DEL- RMS IN

(UTC)

(DEG. N) (DEG. W)

(KM) (KM)

JUN 29 19: $8: 8 \quad 36.710$ 29 19: $9: 15 \quad 36.737$ $29 \quad 19: 10: 53 \quad 36.737$ $29 \quad 19: 11: 54 \quad 36.727$ $29 \quad 19: 12: 15 \quad 36.739$ 29 19:13: 36.729

116.281 116.300 116.290 116.288 116.291

116.265

0.2
1.0
0.3
0.5
0.5
0.2

$\begin{array}{llll}7.08 & 0.2 & 120 & \mathrm{ABI} \\ 7.84 & 1.0 & 123 & \mathrm{BBI}\end{array}$

BI

$\begin{array}{llll}6.60 & 0.3 & 83 & \text { AAI }\end{array}$

$\begin{array}{rrrr}8.44 & 0.4 & 168 & \text { ACI } \\ 7.00 & 0.5 & 53 & \text { AAU }\end{array}$

29 19:13: 36.726

116.265

$29 \quad 19: 16: 40 \quad 36.740$

$29 \quad 19: 25: 23 \quad 36.111$

29 19:28:44 36.710

$29 \quad 19: 29: 36 \quad 36.727$

116.294

117.483

116.280

116.298

29 19:30:12 36.708

116.283

$\begin{array}{lllll}0.2 & 6.55 & 0.4 & 66 & \text { AAI } \\ 0.1 & 7.99 & 0.2 & 85 & \text { AAI }\end{array}$

0.0 6.00* $-\cdots \quad 999$ C P

$\begin{array}{lllll}0.2 & 6.76 & 0.3 & 54 & \text { NAI }\end{array}$

$\begin{array}{lllll}0.6 & 7.39 & 0.5 & 148 & \text { ACI } \\ 0.3 & 6.84 & 0.4 & 120 & \text { ABI }\end{array}$

$29 \quad 19: 30: 59 \quad 36.744$

116.289

$29 \quad 19: 31: 33 \quad 36.712$

116.284

0.7

6.84

116.244

$0.7 \quad 7.56$

$0.6 \quad 105$

$29 \quad 19: 31: 56 \quad 36.717$

$29 \quad 19: 32: 48 \quad 36.709$

$29 \quad 19: 32: 50 \quad 36.703$

$29 \quad 19: 33: 22 \quad 36.737$

116.316

116.294

7.10
7.14

6.37

0.280 AAI

$1.1134 \quad \mathrm{BBI}$

$0.3 \quad 71$ AAI

$29 \quad 19: 33: 36 \quad 36.751$

116.252

$\begin{array}{lllll}0.4 & 7.03 & 0.4 & 129 & \text { ABI }\end{array}$

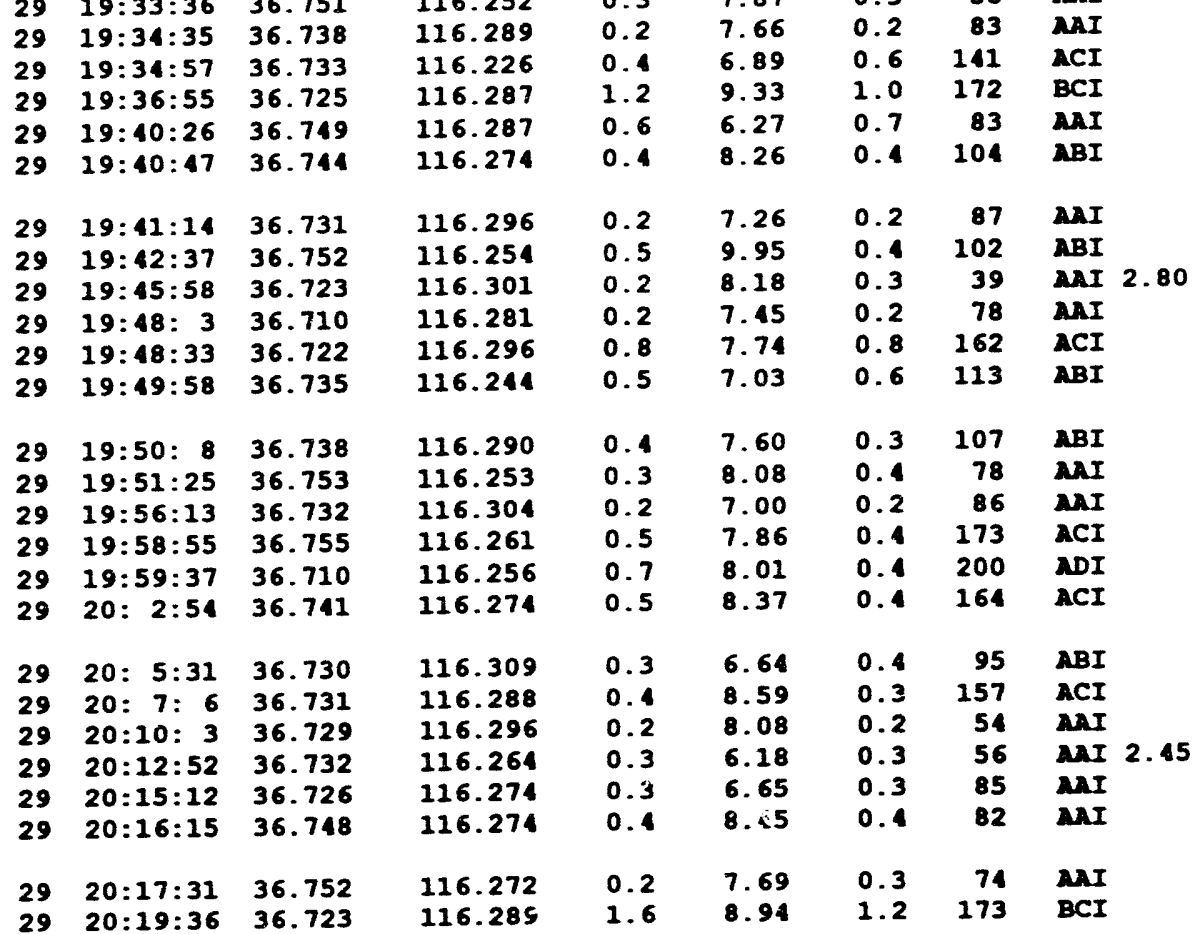

$$
\begin{aligned}
& \begin{array}{lllll}
1.39 & 1.43 & 3.7 & 0.0415 & \text { STRIPED BILLS }
\end{array} \\
& \begin{array}{llllll}
1.36 & 1.03 & 2.5 & 0.14 & 13 & \text { STRIPED BILLS }
\end{array} \\
& \begin{array}{lllllll}
1.98 & 1.84 & 1.5 & 1.7 & 0.07 & 17 & \text { STRIPED } \\
\hline
\end{array} \\
& \begin{array}{lllll}
1.95 & 1.08 & 2.2 & 0.0512 & \text { STRTPRD BITLS }
\end{array}
\end{aligned}
$$

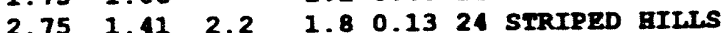

$$
\begin{aligned}
& 2.751 .412 .21 .80 .1324 \text { sTrum } \\
& 2.881 .992 .1 \quad 1.70 .0623 \text { STRIPED BILLS } \\
& 2.442 \quad 2.0 \quad 1.90 .0722 \text { STRIPED gILLS } \\
& 2.14 \quad 1.5 \quad 1.90 .0419 \text { STRIPED GILLS } \\
& 3.20 \text { 3.21 } 23 \text { PANAMINT BOTTE } \\
& \begin{array}{lllllll}
2.38 & 3.2 & 3.7 & 0.08 & 32 & \text { STRIPED EILLS }
\end{array}
\end{aligned}
$$

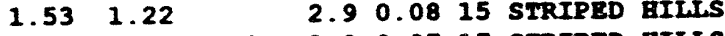

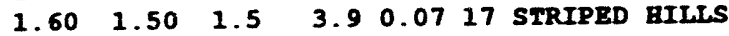

$$
\begin{aligned}
& \begin{array}{llllll}
1.35 & 1.06 \quad & 1.5 & 0.1014 & \text { STRIPED BILLS }
\end{array} \\
& \begin{array}{llllll}
1.91 & 2.0 & 3.6 & 0.05 & 17 & \text { STRIPED BILLS }
\end{array} \\
& \begin{array}{lllll}
1.41 & 1.30 & 3.70 .1513 & \text { SPECTER RANGZ } \\
2.64 & 1.82 & 3.8 & 0.0621 & \text { STRTPED }
\end{array} \\
& \begin{array}{lllllll}
2.64 & 1.82 & & 3.8 & 0.06 & 21 & \text { STRIPED BILLS } \\
3.10 & & 3.1 & 5.9 & 0.13 & 21 & \text { STRIPED }
\end{array} \\
& \begin{array}{lllllll}
3.10 & & 3.1 & 5.9 & 0.13 & 21 & \text { STRIPED BILLS } \\
2.21 & 1.55 & 1.9 & 2.0 & 0.07 & 12 & \text { STRIPED BILLS }
\end{array} \\
& \begin{array}{llllll}
2.64 & 3.1 & 2.0 & 0.10 & 28 & \text { GEORGES WATER } \\
1.87 & 1.9 & 1.6 & 0.04 & 16 & \text { STRIPED BILIS }
\end{array}
\end{aligned}
$$

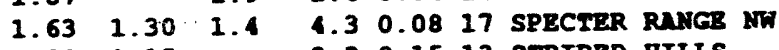

$$
\begin{aligned}
& \begin{array}{llllll}
1.59 & 1.15 & 2.3 & 0.15 & 13 & \text { STRIPED BILLS }
\end{array} \\
& 1.50 .1014 \text { STRIPED RITS } \\
& 1.241 .18 \text { 1.5 } \\
& \begin{array}{lllllll}
1.79 & 1.80 & 1.5 & 2.4 & 0.05 & 18 & \text { STRIPED BILLS }
\end{array}
\end{aligned}
$$

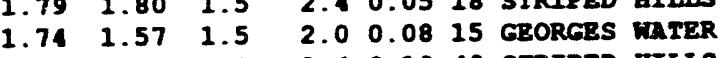

$$
\begin{aligned}
& 3.30303 .1 \quad 3.40 .10 \text { i9 STRIPED BILLS } \\
& 1.79 \quad 1.9 \quad 3.70 .0417 \text { SIRIPED BILLS } \\
& \begin{array}{llllll}
1.46 & 1.18 & 3.1 & 0.1114 & 14 & \text { SIRIPED BILLS }
\end{array}
\end{aligned}
$$

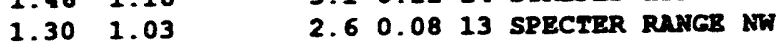

$$
\begin{aligned}
& \begin{array}{llllll}
1.52 & 1.21 & 1.6 & 0.0814 & \text { STRIPED BILLS }
\end{array} \\
& \begin{array}{llllllll}
1.67 & 1.47 & 1.5 & 2.0 & 0.08 & 17 & \text { GBORGES WTER }
\end{array} \\
& 2.501 .862 .0 \quad 3.10 .0520 \text { STRTPED aThIS } \\
& 1.13 \quad 1.01 \quad 2.0 \quad 3.170 .0515 \text { Groress varer } \\
& 1.131 .31 \quad 3.90 .05 \text { S STRIPED } \\
& \begin{array}{llll}
1.27 & 1.01 & 0.2 & 0.0613
\end{array} \\
& \begin{array}{llllllll}
1.81 & 1.28 & 2.0 & 3.6 & 0.07 & 17 & \text { STRIPED BILLS }
\end{array} \\
& \begin{array}{llllll}
1.16 & 0.83 & 1.9 & 0.05 & 14 & \text { STRIPED BILS }
\end{array}
\end{aligned}
$$

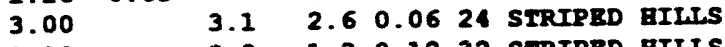

$$
\begin{aligned}
& \begin{array}{llllll}
3.30 & 3.3 & 1.3 & 0.12 & 32 & \text { STRIPED BILLS }
\end{array} \\
& \begin{array}{lllll}
1.28 & 1.38 & 1.9 & 0.0716 & \text { SIRIPED BILLS }
\end{array}
\end{aligned}
$$

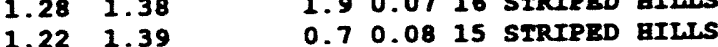

$$
\begin{aligned}
& \begin{array}{lllllll}
1.47 & 1.43 & 1.6 & 1.0 & 0.05 & 17 & \text { GEORGES WATER }
\end{array} \\
& \begin{array}{lllllll}
1.47 & 1.43 & 1.6 & 1.0 & 0.05 & 17 & \text { GEORGES HATER } \\
1.36 & 1.12 & & 2.6 & 0.14 & 12 & \text { SIRIPED HILIS }
\end{array}
\end{aligned}
$$$$
\begin{array}{lll}
29 & 20: 17: 31 & 36.752 \\
29 & 20: 19: 36 & 36.723
\end{array}
$$

116.285 
1992 LOCAL HYPOCENTER SUMAGRY - SGB EARTHQUAKES

\begin{tabular}{|c|c|c|c|c|c|c|c|c|c|c|c|c|c|c|c|c|c|}
\hline \multicolumn{2}{|c|}{$\begin{array}{l}\text { DATE - TIME } \\
\text { (UTC) }\end{array}$} & \multirow{2}{*}{$\begin{array}{c}\text { LATITUDE } \\
\text { (DEG. N) } \\
36.729\end{array}$} & \multirow{2}{*}{$\begin{array}{l}\text { LONGI TUDE } \\
\text { (DEG. W) } \\
116.300\end{array}$} & \multirow{2}{*}{$\begin{array}{c}\text { STAND } \\
\text { ERROR } \\
\text { B (IR) } \\
0.2\end{array}$} & \multirow{2}{*}{$\begin{array}{l}\text { DEPTH } \\
\text { (XO) } \\
7.19\end{array}$} & \multirow{2}{*}{$\begin{array}{c}\text { STAND } \\
\text { ERROR } \\
\text { Z (KN) } \\
0.3\end{array}$} & \multirow{2}{*}{$\begin{array}{c}\text { AZI } \\
\text { GAP } \\
\text { (DEG) } \\
90\end{array}$} & \multirow{2}{*}{$\begin{array}{l}\text { QQD } \\
125 \\
\text { AAI }\end{array}$} & $\begin{array}{l}\text { MAGNITUDE } \\
\text { MCA Md }\end{array}$ & \multicolumn{2}{|c|}{ ESTIMaTES } & MLC & $\begin{array}{l}\text { DEL- } \\
\text { MIX } \\
\text { (RMY) }\end{array}$ & $\begin{array}{l}\text { RYS } \\
\text { RES. } \\
\text { (SEC) }\end{array}$ & $\begin{array}{l}\text { In } \\
\text { PB. }\end{array}$ & \multicolumn{2}{|c|}{$\begin{array}{l}\text { O.s.G.S. } \\
\text { QUNDRAMGLE }\end{array}$} \\
\hline 29 & $20: 19: 52$ & & & & & & & & & 1.41 & 1.20 & & 2.9 & 0.05 & 15 & STRIPED & HILLS \\
\hline 29 & $20: 20: 14$ & 36.733 & 116.298 & 0.3 & 7.48 & 0.3 & 88 & AAI & & 1.60 & 1.59 & 1.5 & 2.5 & 0.06 & 17 & STRIPED & BILLS \\
\hline 29 & $20: 21: 50$ & 36.736 & 116.290 & 0.4 & 7.41 & 0.3 & 86 & AAI & & 1.39 & 1.33 & 1.5 & 1.8 & 0.07 & 16 & STRIPED & AILLS \\
\hline 29 & $20: 22: 43$ & 36.732 & 116.294 & 0.5 & 7.65 & 0.1 & 145 & ACI & & 1.55 & 1.22 & & 2.3 & 0.05 & 14 & STRIPED & HILLS \\
\hline 29 & $20: 23: 53$ & 36.744 & 116.274 & 1.5 & 8.71 & 1.0 & 157 & BCI & & 1.39 & 1.17 & & 0.2 & 0.12 & 11 & STRIPED & BILLS \\
\hline 29 & $20: 24: 59$ & 36.732 & 116.299 & 0.1 & 8.07 & 0.1 & 75 & AAI & & 2.99 & 1.82 & & 2.7 & 0.03 & 24 & STRIPED & BILLS \\
\hline 29 & $20: 27: 16$ & 36.828 & 116.227 & 0.2 & 9.63 & 0.4 & 51 & $\operatorname{AAI}$ & & 1.86 & & & 5.9 & 0.07 & 23 & SKULL MTP & $\mathbf{P N}$ \\
\hline 29 & $20: 28: 20$ & 36.737 & 116.297 & 0.2 & 7.89 & 0.2 & 95 & ABI & & 1.98 & 1.81 & 1.6 & 2.3 & 0.04 & 17 & STRIPED & BILLS \\
\hline 29 & $20: 29: 49$ & 36.717 & 116.306 & 0.4 & 8.00 & 0.5 & 154 & ACI & & & & & 4.1 & 0.05 & 14 & SIRIPED & HILLS \\
\hline 29 & $20: 30: 51$ & 36.739 & 116.291 & 0.4 & 7.75 & 0.3 & 96 & ABI & & 1.06 & 1.11 & & 1.7 & 0.06 & 15 & STRIPED & BILLS \\
\hline 29 & $20: 32: 14$ & 36.717 & 116.277 & 0.1 & 7.94 & 0.2 & 62 & MAI & & 2.33 & 1.77 & & 2.9 & 0.04 & 21 & STRIPED & BILLS \\
\hline 29 & $20: 34: 41$ & 36.765 & 116.259 & 0.3 & 7.47 & 0.3 & 162 & $\mathrm{ACI}$ & & 2.09 & 1.89 & & 2.7 & 0.05 & 17 & GEORGES & WATER \\
\hline 29 & $20: 38: 47$ & 36.723 & 116.300 & 0.4 & 7.42 & 0.4 & 102 & ABI & & & & & 3.3 & 0.08 & 15 & STRIPED & HILLS \\
\hline 29 & $20: 41: 15$ & 36.753 & 116.262 & 0.2 & 7.91 & 0.3 & 43 & AAI & & 3.20 & 2.58 & 3.1 & 1.5 & 0.08 & 37 & GEORGES & WATER \\
\hline 29 & $20: 48: 32$ & 36.705 & 116.302 & 0.3 & 9.89 & 0.2 & 113 & ABI & & 1.64 & 1.33 & & 5.0 & 0.05 & 17 & STRIPED & BILLS \\
\hline 29 & $20: 49: 49$ & 36.733 & 116.274 & 1.2 & 9.14 & 0.9 & 176 & $\mathrm{BCI}$ & & 1.08 & 0.87 & & 1.1 & 0.13 & 10 & SIRIPED & BILLS \\
\hline 29 & $20: 51: 2$ & 36.737 & 116.284 & 0.3 & 7.62 & 0.3 & 95 & $A B I$ & & 0.99 & 1.01 & & 1.3 & 0.06 & 16 & STRIPED & BILLS \\
\hline 29 & $20: 52: 4$ & 36.737 & 116.290 & 0.4 & 8.15 & 0.3 & 136 & ACI & & 1.16 & 0.91 & & 1.7 & 0.05 & 13 & STRIPED & artus \\
\hline 29 & $20: 52: 17$ & 36.690 & 116.274 & 1.2 & 8.58 & 1.4 & 228 & BDI & & 0.96 & 0.89 & & 5.8 & 0.10 & 10 & STRIPED & gILLS \\
\hline 29 & $20: 52: 54$ & 36.741 & 116.274 & 0.5 & 8.17 & 0.5 & 75 & AAI & & 1.02 & 0.96 & & 0.2 & 0.10 & 17 & STRIPED & BILLS \\
\hline 29 & $20: 55: 1$ & 36.744 & 116.282 & 0.5 & 7.26 & 0.3 & 190 & ADI & & 1.16 & 0.63 & & 0.9 & 0.03 & 10 & SIRIPED & arlus \\
\hline 29 & $20: 55: 39$ & 36.744 & 116.274 & 0.6 & 8.10 & 0.4 & 104 & ABI & & 1.24 & 1.15 & & 0.2 & 0.08 & 15 & STRIPED & BILLS \\
\hline 29 & $20: 56: 1$ & 36.813 & 116.255 & 0.8 & 4.99 & 1.5 & 103 & $\mathrm{BBI}$ & & 1.60 & 1.42 & 1.6 & 7.9 & 0.17 & 11 & GRORGES & WATER \\
\hline 29 & $20: 59: 24$ & 36.752 & 116.249 & 0.5 & 9.70 & 0.4 & 182 & ADI & & 1.23 & 0.94 & & 2.3 & 0.07 & 13 & SKOLL MT & $m$ \\
\hline 29 & $20: 59: 43$ & 36.732 & 116.288 & 0.8 & 8.07 & 0.6 & 156 & ACI & & 1.41 & 1.23 & & 1.9 & 0.10 & 13 & STRIRED & BILLS \\
\hline 29 & $21: 1: 23$ & 36.727 & 116.281 & 0.7 & 9.66 & 0.6 & 186 & ADI & & 1.25 & 1.20 & & 1.9 & 0.08 & 10 & SIRIPED & EILLS \\
\hline 29 & 21: 2: 0 & 36.736 & 116.296 & 1.3 & 7.00 & 1.4 & 129 & BBD & & 1.20 & 0.89 & & 2.2 & 0.17 & 13 & STRIPED & BrLLS \\
\hline 29 & $21: 2: 35$ & 36.707 & 116.314 & 0.5 & 9.48 & 0.6 & 154 & ACI & & 1.60 & 1.43 & & 5.5 & 0.07 & 14 & STRIPED & BILLS \\
\hline 29 & $21: 4: 14$ & 36.756 & 116.268 & 0.1 & 8.03 & 0.1 & 55 & AAI & & 2.71 & 1.68 & 2.1 & 1.5 & 0.04 & 34 & GEORGES & WATER \\
\hline 29 & $21: 11: 57$ & 36.749 & 116.257 & 0.7 & 9.98 & 0.5 & 129 & $\mathrm{ABI}$ & & 1.35 & 1.15 & & 1.5 & 0.10 & 15 & SIRIPED & arLLS \\
\hline 29 & $21: 14: 20$ & 36.730 & 116.292 & 0.3 & 8.00 & 0.3 & 153 & $\mathrm{ACI}$ & & 1.46 & 0.98 & & 2.2 & 0.05 & 14 & STRIPED & BILLS \\
\hline 29 & $21: 14: 38$ & 36.647 & 116.341 & 1.1 & 7.00 & 1.3 & 195 & BDD & & 1.37 & 0.79 & & 0.2 & 0.15 & 9 & SERIPED & BILLS \\
\hline 29 & $21: 15: 3$ & 36.696 & 116.307 & 0.4 & 10.25 & 0.5 & 172 & ACI & & 1.36 & 1.09 & & 6.0 & 0.06 & 15 & STRIPED & Bruls \\
\hline 29 & $21: 15: 29$ & 36.744 & 116.272 & 0.8 & 12.40 & 0.8 & 136 & ACI & & 1.38 & 1.29 & & 0.2 & 0.13 & 15 & SIRIPED & artus \\
\hline 29 & $21: 17: 29$ & 36.718 & 116.263 & 2.0 & 9.97 & 0.8 & 316 & BDI & & 0.94 & 0.82 & & 2.8 & 0.11 & 9 & SIRIPED & BILLS \\
\hline 29 & $21: 20: 22$ & 36.731 & 116.293 & 0.3 & 7.86 & 0.3 & 149 & ACI & & 1.76 & 1.26 & & 2.2 & 0.04 & 14 & STRIPED & BILls \\
\hline 29 & $21: 20: 53$ & 36.716 & 116.296 & 0.6 & 8.84 & 0.7 & 170 & $\mathrm{ACI}$ & & 2.41 & 1.20 & 1.6 & 3.6 & 50.09 & 15 & SIRIPED & BILLS \\
\hline 29 & $21: 21: 16$ & 36.710 & 116.289 & 0.2 & 8.58 & 0.2 & 83 & $\operatorname{MAI}$ & & 2.03 & 1.49 & 2.0 & 3.9 & 0.04 & 17 & SIRIPED & BILLS \\
\hline 29 & $21: 22: 8$ & 36.744 & 116.278 & 0.3 & 8.02 & 0.3 & 98 & $\mathbf{A B I}$ & & 1.52 & 1.54 & 1.6 & 0.6 & 0.06 & 16 & STRIPED & BILLS \\
\hline 29 & $21: 22: 27$ & 36.729 & 116.301 & 0.2 & 7.40 & 0.3 & 90 & ABI & & 1.45 & 1.22 & 1.5 & 3.0 & 0.05 & $\begin{array}{l}17 \\
15\end{array}$ & STRIPED & $\begin{array}{l}\text { EILLS } \\
\text { MERR }\end{array}$ \\
\hline 29 & $21: 23: 32$ & 36.751 & 116.257 & 0.8 & 9.35 & 0.5 & 125 & ABI & & $\begin{array}{l}1.39 \\
1.47\end{array}$ & $\begin{array}{l}1.21 \\
1.37\end{array}$ & 1.4 & $\begin{array}{l}1.6 \\
2.2\end{array}$ & 0.11 & 15 & GEORGES & $\begin{array}{l}\text { MATER } \\
\text { MATER }\end{array}$ \\
\hline 29 & $21: 25: 12$ & 36.762 & 116.268 & 0.6 & 9.26 & 0.5 & 148 & ACI & & 1.47 & 1.37 & & 2.2 & 0.08 & 14 & GEORGES & IER \\
\hline $\begin{array}{l}29 \\
29\end{array}$ & $\begin{array}{l}21: 39: 7 \\
21: 30: 35\end{array}$ & $\begin{array}{l}36.722 \\
36.730\end{array}$ & $\begin{array}{l}116.300 \\
116.250\end{array}$ & $\begin{array}{l}0.4 \\
0.3\end{array}$ & $\begin{array}{l}8.96 \\
7.40\end{array}$ & $\begin{array}{l}0.4 \\
0.4\end{array}$ & $\begin{array}{r}155 \\
79\end{array}$ & $\begin{array}{l}\text { ACI } \\
\text { Aar }\end{array}$ & & $\begin{array}{l}1.83 \\
1.55\end{array}$ & $\begin{array}{l}1.41 \\
1.32\end{array}$ & 1.6 & $\begin{array}{l}3.4 \\
2.4\end{array}$ & $\begin{array}{l}0.04 \\
0.07\end{array}$ & $\begin{array}{l}13 \\
16\end{array}$ & $\begin{array}{l}\text { STRIPED } \\
\text { SIRIPED }\end{array}$ & $\begin{array}{l}\text { BILLS } \\
\text { BILLS }\end{array}$ \\
\hline
\end{tabular}


1992 LOCAL BYPOCENTER SUMAMRY - SGB EARTHQUAKES

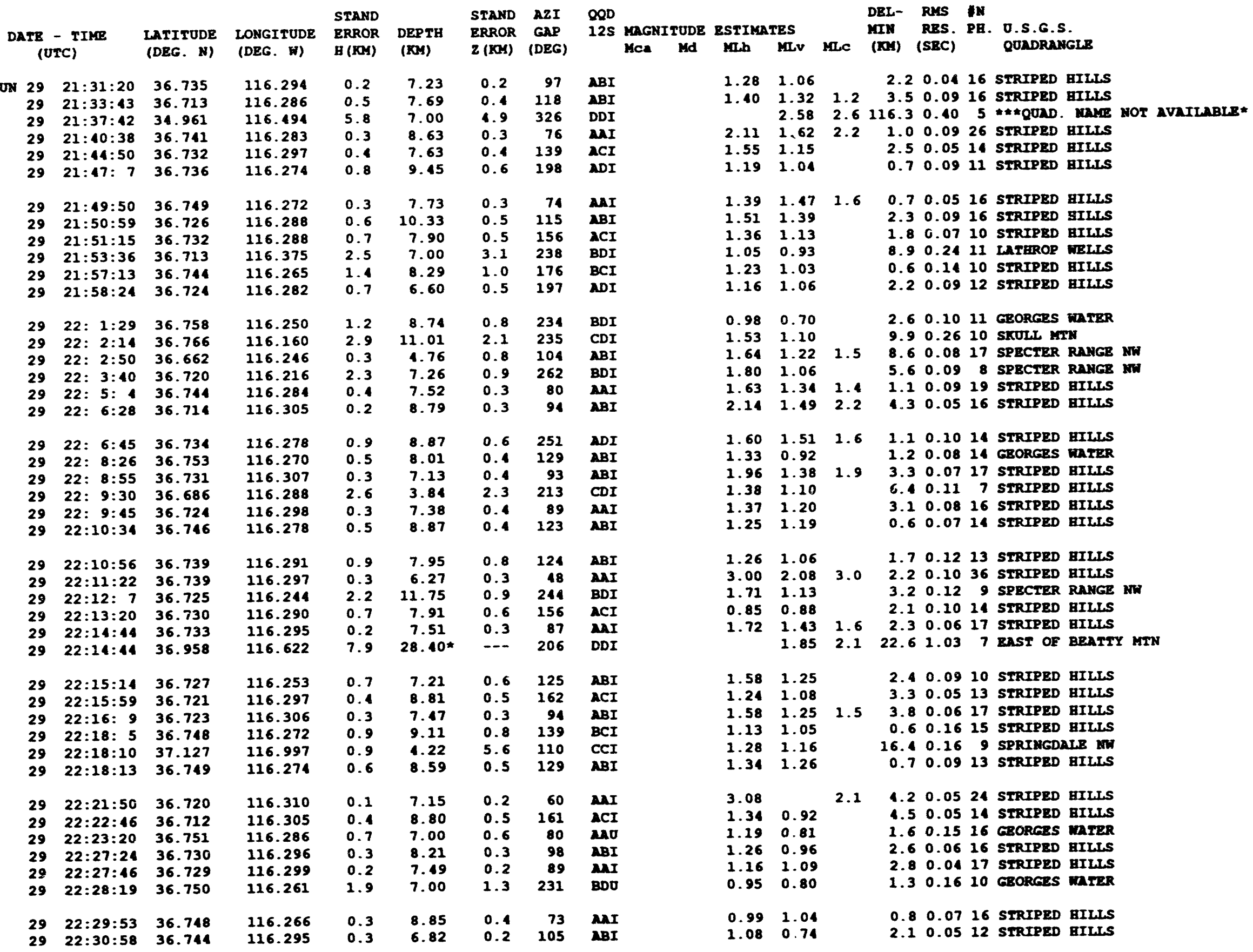


1992 LOCAL HYPOCENFER SUMARAY - SGB RARTHQUAKTS

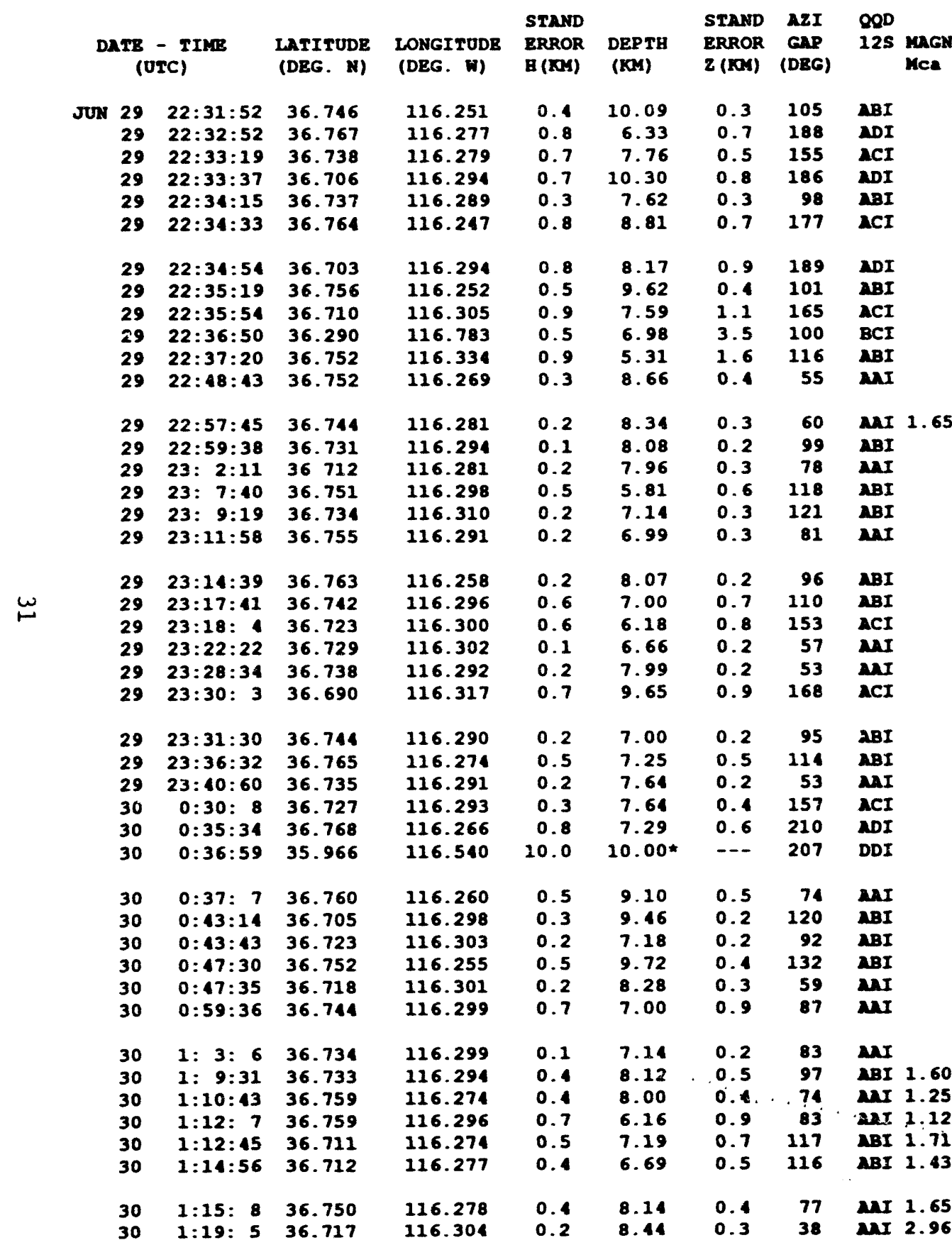

DEL- RYS IN

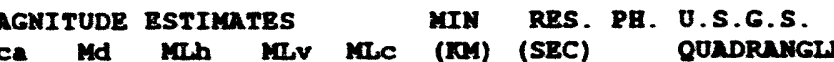

1.90 .0716 STRIPED HILLS 2.70 .0912 GroRGes warer $\begin{array}{llll}0.8 & 0.09 & 13 & \text { SIRIPED GILLS }\end{array}$ 4.50 .0713 SIRIPED GILLS

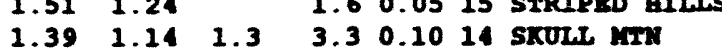
1.04 .

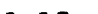

$1.51 \quad 1.24$

1.231 .03

$1.30 \quad 1.14$

4.80 .1014 STRIPED BILLS

$0.96 \quad 0.68$ 2.40 .0916 GRORGRS MTTER

$\begin{array}{llllllll}2.60 & 2.37 & 2.6 & 15.3 & 0.18 & 18 & \text { DEVILS COLF COURSE }\end{array}$

$\begin{array}{llllll}2.65 & 2.2 & 5.6 & 0.13 & 8 & \text { GEORES MATER }\end{array}$

$\begin{array}{llllll}1.86 & 1.72 & 1.6 & 1.1 & 0.11 & 27 \\ \text { GEORGBS WATER }\end{array}$

\begin{tabular}{lllllll}
2.06 & 1.47 & 1.6 & 0.8 & 0.08 & 25 & STRIPED \\
\hline
\end{tabular}

$\begin{array}{lllll}1.36 & 1.09 & 2.3 & 0.03 & 15 \\ \text { STRIPED HILLS }\end{array}$

$\begin{array}{lllllll}1.83 & 1.38 & 1.5 & 3.5 & 0.05 & 19 & \text { STRIPED EILLS }\end{array}$ $\begin{array}{llll}1.13 & 2.5 & 0.049 \text { GEORGES MDTER }\end{array}$ $\begin{array}{lllllll}1.63 & 1.33 & & 3.5 & 0.03 & 12 & \text { STRIPED EILLS } \\ 1.59 & 1.30 & 1.5 & 2.2 & 0.07 & 20 & \text { GRORCES }\end{array}$

$\begin{array}{lllllll}1.96 & 1.73 & 1.9 & 2.6 & 0.06 & 20 & \text { GBORGES MATER }\end{array}$ $\begin{array}{lllll}0.79 & 1.1 & 2.1 & 0.08 & 9\end{array}$ 1.260 .4501 .13 .30 .1011 SIRIPED BILIS

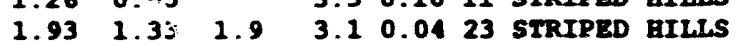
$\begin{array}{lllllll}1.93 & 1.32 & 1.9 & 3.1 & 0.04 & 23 & \text { SIRIPED } \\ 2.08 & 1.30 & 1.6 & 1.8 & 0.04 & 20 & \text { STRIPED BILLS }\end{array}$ $\begin{array}{lllllll}2.08 & 1.30 & 1.6 & 1.8 & 0.04 & 20 & \text { STRIPED BILS } \\ 1.39 & 1.09 & 1.5 & 5.4 & 0.07 & 11 & \text { STRIPED BILSS }\end{array}$

$1.51 \quad 1.35$

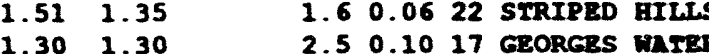
$\begin{array}{lllllll}1.78 & 1.40 & 1.6 & 2.5 & 0.10 & 17 & \text { GEORGES WATER } \\ 1.9 & 0.05 & 25 & \text { STRIPED }\end{array}$

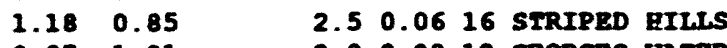

$0.971 .0^{\circ} \quad 2.80 .0912$ GEORGes HATER $\begin{array}{lllll}2.00 & 1.8 & 27.1 & 3.49 & 6 \\ & \text { CONFIDENCE BILIS }\end{array}$

$\begin{array}{lllllll}2.10 & 1.47 & 1.6 & 2.2 & 0.13 & 24 & \text { GEORGES UATER }\end{array}$

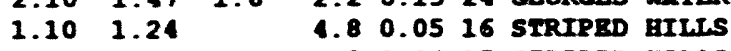
$\begin{array}{llllll}1.82 & 1.33 & 1.9 & 3.5 & 0.0417 & \text { STRIPED HILLS }\end{array}$ $\begin{array}{llll}1.35 & 1.09 & 1.90 .0816 \text { GEORGES MTERR }\end{array}$ $2.29 \quad 2.2 \quad 3.70 .0720$ SIRIPED BILLS $\begin{array}{lllllll}2.60 & 1.80 & 2.1 & 2.4 & 0.14 & 13 & \text { SERIPED HILLS }\end{array}$

$\begin{array}{llllllll}3.07 & 2.15 & 3.2 & 2.6 & 0.06 & 32 & \text { STRIPED gILLS }\end{array}$ $\begin{array}{lllllll}1.82 & 1.17 & 1.2 & 2.2 & 0.10 & 18 & \text { STRIPED BILLS }\end{array}$ $\begin{array}{lllllll}1.41 & 1.28 & 1.5 & 1.8 & 0.11 & 19 & \text { GroRGES MAIER }\end{array}$ $\begin{array}{llllllll}1.41 & 1.28 & 1.5 & 1.8 & 0.11 & 19 & \text { GEORGES MATER } \\ 0.63 & & 2.8 & 0.13 & 13 & \text { GEOREBS MATER }\end{array}$

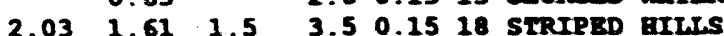
$2.01 \quad 1.35 \quad 1.5 \quad 3.40 .1015$ SIRIPED BILLS $\begin{array}{lllllll}2.07 & 1.97 & 1.9 & 1.0 & 0.1016 \text { GEORGES mTER }\end{array}$ $\begin{array}{lllllll}2.07 & 1.97 & 1.9 & 1.0 & 0.10 & 16 & \text { GEORGES MATER } \\ 3.22 & 3.2 & 1.0 & 0.10 & 44 & \text { STRIPED BILIS (MI ORR) }\end{array}$ 
1992 LOCAL HYFOCENTER SURARRY - SGB EARTBQUAKES

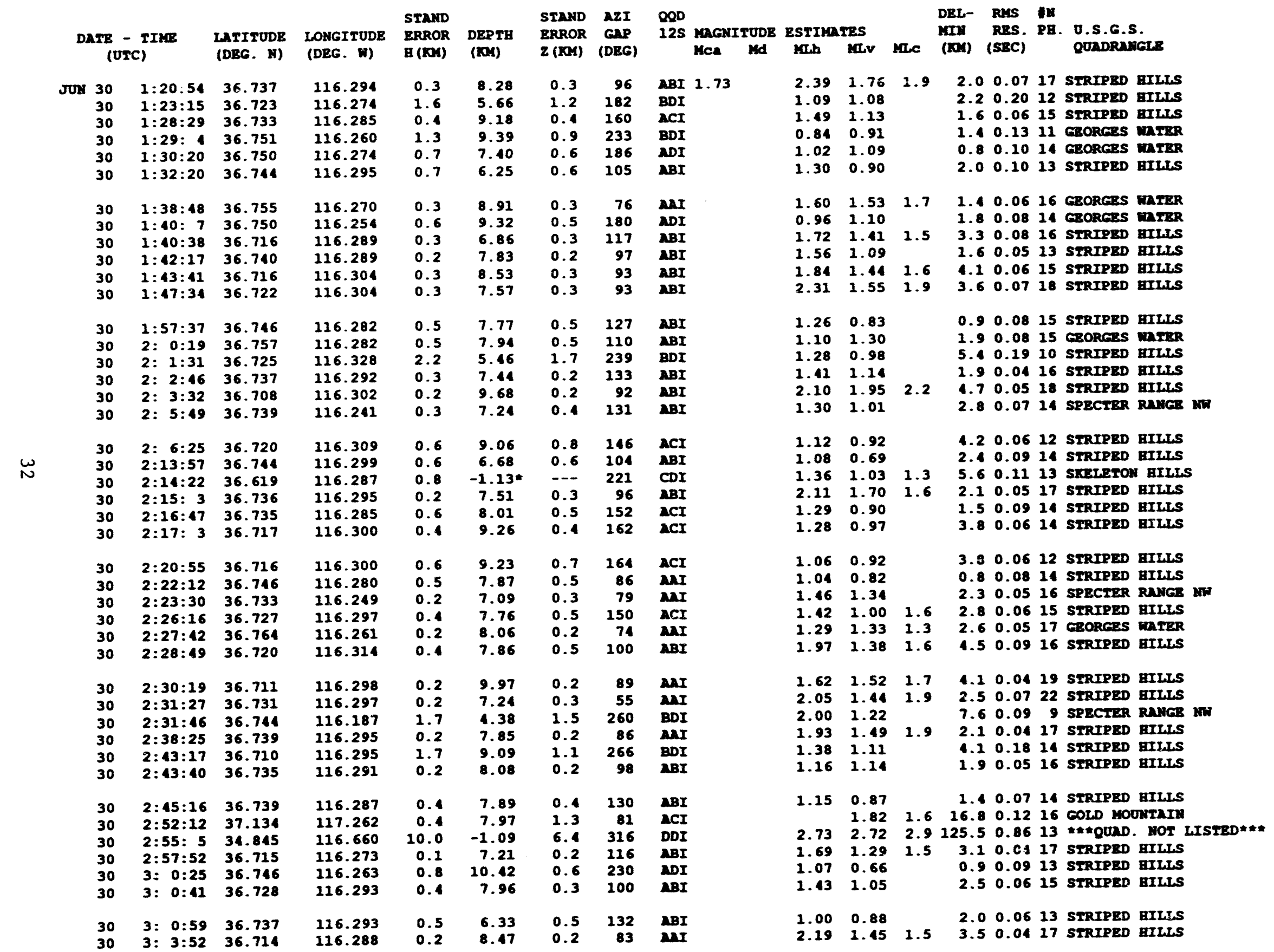


1992 LOCAL HXPOCENTER SURARX - SGB EARTBQUAKRS

ITTFOD LOVGTUDB SPROR (UIC)

(DEG. W) (DEG. W)

DEPTH

$\begin{array}{lll}30 & 3: 6: 58 & 36.737\end{array}$

30 3: $8: 36 \quad 37.146$

$30 \quad 3: 9: 33 \quad 36.741$

$3: 10: 52 \quad 36.756$

117.259

$3: 12: 24 \quad 36.721$

$3: 12: 55 \quad 36.735$

$3: 13: 23 \quad 34.946$

$3: 15: 47 \quad 36.711$

$3: 17: 43 \quad 37.138$

$3: 18: 18 \quad 36.354$

$3: 19: 32 \quad 36.693$

$3: 21: 22 \quad 36.695$

$3: 23: 40 \quad 36.699$

$3: 26: 21 \quad 37.226$

$3: 29: 17$

$3: 37: 19 \quad 37.133$

$\begin{array}{lll}3: 14: 50 & 36.713 & 116.306 \\ 3: 47: 12 & 36.724 & 116.301\end{array}$

$3: 48: 15 \quad 36.744$

$3: 48: 52 \quad 36.712$

3:49: $0 \quad 36.729$

$3: 49: 13 \quad 36.713$

$3.50: 2 \quad 36.730$

$3: 50: 41 \quad 36.753$

$3: 54: 56 \quad 36.740$

$3: 59: 50 \quad 36.717$

1: $0: 25 \quad 36.723$

4: $0: 38 \quad 36.731$

4: $2: 11 \quad 37.139$

4: $2: 22 \quad 36.758$

1: $2: 29 \quad 37.129$

1: 1:14 36.752

4:10: $1 \quad 36.744$

$111: 35 \quad 37.138$

$\begin{array}{ll}1: 15: 37 & 36.740 \\ 1: 36: 16 & 36.744\end{array}$

4.19: $3 \quad 36.748$

$4: 20: 8 \quad 36.758$

STAND AZI COD

S Mantrude estrmates

DEL- RMS AN

$z$ (DO) (DEG)

MTM RES. PB. U.S.G.S

117.621

116.295

117.265

116.293

116.210

$\begin{array}{rr}0.3 & 6.26 \\ 0.6 & 9.29 \\ 0.1 & 8.1 \\ 1.0 & 11.7 \\ 0.3 & 7.64 \\ 0.3 & 6.36\end{array}$

116.272

116.269

116.682

116.276

117.263

117.207

$0.2 \quad 7.16$

0.210 .76

$\begin{array}{ll}0.5 & 0.59 \\ 0.3 & 7.53\end{array}$

0.28 .02

116.294

116.285

116.281

116.251

116.293

116.289

116.296

116.298

116.296

116.289

116.271

116.299

116.296

116.302

116.297

117.278

116.276

117.236

116.271

116.279

117.263

116.251

116.255

$1.0 \quad 7.17$

$0.4 \quad 8.07$

$0.7 \quad 11.23$

9.09

$0.9 \quad 119$ ABI

0.2 81 NII

$2.4154 \quad \mathrm{BCI}$

$\begin{array}{lll}0.3 & 118 & \text { ABI } \\ 0.4 & 108 & \text { ABI }\end{array}$

0.262 MnI

$0.3 \quad 106 \quad$ ABI

4.6327 DDI

0.476 AAI

$\begin{array}{rrr}0.6 & 82 & \text { ACI } \\ 1.0 & 196 & \end{array}$

$\begin{array}{lll}1.1 & 198 & A D I\end{array}$

$\begin{array}{lll}0.5 & 138 & \text { ACI } \\ 0.4 & 141 & A C I\end{array}$

$1.2 \quad 135$ ABI

$\begin{array}{lll}0.2 & 78 & \mathrm{MII}\end{array}$

$\begin{array}{lllll}0.5 & 4.12 & 3.3 & 81 & \text { BCI }\end{array}$

$\begin{array}{lllll}0.3 & 8.85 & 0.4 & 86 & \mathrm{MI}\end{array}$

$\begin{array}{lllll}0.9 & 7.71 & 1.0 & 144 & \text { ACI } \\ 0.5 & 7.11 & 0.5 & 97 & \text { aBI }\end{array}$

$\begin{array}{lllll}0.2 & 7.79 & 0.3 & 95 & \text { ABI }\end{array}$

$\begin{array}{lllll}0.2 & 7.50 & 0.3 & 91 & \text { ABI }\end{array}$

$\begin{array}{lllll}0.3 & 7.86 & 0.2 & 82 & \text { NAI }\end{array}$

$\begin{array}{lllll}0.5 & 9.26 & 0.5 & 176 & \text { ACI }\end{array}$

$0.2 \quad 89$ ANI

$1.0117 \quad$ BBI

0.30 .510 .2 ACI

0.27 .48

1.0

$0.3 \quad 7.78$

$0.8 \quad 13.25$

$0.7 \quad 12.98$

$2.0 \quad 7.00$

0.27 .94

0.48 .56

0.17 .45

$\begin{array}{ll}0.7 & 11.07 \\ 1.4 & 11.91\end{array}$

116.266
116.269

0.2
$1.27 \quad 1.47$

1.47

15.40 .077 rous carrom

$\begin{array}{llllll}1.45 & 1.6 & 2.2 & 0.05 & 26 & \text { STRIPED BILLS } \\ 1.04 & & 17.2 & 0.11 & 7\end{array}$

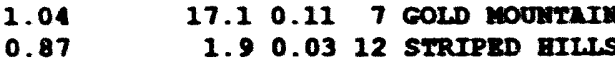

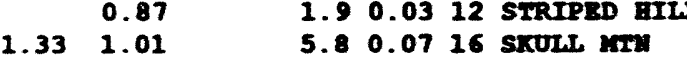

$\begin{array}{lllll}2.14 & 1.70 & 2.4 & 0.0726 & \text { STRIPED BILLS }\end{array}$

$2.58 \quad 2.9114 .2 \quad 0.5711 *$ *OMAD. WOT LISTRD*\#*

$\begin{array}{lllllll}1.77 & 1.52 & 1.5 & 3.6 & 0.08 & 18 & \text { STRIPED BILS }\end{array}$

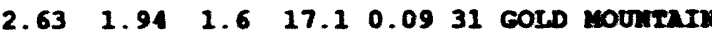
1.741 .410 .50 .10 B Eargenurt pass

$0.93 \quad 1.17$

$1.44 \quad 1.38$

1.231 .11

$1.34 \quad 1.16 \quad 1.5$

$\begin{array}{lllll}1.5 & 6.5 & 0.08 & 14 & \text { STRIPED GILIS } \\ 1.5 & 7 & \end{array}$

$\begin{array}{llllllll}1.45 & 1.24 & 1.4 & 2.5 & 0.05 & 18 & \text { GROB SPRIMG }\end{array}$

0.40 .0617 SIRIPED BIISS

$\begin{array}{llllllll}2.23 & 2.21 & 2.1 & 16.7 & 0.16 & 17 & \text { GOLD MOUnTAIy }\end{array}$

$\begin{array}{lllllll}1.97 & 1.39 & 1.6 & 3.1 & 0.08 & 17 & \text { STRIPED BILLS }\end{array}$

$\begin{array}{lllll}1.16 & 0.98 & 1.0 & 0.0911 & \text { STRIPED GILLS }\end{array}$

$1.110 .91 \quad 3.50 .1015$ STRIPED BILLS

$\begin{array}{lllllll}1.54 & 1.30 & 1.5 & 4.5 & 0.06 & 17 & \text { STRIPED BILLS }\end{array}$

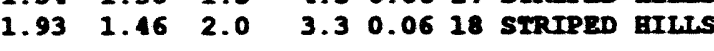

$\begin{array}{lllllll}2.20 & 1.78 & 2.1 & 1.5 & 0.06 & 17 & \text { SIRIPED GILLS }\end{array}$

$1.520 .94 \quad 4.00 .0511$ STRIPED BILLS

$\begin{array}{lllllll}1.99 & 1.84 & 1.6 & 2.8 & 0.05 & 17 & \text { SIRIPED BILLS }\end{array}$

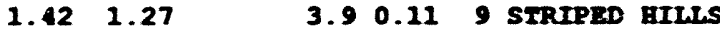

$\begin{array}{lllll}1.11 & 0.97 & 2.0 & 0.08 & 13 \\ \text { STRIPED HILLS }\end{array}$

$1.10 \quad 1.15 \quad 1.20 .0516$ GRORGRS mTER

$\begin{array}{lllllll}3.20 & 2.83 & 3.2 & 2.4 & 0.07 & 36 & \text { STRIPED BILLS }\end{array}$

$\begin{array}{lllll}1.37 & 1.10 & 3.5 & 0.07 & 16 \\ \text { SIRIPED BILS }\end{array}$

$1.501 .25 \quad 3.40 .1615$ SIRIPED gILLS

$\begin{array}{lllllll}1.62 & 1.23 & 1.5 & 2.6 & 0.06 & 16 & \text { STRTPED BILS }\end{array}$

$0.160 .94 \quad 16.60 .098$ GOLD MOUnirark

$0.971 .05 \quad 1.70 .1014$ GeOREs MATER

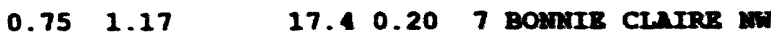

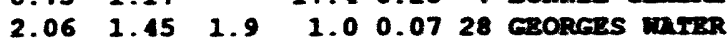

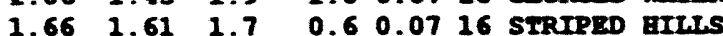

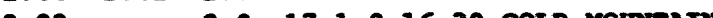

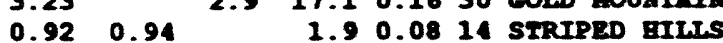

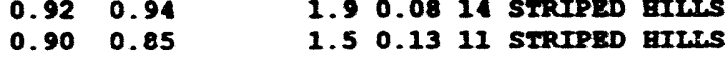

$1.62 \quad 1.50$

$1.22 \quad 1.33$

$\begin{array}{llll}0.8 & 0.05 & 17 & \text { STRIPED BILLS } \\ 1.8 & 0.06 & 16 \text { GBORGES maTER }\end{array}$ 
1992 LOCAL HYPOCENTER SURARY - SGB ENRTHQUAKRS

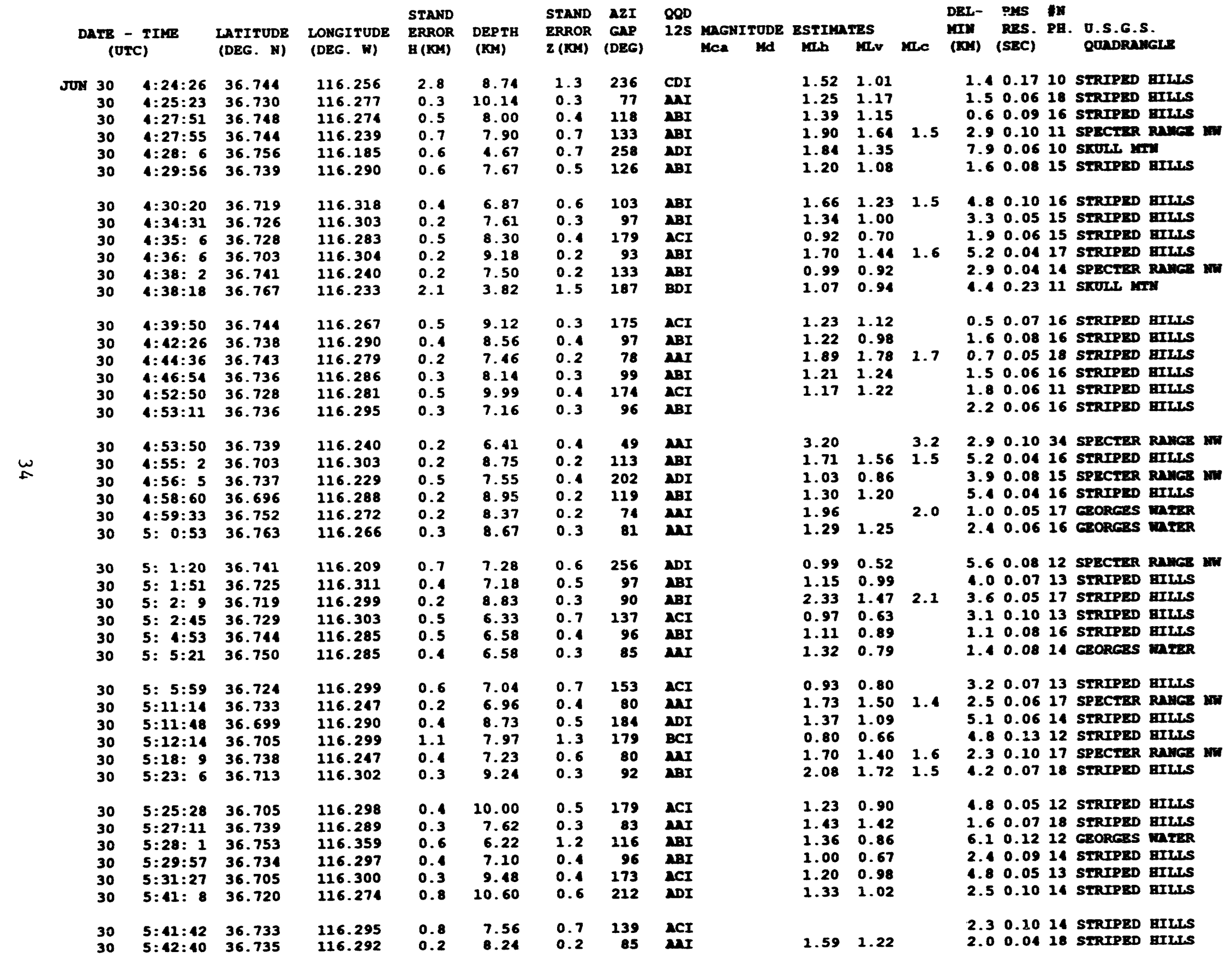


1992 LOCAI HYPOCENTER SUAARY - SGB EARTBQUAKES

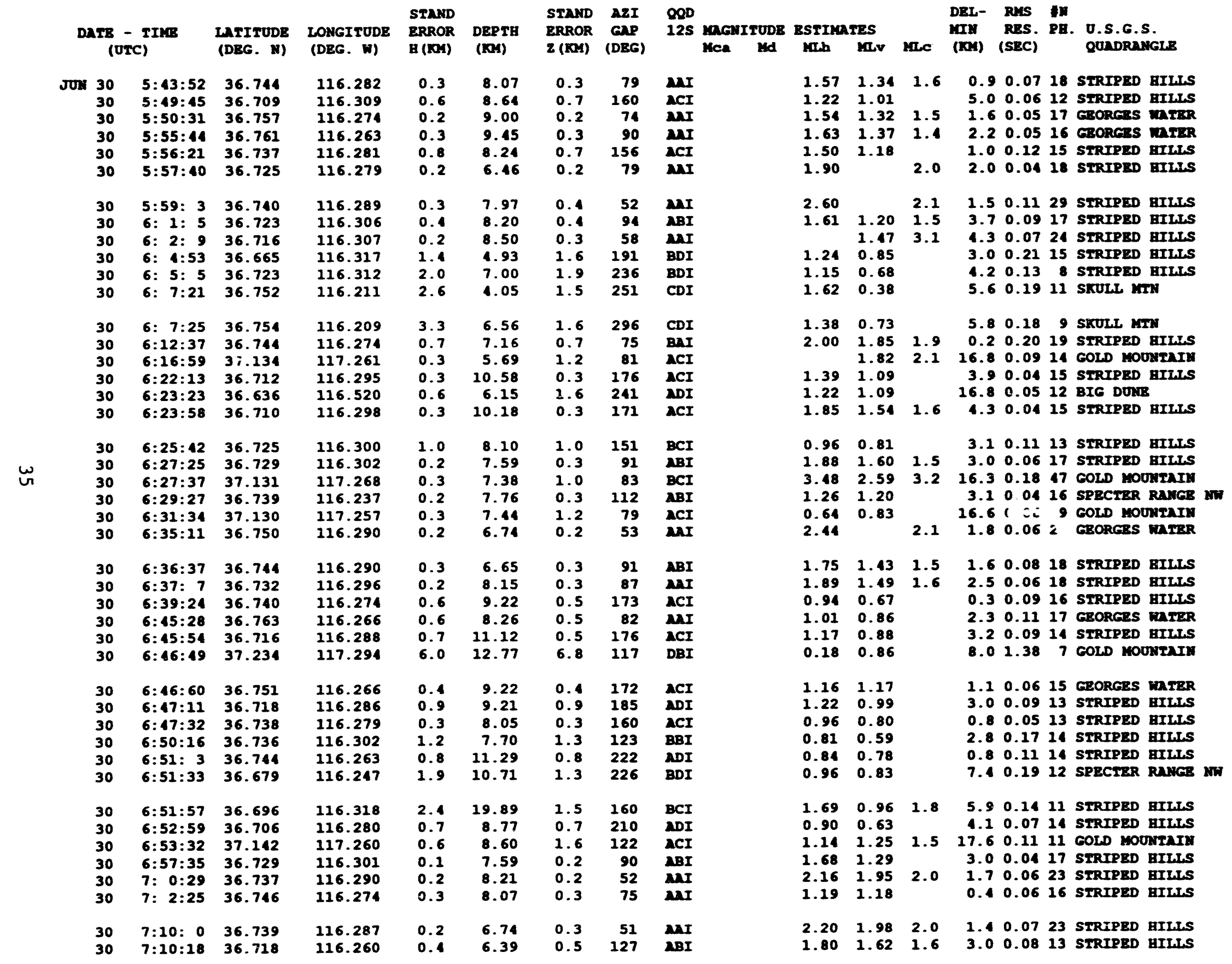


1992 LOCAL HYPOCENTER SURAQRY - SGB EARTHQUAKCES

STAND STAND AZI QQD DEL- RKS IN

DATE - TIME LATITUDE LONGITUDE ERROR DEPTH ERROR GAP 125 MGIITODE ESTIMATES MIN RES. PB. O.S.G.S. (OTC) (DEG. N) (DEG .

BRR DEPTH EFROR CAP Mea Md Mab MLV Ka.C (NM) (SEC)

\begin{tabular}{|c|c|c|c|c|c|c|c|c|}
\hline JOA 30 & $7: 11: 26$ & 36.754 & 116.254 & 0.2 & 9.81 & 0.2 & 58 & $\operatorname{Mar}$ \\
\hline 30 & $7: 12: 15$ & 36.747 & 116.271 & 0.3 & 9.11 & 0.3 & $\begin{array}{r}80 \\
176\end{array}$ & $\begin{array}{l}\operatorname{AAI} \\
\mathrm{ACI}\end{array}$ \\
\hline 30 & $7: 15: 30$ & 36.762 & 116.281 & 0.9 & 7.00 & 0.7 & & ACI \\
\hline 30 & $7: 16: 14$ & 36.739 & 116.287 & 0.3 & 6.68 & 0.3 & $\begin{array}{l}51 \\
83\end{array}$ & $\begin{array}{l}\text { AAI } \\
\text { BBI }\end{array}$ \\
\hline $\begin{array}{l}30 \\
30\end{array}$ & $\begin{array}{l}7: 23: 10 \\
7: 23: 20\end{array}$ & 37.140 & 117.268 & 0.9 & 10.16 & 2.3 & $\begin{array}{r}83 \\
164\end{array}$ & $\begin{array}{l}\mathrm{BBI} \\
\mathrm{ACI}\end{array}$ \\
\hline 30 & $7: 23: 20$ & 36.735 & 116.281 & 0.6 & 6.59 & 0.5 & & $\operatorname{ACI}$ \\
\hline 30 & $7: 25: 9$ & 36.719 & 116.317 & 1.5 & 4.02 & 3.6 & 137 & BCI \\
\hline 30 & $7: 26: 38$ & 36.744 & 116.288 & 1.1 & 12.49 & 1.5 & 105 & BBI \\
\hline 30 & $7: 27: 10$ & 36.725 & 116.303 & 0.3 & 7.33 & 0.3 & 92 & ABI \\
\hline 30 & $7: 32: 54$ & 36.744 & 116.278 & 0.5 & 8.51 & 0.4 & 123 & $A B I$ \\
\hline 30 & $7: 33: 15$ & 36.754 & 116.269 & 0.4 & 7.93 & 0.4 & 80 & MAI \\
\hline 30 & $7: 36: 3$ & 36.715 & 116.300 & 0.3 & 8.97 & 0.4 & 90 & ABI \\
\hline 30 & $7: 36: 14$ & 37.136 & 117.259 & 0.7 & 8.77 & 1.8 & 80 & BBI \\
\hline 30 & $7: 38: 37$ & 36.727 & 116.274 & 0.9 & 9.38 & 0.6 & 208 & ADI \\
\hline 30 & $7: 45: 47$ & 36.737 & 116.294 & 0.2 & 7.87 & 0.2 & 86 & AAI \\
\hline 30 & $7: 46: 36$ & 36.726 & 116.295 & 0.3 & 8.39 & 0.3 & 155 & ACI \\
\hline 30 & $7: 49: 1$ & 36.726 & 116.295 & 0.9 & 7.04 & 0.8 & 244 & ADI \\
\hline 30 & $7: 49: 31$ & 36.724 & 116.267 & 0.9 & 8.59 & 0.6 & 258 & DDI \\
\hline 30 & $7: 50: 56$ & 36.721 & 116.288 & 0.3 & 8.76 & 0.3 & 177 & $\mathrm{ACI}$ \\
\hline 30 & $7: 51: 19$ & 37.135 & 117.269 & 0.5 & 10.11 & 1.4 & 159 & NDI \\
\hline 30 & $7: 51: 32$ & 36.694 & 116.319 & 0.8 & 9.80 & 0.9 & 161 & ACI \\
\hline 30 & $7: 51: 55$ & 36.704 & 116.274 & 0.8 & 9.13 & 0.7 & 220 & ADI \\
\hline 30 & $7: 56: 56$ & 36.749 & 116.277 & 0.5 & 8.01 & 0.4 & 76 & $\boldsymbol{M A I}$ \\
\hline 30 & $8: 3: 10$ & 36.688 & 116.303 & 0.6 & 9.91 & 0.4 & 270 & ADI \\
\hline 30 & $8: 5: 6$ & 37.136 & 117.265 & 0.6 & 8.57 & 1.5 & 82 & ABI \\
\hline 30 & $8: 6: 47$ & 36.692 & 116.280 & 0.6 & 8.63 & 0.6 & 218 & AD \\
\hline 30 & $8: 7: 15$ & 36.727 & 116.291 & 0.5 & 7.75 & 0.4 & 160 & ACI \\
\hline 30 & 8: 8: 4 & 36.744 & 116.270 & 0.5 & 9.33 & 0.4 & 173 & ACI \\
\hline 30 & $8: 9: 26$ & 36.745 & 116.271 & 0.1 & 10.65 & 0.2 & 74 & $\boldsymbol{M I}$ \\
\hline 30 & $8: 9: 32$ & 36.749 & 116.265 & 0.4 & 10.57 & 0.2 & 230 & ADI \\
\hline 30 & $8: 12: 23$ & 36.748 & 116.277 & 0.4 & .43 & 0.4 & 76 & $\boldsymbol{N A I}$ \\
\hline 30 & $8: 18: 7$ & 36.758 & 116.260 & 0.4 & 8.38 & 0.3 & 132 & $\mathbf{A B}$ \\
\hline 30 & $8: 18: 34$ & 36.723 & 116.295 & 0.3 & 8.25 & 0.3 & 162 & ACI \\
\hline 30 & $8: 19: 34$ & 36.741 & 116.289 & 0.2 & 6.37 & 0.2 & 83 & MAI \\
\hline 30 & $8: 19: 49$ & 36.744 & 116.274 & 0.3 & 7.55 & 0.2 & 104 & ABI \\
\hline 30 & $8: 24: 24$ & 36.708 & 116.268 & 0.2 & 7.57 & 0.2 & 72 & $\boldsymbol{M M I}$ \\
\hline 30 & $8: 25: 49$ & 36.744 & 116.281 & 0.2 & 8.14 & 0.2 & 78 & $\operatorname{MaI}$ \\
\hline 30 & $8: 26: 15$ & 37.139 & 117.261 & 0.6 & 8.59 & 1.6 & 81 & BCI \\
\hline 30 & $8: 26: 45$ & 36.744 & 116.241 & 1.4 & 7.00 & 0.7 & 241 & BD \\
\hline 30 & $8: 27: 13$ & 36.735 & 116.277 & 0.4 & 8.58 & 0.3 & 184 & $\boldsymbol{N D I}$ \\
\hline 30 & $8: 30: 48$ & 36.732 & 116.296 & 0.2 & 7.92 & 0.2 & 97 & MBI \\
\hline 30 & $8: 31: 55$ & 36.728 & 116.299 & 0.3 & 7.87 & 0.3 & 145 & ACI \\
\hline $\begin{array}{l}30 \\
30\end{array}$ & $\begin{array}{l}8: 34: 60 \\
8: 36: 32\end{array}$ & $\begin{array}{l}36.722 \\
36.744\end{array}$ & $\begin{array}{l}116.292 \\
116.289\end{array}$ & $\begin{array}{l}0.2 \\
0.6\end{array}$ & $\begin{array}{l}9.37 \\
7.48\end{array}$ & $\begin{array}{l}0.2 \\
0.6\end{array}$ & $\begin{array}{l}169 \\
105\end{array}$ & $\begin{array}{l}\mathrm{ACI} \\
\mathrm{ABI}\end{array}$ \\
\hline
\end{tabular}

$2.06 \quad 1.9 \quad 2.1 \quad 0.0521$ GBORGBS MTRR

$1.141 .06 \quad 0.50 .0717$ STRIPED BILLS $\begin{array}{lllll}1.18 & 1.18 & 2.3 & 0.0811 \text { GRORGES MTRR }\end{array}$ $\begin{array}{lllllll}2.78 & & 2.2 & 1.4 & 0.08 & 23 & \text { STRIPED BILAS } \\ 0.36 & 0.90 & & 17.1 & 0.15 & \text { GOID }\end{array}$ $\begin{array}{rrrrrr}0.36 & 0.90 & 17.1 & 0.15 & 8 & \text { GOLD MOUNTAIR } \\ 0.88 & 0.61 & 1.1 & 0.09 & 17 & \text { STRIPED BILLS }\end{array}$ $0.920 .94 \quad 4.70 .098$ STRIPED BILIS $1.200 .93 \quad 1.40 .1812$ STRIPED BILLS

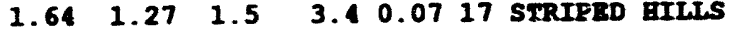
$\begin{array}{llllll}1.07 & 0.95 & 0.5 & 0.08 & 15 & \text { STRIPED BILLS }\end{array}$ $1.171 .19 \quad 1.30 .0917$ GEORGRS IDTER $\begin{array}{llllll}2.10 & 1.40 & 2.1 & 3.9 & 0.07 & 16\end{array}$

$\begin{array}{llllll}1.47 & 1.5 & 17.1 & 0.1511 \text { GOLD MOUNTATY }\end{array}$ $\begin{array}{lllllll}1.12 & 0.87 & 1.8 & 0.11 & 14 & \text { STRIPED GILIS }\end{array}$ $\begin{array}{lllllll}1.61 & 1.47 & 1.5 & 2.1 & 0.04 & 18 & \text { STRIPED BILLS }\end{array}$ $\begin{array}{lllllll}1.73 & 1.24 & 1.6 & 2.7 & 0.05 & 15 & \text { SIRIPED BILLS }\end{array}$ $\begin{array}{lllll}0.81 & 0.63 & 2.8 & 0.1013 & 13 \\ 1 & 0.96 & 2.1 & 0.11 & 15\end{array}$ $\begin{array}{llllll}1.44 & 0.96 & 2.1 & 0.11 & 15 & \text { STRIPED BILLS }\end{array}$

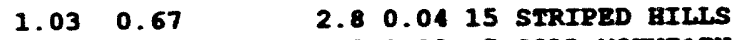
0.62

$1.07 \quad 0.80$

$1.01 \quad 0.76$

$1.27 \quad 1.05$

1.120 .71 16.50 .025 GOLD MOONTAIM 5.70 .1013 SIRIPED BILLS 4.30 .0813 STRIPED GIITS 0.00 .0917 STRTPED GILS 6.60 .0612 SIRTPED artis

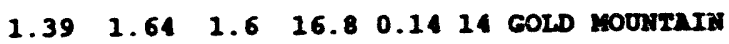
$\begin{array}{lllll}0.98 & 1.07 & 5.6 & 0.0614 & \text { STRIPED BILLS }\end{array}$

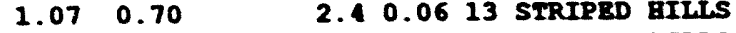
$\begin{array}{llllll}0.85 & 0.73 & 0.3 & 0.0716 & \text { SIRIPED BILIS }\end{array}$ $\begin{array}{lllllll}1.65 & 1.59 & 1.7 & 0.3 & 0.03 & 18 & \text { STRIPED } \\ 1.6 I L L S\end{array}$ $1.801 .99 \quad 1.8 \quad 1.00 .04 \quad 9$ STRIPED BILLS

$\begin{array}{llllllll}1.77 & 1.61 & 1.7 & 0.8 & 0.08 & 18 & \text { SIRIPED BILTS }\end{array}$ $\begin{array}{llllll}1.09 & 0.87 & 2.1 & 0.06 & 14 & \text { GEOREES WMTER }\end{array}$

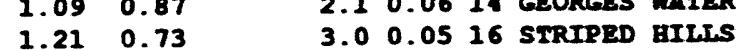
$\begin{array}{llllllll}1.21 & 0.73 & & 3.0 & 0.05 & 16 & \text { STRIPED EILES } \\ 1.87 & 1.52 & 1.6 & 1.5 & 0.04 & 16 & \text { STRIPED }\end{array}$ $\begin{array}{lllllll}1.78 & 1.50 & 1.4 & 0.2 & 0.06 & 16 & \text { STRIPED aILLS }\end{array}$ $2.0720 .2 \quad 3.90 .0417$ STRIPED BILLS

$\begin{array}{lllllll}1.59 & 1.51 & 1.5 & 0.8 & 0.05 & 17 & \text { STRIPED BILLS }\end{array}$ $\begin{array}{lllllll}1.18 & 1.52 & 1.4 & 17.3 & 0.1816 & \text { GOLD HOUTIRIM }\end{array}$ $\begin{array}{lllll}1.03 & 0.91 & 2.8 & 0.1713 \text { SPBCtER RANGE } & \text { wW }\end{array}$ $0.890 .55 \quad 1.00 .0413$ SIRIPED BILLS $1.240 .95 \quad 2.0 .0315$ STRTPED

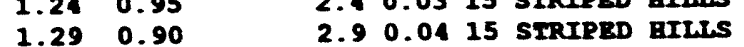

$\begin{array}{lllllll}1.44 & 0.95 & 1.6 & 2.9 & 0.03 & 14 & \text { STRIPED HILLS }\end{array}$ $\begin{array}{llllll}1.78 & 0.65 & 1.5 & 0.0915 & \text { STRIPED BILLS }\end{array}$ 
1992 LOCAI HYPOCENTER SUMAGARY - SGB EARTHQUAKCES

\begin{tabular}{|c|c|c|c|c|c|c|c|c|c|c|c|c|c|c|c|c|c|}
\hline \multicolumn{2}{|c|}{$\begin{array}{l}\text { DATE - TIME } \\
\text { (UTC) }\end{array}$} & \multirow{2}{*}{$\begin{array}{c}\text { LATITUDB } \\
\text { (DEG. N) } \\
36.720\end{array}$} & \multirow{2}{*}{$\begin{array}{l}\text { LONGITUDE } \\
\text { (DEG. W) } \\
116.301\end{array}$} & \multirow{2}{*}{$\begin{array}{c}\text { STAND } \\
\text { ERROR } \\
\text { B (KAY) } \\
0.5\end{array}$} & \multirow{2}{*}{$\begin{array}{l}\text { DEPTH } \\
\text { (KO) } \\
8.32\end{array}$} & \multirow{2}{*}{$\begin{array}{c}\text { STAND } \\
\text { ERROR } \\
\text { Z (KY) } \\
0.6\end{array}$} & \multirow{2}{*}{$\begin{array}{c}\text { AZI } \\
\text { GAP } \\
\text { (DEG) } \\
157\end{array}$} & \multirow{2}{*}{$\begin{array}{l}\text { QOD } \\
125 \\
\text { ACI }\end{array}$} & $\begin{array}{l}\text { MAGNITUDE } \\
\text { Mca Md }\end{array}$ & \multicolumn{2}{|c|}{ ESTIMATES } & MLC & $\begin{array}{l}\text { DEL- } \\
\text { MIN } \\
\text { (NOY) }\end{array}$ & $\begin{array}{l}\text { RYS } \\
\text { RES. } \\
\text { (SEC) }\end{array}$ & $\begin{array}{l}\mathbf{N} \\
\mathbf{P B} .\end{array}$ & \multicolumn{2}{|c|}{$\begin{array}{l}\text { J.S.G.S. } \\
\text { QUADRANGLE }\end{array}$} \\
\hline 30 & $8: 37: 60$ & & & & & & & & & 1.08 & 0.88 & & 3.6 & 0.06 & 14 & STRIPED E & HrLhs \\
\hline 30 & $8: 44: 50$ & $36 . .53$ & 116.269 & 0.2 & 8.09 & 0.3 & 55 & AAI & & 2.20 & & 1.9 & 1.2 & 0.07 & 24 & GEORGES & WATER \\
\hline 30 & $8: 16: 33$ & 36.742 & 116.266 & 0.2 & 10.20 & 0.3 & 74 & AAI & & 0.97 & 0.88 & & 0.6 & 0.05 & 17 & STRIPED & BILLS \\
\hline 30 & $8: 46: 33$ & 37.134 & 117.255 & 0.8 & 6.94 & 2.6 & 79 & BCI & & & 1.02 & 1.4 & 17.0 & 0.15 & 10 & GOLD MOU & NTAIN \\
\hline 30 & $8: 47: 26$ & 36.714 & 116.288 & 0.6 & 8.70 & 0.6 & 187 & ADI & & 1.35 & 0.72 & & 3.5 & 0.07 & 14 & STRIPED E & BILLS \\
\hline 30 & $8: 48: 45$ & 36.725 & 116.301 & 0.4 & 8.07 & 0.4 & 148 & ACI & & 1.13 & 0.89 & & 3.2 & 0.06 & 15 & STRIPED & BILLS \\
\hline 30 & $8: 49: 58$ & 36.730 & 116.307 & 0.3 & 6.80 & 0.3 & 94 & ABI & & 1.79 & 1.41 & 1.9 & 3.4 & 0.06 & 17 & STRIPED & BILLS \\
\hline 30 & $8: 51: 19$ & 36.740 & 116.285 & 0.6 & 7.68 & 0.6 & 128 & ABI & & 0.64 & 0.56 & & 1.1 & 0.10 & 16 & STRIPED E & HILLS \\
\hline 30 & $8: 51: 37$ & 36.757 & 116.274 & 0.5 & 7.95 & 0.5 & 134 & ABI & & 1.05 & 0.98 & & 1.6 & 0.08 & 14 & GEORGES & WATER \\
\hline 30 & $8: 51: 60$ & 36.735 & 116.314 & 0.9 & 5.44 & 1.1 & 115 & ABI & & 1.00 & 0.66 & & 3.8 & 0.14 & 13 & STRIPED E & BILLS \\
\hline 30 & $8: 55: 1$ & 36.718 & 116.297 & 0.5 & 9.00 & 0.5 & 165 & ACI & & 1.54 & 0.80 & 1.6 & 3.5 & 0.06 & 14 & STRIPED & aILLS \\
\hline 30 & $8: 58: 8$ & 37.125 & 117.255 & 0.6 & 6.21 & 2.7 & 81 & $\mathrm{BCI}$ & & & 0.98 & & 16.2 & 0.12 & 9 & GOLD MOU & NTAIN \\
\hline 30 & $8: 59: 28$ & 36.731 & 116.292 & 0.7 & 7.61 & 0.5 & 97 & ABI & & 1.45 & 1.35 & & 2.2 & 0.10 & 14 & STRIPED & HILLS \\
\hline 30 & $9: 1: 15$ & 36.727 & 116.296 & 0.3 & 7.63 & 0.3 & 153 & ACI & & 1.19 & 0.79 & & 2.8 & 0.05 & 15 & STRIPED & BILLLS \\
\hline 30 & $9: 1: 28$ & 36.705 & 116.306 & 0.4 & 9.13 & 0.4 & 168 & ACI & & 1.46 & 1.11 & & 5.1 & 0.06 & 13 & STRIPED & BILLS \\
\hline 30 & $9: 5: 27$ & 36.751 & 116.269 & 0.3 & 7.88 & 0.3 & 85 & AAI & & 1.63 & 1.50 & 1.4 & 1.0 & 0.06 & 16 & GEORGES & WATER \\
\hline 30 & $9: 8: 7$ & 36.726 & 116.295 & 0.4 & 8.06 & 0.4 & 156 & ACI & & 1.10 & 0.77 & & 2.7 & 0.04 & 13 & STRIPED & BILLS \\
\hline 30 & $9: 8: 54$ & 36.730 & 116.296 & 0.3 & 8.07 & 0.3 & 146 & ACI & & 0.79 & 0.52 & & 2.5 & 0.04 & 15 & STRIPED & BILLS \\
\hline 30 & $9: 8: 60$ & 37.135 & 117.257 & 0.5 & 5.00 & 2.3 & 80 & BCI & & & 1.32 & 1.4 & $\begin{array}{r}17.1 \\
2.8\end{array}$ & $\begin{array}{l}0.13 \\
0.06\end{array}$ & $\begin{array}{l}13 \\
18\end{array}$ & GOLD MOU & JTaIN \\
\hline 30 & $9: 13: 6$ & 36.742 & 116.240 & 0.2 & 7.23 & 0.4 & 83 & ARI & & 1.58 & 1.54 & 1.8 & 2.8 & 0.06 & 18 & SPECTER & RANGE \\
\hline 30 & $9: 13: 54$ & 37.130 & 117.253 & 0.5 & 5.32 & 2.2 & 78 & BCI & & & 1.37 & 1.4 & 16.7 & 0.12 & 13 & GOLD MOU & NAmIN \\
\hline 30 & $9: 14: 40$ & 36.718 & 116.301 & 0.1 & 8.40 & 0.2 & 55 & AAI & & 2.78 & & 2.1 & 3.7 & 0.04 & 26 & STRIPED & BrLLS \\
\hline 30 & $9: 16: 6$ & 36.739 & 116.267 & 0.8 & 8.54 & 0.8 & 177 & ACI & & 0.90 & 0.88 & & 0.6 & 0.13 & 16 & STRIPED & BILLS \\
\hline 30 & $9: 17: 18$ & 36.744 & 116.232 & 0.7 & 7.03 & 0.6 & 196 & ADI & & 0.91 & 0.60 & & 3.6 & 0.09 & 12 & SPECTER & RANGE Wh \\
\hline 30 & $9: 18: 10$ & 36.741 & 116.285 & 0.6 & 7.88 & 0.5 & 124 & ABI & & 1.16 & 1.10 & & 1.2 & 0.09 & 14 & STRIPED & BILLS \\
\hline 30 & $9: 18: 10$ & 36.741 & 116.286 & 0.6 & 7.94 & 0.6 & 121 & ABI & & 1.16 & 1.10 & & 1.3 & 0.09 & 14 & STRIPED & BILLS \\
\hline 30 & $9: 21: 52$ & 36.732 & 116.257 & 0.4 & 7.44 & 0.5 & 51 & BAI & & 2.44 & 2.03 & & 1.8 & 0.20 & 43 & STRIPED & BILLS \\
\hline 30 & $9: 23: 35$ & 36.708 & 116.328 & 0.7 & 8.79 & 1.0 & 112 & BBI & 1.43 & 1.45 & 1.15 & 1.5 & 6.3 & 0.16 & 18 & STRIPED & gILLS \\
\hline 30 & $9: 25: 18$ & 36.723 & 116.301 & 1.0 & 8.93 & 1.2 & 99 & BBI & & 1.18 & 1.02 & & 3.4 & 0.24 & 20 & STRIPED & BILLS \\
\hline 30 & $9: 27: 56$ & 36.731 & 116.251 & 0.3 & 7.90 & 0.4 & 113 & ABI & & 0.91 & 0.80 & & 2.2 & 0.06 & 15 & STRIPED & gIns \\
\hline 30 & $9: 29: 18$ & 36.721 & 116.298 & 0.7 & 8.54 & 0.7 & 159 & ACI & & 1.31 & 0.91 & & 3.3 & 0.08 & 12 & STRIPED & HILIS \\
\hline 30 & $9: 30: 2$ & 36.740 & 116.288 & 0.3 & 7.86 & 0.3 & 125 & ABI & & 0.98 & 0.86 & & 1.5 & 0.05 & 15 & STRIPED & HILLS \\
\hline 30 & $9: 30: 12$ & 36.757 & 116.247 & 0.3 & 9.25 & 0.3 & 59 & AAI & & 2.26 & 1.91 & 2.1 & 2.7 & 0.07 & 21 & SKOLL MTI & $\mathbf{F N}$ \\
\hline 30 & $9: 32: 52$ & 36.732 & 116.297 & 0.2 & 7.46 & 0.2 & 140 & ACI & & 1.20 & 0.87 & & 2.5 & 50.04 & 14 & STRIPED & HILISS \\
\hline 30 & $9: 36: 5$ & 36.753 & 116.261 & 0.4 & 8.73 & 0.4 & 100 & ABI & & 1.24 & 1.22 & & 1.6 & 60.08 & 16 & GEORGES & HATER \\
\hline 30 & $9: 36: 55$ & 36.712 & 116.290 & 0.4 & 8.42 & 0.8 & 176 & ACI & & 0.85 & 0.55 & & 3.7 & 70.08 & 14 & STRIPED & BILLS \\
\hline 30 & $9: 37: 9$ & 36.763 & 116.272 & 0.3 & 5.36 & 0.3 & 138 & ACI & & 0.54 & 0.43 & & 2.3 & 0.05 & 11 & GEORGES & WATER \\
\hline 30 & $9: 37: 44$ & 36.708 & 116.306 & 0.6 & 8.99 & 0.7 & 166 & ACI & & 1.12 & 0.68 & & 4.9 & 0.07 & 13 & STRIPED & BILLS \\
\hline 30 & $9: 39: 52$ & 36.706 & 116.302 & 0.2 & 9.98 & 0.2 & 58 & AAI & & 2.15 & 1.78 & 2.2 & 4.8 & 0.04 & 22 & STRIPED & BILLS \\
\hline 30 & $9: 42: 41$ & 36.737 & 116.264 & 0.9 & 10.75 & 0.7 & 181 & ADI & & 1.09 & 0.70 & & 1.0 & 0.12 & 12 & STRIPED & BrLLS \\
\hline 30 & $9: 43: 25$ & 36.729 & 116.297 & 0.2 & 7.93 & 0.2 & 88 & $\operatorname{MAI}$ & & 2.07 & 1.83 & 1.6 & 2.7 & 0.05 & 17 & STRIPED & arths \\
\hline 30 & $9: 45: 48$ & 36.722 & 116.299 & 0.4 & 7.88 & 0.4 & 157 & ACI & & 1.64 & 1.09 & & 3.3 & 30.07 & 17 & STRIPED & Bruls \\
\hline $\begin{array}{l}30 \\
30\end{array}$ & $\begin{array}{l}9: 48: 36 \\
9: 48: 39\end{array}$ & $\begin{array}{l}37.132 \\
36.744\end{array}$ & $\begin{array}{l}117.253 \\
116.270\end{array}$ & $\begin{array}{l}0.7 \\
0.4\end{array}$ & $\begin{array}{l}7.17 \\
8.61\end{array}$ & $\begin{array}{l}2.5 \\
0.4\end{array}$ & $\begin{array}{r}78 \\
173\end{array}$ & $\begin{array}{l}\text { BCI } \\
\text { ACI }\end{array}$ & & 0.95 & $\begin{array}{l}0.78 \\
0.88\end{array}$ & & $\begin{array}{r}17.0 \\
0.2\end{array}$ & $\begin{array}{l}0.17 \\
0.06\end{array}$ & $\begin{array}{l}11 \\
14\end{array}$ & $\begin{array}{l}\text { GOLD MOU } \\
\text { STRIPED }\end{array}$ & $\begin{array}{l}\text { ONTAIN } \\
\text { BILISS }\end{array}$ \\
\hline
\end{tabular}


1992 LOCAL HYPOCENTER SUMAARY - SGB EARTHQUAKES

STAND

DATE - TIKE LATITUDE LONGTTUDE ERRO (UTC)
(DEG. N) (DEG. W)

Jox 30

\section{$\begin{array}{ll}30 & 9: 50: 47 \quad 36.732\end{array}$}

$\begin{array}{ll}30 & 9: 51: 26 \quad 36.693\end{array}$

$9: 51: 45 \quad 36.747$

9:52:25 36.798

$9: 54: 57 \quad 36.767$

$\begin{array}{lll}30 & 9: 55: 52 \quad 36.737\end{array}$

$10: 0.1 \quad 36.689$

10: $1: 18 \quad 36.737$

10: $1: 18$ 36.737

$10: 5: 57 \quad 36.752$

$\begin{array}{lll}30 & 11: 28: 18 & 36.766 \\ 30 & 11: 29: 13 & 37.100\end{array}$

$30 \quad 11: 29: 26 \quad 36.727$

$30 \quad 11: 35: 34 \quad 36.728$

30 11:36: 9 36.750

$30 \quad 11: 37: 26 \quad 36.722$

$30 \quad 11: 38: 33 \quad 37.138$

30 11:39: 937.132

$30 \quad 11: 39: 51 \quad 36.718$

$\underset{\infty}{\omega}$

116.268

116.286

116.140

116.140

116.249

116.280

116.284

116.274

116.254

116.260

117.274

116.298

116.301

116.268

117.260

117.259

116.284

116.274

116.295
9:52:25 36.801

116.294

116.284

116.282

116.300

$30 \quad 11: 46: 44 \quad 36.719$

$30 \quad 11: 46: 58 \quad 36.762$

$30 \quad 11: 48: 48 \quad 36.758$

30 $11: 48: 48,36.758$

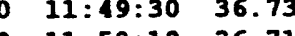

11:50:19 36.711

$\begin{array}{lll}30 & 11: 50: 25 & 36.680 \\ 30 & 11: 51: 7 & 36.754\end{array}$

$30 \quad 11: 54: 11 \quad 36.723$

$30 \quad 11: 55: 50 \quad 36.714$

$30 \quad 11: 56: 49 \quad 36.750$

$30 \quad 11: 57: 6 \quad 36.753$

$30 \quad 11: 57: 37 \quad 36.741$

30 11:59: 5 36.727

$30 \quad 11: 59: 39 \quad 36.719$

30 12: $5: 18 \quad 36.71$

30 12: $6: 9$ 37.140

30 12: $6: 24 \quad 36.707$

30 12: $8: 46 \quad 36.768$

$30 \quad 12: 8: 57 \quad 36.652$

$30 \quad 12: 10: 52 \quad 36.757$

$30 \quad 12: 11: 44 \quad 37.087$
116.264

$\$ 16.245$

116.291

116.326

116.328
116.248

116.303

116.312

116.259

116.289

116.285

116.293

116.290

117.261

116.305

116.250

116.272

117.182
STAND AZI QQD

ERROR GAP 125 MAGNITUDE ESTIMATES

\subsection{2}

$\begin{array}{lrr}0.2 & 98 \quad A B I\end{array}$

$\begin{array}{lll}1.5 & 204 & \text { BDI } \\ 0.6 & 120 & \text { BBI }\end{array}$

$\begin{array}{lllll}1.5 & 6.75 & 1.3 & 249 & \text { BDI } \\ 1.3 & 7.14 & 1.1 & 249 & \text { BDI }\end{array}$

$\begin{array}{lllll}0.4 & 7.70 & 0.4 & 97 & \text { ABI }\end{array}$

$0.3 \quad 9.07$

$1.1 \quad 8.29$

$0.7 \quad 8.12$

$\begin{array}{ll}0.2 & 9.59 \\ 0.5 & 8.88\end{array}$

$\begin{array}{ll}0.5 & 8.88 \\ 1.3 & 0.44\end{array}$

$\begin{array}{lll}1.1 & 196 & \text { BDI }\end{array}$

$0.5195 \quad$ NDI

0.277 NAI

$0.4 \quad 89$ MAI

$0.3 \quad 6.26$

$\begin{array}{ll}0.2 & 7.55 \\ 0.4 & 8.56\end{array}$

$0.5 \quad 8.31$

$0.6 \quad 8.92$

$0.4 \quad 46 \quad$ IAI

$0.2 \quad 90 \quad \mathrm{ABI}$

$\begin{array}{rrr}0.3 & 94 & \text { ABI } \\ 0.6 & 157 & \text { ACI }\end{array}$

$1.4121 \quad$ ABI

$\begin{array}{llllll}0.7 & 8.51 & 0.6 & 189 & \text { ADI }\end{array}$

$0.5 \quad 4.55$

0.47 .58

0.28 .17

$\begin{array}{ll}0.3 & 7.97 \\ 0.3 & 7.94\end{array}$

0.8127

0.4163

$\begin{array}{ll}0.2 & 80 \\ 0.2 & 93\end{array}$

$0.4 \quad 8.98$

$0.3 \quad 6.13$

$0.3 \quad 6.93$

$\begin{array}{rr}0.9 & 7.74 \\ 0.3 & 10.07\end{array}$

0.4

0.3

0.3

$\begin{array}{ll}0.5 & 110 \\ 1.2 & 159\end{array}$

$0.3 \quad 80$

0.27 .54

0.38 .51

$0.3 \quad 92$
$0.4 \quad 150$

$\begin{array}{ll}0.4 & 150 \\ 0.7 & 182\end{array}$

$\begin{array}{ll}0.7 & 182 \\ 0.3 & 176\end{array}$

0.48 .36

0.38 .46

8.49

$\begin{array}{lr}0.3 & 83 \\ 0.3 & 176\end{array}$

$0.3 \quad 171$

$0.3 \quad 8.22$

$\begin{array}{ll}1.3 & 9.22\end{array}$

$\begin{array}{ll}0.5 & 8.17 \\ 0.2 & 9.03\end{array}$

0.48 .30

$1.1 \quad 183$

$\begin{array}{ll}1.5 & 122 \\ 0.3 & 168\end{array}$

$\begin{array}{ll}0.3 & 168 \\ 0.6 & 173\end{array}$

0.9132

$0.3 \quad 8.76$

$\begin{array}{ll}0.2 & 73 \\ 2.0 & 55\end{array}$

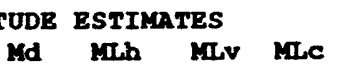

DEL- RYS

MIN RES. PB. U.S.G.S.

$\begin{array}{llllll}1.50 & 1.15 & 2.3 & 0.05 & 18 & \text { STRIPED GILLS }\end{array}$

$\begin{array}{llllll}0.98 & 0.70 & 5.5 & 0.24 & 14 & \text { STRIPED HILLS }\end{array}$

$\begin{array}{llllll}1.51 & 1.16 & 1.3 & 0.16 & 16 & \text { STRIPED BILLS }\end{array}$

$\begin{array}{lllll}1.59 & 1.20 & 6.8 & 0.1611 \text { SKULL MTI }\end{array}$

$1.601 .20 \quad 6.50 .1411$ SKULL MTN

$\begin{array}{lllllll}1.02 & 0.91 & 1.4 & 3.4 & 0.09 & 16 & \text { SKULL MTM }\end{array}$

$\begin{array}{llllll}1.28 & 1.25 & 1.0 & 0.0617 & \text { STRIPED HILLS }\end{array}$

$0.91 \quad 0.67 \quad 6.00 .1415$ STRIPED BILLS

$\begin{array}{lllllll}0.91 & 0.57 & & 0.6 & 0.09 & 14 & \text { STRIPED GILLS } \\ 1.49 & 1.39 & 1.3 & 1.9 & 0.05 & 17 & \text { GRORGES MTTER }\end{array}$

$\begin{array}{llllll}1.21 & 1.04 & 2.8 & 0.0916 \text { GBORGES MATER }\end{array}$ $1.62 \quad 1.3 \quad 13.0 \quad 0.2911$ scotrys CASTLE

$\begin{array}{lllllll}3.20 & 1.99 & 3.2 & 2.9 & 0.13 & 42 & \text { STRIPED BILLS }\end{array}$

$1.50 \quad 1.31 \quad 3 \quad 3.10 .0416$ STRIPED HILLS

$\begin{array}{lllllll}1.40 & 1.39 & 1.4 & 1.0 & 0.07 & 16 & \text { GEORGES WATR }\end{array}$

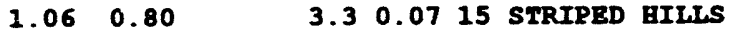
$\begin{array}{llllll}1.29 & 1.4 & 17.3 & 0.15 & 14 & \text { GOLD MOUNTAIM } \\ 1.17 & & 16.7 & 0.10 & 10 & \text { GOLD MOUNTAIN }\end{array}$

$\begin{array}{llllll}0.77 & 0.53 & 2.9 & 0.08 & 13 & \text { STRIPED GILLS }\end{array}$

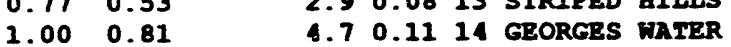

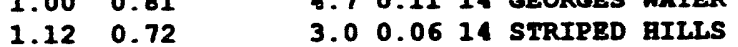

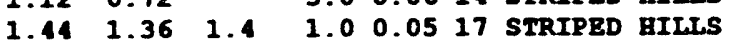

$1.361 .02 \quad 1.0 \quad 1.0516$ STRIPED BILLS

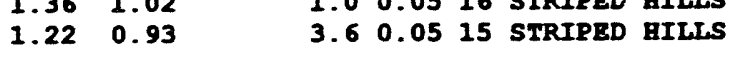

1.040 .93

$1.31 \quad 1.07$

$1.29 \quad 1.13$

$1.39 \quad 1.05$

1.120 .82

2.30 .0815 GEORGES WATER 3.00 .0717 SKOLL MTN 1.70 .0717 STRIPED HILLS 5.90 .0816 STRIPED BILLS $4.0 \quad 0.1010$ STRTPED BILLS 2.50 .0719 SKOLL MTN

$\begin{array}{lllllll}1.62 & 1.27 & 1.5 & 3.5 & 0.05 & 17 & \text { STRIPED HILLS }\end{array}$

$0.940 .51 \quad 1.5 \quad 4.80 .0616$ STRIPED HILLS

$0.720 .61 \quad 2.00 .0915$ GEORGES WATER

$0.820 .66 \quad 1.60 .0716$ GBORGES MATER

$\begin{array}{lllllll}1.92 & 1.59 & 1.6 & 1.5 & 0.07 & 16 & \text { STRIPED BILLS } \\ 1.21 & 0.55 & & 2.1 & 0.05 & 13 & \text { STRIPED BILLS }\end{array}$

$0.90 \quad 0.43$

$1.01 \quad 0.70$

1.01

$1.05 \quad 0.81$

0.590 .45

3.20 .0514 STRIPED HILLS

$3.6 \quad 0.1714$ STRIPED BILLS

17.40 .109 COLD

4.90 .0415 SIRIPBD

3.40 .0612 GEORGRS WATER

7.90 .1314 SIRIPED BILLS

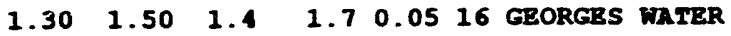
$1.58 \quad 1.4 \quad 17.5 \quad 0.1119$ BONNIB CLAIRE SW 
1992 LOCAL HYPOCENTER SUMAARY - SGB DARTHQUAKES

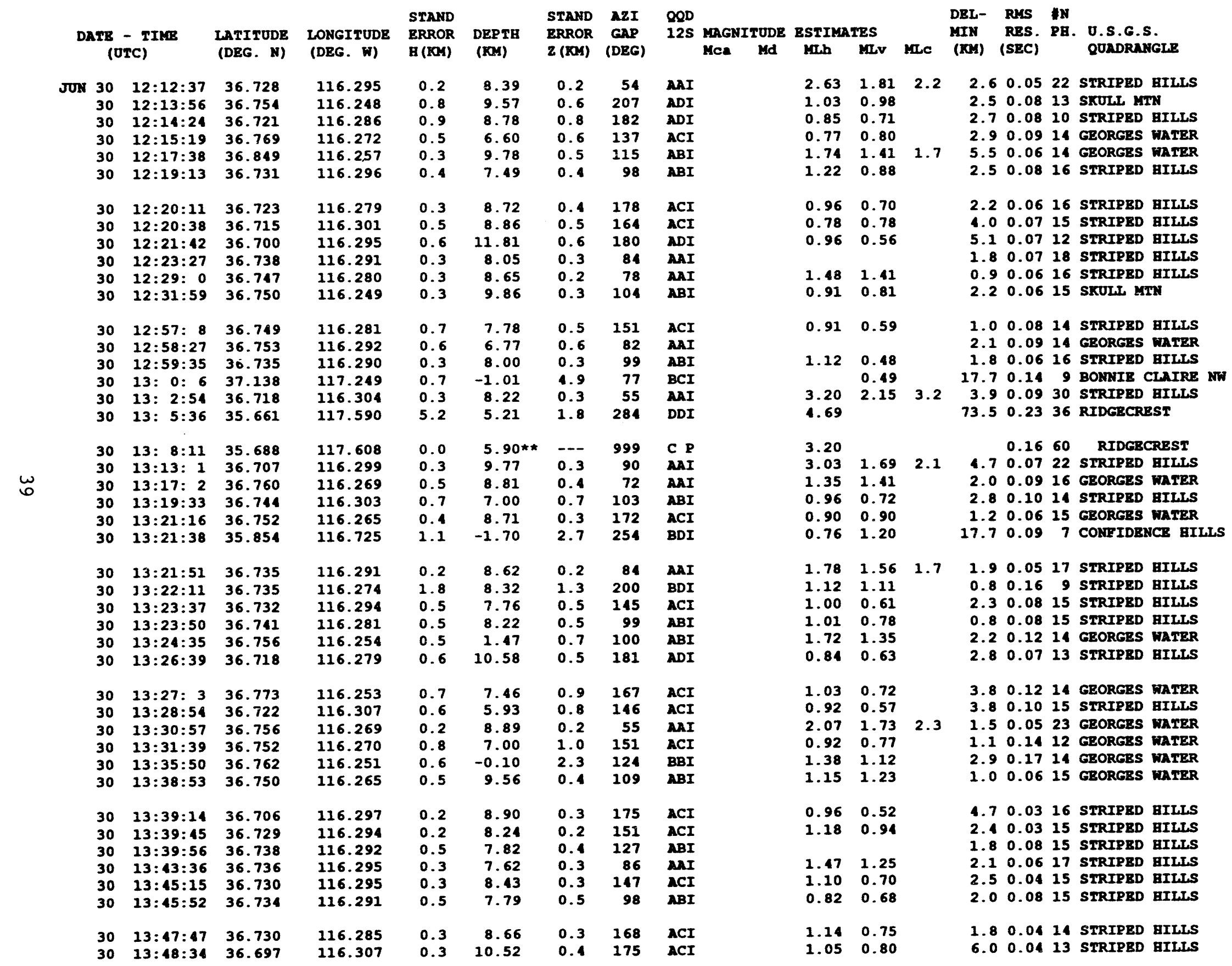


1992 LOCAL HYPOCENTER SUMQMARY - SGB EARTHQUAKES

DATE - IIME LATITUDE LONGITUDE $\begin{array}{lllll} & \text { STAND } & \text { SRROR DEPTH } & \text { STAND AZI } & \text { QQD } \\ \text { ERROR GAP } & \text { 12S MAGNITUDE ESTIMATES }\end{array}$

DEL- RYS IN (OTC)

(DEG. N)

(DEG. W)

\begin{tabular}{ll} 
ERROR & GAP \\
\hline (KOY) & (DEG)
\end{tabular}

Mca

JUN $30 \quad 13: 49: 47 \quad 37.129$

$30 \quad 13: 50: 59 \quad 36.728$

$13: 53: 6 \quad 36.72$

13:53:10 36.755

$30 \quad 13: 53: 38 \quad 36.672$

$0.6 \quad 7.99$

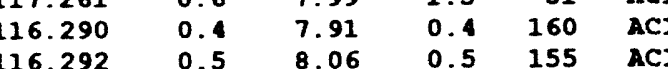

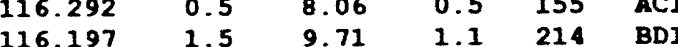

$\begin{array}{llllll}116.263 & 0.4 & 6.01 & 0.7 & 95 & \text { ABI }\end{array}$

$30 \quad 13: 54: 47 \quad 36.719$

116.290

10.78

$0.8 \quad 173 \quad$ BCI

$30 \quad 13: 57: 52 \quad 36.744$

116.305

116.301

$0.5 \quad 5.68$

$\begin{array}{rrr}0.6 & 102 & \mathrm{ABI} \\ 0.3 & 91 & \mathrm{ABI}\end{array}$

$\begin{array}{ll}13: 59: 15 & 36.725 \\ 13: 59: 13 & 36.762\end{array}$

13:59:53 36.772

30 14: $0: 10 \quad 36.730$

30 14: $0: 58 \quad 36.668$

116.269

0.20 .7 .66

$\begin{array}{llll}7.71 & 0.5 & 145 & A C I\end{array}$

$\begin{array}{llllll}116.299 & 1.1 & 8.45 & 0.8 & 139 & \text { BCI }\end{array}$

$\begin{array}{llllll}116.299 & 1.1 & 8.45 & 0.8 & 139 & \text { BCI } \\ 116.260 & 0.5 & 5.99 & 0.7 & 159 & \text { ACI }\end{array}$

30 14: $1: 25 \quad 36.679$

116.225

$0.8272 \quad B D I$

$30 \quad 14: 3: 36 \quad 36.716$

30 14:6:17 36.730

14: 7: 36.670

$14: 8: 15 \quad 36.752$

30 14: $8: 43 \quad 36.747$

116.284

116.312

$0.3 \quad 9.93$

$0.3 \quad 179$ ACI

$\begin{array}{lllll}0.7 & 7.89 & 0.7 & 190 & \text { ADI }\end{array}$

$\begin{array}{llllll}116.287 & 0.5 & 6.75 & 0.5 & 80 & \text { MAI }\end{array}$

$\begin{array}{lllll}0.6 & 10.61 & 0.4 & 235 & \text { ADI }\end{array}$

\begin{tabular}{l}
$30 \quad 14: 9: 22 \quad 36.714$ \\
\hline
\end{tabular}

$30 \quad 14: 10: 15 \quad 36.731$

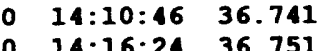

$14: 16: 2436.751$

$30 \quad 14: 17: 35 \quad 36.755$

116.294

116.282

116.289

116.252

116.275
116.260

$0.2 \quad 8.86$

ADI

$\begin{array}{lllll}0.2 & 6.12 & 0.2 & 33 & \text { AAI }\end{array}$

$\begin{array}{lllll}0.9 & 9.04 & 0.8 & 132 & \text { ABI } \\ 1.1 & 7.00 & 0.8 & 140 & \text { BCI }\end{array}$

$14: 19: 5 \quad 36.788$

$14: 19: 54 \quad 36.734$

$14: 23: 10 \quad 36.714$

$14: 24: 18 \quad 36.702$

$14: 25: 31 \quad 36.741$

$30 \quad 14: 30: 31 \quad 37.148$

116.282

116.291

$\begin{array}{rrr}0.3 & 75 & \text { AAI }\end{array}$

$\begin{array}{lll}14: 30: 40 & 36.763 \\ 30 & 14: 45: 50 & 36.753\end{array}$

14:45:50 36.753

14:49: 136.711

$14: 54: 36 \quad 36.734$

14:55:30 36.728

$30 \quad 14: 56: 30 \quad 36.732$

14:57:19 36.746

15: 1:8 36.748

15: $1: 35 \quad 36.726$

$\begin{array}{lll}15: 6: 5 & 36.745 \\ 30 & \end{array}$

116.283

$0.4 \quad 5.42$

$0.7 \quad 116 \quad A B I$

$\begin{array}{llllll}116.284 & 0.5 & 6.13 & 0.4 & 122 & \mathrm{ABI}\end{array}$

117.292

116.266

0.6242 BDI

$9.65 \quad 0.2 \quad 55 \quad$ AAI

$\begin{array}{llllll}116.303 & 0.3 & 9.74 & 0.4 & 113 & \text { ABI }\end{array}$

$\begin{array}{lllll}116.248 & 0.2 & 9.56 & 0.2 & 115 \\ 116.248 & 0.2 & 7.68 & 0.3 & 80\end{array}$

$\begin{array}{llllll}116.294 & 0.3 & 7.85 & 0.3 & 152 & \text { ACI }\end{array}$

$\begin{array}{llllll}116.196 & 1.6 & 11.97 & 2.3 & 123 & \text { BBI }\end{array}$

$\begin{array}{rrrrrr}116.268 & 0.8 & 7.00 & 1.0 & 173 & \text { BCI }\end{array}$

116.268

116.300

116.273

$0.2 \quad 7.77$

$0.3 \quad 90 \quad \mathrm{MAI}$

$\begin{array}{llllll}116.252 & 0.4 & 2.34 & 0.5 & 158 & \text { ACI }\end{array}$

30 15: $6: 33 \quad 36.749$

116.369

$\begin{array}{lllrl}1.2 & -0.70^{\star} & --. & 213 & \mathrm{CDI} \\ 0.4 & 10.66 & 0.5 & 92 & \mathrm{ABI}\end{array}$

\begin{tabular}{|c|c|c|c|c|c|c|}
\hline & 1.09 & & 16.30 .13 & 12 & GOLD HO & UNY \\
\hline 1.13 & 0.84 & & $\begin{array}{lll}2.3 & 0.07\end{array}$ & 14 & STRIRED & grLLs \\
\hline .27 & 1.01 & & 6.90 .17 & 12 & SKULC & ITN \\
\hline 50 & 1.47 & 1.5 & 7.50 .08 & 16 & STRIPED & BILLS \\
\hline & 1.04 & & 3.10 .13 & 14 & SIRIPED & BILLS \\
\hline
\end{tabular}

$\begin{array}{lllllll}1.85 & 1.88 & 1.7 & 3.0 & 0.08 & 13 & \text { STRIPED EILLS }\end{array}$

$\begin{array}{lllllll}1.46 & 1.08 & 1.6 & 3.2 & 0.0617 & \text { STRIPED EILLS }\end{array}$

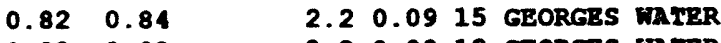

$\begin{array}{lllll}0.83 & 0.93 & 3.3 & 0.0912 & \text { GEORGBS WATER }\end{array}$

$\begin{array}{llllll}1.44 & 1.15 & 2.8 & 0.18 & 16 & \text { STRIPED BILLS }\end{array}$

$1.140 .98 \quad 7.60 .0914$ STRIPED BILLS

$\begin{array}{llllll}0.91 & 0.43 & 8.2 & 0.0812 \text { SPECTER RANGE NW }\end{array}$

$\begin{array}{llllll}1.00 & 0.73 & 3.10 .0412 & \text { STRIPED EILLS }\end{array}$

$\begin{array}{lllllll}1.81 & 1.50 & 1.6 & 1.4 & 0.04 & 21 & \text { STRIPBD } \\ 1.9 I L L S\end{array}$

$\begin{array}{llllll}1.14 & 0.95 & 3.7 & 0.10 & 15 & \text { STRIPED BILLS }\end{array}$

$0.90 \quad 0.54 \quad 1.70 .1016$ GEORGES WATER

$\begin{array}{llllll}0.69 & 0.68 & 1.9 & 0.07 & 14 & \text { STRIPED BILLS }\end{array}$

$\begin{array}{lllllll}0.88 & 0.35 & 3.8 & 0.04 & 14 & \text { STRIPED EILLS }\end{array}$

$\begin{array}{llllll}0.89 & 0.57 & 1.5 & 0.0813 & \text { STRIPED HILLS }\end{array}$

$3.10 \quad 1.50 .0746$ STRIPED HILLS

$\begin{array}{lllllll}0.87 & 0.75 & 2.1 & 0.13 & 13 & \text { GEORGES WATER }\end{array}$

$\begin{array}{lllll}0.94 & 0.63 & 0.3 & 0.21 & 15 \\ \text { STRIPED HILLS }\end{array}$

$\begin{array}{llllll}1.15 & 1.38 & 1.5 & 1.8 & 0.07 & 18 \text { GEORGES WATER }\end{array}$

$\begin{array}{llllll}0.73 & 0.73 & 5.1 & 0.08 & 14 & \text { GEORGES WATER }\end{array}$

$\begin{array}{lllll}1.29 & 1.14 & 1.9 & 0.0518 & \text { STRIPED BILLS }\end{array}$

$\begin{array}{lllllll}1.61 & 1.25 & 1.5 & 3.3 & 0.06 & 16 & \text { STRIPED HILLS }\end{array}$

$\begin{array}{llllll}1.65 & 1.16 & 6.5 & 0.1613 & 13 & \text { SIRIPED BILIS }\end{array}$

$1.200 .86 \quad 1.10 .0916$ STRTPED BILTS

$\begin{array}{llllll}1.46 & 1.6 & 17.2 & 0.08 & 6 & \text { GOLD MOUNTAIN }\end{array}$

$\begin{array}{llll}0.90 & 0.25 \quad 2.4 & 0.07 \quad 8 \text { GEORGES WATER }\end{array}$

$\begin{array}{llllll}2.48 & 2.1 & 1.1 & 0.06 & 21 & \text { GEORGES WATER }\end{array}$

$\begin{array}{lllll}1.12 & 0.89 & 5.0 & 0.0716 & \text { STRIPED gILLS }\end{array}$

$\begin{array}{lllllll}1.71 & 1.42 & 1.6 & 1.1 & 0.05 & 16 & \text { STRIPED BILLS }\end{array}$

$\begin{array}{lllllll}1.93 & 1.32 & 1.9 & 2.4 & 0.05 & 17 & \text { SPBCTER RANGE }\end{array}$

$\begin{array}{lllll}0.96 & 0.83 & 2.5 & 0.04 & 14\end{array}$

$\begin{array}{lllll}1.42 & 0.83 & 6.9 & 0.2612 & \text { SPECTER RANGE NA }\end{array}$

$0.88 \quad 0.82$

$0.98 \quad 0.96$

1.411 .27

3.00 .0617 STRIPED GILLS

$\begin{array}{llll}1.38 & 1.28 & 0.30 .1919 & \text { SIRIPED BILLS }\end{array}$

$8.6 \quad 0.13 \quad 8$ STRIPED GILLS

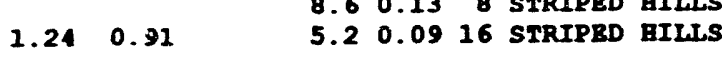

0.60 .1614 STRTPBD

30 15: $7: 43 \quad 36.702$

$\begin{array}{rrrr}10.66 & 0.5 & 92 & A B I\end{array}$

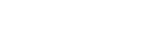


1992 LOCAL HYPOCENTER SUMMARY - SGB EARTHQUAKES

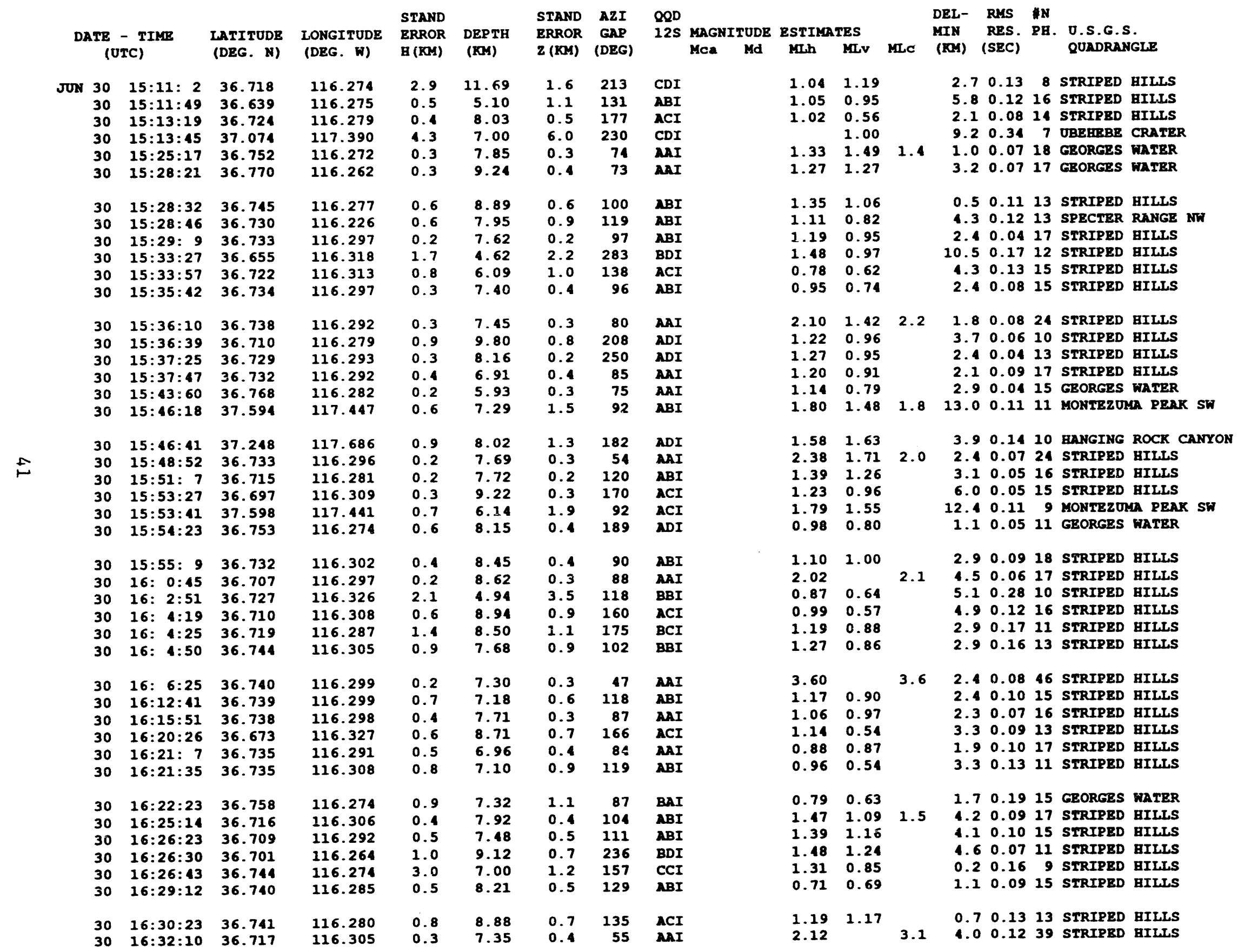


1992 LOCAL HYPOCENTER SUMARRY - SGB EARTBQUAKES

STAND STAND AZI QQD

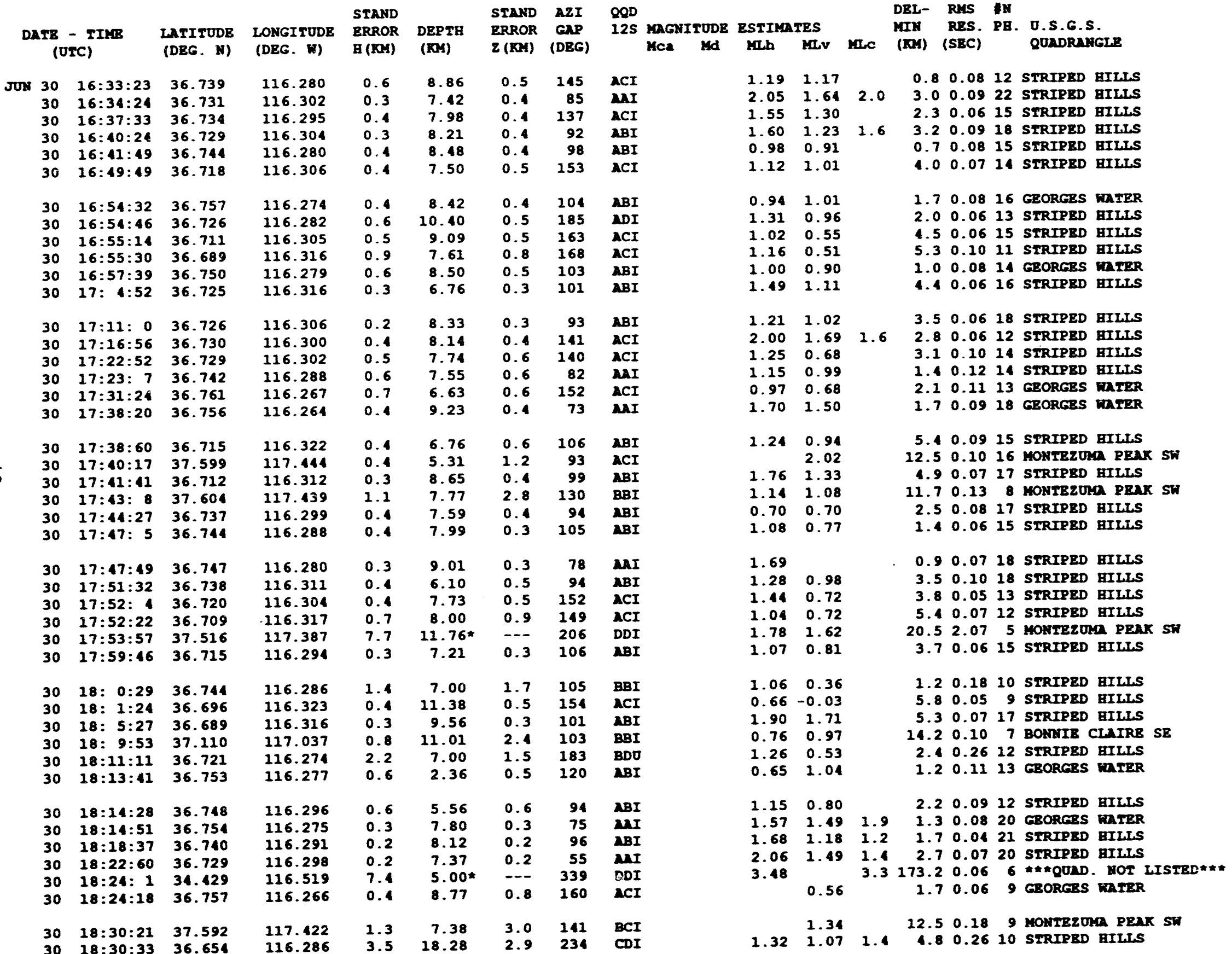


1992 LOCAL HYPOCENTER SURMARY - SGB RARTHQUAKES

STAND STAND AZI QQD

DATE - TIET

(OTC)

IATITUDE (DEG. N)

JUN $30 \quad 18: 32: 42 \quad 37.601$

$30 \quad 18: 35: 21 \quad 37.097$

$30 \quad 18: 38: 19 \quad 36.707$

$30 \quad 18: 12: 28 \quad 37.251$

$30 \quad 18: 12: 32 \quad 36.752$

$30 \quad 18: 43: 55 \quad 37.096$

$30 \quad 18: 45: 16 \quad 36.756$

$30 \quad 18: 48: 14 \quad 36.744$

30 18:50:6 36.713

$18: 51: 3 \quad 35.820$

$18: 51: 3 \quad 35.836$

$30 \quad 18: 52: 20 \quad 35.845$

$30 \quad 18: 53: 12 \quad 36.727$

30 19: 0:40 36.724

30 19: 3: 0 35.750

30 19: 6:47 36.706

$30 \quad 19: 12: 54 \quad 36.701$

$30 \quad 19: 13: 20 \quad 36.730$

$30 \quad 19: 13: 33 \quad 36.703$

$30 \quad 19: 15: 17 \quad 36.757$

$30 \quad 19: 17: 2 \quad 36.744$

19:19: 736.721

$30 \quad 19: 19: 25 \quad 36.728$

$30 \quad 19: 20: 20 \quad 36.725$

$30 \quad 19: 25: 34 \quad 36.733$

30 19:30:8 36.701

30 19:30:44 36.763

30 19:32:3 36.755

19:33:52 36.726

$30 \quad 13: 44: 24 \quad 36.747$

$30 \quad 19: 48: 29 \quad 36.753$

$30 \quad 19: 48: 50 \quad 37.139$

19:49:20 36.731

$\begin{array}{lll}19: 49: 20 & 36.731 \\ 30 & 19: 49: 54 & 36.721\end{array}$

19:52:43 36.737

$30 \quad 19: 59: 38 \quad 36.736$

$30 \quad 20: 4: 4736.726$

$30 \quad 20: 5: 29 \quad 36.706$

$30 \quad 20: 8: 28 \quad 36.749$

$30 \quad 20: 12: 13 \quad 36.746$

$30 \quad 20: 13: 55 \quad 36.732$

$30 \quad 20: 16: 11 \quad 36.767$

$30 \quad 20: 21: 48 \quad 36.746$

$30 \quad 20: 25: 51 \quad 36.724$
LONGITUDE ERROR DEPTH

117.440

117.174

116.305

117.609

116.273

$1.0 \quad 8.65$

$0.4 \quad 2.74$

$0.2 \quad 10.11$

$0.1 \quad 7.86$

$0.4 \quad 4.85$

$\begin{array}{lll}116.261 & 0.4 \quad 9.36\end{array}$

116.307

116.315

116.786

116.774
116.771

$1.1 \quad 7.86$

0.58 .94

$\begin{array}{rr}1.8 & 4.45 \\ 3.5 & -0.98\end{array}$

$1.0 \quad 5.31$

$\begin{array}{lll}116.298 & 0.3 & 7.19 \\ 116.291 & 0.6 & 7.99\end{array}$

116.291

116.256

116.306

116.300

116.304

116.321

116.276

116.289

116.286

116.308

116.277

116.281

116.278

116.267

116.288
116.263

116.282

117.263

116.270

116.303

116.296

116.295

116.304

116.304

116.300

116.296

116.281

$0.3 \quad 9.36$

$0.6 \quad 8.50$

8.49

0.5

0.58 .99

$\begin{array}{rr}0.4 & 6.74 \\ 1.1 & 10.45\end{array}$

0.47 .67

0.37 .98

$0.3 \quad 11.54$

$0.6 \quad 8.06$

$0.4 \quad 6.50$

$\begin{array}{lr}0.7 \quad 7.87 \\ 0.4 & 11.30\end{array}$

$0.4 \quad 11.30$

0.510 .50

$0.6 \quad 8.24$

$\begin{array}{ll}0.2 \quad 7.78 \\ 0.3 & 8.61\end{array}$

$\begin{array}{ll}0.3 & 8.61 \\ 0.4 & 8.28\end{array}$

0.36 .00

$0.6 \quad 8.34$

$\begin{array}{lll}116.284 & 0.2 \quad 8.29\end{array}$

116.314
$0.5 \quad 12.01$

$2.5 \quad 130 \quad \mathrm{BBT}$

$\begin{array}{lll}0.2 & 56 & \text { AAI } \\ 0.5 & 98 & \text { ABI }\end{array}$

$0.2 \quad 74$ AAI

$0.5 \quad 68 \quad$ AAI

$0.991 \quad$ BBI

$0.7 \quad 147$ ACI

$\begin{array}{lll}9.9 & 276 & \text { CDI } \\ 3.4 & 266 & \text { CDI }\end{array}$

3.4266 BDI

$\begin{array}{lll}0.6 & 167 & \text { ACI } \\ 0.3 & 179 & \text { ACI }\end{array}$

$1.0105 \quad \mathrm{BBI}$

$0.3 \quad 175$ ACI

0.392

$0.6 \quad 107$

$\begin{array}{rr}0.4 & 127 \\ 0.3 & 82\end{array}$

0.9184

$0.4 \quad 95$

$0.3 \quad 77$

$0.5 \quad 121$

$\begin{array}{ll}0.6 & 75 \\ 0.4 & 73\end{array}$

0.4115

$\begin{array}{lll}1.4 & 99 & \text { ABI }\end{array}$

$\begin{array}{rrr}0.7 & 82 & \text { ABI } \\ 0.3 & 107 & \text { ABI }\end{array}$

0.2 107 ABI

0.487 AAI

$\begin{array}{lll}0.6 & 132 \quad \mathrm{ABI}\end{array}$

$0.3 \quad 92 \quad A B I$

$0.4 \quad 169$ ACI

$0.579 \quad$ MAI

$\begin{array}{lrr}0.5 & 90 & \text { ABI } \\ 0.6 & 141 & \text { ACI }\end{array}$

$\begin{array}{lllll}0.2 & 8.29 & 0.3 & 77 & \text { MAI } \\ 0.4 & 7.24 & 0.6 & 99 & \text { ABI }\end{array}$
0.47 .00

0.48 .72

$\begin{array}{ll}0.1 & 8.54 \\ 0.4 & 7.25\end{array}$

BRROR GAP 125 MAGNITUDE ESTIMATES

(IOM) (DEG) MCa Md MLh MU MLC (RI) (SEC) QUADRANGLS

RUS

MIN RES. PB. U.S.G.S

$\begin{array}{lll}3.2 & 107 & \mathrm{BCI}\end{array}$

$0.4 \quad 149$ ACI

0.975 ANI

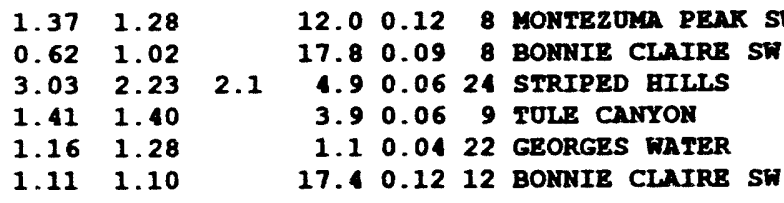

$\begin{array}{lllllll}1.96 & 1.39 & 1.7 & 1.8 & 0.10 & 22 & \text { GEORGES WATER }\end{array}$

$\begin{array}{lllllll}0.99 & 0.70 & 3.1 & 0.16 & 13 & \text { STRIPED BILLS }\end{array}$

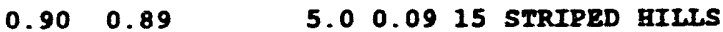

$\begin{array}{lllll}2.13 & 2.1 & 17.6 & 0.24 & 12 \\ \text { TINGATE wASB }\end{array}$

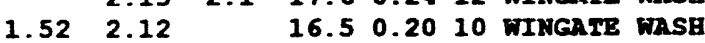

1.841 .781 .515 .80 .1413 WINGATB WASB

$\begin{array}{lll}1.16 & 0.63\end{array}$

1.430 .85

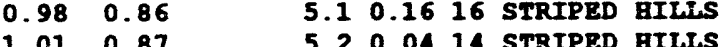

3.10 .0716 STRIPED HILLS

$\begin{array}{ll}1.69 & 1.37\end{array}$

0.710 .80

1.130 .91

1.320 .90

0.980 .81

1.671 .36

$\begin{array}{ll}1.76 & 1.47\end{array}$

1.451 .00

$0.87 \quad 0.81$

$1.06 \quad 1.01$

1.041 .08

$\begin{array}{llll}6.2 & 0.11 & 17 & \text { STRIPED HILLS }\end{array}$ 1.70 .0716 GEORGES MATER $1.5 \quad 0.0716$ STRIPED BILIS 2.70 .1210 STRIPED EILLS 3.60 .0816 SIRIPED BILLS 3.70 .0617 STRIPED BILIS

$1.45 \quad 0.97$

$\begin{array}{llllll}1.70 & 1.6 & 17.2 & 0.1219 & \text { GOLD MOUNTAIN }\end{array}$

$\begin{array}{lllllll}2.05 & 1.63 & 1.6 & 1.3 & 0.08 & 16 & \text { STRIPED BILLS } \\ 2.09 & 1.49 & 1.7 & 3.7 & 0.04 & 21 & \text { STRIPED BILLS }\end{array}$

$0.96 \quad 0.90 \quad 2.20 .0916$ STRIPED HILLS

$0.85 \quad 0.58$

2.20 .1015 STRIPRD HILTS

$1.65 \quad 1.29$

$1.31 \quad 1.37$

$1.30 \quad 0.94$

$1.10 \quad 0.84$

$0.99 \quad 0.64$

3.40 .0618 STRIPED BILLS 5.00 .0615 SIRIPED HILLS 1.20 .1019 STRIPED BIHLS 2.50 .0917 SIRIPED BILIS 2.50 .1115 STRIPED BILLS

$\begin{array}{lllllll}1.70 & 1.33 & 1.5 & 1.2 & 0.07 & 26 & \text { STRIPED HILLS }\end{array}$

$\begin{array}{llllll}1.21 & 0.77 & 4.3 & 0.1015 & \text { STRIPED GILLS }\end{array}$
1.251 .22 
1992 LOCAL HYPOCENTER SUMMARY - SGB EARTHQUAKRS

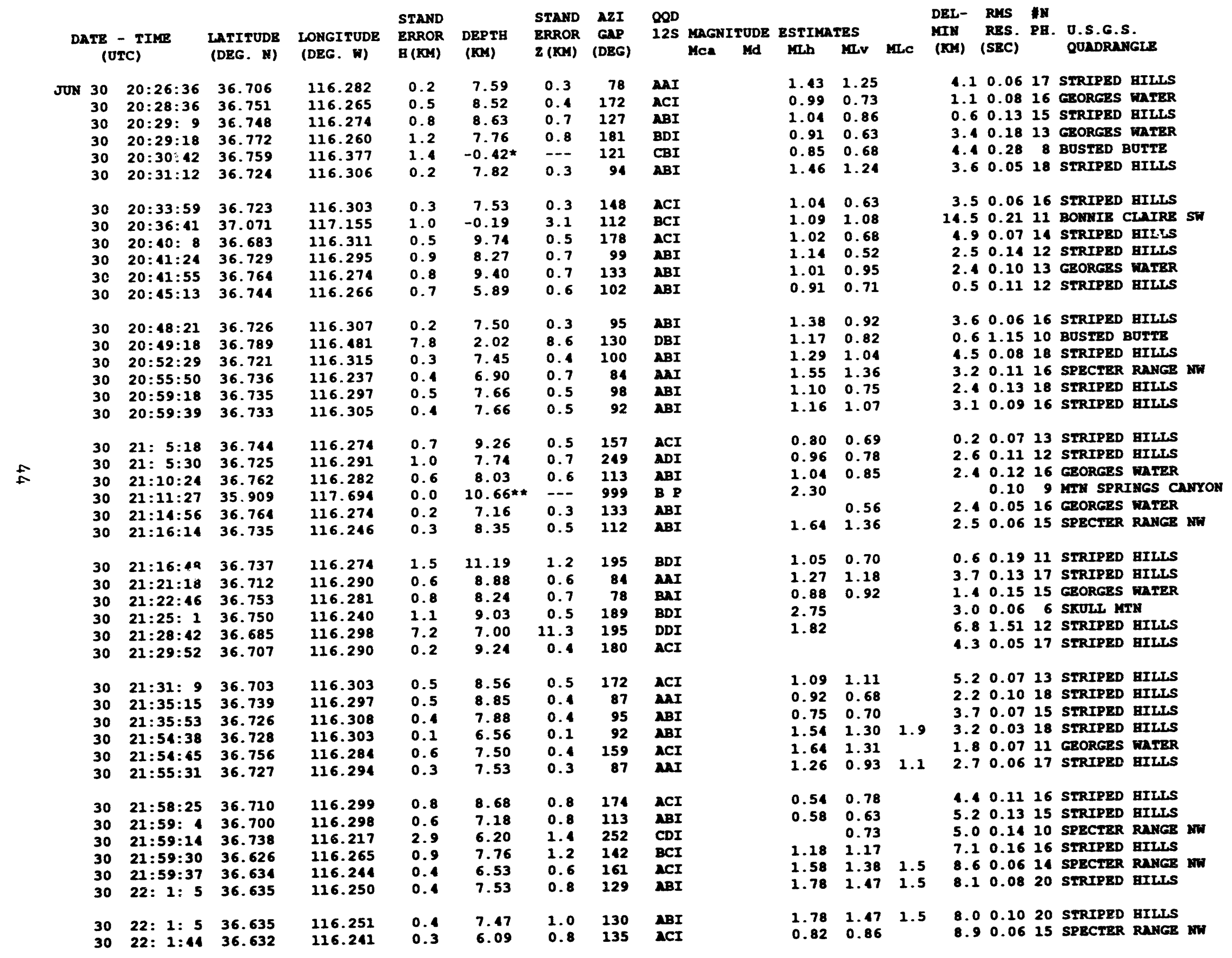




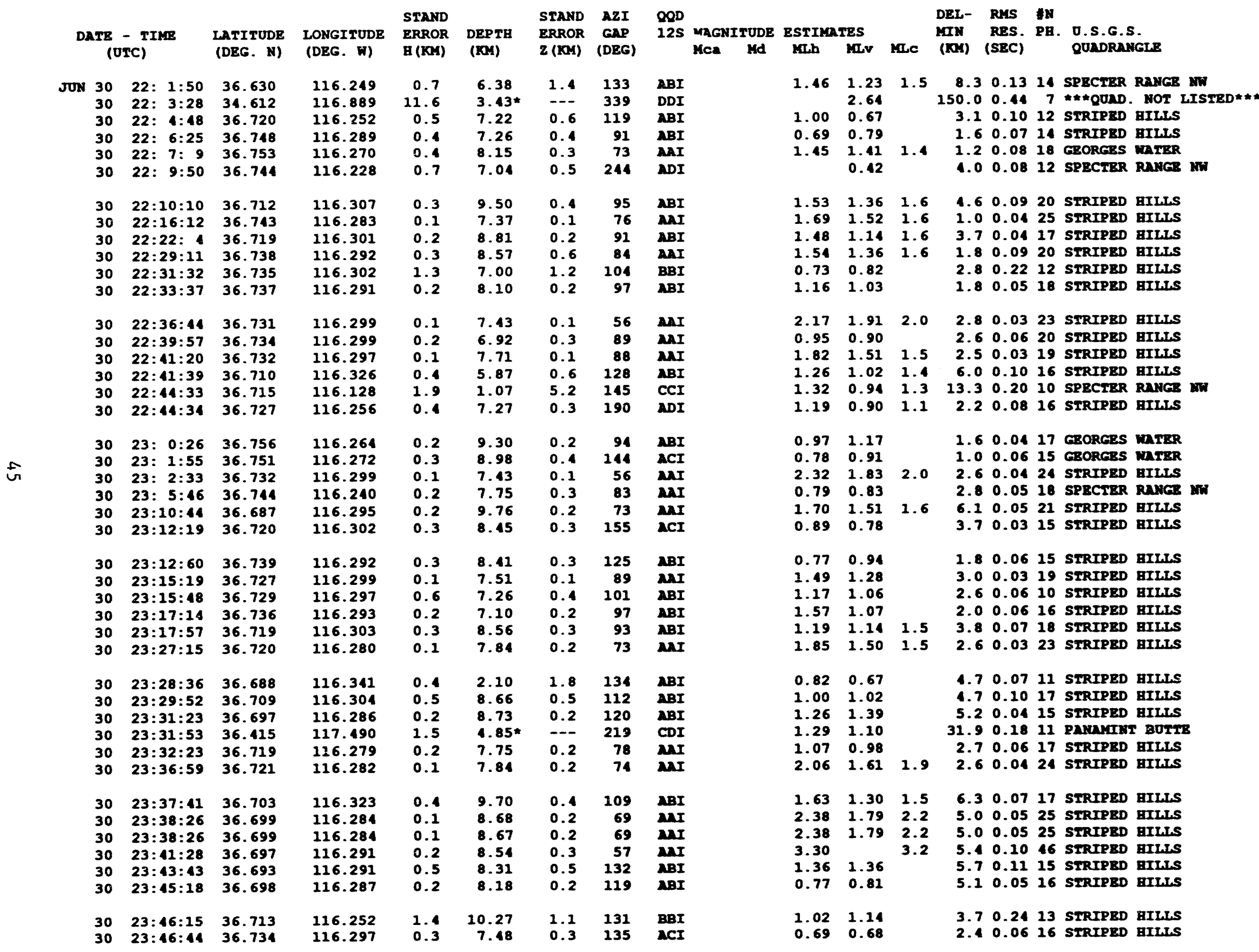


1992 LOCAL HYPOCENTER SUIRARY - SGB EARTHQUAKCS

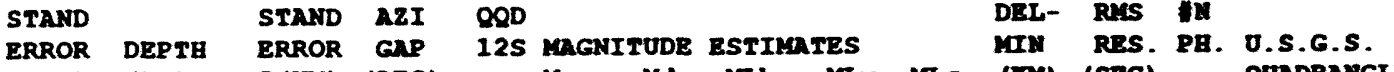

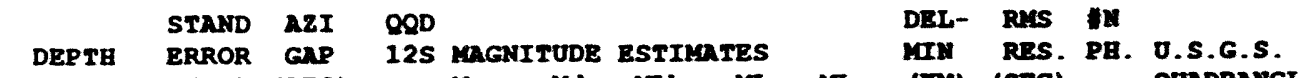

DATE - TIME (urc) (DEG. N)

2 (KM) (DEG)

(Ri) (SEC)

QundRaRGLe

JUN $30 \quad 23: 48: 49 \quad 36.764$

$30 \quad 23: 49: 23 \quad 36.728$

$30 \quad 23: 52: 32 \quad 36.709$

$30 \quad 23: 52: 57 \quad 36.761$

$30 \quad 23: 56: 48 \quad 36.739$

$30 \quad 23: 59: 21 \quad 36.723$

116.213

116.302

116.291

$\begin{array}{lllll}0.2 & 7.76 & 0.3 & 95 & \mathrm{ABI}\end{array}$

$\begin{array}{lllll}0.2 & 8.03 & 0.3 & 177 & \text { ACI }\end{array}$

$\begin{array}{llllll}116.289 & 0.6 & 5.16 & 0.9 & 88 & \mathrm{MI} \\ & 0.24 & 0.3 & 34 & \mathrm{NI}\end{array}$

$\begin{array}{llllll}116.298 & 0.1 & 8.82 & 0.2 & 54 & \mathrm{NMI}\end{array}$

JUL $1 \quad 0: 0: 7 \quad 36.683$

116.291

$0: 6: 45 \quad 36.732$

$0: 8: 27 \quad 36.730$

$0: 14: 39 \quad 36.74$

$0: 18: 57 \quad 36.744$

116.247

116.292

0.48 .97

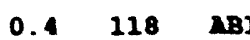

$\begin{array}{lllll}0.6 & 8.37 & 0.8 & 114 & \text { BBI } 0.59 \\ 0.6 & 0.8 & 154 & \text { BCI } 1.15\end{array}$

$\begin{array}{lllllll}0.7 & 7.84 & 0.8 & 105 & \text { BBI } & 1.47\end{array}$

$\begin{array}{llllll}293 & 0.5 & .52 & 0.7 & 94 & \text { BBI } 1.30\end{array}$

$0.5 \quad 8.52$

$\begin{array}{lrrr}0.7 & 94 & \text { BBI } 1.30 \\ 0.6 & 208 & \text { NDI } 1.05\end{array}$

$0: 21: 18 \quad 36.744$

$0: 22: 29 \quad 36.754$

0.58 .05

116.287

$0: 23: 636.756$

$0: 26: 40 \quad 36.761$

$0: 33: 19 \quad 36.729$

$0: 10: 22 \quad 36.694$

116.287

0.9

9.81

$0.5 \quad 105 \quad$ ABI 1.16

116.263

0.7

8.80

0.9168 BCI 1.04

116.297

7.69

$\begin{array}{llll}0.7 & 158 & \text { BCI } & 1.15\end{array}$

$\begin{array}{rrrrrr}116.297 & 0.1 & 7.69 & 0.2 & 88 & \mathrm{MAI} \\ 116.296 & 1.0 & 7.89 & 1.1 & 184 & \mathrm{BDI}\end{array}$

$0: 45: 34 \quad 36.742$

$0: 50: 40 \quad 36.737$

116.293

0.2

36.718

$1: 1: 58 \quad 36.733 \quad 116.296$

$0.3 \quad 94 \quad \mathrm{ABI} 1.57$

$0.5 \quad 9.14$

0.459 MAI 2.02

$0.5 \quad 161 \quad \mathrm{ACI}$

$\begin{array}{llllll}116.307 & 0.4 & 8.56 & 0.6 & 136 & \text { ACI } \\ 116.296 & 0.4 & 9.00 & 0.5 & 138 & \text { ACI } \\ 116.261 & 0.4 & 9.37 & 0.5 & 163 & \text { ACI }\end{array}$

9.37

0.5163 ACI

$1: 13: 29 \quad 36.747$

$1: 13: 55 \quad 36.760$

\begin{tabular}{ll}
$760 \quad 116.267$ \\
\hline
\end{tabular}

$1: 15: 51 \quad 36.712$

$1: 21: 23 \quad 36.239$

$0.2 \quad 8.38$

$\begin{array}{lll}0.2 & 75 & \mathrm{MII}\end{array}$

$\begin{array}{rrrrrr}116.276 & 0.1 & 7.09 & 0.2 & 122 & \text { ABI } \\ 116.299 & 0.1 & 7.91 & 0.1 & 89 & \text { MAI }\end{array}$

$0.3 \quad 7.09$

$0.2213 \quad N D I$

$\begin{array}{llllll}117.115 & 1.3 & 2.77 & 4.8 & 194 & \text { BDI } \\ 116.281 & 0.3 & 8.11 & 0.3 & 142 & \text { ACI }\end{array}$

$1: 22: 55 \quad 36.701$

116.287

$0.1 \quad 8.79$

$1: 23: 34 \quad 36.734$

$1: 25: 936.755$

$1: 25: 24 \quad 36.736$

$1: 26: 38 \quad 36.762$

116.256

0.92 .55

116.252

$3.0 \quad 12.71$

$1: 32: 936.734$

0.28 .16

$\begin{array}{rrr}0.1 & 134 & \text { BBI } \\ 0.4 & 77 & \text { MAI }\end{array}$

$2.2 \quad 240 \quad \mathrm{CDI}$

116.293

7.40

$\begin{array}{rrr}0.2 & 74 & \text { AnI } \\ 0.3 & 138 & \text { ACI }\end{array}$

$1: 34: 40 \quad 36.731$

$1: 45: 35 \quad 36.730$

$1: 48: 8 \quad 37.096$

$1: 48: 18 \quad 36.733$

116.297

$0.1 \quad 7.75$

116.302

0.2

7.75
6.97

$0.1 \quad 98 \quad \mathrm{ABI}$

117.179

0.5

6.97
9.78
7.88

116.301

$\begin{array}{lll}1: 59: 39 & 36.736 & 116.30\end{array}$

0.57 .00

1.9

91 ABI

$\begin{array}{rrrr}7.17 & 0.6 & 111 & \mathrm{NBI}\end{array}$

2: 2:55 36.718

116.273

$0.1 \quad 8.48$

$\begin{array}{lll}0.2 & 63 & \mathrm{MI} \\ 0.2 & 76 & \mathrm{MI}\end{array}$

$\begin{array}{llllllll}0.88 & 1.03 & 1.3 & 5.8 & 0.06 & 19 & \text { skoul }\end{array}$

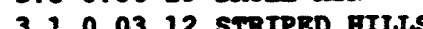
$0.83 \quad 1.10 .0316$ SIRTPED mIns

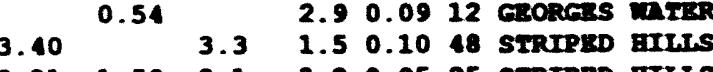

$\begin{array}{lllllll}2.31 & 1.59 & 2.1 & 3.2 & 0.05 & 25 & \text { STRIPED }\end{array}$

$\begin{array}{llllllll}0.87 & 0.99 & & 6.0 & 0.09 & 16 & \text { SIRIPED BILIS } \\ 1.85 & 0.78 & 1.1 & 2.5 & 0.16 & 21 & \text { SPECRER RAMGE }\end{array}$

$1.651 .24 \quad 1.12 .20 .1619$ STRTPED RAMGS

1.360 .921 .101 .90 .1516 STRIPED

1.541 .101301018 STRTPE

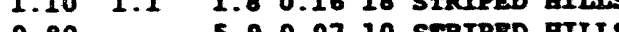

$\begin{array}{lllllll}1.12 & 0.63 & 1.1 & 1.4 & 0.11 & 15 & \text { STRIPED HILS }\end{array}$

$1.490 .72 \quad 1.40 .1818$ GECRERS MAIER 0.9122 .00 .2015 ezorees mitzR

$\begin{array}{lllllll}1.36 & 0.99 & 1.1 & 2.2 & 0.16 & 18 & \text { GEORCRS MmER }\end{array}$

$\begin{array}{lllllll}1.96 & 1.47 & 2.0 & 2.7 & 0.04 & 19 & \text { STRIPED HILLS }\end{array}$

$\begin{array}{llll}1.65 & 1.30 & 5.8 & 0.1212 \text { SIRIPED BIHLS }\end{array}$

\begin{tabular}{lllllll}
1.56 & 0.98 & 1.0 & 1.9 & 0.07 & 22 & STRIPED \\
\hline & HILLS
\end{tabular}

$1.91 \quad 1.34 \quad 1.9 \quad 3.20 .1432$ STRIPED EILLS $\begin{array}{lll}1.00 & 3.6 & 0.0712 \text { STRIPED EILIS }\end{array}$

$\begin{array}{lllllll}1.28 & 1.12 & 1.4 & 3.6 & 0.11 & 19 & \text { SIRIPED BILLS }\end{array}$

$\begin{array}{lllllll}0.79 & 0.70 & 1.1 & 2.4 & 0.09 & 14 & \text { STRIPED GILLS }\end{array}$ 0.5622 .50 .0817 ceOREes mirER

$0.76 \quad 0.93$

$1.29 \quad 1.23$

1.090 .95

1.351 .06

1.351 .06

$1.02 \quad 1.20$

0.68

$1.04 \quad 0.87$
0.56

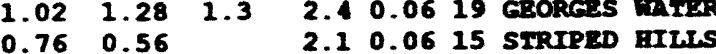

$\begin{array}{llllll}1.75 & 1.13 & 1.1 & 2.5 & 0.02 & 14\end{array}$

$\begin{array}{lllllll}1.40 & 1.39 & 3.0 & 0.06 & 20 & \text { STRIPED BILLS }\end{array}$

$1.451 .46 \quad 18.00 .2219$ BOmis ClaIRe st

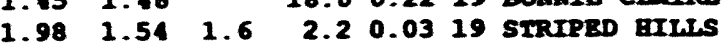

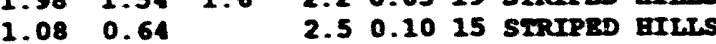

$0.740 .81 \quad 2.90 .0416$ SIRIPED BILIS

$\begin{array}{lllllll}2.17 & 1.67 & 1.6 & 2.7 & 0.04 & 23 & \text { STRIPED HILLS }\end{array}$

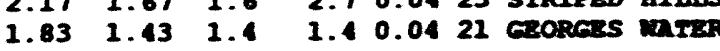


1992 LOCAL HYPOCENTER SUMANRY - SCB EARTHQUAKS

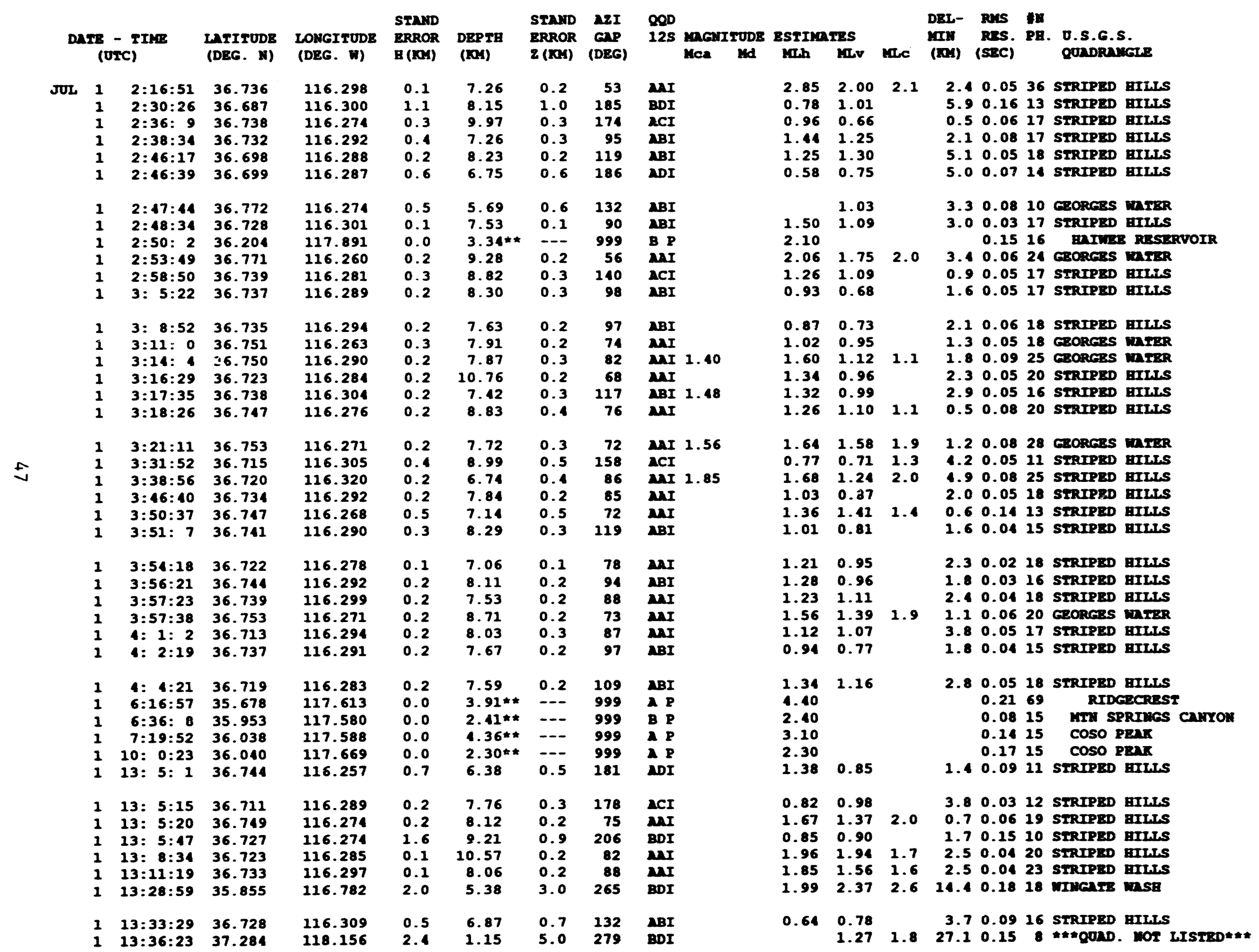


1992 LOCAL HYPOCENTER SUEAARY - SGB RARTHQUAKES

STAND STAND AZI OQD

DATB - TIY (UTC) (DEG. N) (DEG. W) H(KO)

JUL $1 \quad 13: 36: 30 \quad 36.708$ $113: 38: 23 \quad 36.730$ $1 \quad 13: 42: 33 \quad 36.743$ 1 13:14:24 36.851 $13: 45: 13 \quad 36.738$ $1 \quad 13: 45: 3036.742$

$133: 48: 52 \quad 36.741$ $113: 53: 9$ $13: 55: 50 \quad 36.750$ $13.57: 29 \quad 36.738$ 1 13:59: 736.739 1 14: $0: 35$ 36.741

$1 \quad 14: 12: 45 \quad 36.739$ 1 14:17:40 36.752 $1 \quad 14: 18: 35 \quad 36.731$ 1 14:23: 36.727 1 14:25:18 36.748

$14: 27: 33 \quad 36.724$

1 14:28: 236.764
DEL- RES in

ERROR GAP $12 S$ MAGNITUDE ESTIMATES MIN RES. PB. O.S.G.S. Mca Md kIh Mav Mac (INA) (SEC)

4.80 .0418 SIRIPED BILIS $\begin{array}{lll}0.71 & 0.82\end{array}$

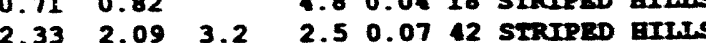

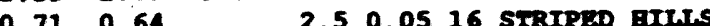
$0.780 .67 \quad 5.10 .1315$ Tanas $0.791 .04 \quad 2.00 .0419$ STRIPED BILIS $1.240 .99 \quad 1.50 .0315$ SIRIPED BILIS

$1.010 .75 \quad 2.00 .0416$ SIRIPED BILLS $\begin{array}{lllllll}1.71 & 1.43 & 1.9 & 1.6 & 0.03 & 18 & \text { SERIPED BILS }\end{array}$ $0.910 .83 \quad 0.90 .0517$ GeORGes WTrER $\begin{array}{lllll}1.30 & 1.27 & 2.0 & 0.0320 & \text { STRIPED EILIS }\end{array}$ $\begin{array}{lllllll}2.36 & 1.70 & 2.1 & 1.3 & 0.05 & 27 & \text { SIRIPED BILLS }\end{array}$ $0.830 .84 \quad 1.00 .0617$ STRTPRD BTITS

$1.47 \quad 1.09 \quad 1.90 .0420$ STRIPED BIWTS $0.830 .86 \quad 1.10 .1019$ GzORess marzo $0.50 \quad 0.54 \quad 2.90 .0315$ SIRIPED aILIS $\begin{array}{lllll}1.85 & 2.1 & 1.9 & 0.0418 & \text { STRIPED BILLS }\end{array}$ $\begin{array}{lllll}0.77 & 0.94 & 0.8 & 0.0517 \text { STRIPED GILES }\end{array}$

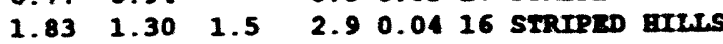

$\begin{array}{llll}0.77 & 0.65 & 3.0 & 0.0516 \text { GeORGES miter }\end{array}$ $\begin{array}{llll}0.68 & 0.92 & 4.6 & 0.0315 \text { SIRIPED aIr.S }\end{array}$ $\begin{array}{lllllll}1.55 & 1.40 & 1.3 & 2.6 & 0.03 & 15 & \text { GgORes }\end{array}$ $\begin{array}{llllllll}1.55 & 1.40 & 1.3 & 2.6 & 0.03 & 15 & \text { comeas matres }\end{array}$

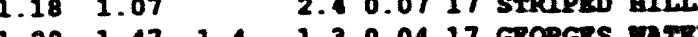
$\begin{array}{lllllll}1.29 & 1.47 & 1.4 & 1.3 & 0.0417 & \text { GEORES MTRR } \\ 0.83 & 0.80 & & 1.0 & 0.0416 & \text { SIRIPED BILLS }\end{array}$

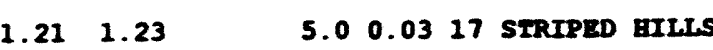
$\begin{array}{lllll}1.49 & 0.97 & 0.5 & 0.1015 & \text { STRIPED BILLS }\end{array}$ $\begin{array}{lllllll}1.45 & 1.26 & 1.6 & 1.8 & 0.04 & 17 & \text { STRTPED } \\ 1.6 I L T S\end{array}$ $\begin{array}{lllllll}1.65 & 1.31 & 1.4 & 1.5 & 0.03 & 15 & \text { GBORGES MnTER }\end{array}$ $2.0122 .0 \quad 1.90 .0319$ STRIPED GILLS $0.77 \quad 1.02 \quad 1.30 .0315$ ezorices betres

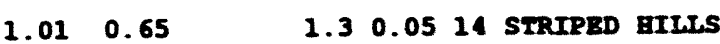
$\begin{array}{llll}0.81 & 0.94 & 2.9 & 0.0712 \text { SIRIPED mILLS }\end{array}$ $\begin{array}{lllllll}0.81 & 0.94 & & 2.9 & 0.07 & 12 & \text { SIRIPED HILLS } \\ 1.54 & 0.99 & 1.5 & 3.7 & 0.09 & 16 & \text { SIRIPED BILLS }\end{array}$ $\begin{array}{llllllll}1.54 & 0.99 & 1.5 & 3.7 & 0.09 & 16 & \text { STRTPED HILS } \\ 1.18 & 0.97 & & 1.9 & 0.06 & 12 & \text { GRORGES EMTER }\end{array}$ $\begin{array}{lllllll}1.34 & 1.20 & 1.6 & 0.8 & 0.04 & 18 & \text { SIRIPED GILLS }\end{array}$

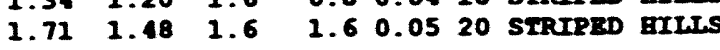
$\begin{array}{llll}1.21 & 0.96 & 1.8 & 0.0717 \text { STRIPED BILLS }\end{array}$ $0.92 \quad 0.82 \quad 1.40 .0714$ GBORGBS mTER $0.97 \quad 0.63 \quad 4.30 .0814$ SIRIPED BI

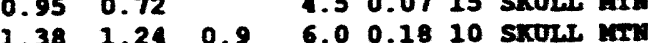

$\begin{array}{lllllll}1.38 & 1.24 & 0.9 & 6.0 & 0.18 & 10 & \text { SKULL MII } \\ 1.13 & 0.78 & & 1.4 & 0.09 & 16 & \text { STRIPED GILLS }\end{array}$ $\begin{array}{lllllll}1.55 & 1.44 & 1.5 & 3.3 & 0.05 & 17 & \text { STRIPED GILIS }\end{array}$

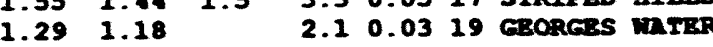


1992 LOCAL HYPOCENTER SUMAQRY - SGB EARTBQUAKRS

DATB - TIME LATITUDE LONGITUDE ERROR (0IC) (DEG. A)

JUL

$\begin{array}{lll}1 & 15: 51: 9 & 36.762 \\ 1 & 16: 3: 40 & 36.746 \\ 1 & 16: 11: 59 & 36.721 \\ 1 & 16: 13: 54 & 36.715 \\ 1 & 16: 17: 30 & 36.748 \\ 1 & 16: 20: 6 & 36.744 \\ & & \\ 1 & 16: 35: 10 & 36.742 \\ 1 & 16: 35: 55 & 36.732 \\ 1 & 16: 37: 6 & 36.719 \\ 1 & 16: 42: 22 & 36.754 \\ 1 & 16: 45: 55 & 36.721 \\ 1 & 16: 52: 29 & 36.755 \\ 1 & 16: 52: 44 & 36.721 \\ 1 & 16: 53: 34 & 36.757 \\ 1 & 16: 53: 50 & 36.755 \\ 1 & 16: 54: 2 & 36.712 \\ 1 & 16: 54: 18 & 36.715 \\ 1 & 16: 58: 15 & 36.634\end{array}$

1 17: 2:11 36.170

\& 1 17: 2:15 36.72

1 17: $3: 28 \quad 36.729$

1 17: 5: 8 36.74

1 17: 6:46 36.732

$177: 17: 47 \quad 36.726$

$177: 17: 59 \quad 36.756$

$17: 22: 31 \quad 36.736$

$17: 22: 12 \quad 36.720$

$\begin{array}{lll}17: 25: 24 & 36.417\end{array}$

$1 \quad 17: 31: 36 \quad 36.716$

$17: 32: 52 \quad 36.742$ $17: 33: 54 \quad 36.739$

$17: 37: 18 \quad 36.736$

$17: 38: 18 \quad 37.093$

$17: 41: 18 \quad 36.756$

$17: 41: 44 \quad 36.737$

$1 \quad 17: 55: 49 \quad 36.731$

$\begin{array}{lll}17: 56: 27 & 36.754\end{array}$

$17: 56: 17$ 36.783

18: $0: 29 \quad 36.739$

$1 \quad 18: 17: 56 \quad 36.727$

$1 \quad 18: 20: 34 \quad 36.735$

$1 \quad 18: 28: 44 \quad 36.740$

$1 \quad 18: 30: 21 \quad 36.692$

\begin{tabular}{|c|c|c|c|c|}
\hline 116.256 & 0.1 & 8.80 & 0.1 & 76 \\
\hline 116.287 & 0.3 & 7.01 & 0.3 & 99 \\
\hline 116.290 & 0.2 & 9.93 & 0.2 & 84 \\
\hline 116.273 & 0.1 & 7.77 & 0.1 & 75 \\
\hline 116.264 & 0.8 & 9.69 & 0.7 & 175 \\
\hline 116.291 & 0.2 & 8.24 & 0.2 & 83 \\
\hline 116.287 & 0.2 & 8.37 & 0.2 & 90 \\
\hline 116.294 & 0.2 & 8.43 & 0.2 & 86 \\
\hline 116.278 & 0.6 & 8. 42 & 0.7 & 181 \\
\hline 116.272 & 0.2 & 8.16 & 0.3 & 46 \\
\hline 116.299 & 0.2 & 8.04 & 0.2 & 52 \\
\hline 116.263 & 0.3 & 7.69 & 0.3 & 121 \\
\hline 116.295 & 0.3 & 8.58 & 0.3 & 103 \\
\hline 116.246 & 0.2 & 8.11 & 0.2 & 101 \\
\hline 116.248 & 0.2 & 8.41 & 0.2 & 79 \\
\hline 116.272 & 0.2 & 7.26 & 0.2 & 74 \\
\hline 116.274 & 0.2 & 7.37 & 0.2 & 99 \\
\hline 116.245 & 0.2 & 7.40 & 0.3 & 129 \\
\hline 116.773 & 1.5 & 10.68 & 2.2 & 119 \\
\hline 116.297 & 0.1 & 8.63 & 0.1 & 89 \\
\hline 116.302 & 0.2 & 6.68 & 0.2 & 91 \\
\hline 116.271 & 0.4 & 8.15 & 0.3 & 173 \\
\hline 116.296 & 0.1 & 7.77 & 0.1 & 87 \\
\hline 116.272 & 0.1 & 8.34 & 0.1 & 74 \\
\hline 116.305 & 0.1 & 6.62 & 0.1 & 93 \\
\hline 116.269 & 0.1 & 8.12 & 0.1 & 72 \\
\hline 116.291 & 0.3 & 8.23 & 0.3 & 84 \\
\hline 116.302 & 0.2 & 8.17 & 0.2 & 92 \\
\hline 117.042 & 1.2 & 9.51 & 1.6 & 117 \\
\hline 116.299 & 0.2 & 9.16 & 0.2 & 90 \\
\hline 116.287 & 0.2 & 6.60 & 0.2 & 81 \\
\hline 116.290 & 0.1 & 7.97 & 0.1 & 53 \\
\hline 116.293 & 0.1 & 7.97 & 0.1 & 85 \\
\hline 117.178 & 0.5 & 4.97 & 4.3 & 82 \\
\hline 116.281 & 0.2 & 8.05 & 0.2 & 77 \\
\hline 116.297 & 0.1 & 7.24 & 0.2 & 87 \\
\hline 116.298 & 0.2 & 7.45 & 0.3 & 90 \\
\hline 116.267 & 0.2 & 8.45 & 0.5 & 89 \\
\hline 116.210 & 2.0 & 9.34 & 2.9 & 192 \\
\hline 116.293 & 0.1 & 7.62 & 0.1 & 54 \\
\hline 116.298 & 0.1 & 8.75 & 0.1 & 89 \\
\hline 116.292 & 0.1 & 8.28 & 0.2 & 85 \\
\hline $\begin{array}{l}116.288 \\
116.344\end{array}$ & $\begin{array}{l}0.3 \\
0.7\end{array}$ & $\begin{array}{l}7.57 \\
1.90\end{array}$ & $\begin{array}{l}0.3 \\
1.4\end{array}$ & $\begin{array}{l}82 \\
94\end{array}$ \\
\hline
\end{tabular}

DEL- RUS IN

MIM RES. PE. O.S.G.S.

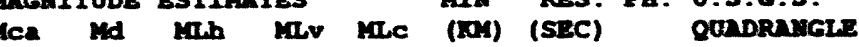

$1.9 \quad 2.6 \quad 0.0319$ GeORGss matra

$\begin{array}{llllll}2.04 & 1.9 & 2.6 & 0.03 & 19\end{array}$

$\begin{array}{llllllll}0.72 & 0.82 & & 1.4 & 0.05 & 15 & \text { STRIPED BII.TS } \\ 1.81 & 1.30 & 1.6 & 2.9 & 0.04 & 19 & \text { STRIPED BILLS }\end{array}$

$\begin{array}{lllllll}1.81 & 1.30 & 1.6 & 2.9 & 0.04 & 19 & \text { STRIPED HIWS } \\ 0.93 & 0.90 & & 3.0 & 0.03 & 17 & \text { STRIPED BILLS }\end{array}$

$0.720 .66 \quad 0.90 .1315$ STRIPED BILLS

$1.060 .90 \quad 1.70 .0516$ SIRIPED BIILS

$\begin{array}{lllllll}1.69 & 1.42 & 1.6 & 1.4 & 0.05 & 19 & \text { STRIPED BILLS } \\ 1.87 & 1.43 & 1.6 & 2.3 & 0.05 & 19 & \text { STRTPED BIILS }\end{array}$

$\begin{array}{llllll}0.77 & 0.96 & 2.6 & 0.11 & 15 & \text { SIRTPED BITS }\end{array}$

$\begin{array}{llllllll}2.44 & 2.37 & 2.4 & 1.2 & 0.11 & 44 & \text { GBORGR MATRR }\end{array}$

$3.20 \quad 3.373 .40 .0954$ STRTPRD

$\begin{array}{lllllll}3.20 & 0.77 & 0.77 & 3.2 & 3.4 & 0.09 & 54\end{array}$

$\begin{array}{llllll}1.30 & 0.85 & 3.1 & 0.07 & 18 & \text { SIRIPED BILLS }\end{array}$

$\begin{array}{llllll}0.96 & 0.62 & 2.8 & 0.0415 & \text { SKOLL Mrn }\end{array}$

$\begin{array}{llllllll}1.18 & 1.12 & 1.3 & 2.5 & 0.04 & 20 & \text { SKOLL MTM }\end{array}$

$\begin{array}{lllllll}1.51 & 1.19 & 1.5 & 3.4 & 0.05 & 18 & \text { STRIPED BILLS }\end{array}$

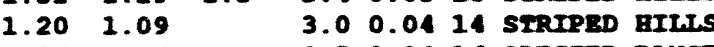

$\begin{array}{llll}0.76 & 0.96 & 8.5 & 0.0416 \text { SPECTER RUMG }\end{array}$

$\begin{array}{llll}1.31 & 1.41 & 9.40 .269 \text { Badenter }\end{array}$

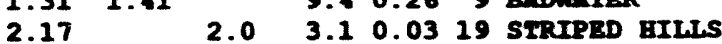

$\begin{array}{lllllll}0.90 & 1.05 & 3.1 & 0.04 & 17 & \text { STRIPED BILLS }\end{array}$

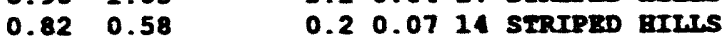

$\begin{array}{lllll}1.67 & 1.37 & 2.40 .0320 & \text { SIRIPED BILLS }\end{array}$

$0.981 .25 \quad 1.00 .0320$ GEORER vITER

$\begin{array}{llllll}1.33 & 0.78 & 3.5 & 0.0219 & \text { STRIPED BILLS }\end{array}$

$\begin{array}{llll}1.48 & 1.47 & 1.5 & 0.0216 \text { GeOReRs MATER }\end{array}$

$1.471 .36 \quad 1.80 .0821$ STRIPED EILLS

$\begin{array}{lllllll}1.90 & 1.57 & 1.6 & 3.7 & 0.05 & 18 & \text { SIRIPED BILLS }\end{array}$

$\begin{array}{llllll}1.27 & 1.2 & 5.9 & 0.09 & 9 & \text { TOCKa mas }\end{array}$

$\begin{array}{llllll}1.98 & 2.1 & 3.8 & 0.04 & 17 & \text { STRTPED BILLS }\end{array}$

$\begin{array}{llllll}1.55 & 1.28 & 1.3 & 0.0418 \text { STRIPED EILS }\end{array}$

$\begin{array}{lllllll}2.08 & 1.52 & 2.0 & 1.7 & 0.03 & 24 & \text { STRIPBD BILLS }\end{array}$

$\begin{array}{lllllll}1.78 & 1.38 & 1.7 & 2.0 & 0.03 & 21 & \text { STRIPED GILLS }\end{array}$

$1.141 .25 \quad 17.70 .1413$ BOMnIB CLaIRE SW

$0.83 \quad 0.95 \quad 1.70 .0518$ GRORGES UATRR

$\begin{array}{llllll}0.82 & 0.60 & 2.7 & 0.04 & 15 & \text { STRIPED HILLS }\end{array}$

$\begin{array}{llllll}0.91 & 0.86 & 1.4 & 0.0514 & \text { GBOREs WATER }\end{array}$

$\begin{array}{llllll}0.91 & 0.86 & 1.4 & 0.05 & 14 \\ 0.67 & 0.50 & 7.2 & 0.22 & \text { STOLI MTI }\end{array}$

$\begin{array}{lllllll}2.06 & 1.69 & 1.6 & 1.9 & 0.03 & 26 & \text { STRIPED BILLS }\end{array}$

$2.01 \quad 1.431 .6 \quad 2.90 .0319$ STRIPED BILLS

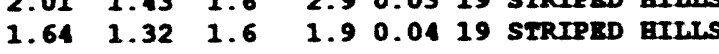

$\begin{array}{llllll}0.83 & 0.91 & 1.4 & 0.09 & 19 & \text { STRIPED GILLS } \\ 0.80 & 5.1 & 0.11 & 10 & \text { STRIPED GILLS }\end{array}$ 
1992 LOCAL HYPOCBNTERR SUAAQRY - SGB EARTBQUNKRS

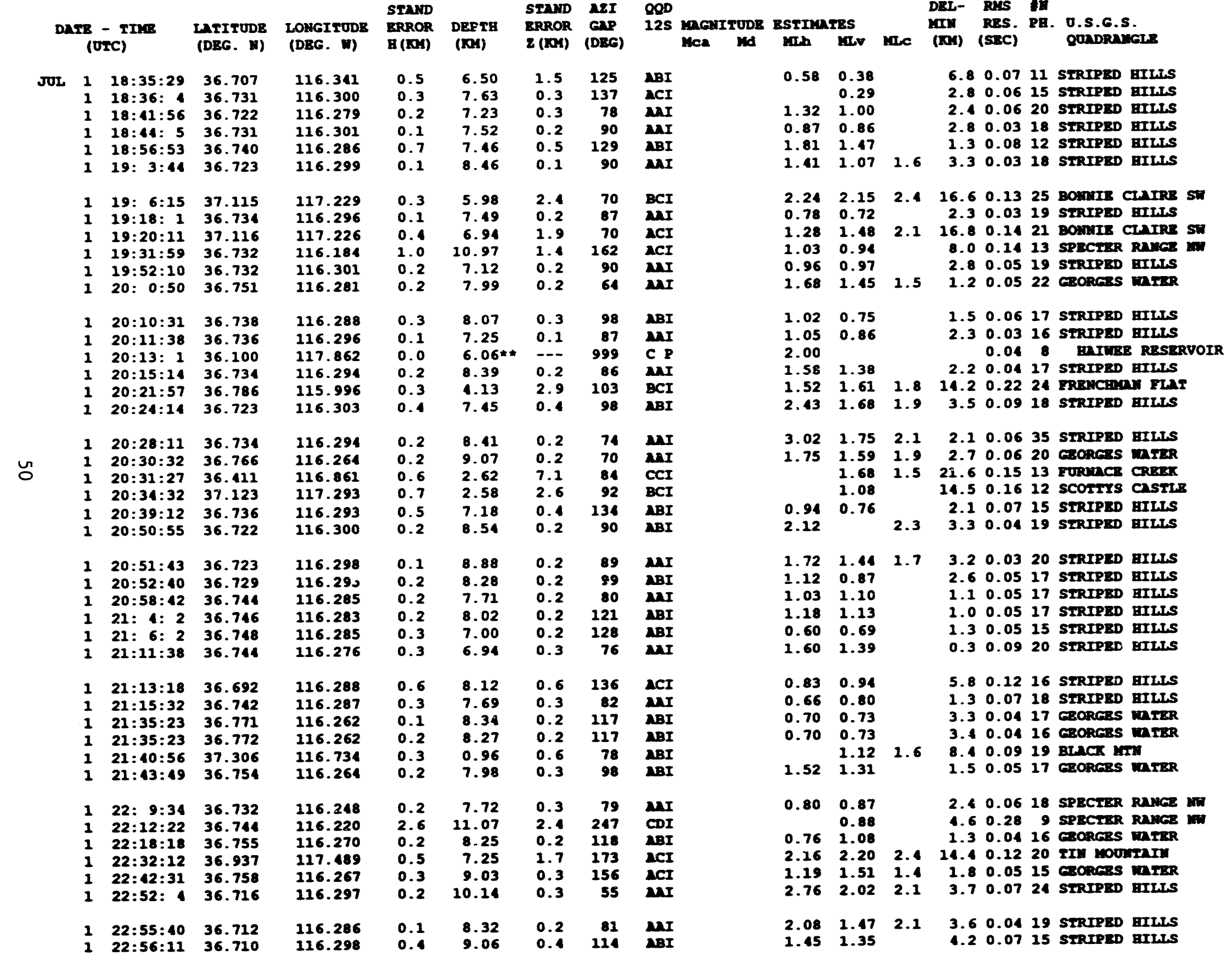


1992 LOCAL HYPOCENTER SUMAARY - SGB EARTHQUAKES

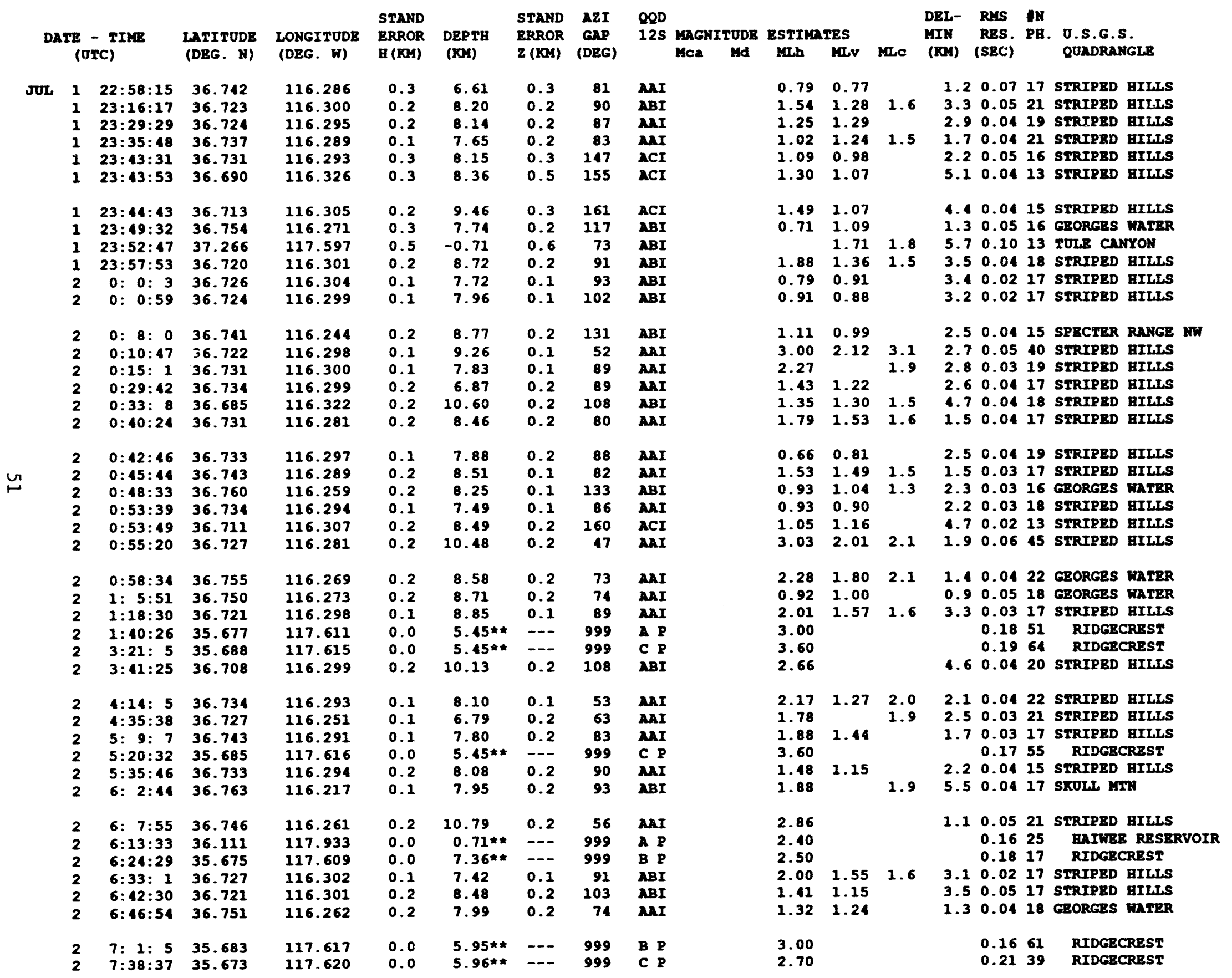


1992 LOCAL HYPOCENTER SUMAARY - SGB EARTHQUAKRS

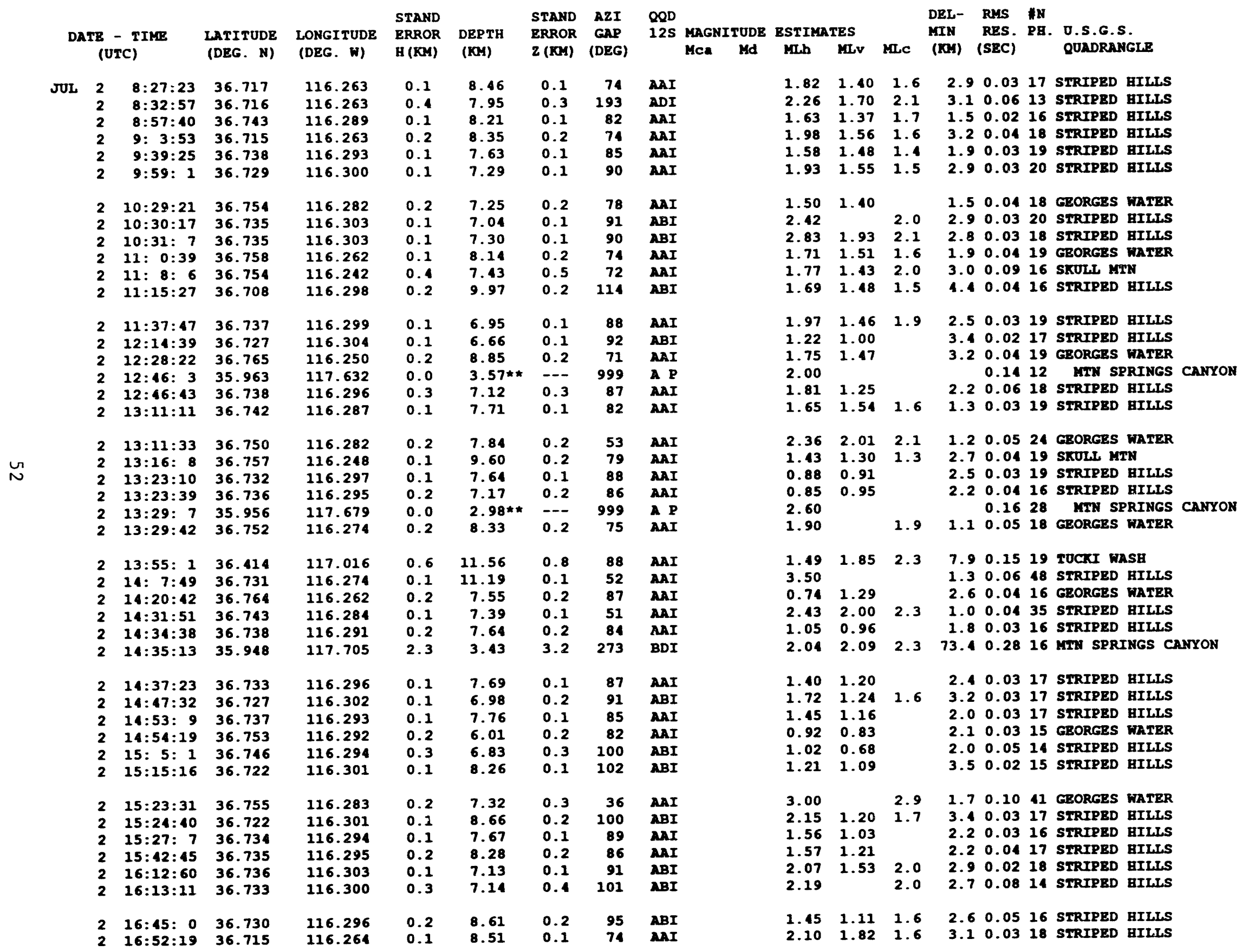


1992 LOCAL HYPOCENTER SUMAMRY - SGB RARTHQUAKES

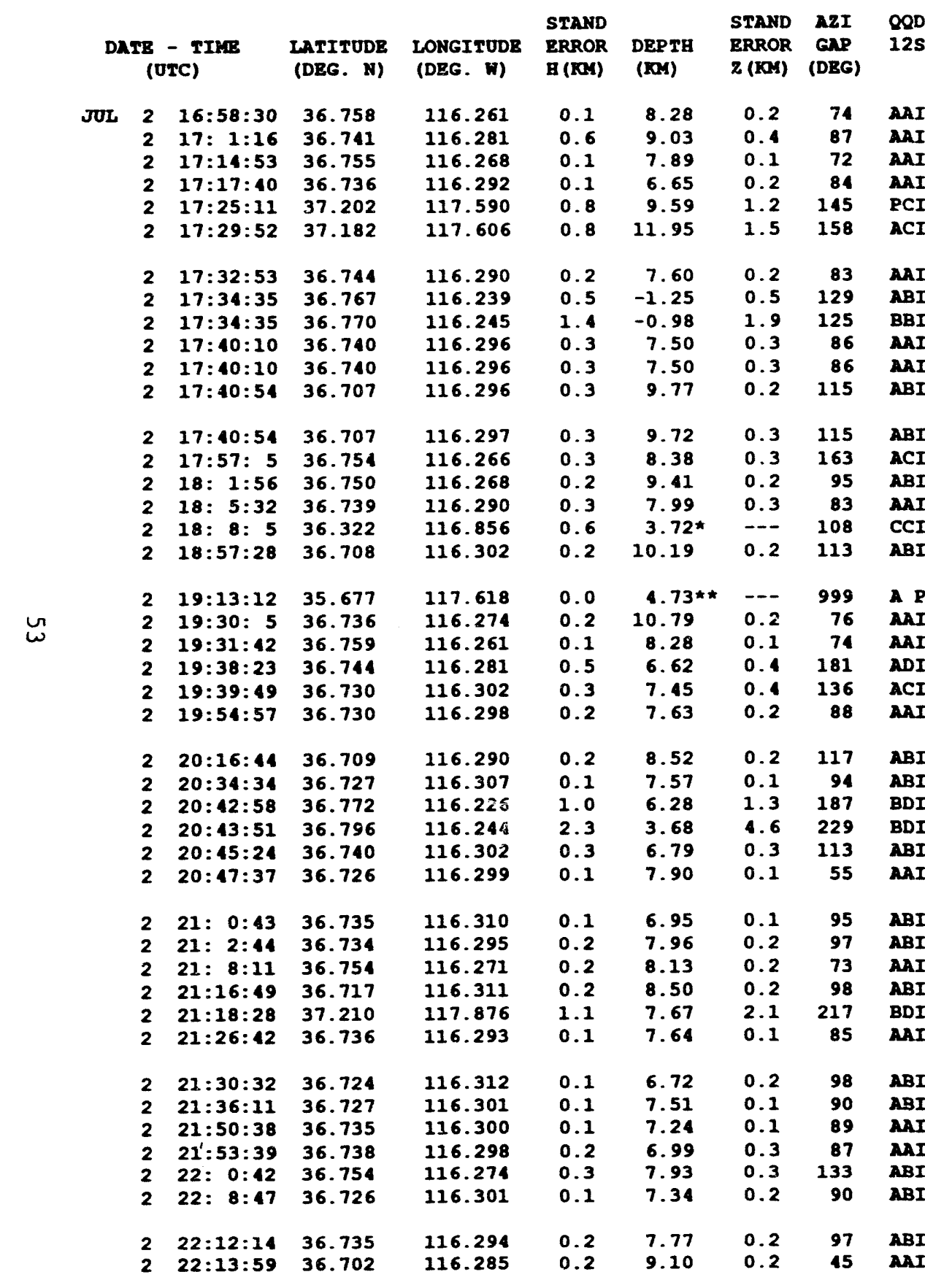

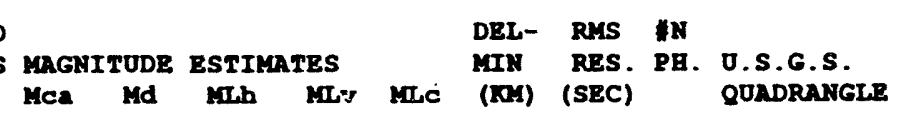

AI

AAI (1) AI II I

BI
1 CI

$\mathbf{P}$

I

II

I

(1)
$\begin{array}{lllllll}1.81 & 1.45 & 1.9 & 2.0 & 0.04 & 19 & \text { GEORGES WATER } \\ 0.95 & 1.01 & & 0.8 & 0.10 & 16 & \text { STRIPED BITSS }\end{array}$ $\begin{array}{lllll}0.95 & 1.01 & 0.8 & 0.10 & 16 \\ 0.98 & 1.19 & 1.5 & 0.0316 \text { GEORGES WATER }\end{array}$
$\begin{array}{lllllll}1.64 & 1.39 & 1.6 & 1.9 & 0.04 & 17 & \text { STRIPED BILLS }\end{array}$
$\begin{array}{llllll}1.36 & 1.73 & 1.7 & 6.1 & 0.2017 \text { SAND SPRTNG }\end{array}$ $\begin{array}{llllll}2.22 & 2.6 & 6.7 & 0.13 & 12 & \text { SAND SPRING }\end{array}$

$1.29 \quad 1.10$

$1.84 \quad 1.30$

1.841 .01

0.890 .73

0.890 .73

0.990 .76

$0.99 \quad 0.76$

$0.83 \quad 1.05$

0.831 .05

$1.21 \quad 1.18$

$\begin{array}{ll}1.43 & 1.33 \\ 1.27 & 1.32\end{array}$

$\begin{array}{lll}1.27 & 1.32\end{array}$

$0.40 \quad 0.92$

$\begin{array}{lll}3.20 & & \\ 1.60 & 1.44 & 1.6\end{array}$

$1.60 \quad 1.44$

0.840 .78

$0.50 \quad 0.95$

$1.46 \quad 1.21$

$0.68 \quad 0.93$

$\begin{array}{llll}0.68 & 0.93 & 1.10 .0416 \text { STRIPED BILLS }\end{array}$

\begin{tabular}{llllll}
1.65 & 1.34 & 1.5 & 3.6 & 0.03 & 17 \\
\hline
\end{tabular}

$1.25 \quad 0.92$

$\begin{array}{llllll}0.94 & 1.03 & 6.5 & 0.11 & 7 & \text { SKOLL MTN } \\ 0 & 2.7 & 0.05 & 14 & \text { STRIPED BILLS }\end{array}$

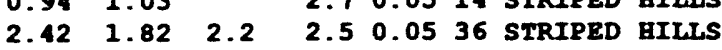

$\begin{array}{lllllll}1.39 & 1.19 & 1.9 & 3.5 & 0.02 & 17 & \text { STRIPED BILLS }\end{array}$

$\begin{array}{llllll}1.17 & 0.86 & 2.3 & 0.04 & 16 & \text { STRIPED HILLS }\end{array}$

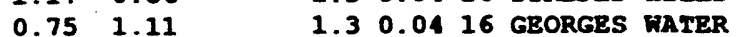

$1.681 .33 \quad 1.50 .0518$ STRTPED GITRS

1.681 .33 T.5 0.0518 SIRIPED BILLS

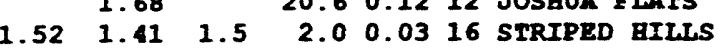

$\begin{array}{lllllll}1.48 & 1.06 & 1.5 & 1.1 & 0.03 & 17 & \text { STRIPED BILS }\end{array}$

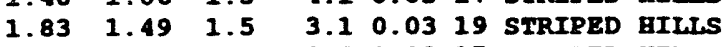

$\begin{array}{llllll}1.91 & 2.0 & 2.7 & 0.02 & 17 & \text { STRIPED BILLS }\end{array}$

$\begin{array}{llllll}0.80 & 0.77 & 2.3 & 0.05 & 16 & \text { STRIPED BILLS }\end{array}$

$\begin{array}{llllll}0.78 & 0.75 & 1.3 & 0.0515 & \text { GEORGES MATER }\end{array}$

$\begin{array}{lllll}1.36 & 1.06 & 3.2 & 0.0316 & \text { STRIPED BILLS }\end{array}$

$\begin{array}{llllll}0.84 & 0.79 & 2.1 & 0.04 & 16 & \text { STRIPED HILLS }\end{array}$

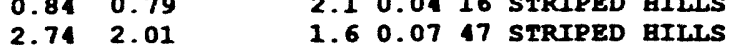




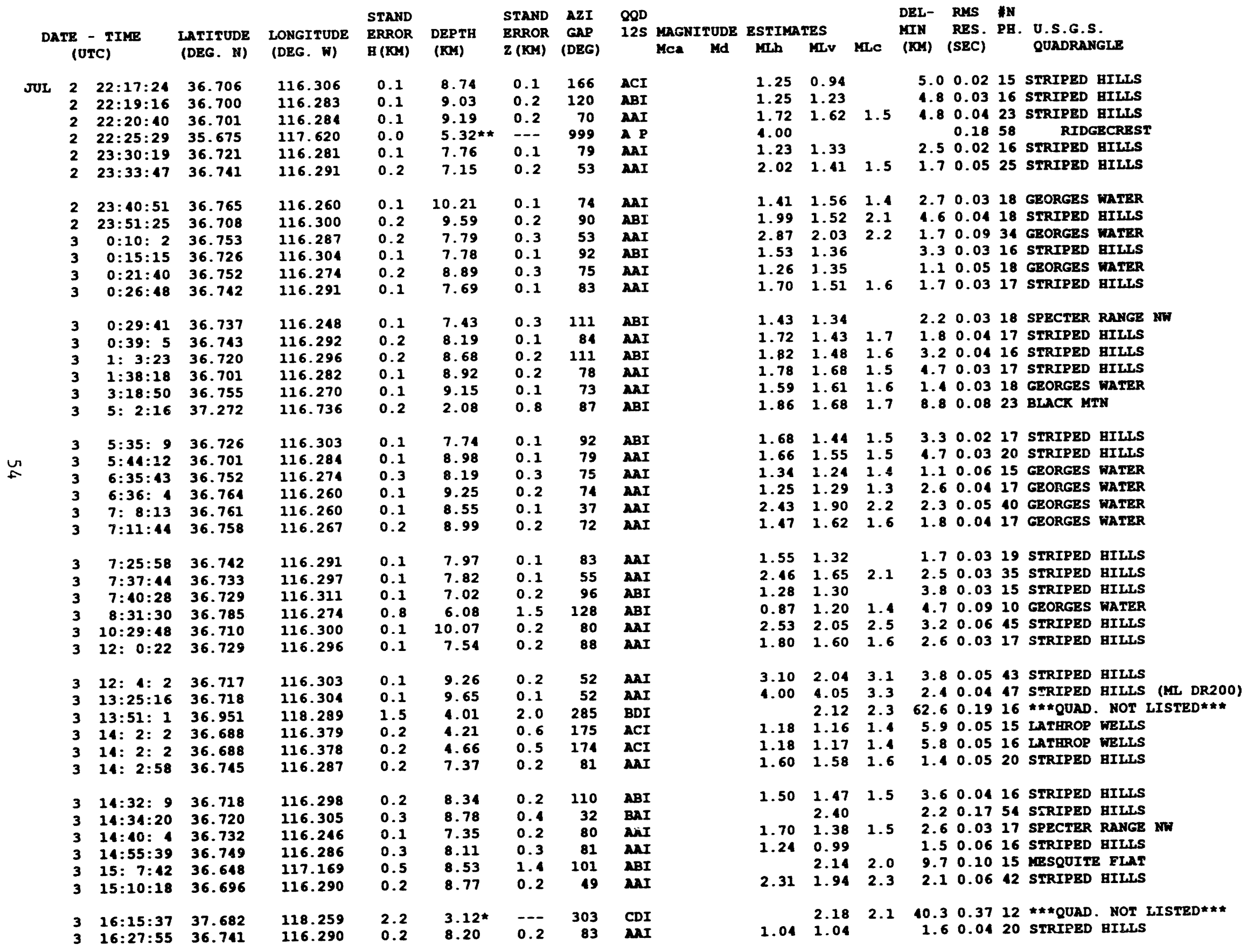


1992 LOCAL HYPOCENTER SUMAARY - SGB EARTHQUAKRS

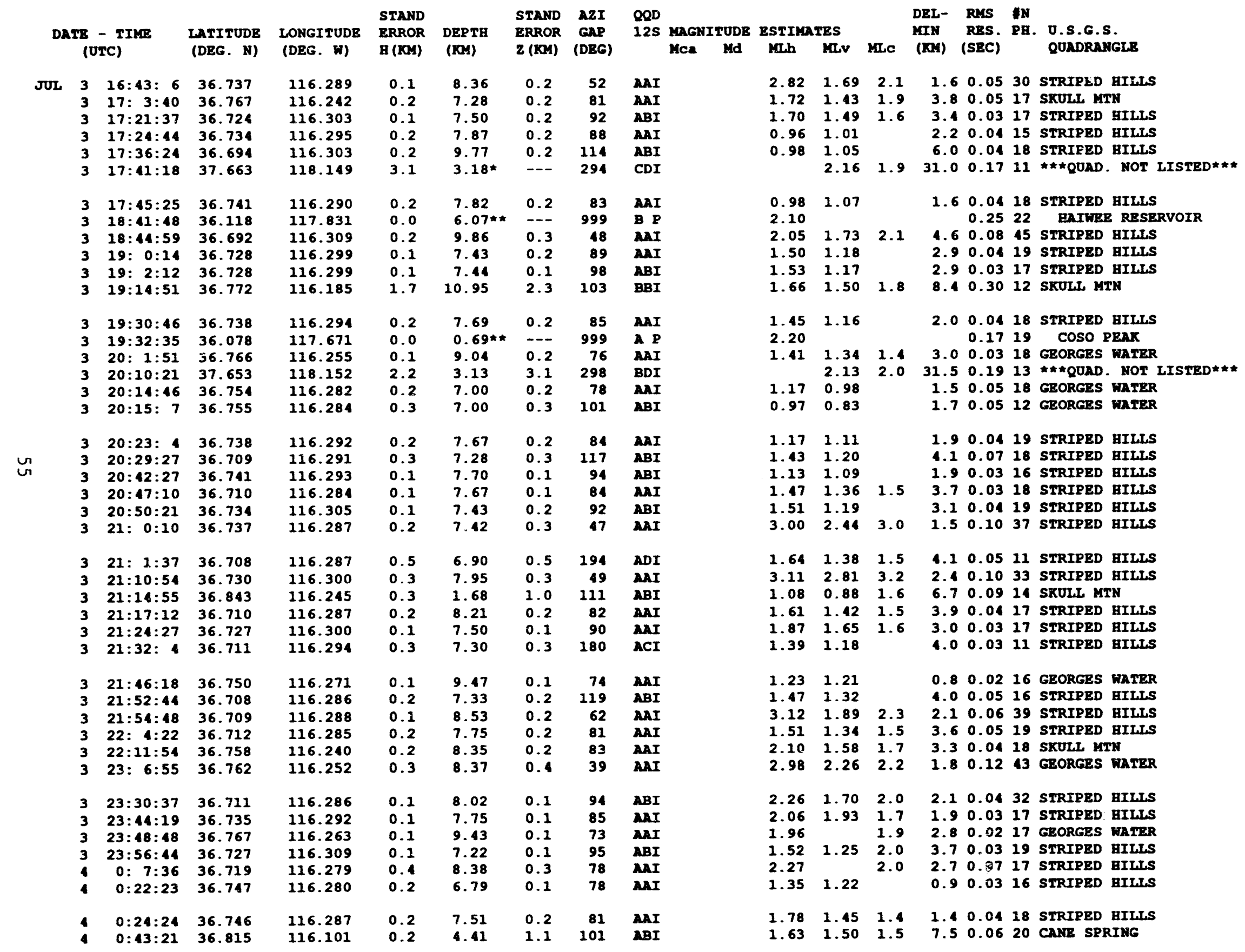


1992 LOCAL HYPOCENTER SUMOMARY - SGB EARTHQUAKES

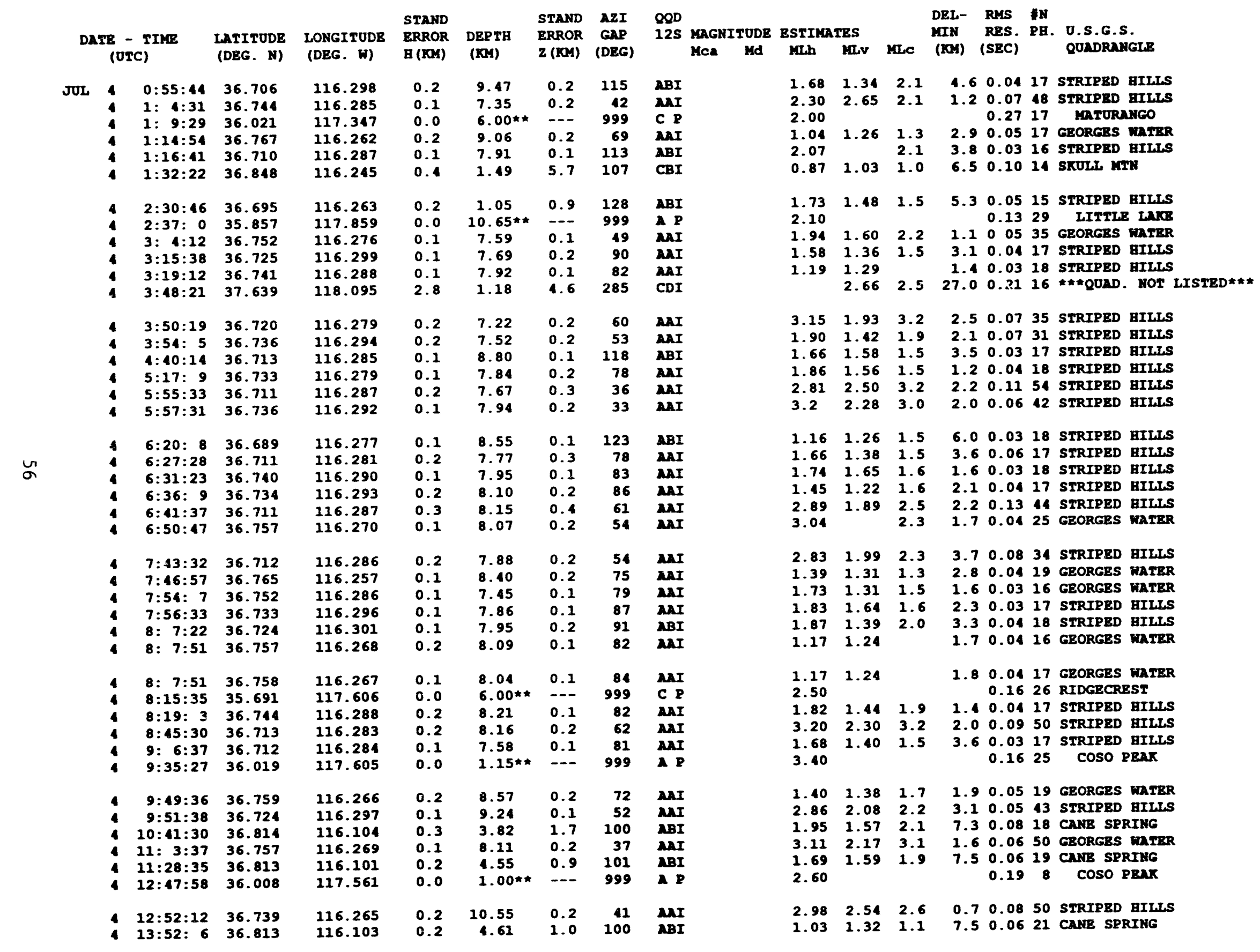




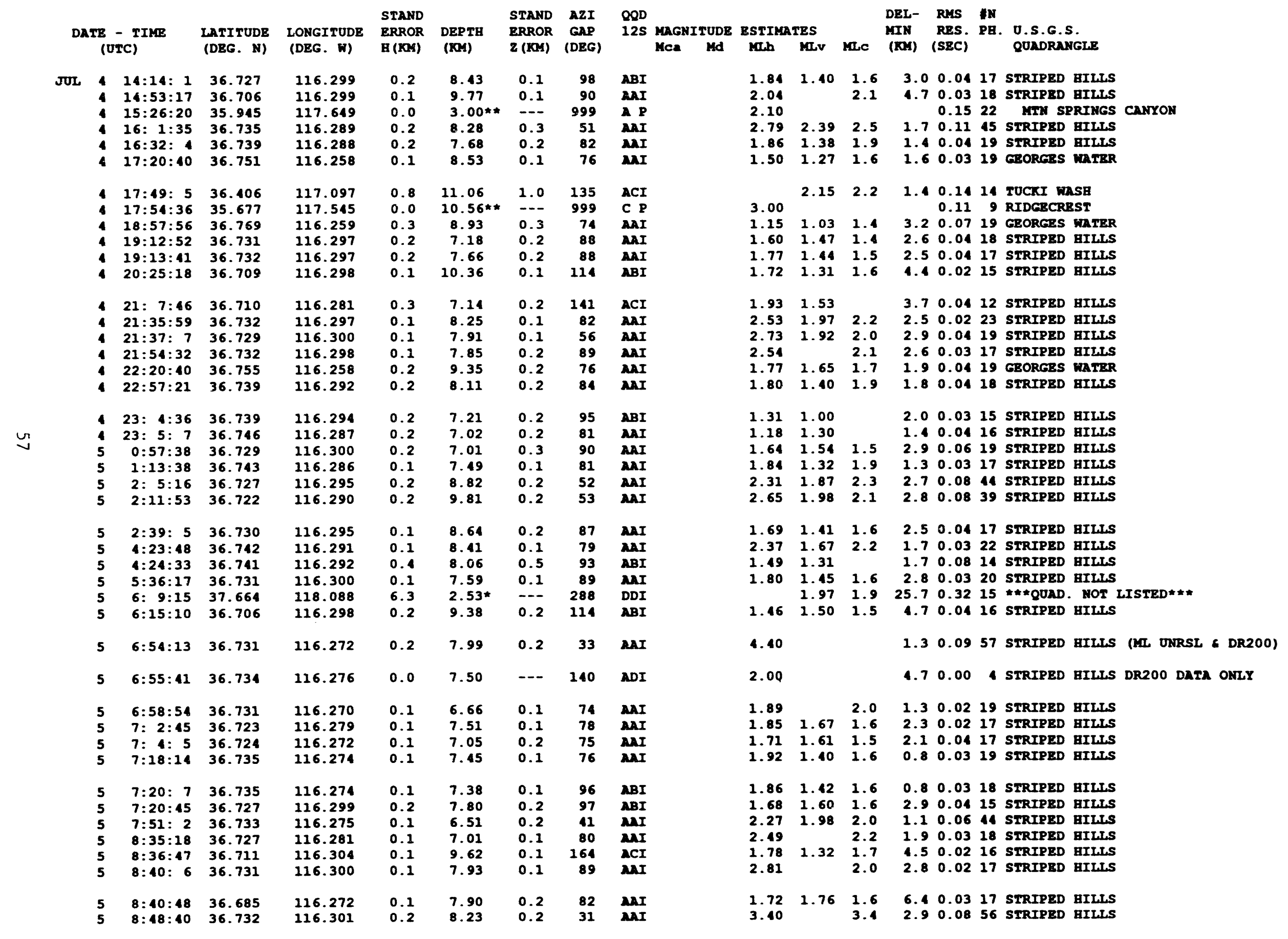


1992 LOCAL HYPOCENTER SUROARY - SGB RARTHQUAKES

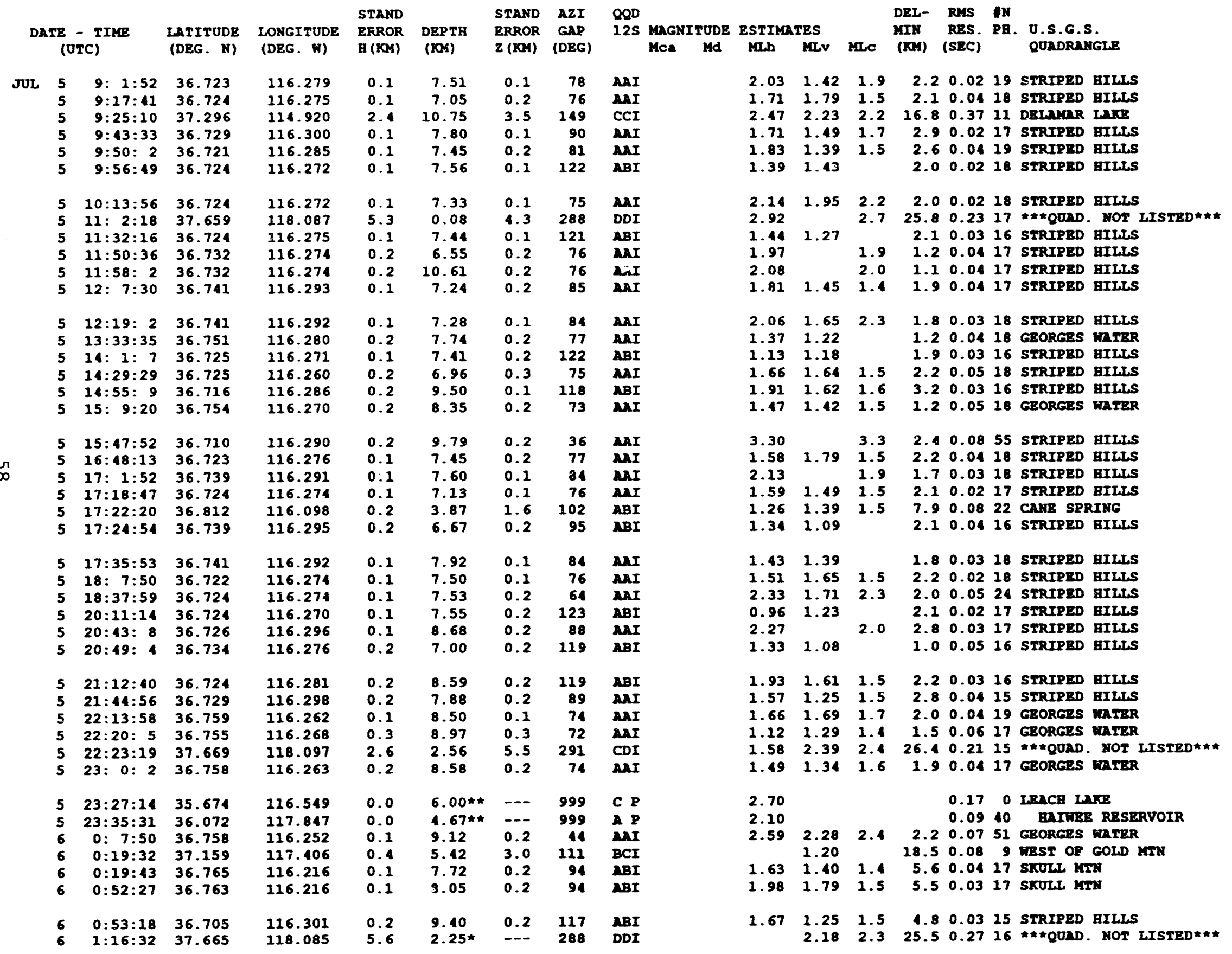


1992 LOCAL HYPOCENTER SURMARY - SGB EARTHQUAKES

DATE - TIME LATITUDE LONGITUDE STAND (OTC)

LATITUDE LONGITUDE

10.67

JuL.

$$
\begin{array}{ll}
1: 11: 21 & 35.679 \\
3: 1: 35 & 36.731 \\
3: 50: 34 & 36.732 \\
3: 58: 10 & 36.719 \\
5: 11: 13 & 36.753
\end{array}
$$

$5: 49: 32 \quad 36.741$

$6: 14: 40 \quad 36: 741$ $6: 31: 33 \quad 36.712$ $7: 3: 48 \quad 36.583$ $7: 14: 36.722$ $7: 57: 25 \quad 36.714$ $8: 39: 9 \quad 36.724$

$6 \quad 9: 3: 51 \quad 36.739$ $9.9 .32 \quad 36.724$ $9: 10: 14 \quad 36.744$ $9: 43: 38 \quad 36.729$ 9:48: $5 \quad 36.755$ $10: 6: 1136.715$

\subsection{3}

116.274

116.288

116.307

116.288

116.292

116.291

116.282

116.250

116.273

116.286

116.300

116.308

116.274

116.236

116.245

116.269

116.304

117.607
116.262

116.262
116.301
116.241

116.290

116.280

$12: 21: 51 \quad 36.734$

$\begin{array}{lll}6 & 13: 11: 54 \quad 37.638\end{array}$

$6 \quad 13: 25: 15 \quad 36.055$

$6 \quad 13: 25: 15 \quad 36.058$

13:39: 736.726

$6 \quad 13: 39: 13 \quad 36.739$

$6 \quad 13: 10: 45 \quad 36.728$

6 13:46: $2 \quad 36.737$

$6 \quad 13: 50: 25 \quad 37.068$

6 14: $5: 3136.728$

6 14:13: 136.739

14:29:41 36.713

6 14:38:32 36.753

$6 \quad 14: 43: 46 \quad 36.757$ $6 \quad 15: 21: 46 \quad 37.784$

$6 \quad 15: 28: 14 \quad 37.672$

$6 \quad 15: 13: 45 \quad 36.589$

15:59: 6 36.734

6 16:3:18 35.715

6 16: 8:19 36.067

118.100 117.579 117.604 116.274 116.310 116.274

116.296 117.932 116.275 116.275 116.292 116.301
116.270

116.273

118.527

118.128

116.233

116.298

117.023
STAND STAND AZI QQD DRROR DEPTE ERROR GNP 125 MENITUDE RSTIMATES $\begin{array}{rlrrr}0.0 & 6.00 * * & -- & 999 & \text { C P } \\ 0.2 & 7.40 & 0.2 & 76 & \text { MNI }\end{array}$ $\begin{array}{lllll}0.2 & 9.34 & 0.3 & 101 & \text { ABI }\end{array}$ $\begin{array}{lllll}0.2 & 7.84 & 0.2 & 95 & \mathrm{ABI}\end{array}$ $\begin{array}{lllll}0.2 & 7.77 & 0.2 & 40 & \mathrm{MNI}\end{array}$

$\begin{array}{lllll}0.2 & 8.12 & 0.2 & 84\end{array}$ $\begin{array}{lllll}0.2 & 8.88 & 0.2 & 119 & A B I\end{array}$ $\begin{array}{lllll}0.2 & 8.32 & 0.7 & 68 & \mathrm{ABI} \\ 0.1 & 7.37 & 0.1 & 75 & \mathrm{MAI}\end{array}$ $\begin{array}{lllll}0.1 & 8.66 & 0.1 & 81 & \mathrm{MAI}\end{array}$ $\begin{array}{lllll}0.2 & 8.10 & 0.2 & 90 & \text { ABI }\end{array}$

$\begin{array}{lllll}0.3 & 6.32 & 0.3 & 112 & \text { ABI }\end{array}$ $\begin{array}{lllll}0.1 & 7.30 & 0.2 & 95 & \mathrm{ABI}\end{array}$

$6.31 * *$

8.35

6.76
7.73

8. 31

8.31
6.58

0.3

0.1

0.2

C P

ABI

MaI

ABI

$\begin{array}{lllll}3.4 & 1.45 & 6.6 & 286 & \mathrm{CDI}\end{array}$

$\begin{array}{llll}1.45 & 6.6 & 286 & \text { CDI } \\ 0.54 * * & --- & 999 & \text { A P }\end{array}$

$\begin{array}{llll}3.57 & 3.4 & 259 & B D I\end{array}$

$\begin{array}{llll}7.53 & 0.1 & 76 & \text { ANI }\end{array}$

$\begin{array}{rrrr}2.79 & 0.6 & 134 & \text { ABI } \\ 7.56 & 0.2 & 76 & \text { AAI }\end{array}$

ABI

$\begin{array}{rlrrr}0.2 & 7.21 & 0.2 & 95 & \mathrm{ABI} \\ 1.3 & 3.11 * & --- & 228 & \mathrm{CDI}\end{array}$

$\begin{array}{lllll}0.1 & 7.26 & 0.2 & 107 & \text { ABI }\end{array}$

$\begin{array}{lllll}0.1 & 7.62 & 0.1 & 84 & \mathrm{MAI}\end{array}$

$\begin{array}{lllll}0.2 & 9.28 & 0.3 & 91 & \text { ABI }\end{array}$

$\begin{array}{llllll}0.2 & 8.13 & 0.2 & 74 & \text { MAI }\end{array}$

$\begin{array}{lllll}3.3 & 3.11 & 1.6 & 327 & \mathrm{CDI}\end{array}$

3.0 3.29* --- 294 CDI

$\begin{array}{rrrrr}0.3 & 0.46 & 0.7 & 131 & \text { ACI } \\ 0.2 & 8.03 & 0.2 & 88 & \text { AAT }\end{array}$

$0.0 \quad 6.00 * \pi-2999$ C P

$\begin{array}{llllll}117.058 & 7.1 & 2.82 * & -- & 311 & \text { DDI } \\ 117.615 & 3.6 & 2.54 * & -- & 259 & \text { CDI }\end{array}$
DEL- RMS IN

RES PH. U.S.G.S Hea Md MLh MLV MLC (NOS) (SEC) QOADRANGLE 
1992 LOCAL HYPOCENTRR SUAYARY - SGB EARTHQUAKES

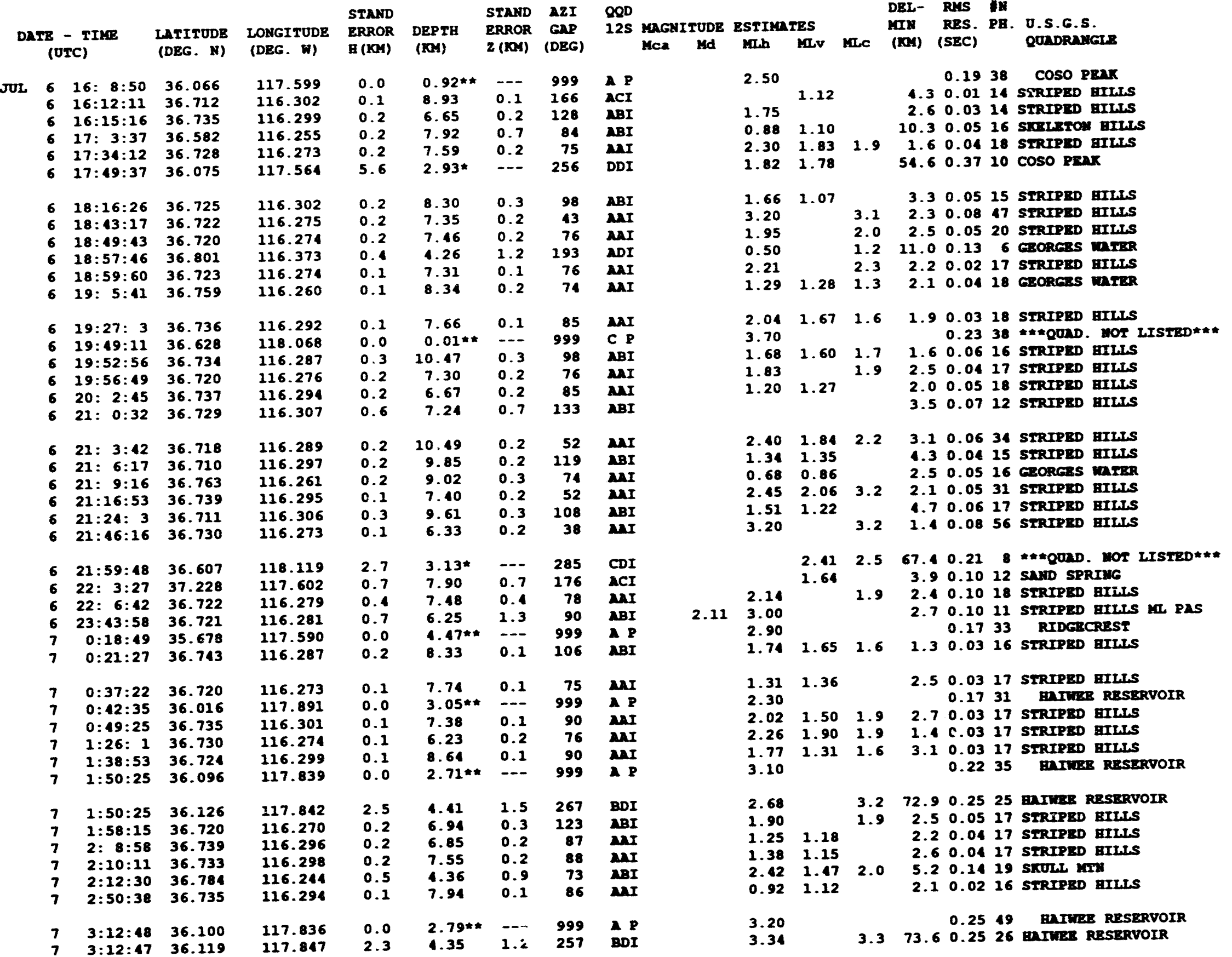


1992 LOCAL HYPOCENTER SURARY - SGB EARTHQOAKES

STAND

DATE - TIMR LATITUDE LONGITUDE ERROR (UrC)
(DEG. N) (DEG

JOL 7

3:28: 136.741 5: $9: 17 \quad 36.707$ $5: 12: 10 \quad 36.722$ $5: 12: 42 \quad 36.720$ $5: 42: 29 \quad 36.709$ 5:17: 36.712

$6: 28: 23 \quad 36.722$

$6: 12: 32 \quad 36.737$

$7: 35: 10 \quad 36.752$

$7: 57: 48 \quad 36.737$

$8: 13: 29 \quad 36.056$

$8: 14: 48 \quad 36.755$

$8: 51: 43 \quad 36.707$

$9: 1: 52 \quad 36.720$

$9: 11: 10 \quad 36.739$

$9: 28: 46 \quad 36.721$

10:8: 9 36.647

7 10:35:55 36.732

7 11:22: $1 \quad 36.724$

$7 \quad 11: 12: 10 \quad 36.710$

7 12:28:42 36.742

7 12:49:8 36.750

7 13: $1: 25 \quad 36.746$

7 13: 7:59 36.755

$7 \quad 13: 32: 20 \quad 36.581$

7 13:41:24 36.580

7 13:50: $8 \quad 36.579$

$7 \quad 14: 11: 58 \quad 36.734$

$7 \quad 14: 42: 13 \quad 37.663$

7 15:21:55 36.724

$7 \quad 15: 24: 35 \quad 36.647$

$17: 20: 55 \quad 36.578$

19: 2:39 36.752

$19: 43: 22 \quad 36.688$

$721: 18: 10 \quad 36.736$

$721: 57: 38 \quad 36.57$

7 22: 4:15 36.580

$722: 5: 38 \quad 36.580$

7 22: 7:19 36.580

$722: 35: 14 \quad 36.741$

$7 \quad 22: 52: 52 \quad 36.581$

0 0:24:30 36.741

$\begin{array}{lll}8 & 0: 24: 17 & 36.741 \\ 8 & 0: 56: 16 & 36.580\end{array}$
116.293

116.299

116.274

116.275

116.298

116.304

116.299

116.293

116.275

116.287

117.604

116.269

116.298

116.275

116.291

116.271

116.265

116.297

116.302

116.284
116.293

116.289

116.290
116.269

116.254

116.250

116.253
116.292

118.086

116.297

118.076

116.260

116.271

116.309

116.292

116.254

116.254

116.255

116.304

116.289

116.290

116.254
(XE) DEPT

0.17 .09

0.38 .87

0.3

7.33
6.59

6.59
10.20

$\begin{array}{rr}0.2 & 10.20 \\ 0.2 & 9.15\end{array}$

$0.1 \quad 8.49$

0.2

0.1

$1.4 \quad 5.97$

$0.2 \quad 9.19$

$0.1 \quad 7.44$

0.17 .69

0.36 .18

$0.1 \quad 8.10$

$0.2 \quad 7.39$

0.17 .10

0.37 .79

7.79
8.39

\subsection{6 .75}

0.1

0.1

8.29

$2.10 *$

STAND AZI OOD

I2S MGNTTODE RSTIMTES DEL- RMS IN

2 (IOY) (DEG)

IIN Res.

U.S.G.s.

\begin{tabular}{|c|c|}
\hline 0.1 & 85 \\
\hline 0.3 & 90 \\
\hline 0.3 & 76 \\
\hline 0.3 & 112 \\
\hline 0.2 & 89 \\
\hline 0.3 & 161 \\
\hline 0.1 & 56 \\
\hline 0.3 & 85 \\
\hline 0.1 & 81 \\
\hline 0.1 & 82 \\
\hline 2.0 & 260 \\
\hline 0.2 & 72 \\
\hline 0.2 & 114 \\
\hline 0.1 & 121 \\
\hline 0.2 & 84 \\
\hline 0.1 & 74 \\
\hline 0.6 & 121 \\
\hline 0.2 & 88 \\
\hline
\end{tabular}

$0.291 \quad A B I$

0.3 84

$0.252 \quad \mathrm{MI}$

0.181 ANI

$\begin{array}{lll}0.2 & 73 & \text { NaI }\end{array}$

$0.06 .00 * \star$

0.27 .17

0.2

$0.7 \quad 7.33$

0.18 .20

0.17 .36

0.27 .62

0.26 .93

$0.3 \quad 6.76$

$\begin{array}{ll}0.1 & 8.35 \\ 0.2 & 8.11\end{array}$

$0.9 \quad 85 \quad \mathrm{ABI}$

$\begin{array}{lll}0.6 & 53 & \mathrm{ABI} \\ 1.4 & 71 & \mathrm{ABI}\end{array}$

$\begin{array}{lll}0.1 & 98 & A B I\end{array}$

$--288 \quad C D I$

--- 999 C P

$0.8 \quad 86 \quad 2 B I$

$\begin{array}{lrl}0.2 & 74 & \text { MAI } \\ 0.9 & 182 & \text { ADI }\end{array}$

$\begin{array}{lll}0.2 & 85 & \text { AMI } \\ 0.7 & 82 & \text { ABI }\end{array}$

$\begin{array}{lll}0.9 & 89 & \text { ABI }\end{array}$

0.7 B ABI

$\begin{array}{lll}0.8 & 89 & \text { ABI }\end{array}$

$0.1 \quad 110 \quad$ ABI

$\begin{array}{lll}0.7 & 85 & \text { MBI } \\ 0.2 & 83 & \mathrm{MII}\end{array}$

$\begin{array}{lllll}0.3 & 7.67 & 0.3 & 83 & \mathrm{MAI} \\ 0.2 & 6.35 & 0.8 & 89 & \mathrm{MBI}\end{array}$ $\begin{array}{lllllll}2.03 & 1.43 & 1.9 & 1.9 & 0.03 & 17 & \text { SIRIPED GILlS }\end{array}$ $\begin{array}{llllll}1.42 & 1.37 & 4.6 & 0.0717 \text { STRIPED BIIS }\end{array}$ $\begin{array}{lllll}1.10 & 1.20 & 2.30 .0719 \text { STRIPED BILLS }\end{array}$ $\begin{array}{llll}0.99 & 0.85 & 2.5 & 0.0616 \text { SIRIPED EILLS }\end{array}$ $1.561 .28 \quad 4.0418$ STRTPED BILLS $1.531 .60 \quad 4.50 .0415$ SIRIPID BILLS

$2.24 \quad 2.0 \quad 3.30 .0321$ STRIPED arLLS $\begin{array}{lllllll}1.49 & 1.30 & 1.7 & 2.0 & 0.07 & 17 & \text { SIRIPED BILLS }\end{array}$ $\begin{array}{lllllll}1.48 & 1.67 & 1.6 & 1.1 & 0.03 & 16 & \text { GeORERS WNTER }\end{array}$ $\begin{array}{lllllll}1.90 & 1.82 & 2.0 & 1.5 & 0.02 & 17 & \text { SIRIPED BILIS }\end{array}$ $\begin{array}{llllllll}2.41 & 2.43 & 2.5 & 58.8 & 0.22 & 20 & \text { coso Pan }\end{array}$

$1.471 .451 .9 \quad 1.40 .0418$ Georess miter

$1.08 \quad 1.18$

$1.10 \quad 1.16$

1.121 .23

$\begin{array}{lllllll}1.47 & 1.51 & 1.4 & 1.7 & 0.0418 & \text { SIRIPED BILLS }\end{array}$

$\begin{array}{lllllll}1.16 & 1.01 & 1.5 & 2.4 & 0.02 & 17 & \text { STRIPED BILLS } \\ 1.5 & 0.08 & 18 & \text { STRIPED BILIS }\end{array}$

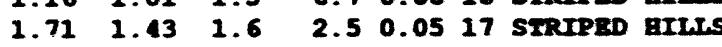

$\begin{array}{llllll}2.02 & 1.42 & 2.0 & 3.4 & 0.0317 & \text { STRIPED GILLS }\end{array}$

$\begin{array}{lllllll}1.71 & 1.39 & 1.5 & 3.8 & 0.05 & 16 & \text { STRIPED } \\ 1.6 I L L S\end{array}$

$\begin{array}{lllllll}2.63 & 1.74 & 2.2 & 1.9 & 0.05 & 38 & \text { SIRIPED GILLS }\end{array}$

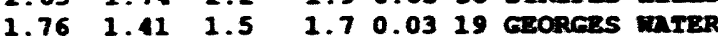

$\begin{array}{lllll}1.31 & 1.12 & 1.6 & 0.0717 \text { STRIPED GILIS }\end{array}$

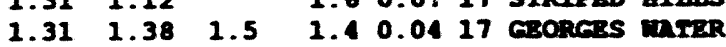

$\begin{array}{llllllll}2.39 & 1.88 & 2.3 & 10.5 & 0.06 & 24 & \text { sxemstom arus }\end{array}$

$\begin{array}{lllllll}3.18 & 2.3 & 10.8 & 0.07 & 37 & \text { skaterom HILLS }\end{array}$

$\begin{array}{lllllll}2.87 & 2.30 & 2.3 & 10.7 & 0.13 & 38 & \text { SKaterom nIL.LS }\end{array}$

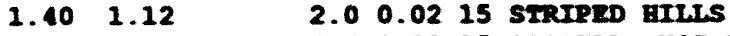
2.132 .525 .60 .2815 **aOND. WOT LISTED***

$\begin{array}{lllllll}1.68 & 1.40 & 1.6 & 3.0 & 0.0417 & \text { SMRIPED GILS }\end{array}$

2.100 .1915 **OOND. HOT LISTED***

$\begin{array}{lllllll}1.98 & 1.97 & 1.7 & 10.3 & 0.07 & 22 & \text { sTunton grus }\end{array}$

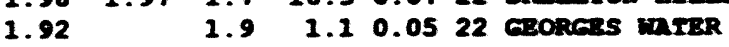

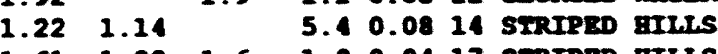

$\begin{array}{lllllll}1.61 & 1.22 & 1.6 & 1.9 & 0.04 & 17 & \text { STRIPED BILLS }\end{array}$

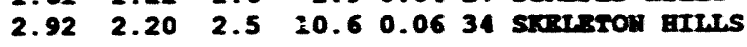

$\begin{array}{lllllll}1.66 & 1.49 & 1.6 & 10.6 & 0.07 & 20 & \text { STELETON HILtS }\end{array}$

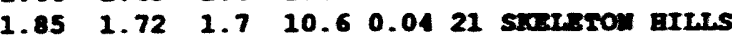

$1.22 \quad 1.31 \quad 10.40 .0619$ skatsrom miLts

$\begin{array}{llllll}0.83 & 0.70 & 2.8 & 0.0415 & \text { SIRTPED BILS }\end{array}$

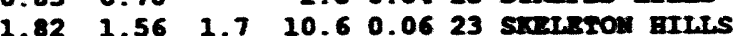

$\begin{array}{llrl}1.78 & 1.9 & 1.5 & 0.0316\end{array}$

$\begin{array}{llllll}1.78 & 1.9 & 1.6 & 0.07 & 17 & \text { STRIPED BILLS }\end{array}$

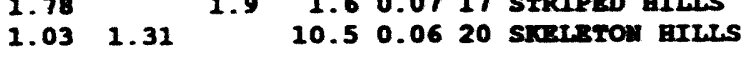


1992 LOCAL HYPOCBNTER SUIANRY - SEB EARTHQUNKS

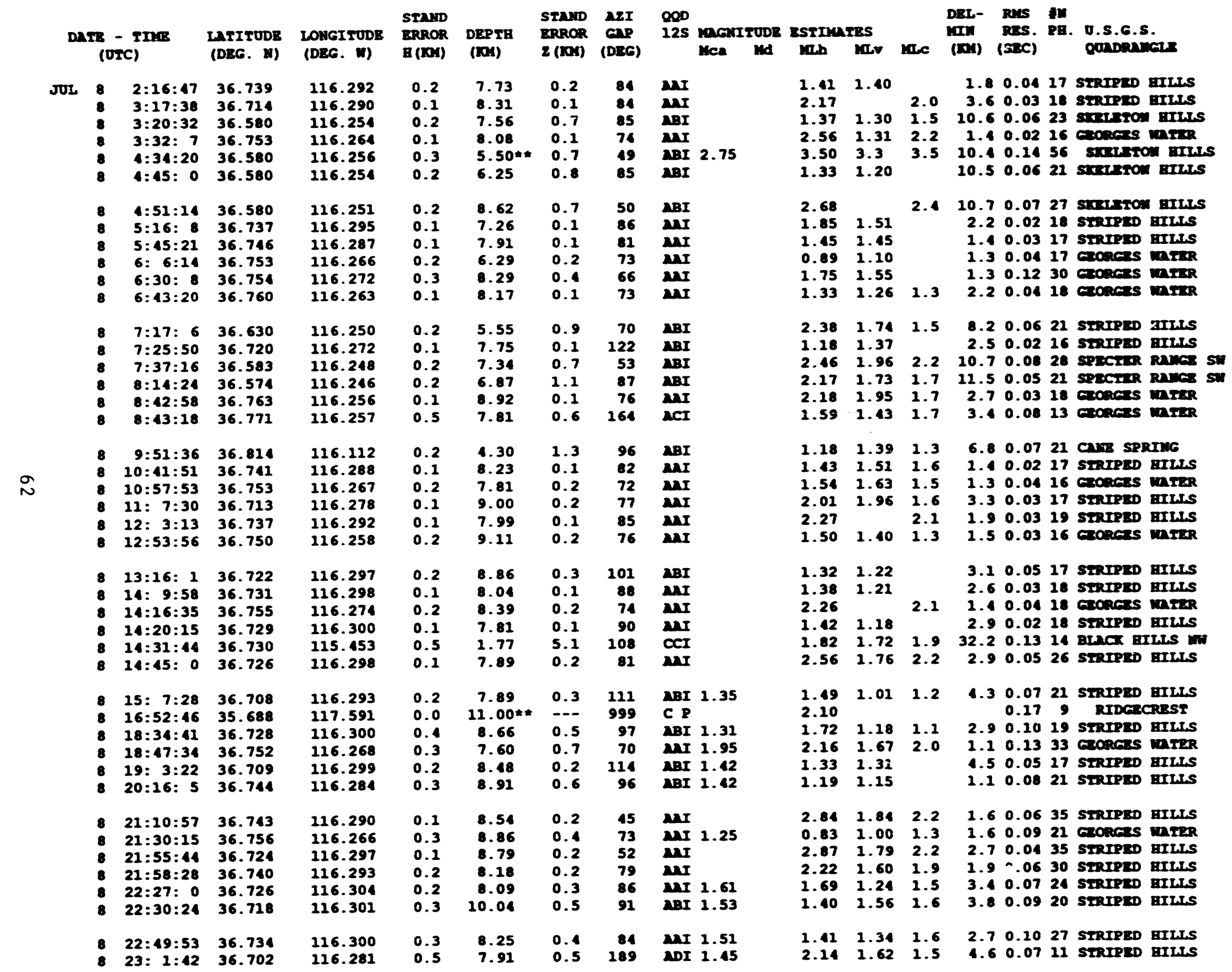


1992 LOCNL BYPOCENTER SUMARY - SGB EARTHQUAKRS

TIVR IATTYDE LOYGITUDB (DEG. W)

116.281

JUL

$$
\begin{array}{lll}
8 & 23: 45: 20 & 36.727 \\
9 & 0: 11: 11 & 36.714 \\
9 & 0: 25: 7 & 36.766 \\
9 & 0: 42: 1 & 36.766 \\
9 & 1: 0: 13 & 36.705 \\
9 & 1: 17: 9 & 37.179 \\
& & \\
9 & 1: 12: 26 & 36.733 \\
9 & 1: 59: 30 & 36.717 \\
9 & 2: 2: 18 & 36.703 \\
9 & 3: 47: 14 & 36.742 \\
9 & 1: 0: 14 & 36.704
\end{array}
$$
4:24: 736.764

1:39: $6 \quad 36.753$ $5: 19: 12 \quad 36.766$ $5: 30: 19 \quad 36.734$ $5: 30: 33 \quad 36.730$ $5: 38: 5 \quad 30.732$ $6: 58: 25 \quad 36.724$

9 7:23:52 36.725 $7: 31: 17 \quad 37.145$ $7: 34: 6 \quad 36.752$ $7: 47: 9 \quad 36.013$ $7: 49: 13 \quad 36.710$ $8: 37: 49 \quad 36.762$

$8: 43: 5136.763$ $8: 48: 11 \quad 36.759$ 9:46:46 36.750 $10: 11: 25 \quad 36.765$ 9 $10: 37: 18 \quad 36.991$ 12:12:10 36.744

15:53:32 37.028 15:54:26 36.692 17:40:46 36.731 18:37:41 36.692 18:53: 136.756 19:19:42 36.732

$9 \quad 19: 31: 29 \quad 36.748$ $20: 26: 29 \quad 36.743$ 20:53:0 36.741 22:34: $5: 36$ $22: 34$ 9 $22: 43: 4736.723$

$10 \quad 1: 9: 52 \quad 36.744$ $10 \quad 2: 17: 53 \quad 36.744$ $\begin{array}{lllll}116.281 & 0.4 & 6.45 & 0.4 & 106\end{array}$

116.257

116.253

116.300

116.296

116.263

116.305

116.288

116.303

116.259

116.260

116.296

16.298

116.299

116.272

116.310

116.599

116.283

116.294

116.249

116.255

116.276

116.278

116.255

117.668

116.270

116.171

116.306

116.294

116.306

116.260

116.295

116.283

116.282

116.280

116.257

116.298

116.274

116.293

116.290
0.47 .41

8.94

9.64

$\begin{array}{ll}0.5 & 168 \\ 0.3 & 70\end{array}$

0.270

0.200

$\begin{array}{rr}0.2 & 90 \\ 7.3 & 115\end{array}$

$90 \mathrm{NN}$

0.17 .99

$$
0.2
$$

0.29 .37

9.37
8.44
9.36

$$
\begin{aligned}
& 0.2 \\
& 0.1 \\
& 0.3
\end{aligned}
$$$$
0.3
$$$$
0.2
$$

\section{$\begin{array}{lllll}0.1 & 7.13 & 0.2 & 96 & \text { ABI }\end{array}$}

$\begin{array}{lllll}0.2 & 1.59 & 0.9 & 61 & \text { ACI } \\ 0.2 & 6.81 & 0.2 & 78 & \text { M I }\end{array}$

$0.0 \quad 3.39 *$ * $-\cdots \quad 999$ AP

$\begin{array}{lllll}0.2 & 7.87 & 0.2 & 86 & \text { NAI }\end{array}$

$\begin{array}{lllll}0.1 & 8.82 & 0.2 & 98 & \text { ABI }\end{array}$

$0.1 \quad 9.06$

0.27 .95

0.28 .14

$\begin{array}{ll}0.1 & 8.86 \\ 0.7 & 2.86\end{array}$

$0.1 \quad 10.16$

$0.280 \quad \mathrm{NAT}$

$\begin{array}{lll}0.2 & 128 \quad A B I\end{array}$

$\begin{array}{lll}0.3 & 77 & \text { MII } \\ 0.2 & 96 & \text { MBI }\end{array}$

$\begin{array}{rrr}0.2 & 96 & \text { ABI } \\ 2.8 & 194 & \text { BDI }\end{array}$

$1.2 \quad 2.95$

0.1

$66 \mathrm{MII}$

$0.1 \quad 9.94$

1.4234 BDI

$\begin{array}{lllll}0.1 & 8.30 & 0.1 & 98 & \text { ABI }\end{array}$

$\begin{array}{lllll}0.1 & 9.83 & 0.2 & 50 & \text { arr } \\ 0.1 & 7.96 & 0.1 & 75 & \text { ant }\end{array}$

0.1

75 NIT

$0.2 \quad 7.57$

0.18 .50$$
\begin{aligned}
& 0.1 \\
& 0.1 \\
& 0.1
\end{aligned}
$$

0.10 .75

$\begin{array}{ll}0.1 & 9.75 \\ 0.2 & 8.53\end{array}$

$0.2 \quad 0.53$

$\begin{array}{lll}0.1 & 79 & \text { NAI } \\ 0.1 & 79 & \text { MI }\end{array}$

$\begin{array}{lll}0.429 & 129 & \text { MBI }\end{array}$

$0.1 \quad 76 \quad \mathrm{MI}$

$\begin{array}{lll}0.2 & 74 & \text { MII } 1.34\end{array}$

$\begin{array}{lllll}0.2 & 7.00 & 0.3 & 80 & \operatorname{Mr} \\ 0.1 & 7.24 & 0.1 & 79 & \operatorname{MI}\end{array}$ 1.65 1.59 II

II

I

I

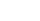

1.34
1.98

DEL- RUS HI

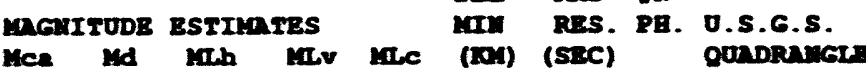

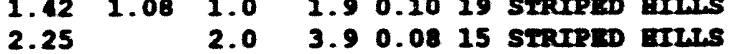

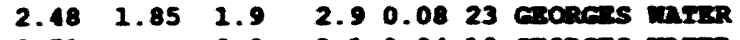
$2.512 \quad 2.0 \quad 3.10 .0419$ ceonces mira $\begin{array}{llllll}2.27 & 2.2 & 4.8 & 0.03 & 18 & \text { STRIPED ETI.S }\end{array}$

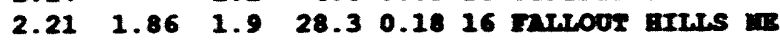
$\begin{array}{lllllll}2.26 & 1.62 & 2.1 & 2.3 & 0.04 & 29 & \text { SIRIPED aIH.S }\end{array}$ $\begin{array}{lllllll}1.50 & 1.19 & 1.6 & 2.9 & 0.02 & 18 & \text { SIRIPED BMLS }\end{array}$

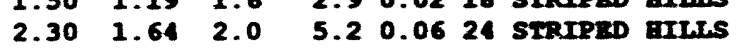
$\begin{array}{llll}1.40 & 1.35 & 1.5 & 0.0517 \text { SERTPED BILLS }\end{array}$

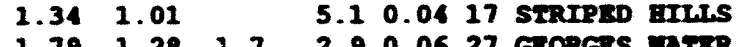

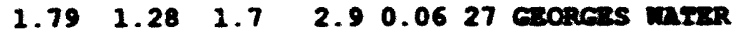

$\begin{array}{lllllll}1.87 & 1.53 & 1.4 & 1.6 & 0.04 & 25 & \text { Geopers mire }\end{array}$ $\begin{array}{llll}1.71 & 1.33 & 2.40 .0317 \text { STRTPED gTurs }\end{array}$ $1.83 \quad 1.61 \quad 1.8 \quad 2.70 .0717$ STmPaD $\begin{array}{llllll}1.56 & 1.19 & 2.70 .0317 \text { SIRIPRD GIILS }\end{array}$

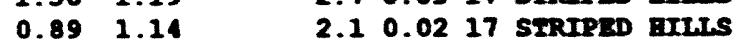

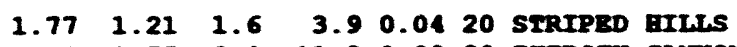
$\begin{array}{lllllll}2.03 & 1.75 & 2.0 & 11.3 & 0.08 & 28 & \text { ImIRST curron }\end{array}$ $\begin{array}{llll}1.51 & 1.25 & 1.40 .0516 \text { GEORCBS MTER }\end{array}$

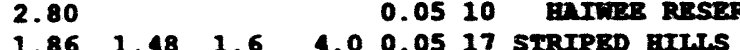

$\begin{array}{llllllll}1.20 & 1.13 & 1.3 & 3.0 & 0.03 & 16 & \text { sxoul }\end{array}$

$\begin{array}{lllllll}1.99 & 1.64 & 1.7 & 2.8 & 0.04 & 22 & \text { esoress mTrR }\end{array}$ $1.601 .351 .4 \quad 1.80 .0415$ GeOReEs mTER $1.51 \quad 1.231 .6 \quad 1.00 .0516$ GeORess miter $\begin{array}{llll}1.71 & 1.41 & 2.9 & 0.0316 \text { Gronces marer }\end{array}$ $\begin{array}{lllllll}1.79 & 1.79 & 1.9 & 26.9 & 0.14 & 19 & \text { SAHTm Pax }\end{array}$ $\begin{array}{lllllll}2.70 & 1.84 & 1.7 & 0.2 & 0.01 & 17 & \text { STRIPED EILLS }\end{array}$

$\begin{array}{lllllll}1.73 & 1.25 & 1.5 & 5.3 & 0.13 & 17 & \text { EIPPIPAB SPRIMG }\end{array}$ $1.090 .89 \quad 2.30 .0315$ STRTPED gItS $2.451 .74 \quad 3.60 .0539$ strTPED 1.451 .201 .43 .60 .0539 stripes mans $\begin{array}{lllllll}1.45 & 1.20 & 1.4 & 1.9 & 0.03 & 17 & \text { GRORGES ETER } \\ 1.51 & 1.09 & & 2.3 & 0.03 & 17 & \text { STRIPED GIILS }\end{array}$

1.191 .09

$1.56 \quad 1.46$ $1.21 \quad 1.19$ $\begin{array}{llllllll}1.24 & 1.37 & & 1.9 & 0.03 & 16 & \text { GEORGRS MTIRR } \\ 2.04 & 1.72 & 1.6 & 3.2 & 0.04 & 17 & \text { STRIPED BILS }\end{array}$ $\begin{array}{lllllllll}0.91 & 0.94 & 0.7 & 1.4 & 0.05 & 23 & \text { Grorers mirr }\end{array}$

\begin{tabular}{lllllll}
1.98 & 1.58 & 1.5 & 1.8 & 0.10 & 31 & STRIPED \\
\hline
\end{tabular}

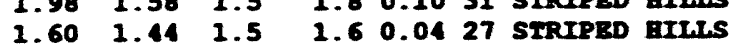


1992 LOCAL HYPOCENTER SUMAARY - SGB EARTHQUAKES

\begin{tabular}{|c|c|c|c|c|c|c|c|c|}
\hline \multicolumn{2}{|c|}{$\begin{array}{l}\text { DAIE - TIME } \\
\text { (UIC) }\end{array}$} & $\begin{array}{l}\text { LATITUDE } \\
\text { (DEG. N) }\end{array}$ & $\begin{array}{l}\text { LONGI TUDE } \\
\text { (DEG. W) }\end{array}$ & $\begin{array}{l}\text { STAND } \\
\text { ERROR } \\
\text { B (KN) }\end{array}$ & $\begin{array}{l}\text { DEPTH } \\
\text { (KO) }\end{array}$ & $\begin{array}{l}\text { STAND } \\
\text { ERROR } \\
\mathrm{Z} \text { (KY) }\end{array}$ & $\begin{array}{c}\text { AZI } \\
\text { GAP } \\
\text { (DEG) }\end{array}$ & $\begin{array}{l}\text { QQD } \\
125\end{array}$ \\
\hline JOL 10 & $7: 47: 22$ & 36.736 & 116.295 & 0.6 & 8.45 & 0.7 & 86 & $\operatorname{BAI}$ \\
\hline 10 & $8: 25: 33$ & 36.734 & 116.290 & 0.1 & 8.14 & 0.1 & 78 & $\mathbf{M I}$ \\
\hline 10 & $8: 57: 1$ & 37.008 & 116.729 & 0.2 & 0.18 & 0.5 & 51 & ACI \\
\hline 10 & $9: 56: 38$ & 36.729 & 116.275 & 0.3 & 7.50 & 0.2 & 39 & MaI \\
\hline 10 & $10: 16: 28$ & 36.079 & 117.730 & 0.0 & $7.28 * *$ & -- & 999 & C P \\
\hline 10 & $12: 20: 44$ & 36.730 & 116.308 & 2.4 & 2.56 & 3.3 & 245 & BDI \\
\hline 10 & $13: 56: 39$ & 35.719 & 118.080 & 0.0 & $2.83 \star \star$ & --- & 999 & $P$ \\
\hline 10 & $17: 3: 48$ & 36.733 & 116.295 & 0.1 & 7.89 & 0.1 & 86 & $\mathbf{M I}$ \\
\hline 10 & $17: 29: 11$ & 36.741 & 116.291 & 0.2 & 7.98 & 0.2 & 83 & NaI \\
\hline 10 & $18: 45: 57$ & 36.744 & 116.289 & 0.1 & 8.11 & 0.2 & 52 & MaI \\
\hline 10 & 19: 1: 6 & 36.734 & 116.295 & 0.2 & 7.19 & 0.2 & 87 & Mar \\
\hline 10 & $19: 31: 9$ & 36.767 & 116.258 & 0.1 & 8.79 & 0.2 & 75 & MAI \\
\hline 10 & $19: 45: 24$ & 36.712 & 116.294 & 0.2 & 9.39 & 0.2 & 86 & anI \\
\hline 10 & 19:54:9 & 36.728 & 116.281 & 0.1 & 6.88 & 0.1 & 79 & MnI \\
\hline 10 & $20: 18: 1$ & 36.737 & 116.293 & 0.1 & 7.64 & 0.2 & 85 & $M I$ \\
\hline 10 & $20: 19: 41$ & 36.724 & 116.299 & 0.1 & 8.19 & 0.2 & 90 & MaI \\
\hline 10 & $20: 25: 60$ & 36.727 & 116.281 & 0.1 & 7.05 & 0.1 & 79 & $\mathrm{MI}$ \\
\hline 10 & $21: 17: 0$ & 36.734 & 116.296 & 0.2 & 7.97 & 0.2 & 50 & MII \\
\hline $\begin{array}{l}10 \\
10\end{array}$ & $21: 59: 6$ & 36.732 & 116.299 & 0.1 & 7.05 & 0.2 & 89 & $\operatorname{lnI}$ \\
\hline $\begin{array}{l}10 \\
10\end{array}$ & $22: 3: 35$ & 36.730 & 116.296 & 0.2 & $\begin{array}{l}8.07 \\
7.97\end{array}$ & 0.2 & $\begin{array}{l}77 \\
87\end{array}$ & $\operatorname{MaI}$ \\
\hline $\begin{array}{l}10 \\
10\end{array}$ & $\begin{array}{l}22: 29: 53 \\
23: 7: 9\end{array}$ & $\begin{array}{l}36.734 \\
36.729\end{array}$ & $\begin{array}{l}116.296 \\
116.275\end{array}$ & $\begin{array}{l}0.2 \\
0.1\end{array}$ & $\begin{array}{l}7.97 \\
6.41\end{array}$ & 0.1 & 76 & NII \\
\hline 10 & $23: 10: 56$ & 36.742 & 116.294 & 0.1 & 7.22 & 0.2 & 48 & $\overline{M I}$ \\
\hline 10 & $23: 26: 36$ & 36.731 & 116.301 & 0.1 & 7.05 & 0.1 & 84 & $\boldsymbol{M I}$ \\
\hline 10 & $23: 39: 23$ & 36.718 & 116.307 & 0.3 & 8.30 & 0.4 & 95 & IBI \\
\hline 10 & $23: 55: 54$ & 36.753 & 116.276 & 0.2 & 8.03 & 0.2 & 58 & $\operatorname{MnI}$ \\
\hline $\begin{array}{l}11 \\
11\end{array}$ & $0: 1: 5$ & 36.711 & 116.285 & 0.2 & 9.01 & 0.1 & 119 & ABI \\
\hline 11 & $0: 3: 33$ & 36.722 & 116.315 & 0.1 & 6.34 & 0.2 & 100 & ABI \\
\hline 11 & $1: 24: 8$ & 36.733 & 116.301 & 0.1 & 8.02 & 0.1 & 95 & ABI \\
\hline 11 & $1: 54: 49$ & 36.729 & 116.284 & 0.2 & 8.16 & 0.2 & 81 & NaI \\
\hline 11 & $2: 19: 26$ & 36.750 & 110 & 0.1 & 7.26 & 0.1 & 78 & $\boldsymbol{M} I$ \\
\hline 11 & $2: 58: 28$ & 36.675 & 116.265 & 0.1 & 6.35 & 0.3 & 93 & ABI \\
\hline 11 & $3: 24: 40$ & 36.058 & 117.576 & 0.0 & $0.60 * *$ & --- & 999 & A P \\
\hline 11 & $3: 24: 44$ & 36.048 & 117.615 & 3.0 & 2.12 & 4.5 & 261 & $\operatorname{CDI}$ \\
\hline 11 & $3: 33: 50$ & 36.723 & 116.299 & 0.2 & 8.38 & 0.2 & 90 & MaI \\
\hline 11 & $3: 38: 53$ & 36.712 & 116.289 & 0.1 & 8.30 & 0.1 & 83 & $\boldsymbol{M I I}$ \\
\hline 11 & $3: 45: 15$ & 36.728 & 116.281 & 0.2 & 6.97 & 0.2 & 79 & MII \\
\hline 11 & 4:9:8 & 36.711 & 116.291 & 0.1 & 8.73 & 0.2 & 54 & $\operatorname{Max}$ \\
\hline 11 & $4: 11: 20$ & 36.712 & 116.288 & 0.2 & 8.44 & 0.2 & 117 & $\mathbf{A B I}$ \\
\hline 11 & $4: 54: 38$ & 36.730 & 116.304 & 0.1 & 7.33 & 0.2 & 92 & ABI \\
\hline 11 & $5: 35: 2$ & 36.725 & 116.294 & 0.2 & 8.22 & 0.2 & 101 & ABI \\
\hline 11 & $5: 47: 10$ & 36.741 & 116.292 & 0.1 & 7.40 & 0.2 & 48 & $\boldsymbol{M I}$ \\
\hline 11 & $6: 17: 22$ & 36.709 & 116.302 & 0.2 & 10.12 & 0.2 & 83 & Tn \\
\hline 11 & $6: 33: 15$ & 36.724 & 116.301 & 0.1 & 8.25 & 0.1 & 91 & $\mathbf{B B}$ \\
\hline
\end{tabular}

DEL- RES In

MTV RES PB, 0.S.6.5

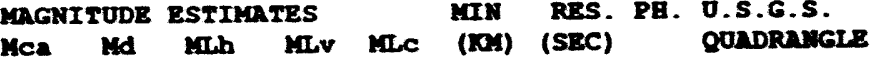

Mca Ma ket

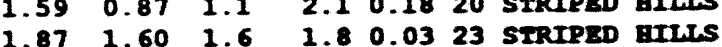

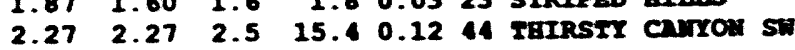

$\begin{array}{llllllll}3.10 & 2.24 & 3.1 & 1.7 & 0.07 & 45 & \text { STRIPED EILWS }\end{array}$

$2.80 \quad 0.067$ coso pex

$2.00 \quad 14.8 \quad 0.1411$ STRTPED aIILS-ODR

$2.70 \quad 0.1531 * *$ *QOND. MOT LISTED***

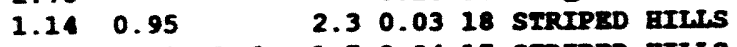

$\begin{array}{lllllll}1.70 & 1.41 & 1.6 & 1.7 & 0.04 & 17 & \text { STRIPED EILS }\end{array}$

$2.711 .71 \quad 1.60 .0535$ SIRIPED gILIS

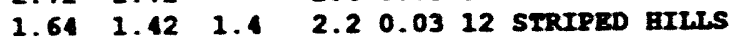

$1.921 .471 .5 \quad 3.00 .0417$ Groress merrs

$\begin{array}{lllllll}1.73 & 1.49 & 1.6 & 3.9 & 0.0417 & \text { STRIPED BILLS }\end{array}$

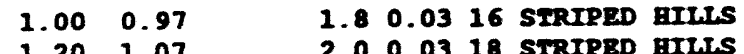

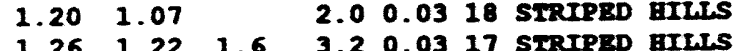

$\begin{array}{lllllll}1.26 & 1.22 & 1.6 & 3.2 & 0.03 & 17 & \text { SIRPTED GILS } \\ 1.64 & 1.45 & 1.5 & 1.8 & 0.03 & 17 & \text { STRIPED BILS }\end{array}$

$2.721 .96 \quad 2.0 \quad 2.30 .0945$ SIRIPED gILIS

$\begin{array}{llll}1.39 & 1.23 & 2.7 & 0.0417 \text { STRIPED BITS }\end{array}$

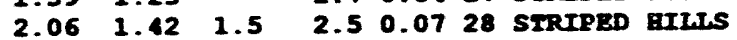

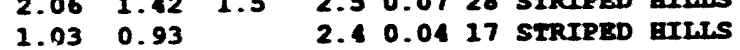

$\begin{array}{lllllll}1.03 & 0.93 & & 2.6 & 0.04 & 17 & \text { STRIPED EILS } \\ 1.90 & & 2.1 & 1.6 & 0.03 & 18 & \text { SIRIPED BILLS }\end{array}$

$2.671 .95 \quad 2.00 .0746$ STRIPED BILLS

$\begin{array}{lllllll}2.02 & 1.43 & 1.5 & 2.8 & 0.03 & 23 & \text { SIRIPED BILS }\end{array}$

$\begin{array}{lllll}1.25 & 0.94 & 4.1 & 0.0718 \text { STRIPRD BILLS }\end{array}$

$\begin{array}{lllllll}2.10 & 1.68 & 1.5 & 1.3 & 0.05 & 32 & \text { GEORGES MATER }\end{array}$

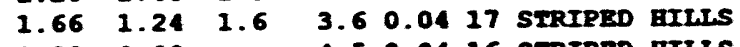

$\begin{array}{llllll}1.28 & 0.99 & 4.5 & 0.0416 & \text { STRIPED BILLS }\end{array}$

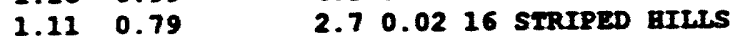

$1.491 .16 \quad 1.90 .0417$ STRIPED HILLS

$\begin{array}{lllll}1.60 & 1.25 & 1.1 & 0.0216 \text { GEORGES mater }\end{array}$

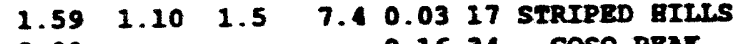

$\begin{array}{lllllll}2.90 & & 0.1634 & \cos 0 \mathrm{PEN}\end{array}$

$\begin{array}{lllllll}1.80 & 1.36 & 1.6 & 3.3 & 0.05 & 18 & \text { STRIPED BILLS }\end{array}$

$1.90 \quad 1.57 \quad 1.5 \quad 3.70 .0317$ sTRIPED BILS

$\begin{array}{lllllll}1.64 & 1.28 & 1.6 & 1.8 & 0.0417 & \text { STRIPED GILLS }\end{array}$

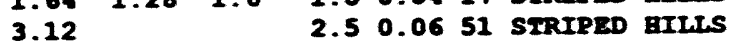

$\begin{array}{llllllll}3.12 & & & 2.5 & 0.06 & 51 & \text { STRIPED BILLS } \\ 1.82 & 1.46 & 1.5 & 3.7 & 0.05 & 17 & \text { STRIPED HILLS }\end{array}$

$\begin{array}{llllllll}1.82 & 1.46 & 1.5 & 3.7 & 0.05 & 17 & \text { STRIPED BILLS } \\ 1.58 & 1.40 & & 3.2 & 0.03 & 17 & \text { STRIPED BILLS }\end{array}$

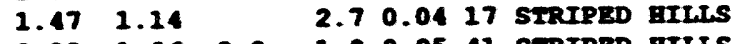

$\begin{array}{lllllll}2.33 & 1.86 & 2.2 & 1.8 & 0.05 & 11 & \text { SERIPED }\end{array}$

$\begin{array}{lllllll}2.05 & 1.24 & & 4.5 & 0.05 & 22 & \text { STRIPED EILLS } \\ 1.54 & 1.26 & 1.6 & 3.3 & 0.03 & 17 & \text { SIRIPED BILLS }\end{array}$ 
1992 LOCAL HYPOCENTER SURAARY - SGB EARTHQUAKES

$\begin{array}{llllll}\text { STAND } & & \text { STAND } & \text { AZI } & \text { QQD } & \text { DEL- RUS WN } \\ \text { ERROR DEPTH } & \text { ERROR } & \text { GAP } & \text { I2S MAGHITUDE ESTIMATES } & \text { MIN RES. PB. U.S.G.S. }\end{array}$

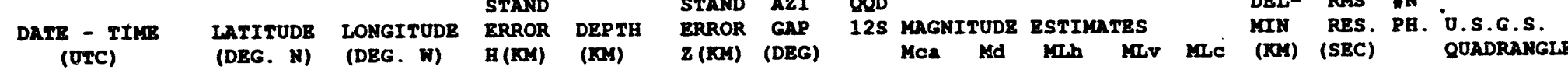

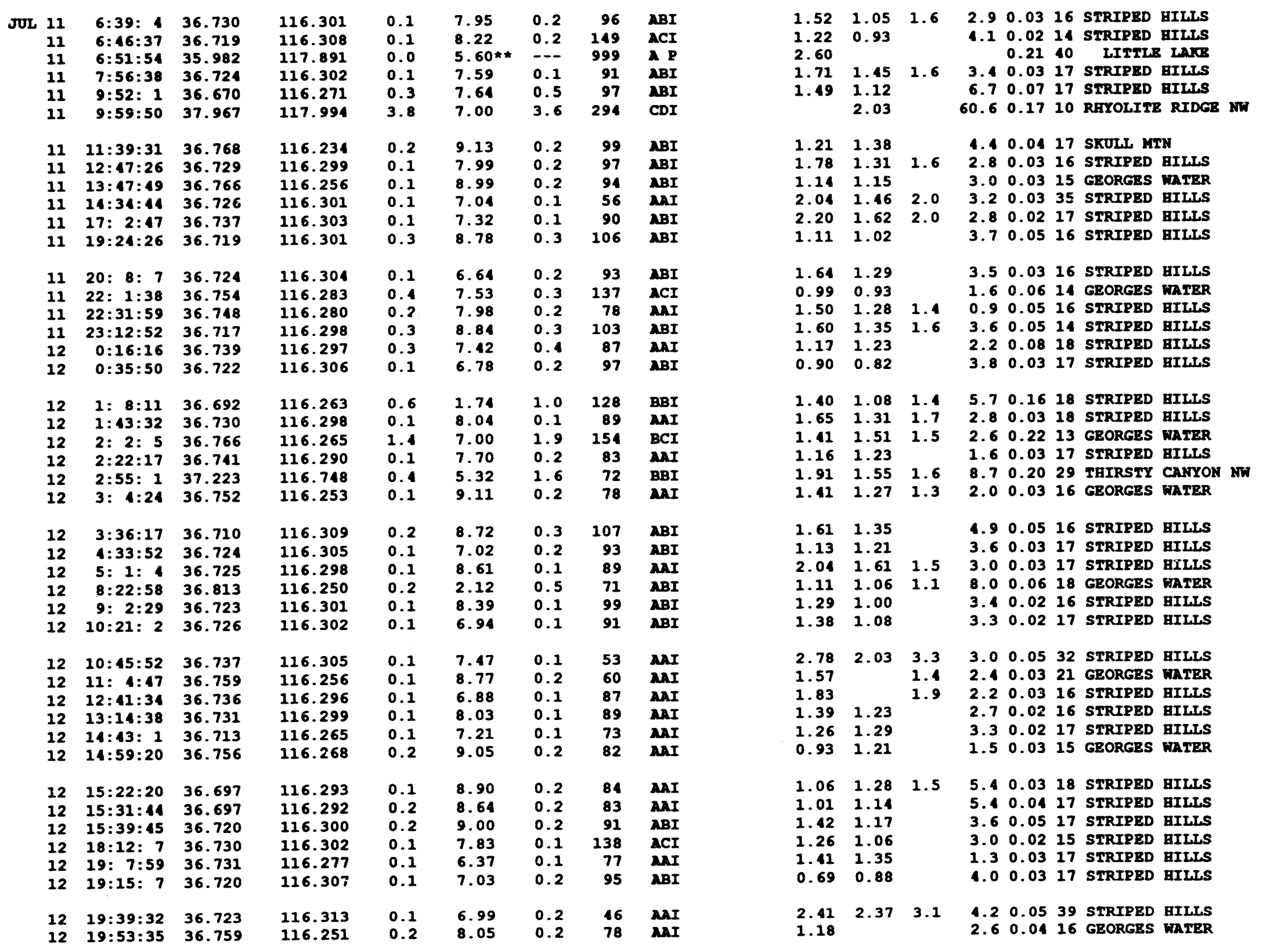


1992 LOCAL HYPOCENTER SURMARY - SGB EARTBQUAKES

STAND STAND AZI QQD

DATR - TIMT (UTC)

LATITUDE LONGITUD
(DEG. N) (DEG. W)

JUL $12 \quad 20: 43: 51 \quad 36.758$

$12 \quad 21: 21: 12 \quad 36.761$

$12 \quad 22: 29: 34 \quad 36.682$

12 23:57:12 36.751

$130.45: 43 \quad 36.721$

3: $2: 57 \quad 36.727$

3: $3: 29 \quad 36.719$

$3: 27: 47 \quad 36.731$

$5: 57: 52 \quad 36.685$

$6: 1: 31 \quad 36.721$

$6: 2: 4236.958$

$6: 19: 29 \quad 36.704$

$7: 18: 2 \quad 36.733$

$8: 25: 50 \quad 36.376$

9: $6: 11 \quad 36.744$

$9.56: 54 \quad 36.648$

$11: 27: 36 \quad 36.710$

$13 \quad 11: 31: 59 \quad 36.733$

\subsection{9}

116.141

116.352

116.219

116.274

116.305

116.295

116.297

116.302

116.35

116.298

114.374

116.307

116.299

117.562

116.288

117.280

116.292

116.296

116.255

116.193

116.304

116.197

116.296

\subsection{2}

116.301

116.252

116.299

116.28

116.304

117.563

117.276

116.293

116.246

116.298

116.266

116.274

116.191

116.269

116.300

116.262

116.289

116.290
116.298
DEPTH

0.28 .61

$\begin{array}{lllll}0.2 & 7.04 & 0.5 & 117 & \text { ABI }\end{array}$

$0.1 \quad 9.15$

0.18 .33

8.91

0.5

0.3

0.2

64

$98 \quad$ AI 1.27

$0.2 \quad 8.10$

0.2

8.75

7.72

8.16

$0.2 \quad 8.75$

0.358

$0.3 \quad 164$

$0.6 \quad 150$

0.253

1.8 259

$0.3 \quad 9.02$

0.37 .02

0.4112

7.02

0.28 .23

7.00 *

0.

0.2

96
271

52

8. 64

0.27 .91

$\begin{array}{ll}0.3 & 9.27 \\ 0.5 & 4.69\end{array}$

$\begin{array}{ll}0.5 & 4.69 \\ 0.2 & 6.78\end{array}$

$\begin{array}{ll}0.3 & -0.78 \\ 0.3 & 7.77\end{array}$

0.255

$\begin{array}{rr}0.4 & 69 \\ 1.9 & 136\end{array}$

$\begin{array}{ll}1.9 & 136 \\ 0.3 & 96\end{array}$

0.466

0.57 .00

$0.2 \quad 9.79$

0.4

94

0.28 .23

0.77 .49

0.46 .38

$0.5 \quad 97$

0.259

0.358

0.9133

$0.4 \quad 83$

6.82

0.2133

$\begin{array}{llllll}0.0 & 4.65 * & -- & 999 & \text { A P }\end{array}$

$\begin{array}{lllll}0.4 & 0.05 & 0.3 & 75 & \text { BBI } 2.14\end{array}$

$\begin{array}{lllll}0.8 & 3.63 & 2.1 & 266 & \text { BDI } \\ 0.5 & 10.05 & 0.4 & 198 & \end{array}$

$\begin{array}{lllll}0.5 & 10.05 & 0.4 & 197 & \text { ADI } 1.49\end{array}$

$1.0 \quad 10.87$

$\begin{array}{rr}0.9 & -1.57 \\ 0.5 & 5.80\end{array}$

$0.5 \quad 8.33$

0.48 .49

8.49
7.76

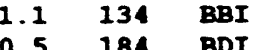

$\begin{array}{llll}0.5 & 184 & \text { BDI } & 1.28 \\ 1.0 & 114 & \text { NBI } 0.93\end{array}$

$0.4 \quad 152 \quad$ ACI 3.64

$\begin{array}{rrr}0.4 & 96 & \text { ABI } \\ 0.1 & 188 & \text { ADI }\end{array}$

$\begin{array}{rrrrr}0.1 & 8.34 & 0.2 & 206 & \text { ADI } \\ 0.2 & 20.52 & 0.2 & 119 & \text { ABI }\end{array}$
DEL- RMS N

MIN RES. PG. O.S.G.S.

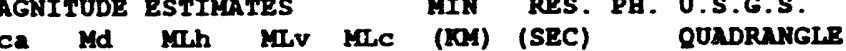

$\begin{array}{llllll}1.30 & 1.25 & 2.1 & 0.0518 & \text { GBORGES GATRR }\end{array}$

1.63

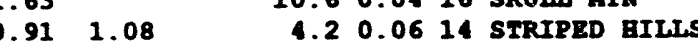

$\begin{array}{lllllll}1.75 & 1.29 & 1.7 & 5.1 & 0.04 & 24 & \text { SKOLL } \\ 2 & \mathrm{mTH}\end{array}$

$\begin{array}{lllllll}2.29 & 1.70 & 2.2 & 0.9 & 0.06 & 34 & \text { GRORGRS MATRR }\end{array}$

$\begin{array}{llll}1.04 & 0.85 & 3.7 & 0.0417 \text { STRIPED BILLS }\end{array}$

$\begin{array}{lllllll}1.43 & 1.39 & 1.5 & 2.7 & 0.05 & 23 & \text { STRIPED BILLS }\end{array}$

$\begin{array}{lll}1.11 & 0.92\end{array}$

\begin{tabular}{lllllll}
1.46 & 1.27 & 1.1 & 3.0 & 0.07 & 29 & STRIPRD \\
\hline & aILLS
\end{tabular}

$\begin{array}{lllllll}1.45 & 1.15 & 0.8 & 4.6 & 0.07 & 16 & \text { STRIPED GILLS }\end{array}$

\begin{tabular}{lllllll}
2.91 & 1.83 & 2.1 & 3.3 & 0.06 & 33 & STRIPED \\
\hline & $2.4 I L L S$
\end{tabular}

$2.422 .276 .0 \quad 0.159 *$ Q 2.202 . HOT LISTED***

$\begin{array}{lllllll}1.59 & 1.34 & 1.6 & 5.3 & 0.10 & 22 & \text { STRIPED HILLS }\end{array}$

$\begin{array}{lllllll}1.43 & 0.84 & 1.1 & 2.6 & 0.09 & 21 & \text { STRIPED BILS }\end{array}$ $2.439 .3 \quad 0.7512$ DARKIN

$\begin{array}{lllllll}2.68 & 1.76 & 2.0 & 1.5 & 0.07 & 31 & \text { STRIPED BILLS }\end{array}$

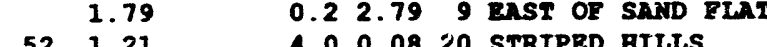

$\begin{array}{llll}1.52 & 1.21 & 4.0 & 0.0820 \text { STRIPED BILLS }\end{array}$

$\begin{array}{lllllll}1.70 & 1.14 & 1.5 & 2.4 & 0.06 & 28 & \text { STRIPED BILLS }\end{array}$ $\begin{array}{lllllll}1.47 & 1.33 & 1.6 & 2.2 & 0.10 & 32 & \text { GEORGES WATER }\end{array}$ $\begin{array}{lllllll}2.48 & 1.75 & 1.8 & 7.9 & 0.14 & 15 & \text { SPECTER RANGE }\end{array}$

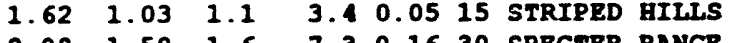

$\begin{array}{lllllll}2.09 & 1.50 & 1.6 & 7.3 & 0.16 & 30 & \text { SPECTER RANGE NH }\end{array}$

$\begin{array}{lllllll}2.19 & 1.59 & 2.0 & 2.1 & 0.12 & 24 & \text { STRIPED BILLS }\end{array}$

$\begin{array}{lllllll}1.80 & 1.15 & 1.6 & 1.9 & 0.15 & 19 & \text { STRIPED BILLS }\end{array}$ $\begin{array}{lllllll}2.43 & 1.55 & 2.2 & 5.1 & 0.06 & 30 & \text { STRIPED BILLS }\end{array}$ $\begin{array}{lllllll}1.76 & 1.50 & 1.7 & 2.8 & 0.09 & 31 & \text { GBORGBS WATER }\end{array}$ $\begin{array}{llllll}0.33 & 1.0 & 2.6 & 0.14 & 11 & \text { STRIPED BILLS }\end{array}$ $1.270 .81 \quad 1.130 .1023$ STRIPED BILLS $\begin{array}{llllll}2.27 & 1.72 & 1.9 & 3.6 & 0.05 & 24 \\ \text { STRIPED BILLS }\end{array}$

2.00

$\begin{array}{llllll}2.31 & 1.87 & 2.0 & 0.1621 & \text { RIDGECREST }\end{array}$

$\begin{array}{lllllll}1.19 & 0.56 & 1.0 & 6.9 & 0.16 & 24 & \text { COLD MOUTIAIN }\end{array}$

$\begin{array}{llllllll}0.98 & 0.98 & 1.1 & 3.0 & 0.14 & 11 & \text { STRIPED EILLS }\end{array}$

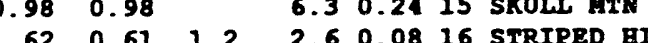

$0.371 .2 \quad 2.90 .1213$ GEORGES WATER

$\begin{array}{lll}0.46 \quad 1.6 & 0.1614 \text { GEORGES WMTER }\end{array}$

$\begin{array}{llllllll}1.52 & 0.76 & 1.1 & 7.7 & 0.16 & 18 & \text { SPECTER RANGB }\end{array}$ $\begin{array}{llllll}0.75 & 1.2 & 6.3 & 0.13 & 14 & \text { GEORGES MATER }\end{array}$

$\begin{array}{lllllll}1.84 & 1.14 & 1.1 & 2.7 & 0.11 & 20 & \text { STRIPED BILLS }\end{array}$

$\begin{array}{llll}1.28 & 1.17 & 2.0 & 0.0612 \text { GRORGES WATER }\end{array}$

$\begin{array}{lllllll}2.06 & 1.86 & 1.5 & 1.5 & 0.03 & 13 & \text { STRIPED BILLS }\end{array}$

$\begin{array}{lllllll}1.55 & 1.33 & & 3.7 & 0.04 & 13 & \text { STRIPED BILLS } \\ 3 & 3.3 & 3.3 & 3.5 & 0.07 & 31 & \text { STRIPED BILLS }\end{array}$ 
1992 LOCAL HYPOCBNTER SURAMARY - SGB EARTBQUAKRS

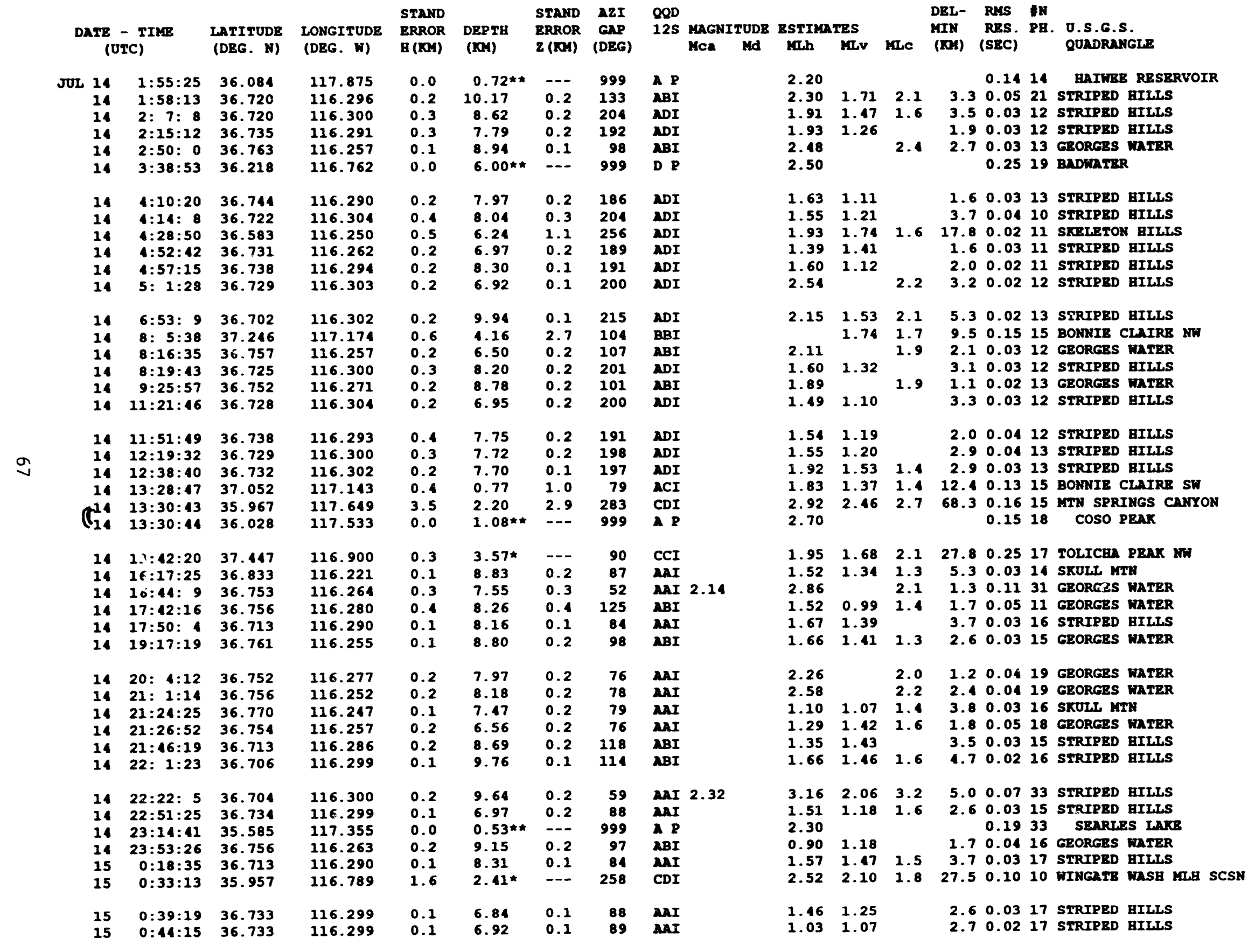


1992 LOCAL HYPOCENTER SUMAARY - SGB EARTHQUAKES

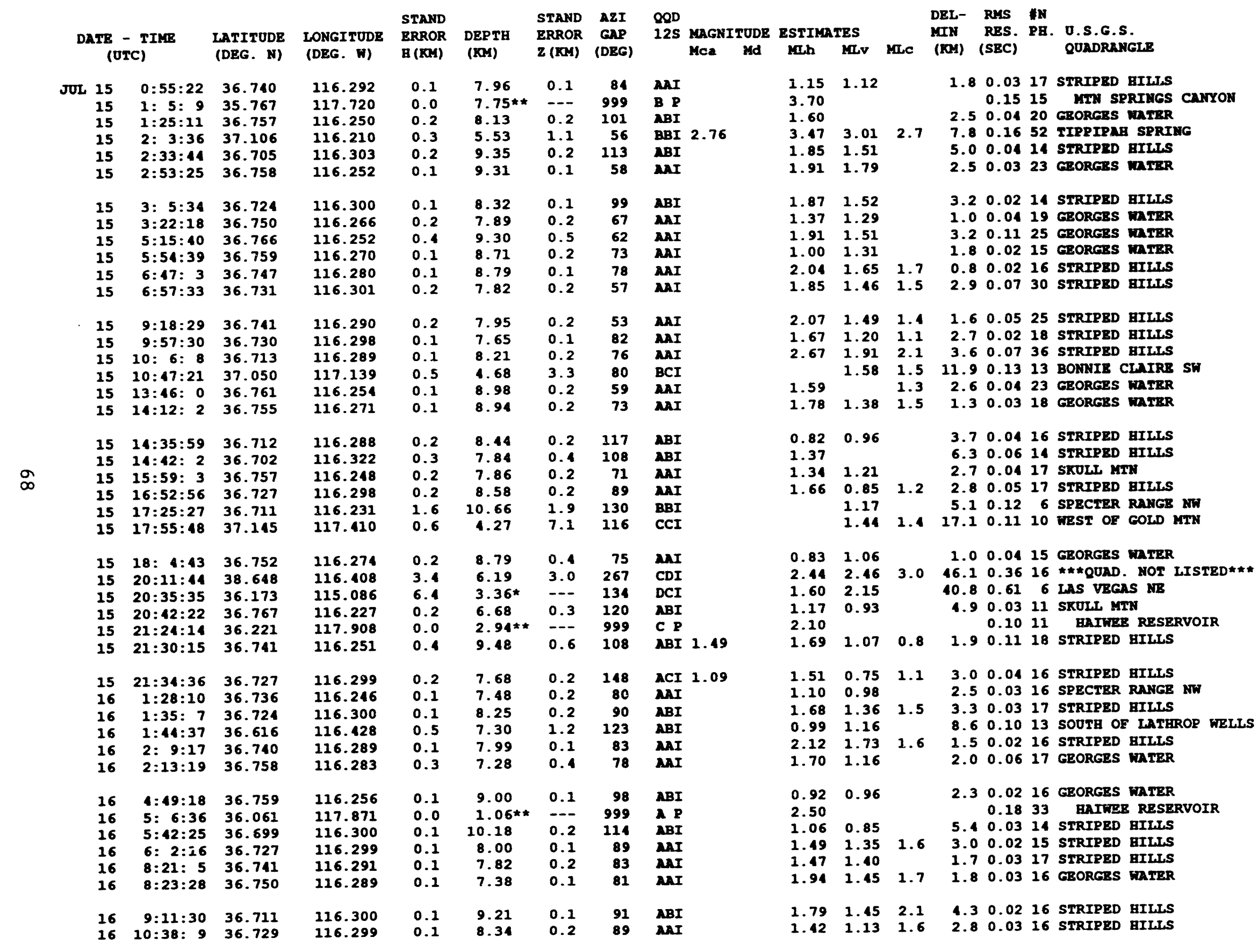


1992 LOCAL HYPOCENTER SUIARARY - SGB EARTHQUAKES

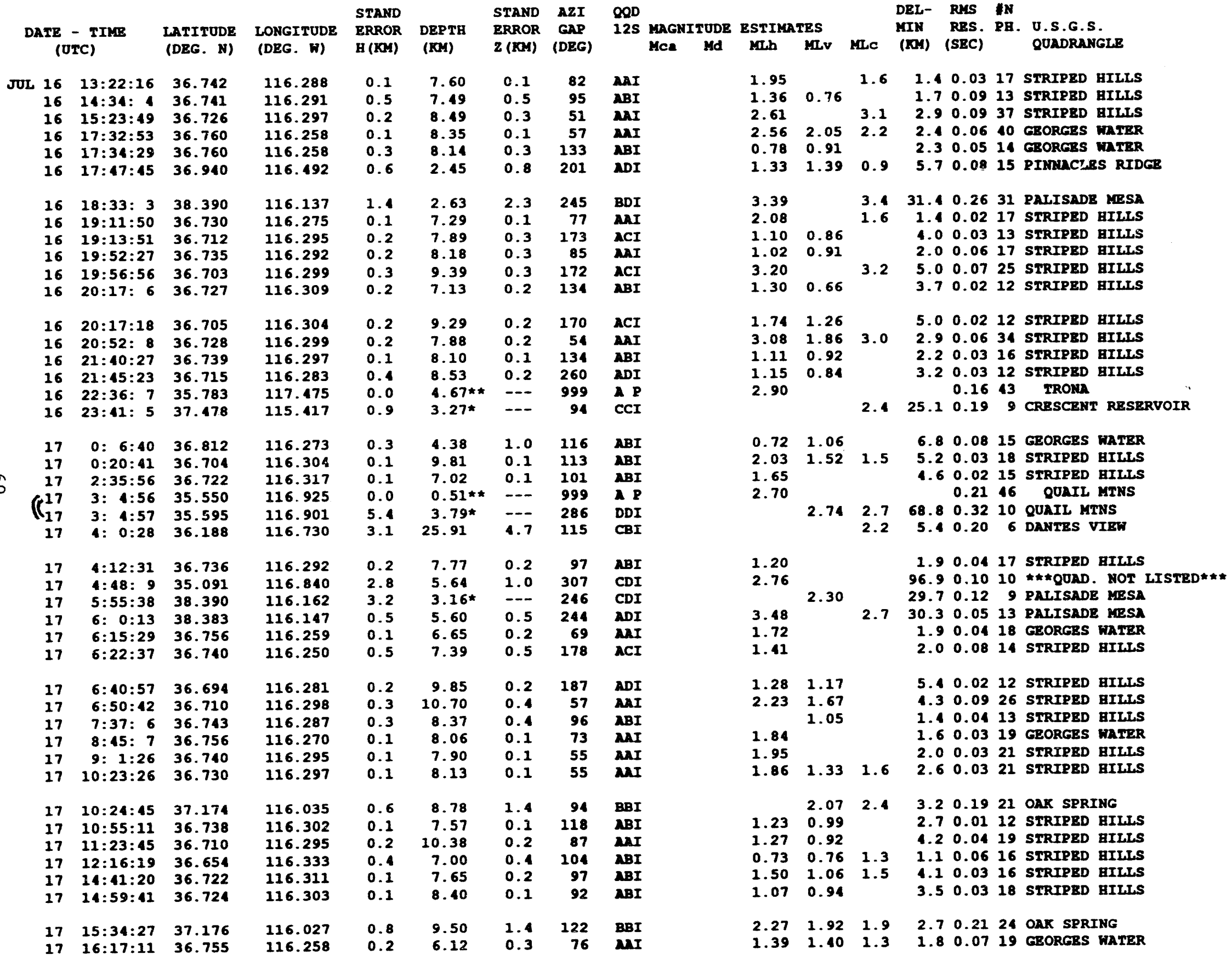


1992 LOCAL HYPOCENTER SUMAARY - SGB EARTBQOAKTS

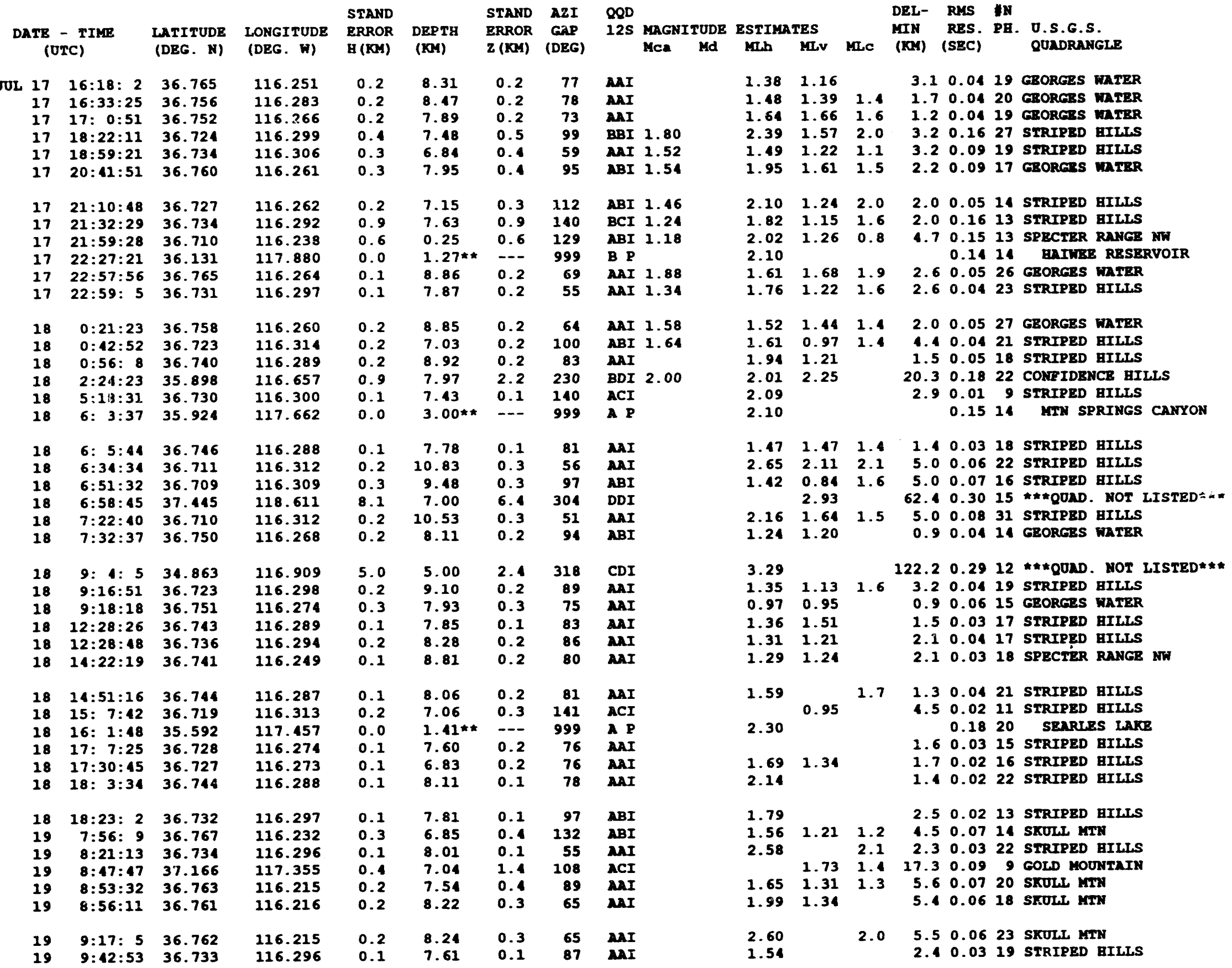


1992 LOCAL HYPOCENTER SUMAARY - SGB EARTHQUAKES

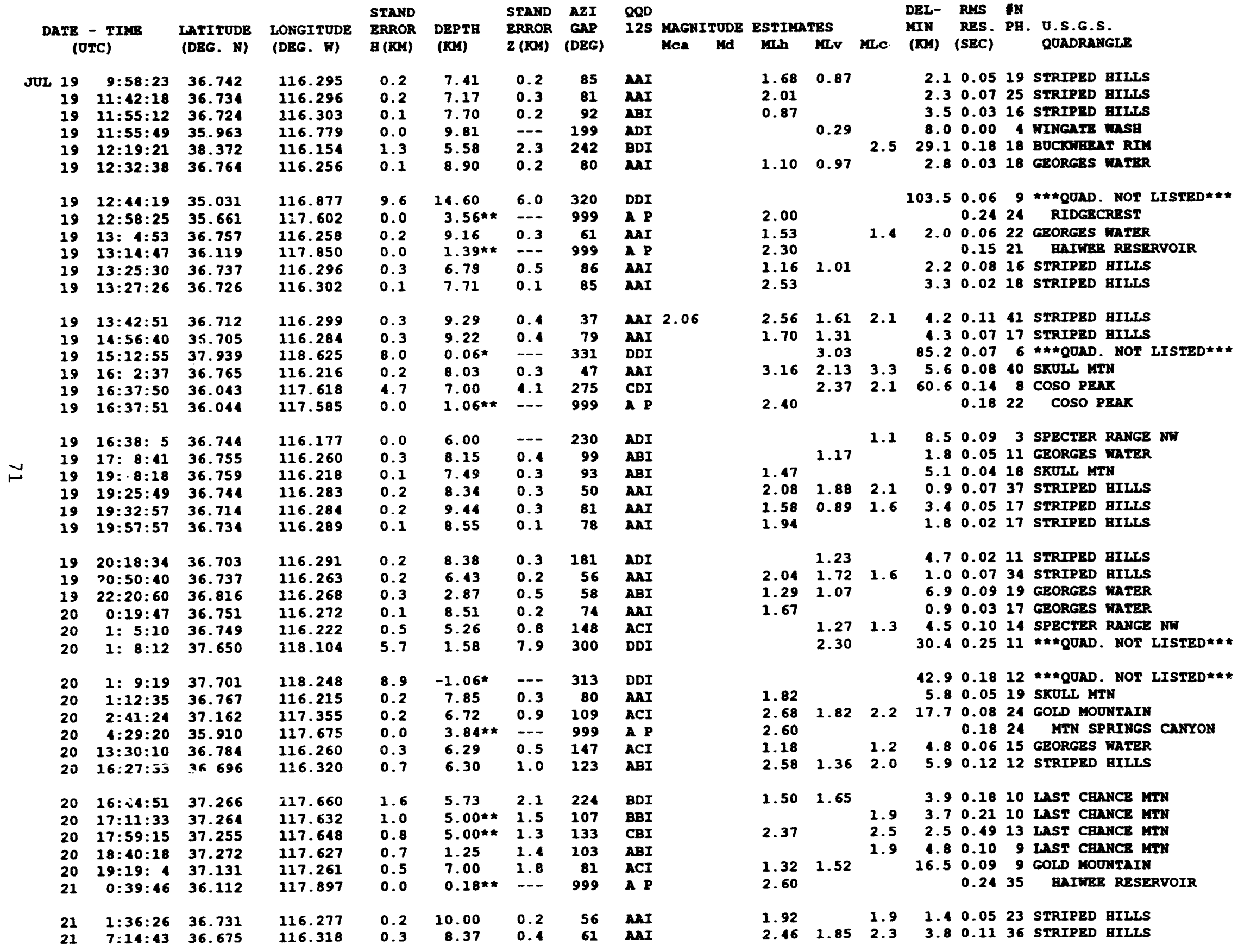


1992 LOCAL HYPOCENTER SUMARAY - SGB EARTHQUAKES (UTC) (DEG. N) (DEG. W)

JUL $21 \quad 11: 11: 43 \quad 36.723$ 21 12: $1: 43$ 21 12: $1: 12 \quad 35.786$ $21 \quad 13: 34: 33 \quad 36.729$ $21 \quad 14: 0.20 \quad 36.051$

$21 \quad 20: 57: 58 \quad 36.744$ $21 \quad 20: 58: 14 \quad 38.924$ $21 \quad 22: 11: 56 \quad 36.752$ $22 \quad 1: 27: 14 \quad 36.729$ $2: 48: 52 \quad 36.748$ $3: 19: 37 \quad 36.701$

$22 \quad 3: 26: 19 \quad 36.718$ $3: 37: 49 \quad 36.751$ $3: 50: 36 \quad 36.737$ 9:19 37.276 :.33: $5 \quad 36.751$ 7: $2: 16 \quad 36.728$

$228: 27: 31 \quad 36.810$ $22 \quad 9: 5: 17 \quad 36.739$ $\begin{array}{rrr}22 & 9: 17: 35 & 36.742 \\ 22 & 12: 41: 55 & 36.737\end{array}$ $22 \quad 16: 25: 32 \quad 36.738$ $22 \quad 16: 46: 43 \quad 36.704$

$22 \quad 16: 47: 26 \quad 36.699$ $22 \quad 16: 51: 55 \quad 36.688$ 22 17:35:24 36.707 22 18: 2:41 36.766 22 19:11:29 36.740 $22 \quad 19: 26: 17 \quad 36.053$

$22 \quad 19: 35: 48 \quad 35.968$ $22 \quad 22: 11: 6 \quad 36.721$ $22 \quad 23: 20: 40 \quad 36.748$

$23 \quad 0: 11: 8 \quad 36.689$

$23 \quad 3: 40: 3 \quad 36.689$ $3: 44: 28 \quad 36.686$

23 3:49:4 36.733

23 8: 8:12 36.724 9: $8: 25 \quad 36.711$ $9: 27: 49 \quad 36.735$ 23 14:33: $1 \quad 36.747$ $23 \quad 19: 18: 20 \quad 36.722$

$2320: 4: 25 \quad 36.707$ $23 \quad 20: 26: 41 \quad 36.731$

\begin{tabular}{|c|c|c|c|c|c|c|}
\hline 116.303 & 0.1 & 7.80 & 0.2 & 92 & $\mathrm{ABI}$ & \\
\hline 117.622 & 0.0 & $4.55 * \star$ & --- & 999 & $\mathbf{A} \mathbf{P}$ & \\
\hline 117.617 & 0.0 & $4.76 \star \star$ & $-\cdots$ & 999 & A $P$ & \\
\hline 116.298 & 0.2 & 8.08 & 0.2 & 89 & MAI & \\
\hline 117.708 & 0.0 & $2.71 * \star$ & -- & 999 & A P & \\
\hline 116.261 & 0.2 & 6.12 & 0.2 & 12 & $\operatorname{AaI}$ & \\
\hline 116.256 & 0.2 & 6.32 & 0.3 & 65 & AAI & \\
\hline 115.664 & 0.0 & 3.61 & $\ldots$ & 315 & ADI & \\
\hline 116.268 & 0.4 & 10.65 & 0.6 & 90 & MAI & \\
\hline 116.303 & 0.4 & 7.83 & 0.5 & 95 & $A B I$ & 1.46 \\
\hline 116.294 & 0.4 & 8.15 & 0.5 & 92 & ABI & 1.40 \\
\hline 116.278 & 0.4 & 8.60 & 0.5 & 122 & $A B I$ & 1.57 \\
\hline 116.304 & 0.4 & 9.66 & 0.5 & 93 & ABI & 1.83 \\
\hline 116.268 & 0.4 & 8.58 & 0.4 & 164 & ACI & 1.60 \\
\hline 116.300 & 0.4 & 7.88 & 0.5 & 100 & ABI & 1.17 \\
\hline 117.619 & 0.5 & -0.03 & 0.5 & 96 & BBI & 1.89 \\
\hline 116.260 & 0.6 & 11.03 & 0.8 & 68 & BAI & \\
\hline 116.251 & 0.2 & 8.39 & 0.5 & 115 & ABI & 1.62 \\
\hline 116.275 & 0.5 & 4.75 & 1.5 & 116 & BBI & 1.21 \\
\hline 116.296 & 0.2 & 7.03 & 0.2 & 86 & AAI & \\
\hline 116.292 & 0.4 & 8.16 & 0.4 & 95 & ABI & 1.23 \\
\hline 116.301 & 0.3 & 7.90 & 0.4 & 93 & $\mathrm{ABI}$ & 1.26 \\
\hline 116.295 & 0.3 & 7.69 & 0.3 & 95 & ABI & 1.80 \\
\hline 116.309 & 0.6 & 9.25 & 0.6 & 166 & $\mathrm{ACI}$ & 1.42 \\
\hline 116.305 & 0.3 & 9.61 & 0.4 & 110 & ABI & 1.81 \\
\hline 116.332 & 1.1 & 12.66 & 1.3 & 120 & BBI & 1.36 \\
\hline 116.320 & 0.7 & 7.55 & 0.9 & 106 & BBI & 1.47 \\
\hline 116.257 & 0.3 & 8.95 & 0.4 & 57 & $\operatorname{AAI}$ & 2.22 \\
\hline 116.299 & 0.5 & 8.18 & 0.7 & 93 & ABI & 1.47 \\
\hline 117.592 & 3.3 & 0.80 & 2.6 & 273 & $\mathrm{CDI}$ & 2.33 \\
\hline 114.801 & 1.2 & 4.31 & 1.2 & 149 & $\mathrm{BCI}$ & 2.58 \\
\hline 116.285 & 0.5 & 6.97 & 0.5 & 107 & $A B I$ & 1.26 \\
\hline 116.285 & 0.4 & 8.35 & 0.5 & 88 & AAI & 1.17 \\
\hline 116.311 & 0.5 & 6.86 & 0.7 & 174 & ACI & 1.25 \\
\hline 116.314 & 0.6 & 5.64 & 1.4 & 170 & ACI & 1.22 \\
\hline 116.302 & 0.5 & 8.08 & 0.6 & 87 & AAI & 1.51 \\
\hline 116.309 & 0. & 8.09 & 0.7 & 94 & $\mathrm{BBI}$ & 1.19 \\
\hline 116.277 & 0.2 & 7.68 & 0.2 & 77 & NAI & \\
\hline 116.276 & 0.2 & 7.88 & 0.3 & 65 & MAI & \\
\hline 116.292 & 0.3 & 7.80 & 0.3 & 53 & $\max$ & \\
\hline 116.285 & 0.2 & 7.71 & 0.2 & 80 & $\operatorname{MAI}$ & \\
\hline 116.300 & 0.2 & 8.55 & 0.2 & 90 & $\overline{A B I}$ & \\
\hline 16. & & 8.19 & 0.4 & 214 & ADI & \\
\hline 116.297 & 0.2 & 7.49 & 0.4 & 53 & MAI & \\
\hline
\end{tabular}

116.308
116.297

$\begin{array}{llllll} & & \text { STAND } & \text { STAND AZI QQD } \\ \text { DATB - TIME LATITUDE LONGITUDE } & \text { ERROR DEPTH } & \text { ERROR GAP } & \text { 12S MAGNITUDE FSTIMATES }\end{array}$

DEL- RHS H

MIN RES. PH. U.S.G.S. Hea Mdh MLV MLC (KA) (SEC) OUNDRANGLB

2.01

$\begin{array}{lllll}2.0 & 3.5 & 0.03 \quad 17 & \text { STRIPED BILLS }\end{array}$

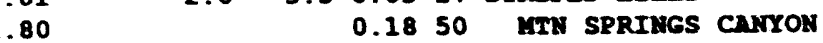

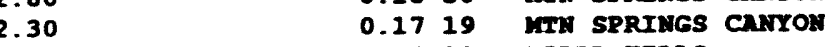

$\begin{array}{lllllll}1.71 & 1.38 & 1.6 & 2.7 & 0.04 & 19 & \text { STRIPED EILLS }\end{array}$

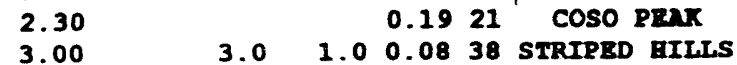

$\begin{array}{llllll}2.57 & 2.2 & 1.4 & 0.07 & 21 & \text { STRIPED HILLS }\end{array}$

$0.93 \quad 102.00 .08 \quad * *$ QUAD. NOT LISTED***

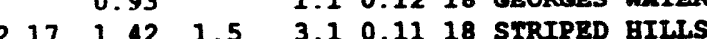

$\begin{array}{lllllll}1.65 & 1.34 & 1.5 & 2.0 & 0.12 & 19 & \text { STRIPED BILS }\end{array}$

$\begin{array}{lllllll}1.65 & 1.34 & 1.5 & 1.5 & 4.6 & 0.11 & 19\end{array}$

$2.24 \quad 1.452 .0 \quad 3.90 .1423$ STRIPED HILLS

$\begin{array}{lllllll}1.97 & 1.74 & 1.4 & 1.1 & 0.08 & 14 & \text { GRORGES MATER }\end{array}$

$\begin{array}{lllllll}1.67 & 1.00 & 1.1 & 2.6 & 0.10 & 15 & \text { STRTPED BILLS }\end{array}$

$2.48 \quad 1.73 \quad 2.4 \quad 5.40 .1624$ TOLE Caxyon

$\begin{array}{llllllll}1.43 & 0.89 & 1.2 & 1.5 & 0.16 & 24 & \text { GEORGES MDTER }\end{array}$

$\begin{array}{llllll}2.18 & 1.75 & 2.5 & 0.08 & 20 & \text { STRIPED BILLS }\end{array}$

$$
0.87 \quad 7.0 \quad 0.1617 \text { GBORGES WATER }
$$

$\begin{array}{lllllll}1.48 & 1.16 & 1.4 & 2.2 & 0.04 & 17 & \text { STRIPED BILLS }\end{array}$

$\begin{array}{lllllll}1.31 & 0.89 & 1.1 & 1.8 & 0.12 & 19 & \text { STRIPED HILLS }\end{array}$

$\begin{array}{lllllll}1.66 & 1.22 & 1.1 & 2.7 & 0.10 & 18 & \text { STRIPED HILLS }\end{array}$

$\begin{array}{lllllll}2.39 & 1.05 & 2.0 & 2.1 & 0.08 & 18 & \text { STRIPED BILLS }\end{array}$

$\begin{array}{llllll}1.78 & 1.45 & 1.2 & 5.4 & 0.11 & 15\end{array}$

$\begin{array}{llllllll}2.49 & 1.83 & 2.1 & 5.7 & 0.07 & 14 & \text { STRIPED BILLS }\end{array}$ $\begin{array}{lllllll}1.70 & 1.23 & 1.4 & 4.8 & 0.22 & 16 & \text { STRIPED BILLS }\end{array}$

$\begin{array}{lllllll}1.65 & 1.23 & 1.5 & 5.8 & 0.18 & 16 & \text { STRIPED HILLS }\end{array}$

$2.81 \quad 1.92 .90 .1230$ GEORGES WATER

$\begin{array}{lllllll}1.77 & 1.51 & 1.4 & 2.4 & 0.14 & 16 & \text { STRIPED BILLS }\end{array}$ $\begin{array}{llllll}2.51 & 2.7 & 58.1 & 0.22 & 15 & \text { coso PEAK }\end{array}$

$\begin{array}{lllllll}2.91 & 2.47 & 2.6 & 6.6 & 0.23 & 19 & \text { BOULDER CIIY }\end{array}$

$\begin{array}{lllllll}1.40 & 1.25 & 1.5 & 2.7 & 0.12 & 16 & \text { STRIPED BILLS }\end{array}$

$\begin{array}{lllllll}1.60 & 1.11 & 1.1 & 1.3 & 0.12 & 17 & \text { STRIPED BILLS }\end{array}$

$1.321 .11 \quad 5.50 .0915$ STRIPED BILLS

$\begin{array}{llllll}0.84 & 0.8 & 5.4 & 0.08 & 14 & \text { STRIPED BILLS }\end{array}$

$\begin{array}{lllllll}0.85 & 1.16 & 0.8 & 5.7 & 0.14 & 21 & \text { STRIPED aILLS }\end{array}$

$\begin{array}{lllllll}1.50 & 0.80 & 0.7 & 3.4 & 0.15 & 23 & \text { SIRIPED HILLS }\end{array}$

$\begin{array}{lllllll}1.61 & 1.25 & 1.6 & 2.2 & 0.04 & 17 & \text { STRIPED gIL.S }\end{array}$

$\begin{array}{lllllll}2.04 & 1.58 & 1.6 & 3.5 & 0.07 & 24 & \text { SIRIPED } \\ 2.04 L L S\end{array}$

$\begin{array}{lllll}1.51 & 1.29 & 2.0 & 0.07 & 21 \\ \text { STRIPED HILLS }\end{array}$

$\begin{array}{lll}16 & 1.23 & 1.30 .0518 \text { STRIPED gIIIS }\end{array}$

$1.441 .15 \quad 3.30 .0417$ STRIPED HILLS

$\begin{array}{lllllll}1.54 & 1.19 & 1.1 & 5.1 & 0.06 & 13 & \text { STRIPED GILLS } \\ 3.10 & & 3.1 & 2.5 & 0.09 & 35 & \text { STRIPED BILLS }\end{array}$ 


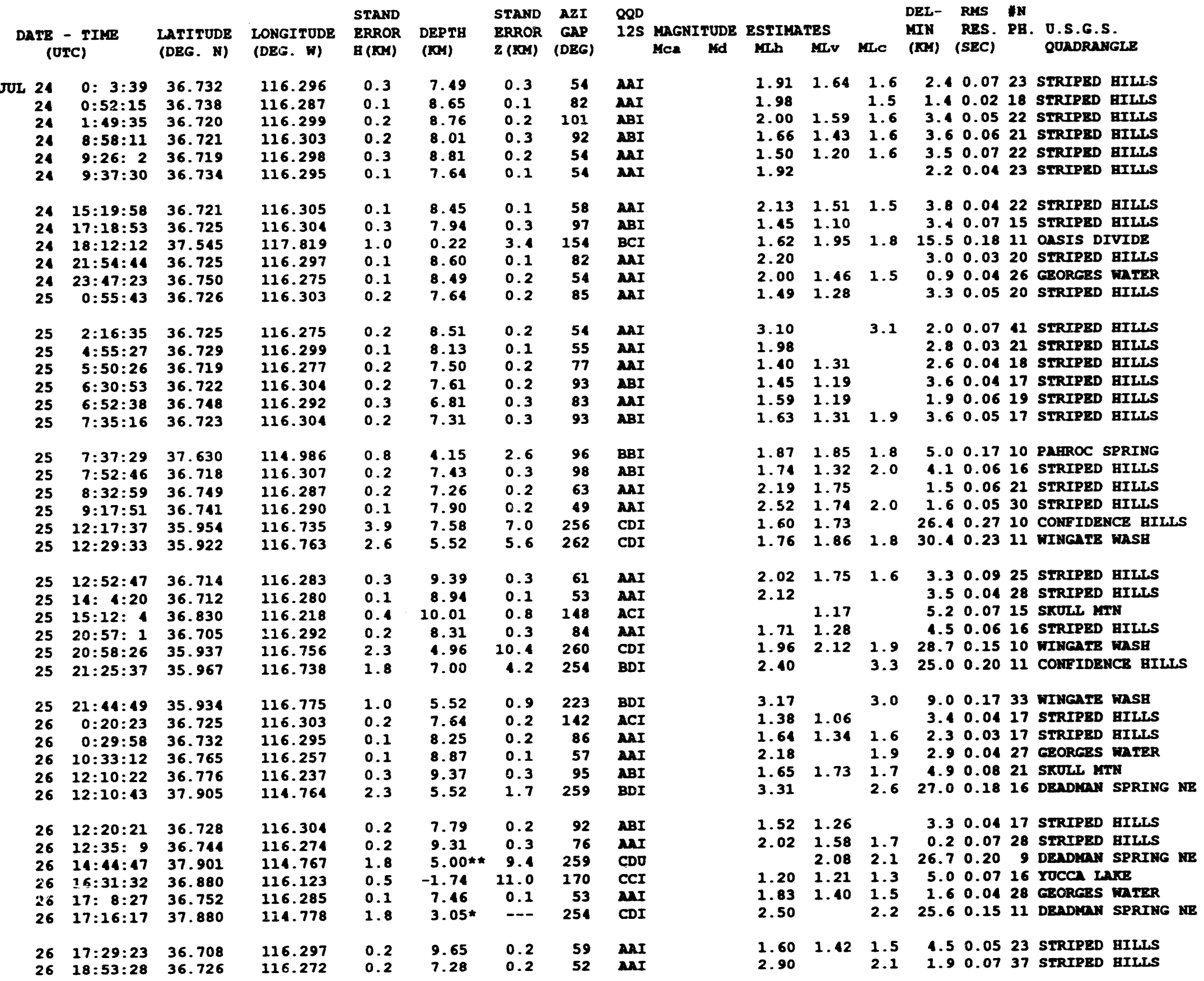


1992 LOCAL HYPOCENTER SULEARY - SGB EARTBQUAKES

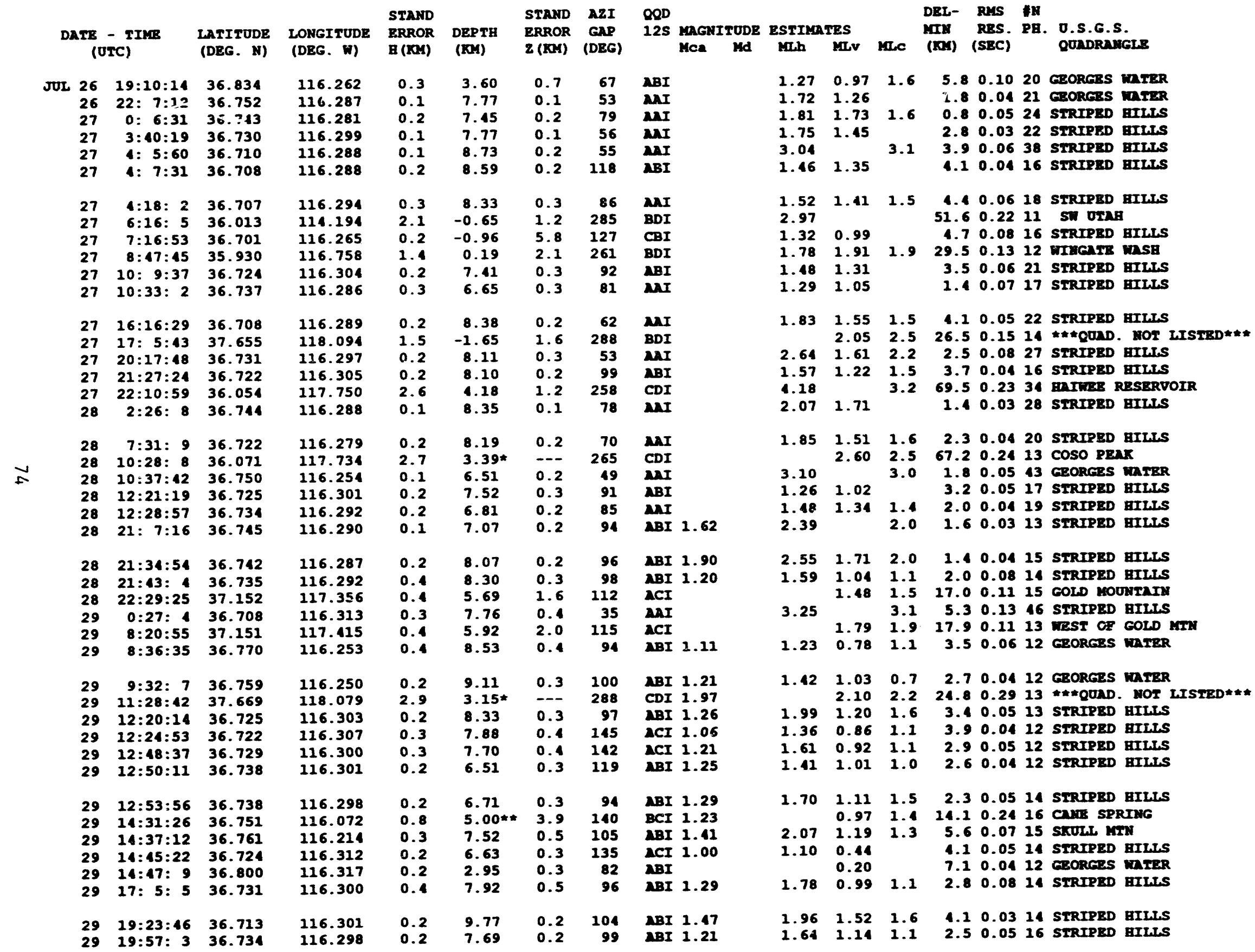


1992 LOCAI HYPOCENTER SUMGNRY - SGB EARTHQOAKRS

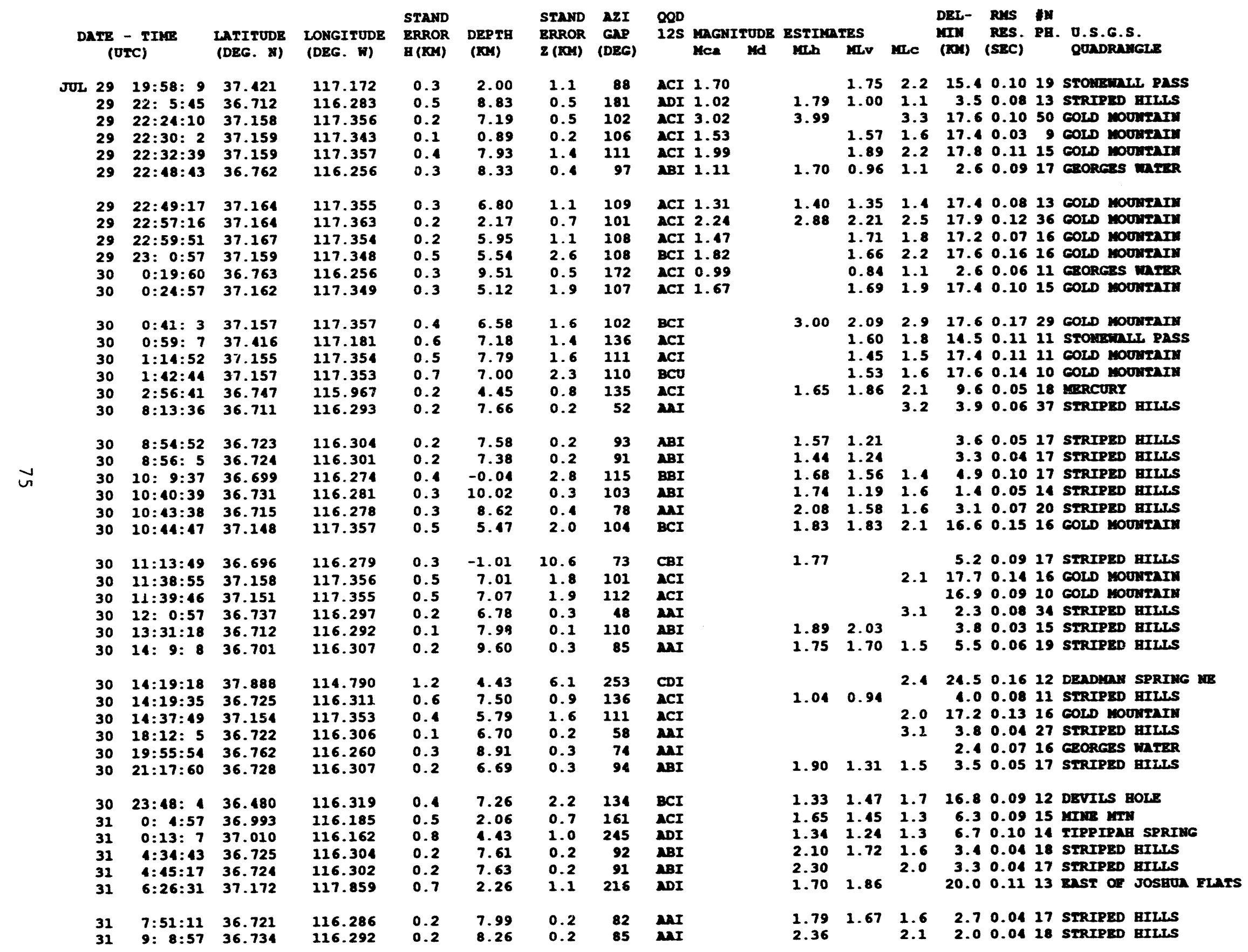


1992 LOCAL HYPOCENTER SURAARY - SGB RARTHQUNKES

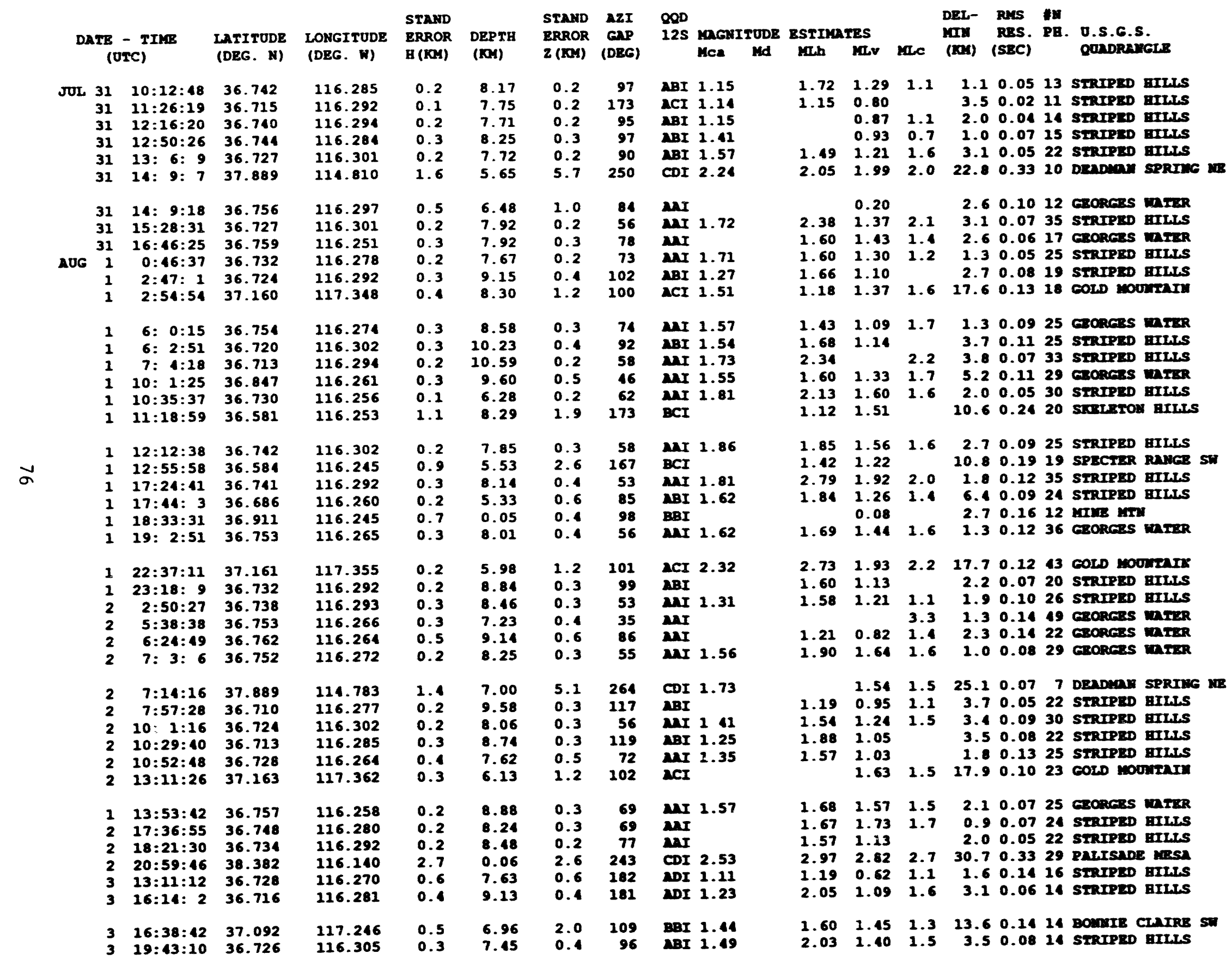


1992 LOCAL HYPOCENTER SUIARRY - SGB ENRTBQUNKES

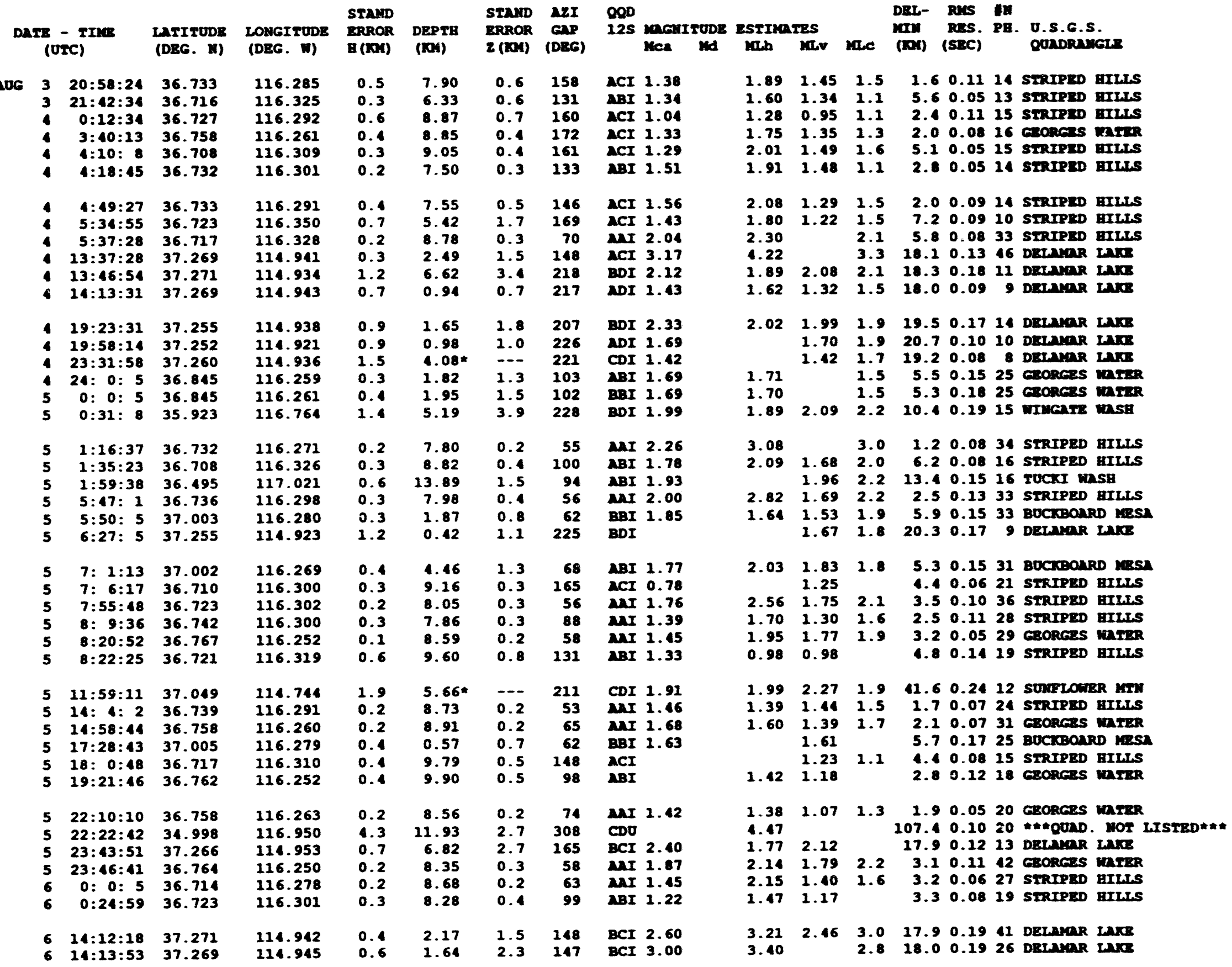


1992 LOCAL HYYOCENTER SULAGARY - SGB EARTHQUAKES

$\begin{array}{llll} & \text { STAND } & \text { STAND AZI QQD }\end{array}$ (0Tc) (DEG. W) (DEG. W) B (KOH) (KOA) 6 $14: 13: 53 \quad 37.269$

$15: 16: 57 \quad 36.740$

$6 \quad 16: 32: 57 \quad 37.269$

$17: 14: 25 \quad 37.269$

$6 \quad 19: 36: 59 \quad 36.724$

69:11:16 36.439

20: $6: 10 \quad 36.730$

20:32: 6 37.265

$23: 59: 51 \quad 36.756$

$6: 35: 37 \quad 36.762$

$9: 53: 33 \quad 37.278$

$7 \quad 10: 26: 41 \quad 36.725$

$711: 0: 59 \quad 36.724$

$11: 56: 32 \quad 36.705$

$12: 23: 16 \quad 36.763$

15:19: 236.091

7 15:22:11 36.763

$15: 32: 60 \quad 36.727$

7 16:24:31 36.706

$\begin{array}{rrr}19: 13: 10 & 36.765 \\ 0: 40: 6 & 36.721\end{array}$

2.44: $7 \quad 36.727$

$3: 29: 836.759$

B $3: 31: 29 \quad 36.756$

$5: 33: 3 \quad 36.761$

8:29:26 36.739

11:22:19 37.730

14:29:28 36.713

8 16:58:53 37.267

$18: 14: 59 \quad 36.704$

$18: 21: 23 \quad 36.747$

$21: 11: 39 \quad 37.290$

$\begin{array}{ll}1: 13: 27 & 36.725\end{array}$

$1: 28: 52 \quad 37.007$

4:53:49 36.708

$5: 18: 28 \quad 36.736$ 8: $0: 50 \quad 36.709$ 8: 7: $8 \quad 36.736$ $15: 45: 56 \quad 36.259$ 15:55:17 36.781

$16: 43: 1436.745$

114.942

114.945

$\begin{array}{ll}0.4 & 2.17 \\ 0.6 & 1.64\end{array}$

$\begin{array}{ll}0.6 & 1.6 \\ 0.3 & 6.28\end{array}$

$2.7 \quad 10.69$

114.947

116.303

$\begin{array}{ll}2.7 & 0.36\end{array}$

(III) (DEG)

25 mantrude estrutes

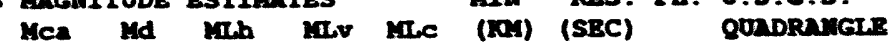

$\begin{array}{llllll}0.3 & 8.11 & 1.4 & 78 & \text { ACI } 2.11\end{array}$

116.284

114.952

116.263

116.260

114.987

116.312

116.312

116.307

116.280

116.253

116.301

116.243

116.244

116.329

116.307

116.255

$\begin{array}{lll}1.5 & 148 \quad \text { BCI } 2.60\end{array}$

$\begin{array}{lrr}147 & \text { BCI } 3.00\end{array}$

3.4214 CDI

CDI 2.39

BII

$3.21 \quad 2.46$

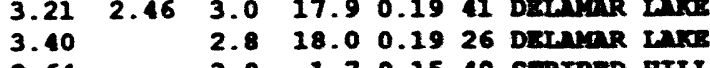

$3.0 \quad 1.70 .1540$ STRIPED mIL.

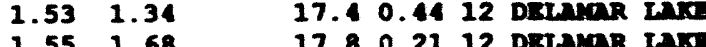

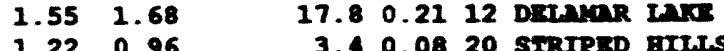

$\begin{array}{llll}8.20 & 0.3 & 81 & \text { MAI }\end{array}$

$1.2 \quad 2.61$

$0.3 \quad 9.09$

$\begin{array}{rr}5.0 & 182 \\ 0.3 & 70\end{array}$

$0.3 \quad 70$

0.5204

BDI 1.64

MAI 1.48

NAI 1.47
ADI 1.46

$\begin{array}{lllll}0.2 & 6.99 & 0.2 & 61 & \text { NaI } 1.98\end{array}$

0.262

NAI 1.64

ABI 1.45

MI 1.28

CDI 2.01

ABI 1.73

116.259

116.260

116.254

115.012

116.325

117.659

116.292

116.284

114.968

116.313

116.276

116.301

116.294

116.335

116.295

116.208

116.234

116.287

116.238

116.272

ABI 1.14

BaI 1.99

ABI 1.83

ABI 1.39

ABI 1.50

$\begin{array}{lllllll}2.50 & 2.11 & 2.2 & 17.1 & 0.14 & 38 & \text { WEST OR FURACB CRERX }\end{array}$

$\begin{array}{lllllll}1.37 & 1.09 & 0.8 & 1.7 & 0.07 & 20 & \text { STRIPED BILLS }\end{array}$

$\begin{array}{lllllll}1.37 & 1.51 & 1.8 & 18.1 & 0.21 & 13 & \text { DELMMR L LXe }\end{array}$

$\begin{array}{lllllll}1.59 & 1.52 & 1.4 & 1.7 & 0.07 & 23 & \text { GzORER mitrR }\end{array}$

$\begin{array}{lllllll}1.53 & 1.34 & 1.3 & 2.4 & 0.11 & 30 & \text { ERORers MATRR }\end{array}$

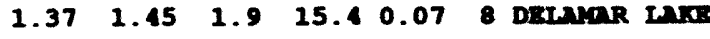

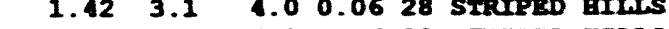

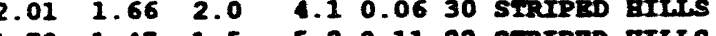

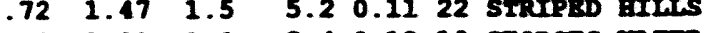

$\begin{array}{lllllll}1.69 & 1.01 & 1.0 & 2.4 & 0.1318 & \text { GEORGS MATER }\end{array}$

$\begin{array}{lllllll}2.20 & 1.56 & 1.9 & 2.8 & 0.09 & 18 & \text { eroners mmR }\end{array}$

$\begin{array}{lllllll}1.81 & 1.18 & 1.5 & 3.1 & 0.10 & 17 & \text { STRIPED BILLS }\end{array}$

$2.431 .761 .6 \quad 4.80 .1926$ SPECTER RMNGE $\mathrm{WH}$

$\begin{array}{lllllll}1.86 & 1.84 & 1.7 & 3.6 & 0.07 & 24 & \text { SKOLL MTH }\end{array}$

$\begin{array}{lllllll}1.18 & 1.02 & 1.2 & 5.6 & 0.05 & 21 & \text { STRIPED BILLS }\end{array}$

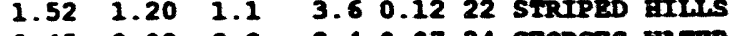

$\begin{array}{lllllll}2.45 & 2.02 & 2.2 & 2.4 & 0.07 & 34 & \text { GEORGES MATER }\end{array}$

ABI 1.61

MII 1.39

$\max 1.56$

BCI 1.71

BCI 1.74

$\begin{array}{lllllll}1.69 & 1.28 & 1.7 & 2.0 & 0.08 & 21 & \text { GRORGES MTER }\end{array}$

$\begin{array}{lllllll}1.58 & 1.29 & 1.7 & 2.4 & 0.11 & 25 & \text { Georas }\end{array}$

$2.141 .341 .9 \quad 1.70 .0926$ STRIPED BILLS

1.5010 .90 .1911 grro

$\begin{array}{lllrlll}1.18 & 1.25 & 1.1 & 5.7 & 0.11 & 23 & \text { STRIPED aILLS }\end{array}$

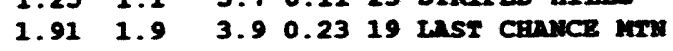

$\begin{array}{lllll}0.3 & 10.00 & 0.5 & 180 & \text { ACI } 1.28\end{array}$

NaI 1.42

BDI

$\begin{array}{llll}1.81 & 1.43 & 4.70 .0718 \text { SIRTPED GILLS }\end{array}$

$\begin{array}{lllllll}1.77 & 1.40 & 1.5 & 1.2 & 0.08 & 28 & \text { STRTPRD BILLS }\end{array}$

$1.841 .35 \quad 14.90 .137$ DELryar WKe

$\begin{array}{llll}1.17 & 1.18 & 4.20 .0621 & \text { STRIPBD BII.S }\end{array}$

$\begin{array}{lllllll}1.22 & 1.53 & 1.6 & 5.4 & 0.1626 \text { BOCKBOARD MESA }\end{array}$

21.37

NaI 1.45

$2.00 \quad 1.49 \quad 1.6$

$1.25 \quad 1.09$

NBI

MAI 3.14

BCI 2.55

BAI 1.83

$\begin{array}{lllllll}3.50 & 3.02 & 3.0 & 2.2 & 0.12 & 52 & \text { STRIPBD } \\ & 1.08 & & 15.4 & 0.26 & 13 & \text { MILI }\end{array}$

1.08

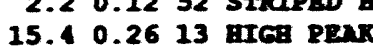

2.541 .921 .95 .40 .2720 SkणLI $\mathrm{km}$

$\begin{array}{lll}1.42 & 0.95 & 1.30 .0818 \text { STRIPED BILLS }\end{array}$

$18: 27: 47 \quad 36: 761$

ABI 1.33

$\begin{array}{lll}1.73 & 0.97\end{array}$

$\begin{array}{lllllll}1.73 & 0.97 & & 3.7 & 0.08 & 13 & \text { SKULL MTH } \\ 1.40 & 1.34 & 1.6 & 1.6 & 0.12 & 19 & \text { STRIPED BILSS }\end{array}$

$0.5 \quad 9.35$

$0.6 \quad 184 \quad$ ADI 1.81 
1992 LOCAL HYPOCENYER SURAARY - SGB EARTHQUAKRS

STAND

DATE - TIME LATITUDE LONGITUDE BRR (OTC)

$922: 3: 36 \quad 36.733$

$6: 12: 42 \quad 36.735$

$10 \quad 10: 21: 52 \quad 36.767$

$10 \quad 10: 54: 15 \quad 36.784$

10 11:25: $7 \quad 36.782$

$10 \quad 18: 28: 15 \quad 36.725$

$10 \quad 19: 16: 29 \quad 36.716$

10 21: $3: 35 \quad 36.715$

$10 \quad 21: 32: 20 \quad 36.729$

$11 \quad 2: 41: 55 \quad 36.720$

$113: 51: 16 \quad 36.767$

$\begin{array}{lll}11 & 6: 23: 45 & 36.794 \\ 11 & 8: 48: 9 & 36.743\end{array}$

$\begin{array}{rrr}11 & 8: 48: 9 & 36.743 \\ 11 & 10: 18: 45 & 36.713\end{array}$

11 21:28: 936.740

116.291

116.308

116.298

116.241

116.252

116.224

116.190

116.243

116.292

116.291

116.298

116.274

116.271

116.278

116.289

116.289

116.302

116.289

116.289 $\begin{array}{lll}23: 17: 48 & 36.718 \\ 20 & 6: 7: 58 & 36.738\end{array}$

$\begin{array}{lll}10 & 6: 42: 42 & 36.755 \\ 10 & 7: 23: 54 & 36.757\end{array}$

11 14:52:31 36.73e

116.261

116.306

116.311

116.279

116.300

116.256

116.261

116.300

116.301
116.295

$1: 30: 32 \quad 36.728$

$5: 38: 47 \quad 36.762$

9:9:1 36.714

$15: 10: 29 \quad 35.855$

$13 \quad 15: 22: 18 \quad 36.746$

$\begin{array}{lll}13 & 15: 43: 34 & 36.721 \\ 13 & 17: 7: 56 & 36.750\end{array}$

$14 \quad 2: 26: 44 \quad 36.729$

$14 \quad 4: 55: 14 \quad 35.816$

$146: 6: 16 \quad 36.727$

$14 \quad 10: 22: 1 \quad 36.629$

12: 6: $5 \quad 36.725$

$1412: 11: 636.724$

$14 \quad 12: 58: 24 \quad 36.762$

14 14: 1: 636.713 116.281

116.285 117.669 116.272

116.068

116.303
STAND AZI QOD

PRPOR GAP 125 MAGNITUDE ESTIMATES

2 (RO) (DEG)

Ma. Md

DEL- RKS N

MIY RES PH. O.S.G.S.

AAI 1.45 1.50 $1.02 \quad 1.202 .00 .1022$ STRTPED

$\begin{array}{lllll}0.3 & 8.88 & 0.4 & 52 & \text { AAI } 1.45 \\ 0.1 & 7.78 & 0.2 & 98 & \text { ABI } 1.21\end{array}$

$0.4 \quad 125 \quad \mathrm{ABI} 1.23$

$\begin{array}{llllll}0.3 & 6.14 & 0.5 & 103 & \text { ABI } 1.19\end{array}$

$\begin{array}{llllll}0.4 & 9.64 & 0.6 & 100 & \text { ABI } 1.38\end{array}$

$\begin{array}{lllll}1.2 & 5.45 & 1.5 & 273 & \text { BDI } 1.32\end{array}$

$\begin{array}{lllll}0.4 & 2.60 & 0.7 & 91 & \text { ABI } \\ 0.7 .74\end{array}$

$\begin{array}{llllll}0.5 & 8.59 & 0.6 & 102 & \text { ABI } 1.39\end{array}$

$0.6 \quad 7.58$

$0.2 \quad 7.51$

$\begin{array}{llllll}0.3 & 7.72 & 0.5 & 99 & \text { ABI } 1.29\end{array}$

$\begin{array}{llllll}0.4 & 7.69 & 0.6 & 112 & \text { ABI } 1.32\end{array}$

$0.3 \quad 11.79$

$0.3 \quad 2.20$

$0.2 \quad 7.88$

$0.3 \quad 6.65$

$\begin{array}{rr}0.6 & 112 \\ 0.5 & 72\end{array}$

$\begin{array}{ll}0.5 & 72 \\ 0.9 & 75\end{array}$

75 AnT 1.40

AAI 1.65

MAI 1.96

ABI 1.77

$\begin{array}{lll}1.50 & 1.02 & 1.2\end{array}$

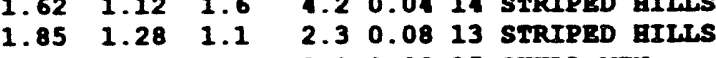

$2.08 \quad 1.9 \quad 3.10 .0915$ SKULL KTN

$\begin{array}{lllllll}2.62 & 1.00 & 1.4 & 2.4 & 0.09 & 13 & \text { GBORGES MAIER } \\ & & 2.2 & 5.1 & 0.20 & 15 & \text { SKOLL MPN }\end{array}$

$\begin{array}{llllllll}2.45 & 1.64 & 1.2 & 8.6 & 0.11 & 10 & \text { SKOLL MTN }\end{array}$

$\begin{array}{llllll}2.62 & 1.9 & 5.1 & 0.11 & 14 & \text { SXOLL MTN }\end{array}$

$\begin{array}{lllllll}1.93 & 1.30 & 1.6 & 2.6 & 0.13 & 17 & \text { STRIPED BILLS }\end{array}$ $\begin{array}{lllll}0.51 & 3.4 & 0.07 & 8 & \text { STRIPED gILLS }\end{array}$

$\begin{array}{lllllll}1.89 & 1.32 & 0.8 & 3.8 & 0.06 & 15 & \text { STRIPED HILLS } \\ 1.82 & 1.11 & 1.5 & 2.5 & 0.09 & 15 & \text { STRIPED BILLS }\end{array}$

$\begin{array}{lllllll}1.48 & 1.12 & 1.2 & 2.5 & 0.12 & 15 & \text { STRIPED BILLS }\end{array}$ \begin{tabular}{lllll}
1.05 & 1.2 & 2.8 & 0.08 & 13 \\
\hline
\end{tabular} $\begin{array}{lllllll}1.28 & 1.07 & 1.5 & 5.7 & 0.15 & 23 & \text { GRORGES WATRR }\end{array}$ $\begin{array}{lllllll}2.35 & 1.85 & 2.1 & 1.5 & 0.06 & 36 & \text { STRIPED BILLS }\end{array}$ $2.461 .882 .2 \quad 3.60 .1241$ STRIPED

$\begin{array}{lllllllll}1.89 & 1.33 & 1.5 & 2.8 & 0.07 & 14 & \text { STRIPBD }\end{array}$

$\begin{array}{llllll}1.98 & 1.9 & 1.5 & 0.07 & 16 & \text { STRIPED BILLS }\end{array}$ $\begin{array}{lllllll}1.86 & 1.39 & 1.1 & 1.5 & 0.06 & 13 & \text { STRIPED HILLS }\end{array}$ $\begin{array}{lllllll}1.82 & 1.43 & 1.2 & 1.0 & 0.12 & 16 & \text { STRIPED BILLS }\end{array}$ $\begin{array}{lllllll}1.75 & 1.16 & 1.2 & 4.9 & 0.11 & 15 & \text { STRIPED HILLS }\end{array}$ $\begin{array}{lllllll}2.54 & 2.09 & 2.0 & 4.0 & 0.09 & 31 & \text { STRIPED BILLS }\end{array}$ $\begin{array}{lllllll}2.32 & 1.36 & 1.6 & 2.7 & 0.06 & 15 & \text { STRIPED BILLS }\end{array}$

\begin{tabular}{lllllll}
1.60 & 0.99 & 1.1 & 3.9 & 0.17 & 11 & STRIPED \\
\hline
\end{tabular} $1.68 \quad 1.16 \quad 1.3 \quad 2.10 .0914$ GRORGES 1.931 .331 .71 .90 .0915 GEORGRS UnIRR 1.91 .08 1.7 1.90 .0722 STRTPED $1.771 .21 \quad 3.00 .0495$ $\begin{array}{lllllll}1.52 & 0.99 & 1.5 & 2.9 & 0.05 & 17 & \text { SIRIPED BILLS }\end{array}$ 117.692 116.286 116.306
116.279

8.40
8.45

ABI 1.23 ABI 1.24 AAI 1.79 ABI 1.50 $\begin{array}{ll}0.1 & 7.90 \\ 6.7 & 2.54\end{array}$ 7.46
11.08

BBI 1.08 ABI 1.18 AAI

ABI 2.55

ABI

$\begin{array}{lllllll}1.68 & 1.26 & 1.3 & 2.9 & 0.06 & 21 & \text { GEORGES WATER }\end{array}$

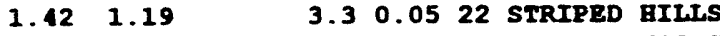

AAI 1.26 MaI 1.26 BDI 2.19 ABI 1.34 AAI 1.73

$1.56 \quad 2.26$ $\begin{array}{llllll}26 & 2.6 & 75.4 & 0.20 & 14 & \text { MTN SPRINGS CANYON }\end{array}$

$\begin{array}{lllllll}1.56 & 1.17 & 1.1 & 1.3 & 0.06 & 15 & \text { STRIPED BILLS }\end{array}$ $\begin{array}{lllllll}1.90 & 1.37 & 1.5 & 3.9 & 0.06 & 21 & \text { SIRIPED BILLS }\end{array}$ $\begin{array}{llllllll}1.71 & 1.36 & 1.6 & 1.0 & 0.10 & 34 & \text { GRORGES WATER }\end{array}$

$\begin{array}{lllllll}1.21 & 1.16 & 1.1 & 1.9 & 0.03 & 17 & \text { STRIPED BILLS }\end{array}$ $\begin{array}{lllllll}2.17 & 2.14 & 2.3 & 74.2 & 0.15 & 10 & \mathrm{kTM} \text { SPRINGS CAYYON }\end{array}$ $\begin{array}{llllllll}2.31 & 1.41 & 1.9 & 1.7 & 0.02 & 15 & \text { STRIPED EILLS }\end{array}$ $\begin{array}{lllllll}1.38 & 1.40 & 1.2 & 10.2 & 0.10 & 18 & \text { CMYP DESERT ROCK }\end{array}$ $\begin{array}{llllllll}2.13 & 1.47 & 1.5 & 3.4 & 0.06 & 15 & \text { STRIPED BILLS }\end{array}$ $\begin{array}{llllllll}2.23 & 1.57 & 1.6 & 2.2 & 0.03 & 14 & \text { STRIPED BILLS }\end{array}$

$\begin{array}{llllllll}1.87 & 1.60 & 1.7 & 2.6 & 0.09 & 32 & \text { GEORGES WATER }\end{array}$

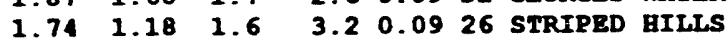


1992 LOCAL HYPOCENTER SUMMARY - SGB EARTHQUAKES

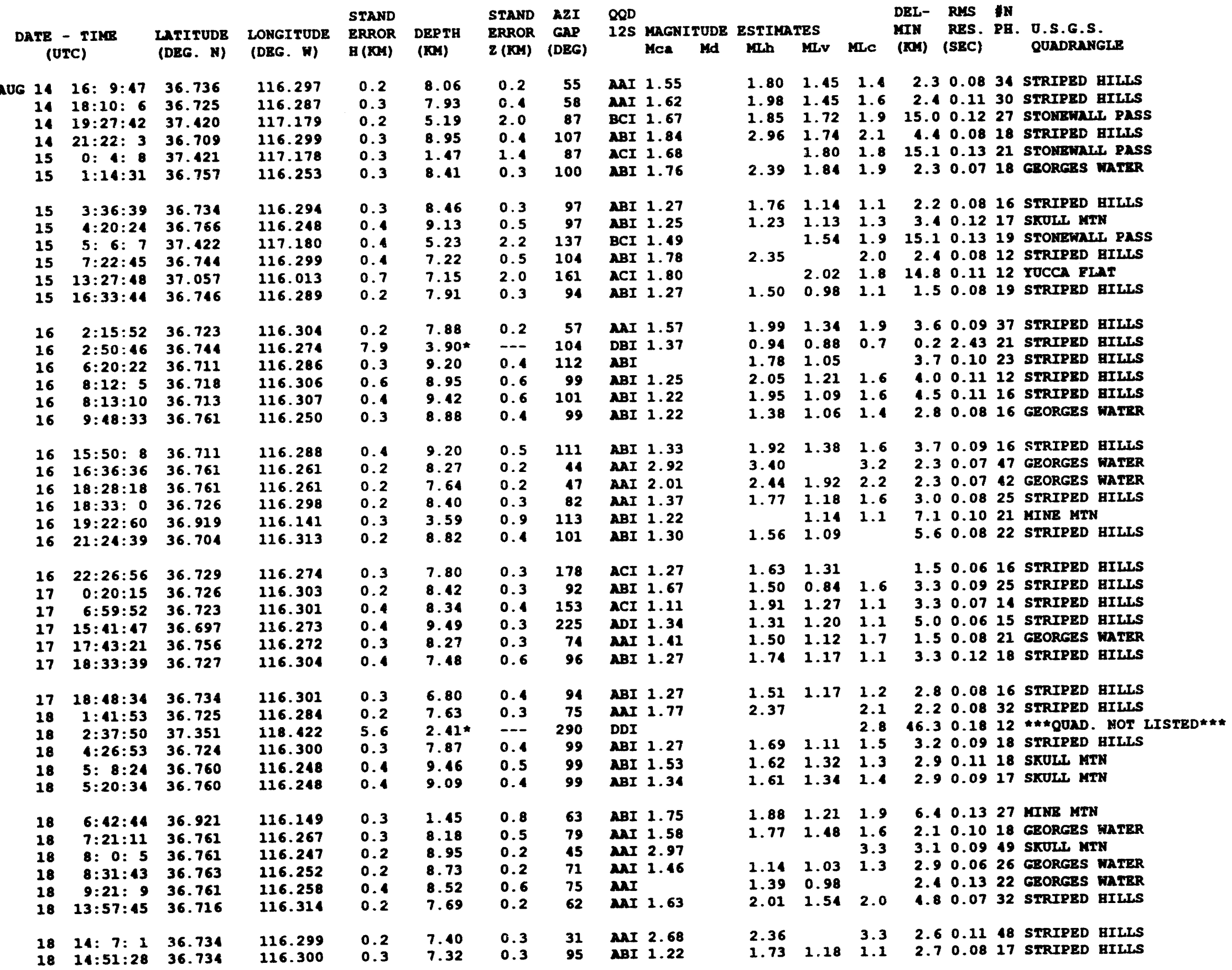


1992 LOCAL HYPOCENTER SUMDEARY - SGB EARTHQUAKES

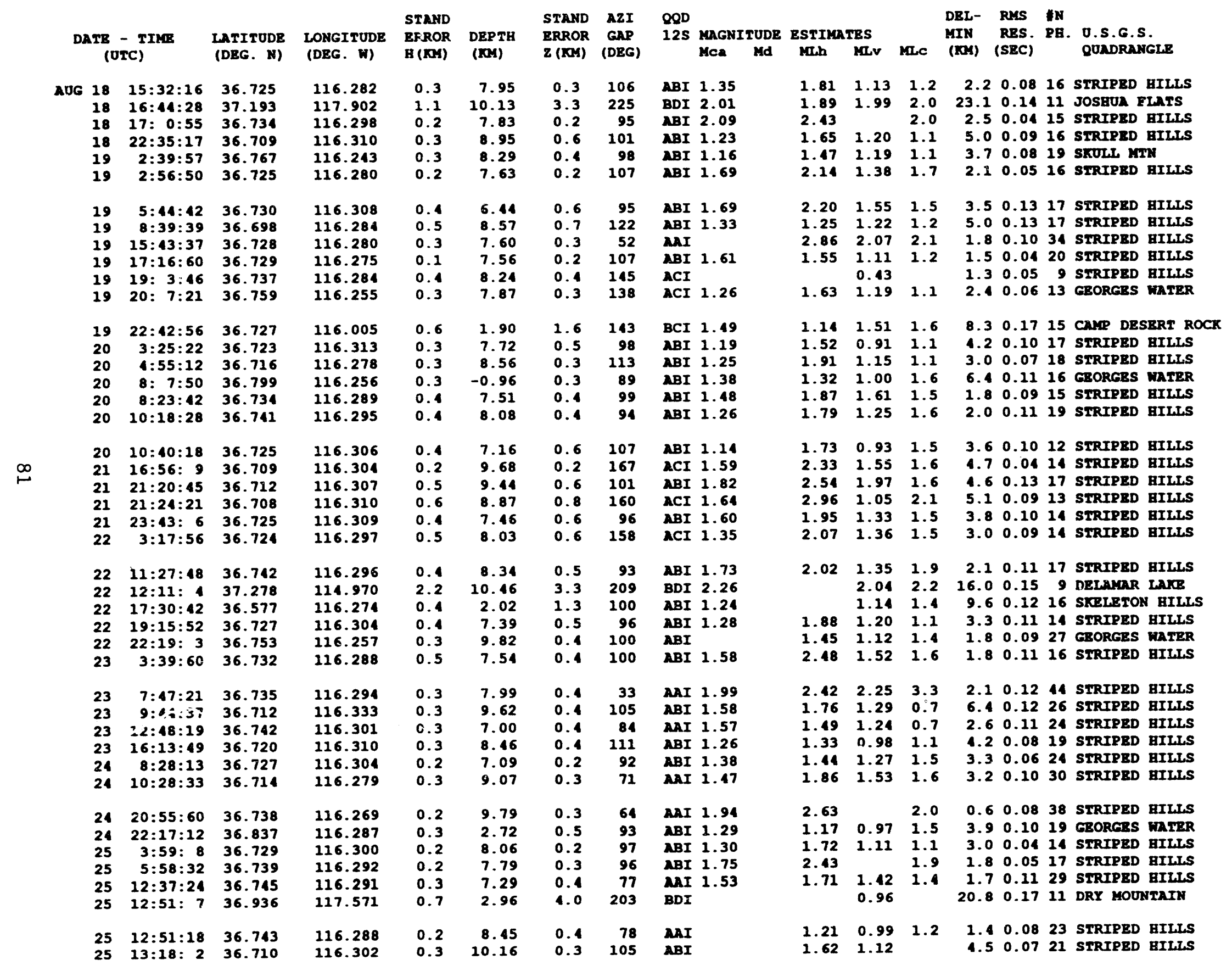


1992 LOCAL HYPOCENTER SULAYARY - SGB EARTHQUAKES

$\begin{array}{lll}\text { STAND } & \text { STAND AZI QOD }\end{array}$ $\begin{array}{lllllll}\text { (UTC) } & \text { (DEG. N) } & \text { (DEG. W) } & \text { H (KO) } & \text { (KOY) } & \text { Z (IOY) } & \text { (DEG) }\end{array}$

AUG 25 14: $7: 21 \quad 36.753$ $25 \quad 23: 35: 33 \quad 36.582$ $26 \quad 13: 11: 48 \quad 36.754$ 26 22: $0.15 \quad 36.873$ 26 23: $0: 27 \quad 36.721$ $26 \quad 13: 11: 48 \quad 36.755$

$27 \quad 2: 55: 24 \quad 36.736$ $27 \quad 5: 5: 58 \quad 36.744$ $27 \quad 6: 35: 22 \quad 36.754$ $27 \quad 20: 48: 57 \quad 36.747$ $27 \quad 22: 32: 12 \quad 36.716$ $27 \quad 22: 48: 16 \quad 36.722$

$27 \quad 23: 16: 8 \quad 35.721$ 7:23:27 36.754 $10: 14: 43 \quad 36.029$ $28 \quad 11: 49: 48 \quad 36.708$ $28 \quad 16: 58: 41 \quad 37.208$

116.291 116.256 116.267 116.176 116.305

116.285 116.269 6.263 116.274 116.274 116.281

116.309 116.283 116.274 114.909 114.909 116.299 116.179

116.308 116.267

1.57 .00
$0.5 \quad 10.65$ $\begin{array}{ll}0.3 & 7.93 \\ 2.5 & 3.20\end{array}$ $2 S$ kAGNITUDE ESTIMATES

DEL- RMS IN 117.635 117.648 116.199 116.267

116.255 116.261 116.27 116.214 116.140 116.261 116.305 116.274 116.291 116.292
116.253 116.253
116.251

$\begin{array}{lll}1.5 & 156 \quad \mathrm{BCI}\end{array}$

$13 \cdot 43: 1$

$\begin{array}{ll}15: 37: 16 & 36.767\end{array}$

$20: 35: 21 \quad 36.728$

$22: 20: 5936.727$

$22: 31: 59 \quad 36.760$

22:31:59 36.821

1:56: $3 \quad 37.165$
116.281

116.305

116.285

116.247

116.299

117.347

116.263

116.280
21:58: $3 \quad 36.738$

1:59:9 36.713

$5: 58: 49 \quad 36.725$ $0.3 \quad 7.35$ $0.4 \quad 9.31$ 0.37 .39 0.3 $0.7 \quad 8.89$ $0.5 \quad 9.27$ $\begin{array}{ll}0.5 & 8.04 \\ 0.4 & 7.88\end{array}$

$0.5 \quad 7.42$ 0.18 .01 $0.3 \quad 8.09$ $0.7-1.77$ 0.56 .91

8.11 0.89 .55

$\begin{array}{ll}1.1 & 89 \\ 0.4 & 72\end{array}$ 0.5103 0.155 0.8149 0.6101 $0.5 \quad 99$ $\begin{array}{ll}0.9 & 75 \\ 0.7 & 115\end{array}$ 0.4108

0.696 0.5108 0.380 0.9145 $0.6 \quad 81$

$0.6 \quad 95$

0.485

$3 .-288$

1.4152

8.32

0.5

0.6

0.9

4.47
2.78

2.78
9.04

$0.5 \quad 7.89$

0.57 .84

ABI 1.77

AaI 1.75

ABI 1.54

$\operatorname{MAI} 2.23$

MAI 1.75

ACI 1.74 ABI 1.19 ABI 1.40 AAI 1.24 ABI 1.92 ABI 1.37

ABI 1.42 ABI 1.59

BAI 1.40

BaI 1.44

BCI 1.62

ABI 1.26

AAI 1.28

CDI 2.37

BDI 1.82

AaI 2.38

BBI 1.31

ABI 1.52

AAI 1.27

BCI 1.26

ACI 1.44

ABI 1.57

ABI 1.25

AAI 1.67

ABI 1.30

ABI 1.19

ABI 1.19

ABI 2.21

ABI 1.45

ABI 1.30

ABI 1.34

ACI 1.56

$\begin{array}{rrrr}9.03 & 0.2 & 171 & \text { ACI } \\ 4.47 & 0.5 & 92 & \text { ABI } 1.33 \\ 6.42 & 1.7 & 106 & \text { ACI } 1.63\end{array}$

$\begin{array}{lllll}0.2 & 8.21 & 0.3 & 120 & \text { ABI } 1.35 \\ 0.1 & 7.90 & 0.1 & 107 & \text { ABI } 1.37\end{array}$
MTN RES. PB. O.S.G.S

QUADRANGL
2.00 .1910 GEORGES WATER

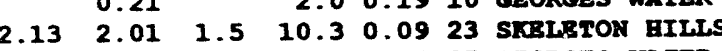
$\begin{array}{lllllll}1.81 & 1.28 & 1.9 & 1.3 & 0.12 & 27 \text { GrORGRS WATK }\end{array}$ $\begin{array}{llllll}1.38 & 1.3 & 2.2 & 0.10 & 16 & \text { SKULL MTI }\end{array}$

$\begin{array}{lllllll}2.82 & 1.90 & 1.7 & 3.7 & 0.13 & 36 & \text { STRIPED HILLS }\end{array}$ $\begin{array}{llllll}1.81 & 1.29 & 1.9 & 1.8 & 0.11 \quad 27 \text { GBORGES WALER }\end{array}$

$2.32 \quad 2.0 \quad 1.40 .1313$ STRIPED HILLS $0.88 \quad 0.40 .1013$ STRIPED BILLS 1.101 .10 .60 .1314 STRIPED DITS $2.40 \quad 2.0 \quad 3.00 .1316$ STRIPED BILLS $\begin{array}{lllllll}1.83 & 1.40 & 1.6 & 2.4 & 0.10 & 17 & \text { STRIPED BILLS }\end{array}$ \begin{tabular}{lllllll}
1.83 & 1.29 & 1.5 & 4.0 & 0.12 & 15 & STRIPED \\
\hline & aILLS
\end{tabular} $\begin{array}{lllllll}2.11 & 1.80 & 1.6 & 2.5 & 0.11 & 16 & \text { STRIPED BILLS }\end{array}$ $\begin{array}{llllll}1.84 & 1.54 & 1.3 & 1.3 & 0.07 & 15 \\ 2.58 & 2.39 & 2.0 & 18.3 & 0.16 & 22 \\ \end{array}$ $\begin{array}{lllllll}1.58 & 2.39 & 2.0 & 18.3 & 0.16 & 22 & \text { GENDERSON }\end{array}$

$\begin{array}{llllllll}1.57 & 1.33 & 1.6 & 4.6 & 0.16 & 27 & \text { STRIPED HILLS }\end{array}$

$92 \quad 1.15 \quad 1.5 \quad 3.60 .1318$ STRIPED HILLS $\begin{array}{lllllll}2.43 & 1.87 & 1.9 & 1.7 & 0.09 & 17 & \text { GEORGES MATER }\end{array}$ $\begin{array}{llllll}2.56 & 2.5 & 61.7 & 0.22 & 9 \\ & \text { coso PEAK }\end{array}$ $\begin{array}{llllll}1.99 & 2.1 & 25.9 & 0.17 & 14 & \text { SALINB PEAK }\end{array}$ $\begin{array}{llllll}1.26 & 1.4 & 3.1 & 0.15 & 14 & \text { SKULL } \\ & 3.2 & 3.1 & 0.13 & 37\end{array}$ $\begin{array}{lllll}3.2 & 3.1 & 0.13 & 37 & \text { STRIPED GILLS }\end{array}$

$\begin{array}{lllllll}1.22 & 0.84 & 1.7 & 5.6 & 0.17 & 19 & \text { GRORGES WATER }\end{array}$ $\begin{array}{lllll}1.24 & 1.4 & 1.4 & 0.1416 & \text { GEORGES WATER }\end{array}$ $\begin{array}{llllll}1.59 & 1.44 & 1.5 & 0.9 & 0.1016 & 16 \text { GORGES WATER }\end{array}$ $\begin{array}{lllllll}1.29 & 1.01 & 1.0 & 4.6 & 0.19 & 15 & \text { SKULL MTN }\end{array}$ $\begin{array}{lllllll} & 0.99 & 0.9 & 3.6 & 0.13 & 14 & \text { MINE MTN } \\ 1.93 & & 1.9 & 2.0 & 0.08 & 15 & \text { GEORGES WATER }\end{array}$ $\begin{array}{llllllll}1.71 & 1.21 & 1.5 & 3.4 & 0.13 & 16 & \text { STRIPED BILIS }\end{array}$ $\begin{array}{lllllll}1.86 & 1.85 & 1.7 & 1.0 & 0.12 & 18 & \text { GEORGES WATER }\end{array}$ $\begin{array}{lllllll}2.13 & 1.69 & 1.5 & 2.4 & 0.13 & 16 & \text { SIRIPED BILS }\end{array}$ $\begin{array}{lllllll}2.08 & 1.15 & 1.5 & 4.2 & 0.11 & 15 & \text { STRIPED BILLS }\end{array}$ $\begin{array}{llllllll}1.55 & 1.37 & 1.7 & 2.7 & 0.16 & 20 & \text { GEORGES WATER }\end{array}$ 3.143 .13 .30 .1315 GBORGES WATER

$\begin{array}{llllllll}1.69 & 1.06 & 1.7 & 1.7 & 0.14 & 15 & \text { STRIPED BILLS }\end{array}$ $\begin{array}{lllllll}1.65 & 1.27 & 1.1 & 3.0 & 0.08 & 14 & \text { STRIPED BILLS }\end{array}$ $\begin{array}{lllllll}1.71 & 1.22 & 1.7 & 2.1 & 0.11 & 15 & \text { STRIPED HILLS }\end{array}$ $\begin{array}{lllllll}1.93 & 1.83 & 1.7 & 3.0 & 0.04 & 14 & \text { SKULL MTN }\end{array}$

$\begin{array}{llllll}1.12 & 0.90 & 1.5 & 4.9 & 0.07 & 20 \\ & 1.76 & 1.5 & 17.0 & 0.11 & 14 \\ \end{array}$

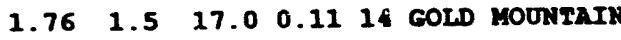
$\begin{array}{lllllll}2.14 & 1.54 & 1.6 & 3.4 & 0.06 & 15 & \text { STRIPED BILLS } \\ 1.77 & 1.42 & 1.2 & 2.0 & 0.02 & 13 & \text { STRIPED BILLS }\end{array}$ 
1992 LOCAL HYPOCENTER SURQAARY - SGB RARTHQUAKES

$\begin{array}{llllll}\text { STAND } & \text { STAND AZI } & \text { QQD } & \text { DEL- RMS HN } \\ \text { BRROR DEPTH } & \text { ERROR GAP } & \text { I2S MAGNITUDE ESTIMATES } & \text { MIN RES. PB. D.s.G.s. }\end{array}$

DATE - TIME LATITUDE LONGITUDE ERROR DEPTH ERROR GAP 12S MAGNTTUDE ESTIMATES MES MIN RES. PB. D.S.G.S.

(UTC)

(DEG. N)

$\begin{array}{llll}\text { AUG } 31 \quad 9: 11: 26 & 36.726 & 116.295\end{array}$

$21: 37: 59 \quad 36.724 \quad 116.276$

$2: 24: 43 \quad 36.733$

$\begin{array}{ll}0.4 & 7.34 \\ 0.4 & 8.08 \\ 0.3 & 6.90\end{array}$

37

$\begin{array}{lllllll}2.09 & 1.69 & 1.5 & 2.7 & 0.10 & 15 & \text { STRIPED GILLS }\end{array}$

$\begin{array}{lllllll}2.21 & 1.88 & 1.7 & 2.1 & 0.10 & 16 & \text { STRIPED BILLS }\end{array}$

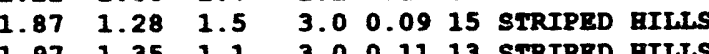

$\begin{array}{lllllll}1.97 & 1.35 & 1.1 & 3.0 & 0.11 & 13 & \text { STRIPED BILLS }\end{array}$

$1.541 .37 \quad 1.4 \quad 1.30 .1014$ STRIPED BILLS

$\begin{array}{rrrrrrrr}5: 41: 12 & 36.740 & 116.286 & 0.4 & 8.49 & 0.5 & 98 & \text { ABI } 1.33\end{array}$

$12: 53: 10 \quad 37.109$

117.046
116.277

116.277

$0.4 \quad 10.53$

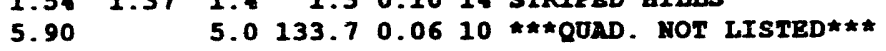

$3: 36: 36$

$8: 32: 52 \quad 37.414$

116.263

0.47 .94

$0.2 \quad 9.19$

$\begin{array}{llll}1.6 & 102 & A B I & 1.57\end{array}$

$\begin{array}{lllllll}1.37 & 1.30 & 1.9 & 14.1 & 0.14 & 16 & \text { BOMaIB CLAIRE SE }\end{array}$

$\begin{array}{lllllll}1.73 & 1.29 & 1.3 & 1.9 & 0.08 & 15 & \text { STRIPED HILLS }\end{array}$

$\begin{array}{lllllll}1.76 & 1.16 & 1.6 & 3.2 & 0.08 & 23 & \text { SIRIPED BILLS }\end{array}$

$\begin{array}{lllllll}2.31 & 1.72 & 1.7 & 3.1 & 0.03 & 24 & \text { STRIPED BILLS }\end{array}$

$\begin{array}{llllllll}2.27 & 1.73 & 2.1 & 9.0 & 0.15 & 26 & \text { LIDA }\end{array}$

$\begin{array}{lllllll}2.27 & 1.73 & 2.1 & 9.0 & 0.15 & 26 & \text { LIDA } \\ 1.83 & 1.12 & & 3.3 & 0.09 & 20 & \text { STRIPED BILLS }\end{array}$

$\begin{array}{rr}0.3 & -0.68 \\ 0.3 & 8.97\end{array}$

$\begin{array}{rrrr}0.3 & 63 & \text { ABI } 1.95 \\ 0.5 & 114 & \text { ABI } 1.20\end{array}$

$\begin{array}{llllll}116.217 & 0.5 & 10.07 & 0.8 & 79 & \text { BAI } 1.61\end{array}$

116.300

$\begin{array}{rr}0.5 & 10.07 \\ 0.2 & 6.76\end{array}$

$0.3 \quad 89$ AAI 1.29

11: $5: 24 \quad 36.828$

$\begin{array}{ll}17: 57: 51 & 36.728\end{array}$

$21: 11: 31 \quad 36.357$

$21: 15: 35 \quad 36.779$

$23: 11: 29 \quad 36.721$

116.752

8.54

$0.3 \quad 55 \quad \operatorname{AAI} 1.68$

19.63
5.76

110 DBI 2.08

116.309

$0.3 \quad 7.73$

1.7 171 BCI 1.48

$\begin{array}{llllll}116.248 & 0.4 & 5.81 & 1.1 & 105 & \text { BBI } 1.34\end{array}$

116.302

$\begin{array}{ll}0: 54: 28 & 36.694 \\ 8: 47: 18 & 36.719\end{array}$

$\begin{array}{ll}8: 47: 18 & 36.719 \\ 13: 22: 34 & 36.724\end{array}$

116.296

$0.3 \quad 9.04$

$0.3 \quad 178$ ACI 1.55

$\begin{array}{llllll}116.286 & 0.4 & 7.93 & 0.5 & 82 & \text { AAI } 1.71\end{array}$

$\begin{array}{llllll}116.296 & 0.4 & 7.84 & 0.5 & 88 & \text { AAI } 1.61\end{array}$

$\begin{array}{lll}13: 25: 38 & 36.722 & 116.296\end{array}$

$1.0 \quad 5.35$

$\begin{array}{lll}1.5 & 136 & \text { BDI } 3.12\end{array}$

$15: 53: 52 \quad 37.415$

117.395

$0.6 \quad 4.69$

$2.6 \quad 100$ BBI 1.92

$16: 23: 13 \quad 36.719$

$18: 18: 44 \quad 36.779$

$22: 49: 58 \quad 36.723$

$\begin{array}{ll}5: 28: 52 & 36.736 \\ 9: 27: 45 & 37.414\end{array}$

116.271

116.237

$0.3 \quad 8.24$

$2.6 \quad 35.40$

116.305

0.26 .90

$0.3 \quad 74$ AAI 1.62

$3.1 \quad 176$ CCI

$0.3 \quad 106 \quad A B I$

117.402

$-0.78$

$\begin{array}{lll}0.3 & 92 & \text { ABI } \\ 0.4 & 63 & \text { ABI }\end{array}$

12:41: $5 \quad 36.710$

$16: 39: 436.730$

$19: 51: 11 \quad 37.094$

20:22: 36.758

$0: 18: 38 \quad 36.714$

116.296
116.305

c.3 8.69

0.488 AAI

117.980

$0.1 \quad 7.08$

$0.2 \quad 54 \quad$ AAT 1.78

$\begin{array}{ll}1.2 & 1.31 \\ 0.2 & 8.00\end{array}$

235 BDI 2.09

$\begin{array}{ll}0.6 & 91 \\ 0.3 & 77\end{array}$

ABI 1.50

AAI

$\begin{array}{lllllll}1.62 & 1.27 & 1.4 & 5.3 & 0.21 & 33 & \text { SKULL MTN }\end{array}$

$\begin{array}{lllllll}1.86 & 1.17 & 1.5 & 2.7 & 0.08 & 24 & \text { STRIPED BILLS }\end{array}$

$\begin{array}{lllllll}2.08 & 1.73 & 1.5 & 2.8 & 0.08 & 27 & \text { STRIPED } \\ 1.5 I L L S\end{array}$

$\begin{array}{lllllll}1.49 & 1.66 & 2.1 & 20.3 & 0.88 & 12 & \text { DEVIISS GOLF COURSE }\end{array}$

$\begin{array}{lllllll}1.40 & 0.97 & 4.8 & 0.2413 & \text { SKULL MTW }\end{array}$

$\begin{array}{lllllll}1.46 & 1.55 & 1.5 & 4.1 & 0.08 & 20 & \text { STRIPED BILLS }\end{array}$

$\begin{array}{lllllll}1.19 & 1.16 & 1.3 & 6.3 & 0.17 & 23 & \text { SKULL MTN }\end{array}$

$\begin{array}{lllll}1.46 & 1.18 & 6.0 & 0.0620 & \text { STRIPED HILLS }\end{array}$

$\begin{array}{llllll}2.05 & 1.52 & 1.5 & 3.3 & 0.11 & 23 \\ \text { SIRIPED BILLS }\end{array}$

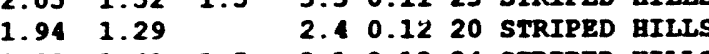

$\begin{array}{rrrrrrr}1.99 & 1.41 & 1.5 & 3.1 & 0.13 & 24 & \text { STRIPED GILLS } \\ 3.77 & & & 75.9 & 0.28 & 29 & \text { HAIMRE RESERVOIR }\end{array}$

$\begin{array}{rrrrrr}116.277 & 0.2 & 8.95 & 0.3 & 77 & \text { AAI } \\ 116.214 & 0.4 & 7.70 & 0.7 & 103 & \text { ABI } 1.70\end{array}$

116.299

$\begin{array}{rrrr}0.4 & 108 & \text { ABI } & 1.91 \\ 0.3 & 32 & \text { AAI } & 2.18\end{array}$

$6 \quad 1: 23: 30 \quad 36.708$

$6 \quad 5: 0: 0036.735$

116.298

$0.3 \quad 8.22$

$\begin{array}{lllllll}116.278 & 0.2 & 8.07 & 0.2 & 106 & \text { ABI } 1.28\end{array}$

$\begin{array}{llll}6.80 & 0.6 & 116 & \text { ABI } 1.19\end{array}$

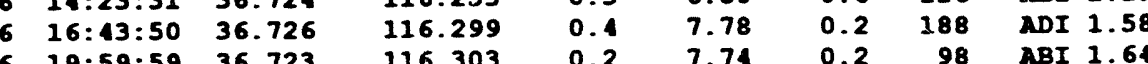

$619: 59.59 \quad 36.723$

116.303

$0.4 \quad-1.27$

$0.7 \quad 141 \quad$ BCI 2.67

$\begin{array}{lllllll}1.66 & 2.1 & 9.7 & 0.24 & 17 & \mathrm{LIDA}\end{array}$

$\begin{array}{lllllll}2.00 & 1.22 & 1.5 & 2.7 & 0.08 & 21 & \text { STRIPED HILLS }\end{array}$

1.541 .29

1.471 .06

$2.07 \quad 1.9 \quad 9.10 .1319$ LIDA

$\begin{array}{lllllll}1.51 & 1.28 & 1.2 & 4.2 & 0.12 & 25 & \text { STRIPED BILLS }\end{array}$

$2.71 \quad 2.0 \quad 3.30 .0631$ STRIPED BILLS

$2.00 \quad 2.2 \quad 33.40 .1418$ WAUCOBA SPRING

$\begin{array}{lllllll}1.33 & 1.02 & 1.3 & 4.9 & 0.11 & 28 & \text { SKULL MTA }\end{array}$

$\begin{array}{llllll}1.58 & 1.08 & 3.2 & 0.07 & 23 & \text { STRIPED BILLS }\end{array}$

$\begin{array}{llllll}2.73 & 2.0 & 5.8 & 0.11 & 18 & \text { SKULL MTN }\end{array}$

$\begin{array}{llllll}2.50 & 2.1 & 4.5 & 0.0919 & \text { STRIPED BILLS }\end{array}$

$\begin{array}{lllllll}2.78 & 2.02 & 3.3 & 2.5 & 0.10 & 14 & \text { SIRIPED BILLS }\end{array}$

$\begin{array}{lllllll}1.38 & 1.05 & 1.2 & 1.7 & 0.04 & 14 & \text { STRIPED BILLS }\end{array}$

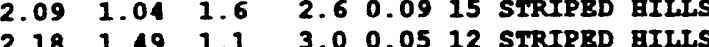

$2.18 \quad 1.49 \quad 1.1 \quad 3.00 .0512$ SIRIPED BILLS

$\begin{array}{lllllll}2.81 & 1.06 & 1.9 & 3.5 & 0.05 & 16 & \text { STRIPED BILLS }\end{array}$

$1: 11: 55 \quad 37.315$

114.965

$0.3 \quad 8.76$

$\begin{array}{lllrlll}2.95 & & 2.7 & 12.6 & 0.15 & 32 & \text { DELARAR LAKE } \\ 1.31 & 0.75 & 1.3 & 2.9 & 0.08 & 21 & \text { GEORGES WATER }\end{array}$ 
1992 LOCAL HYPOCENTER SUMAARY - SGB EARTHQUAKES

STMND (UTC)

LATITUDE LONGITUDE ERROR DEPTH

(DEG. N) (DEG. W) B(KY) (NO)

STAND AZI QQD

$\begin{array}{lll} & \text { DEL- RYS } \\ \text { MIN } & \text { RES. PB }\end{array}$

Mca Md Mah MI

MIV RES PH D.S.G.S.

SEP

$7 \quad 8: 52: 15 \quad 37.070$

115.289

$0.6 \quad 4.92$

$\begin{array}{lll}5.4 & 130 & \text { CCI } \\ 8.8 & 289 & \text { CDI }\end{array}$

$7 \quad 9: 11: 1936.024$

$710: 28: 53 \quad 36.768$

$710: 51: 8 \quad 36.739$

$\begin{array}{lll}7 & 13: 26: 47 & 36.727 \\ 7 & 13: 32: 52 & 36.814\end{array}$

116.255

0.

4.92
6.41
8.86

116.291

$0.1 \quad 8.86$

$\begin{array}{llll}0.2 & 94 & \text { ABI } 1.46 \\ 0.5 & 97 & \text { ABI } 1.14\end{array}$

$\begin{array}{llllll}116.281 & 0.2 & 7.40 & 0.2 & 106 & \text { ABI } 1.58\end{array}$

$13: 47: 59 \quad 36.715$

19:53: $6 \quad 36.713$

$21: 27: 53 \quad 36.765$

$7 \quad 21: 34: 45 \quad 36.721$

$7 \quad 23: 20: 59 \quad 36.715$

$723: 23: 41 \quad 36.715$

116.284

$\begin{array}{rrr}0.2 & 97 & \text { BBI } 1.46\end{array}$

116.268

$0.3 \quad 8.53$

0.4111

BBI 1.46

116.256

$0.4 \quad 8.75$

$\begin{array}{lll}116.271 & 0.2 & 7.28 \\ 116.276 & 0.3 & 7.67\end{array}$

$0.6 \quad 96$

ABI 1.15

ABI 1.10

ABI 1.27

AAI 2.2

AAI 1.60

116.271

$0: 10: 50 \quad 36.716$

$1: 52: 25 \quad 36.733$

1:56: $6 \quad 36.718$

$4: 38: 28 \quad 36.82$

$\begin{array}{ll}\text { 6:57: } 9 & 36.726 \\ 7: 1: 43 & 36.729\end{array}$

0.3

7.88

116.274

0.38 .54

$\begin{array}{lll}16.274 & 0.4 & 3.75\end{array}$

116.27

0.37 .6

116.300

0.2

7.64
8.73

AAI 1.63

ABI 1.44

AAI 1.75

BBI 1.48

ABI 2.19

ABI 1.22

$\begin{array}{llllll}116.289 & 0.2 & 9.23 & 0.3 & 75 & \text { AAI } 1.50\end{array}$

116.258

$0.2 \quad 0.57$

$\stackrel{\infty}{\infty}$

$\begin{array}{rr}7: 24: 25 & 36.708 \\ 8: 22: 38 & 36.792 \\ 8: 40: 12 & 36.787 \\ 12: 33: 57 & 36.744 \\ 15: 5: 21 & 36.721\end{array}$

116.259

0.3

3.73

$0.3 \quad 72$

ABI 1.43

BBI 0.99

ABI 1.34

$\begin{array}{llllll}116.308 & 0.3 & 8.14 & 0.5 & 95 & \text { ABI } 1.25\end{array}$

8 15:36:31 36.742

116.287

8.14

0.482

AAI 1.44

$8 \quad 17: 58: 5 \quad 36.721$

8 18: 6:21 37.061

$818: 26: 38 \quad 36.856$

$19: 57: 53 \quad 36.710$

20:15:14 36.741

116.292

117.462

116.159

0.37 .68

0.485

AAI 1.47

CDI 2.47

BCI 1.67

ABI 1.29

$\begin{array}{rrrrrr}116.293 & 0.3 & 8.09 & 0.3 & 95 & \text { ABI } 1.24 \\ 116.298 & 0.6 & 8.93 & 0.8 & 114 & \text { ABI } 1.56\end{array}$

$0: 14: 27 \quad 36.864$

$1: 17: 33 \quad 36.753$

2:59:13 36.722

4:53: $6 \quad 36.716$

$\begin{array}{ll}6: 7: 16 & 37.046 \\ 8: 50: 12 & 36.703\end{array}$

116.266

116.298

116.311

$0.4 \quad-1.00$

116.314
117.462

0.27 .70

0.486

AAI 1.34

AAI 1.24

ABI 1.00

ABI 1.26

$0.3 \quad 98$

3.0153

116.291

$0.4 \quad 4.83$

0.6198

BCI 2.24

116.305

0.48 .05

$0.5 \quad 158$

$9 \quad 11: 41: 9 \quad 36.715$

9 13:14:57 37.313

16.301

7.31

0.496

ACI 1.39

114.986

$\begin{array}{ll}0.9 & 9.45 \\ 0.2 & 8.23\end{array}$

$23: 34: 48 \quad 36.727$

1:53:47 37.670

114.879

$0.2 \quad 8.23$

ABI 1.57

BCI 2.50

$\begin{array}{llllll}116.300 & 0.2 & 7.10 & 0.3 & 88 & \text { MAI } 1.29\end{array}$

$10 \quad 3: 54: 8 \quad 36.722$

116.317

$0.5 \quad 6.64$

$0.7 \quad 133$

$\operatorname{ABI}$
$\operatorname{MAI} 1.26$

$2.221 .992 .3 \quad 14.20 .2017$ DESERE $\begin{array}{llllll}1.99 & 2.3 & 14.2 & 0.20 & 17 & \text { DESERT HI } \\ 1.87 & & 84.9 & 0.36 & 16 & \text { matURanco }\end{array}$

$\begin{array}{lllllll}1.56 & 1.02 & 1.3 & 3.2 & 0.04 & 20 & \text { GRORGRS WATER }\end{array}$

$\begin{array}{lllllll}1.49 & 1.11 & 1.2 & 1.7 & 0.11 & 15 & \text { SIRTIPED BILLS }\end{array}$

$\begin{array}{lllllll}2.06 & 1.26 & 1.6 & 1.9 & 0.04 & 12 & \text { STRIPED BILLS }\end{array}$

$\begin{array}{llll}1.27 & 1.02 & 4.5 & 0.1818 \mathrm{SKJLI} \\ \mathrm{MTN}\end{array}$

$\begin{array}{lllllll}1.62 & 1.20 & 1.1 & 3.2 & 0.08 & 14 & \text { STRIPED BILLS }\end{array}$

$\begin{array}{lllllll}2.02 & 1.55 & 1.6 & 3.3 & 0.08 & 27 & \text { STRIPED HILLS }\end{array}$

$\begin{array}{lllllll}1.16 & 0.78 & 1.1 & 2.9 & 0.09 & 12 & \text { GEORGES MATER }\end{array}$

$\begin{array}{llllllll}1.50 & 1.21 & 1.1 & 4.1 & 0.04 & 12 & \text { STRIPED HILLS }\end{array}$

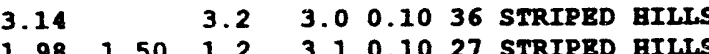

$\begin{array}{lllllll}1.98 & 1.50 & 1.2 & 3.1 & 0.10 & 27 & \text { STRIPED EILIS }\end{array}$

$\begin{array}{lllllll}1.47 & 1.28 & 1.6 & 3.0 & 0.10 & 24 & \text { STRIPED HILLS }\end{array}$

$\begin{array}{lllllll}1.24 & 1.03 & 0.7 & 2.6 & 0.08 & 21 & \text { STRIPED BILTS }\end{array}$

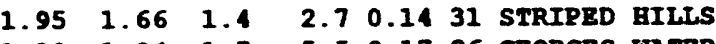

$\begin{array}{llllllll}1.30 & 1.04 & 1.7 & 5.5 & 0.17 & 26 & \text { GRORGES WATRR }\end{array}$

$2.54 \quad 2.3 \quad 1.90 .1241$ SIRIPED BILLS

$1.450 .86 \quad 2.90 .0622$ STRIPED HILLS

$\begin{array}{lllllll}1.78 & 1.56 & 1.5 & 4.2 & 0.09 & 29 & \text { STRIPED BILLS }\end{array}$ $\begin{array}{lllll}1.12 & 1.6 & 5.6 & 0.08 & 23 \\ 08 O R G E S & \text { WATER }\end{array}$ 0.821 .15 .00 .1518 GEORGES WATER

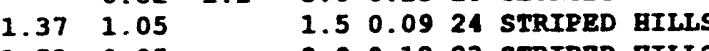

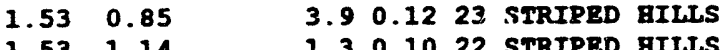

$1.531 .14 \quad 1.30 .1022$ STRIPED BILLS

$\begin{array}{lllllll}1.52 & 0.96 & 1.6 & 2.9 & 0.10 & 22 & \text { STRIPED BILLS }\end{array}$ $\begin{array}{llllll}1.99 & 1.9 & 25.1 & 0.39 & 17 & \text { UBEHBBE CRATER }\end{array}$ $\begin{array}{llrl}1.19 & 1.4 & 0.9 & 0.1717 \text { SKULL MTN }\end{array}$

$\begin{array}{lllll}1.06 & 3.8 & 0.1318 & 18 & \text { STRIPED BILLS }\end{array}$

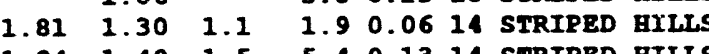

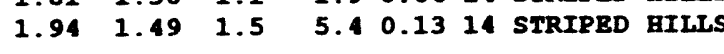

$\begin{array}{lllllll}1.51 & 1.04 & 1.3 & 4.5 & 0.13 & 17 & \text { GEORGES WATER }\end{array}$

$\begin{array}{lllllll}1.41 & 1.06 & 1.2 & 2.6 & 0.09 & 12 & \text { GBORGES WATER }\end{array}$

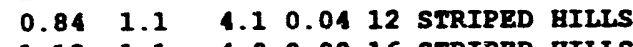

$\begin{array}{llllllll}1.65 & 1.13 & 1.1 & 4.8 & 0.08 & 16 & \text { STRIPED GILLS }\end{array}$ $\begin{array}{llllll}2.24 & 2.1 & 11.6 & 0.13 & 16 & \text { UBEHEBE CRATER }\end{array}$

$\begin{array}{lllllll}1.92 & 1.52 & 1.5 & 4.6 & 0.10 & 14 & \text { SIRIPED }\end{array}$

$\begin{array}{lllllll}1.58 & 0.91 & 1.1 & 4.3 & 0.07 & 14 & \text { STRIPED HILLS }\end{array}$

$2.7122 .0 \quad 2.90 .08$ 14 STRIPED HILTS

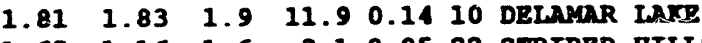

$\begin{array}{lllllll}1.65 & 1.16 & 1.6 & 3.1 & 0.05 & 22 & \text { STRIPED BILLS }\end{array}$

$\begin{array}{lllllll}2.10 & 2.12 & 2.7 & 5.5 & 0.24 & 18 & \text { PAEROC SPRING }\end{array}$

$\begin{array}{lllllll}1.52 & 1.10 & 1.5 & 2.5 & 0.08 & 22 & \text { STRIPED BILLS }\end{array}$

$\begin{array}{llllllll}1.21 & 0.78 & 1.1 & 4.6 & 0.08 & 14 & \text { STRIPED BILLS } \\ 1.05 & 0.85 & & 1.1 & 0.06 & 21 & \text { STRIPED BILLS }\end{array}$ 
1992 LOCAL GYPOCENTER SULMARY - SGB EARTHQUAKES

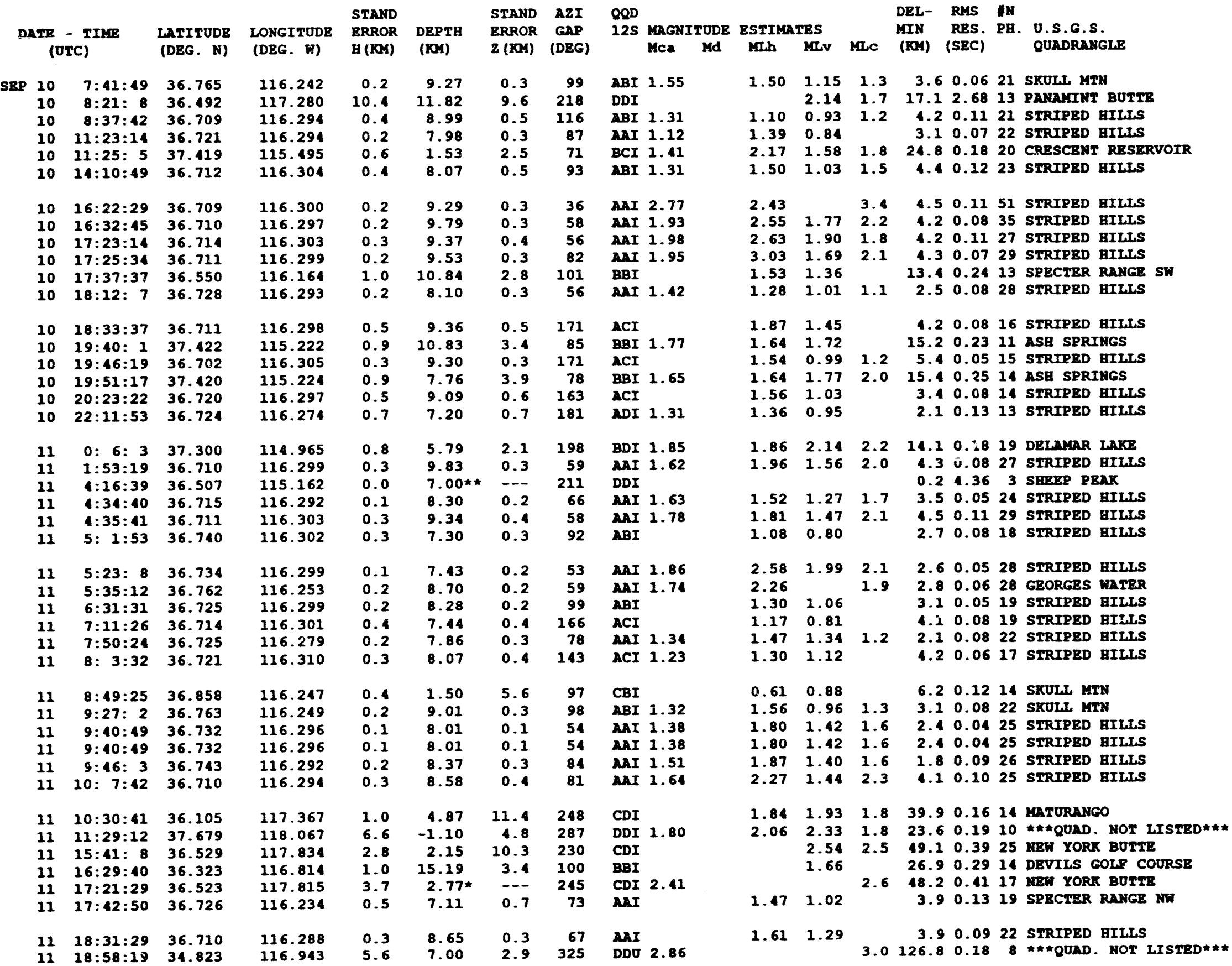


1992 LOCAL HYPOCENTER SUMAARY - SGB EARTHQUAKES

TONGITODE STAND (uTC)

SEP

$\begin{array}{rrr}11 & 23: 38: 51 & 36.788 \\ 12 & 2: 41: 22 & 37.672 \\ 12 & 6: 8: 17 & 36.721 \\ 12 & 6: 51: 50 & 36.698 \\ 12 & 7: 49: 32 & 36.705 \\ 12 & 9: 16: 35 & 36.728 \\ & & \\ 12 & 10: 17: 19 & 36.104 \\ 12 & 10: 44: 43 & 36.693 \\ 12 & 13: 0: 33 & 36.747 \\ 12 & 13: 2: 34 & 36.783 \\ 12 & 15: 37: 58 & 37.151 \\ 12 & 17: 56: 36 & 36.348 \\ 12 & 18: 34: 35 & 36.692 \\ 12 & 21: 1: 28 & 36.712 \\ 12 & 21: 40: 1 & 37.664 \\ 12 & 22: 34: 9 & 36.712 \\ 13 & 1: 50: 8 & 36.735 \\ 13 & 1: 58: 37 & 36.744 \\ & & \end{array}$

116.245

118.131

116.308

116.370

116.363

116.304

117.377

116.310

116.248

116.279

116.611

116.277

116.288

118.075

116.309

116.305

116.271

\begin{tabular}{|c|c|c|c|c|c|c|c|c|c|}
\hline 13 & $3: 12: 37$ & 6. & 116.2 & 0.6 & 1.57 & 0.8 & 195 & ADI & \\
\hline 13 & 4: $1: 53$ & 36.729 & 116.302 & 0.3 & 7.60 & 0.5 & 85 & aAI & \\
\hline 13 & $4: 53: 32$ & 35.939 & 117.711 & 3.2 & 4.12 & 7.7 & 288 & CDI & \\
\hline 13 & $6: 12: 13$ & 36.763 & 116.224 & 0.3 & 8.21 & 0.6 & 77 & $\operatorname{AaI}$ & \\
\hline 13 & 9: 9:41 & 36.161 & 116.743 & 0.9 & 12.36 & 1.5 & 113 & CBI & \\
\hline 13 & $10: 22: 53$ & 36.742 & 116.291 & 0.3 & 8.60 & 0.4 & 83 & ANI & \\
\hline 3 & $11: 46: 21$ & 36.732 & 116.299 & 0.2 & 7.41 & 0.3 & 32 & NAI & \\
\hline 13 & $11: 51: 12$ & 36.759 & 116.333 & 0.3 & -1.84 & 0.3 & 111 & $\mathbf{A B I}$ & \\
\hline 13 & $11: 53: 17$ & 36.703 & 116.321 & 1.7 & 7.74 & 2.4 & 149 & BCI & \\
\hline 13 & $11: 57: 1$ & 36.726 & 116.313 & 0.4 & 6.85 & 0.6 & 92 & ABI & \\
\hline 13 & $11: 57: 24$ & 36.713 & 116.333 & 3.0 & 9.64 & 3.6 & 125 & CBI & \\
\hline 13 & $12: 0: 42$ & 36.716 & 116.318 & 0.2 & 7.00 & 0.3 & 94 & $\mathrm{ABI}$ & \\
\hline 3 & $12: 1: 33$ & 36.737 & 116.295 & 0.3 & 7.59 & 0.3 & 81 & AAI & \\
\hline 13 & $12: 3: 23$ & 36.722 & 116.306 & 0.4 & 7.48 & 0.4 & 97 & ABI & \\
\hline 13 & $12: 5: 46$ & 36.735 & 116.311 & 0.4 & 6.73 & 0.6 & 95 & ABI & \\
\hline 13 & $12: 6: 45$ & 36.728 & 116.292 & 0.7 & 8.21 & 0.8 & 159 & ACI & \\
\hline 13 & 12: $7: 9$ & 36.723 & 116.294 & 0.3 & 8.10 & 0.3 & 111 & ABI & \\
\hline 13 & $12: 11: 3$ & 36.712 & 116.322 & 0.4 & 6.80 & 0.6 & 107 & $\mathbf{A B I}$ & \\
\hline 13 & $12: 13: 11$ & 36.741 & 116.292 & 0.4 & 8.72 & 0.5 & 84 & MAI & \\
\hline 13 & $12: 16: 0$ & 36.729 & 116.306 & 0.5 & 6.82 & 0.7 & 94 & BBI & \\
\hline 13 & $12: 16: 41$ & 36.719 & 116.309 & 0.4 & 7.12 & 0.5 & 97 & ABI & \\
\hline 13 & $12: 17: 1$ & 36.729 & 116.307 & 0.4 & 7.02 & 0.6 & 133 & ABI & \\
\hline 13 & $12: 19: 24$ & 36.729 & 116.303 & 0.4 & 7.74 & 0.5 & 95 & ABI & \\
\hline 13 & $12: 22: 55$ & 36.759 & 116.288 & 0.9 & 7.91 & 1.3 & 137 & $\mathbf{A C I}$ & \\
\hline & $12: 23: 56$ & 6.728 & 116.310 & 0.5 & 6.71 & 0.7 & 96 & ABI & \\
\hline & $12: 27: 5$ & 36.725 & 116.315 & 0.3 & 6.70 & 0.5 & 100 & ABI & \\
\hline
\end{tabular}

H(XI) DEPTH

STAND AZI QOD

Magnitude ESTIMATES

DEL- RMS IN

$z$ (BY) (DEG)

$\begin{array}{lllrl}0.5 & 1.71 & 1.9 & 163 & \text { ACI } \\ 1.5 & 3.18 * & -- & 294 & \text { CDI } \\ 0.2 & 8.49 & 0.3 & 97 & \text { ABI }\end{array}$

$\begin{array}{rrrrr}0.2 & 8.49 & 0.3 & 97 & \text { ABI } \\ 0.4 & 5.79 & 0.8 & 158 & \text { ACI }\end{array}$

$0.4 \quad 5.52$

6.95

0.2

$95 \quad \mathrm{BBI} 1.48$

$1.4 \quad 2.75$

$5.7 \quad 250$

0.361 AAT 1.53

0.471 AAI 1.64

$0.9 \quad 121 \quad$ ABI 1.34

BBI 1.61

BBI 1.93

$\begin{array}{lllll}0.3 & 0.97 & 0.4 & 115 & \text { ABI }\end{array}$

$\begin{array}{llllll}0.3 & 8.48 & 0.5 & 75 & \text { MAI } 1.75\end{array}$

$\begin{array}{llll}1.39 & 2.2 & 286 & \text { BDI } 1.74\end{array}$

ABI 1.39

ABI 1.24

ABI 1.16

2.40

CBI 1.55

1.38

1.48

ABI 1.68

CBI

$\operatorname{MaI} 1.54$

ABI 1.58

ACI 1.44

ABI 1.38

MAI 1.36

BBI 1.23

ABI 1.20

ACI 1.69

1.630 .69

$1.27 \quad 1.09$

$\begin{array}{lllllll}0.76 & 1.0 & 5.6 & 0.10 & 13 & \text { SKOLL MTH }\end{array}$

$\begin{array}{lllllll}1.07 & 0.81 & & 4.0 & 0.0417 & \text { STRTPED } & 0.75 T s\end{array}$

$1.031 .01 \quad 6.50 .0611$ STRIPED BILIS

$\begin{array}{llll}.11 & 0.80 & 6.90 .0816 \text { STRIPED BILLS }\end{array}$

$1.701 .19 \quad 3.30 .0420$ STRIPED BILLS

$\begin{array}{llllllll}2.50 & 2.10 & 2.2 & 40.6 & 0.16 & 20 & \text { Maturango }\end{array}$

$\begin{array}{lllllll}1.64 & 1.22 & 1.5 & 5.9 & 0.08 & 26 & \text { STRIPBD BILLS }\end{array}$

$\begin{array}{lllllll}1.55 & 1.42 & 1.5 & 2.2 & 0.11 & 23 & \text { SPECTER RAMGE }\end{array}$

4.50 .1418 GEORGES MATER

$\begin{array}{llllll}66 & 1.23 & 10.2 & 0.16 & 23 & \text { THIRSTY CANYON }\end{array}$

$\begin{array}{lllllll}1.68 & 1.81 & 1.8 & 20.5 & 0.18 & 27 & \text { DEVILS SPERDWAY }\end{array}$

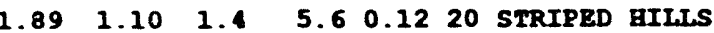

\begin{tabular}{llllll}
1.19 & 1.74 & 1.5 & 3.7 & 0.1326 & 26 \\
\hline
\end{tabular}

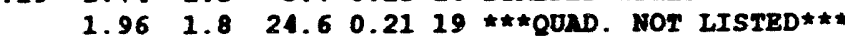

$\begin{array}{lllllll}1.27 & 1.22 & 1.2 & 4.8 & 0.11 & 21 & \text { STRIPED BILLS }\end{array}$

$\begin{array}{llllll}1.32 & 0.68 & 3.1 & 0.07 & 20 & \text { STRIPED GILLS }\end{array}$

$\begin{array}{lllll}1.32 & 0.90 & 0.2 & 0.1422 & \text { STRIPED BILLS }\end{array}$

$1.48 \quad 2.90 .0912$ STRIPED HILLS

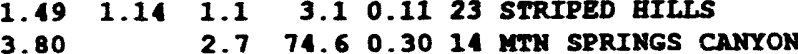

$\begin{array}{rrrrrrr}1.80 & & 2.7 & 74.6 & 0.30 & 14 & \text { MTR SPRINGS } \\ 1.47 & 1.16 & 1.3 & 4.8 & 0.14 & 26 & \text { SKULL MTY }\end{array}$

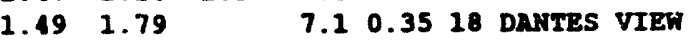

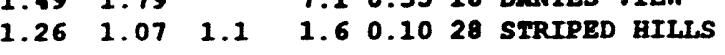

$4.10 \quad 2.70 .1153$ STRIPED HILLS

$\begin{array}{llllllll}1.78 & 1.29 & 1.8 & 5.7 & 0.13 & 11 & \text { GEORGES WATER }\end{array}$

$\begin{array}{lllllll}2.24 & 1.58 & 1.5 & 6.2 & 0.27 & 11 & \text { STRIPED HILLS }\end{array}$

$\begin{array}{lllllll}2.26 & 1.36 & 2.1 & 4.1 & 0.13 & 23 & \text { STRIPED HILLS }\end{array}$

2.7520 .25 .40 .5011 STRTPED HILIS

$40 \quad 1.09 \quad 1.5 \quad 5.10 .0825$ STRTPED MILIS

$\begin{array}{lllllll}1.89 & 1.57 & 1.4 & 2.2 & 0.10 & 19 & \text { STRIPED HILLS }\end{array}$

$\begin{array}{lllllll}1.97 & 1.60 & 1.5 & 3.8 & 0.10 & 15 & \text { STRIPED HIL.LS }\end{array}$

$\begin{array}{lllllll}1.77 & 1.39 & 1.9 & 3.5 & 0.10 & 15 & \text { STRIPED } \\ 1.9 I L L S\end{array}$

$\begin{array}{lllllll}1.64 & 1.35 & 1.2 & 2.4 & 0.14 & 14 & \text { STRIPED HILLS }\end{array}$

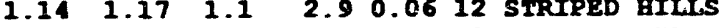

$\begin{array}{lllllll}2.23 & 1.20 & 2.0 & 5.6 & 0.11 & 13 & \text { STRIPED BILLS }\end{array}$

1.80 .1122 STRIPED HILIS

$\begin{array}{lllllll}1.34 & 1.19 & 1.6 & 3.4 & 0.15 & 18 & \text { STRIPED HILLS }\end{array}$

1.731 .013 .10 .1013 STRTPED

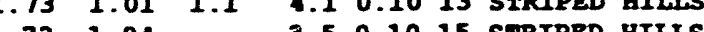

$1.731 .04 \quad 3.50 .1015$ STRIPED HILLS

$\begin{array}{lllllll}1.28 & 1.29 & 1.4 & 2.3 & 0.14 & 10 & \text { GEORGES WATER }\end{array}$

$\begin{array}{lllllll}1.57 & 1.00 & 1.5 & 3.8 & 0.13 & 17 & \text { STRIPED HILLS }\end{array}$

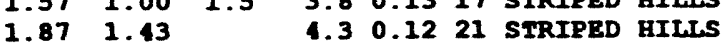


1992 LOCAL HYPOCENYER SUREARY - SG3 EARTHQUAKES

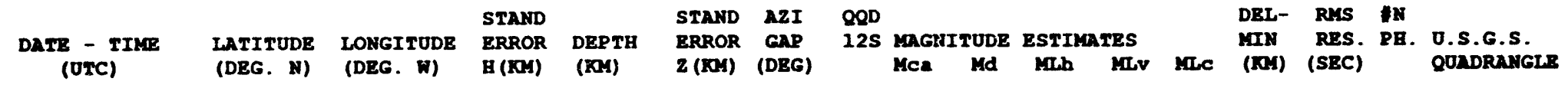

SEP $13 \quad 12: 29: 49 \quad 36.721$ $13312: 31: 15 \quad 36.721$ $13 \quad 12: 31: 15 \quad 36.733$ 13 12:40:23 36.720 13 18:30:46 36.716 13 19:23:7 36.725 $1320: 37: 13 \quad 36.723$

$13 \quad 21: 27: 7 \quad 36.728$ $13 \quad 21: 54: 45 \quad 36.733$ $13 \quad 21: 59: 52 \quad 36.705$ $13 \quad 23: 15: 58 \quad 36.709$ $14 \quad 0.29: 57 \quad 36.724$

$14 \quad 0: 29: 57 \quad 36.724$

$14 \quad 1: 57: 52 \quad 36.720$

$14 \quad$ 1: $0: 34 \quad 36.735$

$14 \quad$ : $: 3: 34 \quad 36.728$

14 : $5: 5: 32 \quad 36.729$

$14 \quad 5: 35: 52 \quad 36.724$

116.318
116.303
116.308
116.330
116.303
116.313
116.306
116.304
116.320
116.287
116.304
116.304
116.306
116.295
116.305
116.303
116.314
116.303

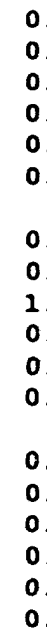

116.305

116.302

116.304

116.290

116.288

116.299

116.305

116.284

116.299

116.286

116.318

116.305

116.296

116.296

116.299

116.302

116.304

116.291

116.303

116.301

116.303

116.303

117.405
117.469

116.298

(RA)

6.99

7.45

8. 13

$0.3 \quad 7.76$

6.41

0.7193

ADI 1.78

NAI 2.01

BBI 1.32

ABI

ABI 1.18

ABI 1.17

\section{$\begin{array}{llll}6.95 & 0.4 & 95 & \mathrm{ABI}\end{array}$}

$\begin{array}{llll}7.72 & 0.2 & 47 \text { MAI } 1.87\end{array}$

$\begin{array}{llll}8.56 & 0.8 & 202 & \text { BDI } 1.64 \\ 7.53 & 0.3 & 74 & \text { ADI } 1.52\end{array}$

$\begin{array}{llll}7.70 & 0.4 & 97 & \text { ABI }\end{array}$

$\begin{array}{llll}7.88 & 0.4 & 82 & \text { AaI } 1.74\end{array}$

8. 51

7.74

7.55

7.87

7.24
7.00

0.4

Aar 1.82

AAI 1.82

ABI 1.26

ABI 1.33

BBI 1.57

AaI 2.59

$0.1 \quad 6.82$

$0.2 \quad 7.08$

$\begin{array}{rr}0.2 & 7.67 \\ 0.1 & -1.34\end{array}$

$0.4-1.11$

$0.7 \quad 4.48$

$\begin{array}{llll}0.7 & 4.52 & 1.4 & 113\end{array}$

$\begin{array}{llll}0.6 & 6.63 & 1.0 & 93 \\ 0.4 & 7.41 & 0.6 & 77\end{array}$

$\begin{array}{llll}0.4 & 8.39 & 0.5 & 195\end{array}$

$\begin{array}{llll}0.2 & 7.95 & 0.3 & 103\end{array}$

0.1

6.66

$\begin{array}{ll}0.1 & 8.66 \\ 0.1 & 7.82\end{array}$

0.27 .79

$0.2 \quad 7.36$

$\begin{array}{ll}0.2 & 7.25 \\ 0.3 & 8.51\end{array}$

$0.3 \quad 8.55$

0.27 .00

0.27 .48

0.92 .43

$\begin{array}{ll}0.5 & 8.60 \\ 0.2 & 7.88\end{array}$

0.2

116.306

AAI 1.67 ABI

AAI 2.00 BBI 1.79 BBI 1.79 BBI 1.63

BBI 1.63 BBI 1.43 ADI

ABI

MaI 1.76

ABI 1.40

$\operatorname{AaI} 1.66$

AaI 1.45

$\operatorname{anI} 1.42$

ABI 1.26

$\operatorname{MaI} 1.70$

ADI 1.15

Aar 2.06

ABI 1.31

BBI

NDI

NBI 1.27 $\begin{array}{llllll}2.34 & 2.0 & 4.7 & 0.12 & 14 & \text { SIRIPED BILLS }\end{array}$

$3.02 \quad 2.1 \quad 3.00 .1230$ STRIPED BILLS

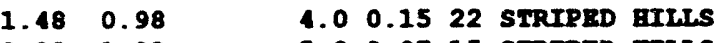
$\begin{array}{llllll}0.82 & 1.03 & 5.9 & 0.07 & 15 & \text { STRIPED BILLS } \\ 1.67 & 1.03 & 1.1 & 3.4 & 0.07 & 16\end{array}$ $\begin{array}{lllllll}1.67 & 1.03 & 1.1 & 3.4 & 0.07 & 16 & \text { STRIPED BILLS }\end{array}$

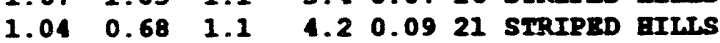

$1.23 \quad 1.05$ 2.013 .1

3.40 .0716 SIRIPED BILLS $\begin{array}{llllll}3.1 & 3.1 & 0.07 & 33 & \text { STRIPED BILLS }\end{array}$ $\begin{array}{rllllll}1.60 & 1.33 & 1.9 & 3.9 & 0.10 & 9 & \text { SIRIPED BILLS } \\ 1.0 & 3.9 & 0.08 & 26 & \text { SIRIPED BILLS }\end{array}$ $\begin{array}{llll}1.42 & 0.89 & 3.50 .0916 \text { STRIPED BILLS }\end{array}$ $\begin{array}{llllll}2.17 & 1.90 \quad 2.0 & 3.4 & 0.1034 & \text { STRTPED gITLS }\end{array}$ $\begin{array}{lllllll}2.90 & 1.58 & 2.0 & 3.9 & 0.14 & 36 & \text { STRIPED EILLS }\end{array}$ $\begin{array}{lllllll}2.69 & 1.72 & 2.1 & 2.2 & 0.13 & 33 & \text { STRIPED BIILS }\end{array}$ $\begin{array}{lllllll}1.18 & 0.78 & 3.4 & 0.10 & 26 & \text { SIRIPED BILLS }\end{array}$ $\begin{array}{lllllll}1.40 & 1.10 & 1.1 & 3.1 & 0.08 & 26 & \text { STRIPED HILLS }\end{array}$

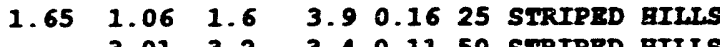
$\begin{array}{llllll}3.01 & 3.2 & 3.4 & 0.11 & 50 & \text { STRIPED aIILS }\end{array}$

$2.04 \quad 1.37 \quad 1$ 3.70 .0526 SIRIPED BILLS

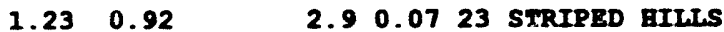
$\begin{array}{lllllll}1.80 & 1.38 & 1.5 & 3.0 & 0.07 & 26 & \text { STRIPED HILLS }\end{array}$ \begin{tabular}{lllllll}
1.90 & 1.79 & 2.0 & 5.8 & 0.18 & 25 & SIRIPRD \\
\hline & HILLS
\end{tabular} $\begin{array}{lllllll}1.88 & 1.79 & 2.0 & 5.5 & 0.17 & 25 & \text { STRTPED HILLS }\end{array}$ $1.351 .51 \quad 1.4 \quad 5.90 .1812$ SIRIPED BILLS

$\begin{array}{lll}1.36 & 1.52 \quad 1.4\end{array}$ $\begin{array}{lllllll}1.23 & 1.19 & & 5.7 & 0.1720 & \text { STRIPED EILLS } \\ 1.60 & 1.43 & 1.6 & 5.9 & 0.14 & 22 & \text { STRTPED BILLS }\end{array}$

6.10 .1812 STRIPED HILIS

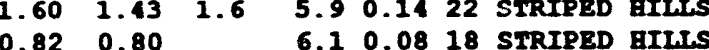
$\begin{array}{llllllll}1.58 & 1.10 & 1.1 & 5.2 & 0.07 & 24 & \text { STRIPED } & 5 T H S\end{array}$

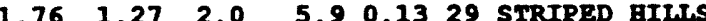

$\begin{array}{lllllll}1.54 & 1.21 & 1.1 & 2.7 & 0.05 & 21 & \text { STRIPED BILLS }\end{array}$ $\begin{array}{lllllll}1.81 & 1.45 & 1.9 & 2.3 & 0.06 & 35 & \text { STRIPED BILLS }\end{array}$ $\begin{array}{llllll}1.34 & 0.97 & 2.8 & 0.06 & 23 & \text { SIRIPED BILLS }\end{array}$ $\begin{array}{lllllll}1.95 & 1.23 & 1.9 & 3.1 & 0.08 & 31 & \text { STRIPED } \\ 1.53 & 0.99 & & 3.3 & 0.06 & 20 & \text { SIRIPED }\end{array}$ $\begin{array}{llllll}1.53 & 0.99 & 3.30 .06 & 20 & \text { STRIPED BILLS }\end{array}$ $\begin{array}{lllllll}1.35 & 1.12 & 1.5 & 5.6 & 0.11 & 27 & \text { SIRIPED HILLS }\end{array}$ $0.9166 .0 \quad 0.0515$ SIRIPED BILIS \begin{tabular}{lllllll}
2.96 & 2.05 & 2.2 & 2.8 & 0.09 & 43 & SIRIPED \\
\hline & aILLS
\end{tabular} $\begin{array}{lllllll}1.58 & 1.03 & 1.1 & 3.3 & 0.08 & 21 & \text { STRIPED BILS }\end{array}$ $\begin{array}{llllll}1.62 & 1.6 & 9.4 & 0.27 & 16 & \mathrm{LIDA}\end{array}$ $\begin{array}{llllll}1.62 & 1.6 & 9.4 & 0.27 & 16 & \text { IIDA } \\ 1.29 & 1.3 & 11.4 & 0.04 & 9 & \text { IIM MOUNTrY }\end{array}$ $\begin{array}{ll}1.59 & 0.97\end{array}$ $\begin{array}{lll}2.8 & 0.0519 & \text { SIRIPED BILLS }\end{array}$

$\begin{array}{llllllll}1.78 & 1.40 & 1.6 & 5.7 & 0.06 & 26 & \text { STRIPED } & \text { BILLS }\end{array}$ $\begin{array}{lllllll}1.64 & 1.06 & 0.7 & 3.7 & 0.08 & 20 & \text { STRIPED BILLS }\end{array}$ 
1992 LOCAL HYPOCENTER SUIGARY - SGB EARTHQUAKCS

DATE - IIRE LATITUDE LONGITUDE ERROR DEPTH ERROR GAP 12S (एTC)

SEP

ERROR DEPTH ERROR GAP 125 MAGNITUDE BSTIMATES

DEL- RES N

MLC RES. PB. O.S.G.S.

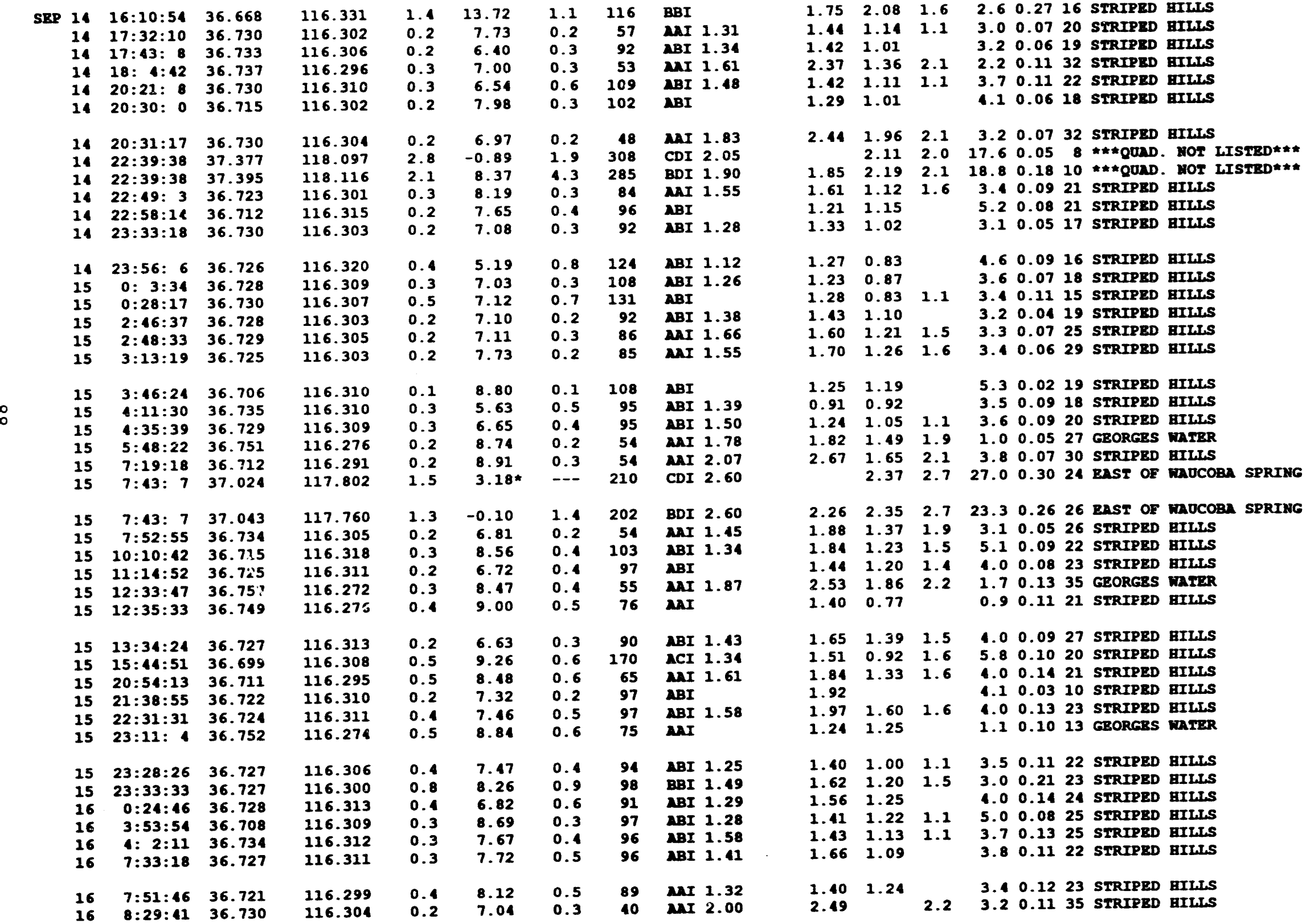


1992 LOCAL HYPOCENTER SUIAERY - SGB EARTHQUAKES

DATE - STAND STAND AZI OOD (OTC)

$\begin{array}{llll} & \text { SRP } 16 \quad 8: 55: 58 & 36.734\end{array}$ $16 \quad 9: 14: 15 \quad 36.730$ $16 \quad 10: 22: 336.730$ 16 10:22:1 36.730 16 16:5: 36.717 $16 \quad 18: 49: 46 \quad 36.728$

$16 \quad 19: 29: 10 \quad 36.733$ $16 \quad 21: 36: 37 \quad 36.713$

$\begin{array}{lll}16 & 22: 10: 17 & 36.815\end{array}$ $\begin{array}{lll}16 & 22: 23: 10 & 36.882\end{array}$ $16 \quad 23: 23: 3 \quad 36.730$

$17 \quad 1: 58: 16 \quad 36.724$

$17 \quad 3: 37: 30 \quad 36.725$ $17 \quad 3: 52: 58 \quad 36.713$ 17 : : $2: 52 \quad 36.726$ $17 \quad 10: 25: 52 \quad 36.722$ $17 \quad 11: 26: 46 \quad 36.724$

$17 \quad 11: 17: 46 \quad 36.726$ $0.3 \quad 8.60$

1.4102

ABI

ABI

BCI
ERROR (KO)

116.300 116.306 116.301 116.304 116.290 116.301

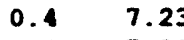

0.47 .31 0.58 .13 0.46 .79 8.21

116.299 116.293 116.206 116.162 116.300 116.302

116.301 116.292 116.303 16.303 116.307 116.278

117.391 117.390 116.316 116.314 116.288
114.967 116.308 116.298 116.296 116.319 116.311

116.274 116.262 117.419 116.290 116.293 116.267

116.287 116.287 116.303 116.300 116.293 116.276 115.064

\subsection{8 .08} $\begin{array}{rr}1.0 & -1.92 \\ 0.7 & 0.80\end{array}$ 0.36 .81 0.27 .05

\subsection{8 .23} $0.3 \quad 8.64$ 0.27 .02 $0.1 \quad 7.67$ 7.19 0.37 .73

$0.3 \quad 4.86$

3.05* $\begin{array}{ll}\text { ERROR } & \text { GAP } \\ z \text { (KE) } & \text { (DEG) }\end{array}$

0.5

0.6

0.5

0.3

0.3

$0.3 \quad 89$

$\begin{array}{ll}0.4 & 1 \\ 0.9 & 170\end{array}$

0.3163

0.4102

0.254

0.3109

0.2

0.2

0.3

$1.8 \quad 103$

$\begin{array}{ll}0.3 & 92 \\ 0.5 & 99\end{array}$

$0.2 \quad 91$

-.- 202

0.27 .11

0.27 .05

7.01

$\begin{array}{ll}0.7 & 5.88 \\ 0.2 & 7.58\end{array}$

0.2

$\begin{array}{lr}0.3 & 136 \\ 0.2 & 55\end{array}$

0.25

0.9278

0.260

0.275

0.27 .8

0.18 .00

$\begin{array}{ll}0.7 & 1.00 \\ 0.2 & 7.75\end{array}$

8.51

0.375

0.256

$\begin{array}{rr}1.9 & 182 \\ 0.3 & 96\end{array}$

$\begin{array}{ll}0.3 & 96 \\ 0.2 & 95\end{array}$

$\begin{array}{ll}0.2 & 95 \\ 0.8 & 78\end{array}$

$0.6 \quad 9.35$

$\begin{array}{ll}0.8 & 94 \\ 0.2 & 58\end{array}$

$\begin{array}{rr}0.2 & 58 \\ 0.1 & 171\end{array}$

$\begin{array}{rr}0.1 & 171 \\ 0.3 & 96\end{array}$

0.277

$\begin{array}{ll}9.8 & 121\end{array}$

6.35

$6.2-0.47$

116.293

116.179

$2 S$ mgNitude ESTIMATES

DEL- RYS

Mca Md Mab ML MLC (KM) (SEC) PB. O.S.G.S.

BAI 1.90

ABI 1.44

BAI 1.33

arr 1.43

$\mathrm{NT} 1.85$

$\begin{array}{lllllll}2.54 & 1.94 & 2.1 & 2.7 & 0.16 & 40 & \text { STRIPED BILLS }\end{array}$

$\begin{array}{llllll}0.89 & 1.20 & 3.4 & 0.1019 & \text { STRIPED BILS }\end{array}$

$\begin{array}{lllllll}1.51 & 1.07 & 1.6 & 2.9 & 0.15 & 23 & \text { SIRIPED GILLS }\end{array}$

$\begin{array}{llllll}1.29 & 0.92 & 3.1 & 0.11 & 19 & \text { STRIPED BILSS }\end{array}$

\begin{tabular}{lllllll}
60 & 1.20 & 1.5 & 3.2 & 0.09 & 23 & STRIPED \\
\hline IIILS
\end{tabular}

$\operatorname{AnI} 1.34$

ABI 1.24

AnI 1.35

$\begin{array}{lllllll}06 & 1.46 & 1.6 & 3.0 & 0.09 & 31 & \text { STRIPED BILLS }\end{array}$

$\operatorname{AaI} 1.55$

ABI 1.24

ABI 1.42

MaI 1.47

$\operatorname{MaI} 1.37$

$1.33 \quad 0.99$

1.08
0.81

2.60 .0622 STRIPED HILLS

3.70 .0918 STRIPRD BILLS

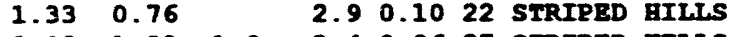

$\begin{array}{llllll}2.02 & 1.23 & 1.9 & 3.4 & 0.06 & 27 \\ \text { STRIPED HILLS }\end{array}$

$\begin{array}{lllllll}2.00 & 1.67 & 2.0 & 3.2 & 0.07 & 36 & \text { STRIPED BILLS }\end{array}$

$\begin{array}{llllll}1.24 & 0.84 & 3.7 & 0.0720 & \text { SIRIPED BILLS }\end{array}$

$\begin{array}{lllllll}1.40 & 1.07 & 0.7 & 3.4 & 0.04 & 19 & \text { SIRIPED BILCS }\end{array}$

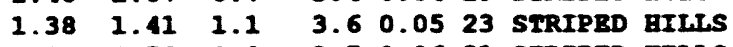

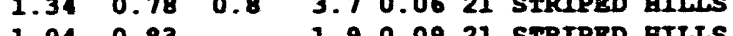

$1.040 .83 \quad 1.90 .0921$ STRIPED BILLS

ACI 1.57

ACI 1.83

ABI 1.31

ABI 1.40

AAI 1.72

$\begin{array}{lllllll}1.69 & 1.5 & 17.9 & 0.11 & 22 & \text { WEST OF GOLD MTN }\end{array}$

$\begin{array}{lllllll}2.64 & 1.93 & 1.9 & 17.5 & 0.11 & 33 & \text { WEST OF GOLD MTN }\end{array}$

$\begin{array}{lllllll}1.39 & 1.16 & 1.5 & 1.4 & 0.05 & 21 & \text { STRIPED } \\ 1.3 I L L S\end{array}$

$1.35 \quad 0.97$

$\begin{array}{lll}3 & 0.1021 & \text { STRIPED GILLS }\end{array}$

$\begin{array}{lllllll}1.87 & 1.45 & 1.6 & 1.5 & 0.05 & 23 & \text { STRIPED HILLS }\end{array}$

$\begin{array}{llllllll}1.54 & 1.96 & 1.9 & 13.8 & 0.17 & 15 & \text { DELAMar LAKE }\end{array}$

ACI

AAI 1.51

AnI 1.46

ADI

1.110 .93

3.60 .0416 STRIPED BILLS

$\begin{array}{lllllll}1.66 & 1.31 & 1.9 & 2.5 & 0.06 & 23 & \text { STRIPED HILLS }\end{array}$

$\begin{array}{lllllll}1.79 & 1.48 & 1.9 & 2.3 & 0.06 & 22 & \text { STRIPBD HILLS }\end{array}$

$\begin{array}{llll}1.31 & 1.44 & 6.30 .0811 & \text { STRIPED BILLS }\end{array}$

$\begin{array}{llllll}2.60 & 2.0 & 1.5 & 0.05 & 25 & \text { SIRIPED BILLS }\end{array}$

NAI 1.19

1.231 .11

1.30 .0520 STRIPED BILLS

AaI 1.41

$0.90 \quad 0.97$

0.90 .0618 GBORGES MATER

AnI 1.67

ABI

ABI 1.18

$\begin{array}{lllllll}1.82 & 1.56 & 1.9 & 2.1 & 0.05 & 30 & \mathrm{GBOR}\end{array}$

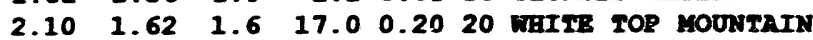

$\begin{array}{lllll}1.20 & 0.87 & 1.6 & 0.06 & 21 \\ \text { STRIPED BIILS }\end{array}$

$\begin{array}{llllllll}0.98 & 0.70 & 1.2 & 1.9 & 0.04 & 18 & \text { sTRIPED Mruns }\end{array}$

$\begin{array}{lllllll}0.92 & 0.88 & 0.8 & 2.4 & 0.16 & 20 & \text { GBORGES MATER }\end{array}$

BBI

Aar 1.70

ACI

$\begin{array}{lllllll}1.37 & 0.94 & 1.2 & 3.3 & 0.18 & 17 & \text { STRIPED HILLS }\end{array}$ $\begin{array}{llllllll}2.16 & 1.56 & 1.6 & 4.4 & 0.04 & 28 & \text { SIRIPED HIL.S }\end{array}$

$\begin{array}{lllllll}1.28 & 0.89 & 1.2 & 4.6 & 0.02 & 16 & \text { SIRIPED EILS }\end{array}$

$\begin{array}{llllll}1.28 & 0.88 & 2.0 & 0.07 & 21 & \text { SIRIPED BILLS }\end{array}$

1.310 .91

1.0 0.0721 SIRTPED kins

AnI

$0.81 \quad 1.21$

9.00 .936 ANMHO NB

$\begin{array}{llllllll}89 & 1.39 & 2.0 & 3.8 & 0.06 & 28 & \text { SIRIPED HILLS }\end{array}$

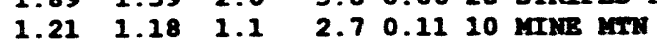


1992 LOCAL HYPOCENTER SUIMARY - SGB EARTHQUAKRS

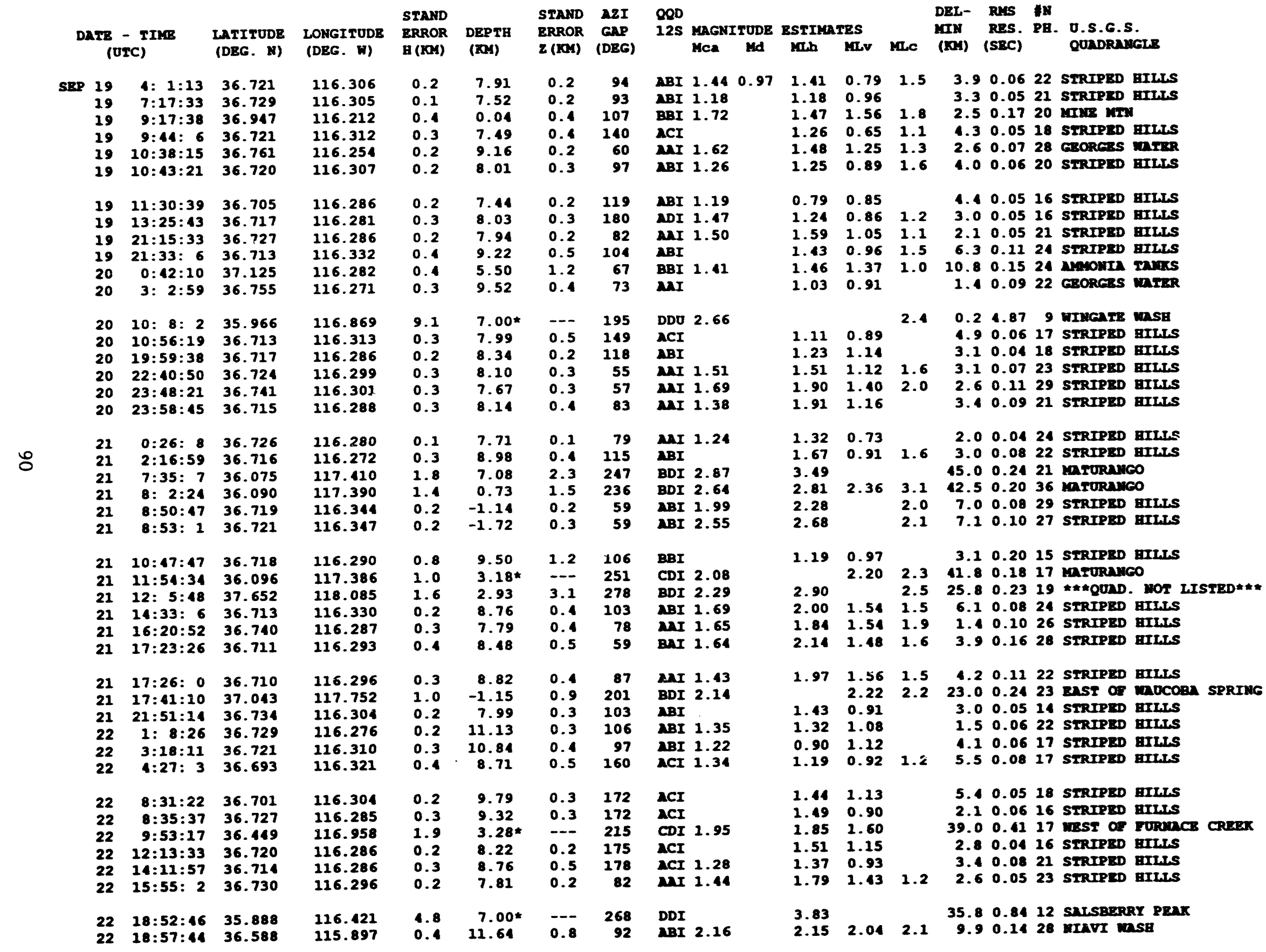


1992 LOCAL HYPOCENTER SURARY - SCB ENRTBQUNKES

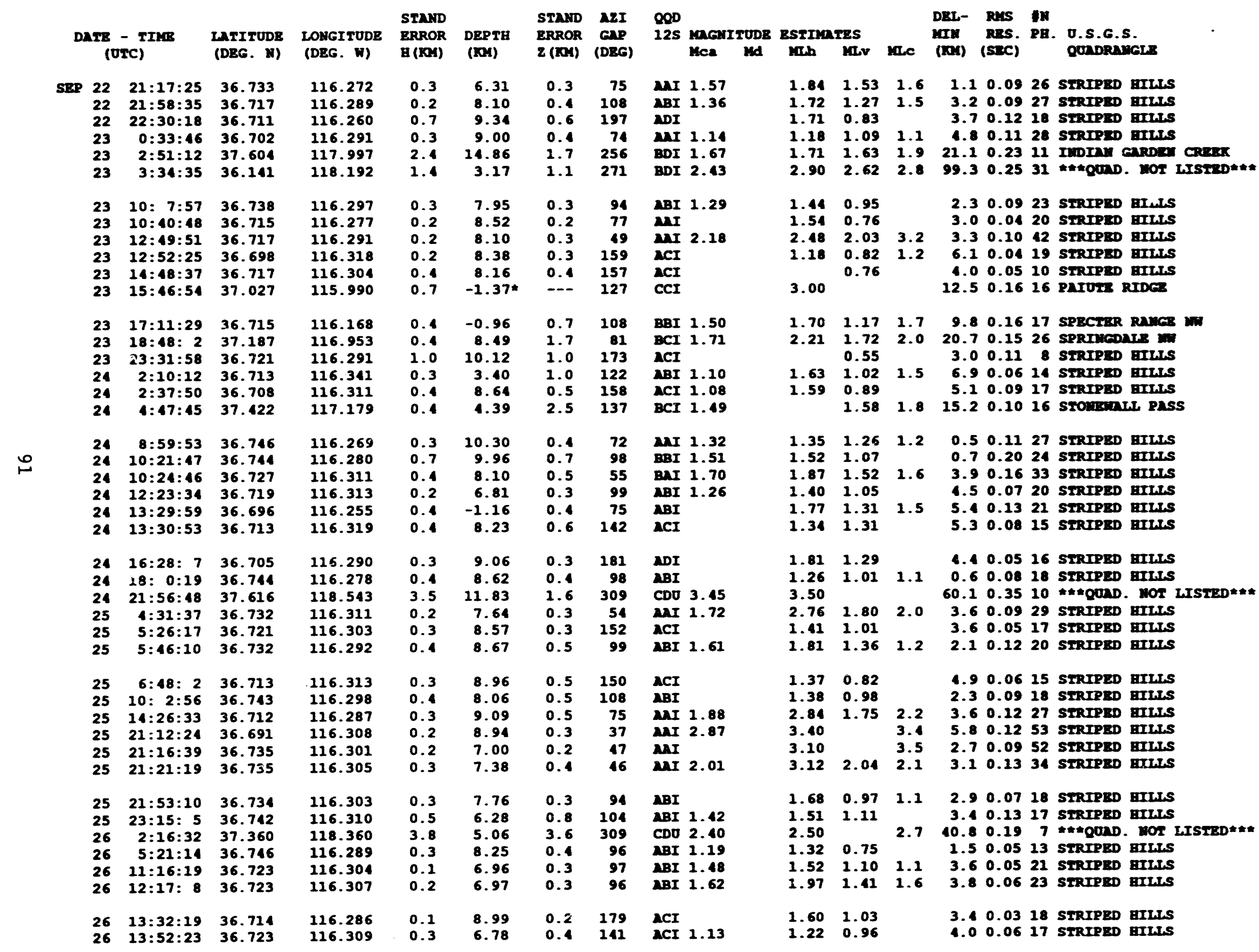


1992 LOCAL HYPOCENTER SUARARY - SGB EARTHQUNKRS

STAND STAND AZI OQD DEL- RES II

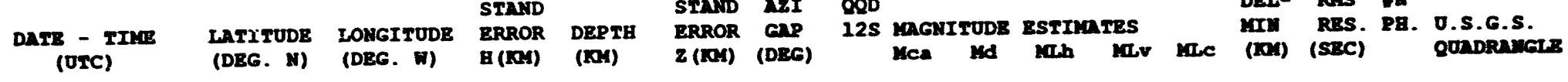

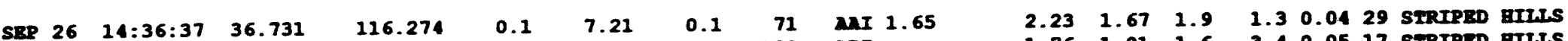

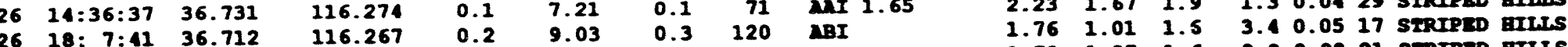
$\begin{array}{lllllllll}26 & 20: 17: 12 & 36.736 & 116.304 & 0.3 & 7.54 & 0.4 & 86 & \text { Mar } 1.42\end{array}$

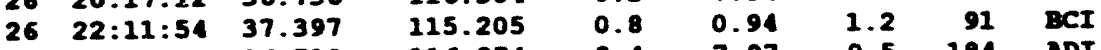

$\begin{array}{rrrrrrrrr}26 & 22: 49: 25 & 36.718 & 116.274 & 0.4 & 7.97 & 0.5 & 184 & \text { ADI } \\ 27 & 0: 7: 30 & 36.735 & 116.303 & 0.3 & 7.07 & 0.4 & 102 & \text { ABI } 1.12\end{array}$

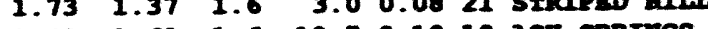
$\begin{array}{lllllll}1.48 & 1.62 & 1.6 & 13.7 & 0.19 & 10 & \text { asd serums }\end{array}$

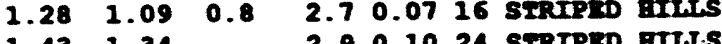
$1.431 .34 \quad 2.90 .1024$ SIRIPED BITLS

$\begin{array}{lllllllll}27 & 6: 53: 54 & 36.729 & 116.312 & 0.4 & 7.00 & 0.5 & 127 & \text { ABI }\end{array}$

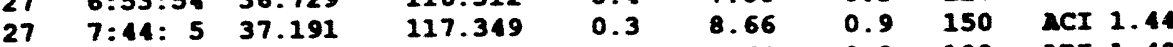
27 12:52:13 36.74 27 12:52:13 36.748 $27 \quad 18: 16: 18$ 36.721 $27 \quad 23: 44: 60 \quad 36.726$ 116.304 116.295 $\begin{array}{lllll}0.2 & 9.64 & 0.2 & 100 & \text { ABI } 1.40\end{array}$ $\begin{array}{llll}8.00 & 0.5 & 98 & \mathrm{ABI}\end{array}$ $\begin{array}{rrrr}8.00 & 0.5 & 98 & \mathrm{ABI} \\ 9.24 & 0.4 & 164 & \mathrm{ACI}\end{array}$

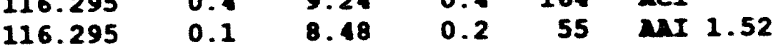

$\begin{array}{lllllll}1.19 & 1.03 & 1.1 & 3.8 & 0.07 & 16 & \text { STRIPED BILS }\end{array}$ $\begin{array}{lllllll}1.63 & 1.5 & 14.6 & 0.09 & 14 & \text { GOID mounraIx }\end{array}$ $\begin{array}{lllll}1.68 & 0.98 & 0.8 & 0.05 & 21 \\ 1.52 R I P E D & 1.12 & \text { aILLS }\end{array}$ $\begin{array}{lllllll}1.59 & 1.12 & 1.1 & 3.8 & 0.12 & 22 & \text { SIRTPED BILS }\end{array}$ $1.301 .03 \quad 1.13 .10 .0515$ STRTPED ATI.TS $1.893 .28 \quad 1.6 \quad 2.70 .0523$ STRTPED GTITS $\begin{array}{llllll}117.697 & 0.6 & 1.76 & 2.1 & 101 & \text { BCI } 1.67\end{array}$ $\begin{array}{llllll}116.304 & 0.3 & 8.45 & 0.5 & 93 & \text { ABI } 1.48\end{array}$ 116.311 $3: 11: 3 \quad 37.572$ $7: 38.50536 .721$ $7: 44: 5336.810$ 9: $9: 5736.733$ $9: 29: 46$
$9: 36.743$ 116.297

\begin{tabular}{|c|c|c|c|}
\hline 28 & $11: 15: 29$ & 36.737 & 216.305 \\
\hline 28 & $13: 30: 21$ & 36.721 & 116.289 \\
\hline 28 & $16: 40: 27$ & 36.719 & 116.295 \\
\hline 88 & $18: 14: 11$ & 36.792 & 116.262 \\
\hline $\begin{array}{l}28 \\
28\end{array}$ & $\begin{array}{l}18: 17: 48 \\
20: 39: 33\end{array}$ & $\begin{array}{l}36.795 \\
36.426\end{array}$ & $\begin{array}{l}116.259 \\
115.388\end{array}$ \\
\hline 88 & $22: 13: 45$ & 36.741 & 116.304 \\
\hline 18 & $22: 23: 51$ & 36.753 & 116.268 \\
\hline 88 & $23: 9: 24$ & 36.723 & 116.305 \\
\hline 38 & $23: 56: 24$ & 35.480 & 117.519 \\
\hline & $0: 40: 30$ & 36.722 & 116.307 \\
\hline 9 & $1: 40: 46$ & 36.707 & 116.280 \\
\hline 29 & 4:33 & 36.731 & 116.305 \\
\hline 9 & $11: 44: 22$ & 36.721 & 116.310 \\
\hline 29 & $11: 59: 25$ & 36.852 & 116.101 \\
\hline 9 & $18: 31: 1$ & 36.723 & 116.295 \\
\hline 29 & $20: 10: 15$ & 36.827 & 116.309 \\
\hline 29 & $21: 14: 25$ & 36.730 & 116.305 \\
\hline 30 & $1: 27: 5$ & 36.739 & 116.291 \\
\hline 30 & $6: 52: 43$ & 35.826 & 117.654 \\
\hline 30 & $8: 50: 16$ & 36.726 & 116.292 \\
\hline 80 & $11: 5 E: 48$ & 36.647 & $\begin{array}{r}116.329 \\
116.300\end{array}$ \\
\hline & $\begin{array}{l}12: 22: 25 \\
14: 57: 53\end{array}$ & $\begin{array}{l}36.721 \\
36.732\end{array}$ & 116.300 \\
\hline
\end{tabular}

$\begin{array}{lllllllll}1.86 & 2.06 & 2.0 & 18.5 & 0.20 & 18 & \text { LIDA mSB SW }\end{array}$

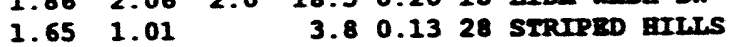
$\begin{array}{lllllll}1.62 & 1.11 & 1.7 & 5.9 & 0.18 & 18 & \text { GBORGES INIER }\end{array}$

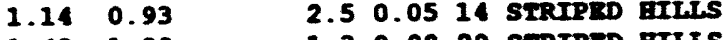
$1.431 .00 \quad 1.30 .0820$ STRIPED BILLS $\begin{array}{lllllll}1.72 & 1.21 & 1.7 & 1.8 & 0.11 & 27 & \text { SIRIPED }\end{array}$ $\begin{array}{lllllll}2.34 & 1.49 & 2.2 & 3.0 & 0.08 & 28 & \text { STRIPED GILLS }\end{array}$

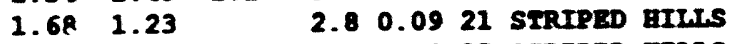
$\begin{array}{lllllll}1.77 & 1.22 & 1.1 & 3.3 & 0.08 & 25 & \text { SIRIPED MILS }\end{array}$

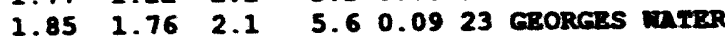
$2.642 .762 .0 \quad 5.90 .1129$ Grorers $m$ mrzR $\begin{array}{lllllll}1.22 & 0.98 & 21.4 & 0.40 & 10 & \text { CORM CREEK SPRIMES MH }\end{array}$

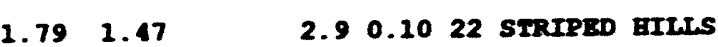
$\begin{array}{lllllll}1.44 & 1.16 & 1.4 & 1.2 & 0.12 & 23 & \text { GBOREES MATER }\end{array}$ $\begin{array}{lllllll}2.05 & 1.51 & 1.5 & 3.7 & 0.10 & 21 & \text { STRIPED BILTS }\end{array}$

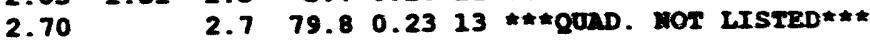
$\begin{array}{llll}1.59 & 1.12 & 3.8 & 0.0719 \text { STRIPED BILLS }\end{array}$ $\begin{array}{lllll}1.52 & 0.81 \quad 1.2 & 0 & 0.1119 & 19 \\ \text { STRTPED BITLS }\end{array}$

$\begin{array}{lll}1.47 & 0.94 & 3.20 .0717 \text { STRIPED BILLS }\end{array}$ $\begin{array}{lllllll}2.33 & 1.67 & 1.5 & 1.1 & 0.07 & 26 & \text { SERTPED BIISS }\end{array}$ $\begin{array}{lllllll}2.33 & 1.67 & 1.5 & 1.1 & 0.07 & 26 & \text { SERTPED HIWG } \\ & 1.18 & 1.4 & 6.1 & 0.12 & 19 & \text { CNII SPRIMG }\end{array}$

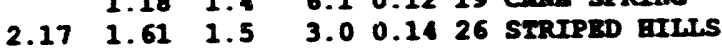
$\begin{array}{lllllll}1.60 & 1.23 & 1.2 & 4.1 & 0.13 & 22 & \text { Grosers WTER }\end{array}$

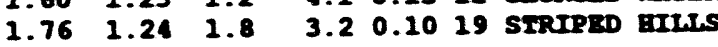

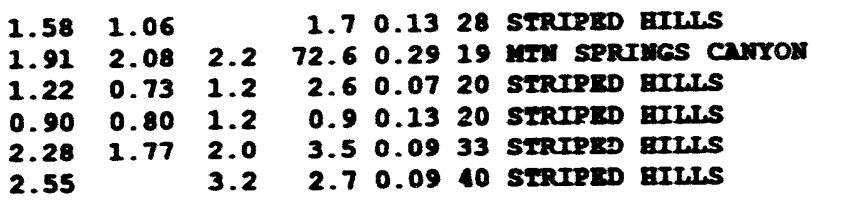



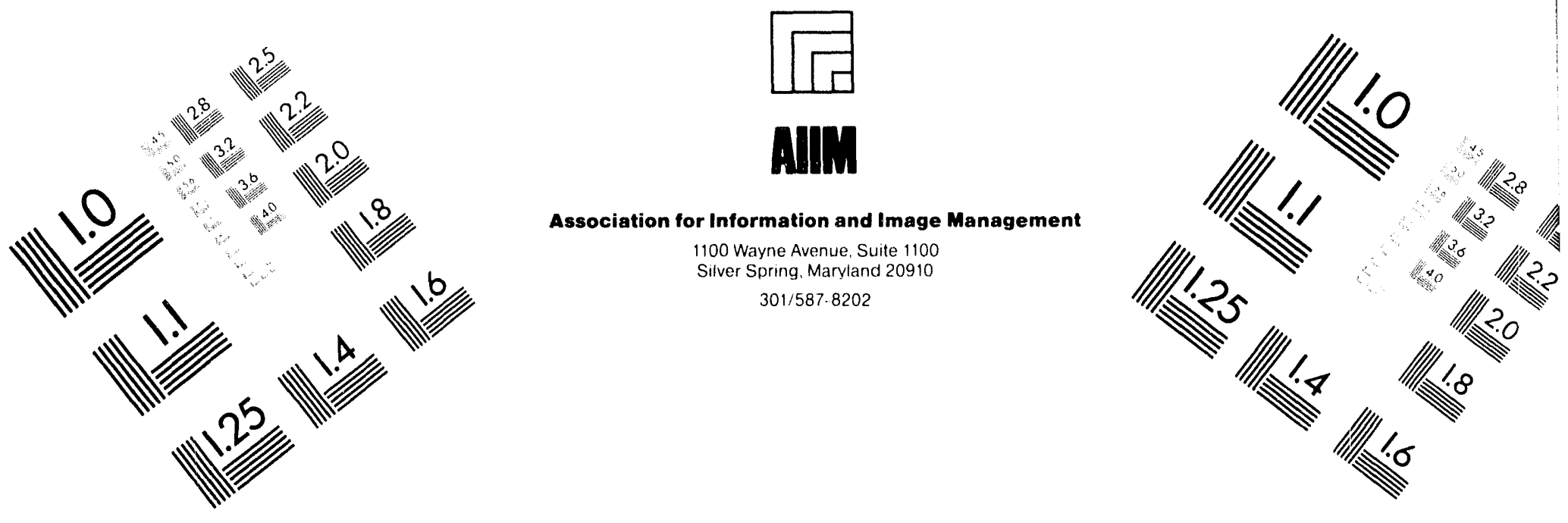

\section{Centimeter}

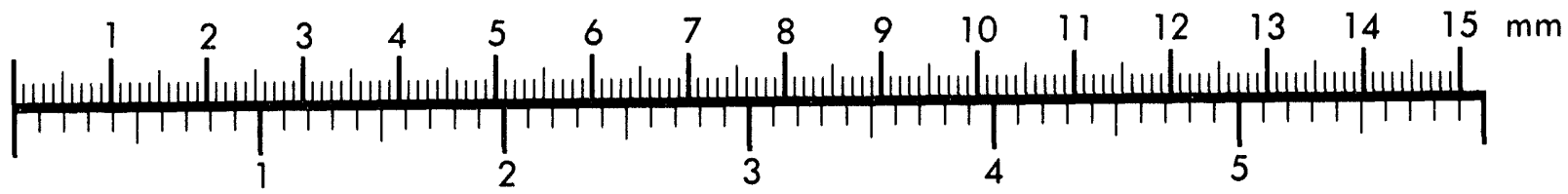
Inches
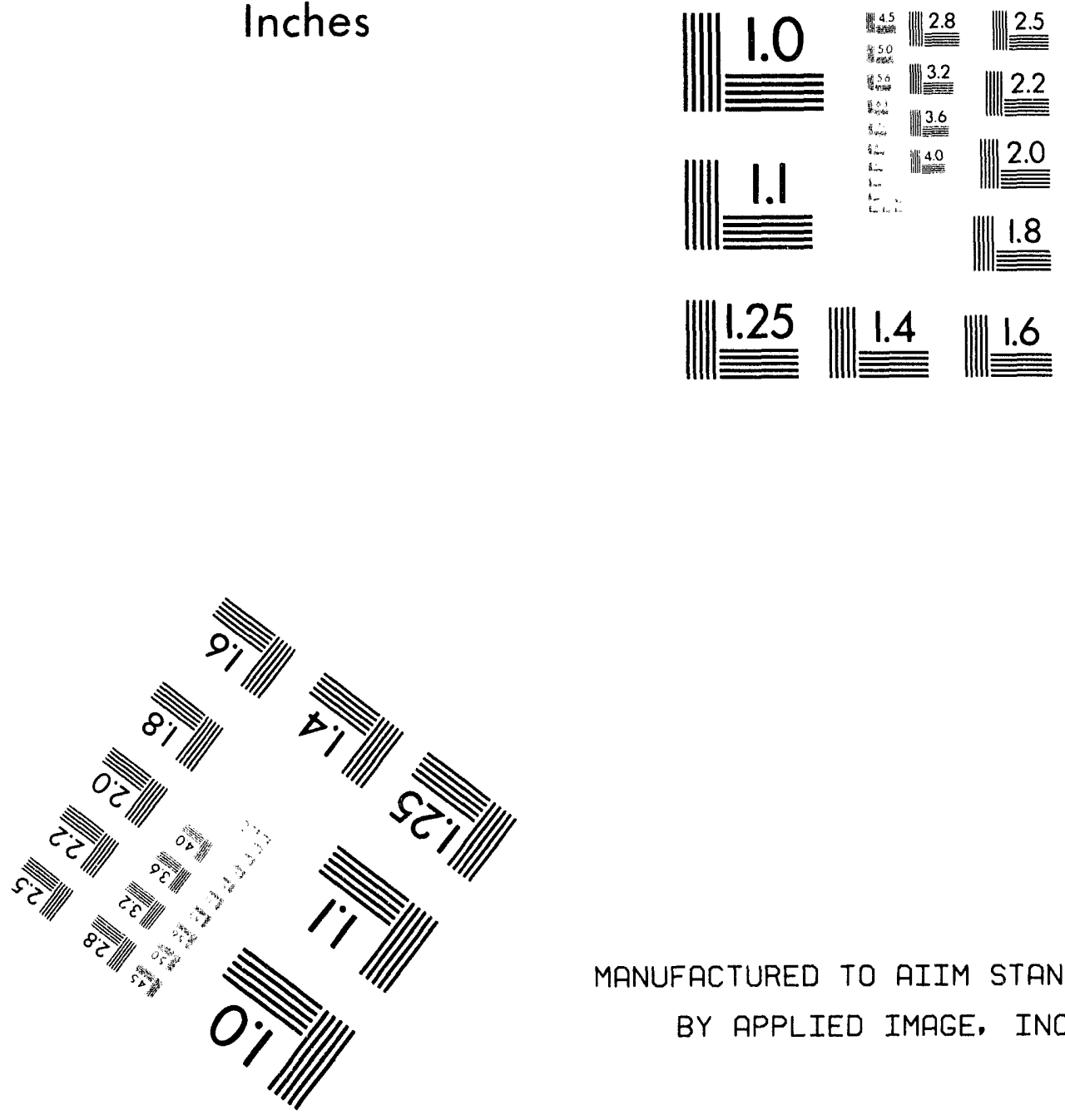

MANUFACTURED TO AIIM STANDARDS BY APPLIED IMAGE, INC.

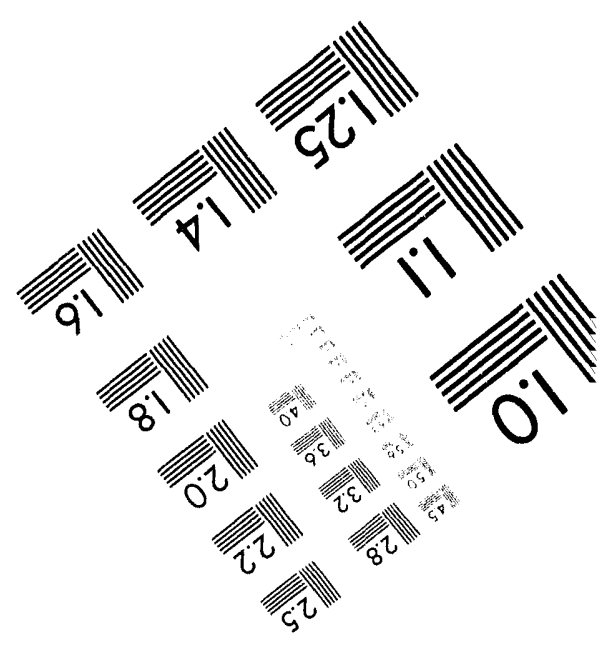



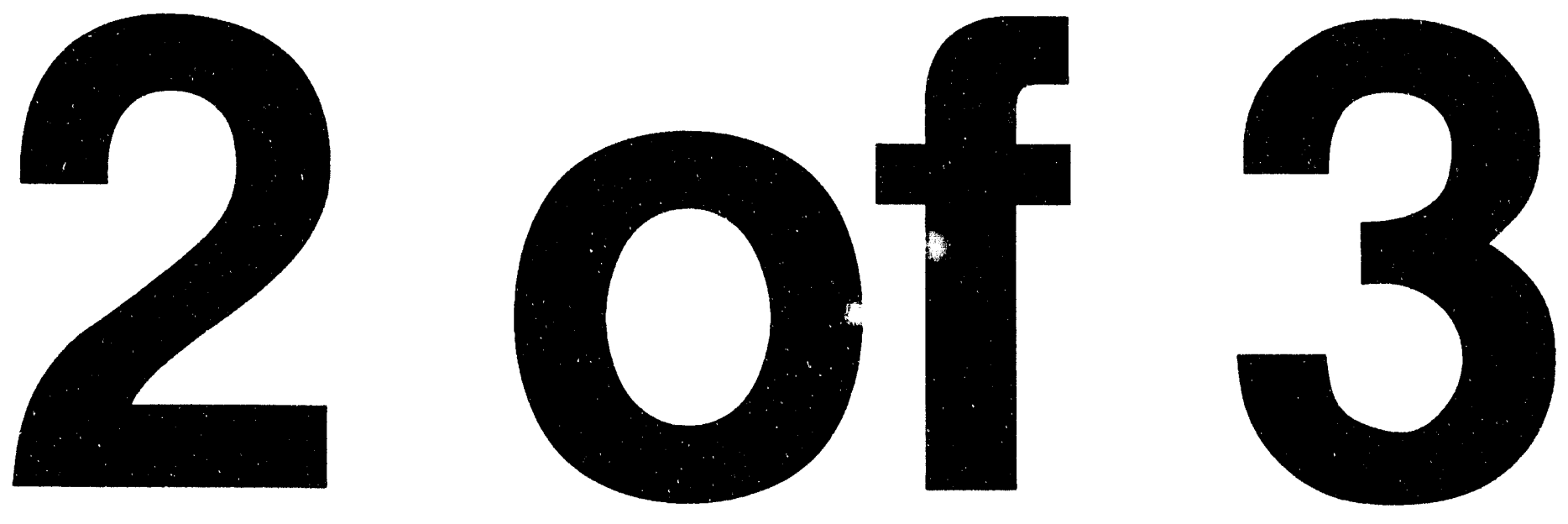


\section{Appendix B}

Preliminary SGB chemical and nuclear explosion location data, January 1 to September 30, 1992

Known and suspected chemical explosions for the first nine months of 1992 are listed in Appendix B. A partial list of companies engaged in mining-related or construction-related blasting in the SGB is included in Harmsen (1993). The depth of commercial chemical blasts is at the earth's surface (plus $<100$ feet, usually), but in many instances, hypocenters have been located with depth as a free parameter, to examine the location algorithm and velocity model. If the hypocenter depth is reported as -1.00 to $-1.10 \mathrm{~km}$, it was fixed at that value during hypocenter determination. All other depths are freely determined. If the letters "PB" follow the depth estimate, the event is a probable blast, but just enough ambiguity was present in the seismograms to prevent a certain judgment. Data from known chemical explosion sources are not scaled from Develocorder films, so "gaps" in the explosion record may exist for periods of seismic computer downtime. The US Air Force conducts gunnery practice in the Spotted Range and Indian Springs Valley east of Frenchman Flat (P. T. Finke, USAF, written commun., 1992) in an area of active natural seismicity. Although bombs having TNT-equivalent size $\leq 2000$ lbs are detonated, blasting at those sites has not been identified by our seismic analysis. Whether any such explosions contaminate the SGBSN natural seismicity catalog is unknown, and likelihood estimates should await further research into source and site characteristics. 100 lb charges detonated in the Amargosa Desert for a seismic reflection feasibility study (Brocher and others, 1990) triggered the SGBSN detection computer, so that, other factors being equal, a seismic coupling $\geq 5 \%$ for the larger Indian Springs Valley explosions also should have resulted in a seismic detection.

Announced US Department of Energy nuclear device tests at northern NTS for January 1, 1992, through September 30, 1992, are listed in table B1. Magnitudes are those reported in the USCS National Earthquake Information Center Prelimination Determination of Epicenters monthly bulletins. Depths are the reported working point depths $(\mathrm{km}$, with a negative sign to signify above sea level). Chenical and nuclear device explosion epicenters in the SGB for the first nine months of 1992 are shown in figure B1.

Table B1. Announced nuclear-device tests at Nevada Test Site for the first nine months of 1992.

\begin{tabular}{|c|c|c|c|c|c|c|}
\hline YRMODA & $\begin{array}{c}\text { HR MN SEC. } \\
\text { UTC }\end{array}$ & $\begin{array}{c}M_{L} \text { or } M_{b} \\
\text { SRC }\end{array}$ & $\begin{array}{c}\text { Latitude } \\
{ }^{\circ} \mathrm{N}\end{array}$ & $\begin{array}{c}\text { Longitude } \\
{ }^{\circ} \mathrm{W}\end{array}$ & $\begin{array}{c}\text { Depth } \\
(\mathrm{km})\end{array}$ & NAME \\
\hline 920326 & 163000.001 & $5.5 \mathrm{BRK}$ & 37.2725 & 116.3598 & -1.42 & JUNCTION \\
920623 & 150000.072 & $4.2 \mathrm{BRK}$ & 37.1239 & 116.0312 & -1.01 & GALENA \\
920918 & 170000.078 & $4.4 \mathrm{BRK}$ & 37.2069 & 116.2100 & -1.85 & HUNTERS TROPHY \\
920923 & 150400.000 & $4.3 \mathrm{BRK}$ & 37.0207 & 115.9879 & -0.81 & DIVIDER \\
\hline
\end{tabular}

Low-frequency induced seismicity recorded by the SGBSN from the above tests was archived on magnetic tapes separate from the earthquake and chemical explosion data tapes. The low-frequency event tapes are stored at USGS Branch of Earthquake and Landslide Hazards, Golden, Co. 


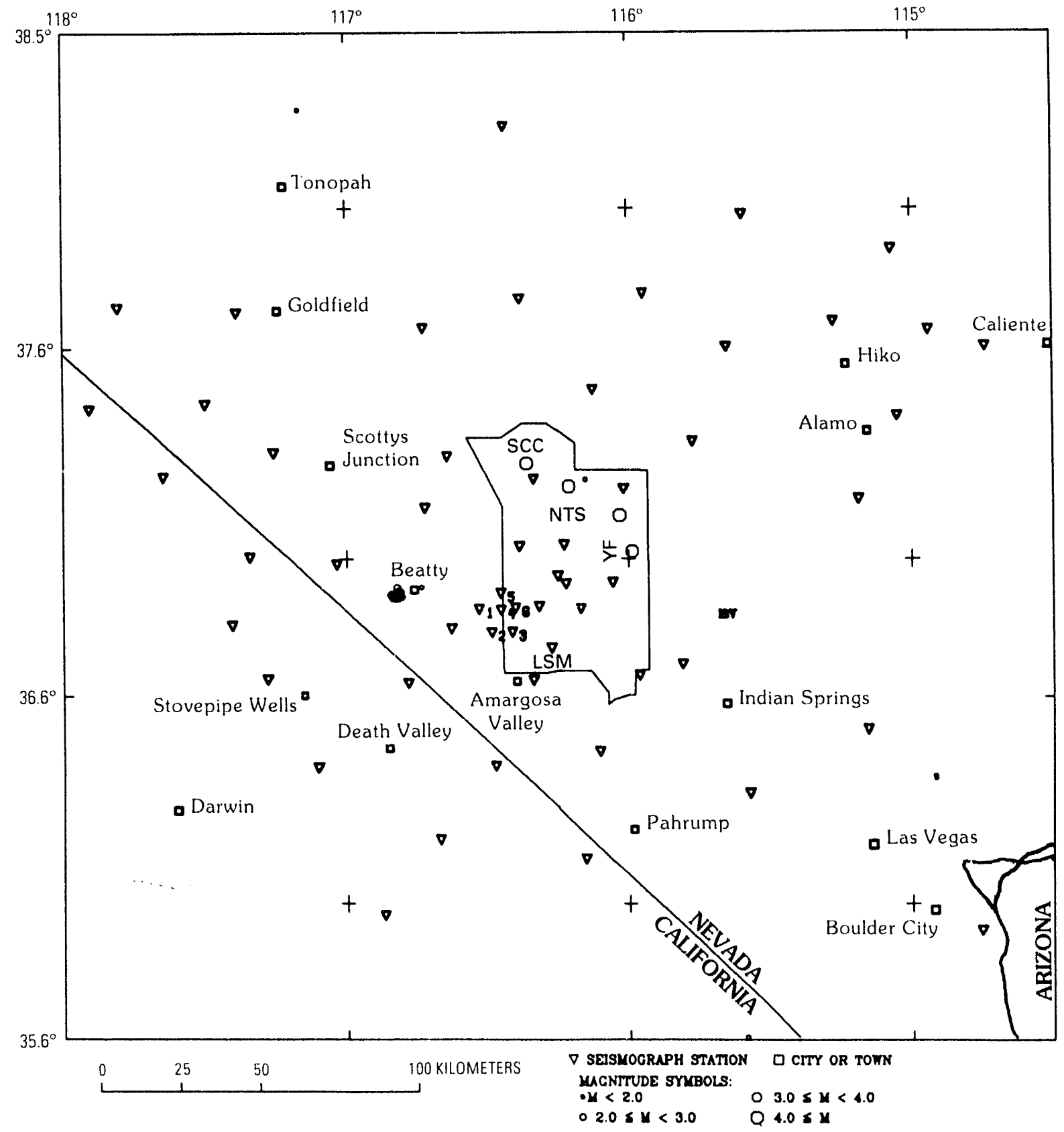

Figure B1.- Map of epicenters for chemical explosions and nuclear device tests in the southern Great Basin of Nevada and California for the period January 1, 1992 to September 30, 1992. 
1992 LOCAL HYPOCENTER SUMAYARY - SGB CHEMICAL EXPLOSIONS

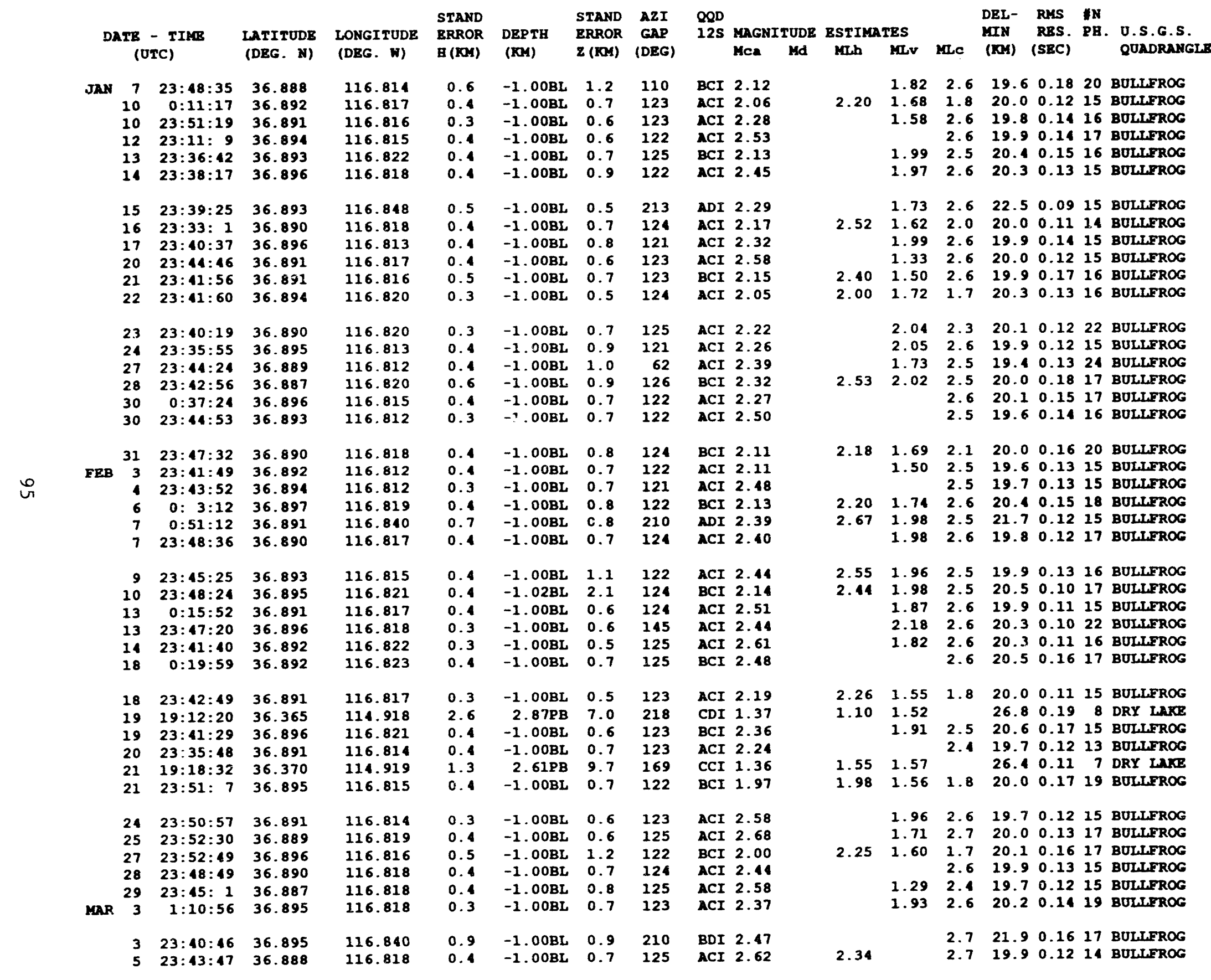


1992 LOCAL HYPOCENTER SUMMARY - SGB CHEMICAL EXPLOSIONS

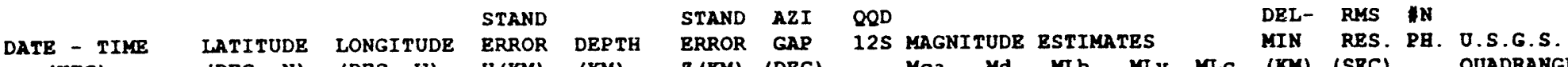
$\begin{array}{llllllllll}\text { (UTC) } & \text { (DEG. N) } & \text { (DEG. W) } & \text { H(KM) } & \text { (KM) } & \text { Z(KM) } & \text { (DEG) } & \text { MCa Md MLh MIV MLC (KM) (SEC) } & \text { QUADRANGL }\end{array}$

$\begin{array}{lllllllllll}\text { MAR } 9 & 23: 56: 56 & 36.889 & 116.817 & 0.3 & -1.00 \mathrm{BL} & 0.5 & 124 & \text { ACI } 2.37\end{array}$ $\begin{array}{llllllllll}10 & 23: 37: 3 & 36.899 & 116.820 & 0.4 & -1.00 B L & 0.8 & 122 & \text { ACI } 2.50\end{array}$

$\begin{array}{lllllllll}11 & 23: 38: 25 & 36.893 & 116.825 & 0.5 & -1.00 B L & 0.9 & 125 & \text { BCI } 2.27\end{array}$

$\begin{array}{lllllllll}12 & 23: 48: 14 & 36.896 & 116.841 & 0.7 & -1.00 \text { BL } & 0.6 & 210 & \text { ADI } 1.74\end{array}$

$\begin{array}{lllllllll}12 & 23: 48: 44 & 36.896 & 116.841 & 0.7 & -1.00 B L & 0.6 & 210 & \text { ADI } 1.74 \\ 13 & 22: 10: 43 & 36.701 & 115.510 & 1.3 & 0.00 B L & 1.7 & 187 & \text { BDI } 1.74\end{array}$

$\begin{array}{lllllllll}13 & 23: 48: 18 & 36.898 & 116.823 & 0.5 & -1.00 \mathrm{BL} & 0.8 & 123 & \text { ACI } 2.30\end{array}$

$\begin{array}{lllllllll}16 & 23: 40: 6 & 36.894 & 116.810 & 0.7 & -1.00 \mathrm{BL} & 2.2 & 109 & \text { BCI } 1.72\end{array}$

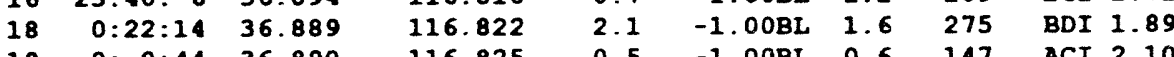

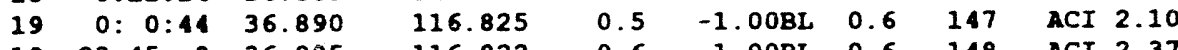

$\begin{array}{lllllllll}19 & 23: 45: 8 & 36.885 & 116.822 & 0.6 & -1.00 B L & 0.6 & 148 & \text { ACI } 2.37\end{array}$

$\begin{array}{lllllllll}20 & 23: 44: 56 & 36.885 & 116.834 & 0.9 & -1.00 \mathrm{BL} & 0.9 & 147 & \text { BCI } 2.22 \\ 23 & 15.36: 20 & 37.291 & 115.423 & 0.6 & 0.00 \mathrm{BL} & 0.9 & 167 & \text { ACI } 1.73\end{array}$

$23 \quad 23: 44: 12 \quad 36.894$

$0: 18: 54 \quad 36.888$

$0: 12: 22 \quad 36.889$

$23: 13: 24 \quad 36.892$

23:10:1 36.890

$31 \quad 23: 38: 21 \quad 36.896$

116.816

$0.3 \quad-1.00 \mathrm{BL} \quad 0.7$

122 ACI 2.10

$\begin{array}{llllll}116.821 & 0.6 & -1.00 B L & 0.9 & 149 & \text { ACI } 1.98\end{array}$

$116.817 \quad 0.4 \quad-1.008 \mathrm{~B} \quad 0.8 \quad 124 \quad A C T 2.36$

$116.8130 .5-1.00 \mathrm{BL} 0.7 \quad 122$ ACI 2.22

$\begin{array}{lllllll}116.821 & 0.4 & -1.00 B L & 0.7 & 125 & \text { BCI } 2.30\end{array}$ $\begin{array}{llllll}116.821 & 0.1 & -1.00 B L & 0.7 & 125 & \text { BCI } 1.30 \\ 116.815 & 0.6 & -1.00 B L & 1.7 & 122 & \text { BCI } 1.99\end{array}$

$\begin{array}{lll}\text { APR } 2 & 0: 4: 31 & 36.893 \\ 2 & 23: 50: 3 & 36.893\end{array}$ $223: 50: 3 \quad 36.893$ $60: 23: 0036.890$ $722: 53: 17 \quad 36.889$

8 $22: 46: 52 \quad 36.891$

116.813

$\begin{array}{llllll}116.820 & 0.4 & -1.00 \mathrm{BL} & 0.6 & 122 & \text { ACI } 2.14\end{array}$

$\begin{array}{lllllll}116.814 & 0.4 & -1.00 B L & 1.1 & 123 & \text { ACI } 2.58\end{array}$

$\begin{array}{llllll}116.819 & 0.4 & -1.00 B L & 0.7 & 123 & \text { ACI } 2.19\end{array}$

$\begin{array}{lllllll}116.823 & 0.5 & -1.00 B L & 0.9 & 126 & \text { BCI } 2.37\end{array}$

$\begin{array}{llllll}116.813 & 0.4 & -1.00 B L & 0.9 & 123 & \text { ACI } 2.26\end{array}$

$10 \quad 22: 48: 23 \quad 36.889$

$13 \quad 22: 50: 23 \quad 36.892$

14 22:46:1 36.892

$15 \quad 22: 50: 38 \quad 36.893$

$\begin{array}{lll}16 & 23: 2: 30 & 36.888 \\ 17 & 22: 42: 38 & 36.892\end{array}$

116.813

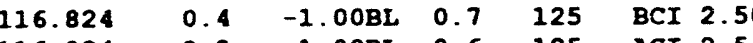

$\begin{array}{llllll}116.824 & 0.3 & -1.00 B L & 0.6 & 125 & \text { ACI } 2.54\end{array}$

$116.814 \quad 0.5 \quad-1.008 L \quad 0.9 \quad 122$ BCI 2.18

$\begin{array}{llllll}116.820 & 0.4 & -1.008 L & 0.6 & 125 & \text { ACI } 2.32\end{array}$

$20 \quad 22: 51: 38 \quad 36.898$

$22 \quad 22: 56: 10 \quad 36.890$

$24 \quad 23: 33: 60 \quad 36.894$

27 23: $3: 5 \quad 36.890$

28 23:13: $6 \quad 36.889$

$29 \quad 22: 37: 3 \quad 35.610$

$-1.00 B L \quad 0.9$

ACI 2.38

$\begin{array}{lllllll}116.818 & 0.4 & -1.00 B L & 0.7 & 122 & \text { BCI } 2.22\end{array}$

$\begin{array}{llll}1.00 B L & 0.6 & 125 & \text { ACI } 1.98 \\ -1.00 B L & 1.0 & 123 & \text { BCI } 2.26\end{array}$

$116.8160 .301 .00810 .6 \quad 124$ BCI 2.26

116.8160 .3

$\begin{array}{cccccc}116.805 & 0.7 & -1.00 \mathrm{BL} & 1.4 & 121 & \text { BCI } 2.56 \\ 115.585 & 3.8 & 2.13 * & \ldots & 223 & \text { CDI }\end{array}$

$29 \quad 22: 11: 28 \quad 36.892$

$30 \quad 16: 29: 60 \quad 37.225$

$30 \quad 22: 38: 37 \quad 36.888$

MaY $122: 44: 36 \quad 36.893$

23:45:37 36.891

116.813

$\begin{array}{llllll}1.80 & 2.6 & 19.9 & 0.11 & 15 & \text { BULLFROG } \\ 1.79 & 2.5 & 20.7 & 0.14 & 17 & \text { BULLFROG }\end{array}$

$\begin{array}{lllllll}1.79 & 2.5 & 20.7 & 0.14 & 17 & \text { BULLFROG } \\ 2.03 & 2.6 & 20.6 & 0.21 & 18 & \text { BULLFROG }\end{array}$

$\begin{array}{lllllll}2.14 & 1.61 & 2.0 & 22.1 & 0.10 & 14 & \text { BULLFROG }\end{array}$

$\begin{array}{lllllll}1.42 & 1.71 & 1.8 & 26.8 & 0.20 & 13 & \text { BRAVENS WELL }\end{array}$

$\begin{array}{llllllll}2.55 & 1.93 & 2.6 & 20.8 & 0.14 & 14 & \text { BULLFROG }\end{array}$

$\begin{array}{llllll}1.82 & 1.9 & 19.6 & 0.15 \quad 10 & \text { BULLFROG }\end{array}$

$2.11 \quad 1.70 \quad 1.2 \quad 20.2 \quad 0.0912$ BULLFROG $\begin{array}{llllll}2.06 & 2.4 & 20.5 & 0.11 & 15 & \text { BULLFROG }\end{array}$ $2.012 .6 \quad 19.9 \quad 0.1214$ BULLFROC

$\begin{array}{llllll}2.56 & 2.16 & 2.1 & 20.9 & 0.20 & 15 \\ & 1.4 U L I F R O G\end{array}$

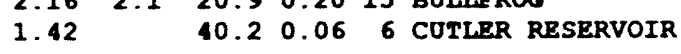

$\begin{array}{lllllll}2.36 & 1.70 & 1.7 & 20.1 & 0.10 & 13 & \text { BULLFROG }\end{array}$ $\begin{array}{lllllll}2.02 & 1.72 & 1.7 & 20.1 & 0.12 & 12 & \text { BULLFROG }\end{array}$ $\begin{array}{llllll}2.5 & 19.8 & 0.12 & 13 & \text { BULLFROG }\end{array}$

$2.46 \quad 1.71 \quad 1.9 \quad 19.7 \quad 0.1414$ BULLFROG

$\begin{array}{llllll}1.86 & 2.6 & 20.2 & 0.15 & 15 & \text { BULLFROG }\end{array}$

$\begin{array}{llllllll}2.31 & 1.66 & 1.9 & 20.1 & 0.15 & 12 & \text { BULLFROG }\end{array}$

$\begin{array}{llllllll}2.46 & 1.66 & 1.7 & 19.8 & 0.12 & 16 & \text { BULLFROG }\end{array}$

$2.45 \quad 1.75 \quad 2.420 .3 \quad 0.1416$ BULLFROG

$\begin{array}{llllllll} & & & 2.7 & 19.7 & 0.12 & 14 & \text { BULLFROG }\end{array}$

$\begin{array}{lllllll}1.95 & 2.0 & 20.4 & 0.14 & 14 & \text { BULLFROG } \\ 1.95 & 2.7 & 20.3 & 0.18 & 16 & \text { BULLFROG }\end{array}$

$\begin{array}{llllll}1.57 & 2.5 & 19.6 & 0.12 \quad 13 & \text { BULLFROG }\end{array}$

$\begin{array}{lllllll}2.10 & 2.6 & 19.5 & 0.14 & 14 & \text { BULLFROG }\end{array}$

$\begin{array}{llllll}1.62 & 2.7 & 20.5 & 0.16 & 16 & \text { BULLFROG }\end{array}$

$\begin{array}{llllllll}2.62 & 1.91 & 2.2 & 20.5 & 0.14 & 17 & \text { BOLLFROG }\end{array}$

$\begin{array}{llllllll}2.42 & 1.75 & 2.4 & 19.8 & 0.17 & 16 & \text { BULLFROG }\end{array}$

$\begin{array}{lllllll}1.53 & 2.6 & 20.0 & 0.13 & 16 & \text { BOLLLROG }\end{array}$ $2.5 \quad 19.7 \quad 0.1415$ BULLFROG

$\begin{array}{lllllll}2.40 & 1.71 & 1.9 & 20.4 & 0.20 & 18 & \text { BULLFROG }\end{array}$ $\begin{array}{llllllll}2.39 & 1.72 & 1.8 & 20.2 & 0.14 & 13 & \text { BULLFROG }\end{array}$ $\begin{array}{llllllll}2.34 & 1.75 & 2.0 & 20.2 & 0.21 & 17 & \text { BULLFROG }\end{array}$

$\begin{array}{llllllll}2.32 & 1.74 & 1.6 & 19.8 & 0.10 & 19 & \text { BULLFROG }\end{array}$ 2.718 .90 .2616 BULLFROG

$77.0 \quad 0.28 \quad 8$ CLARK MTN

$\begin{array}{rrrrrr}0.5 & -1.00 B L & 1.0 & 109 & \text { BCI } 2.45 \\ 0.4 & -1.30 \mathrm{~PB} & 0.7 & 94 & \text { ACI } & 1.23\end{array}$

$\begin{array}{lllllll}116.817 & 1.7 & -1.00 \mathrm{BL} & 1.3 & 274 & \text { BDI } 2.00\end{array}$

$\begin{array}{llllll}116.817 & 0.4 & -1.00 \mathrm{BL} & 0.6 & 123 & \text { ACI } 2.44\end{array}$

$\begin{array}{llllll}116.825 & 0.5 & -1.00 B L & 0.8 & 126 & \text { BCI } 2.49\end{array}$

$\begin{array}{llllll}116.821 & 0.3 & -1.00 B L & 0.6 & 125 & \text { ACI } 2.22\end{array}$

$623: 16: 37 \quad 36.896$

116.836

$\begin{array}{rrrrrr}0.3 & -1.00 B L & 0.6 & 127 & \text { ACI } & 2.64 \\ 0.5 & 3.68 \mathrm{~PB} & 55 & 217 & \text { CDI } & 1.13\end{array}$

$0: 49: 32 \quad 36.916$

116.734

$0.5 \quad 3.68 \mathrm{~PB} 5 \mathrm{5}$

$\begin{array}{lllllll}1.86 & 2.5 & 19.7 & 0.1919 & \text { BULLFROG }\end{array}$

$\begin{array}{llllllll}1.25 & 0.80 & 1.3 & 12.1 & 0.14 & 15 & \text { RAINIER MRSA }\end{array}$

$\begin{array}{lllllll}2.31 & 1.60 & 2.0 & 19.8 & 0.08 & 13 & \text { BUL.LFROG }\end{array}$

$2.10 \quad 2.5 \quad 20.1 \quad 0.1417$ BEATTY

$\begin{array}{lllllll} & 2.14 & 2.7 & 20.5 & 0.17 & 18 & \text { BEATTY } \\ 2.30 & 1.76 & 2.5 & 20.2 & 0.13 & 15 & \text { BEATTY }\end{array}$

$\begin{array}{llllll}1.05 \quad 0.64 & 2.5 & 20.2 & 0.07 & 9 & \text { BEATTY }\end{array}$ 
1992 LOCAL HYPOCENTER SOMAARY - SGB CHEMICAL EXPLOSIONS

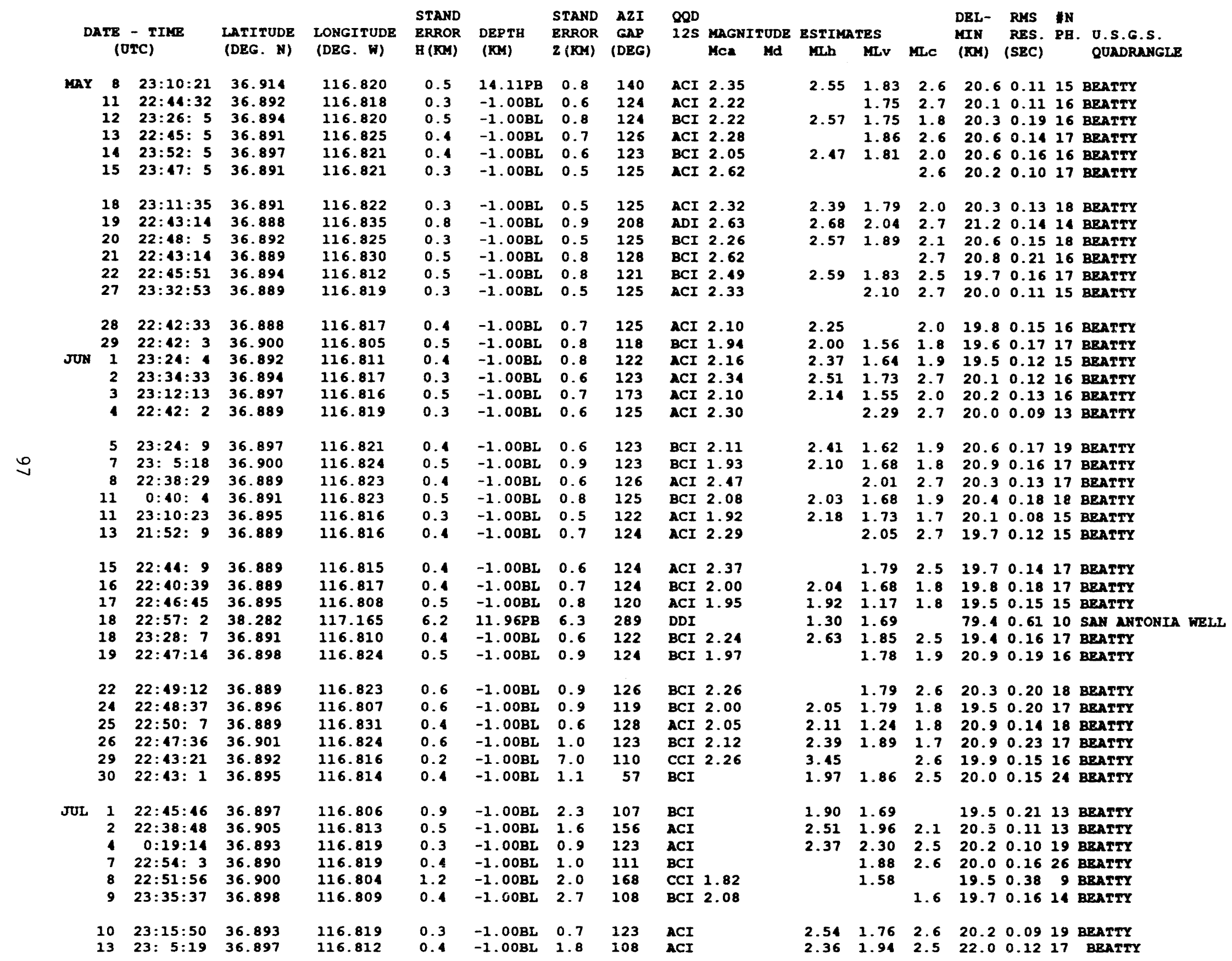


1992 LOCAL HYPOCENTER SURARY - SGB CHEMICAL EXPLOSIONS

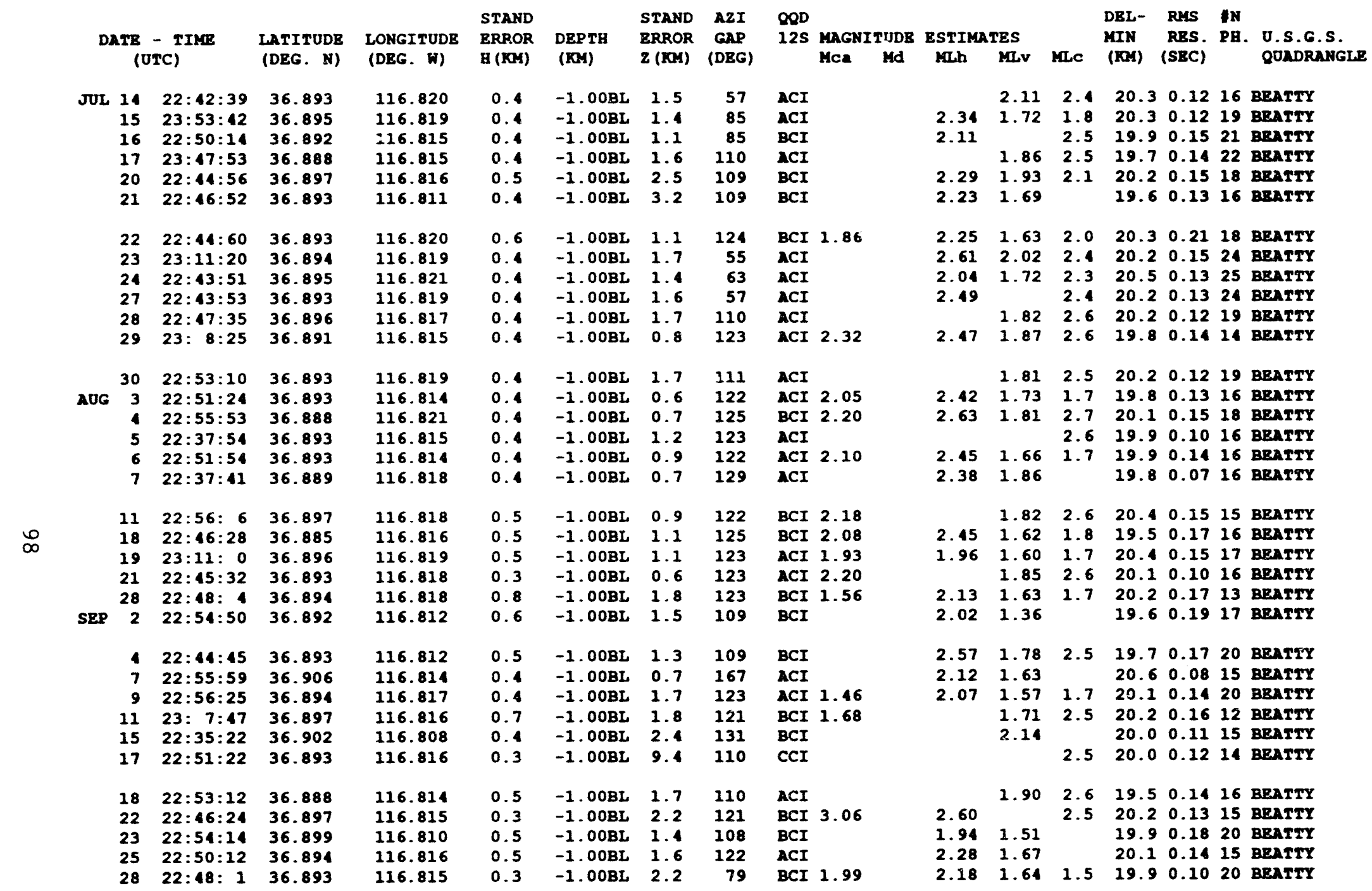




\section{Appendix C}

Southern Great Basin preliminary earthquake focal mechanisms, January to September, 1992

110 focal mechanism solutions for SGB earthquakes for the period January through September, 1992, are presented in chronological order. These focal mechanisms were obtained by selecting the best-fitting solution(s) from the application of the computer program "FOCMEC" (Snoke and others, 1984) to preliminary hypocenter ray data scaled from USGS/SGBSN seismograms. A more complete set of seismograms for many of these earthquakes is now being assembled, merging USGS and UNRSL data (K. Smith, oral commun., 1993). I plot polarity data using the equal-area projection. The symbols represent first-motion $\mathbf{P}$-polarities, and their positions represent the points where the theoretical raypaths intersect the lower focal hemisphere. The darkened circles represent impulsive compressional arrivals, the + symbols represent emergent compressionals, the open circles represent impulsive dilatationals, the - symbols represent emergent dilatationals, and the $x$ symbols represent indeterminate or nodal readings. The + symbol at the center of each mechanism is not a compression; it is merely a point of reference. SGBSN station names are printed adjacent to the first-motion symbol for most of the solutions presented in Appendix C. For several focal mechanisms computed for earthquakes in the Little Skull Mountain aftershock period June 30 through July 14, 1992, data from the temporary seismic net deployed by the USGS are used; details of the deployment are contained in Meremonte and others (1993). The temporary station coordinates are also listed in Table $\mathrm{C} 1$, below, for reference. Station code TCR shown in several mechanisms below refers to station TCT, with polarity corrected to true ground motion (M. Meremonte, oral commun., 1993). Further focal mechanism details may be obtained by E-mail from the author at harmsen@gldrtv.cr.usgs.gov.

Figure $\mathrm{C} 1$ is a map of epicenters corresponding to the earthquake focal mechanisms of Appendix C. Focal mechanisms for six SGB earthquakes having origin times in June through September, 1992. and $M_{L} \geq 4.0$ are also shown in Figure $\mathrm{C} 1$ with leaders to their epicenters. Small-font figure captions contain some abbreviations: FM=focal mechanism; LSM=Little Skull Mountain; UNRSL=University of Nevada Seismic Laboratory; and NEIC=National Earthquake Information Center (Golden, Co.). In the following figures the $\mathbf{P}$ and $\mathbf{T}$ symbols represent the focal mechanism pressure and tension axes, respectively. Primed $\mathbf{P}$ and $\mathbf{T}$ symbols are the respective vectors for alternate (dashed) solutions when they are presented. $\mathbf{X}$ and $\mathbf{Y}$ symbols represent slip vectors for each nodal plane, and $\mathbf{B}$ is the null axis. In this open-file report, no attempt is made to select preferred and auxiliary nodal planes. The author submitted a research paper on the Little Skull Mountain aftershock series focal mechanisms and stress field implications to the Seismological Society of America Bulletin. In that paper, preferred nodal planes for 80 LSM earthquakes are given. 
Table C1. Locations and elevations of USGS temporary seismic network stations in southwestern NTS (Meremonte and others, 1993)

\begin{tabular}{|c|c|c|c|}
\hline Sta & Latitude & Longitude & Elovations \\
\hline$\overline{S P E}$ & $36^{\circ} 39.22^{\prime} \mathrm{N}$ & $116^{\circ} 7.09^{\prime} \mathrm{W}$ & $1076 \mathrm{~m}$ \\
\hline SPW & $36^{\circ} 41.30^{\prime} \mathrm{N}$ & $116^{\circ} 10.69^{\prime} \mathrm{W}$ & $1073 \mathrm{~m}$ \\
\hline $\mathrm{COM}$ & $36^{\circ} 43.72 \mathrm{~N}$ & $116^{\circ} 13.41^{\prime} \mathrm{W}$ & $1169 \mathrm{~m}$ \\
\hline $\mathrm{CAL}$ & $36^{\circ} 51.42^{\prime} \mathrm{N}$ & $116^{\circ} 17.45^{\prime} \mathrm{W}$ & $1268 \mathrm{~m}$ \\
\hline TOW & $36^{\circ} 47.27^{\prime} \mathrm{N}$ & $116^{\circ} 19.70^{\prime} \mathrm{W}$ & $1111 \mathrm{~m}$ \\
\hline JAC. & $36^{\circ} 45.84^{\prime} \mathrm{N}$ & $116^{\circ} 22.43^{\prime} \mathrm{W}$ & $949 \mathrm{~m}$ \\
\hline LAT & $36^{\circ} 40.65^{\prime} \mathrm{N}$ & $116^{\circ} 22.84^{\prime} \mathrm{W}$ & $849 m$ \\
\hline LSD & $36^{\circ} 43.81^{\prime} \mathrm{N}$ & $116^{\circ} 19.61^{\prime} \mathrm{W}$ & $1177 \mathrm{~m}$ \\
\hline CLO & $36^{\circ} 50.32^{\prime} \mathrm{N}$ & $116^{\circ} 20.29^{\prime} \mathrm{W}$ & $1177 \mathrm{~m}$ \\
\hline $\mathrm{H} 50$ & $36^{\circ} 51.40^{\prime} \mathrm{N}$ & $116^{\circ} 27.94^{\prime} \mathrm{W}$ & $1475 \mathrm{~m}$ \\
\hline AR0 & $36^{\circ} 49.74^{\prime} \mathrm{N}$ & $116^{\circ} 16.26^{\prime} \mathrm{W}$ & $1160 \mathrm{~m}$ \\
\hline MVR & $36^{\circ} 51.65^{\prime} \mathrm{N}$ & $116^{\circ} 13.60^{\prime} \mathrm{W}$ & $1326 \mathrm{~m}$ \\
\hline TCT & $36^{\circ} 48.34^{\prime} \mathrm{N}$ & $116^{\circ} 8.95^{\prime} \mathrm{W}$ & $1352 \mathrm{~m}$ \\
\hline TWR & $36^{\circ} 46.43^{\prime} \mathrm{N}$ & $116^{\circ} 14.29^{\prime} \mathrm{W}$ & $1109 m$ \\
\hline TPP & $36^{\circ} 43.37^{\prime} \mathrm{N}$ & $116^{\circ} 7.74^{\prime} \mathrm{W}$ & $1158 \mathrm{~m}$ \\
\hline SLS & $36^{\circ} 42.00^{\prime} \mathrm{N}$ & $116^{\circ} 16.02^{\prime} \mathrm{W}$ & $1045 \mathrm{~m}$ \\
\hline ARY & $36^{\circ} 49.74^{\prime} \mathrm{N}$ & $116^{\circ} 16.26^{\prime} \mathrm{W}$ & $1160 \mathrm{~m}$ \\
\hline ARX & $36^{\circ} 49.74 \mathrm{~N}$ & $116^{\circ} 16.26^{\prime} \mathrm{W}$ & $1160 \mathrm{~m}$ \\
\hline
\end{tabular}




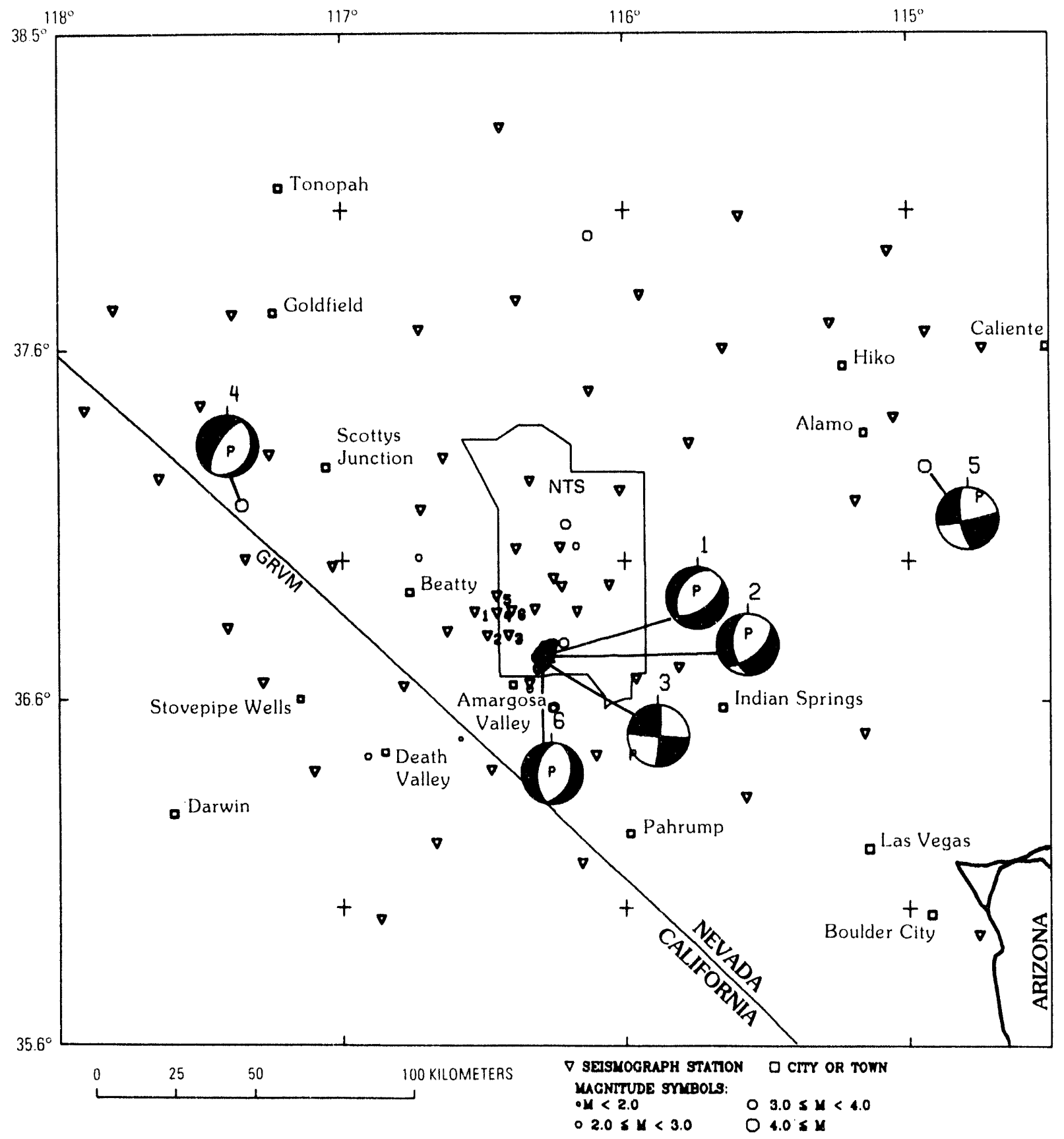

Figure C1. Earthquake epicenters (circles) for 110 earthquakes for which focal mechanisms were computed for the period January 1, 1992 to September 30, 1992. Lower-hemisphere projections of the focal mechanisms for the six largest of these earthquakes with magnitude $M_{L} \geq 4.0$ are shown. The origin times of these six earthquakes are (1) June 29, 10:14 (Little Skull Mtn. mainshock), (2) July 3, 13:25, (3) July 5 06:54, (4) July 29, 22:24, (5) August 4, 13:37, and (6) September 13, 11:46. 


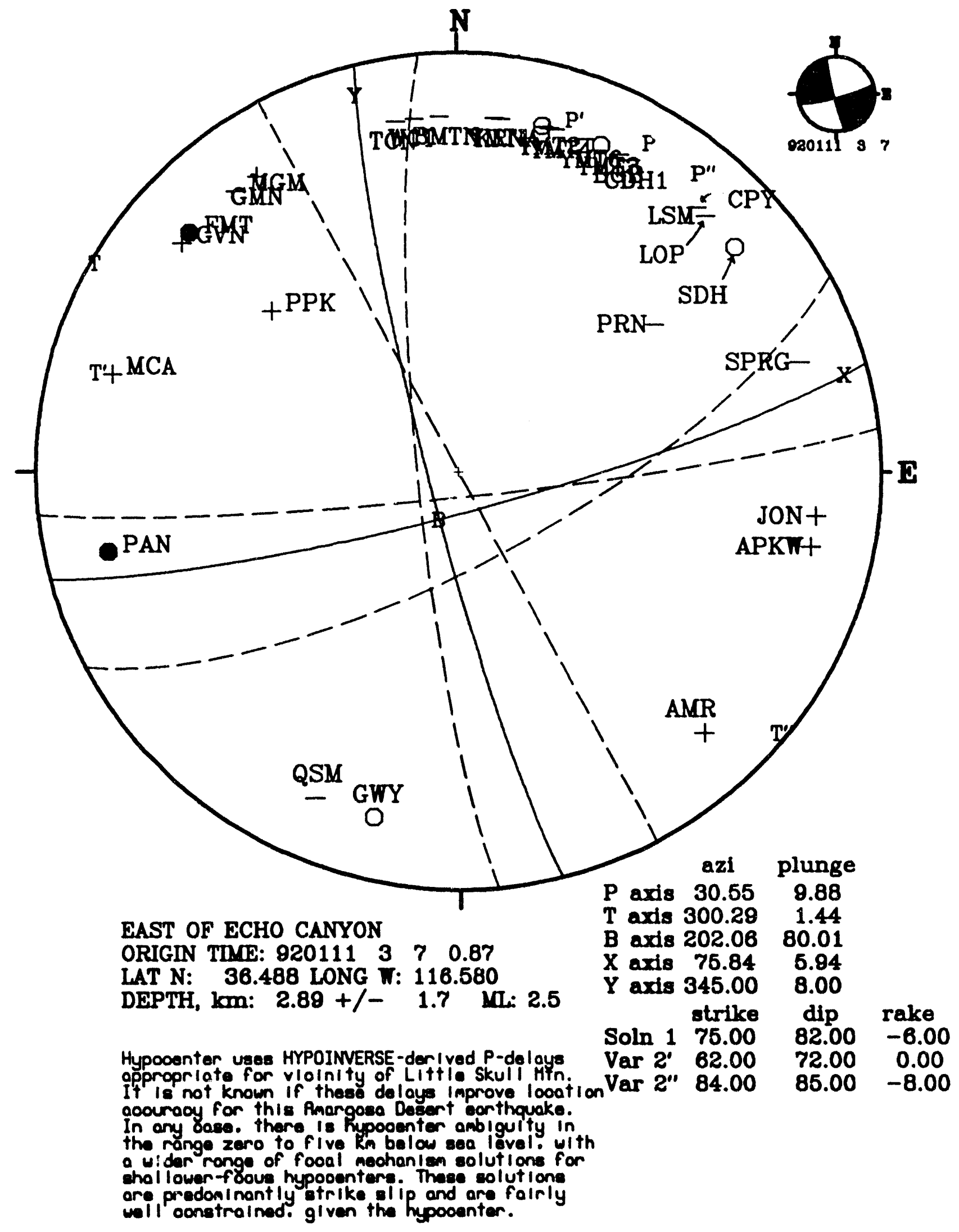




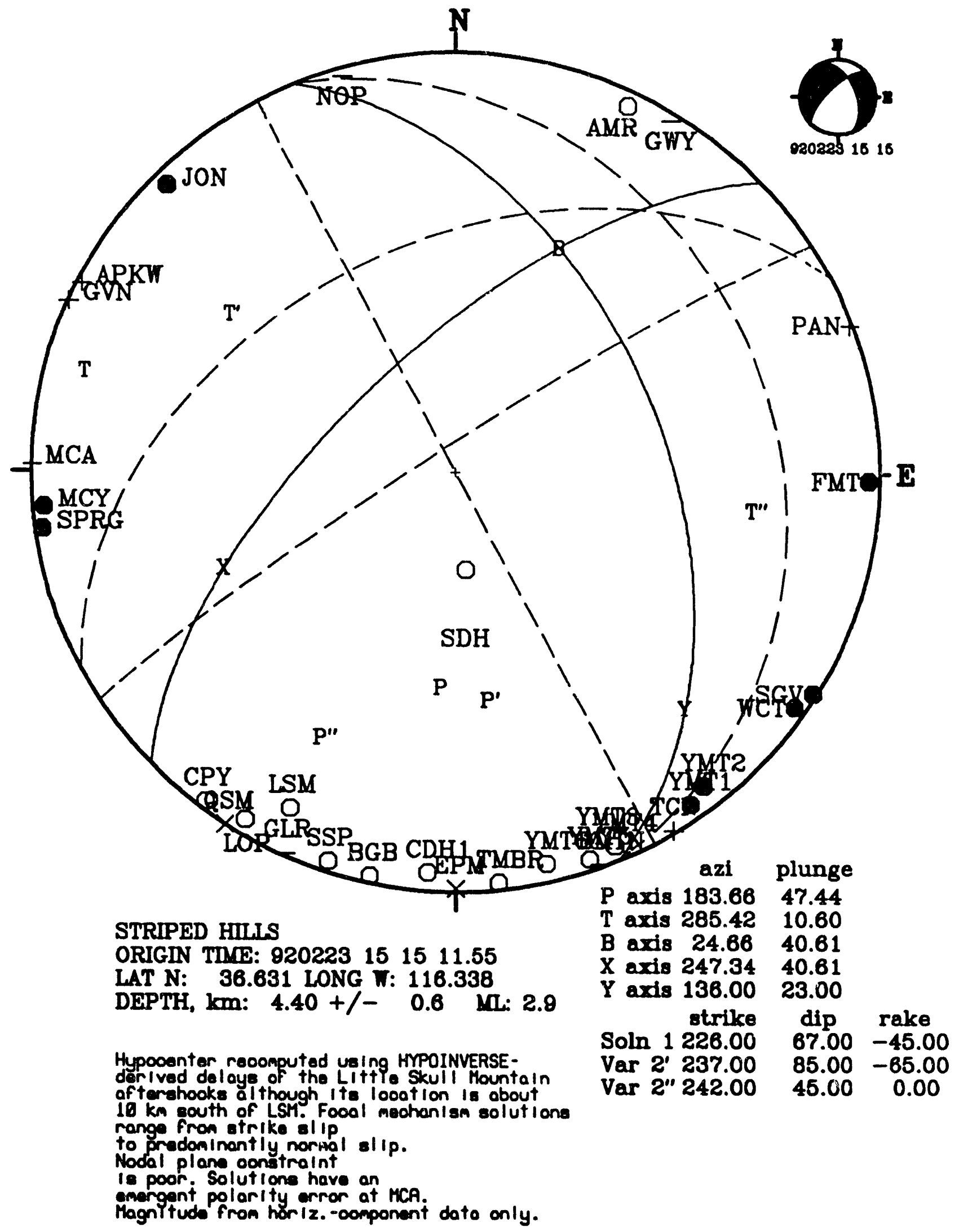




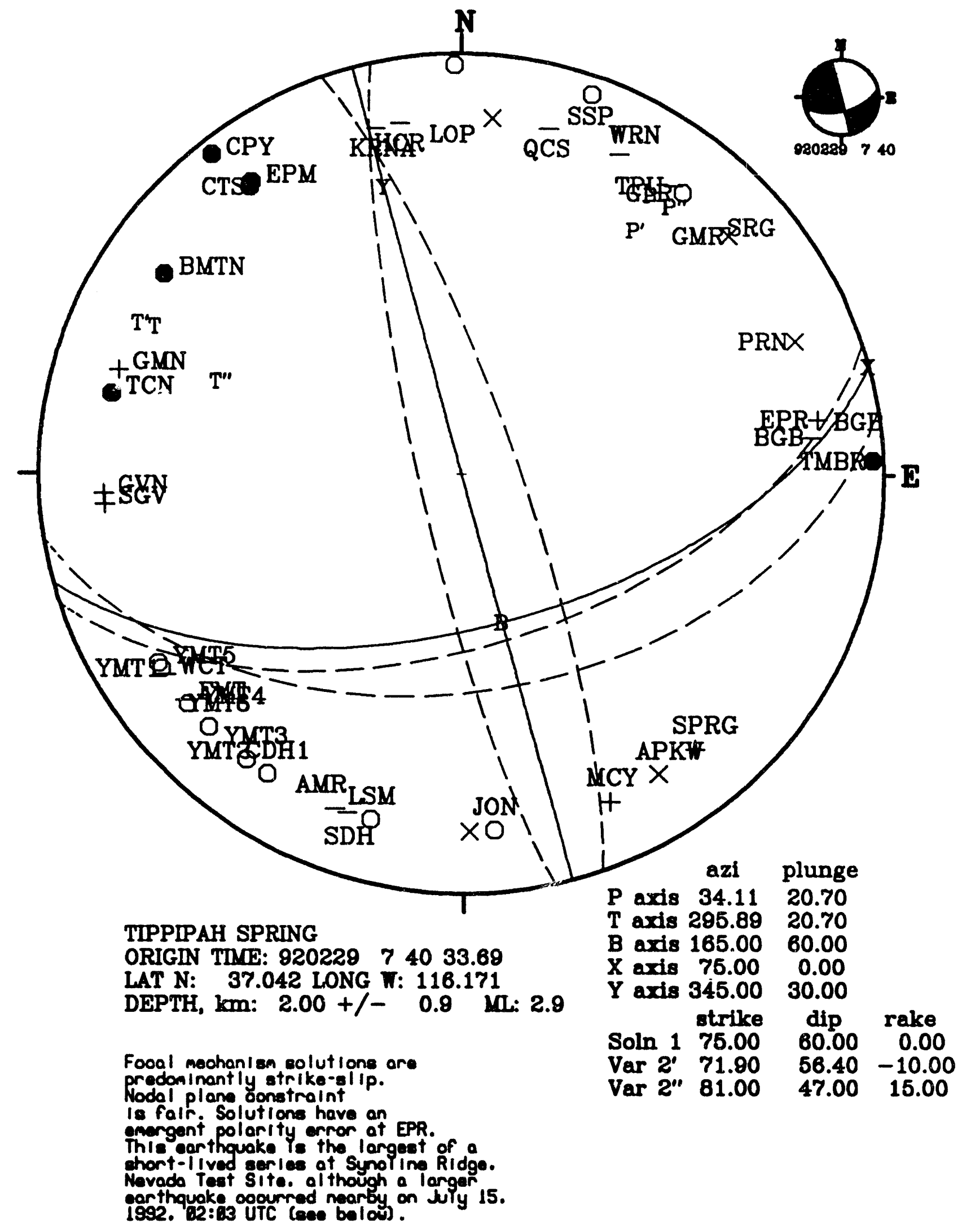




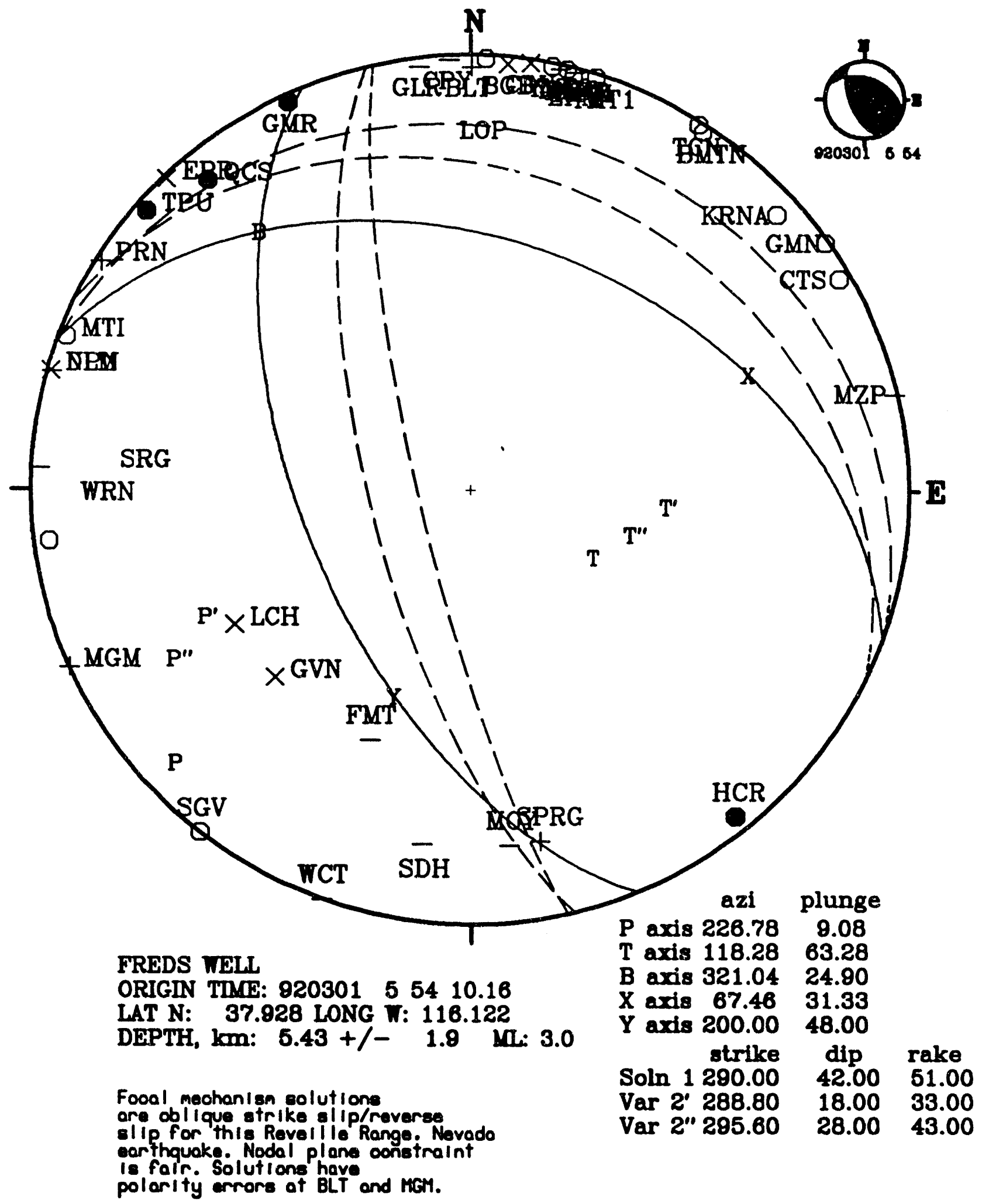




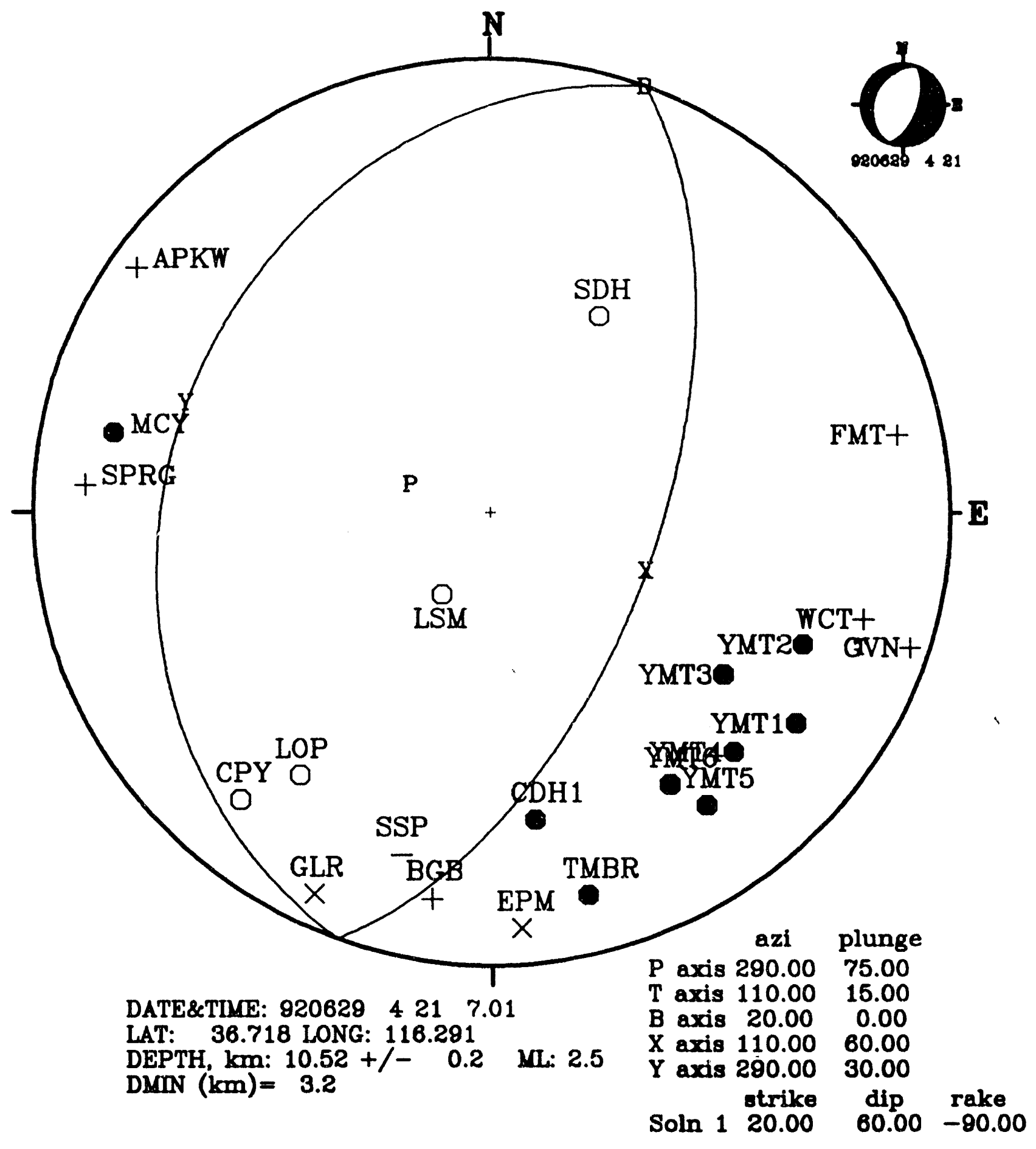




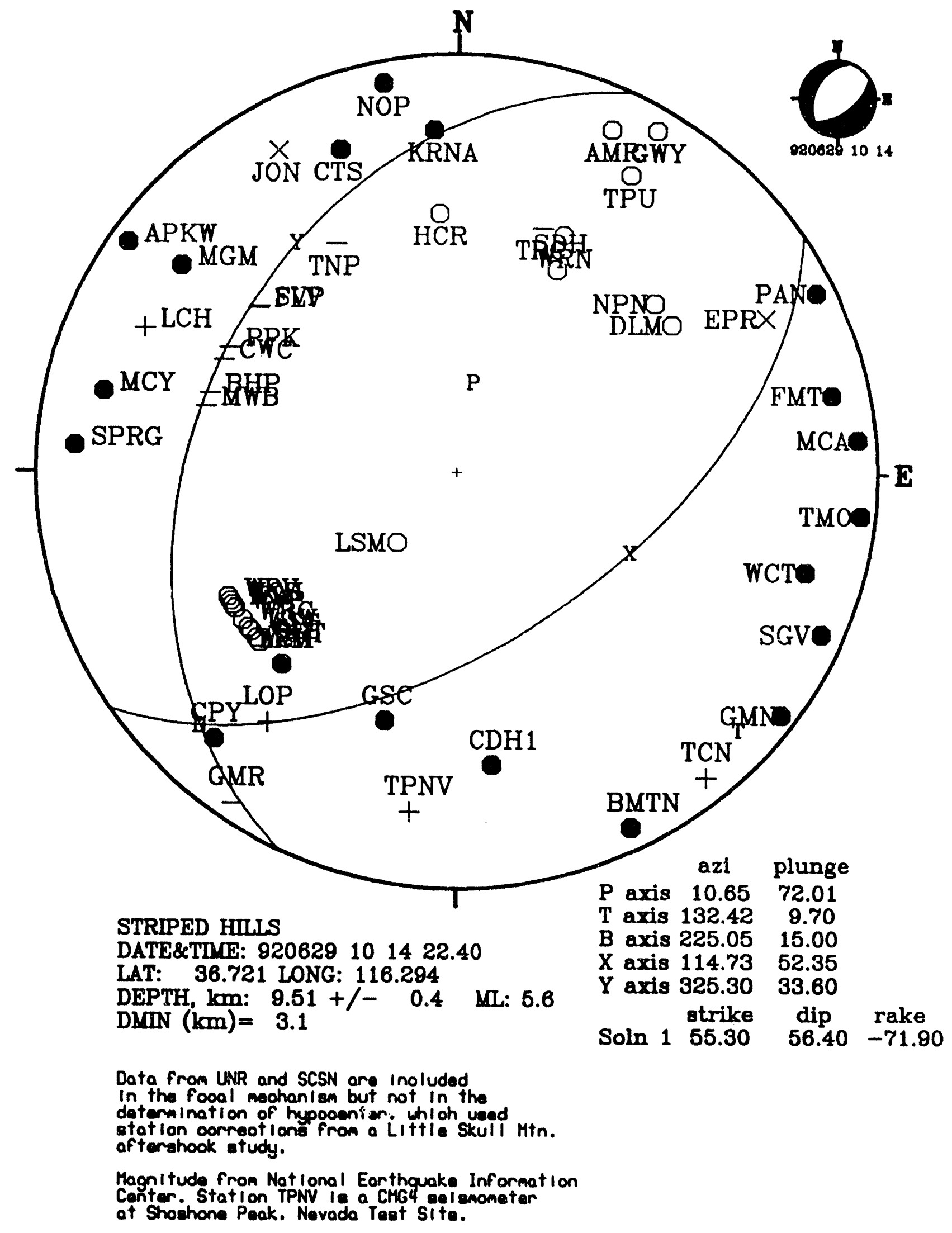




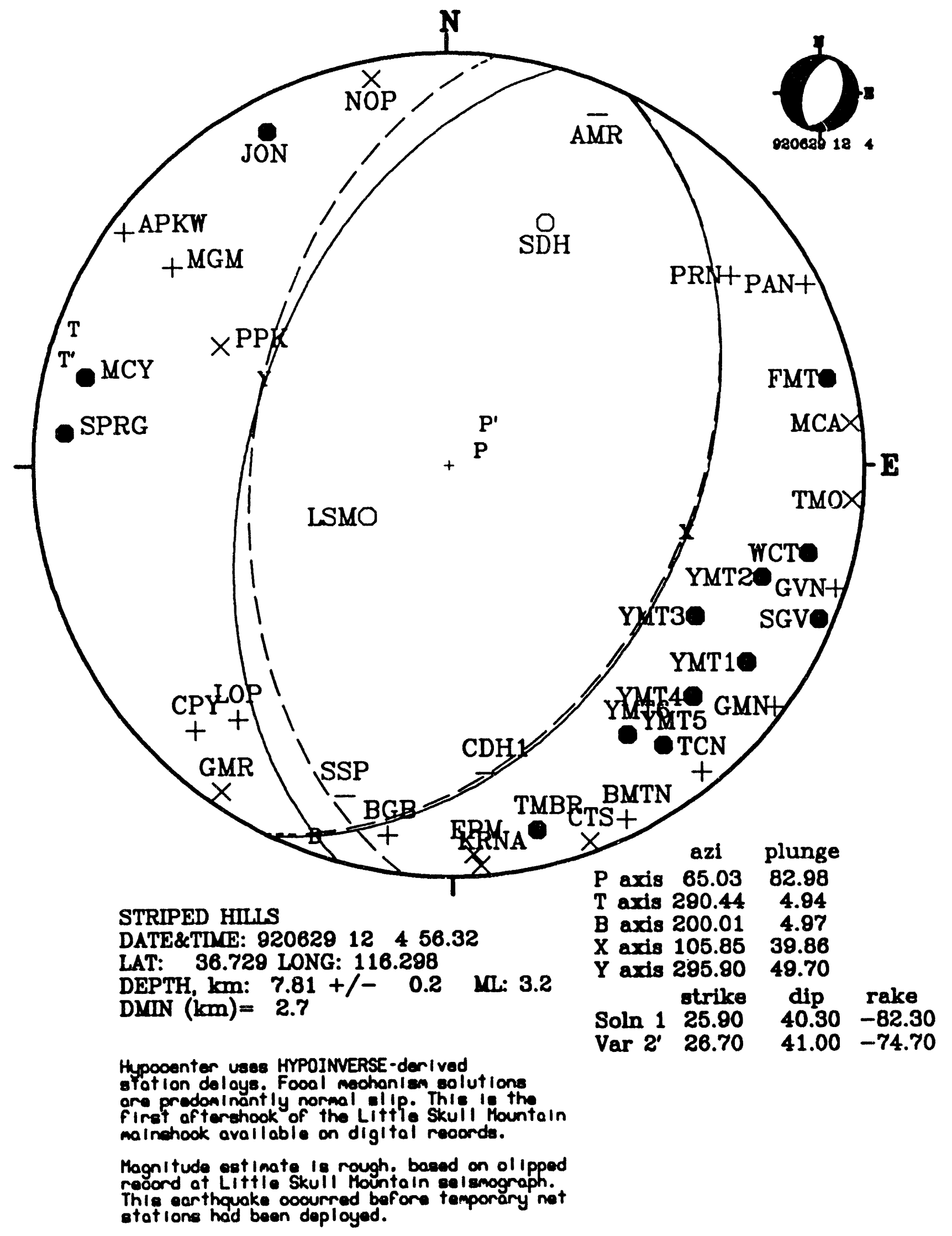




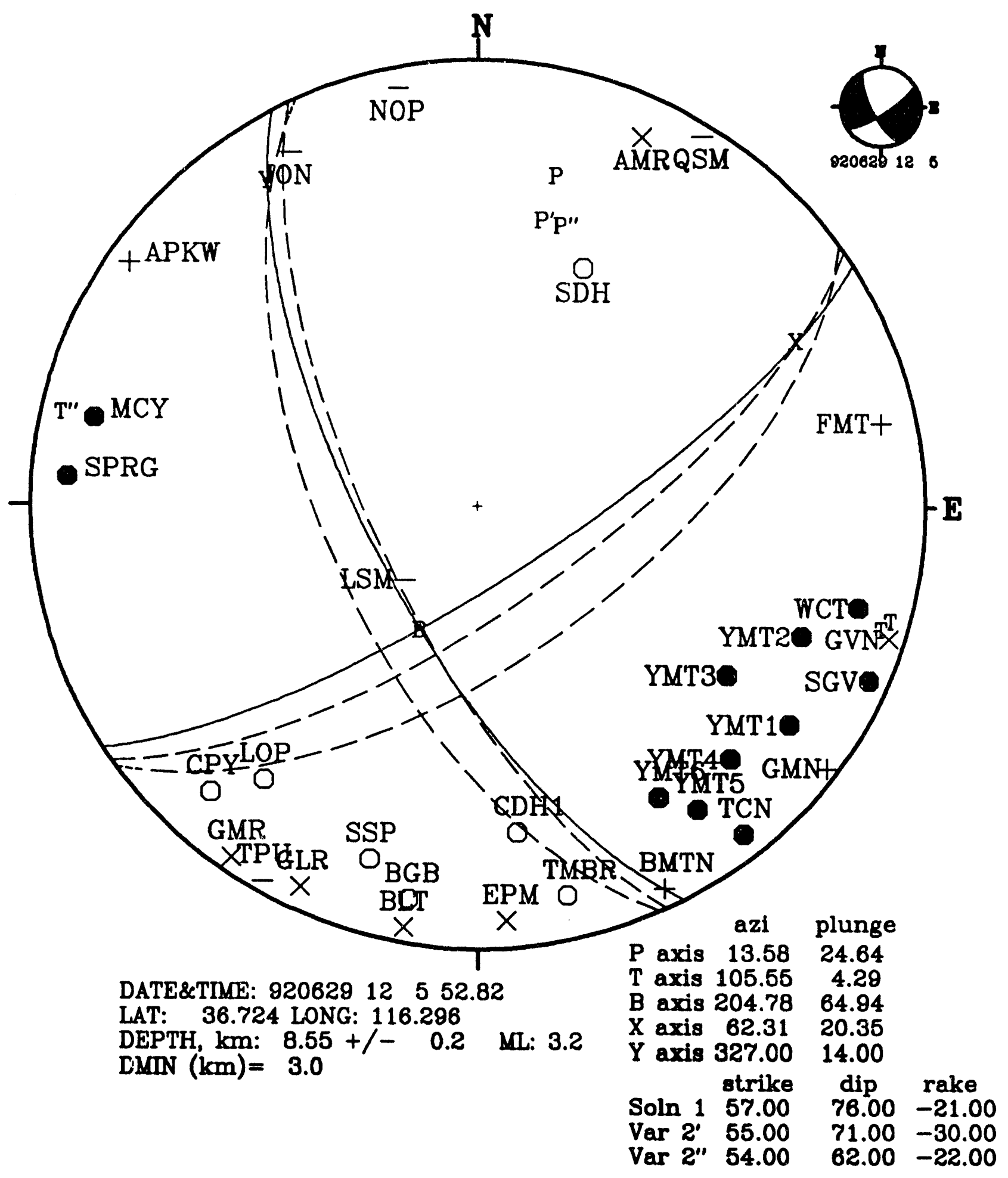




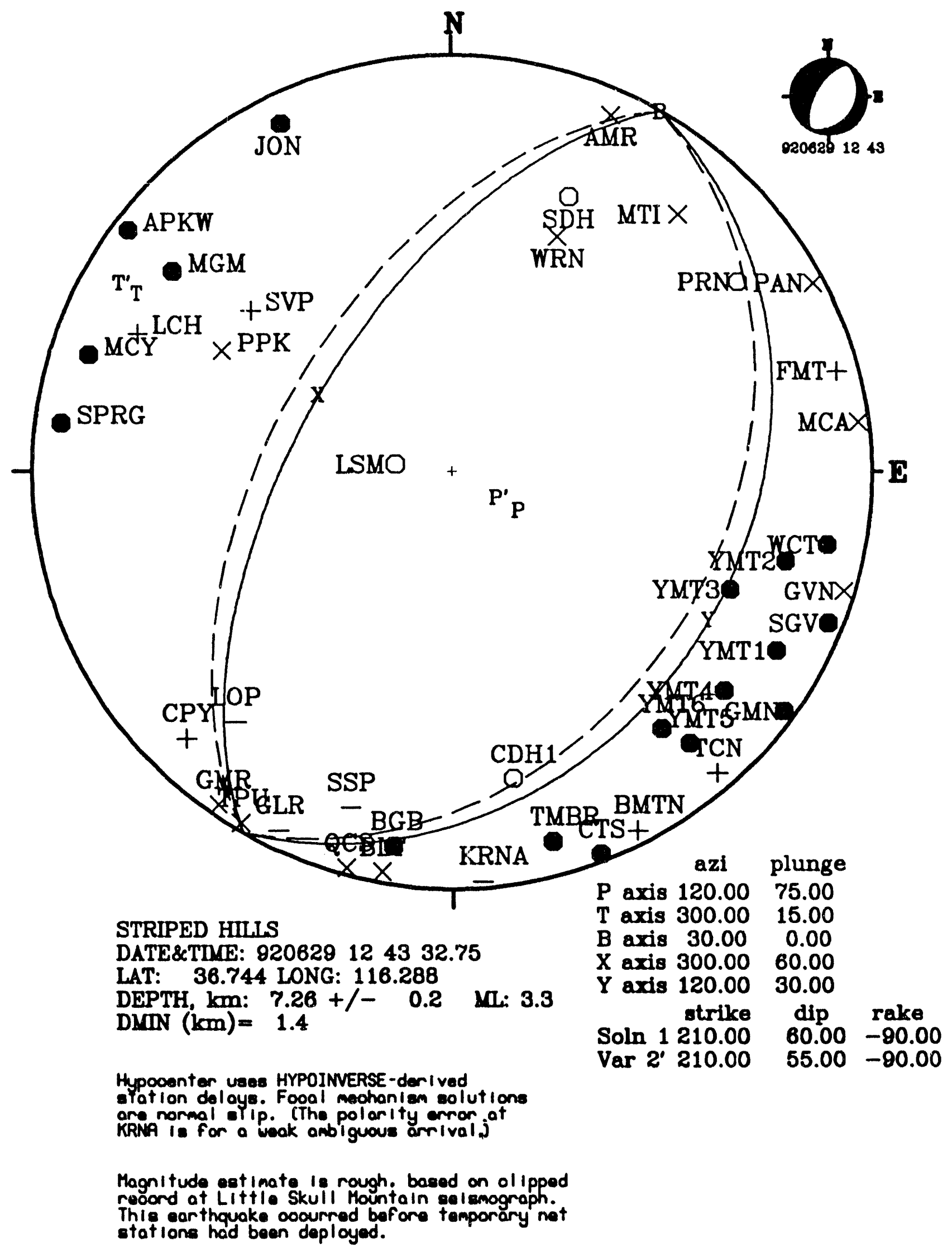




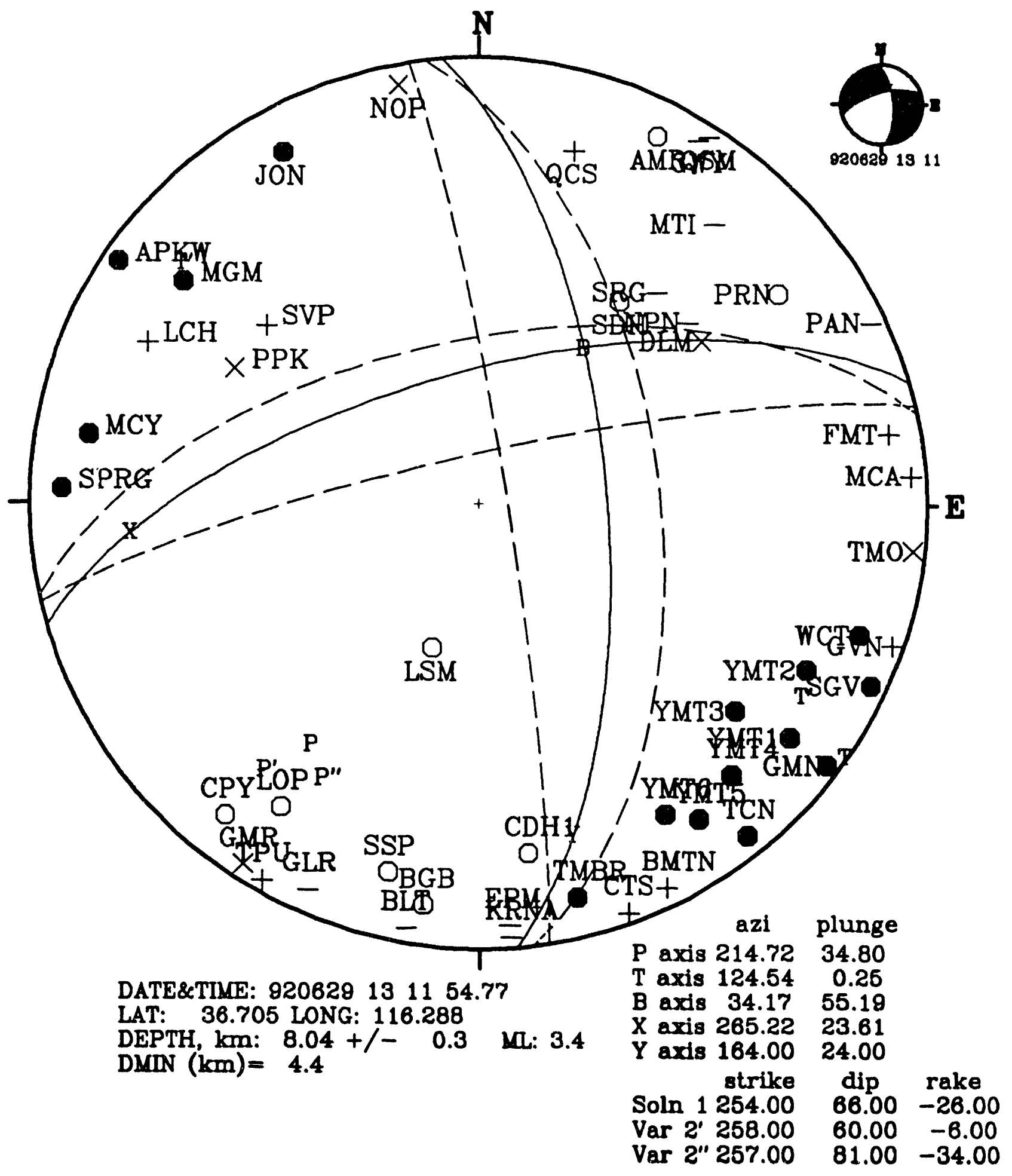




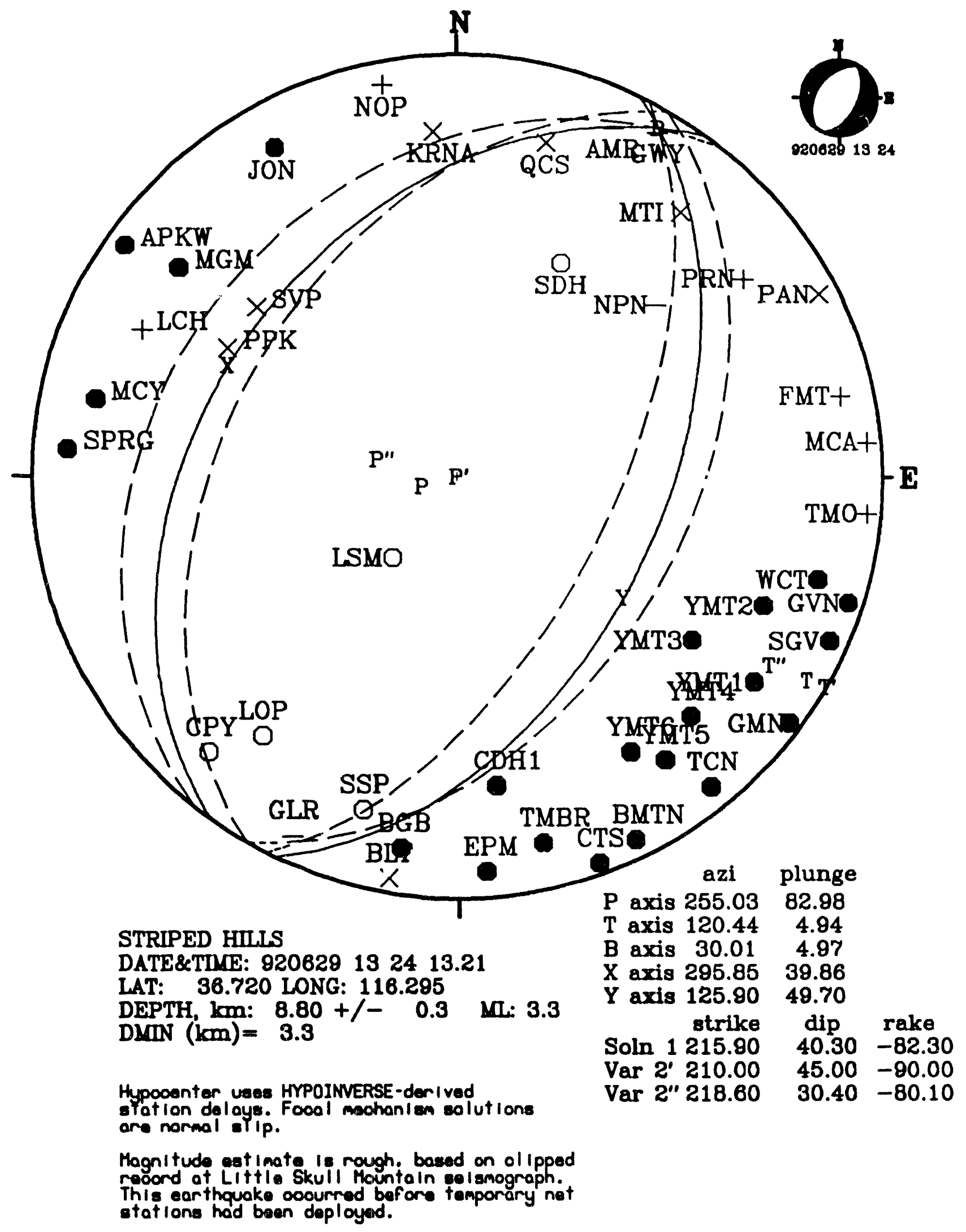




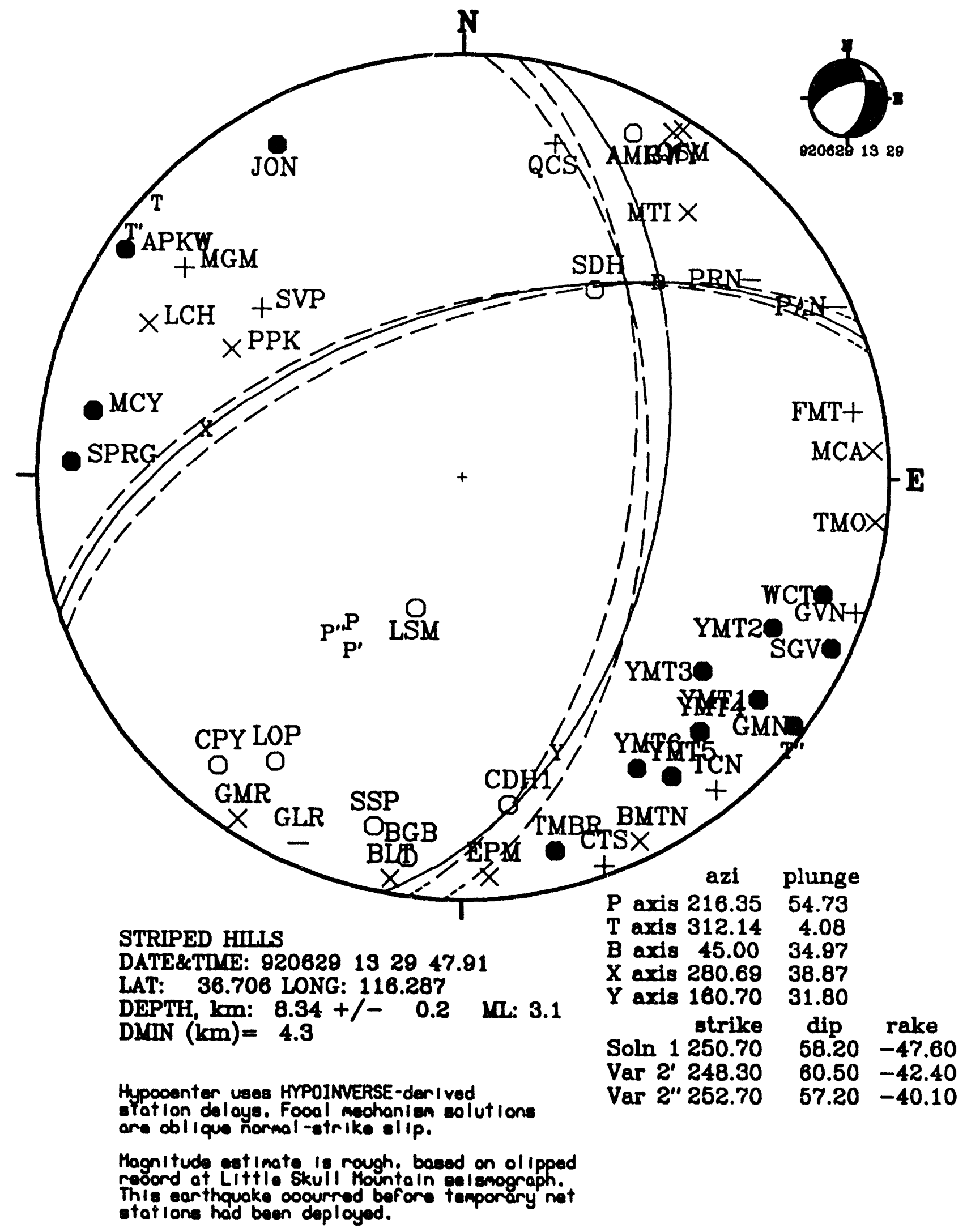




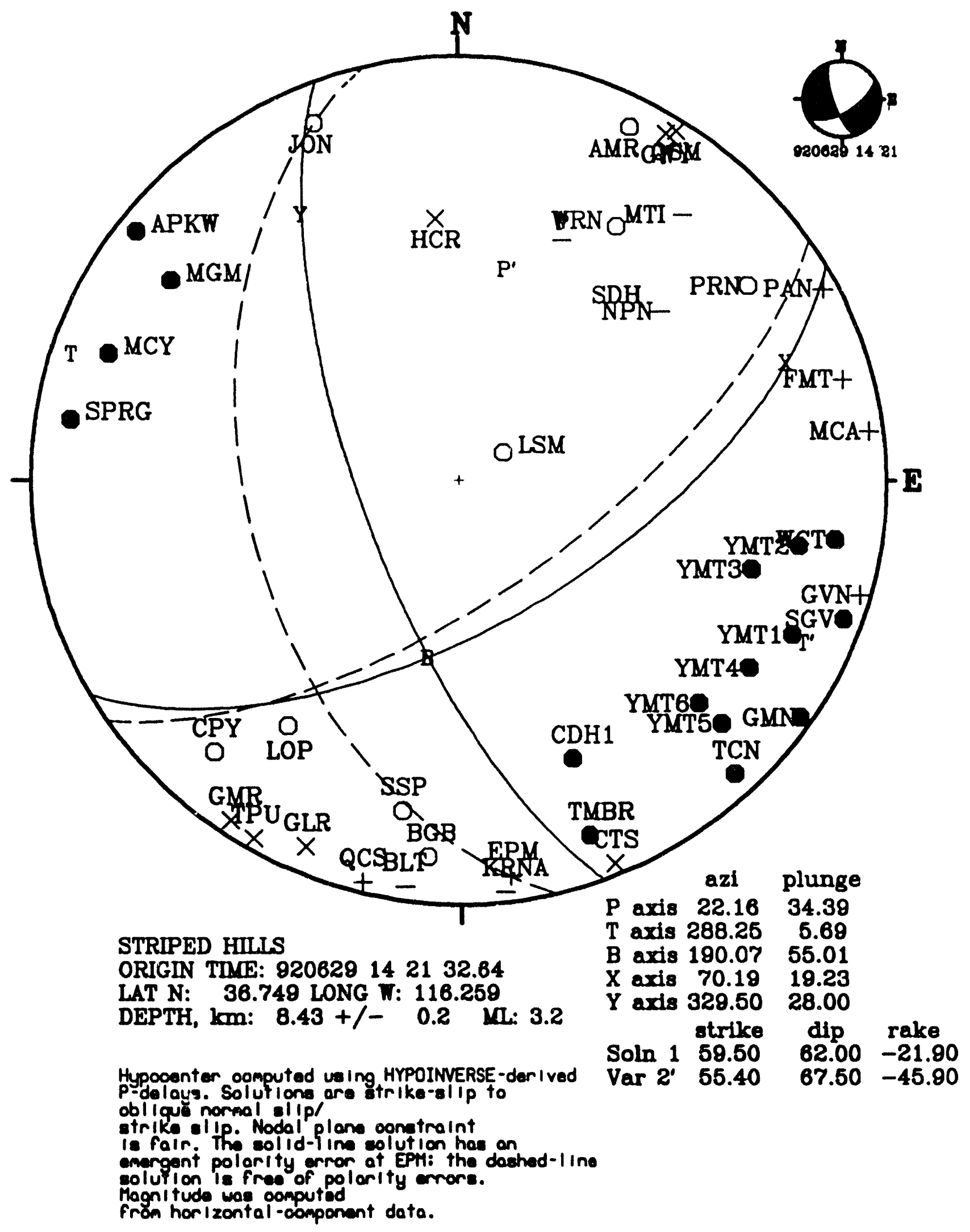




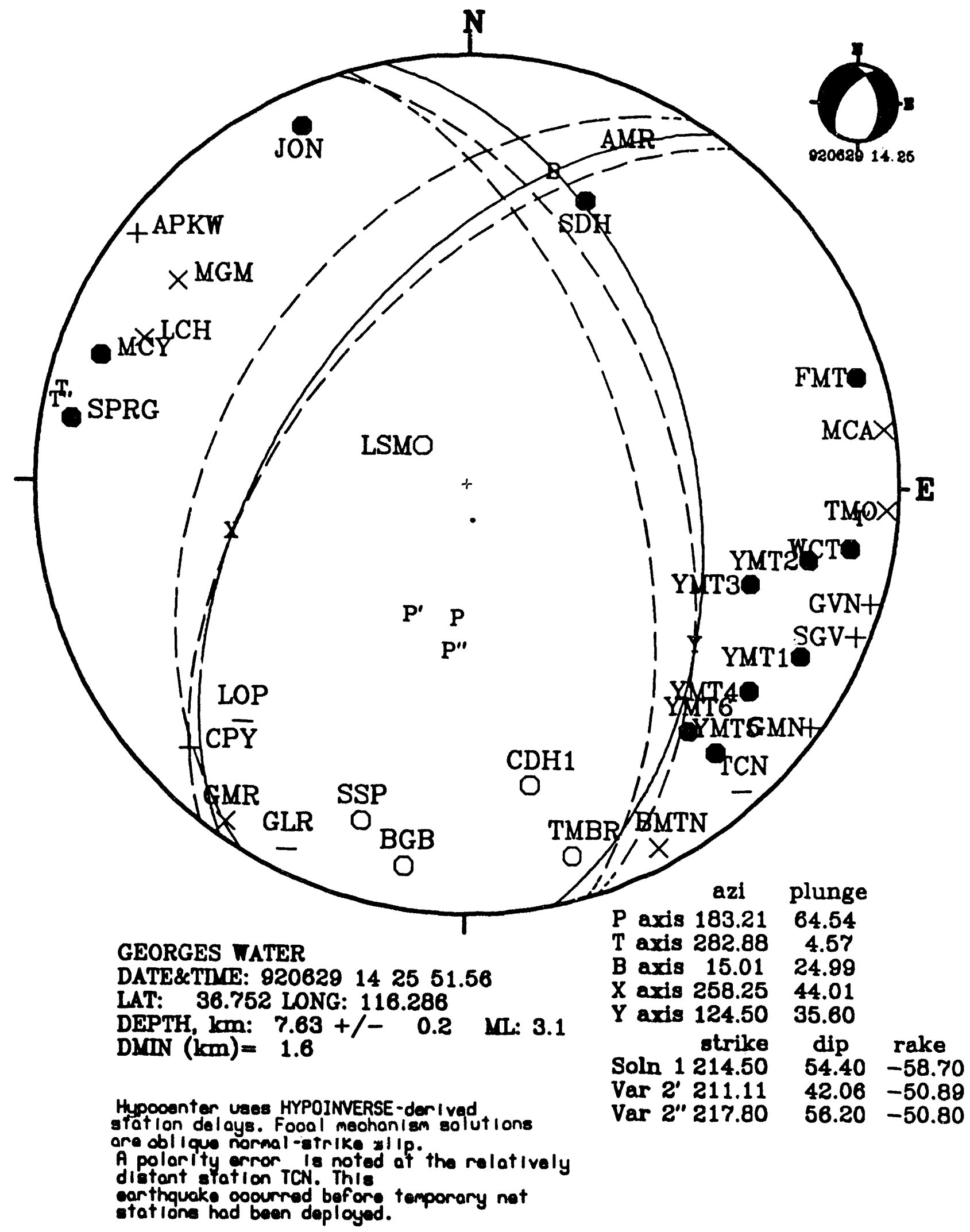




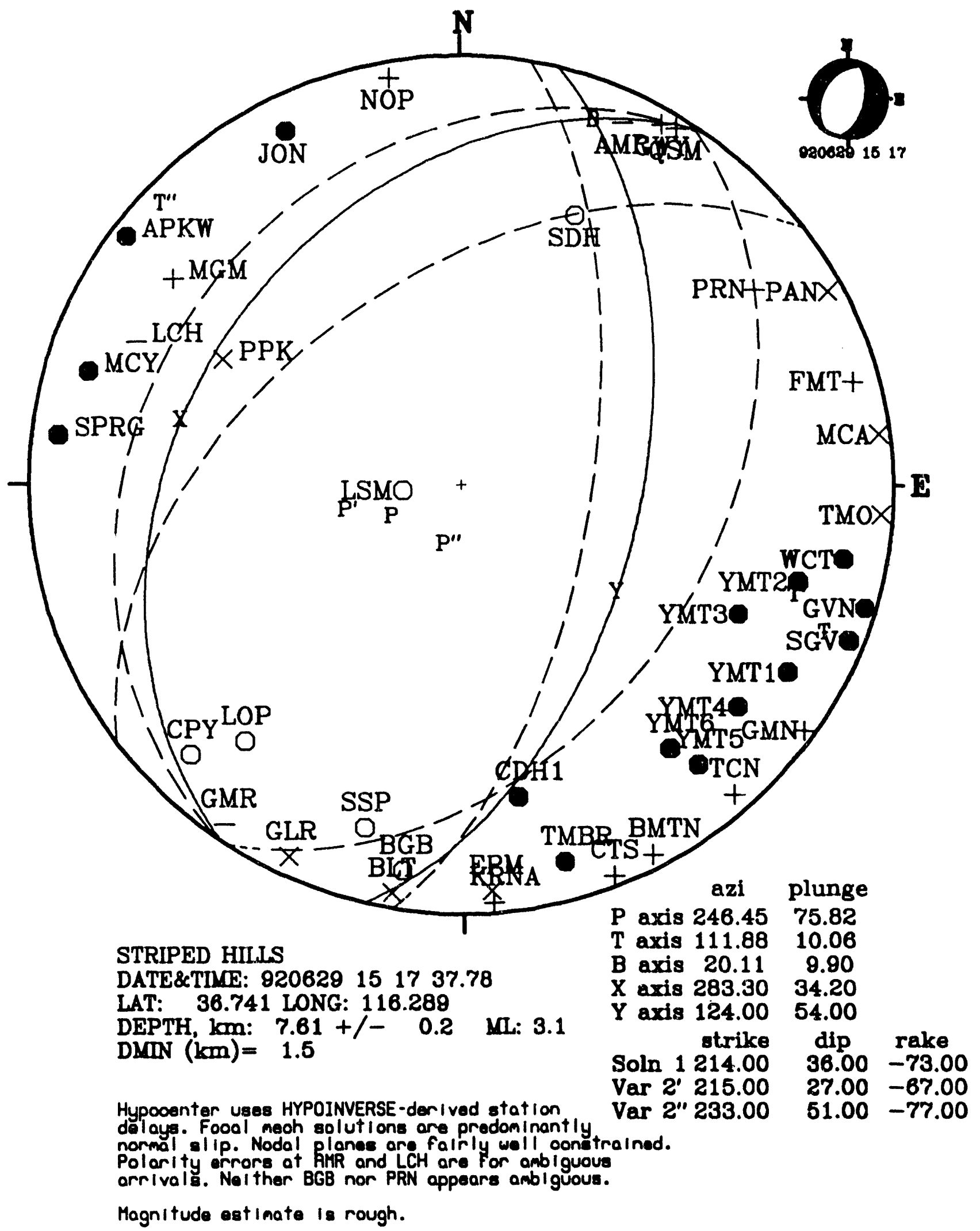




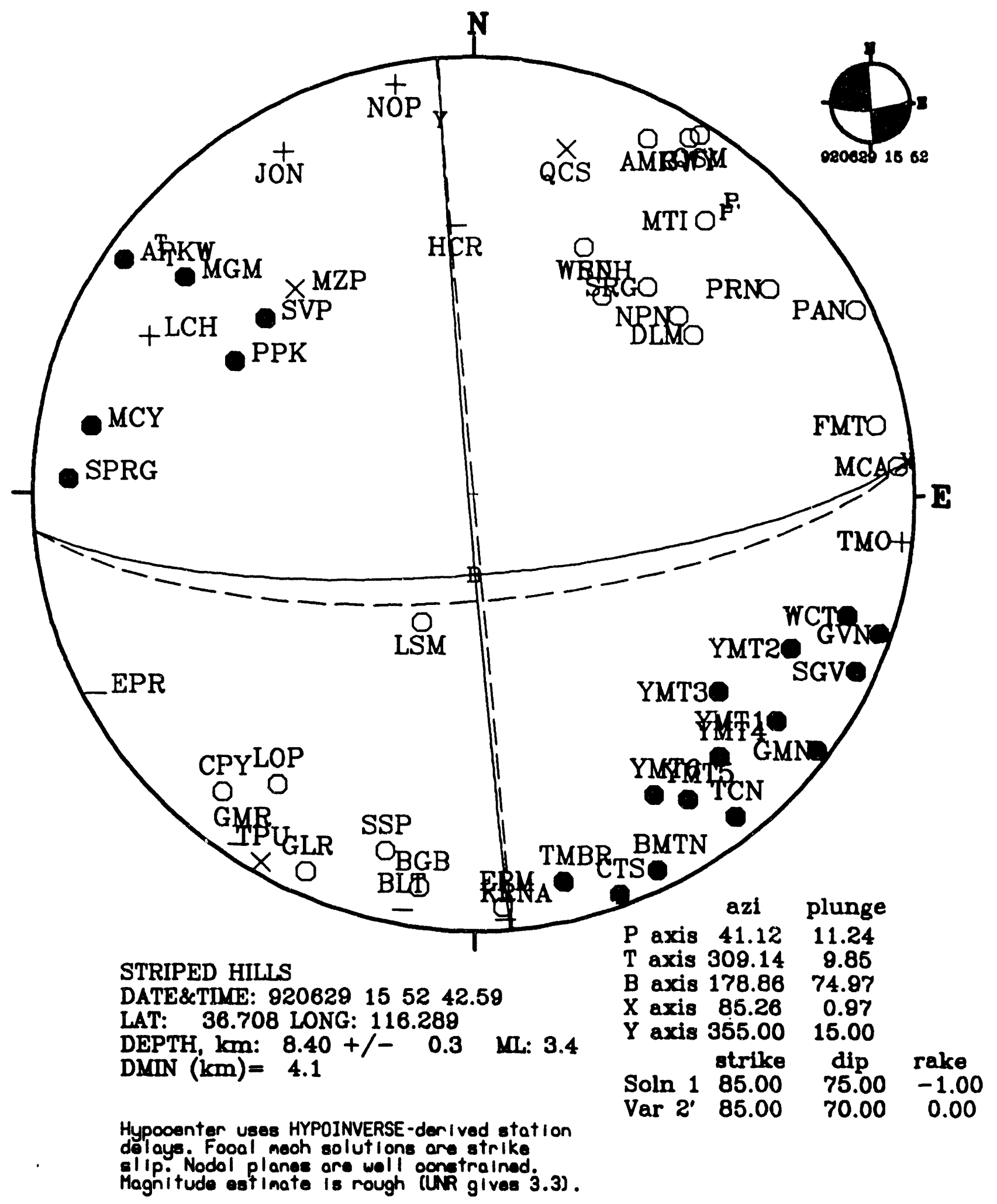




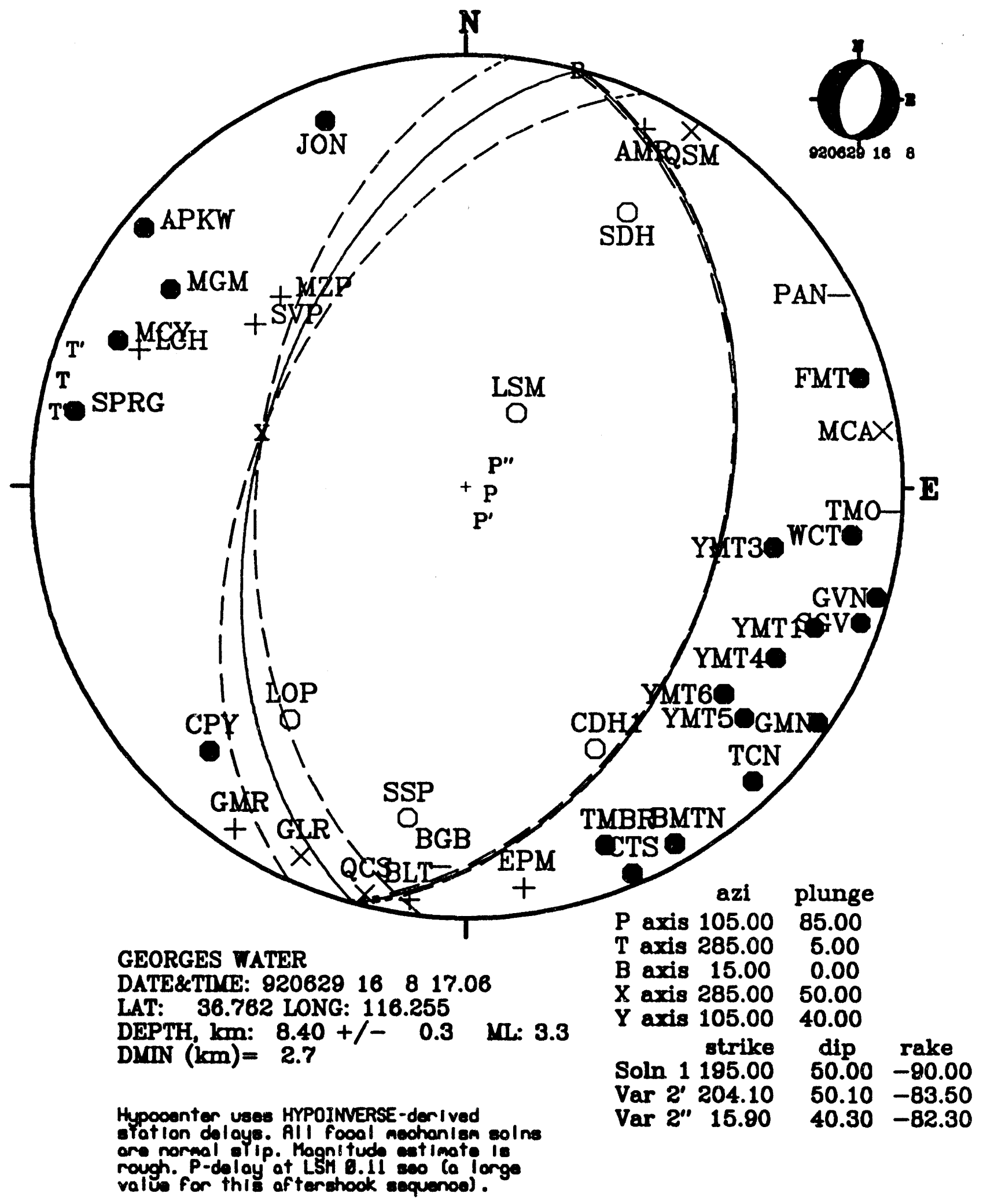




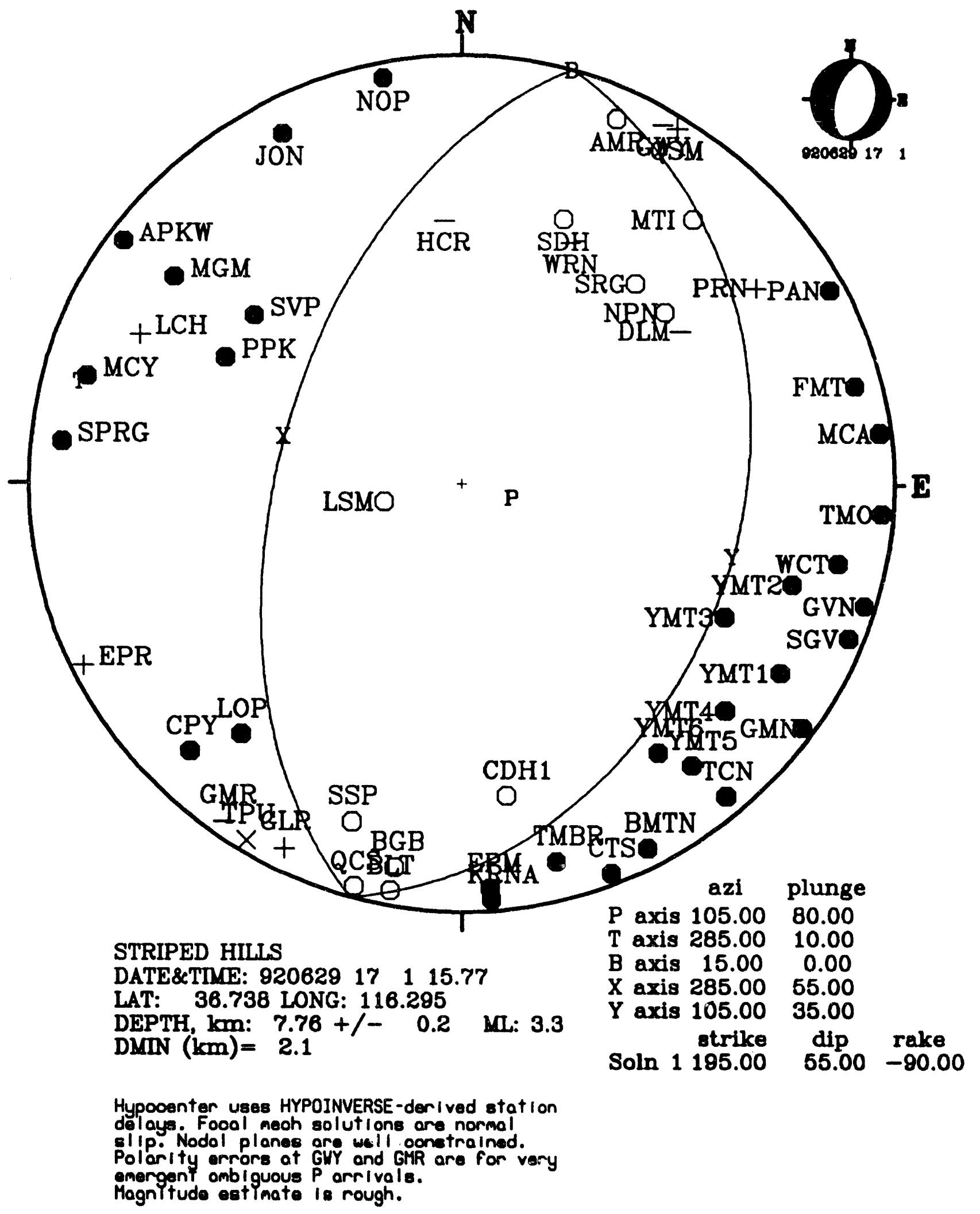




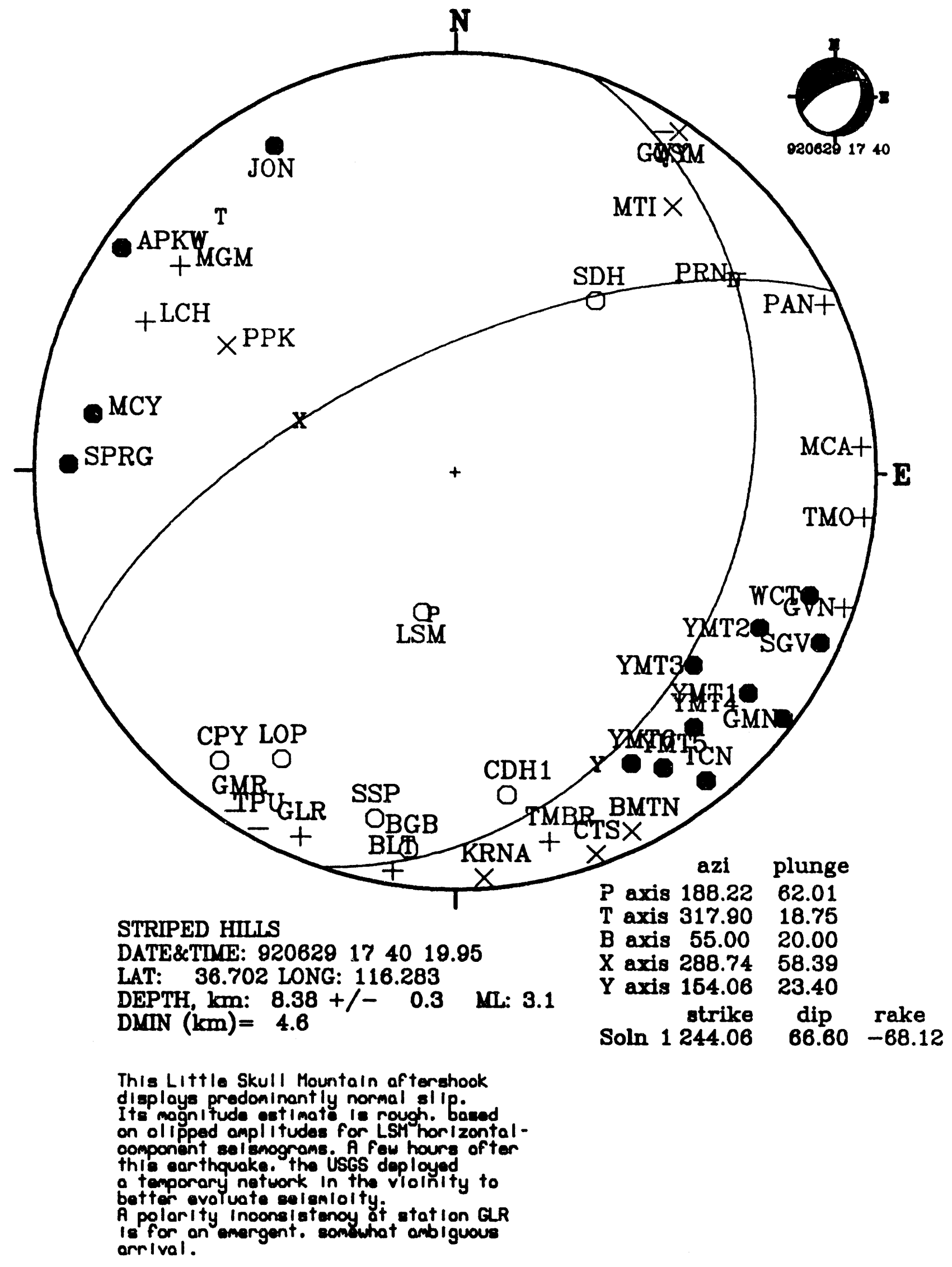




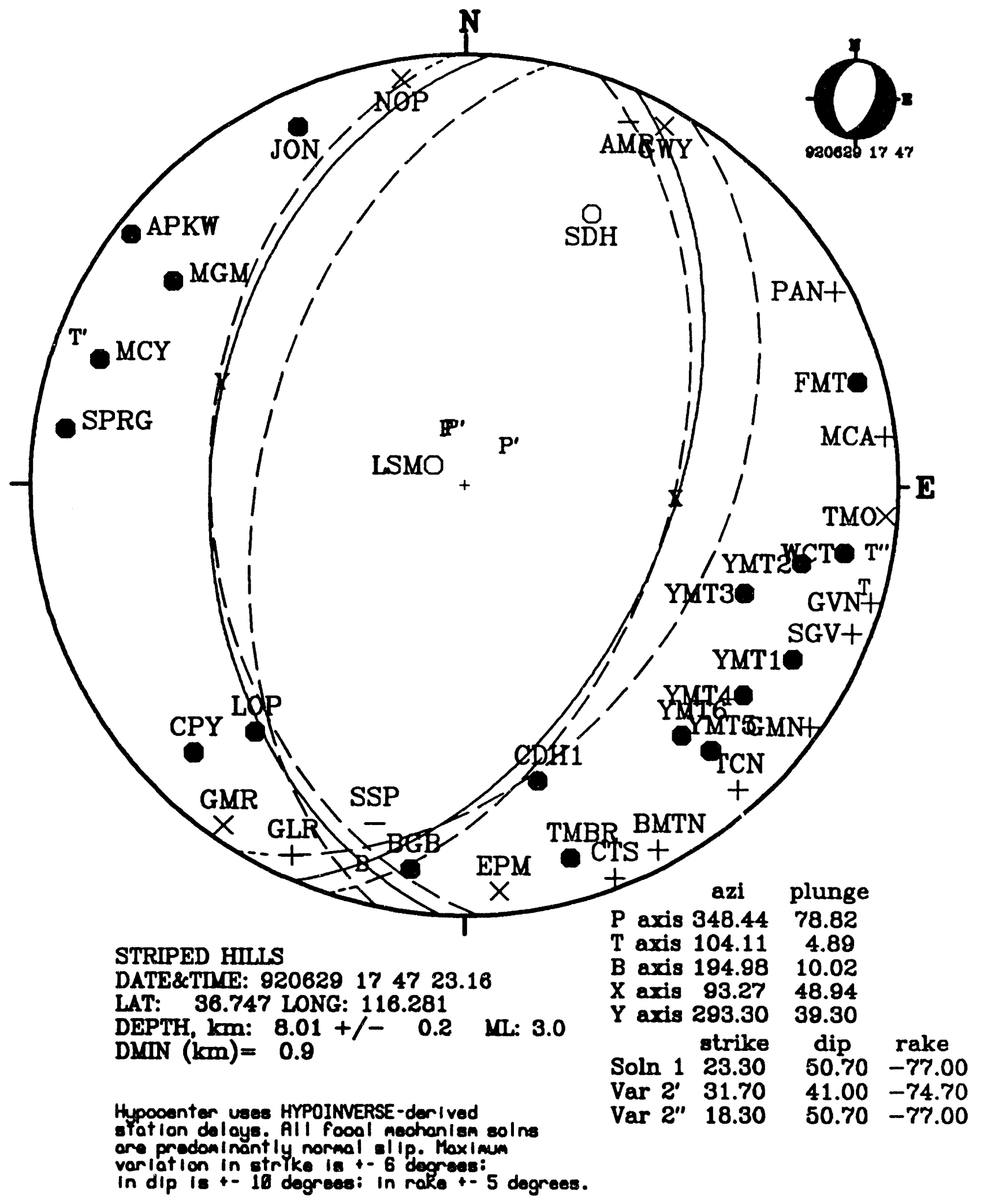




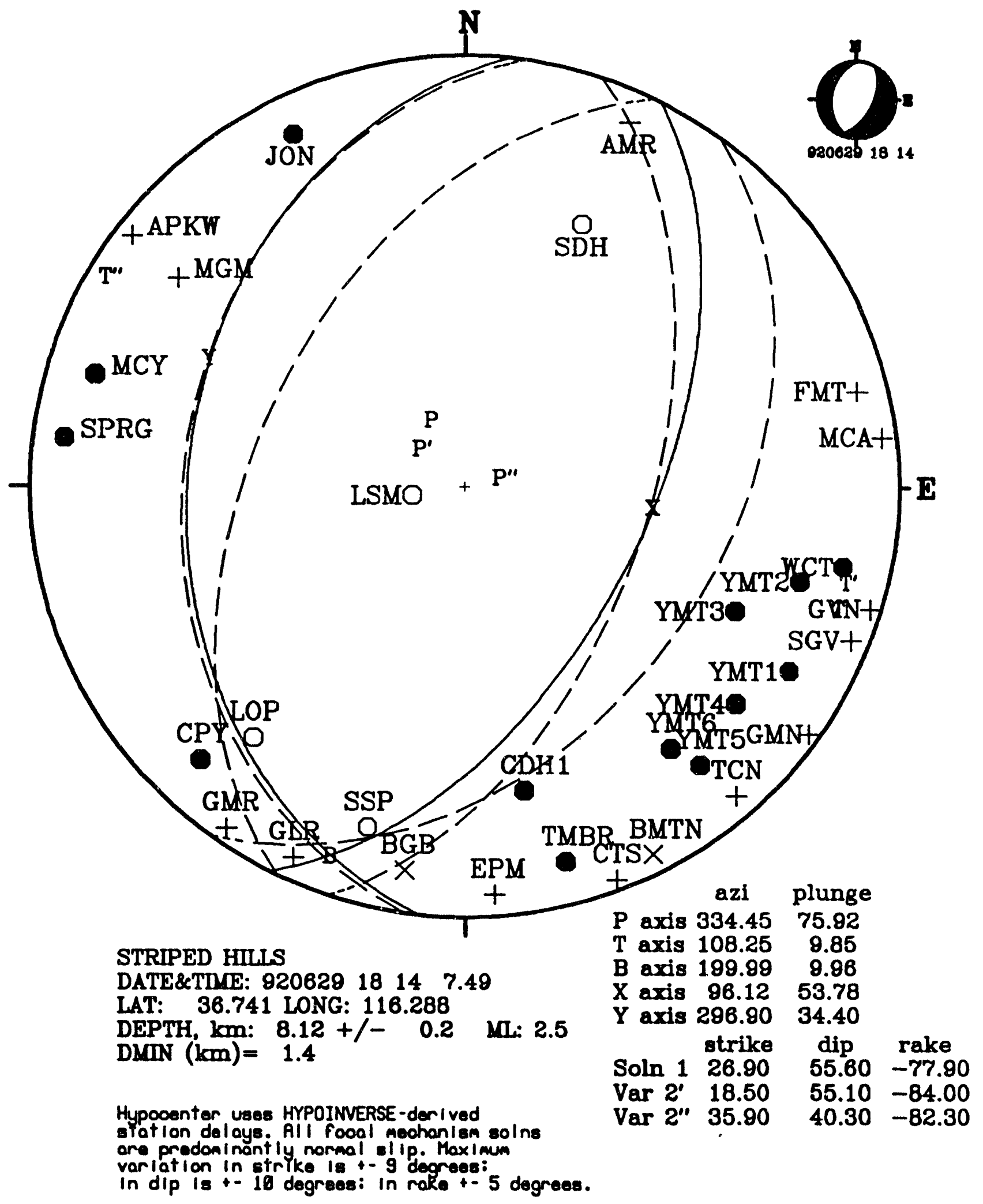




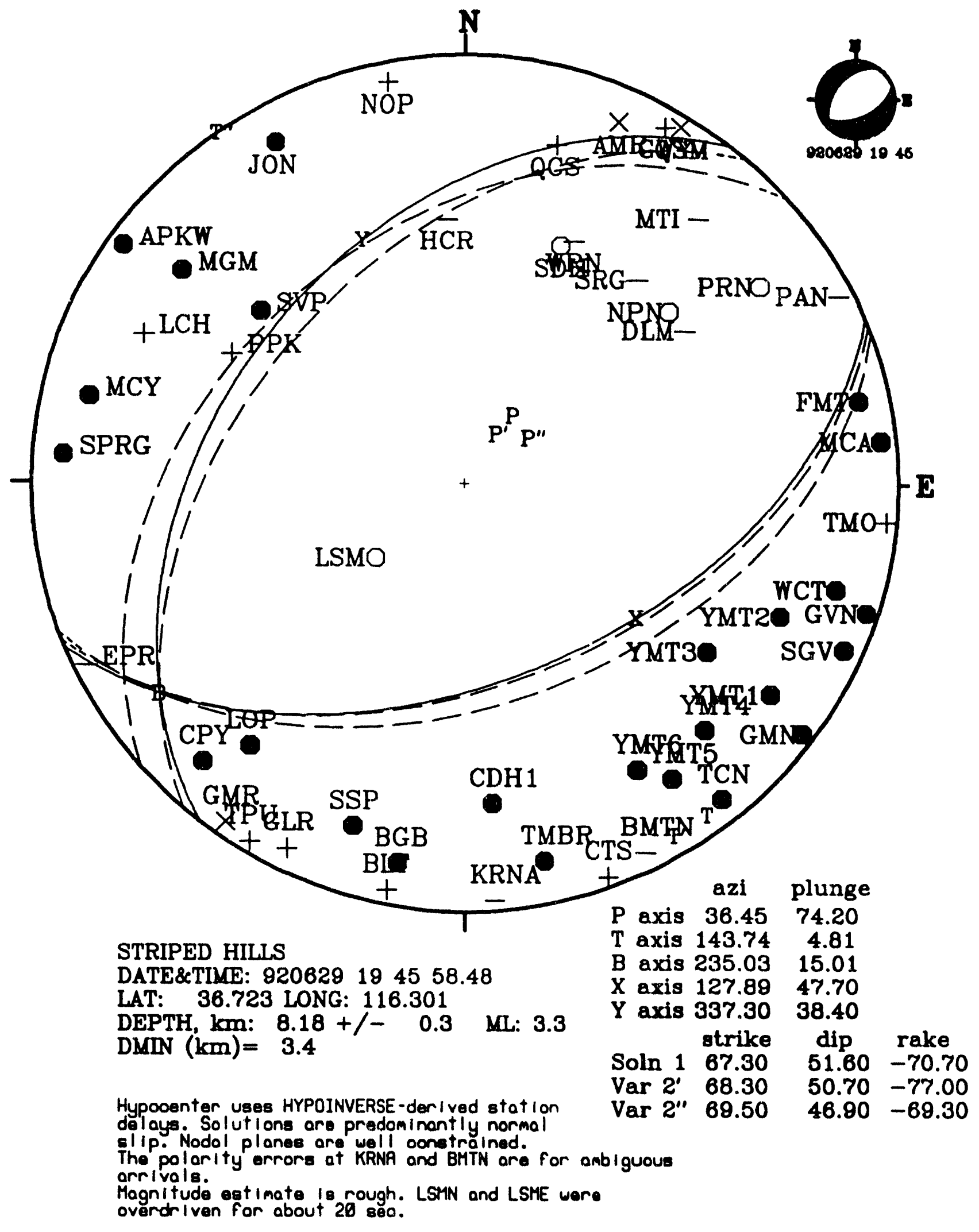




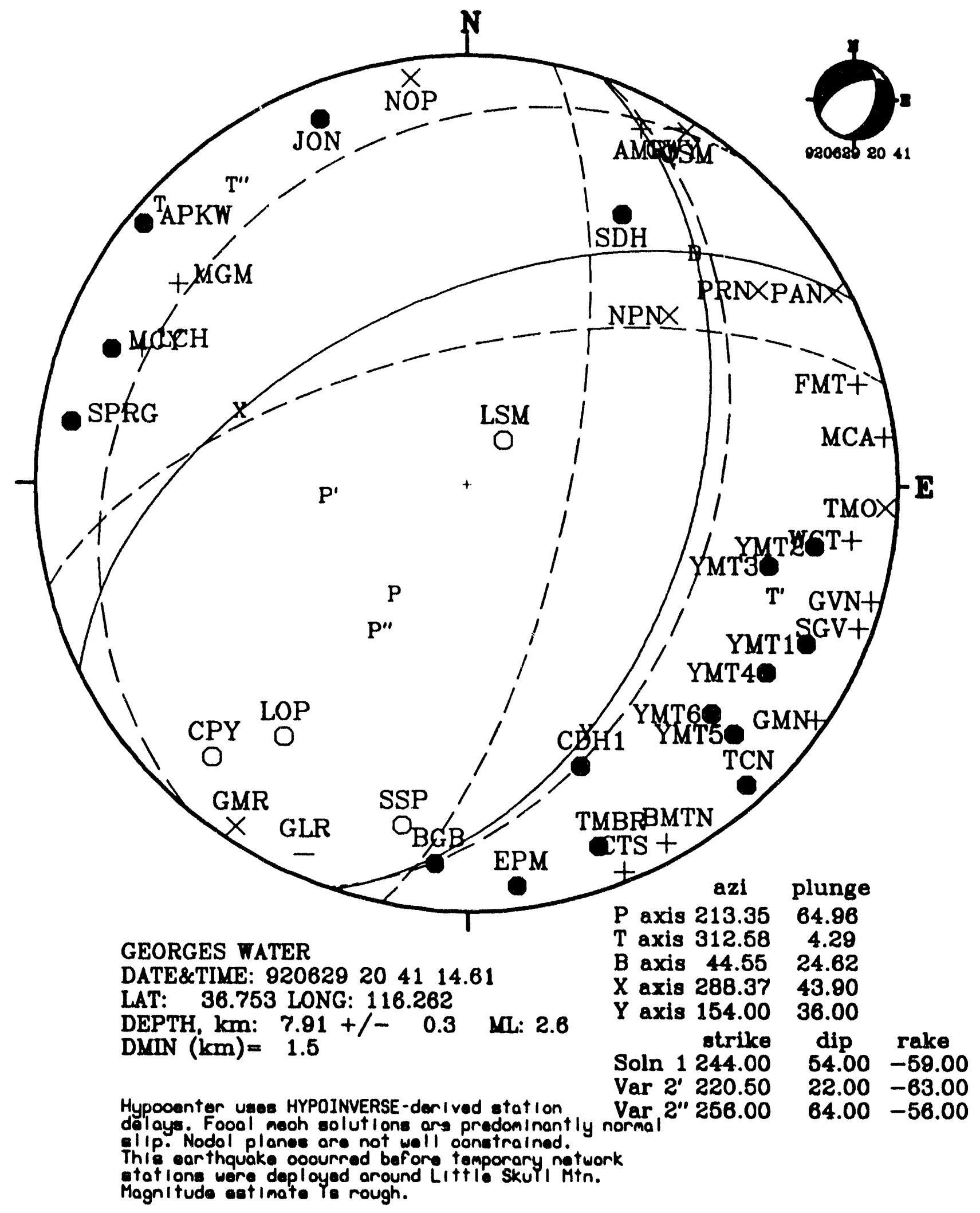




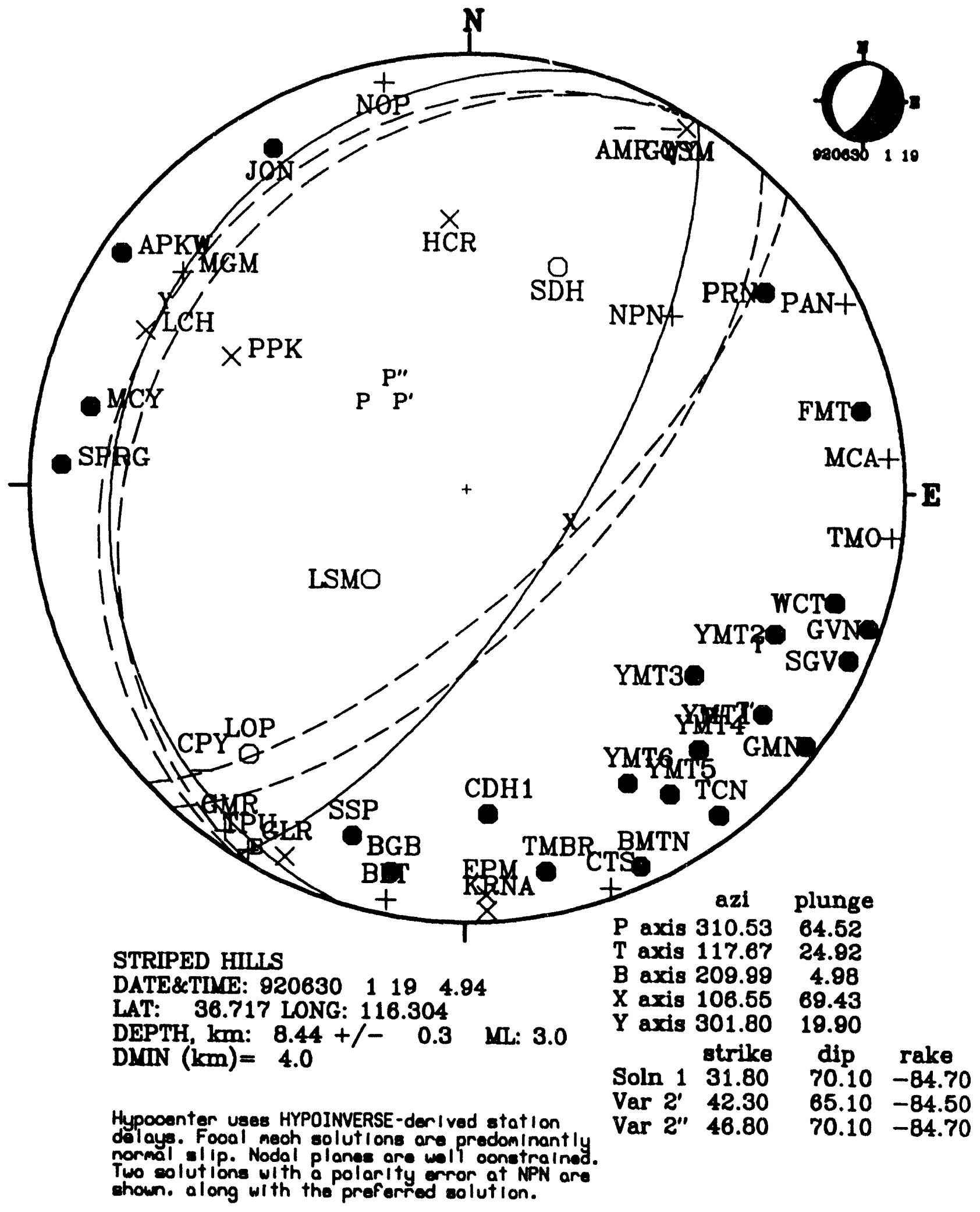

UNRSL magnitude estimate is ML 3.09. 


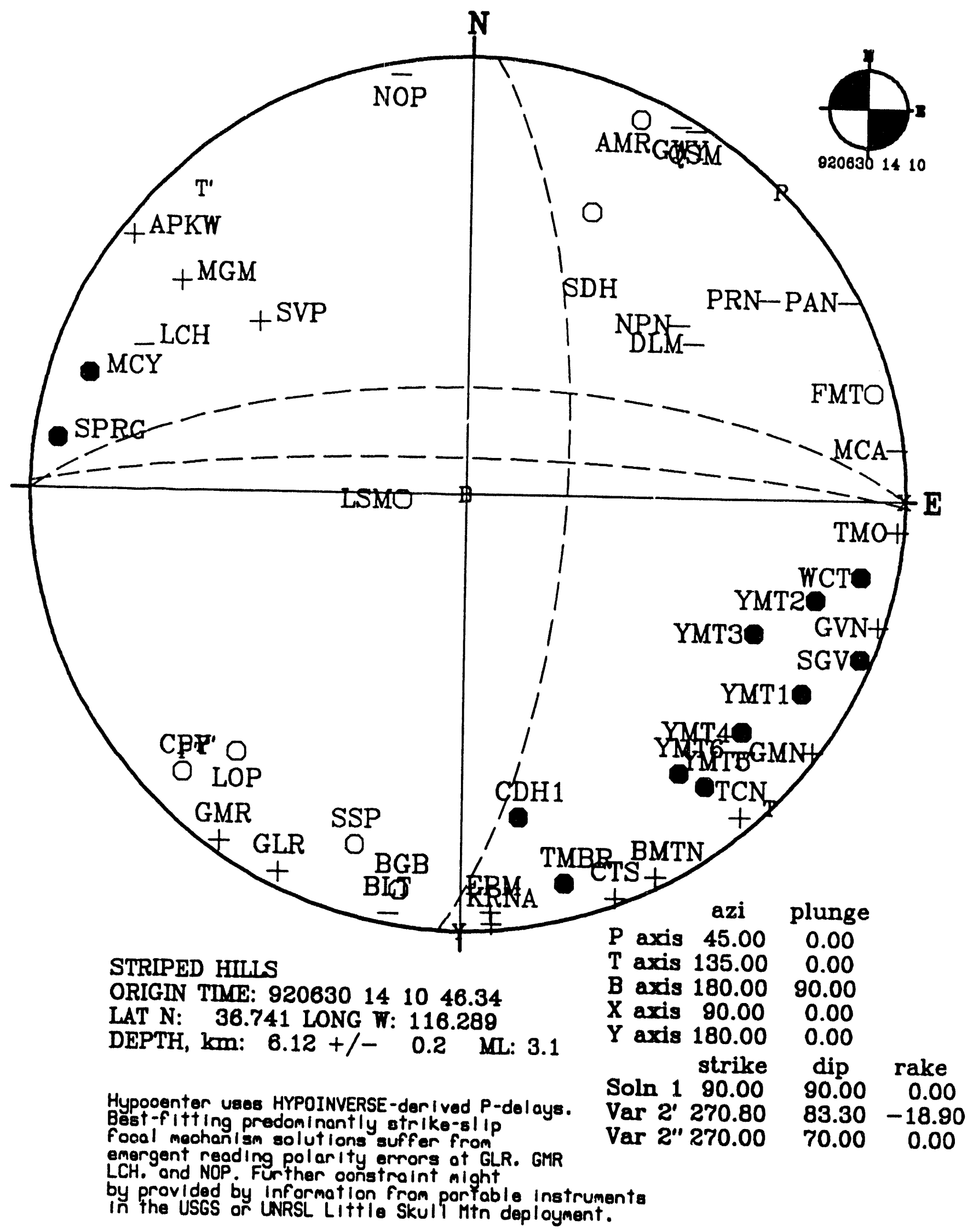




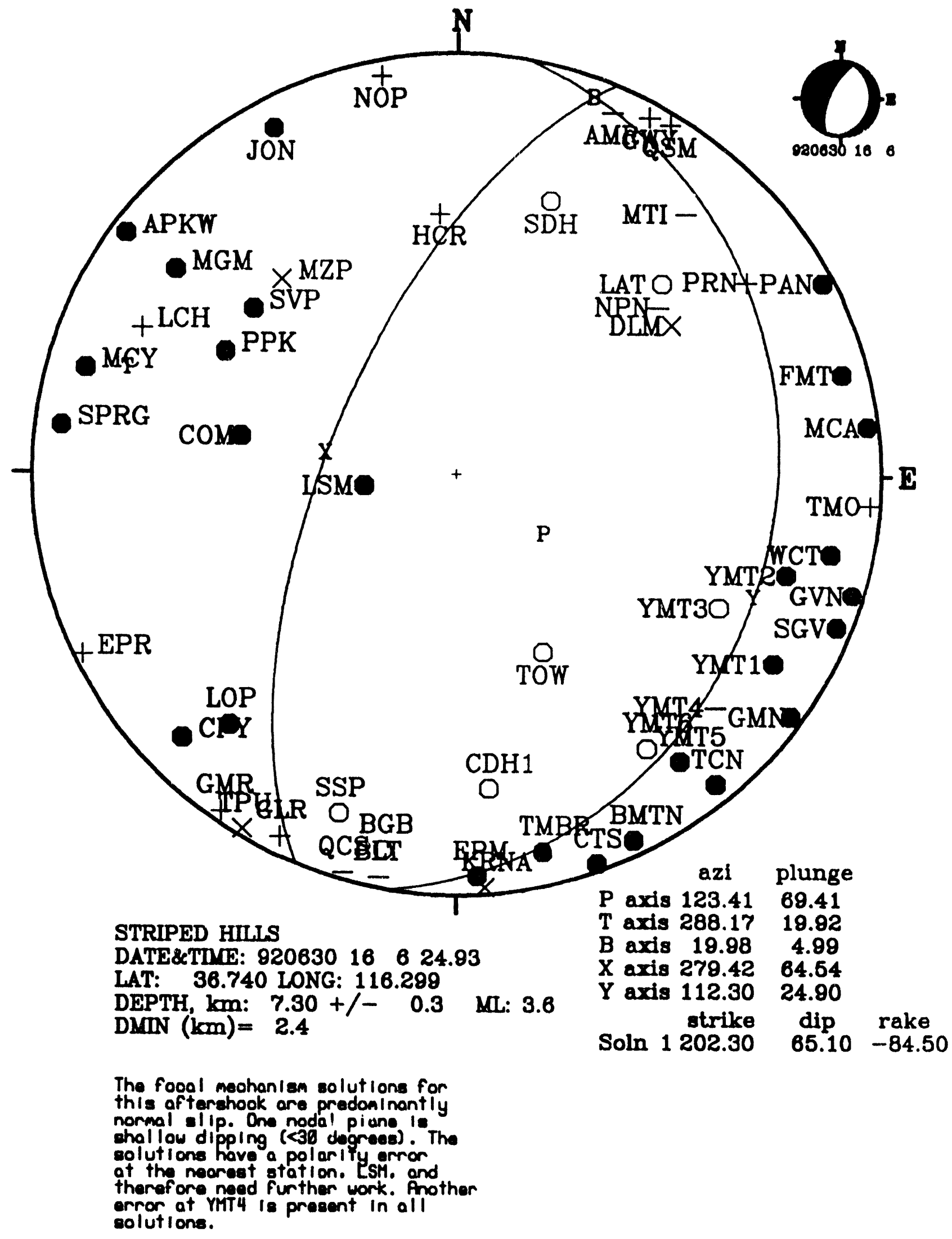




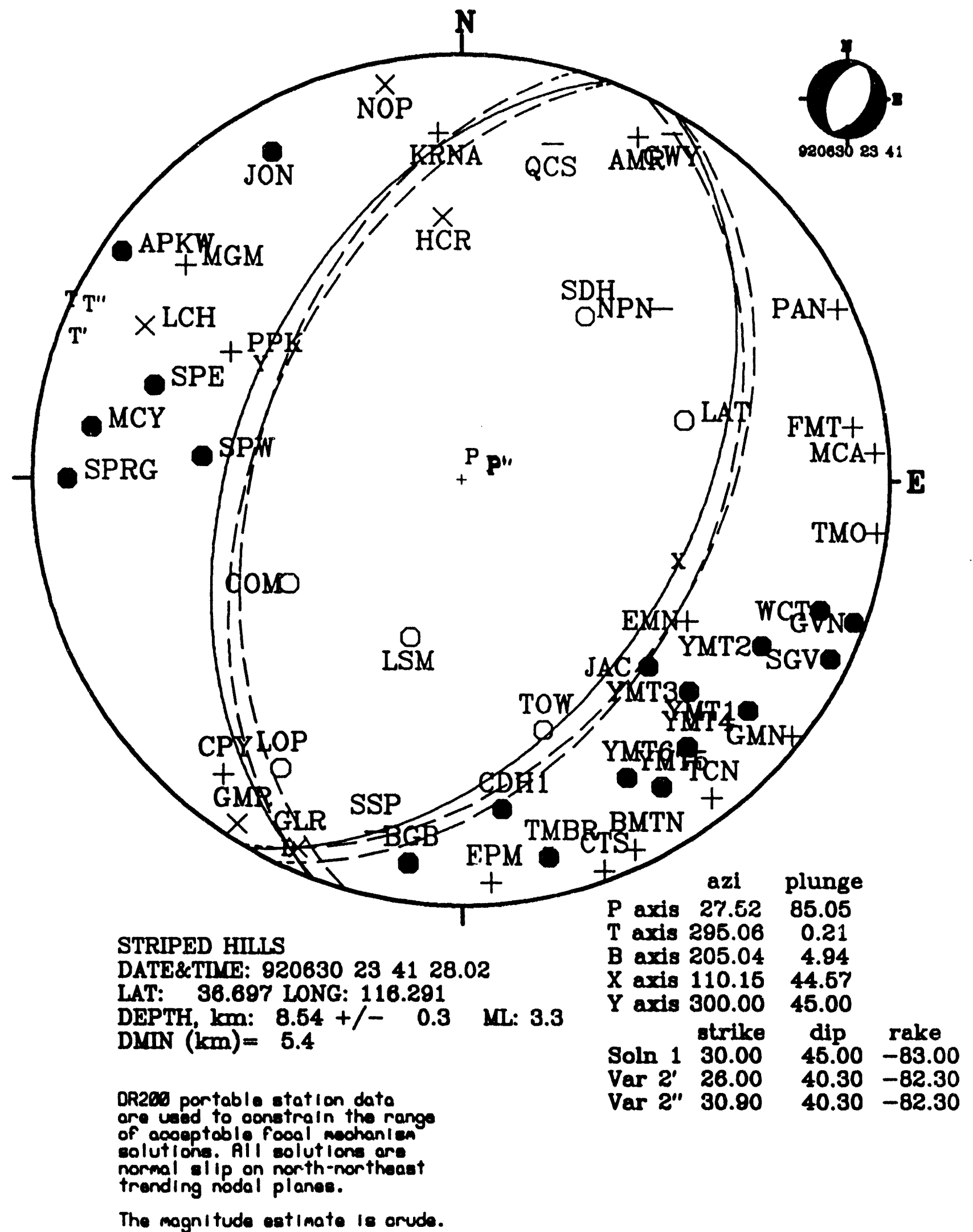




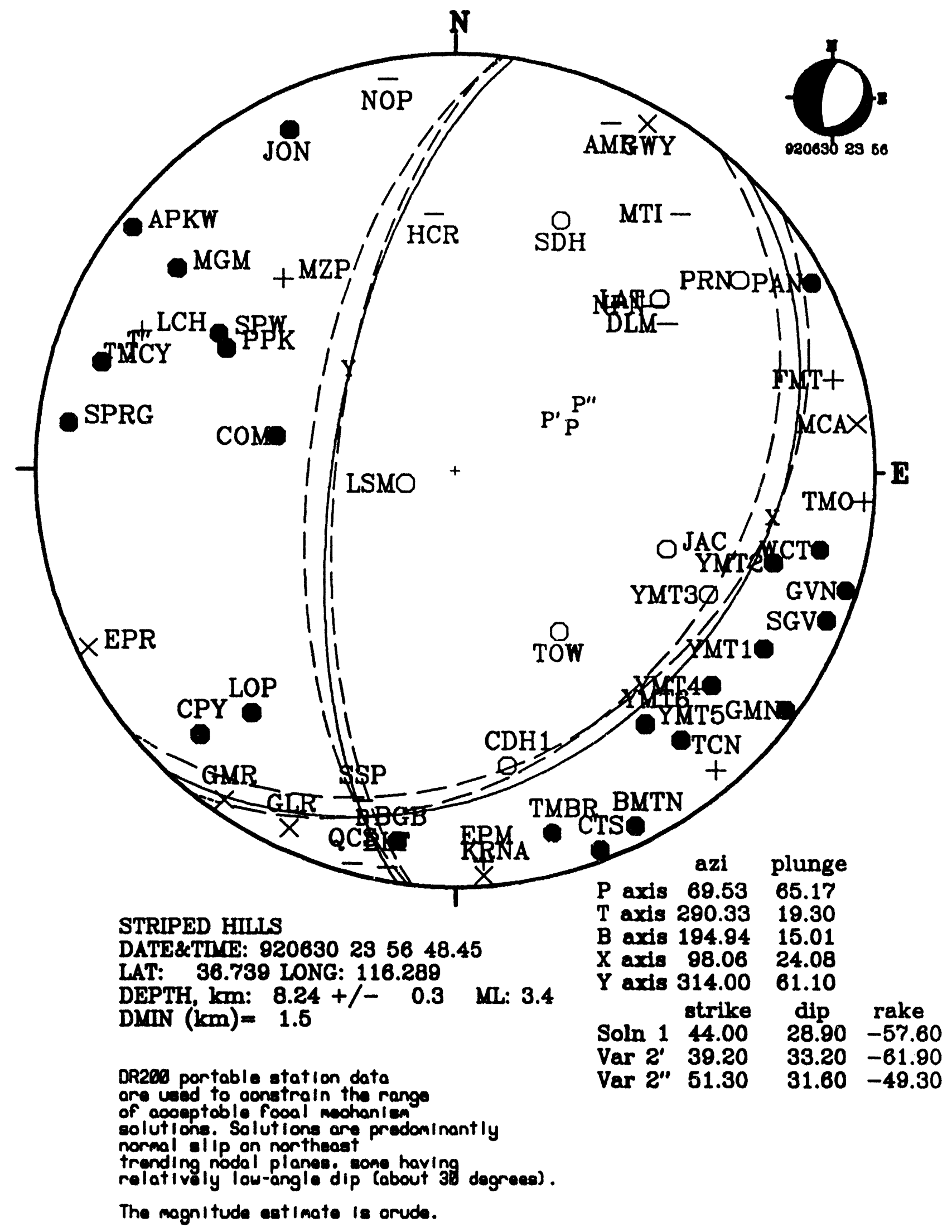




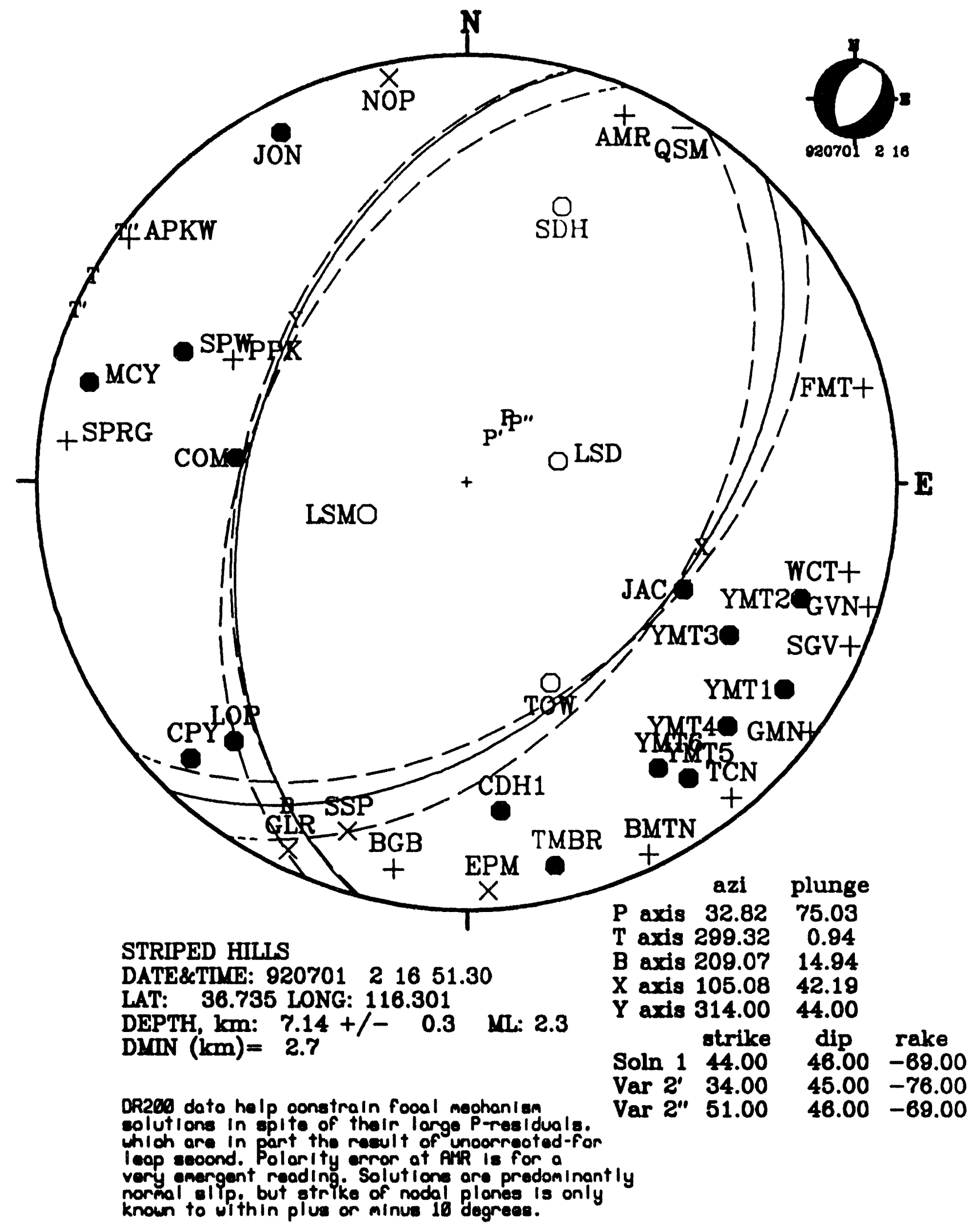




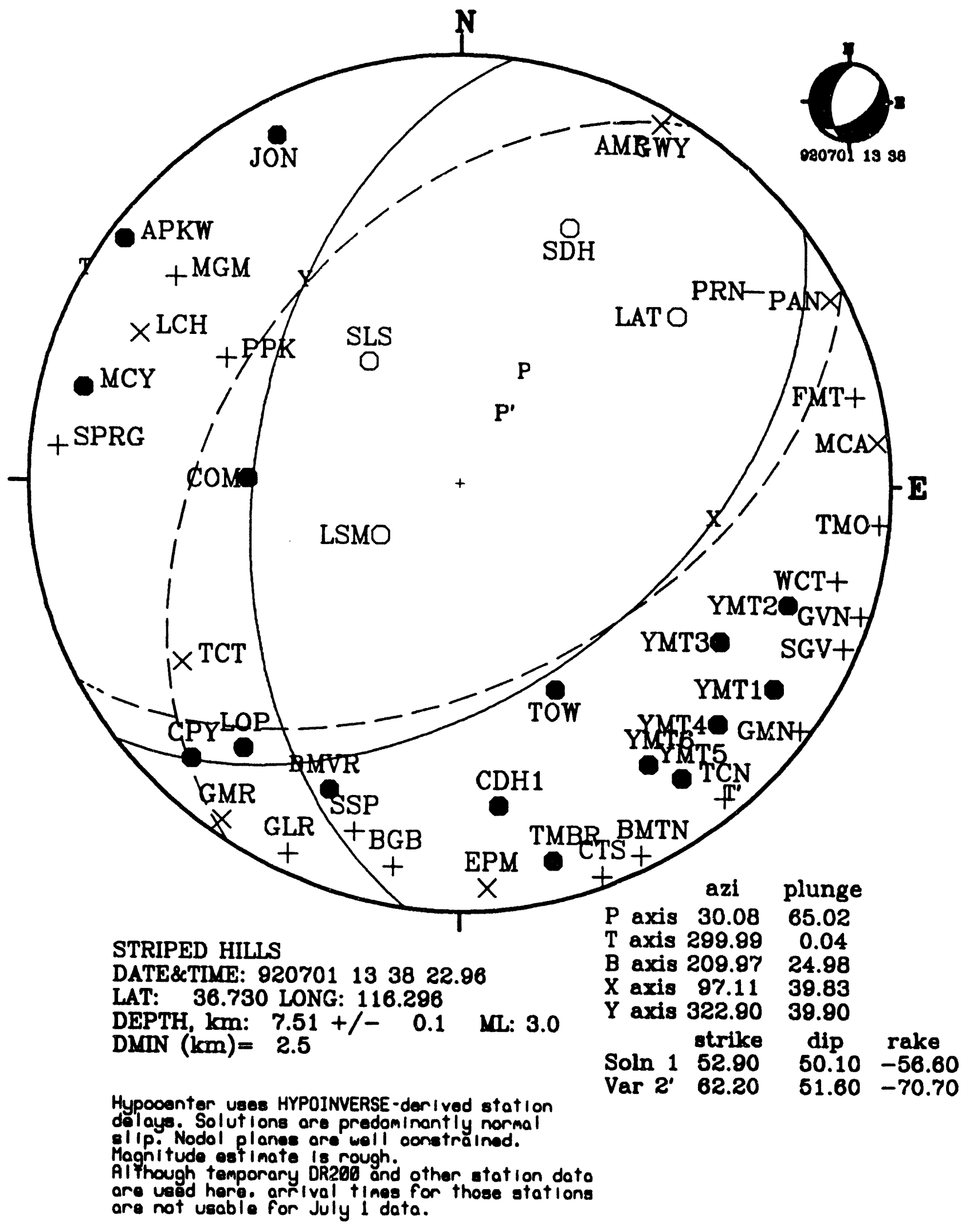




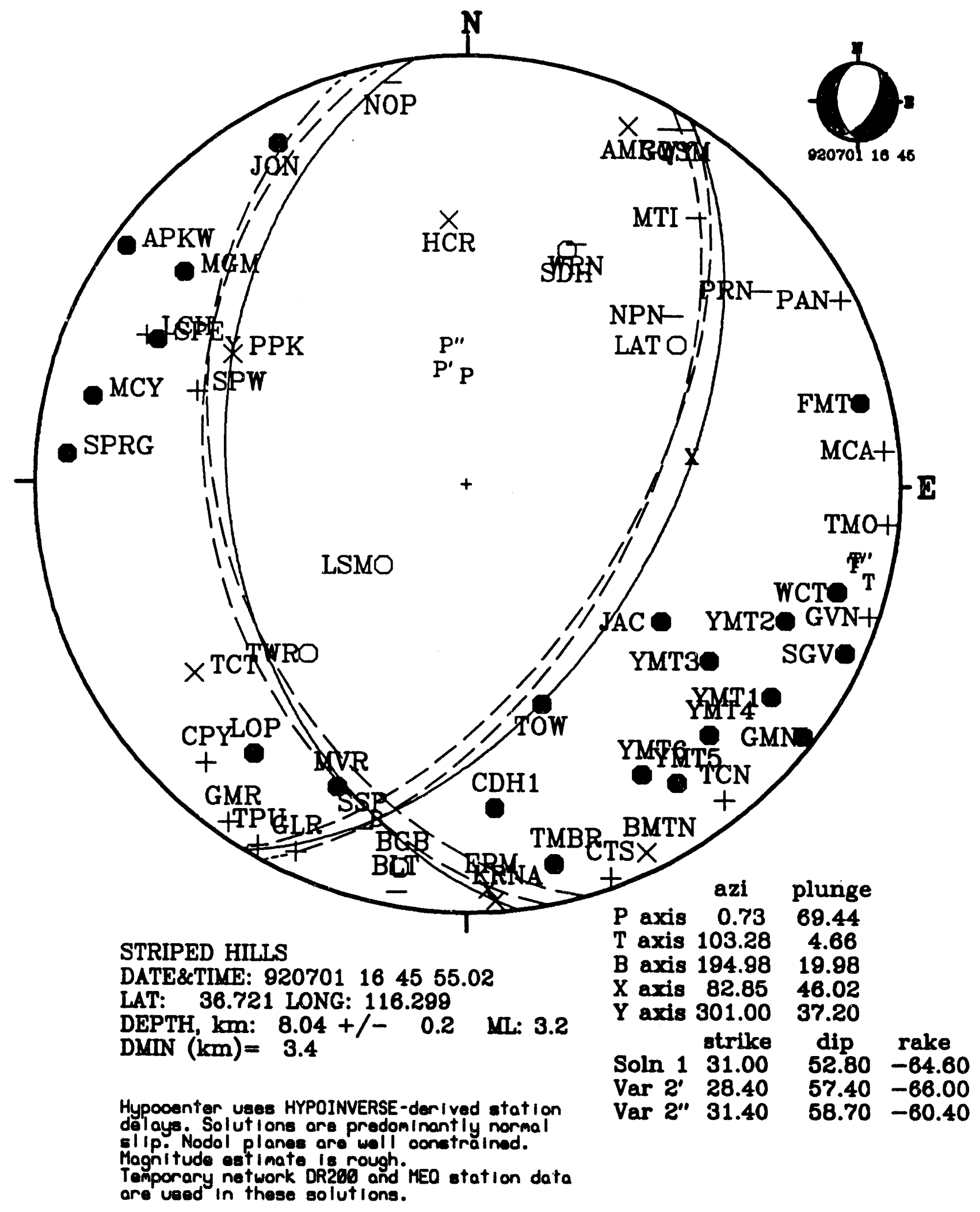




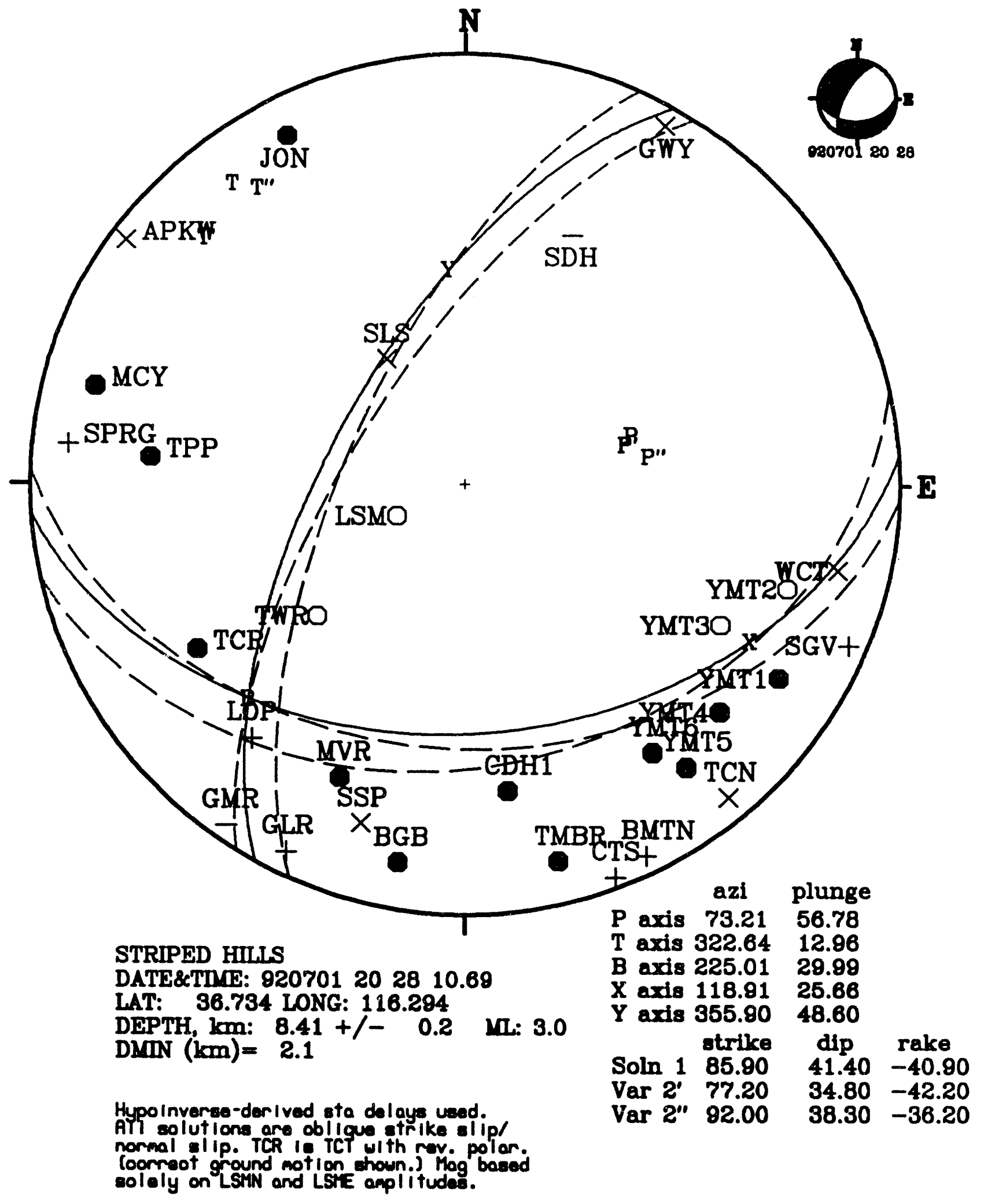




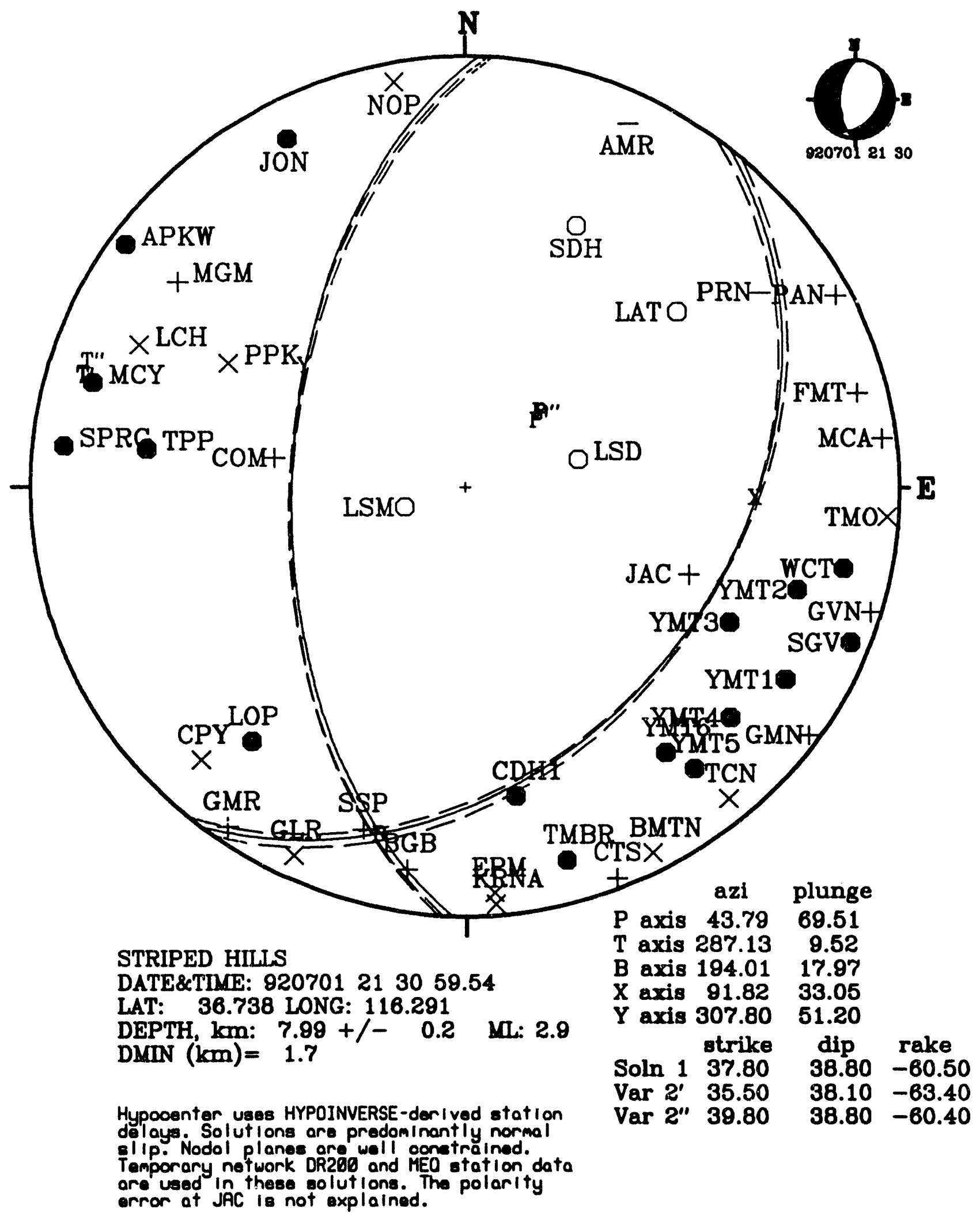




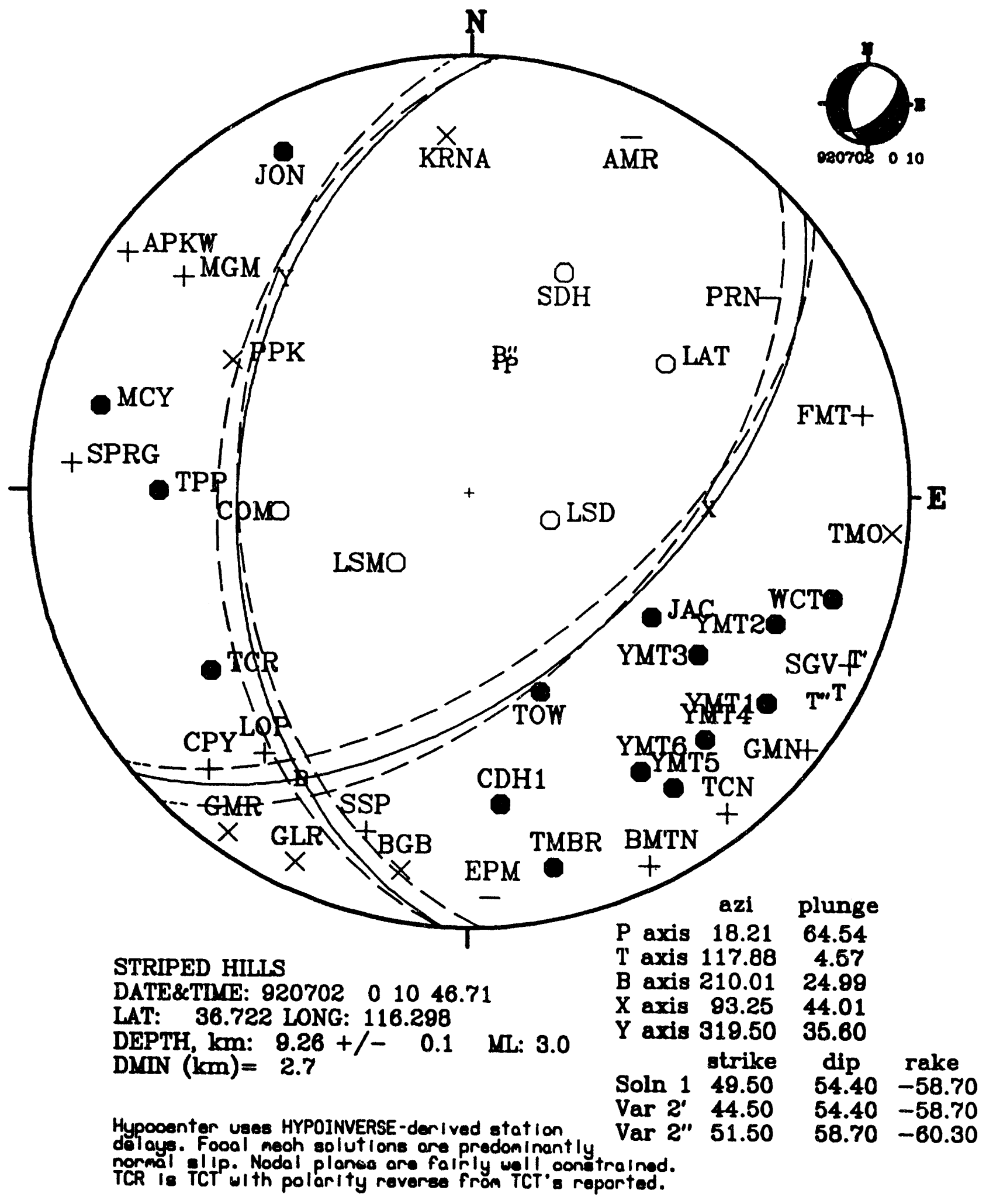




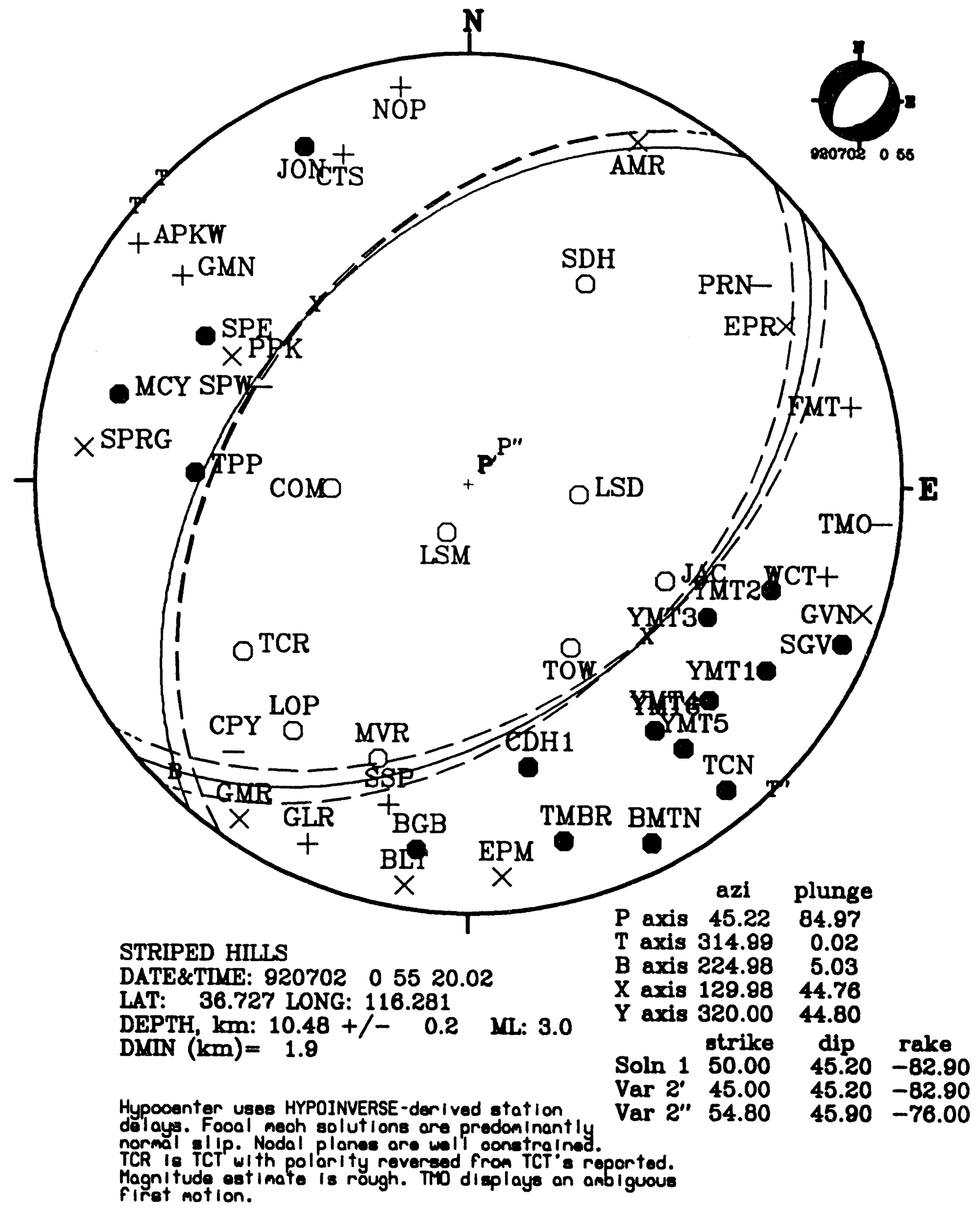




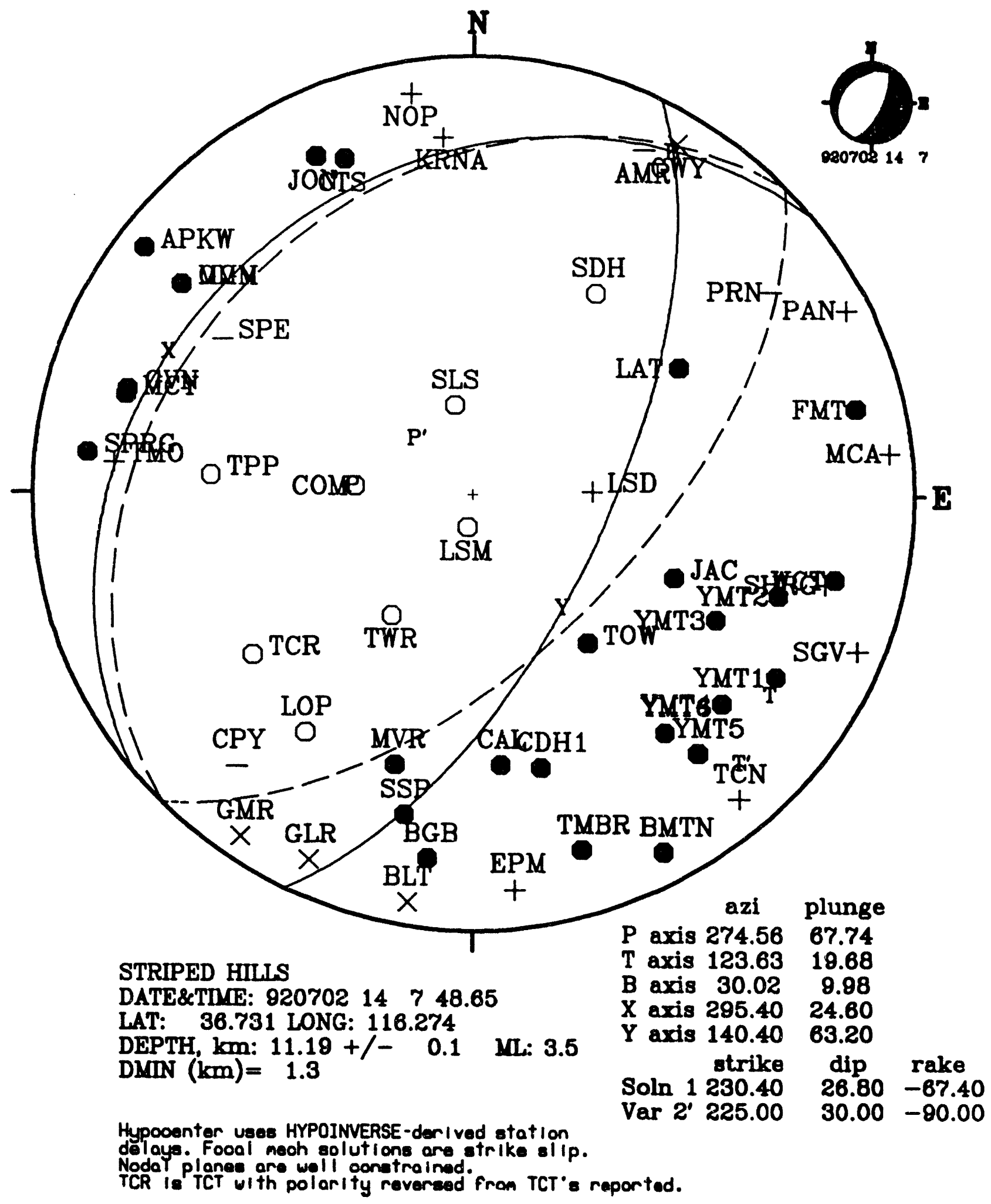




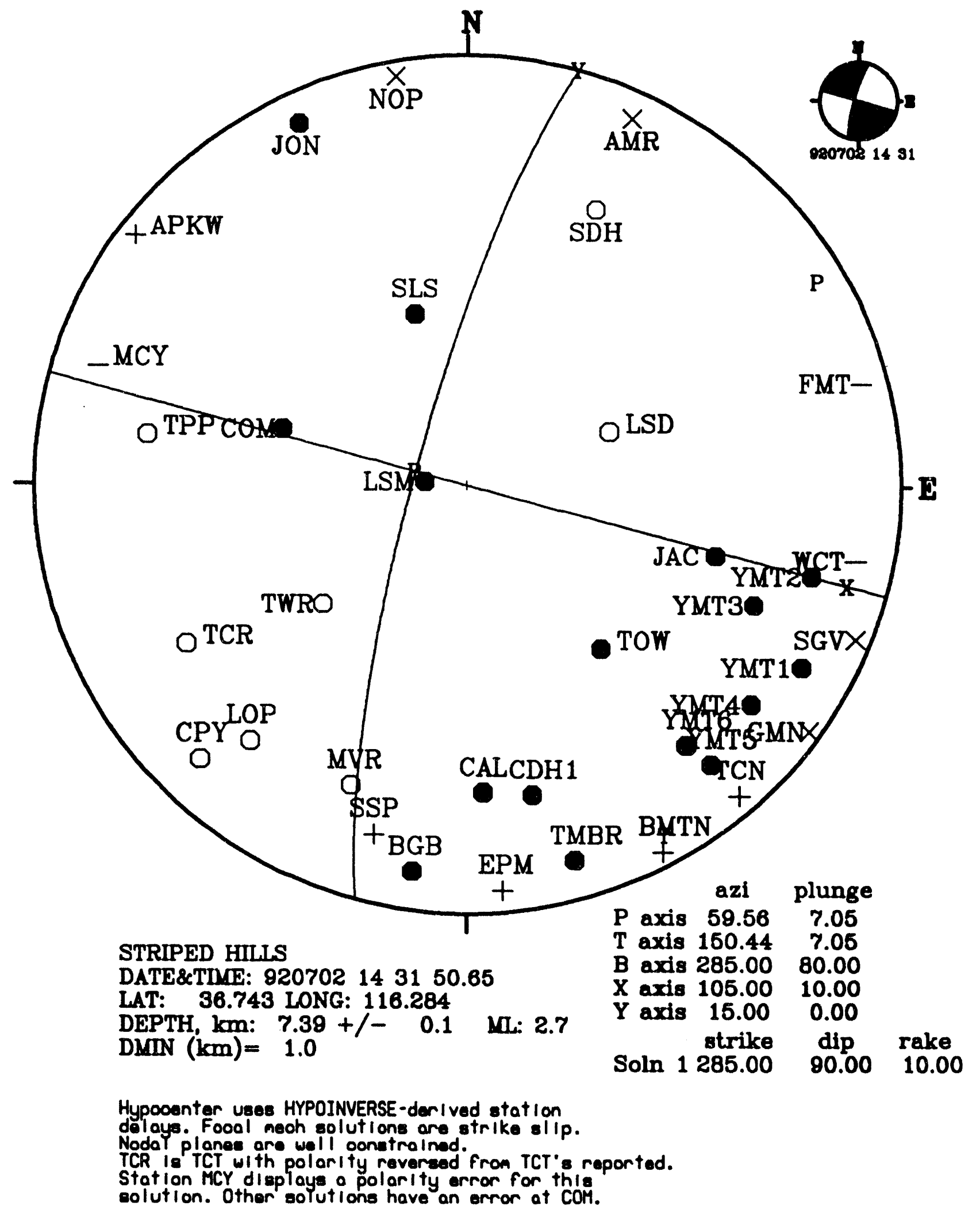




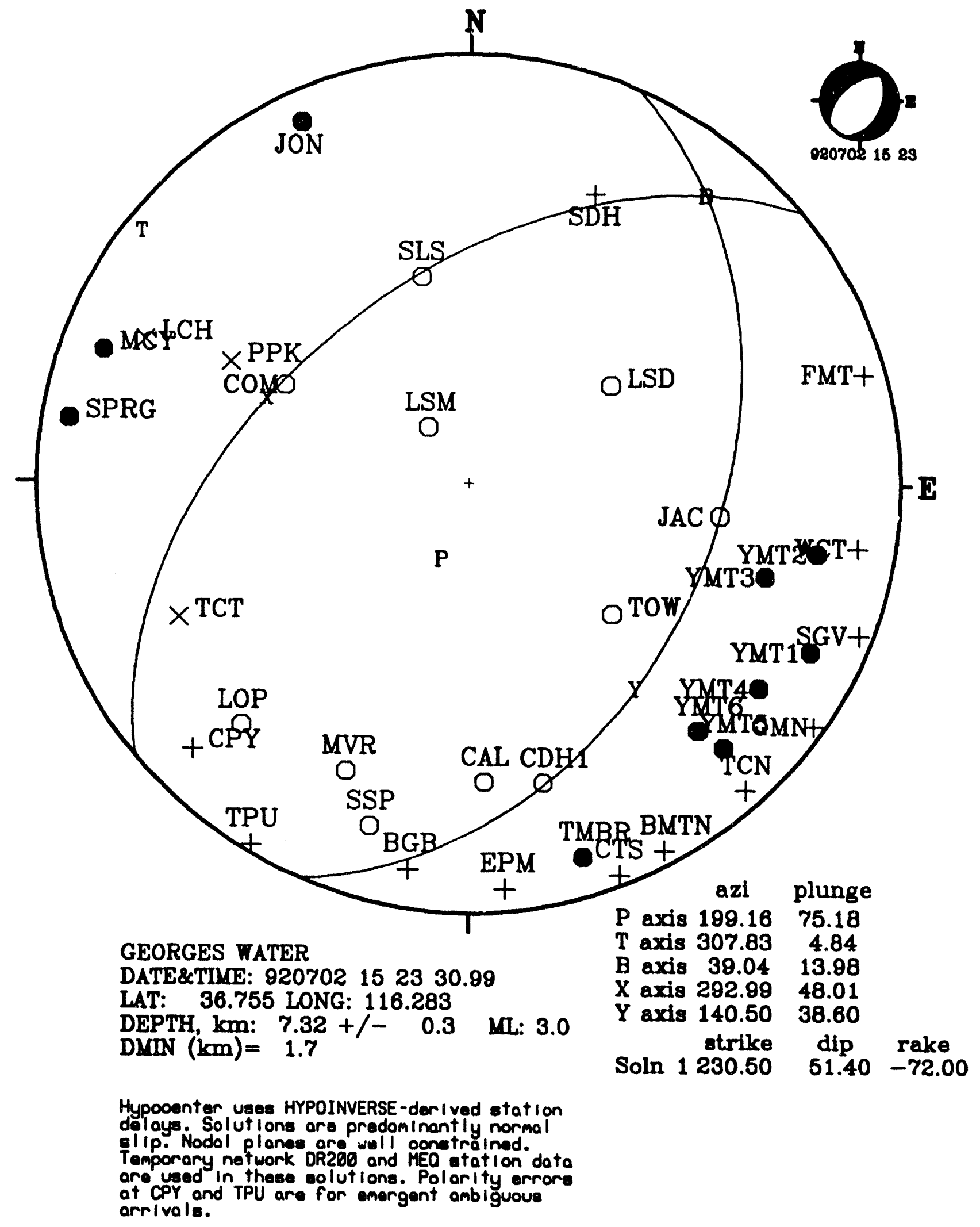




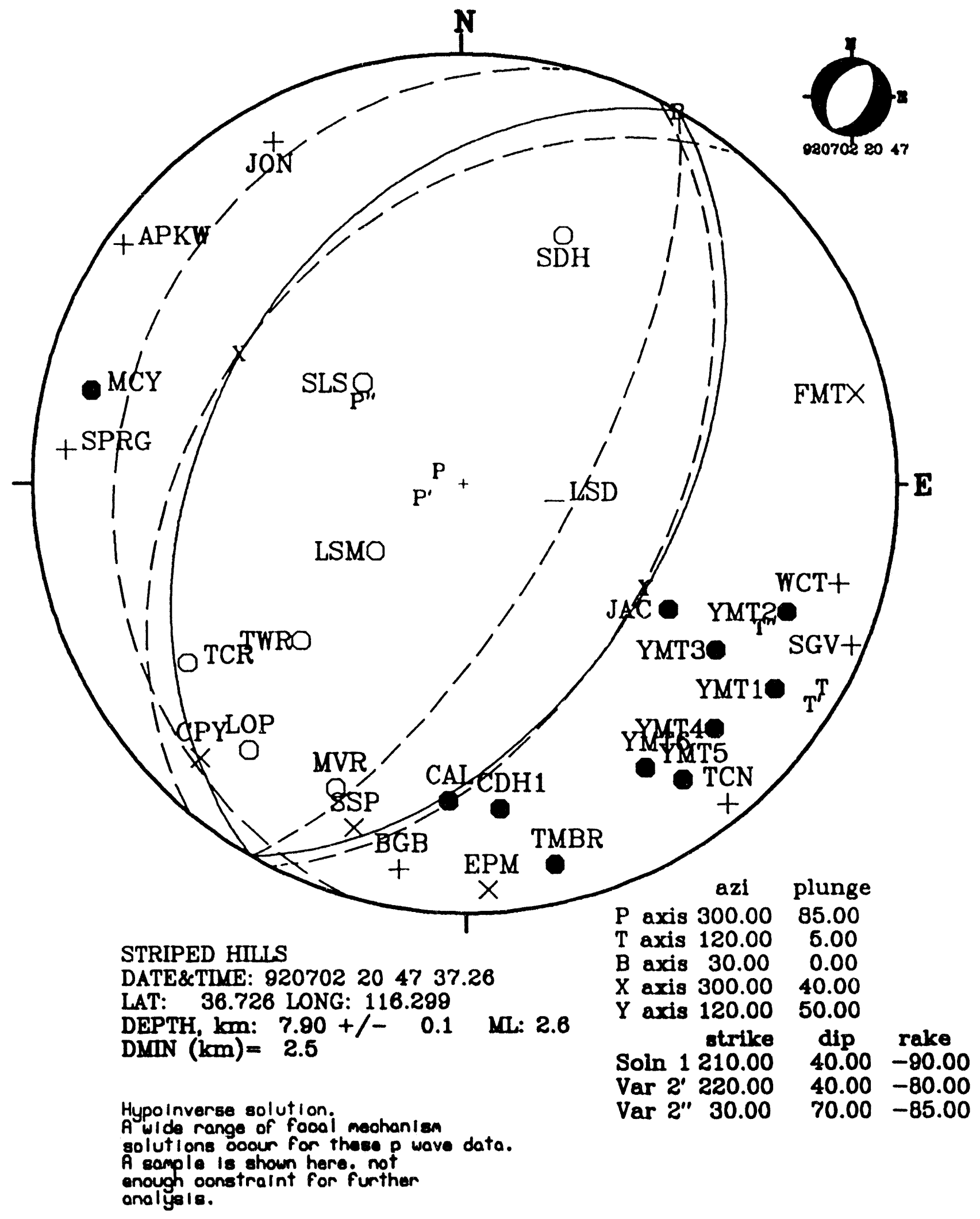




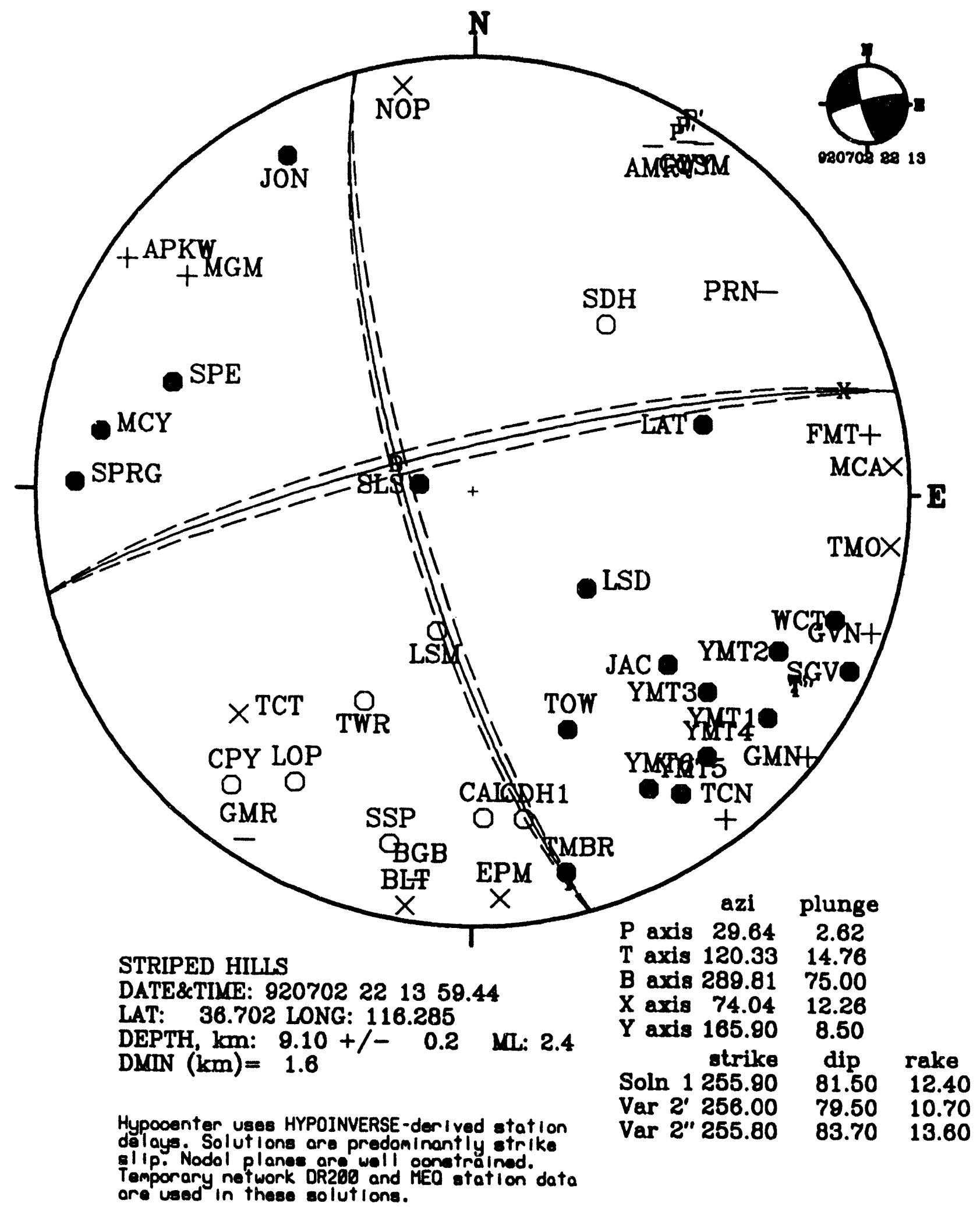




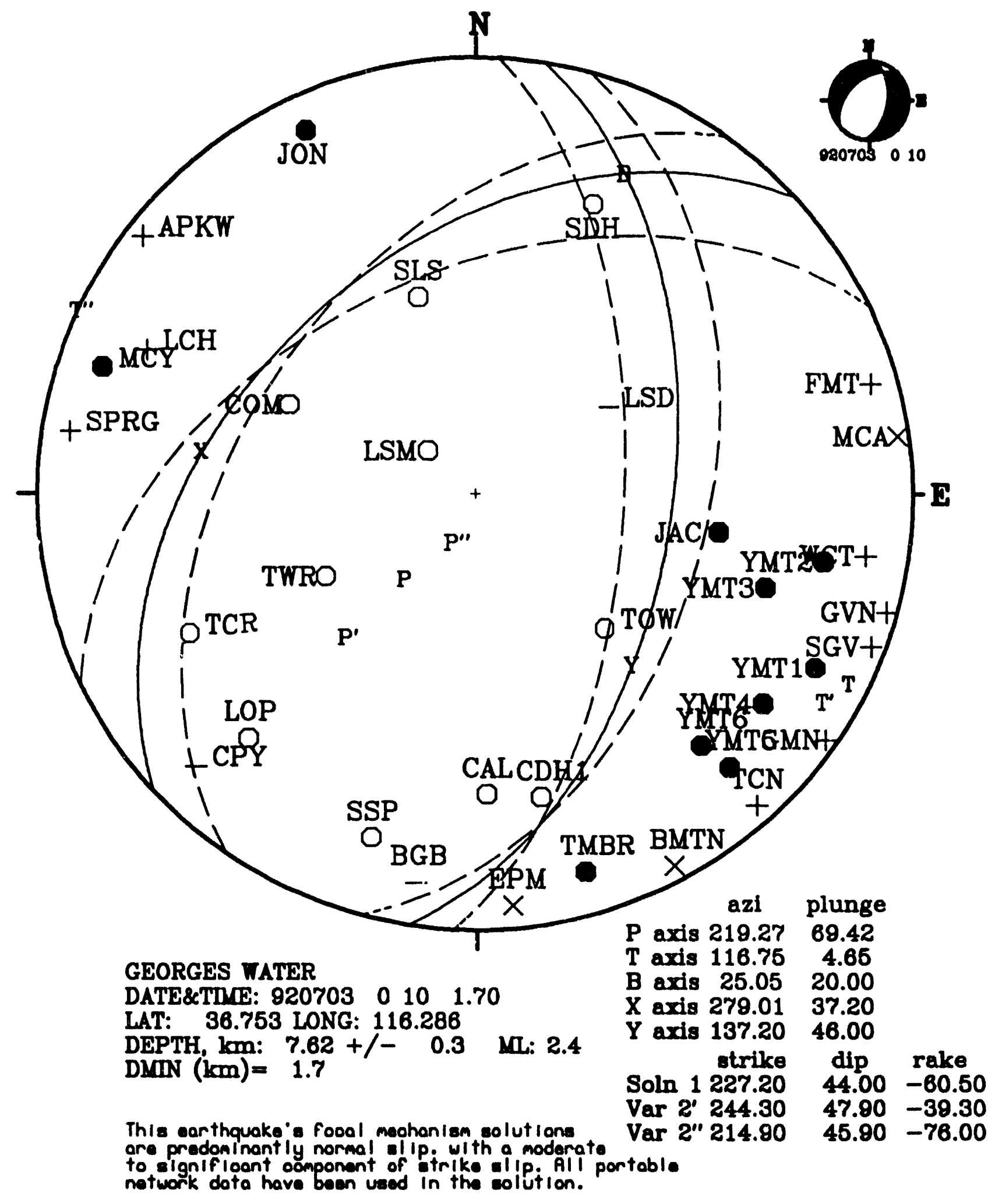




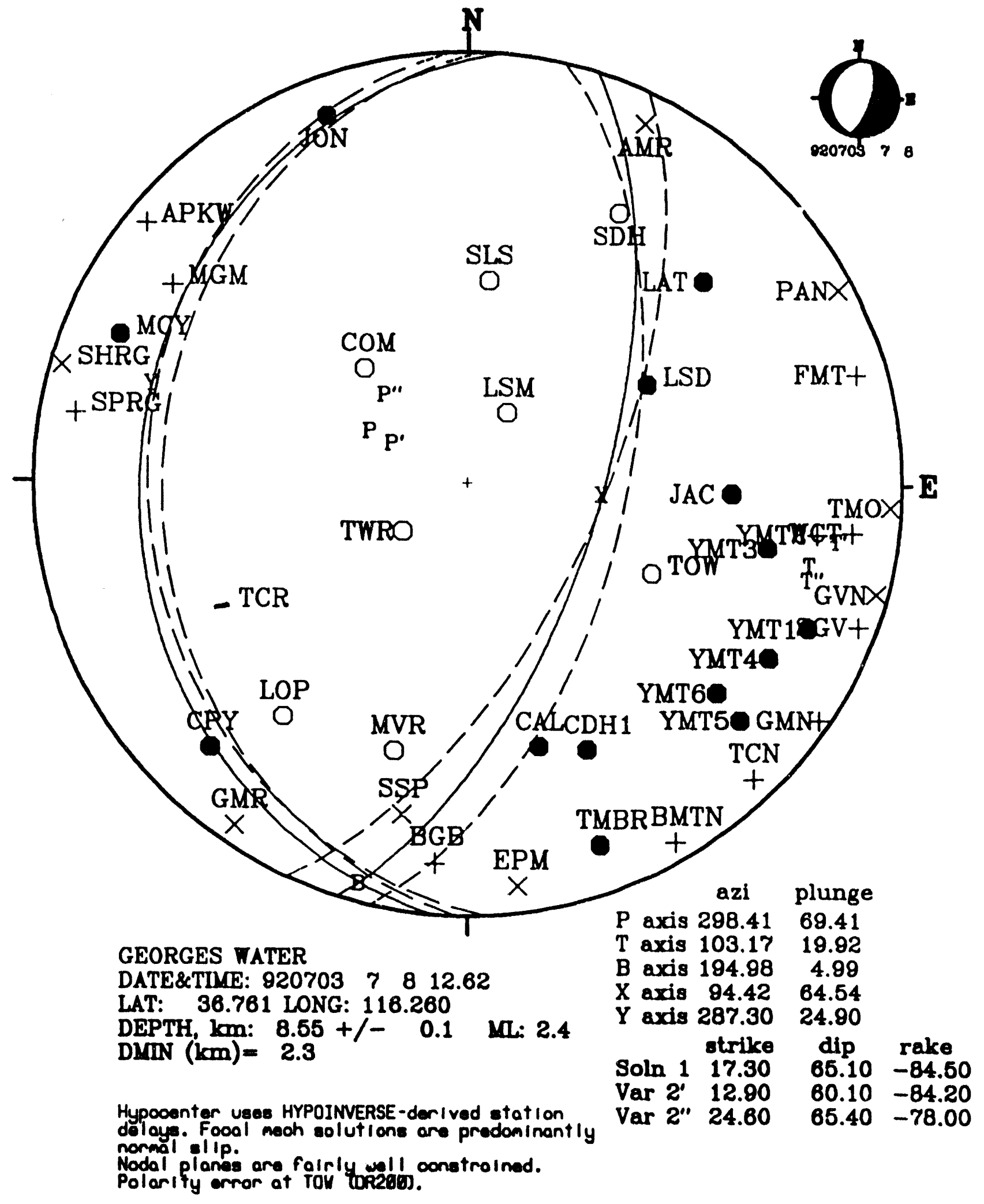




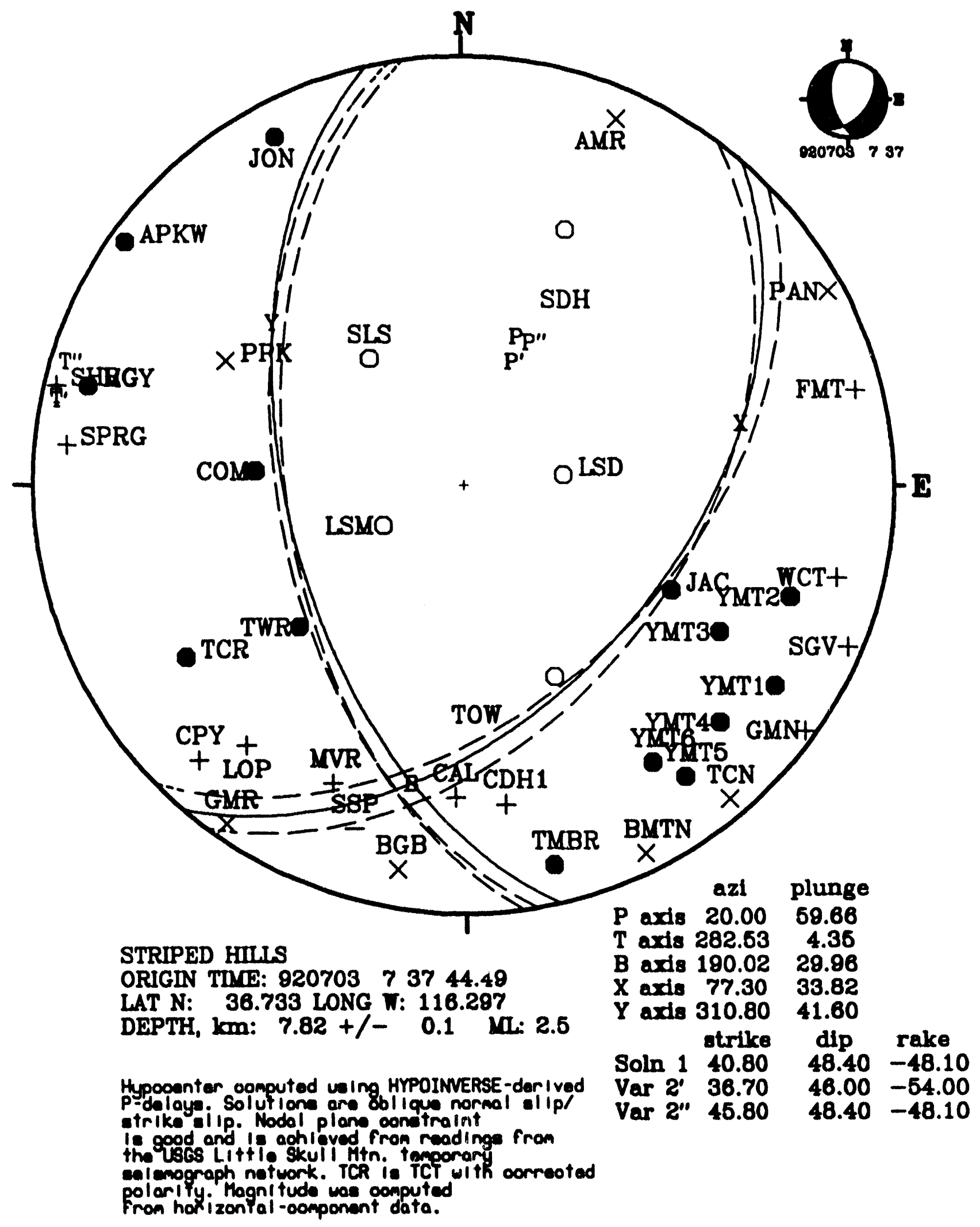




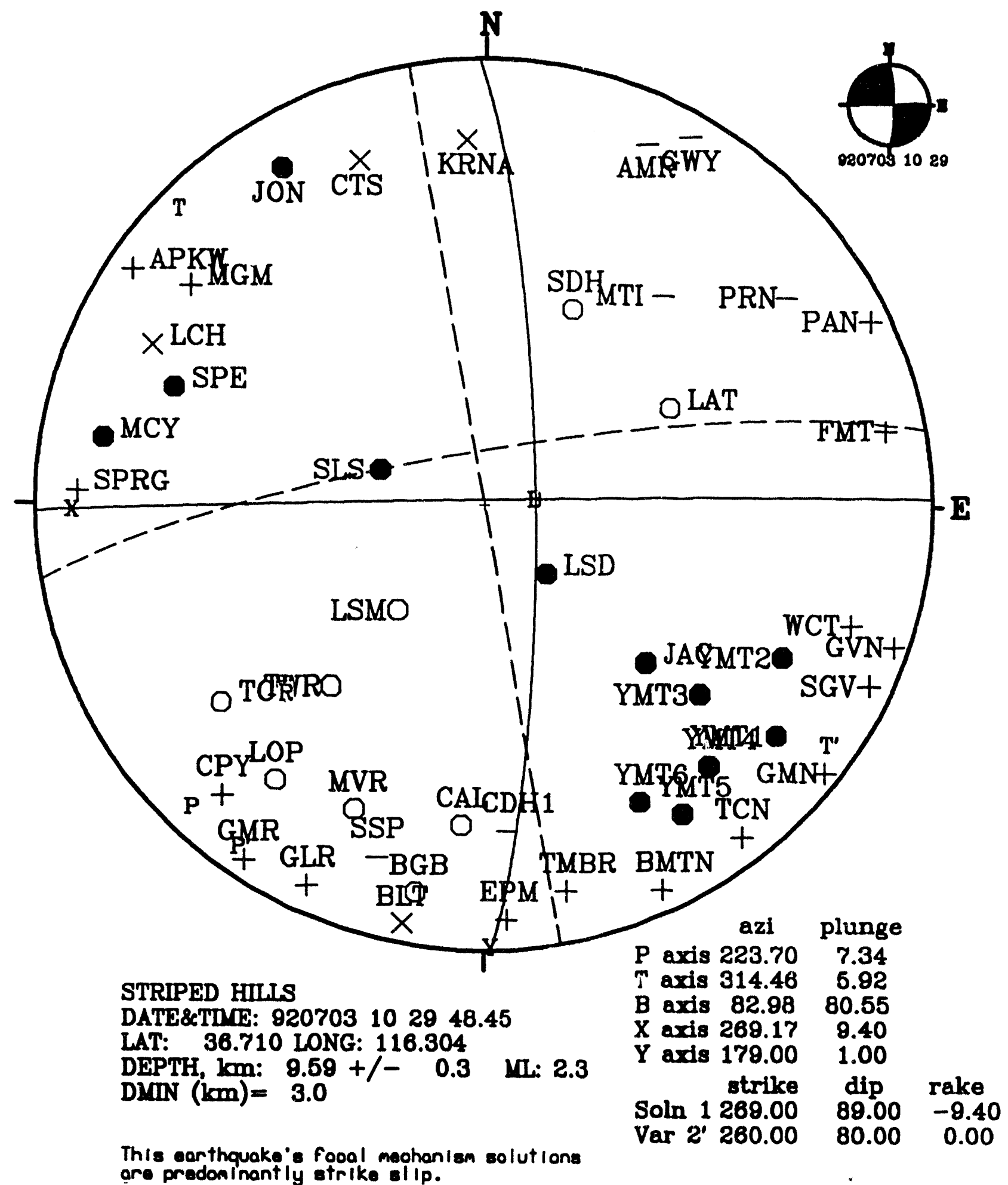




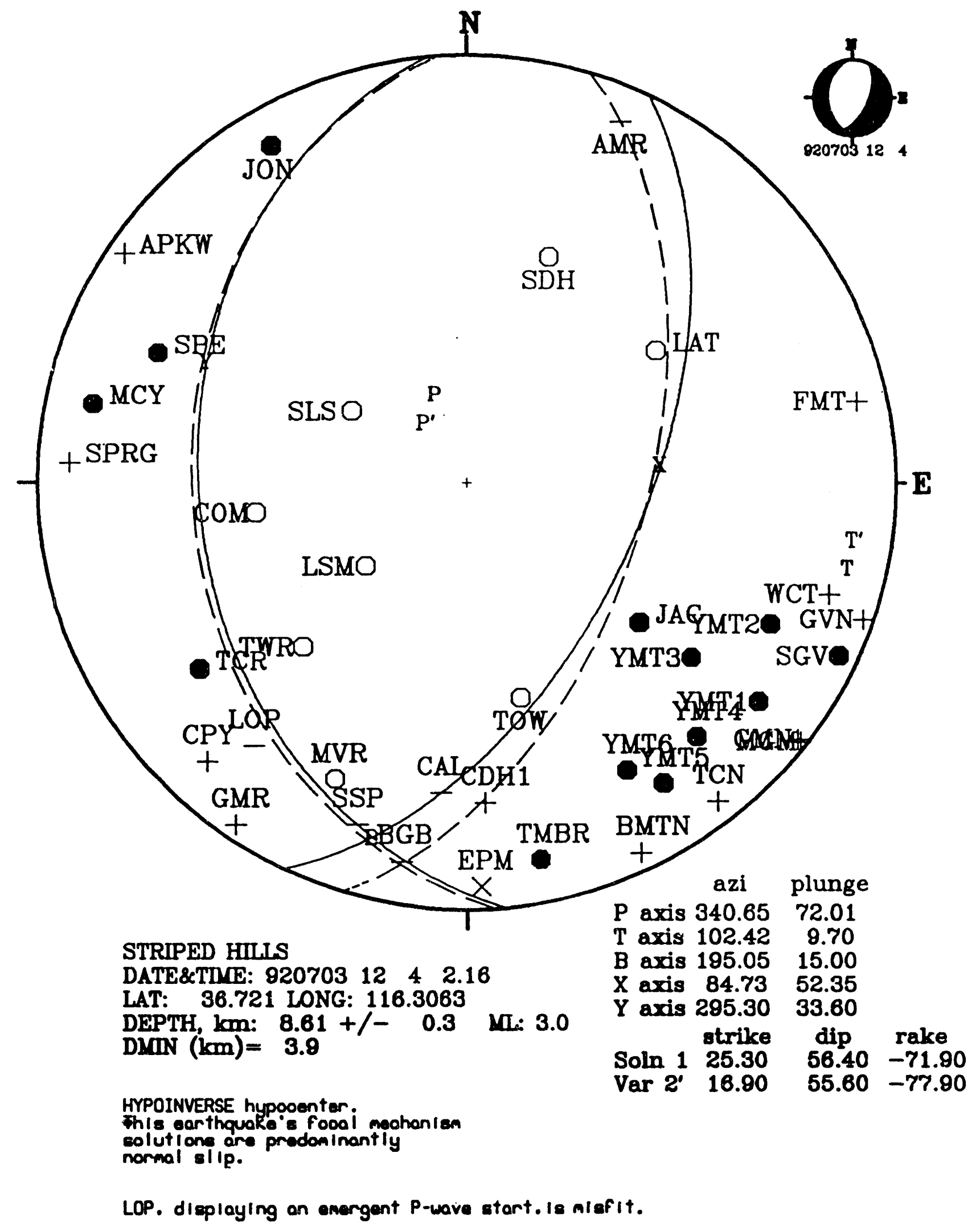




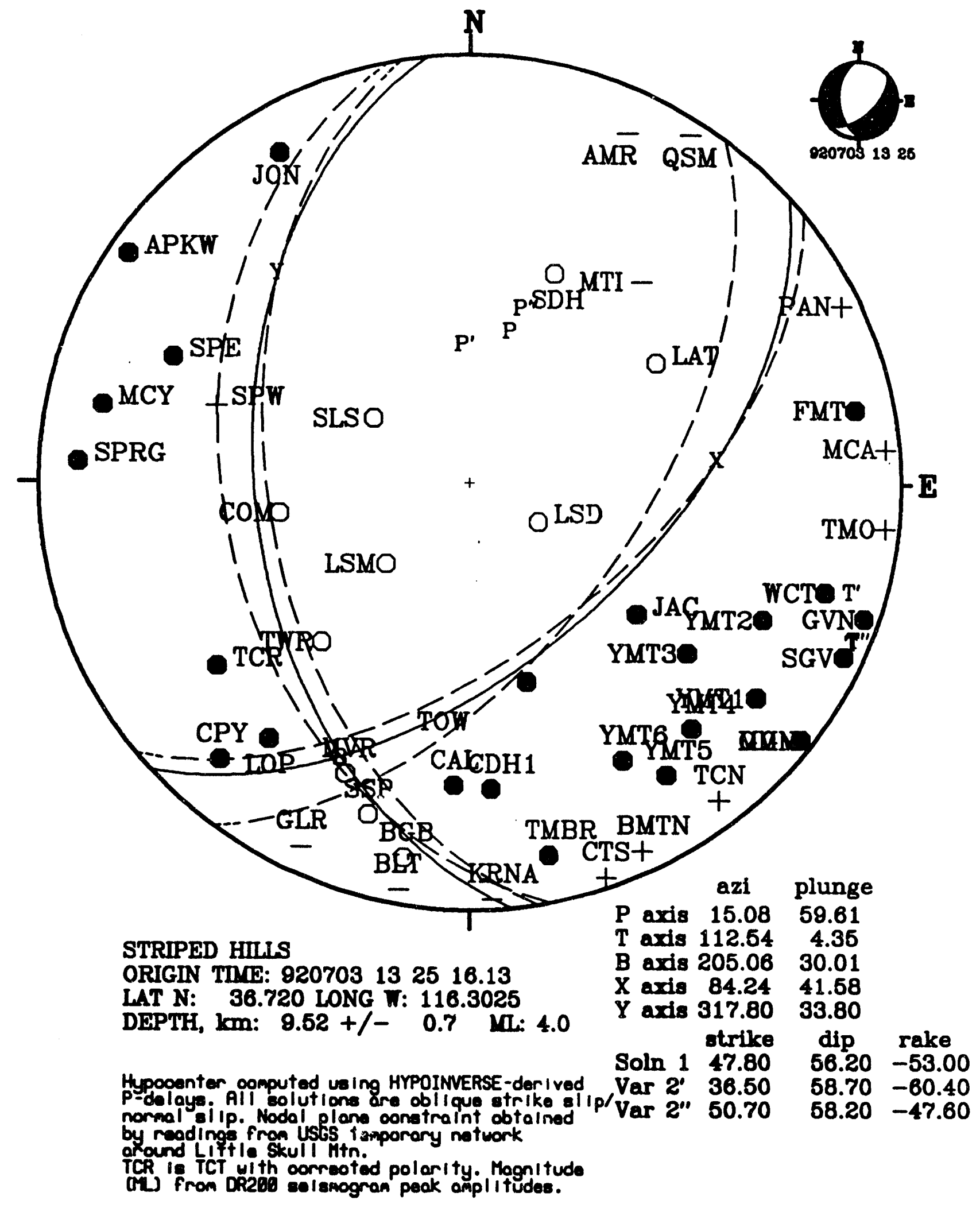




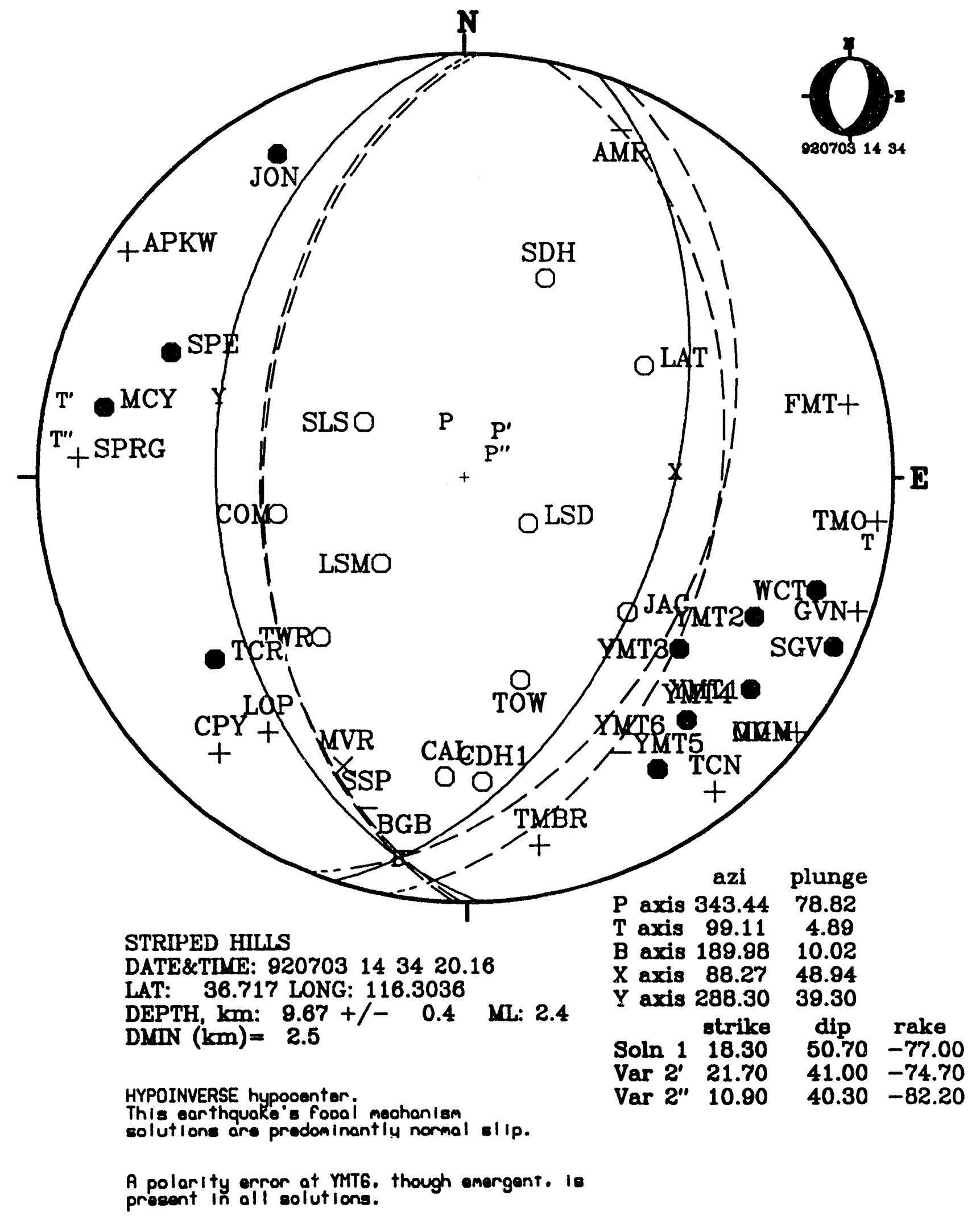




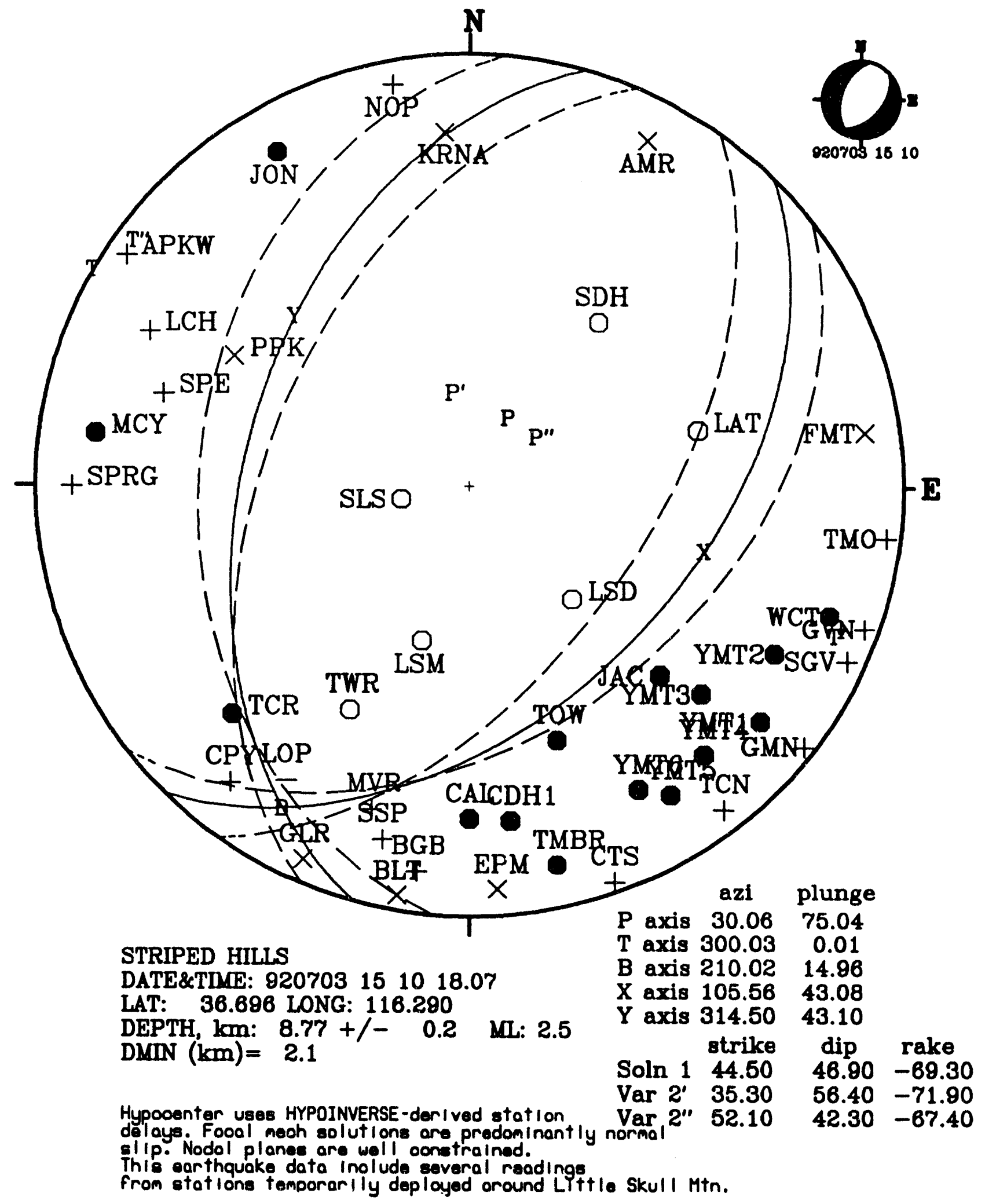




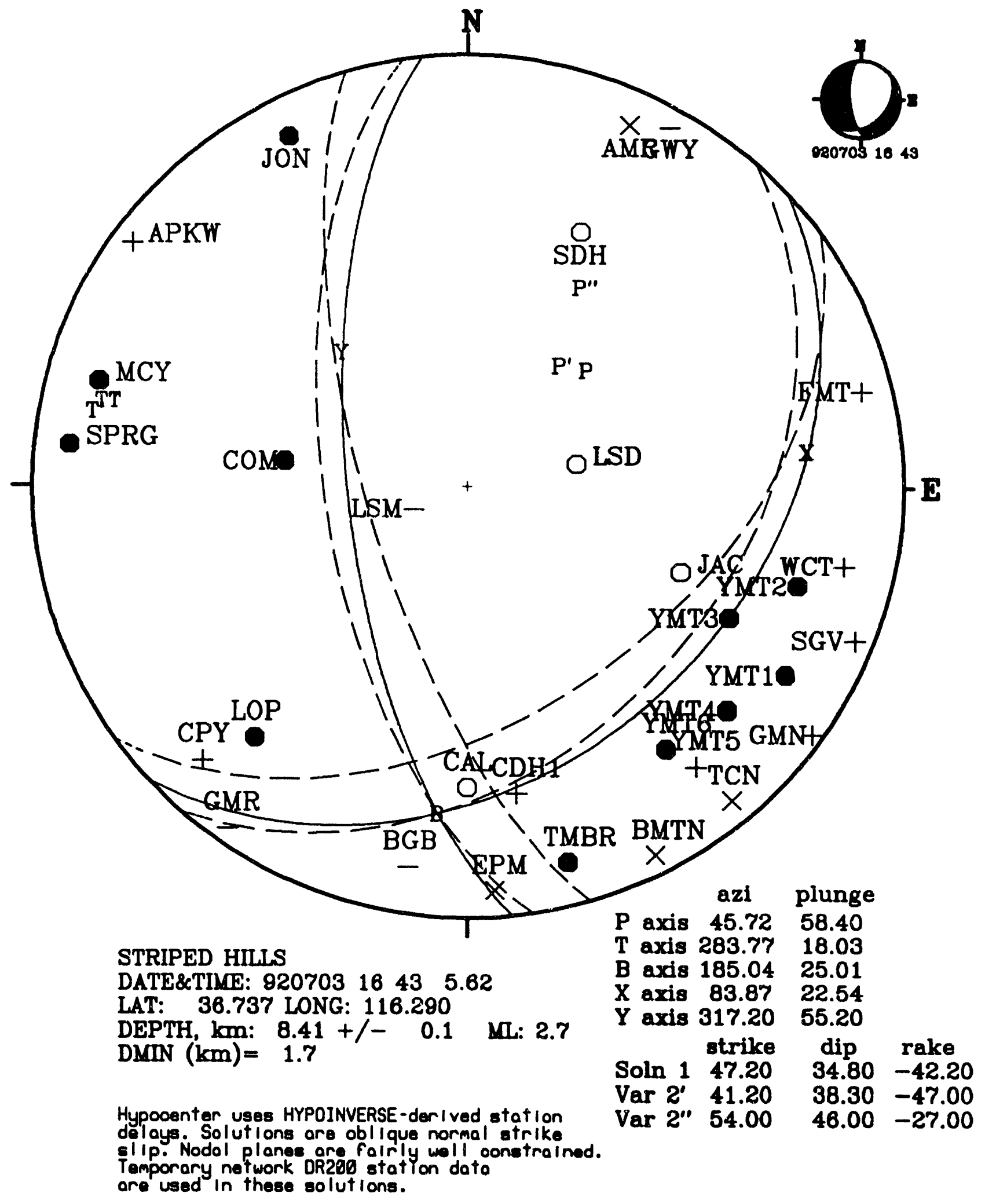




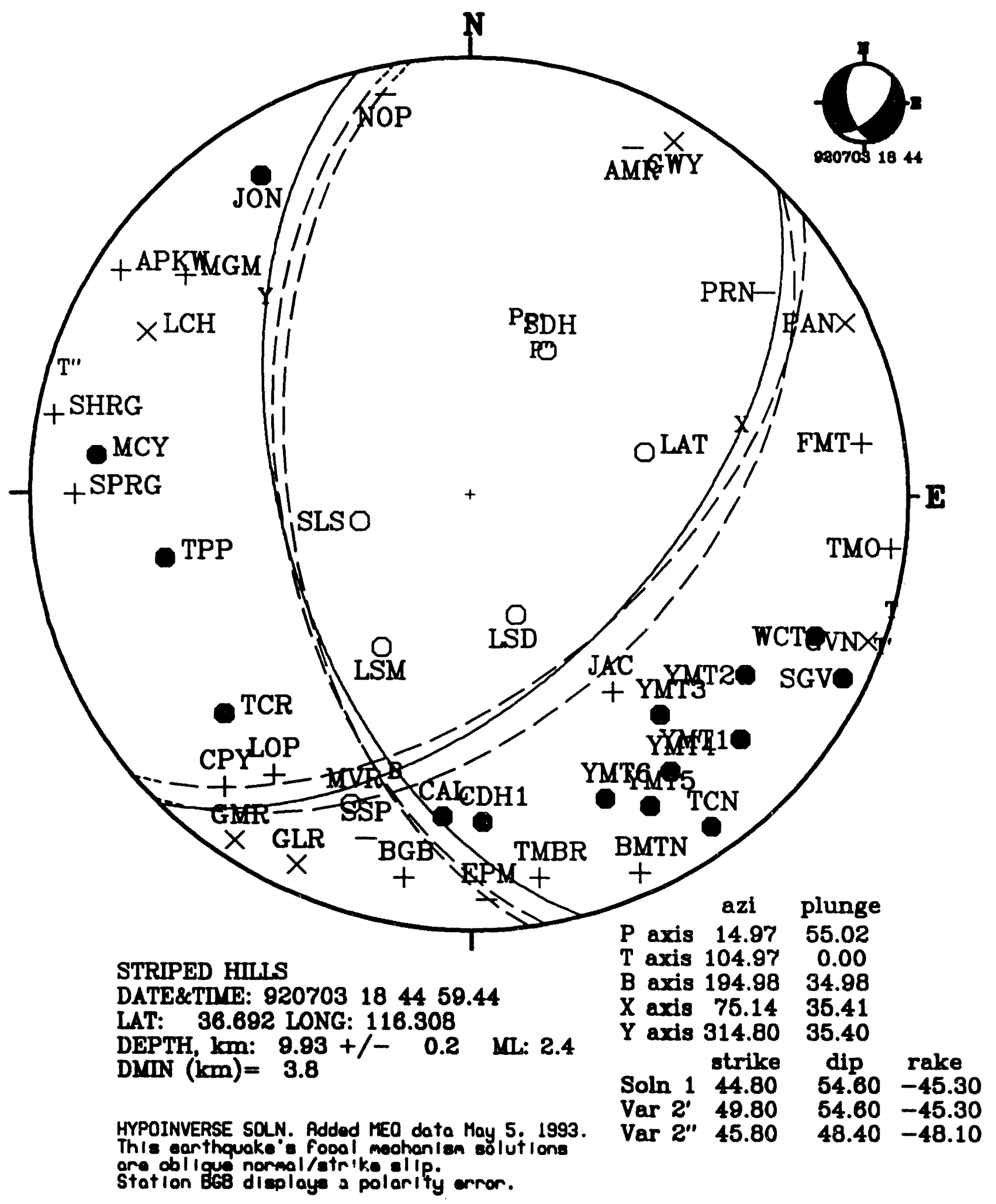




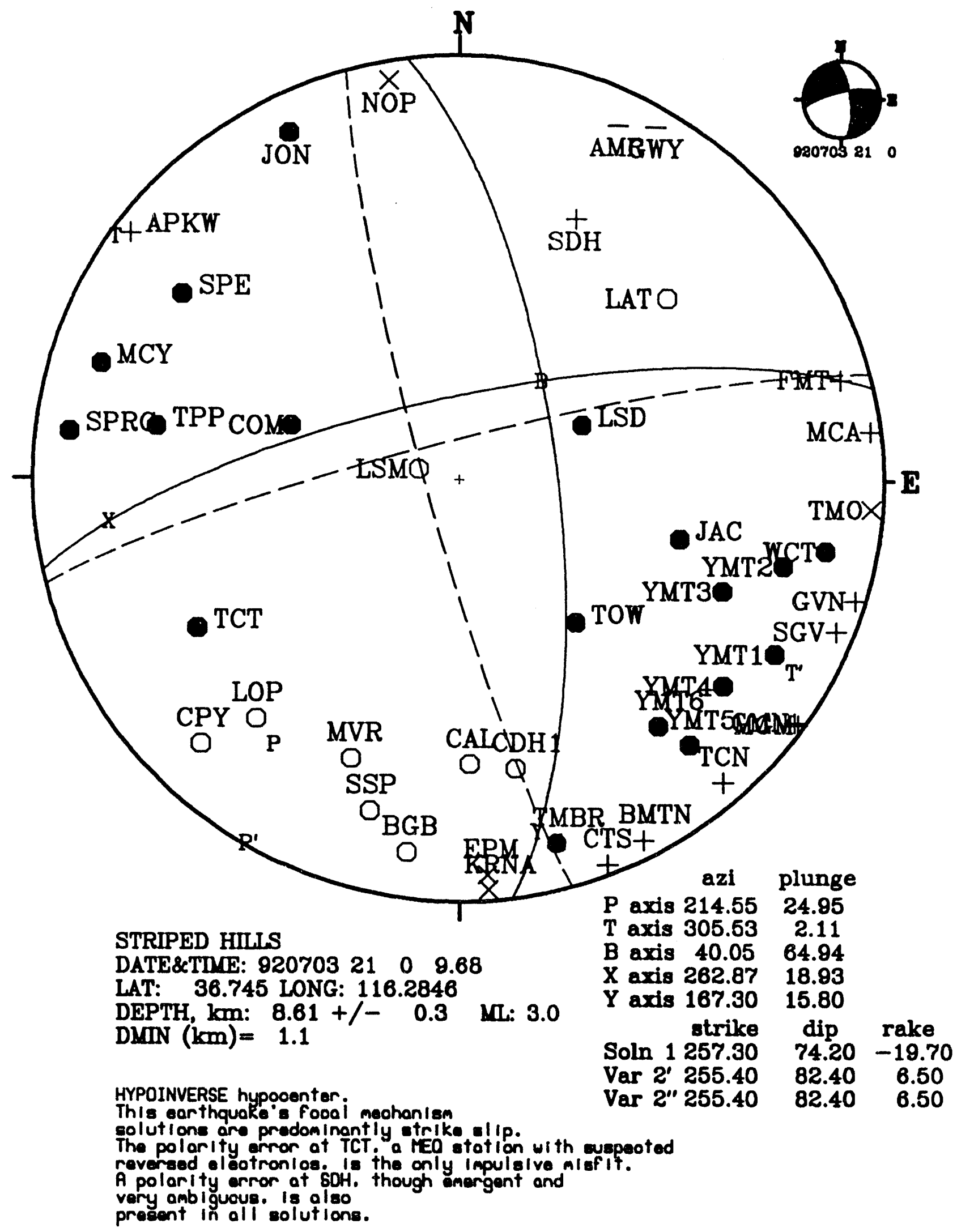




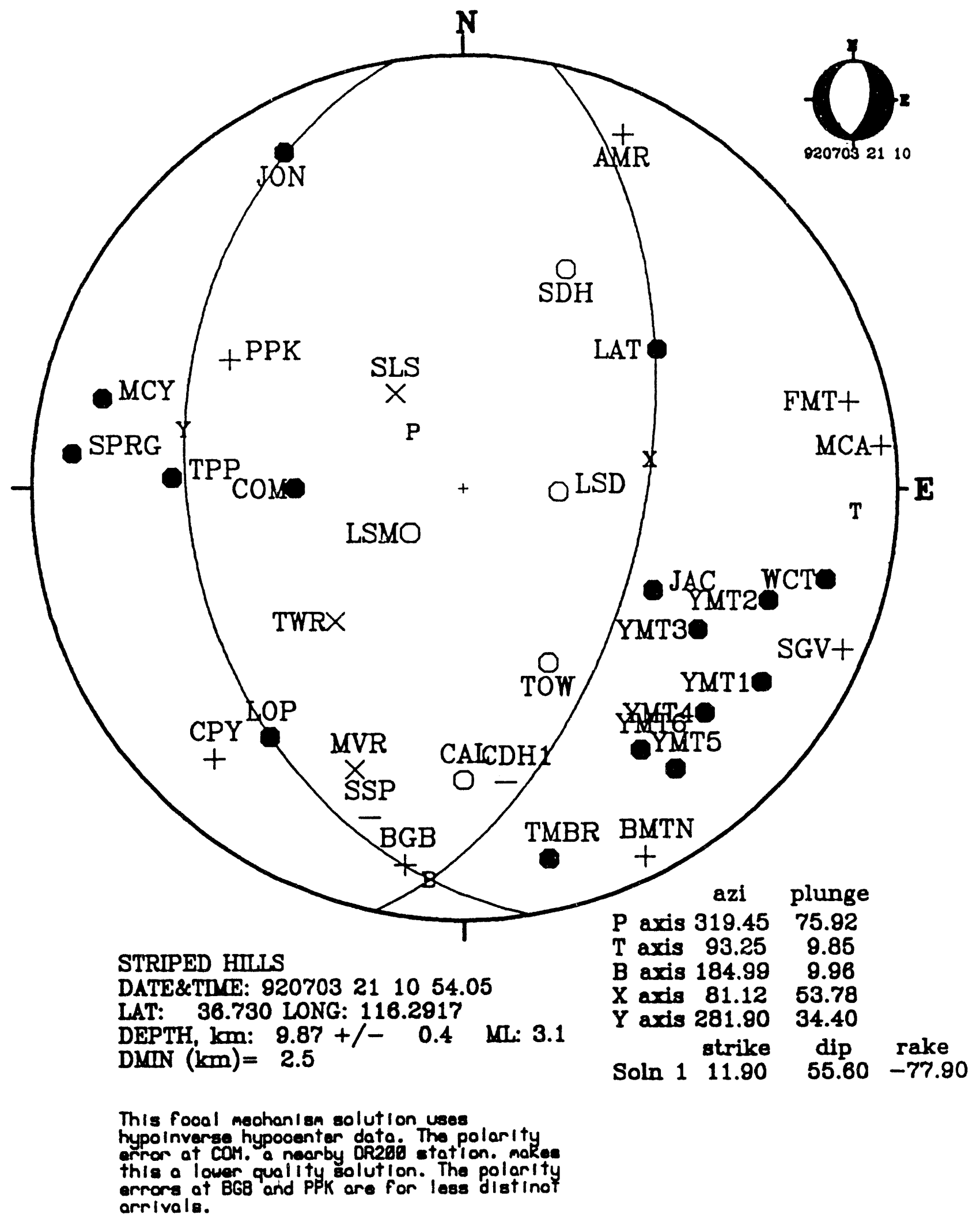




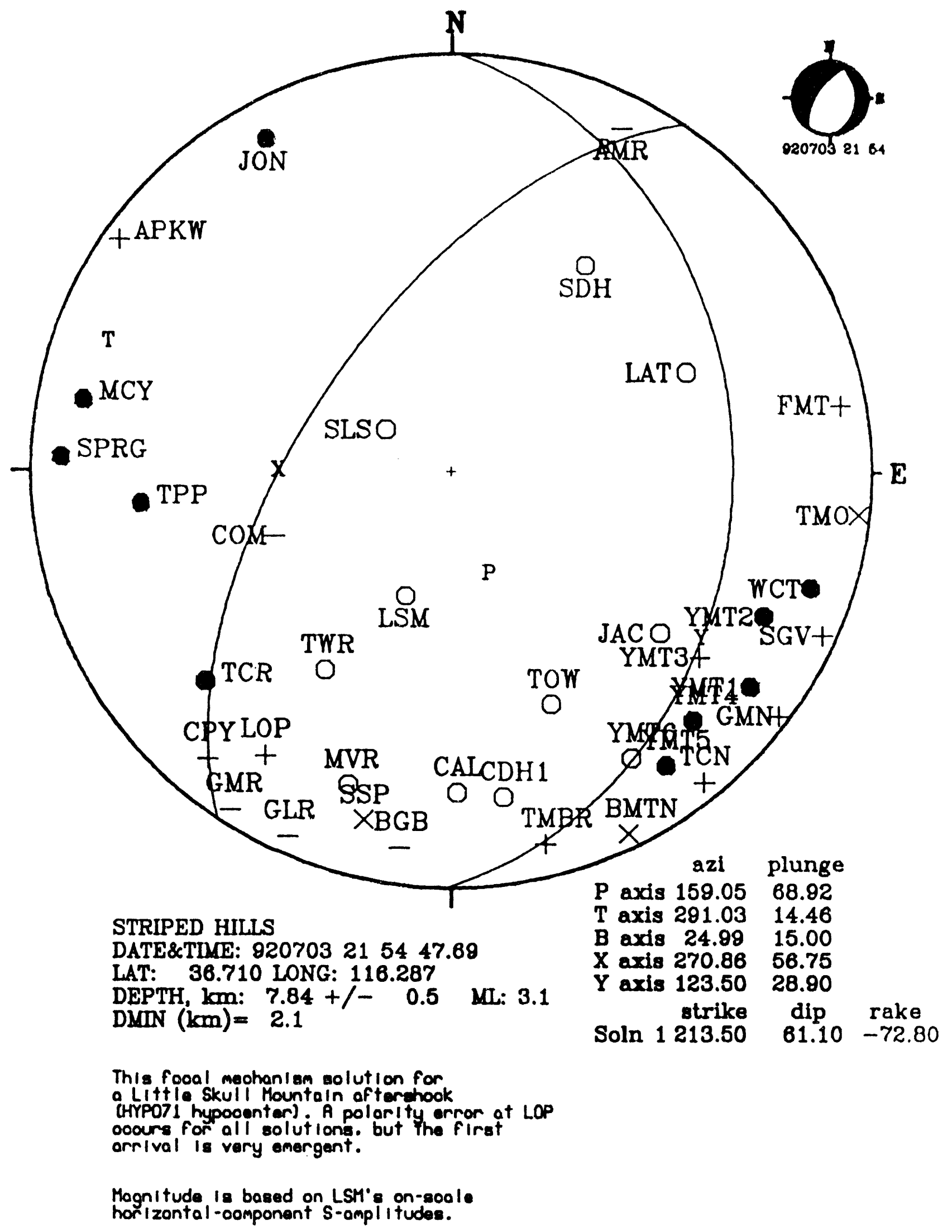




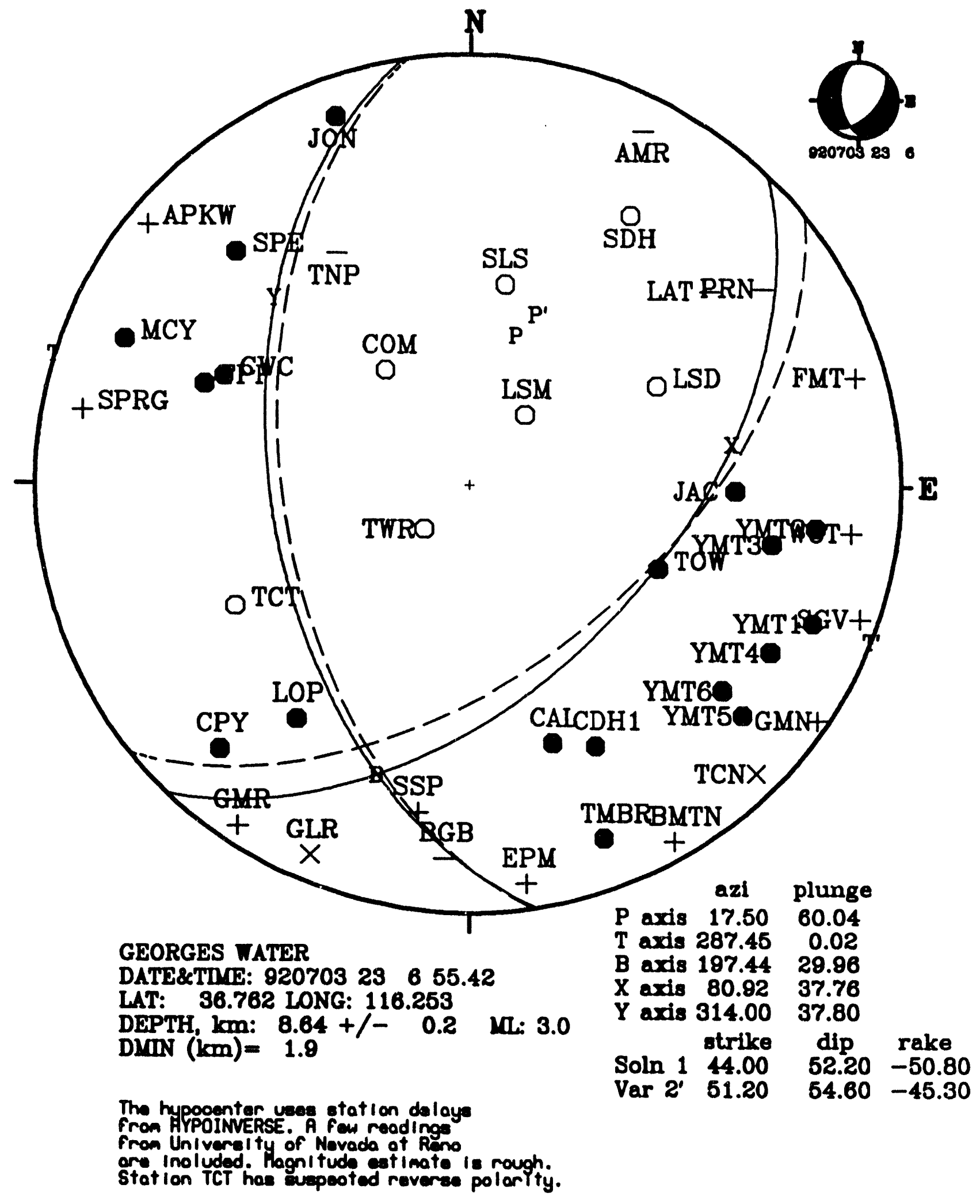




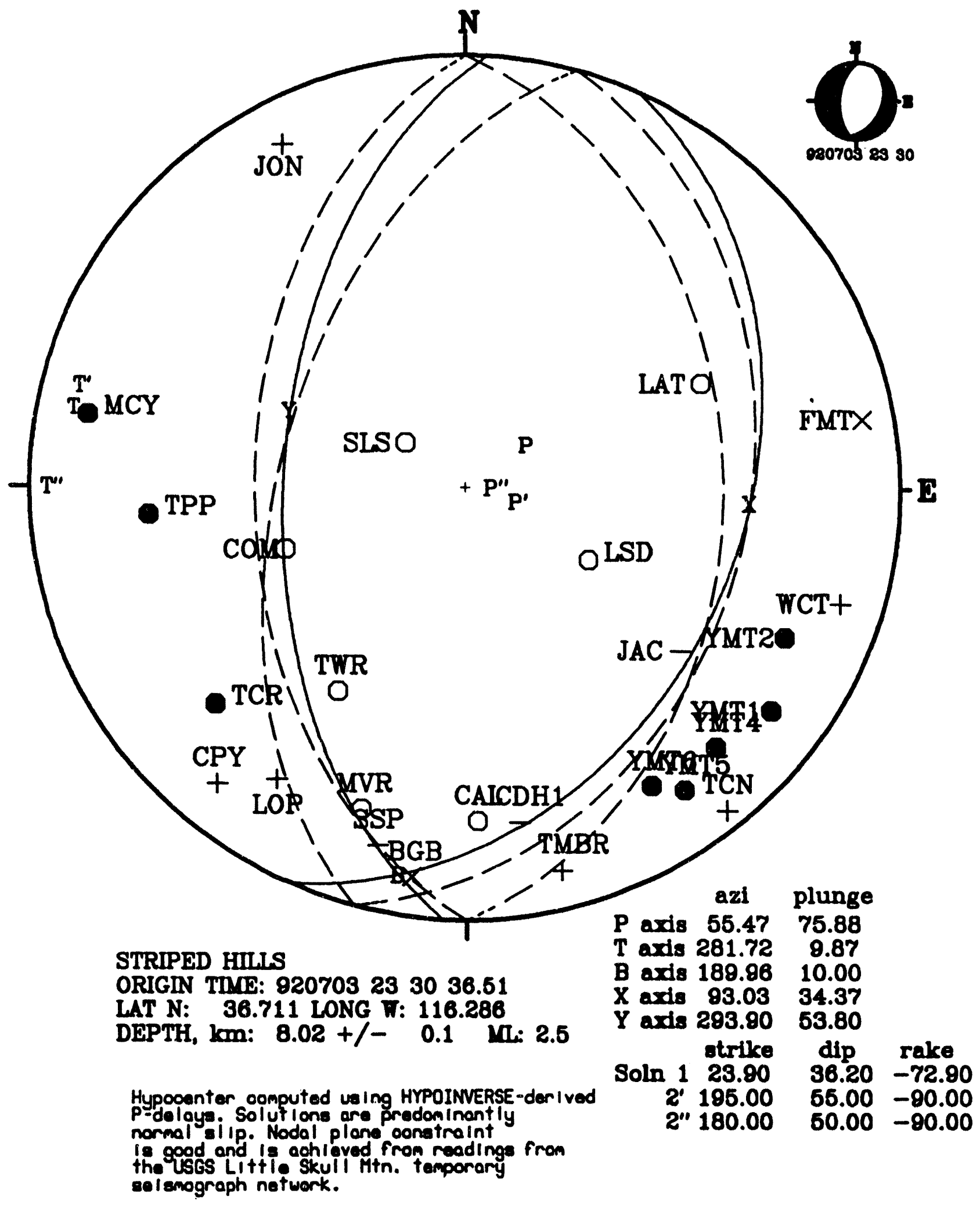




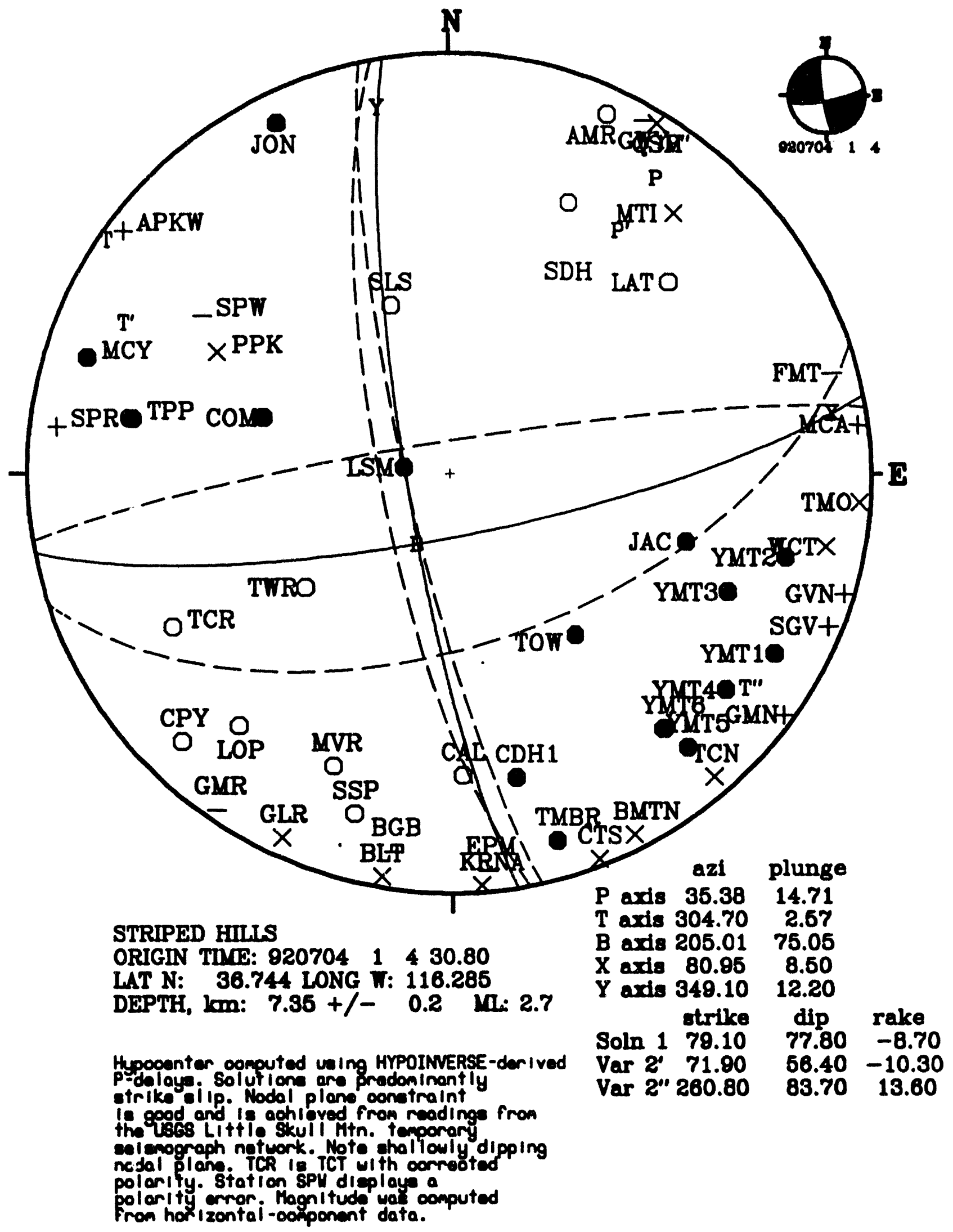




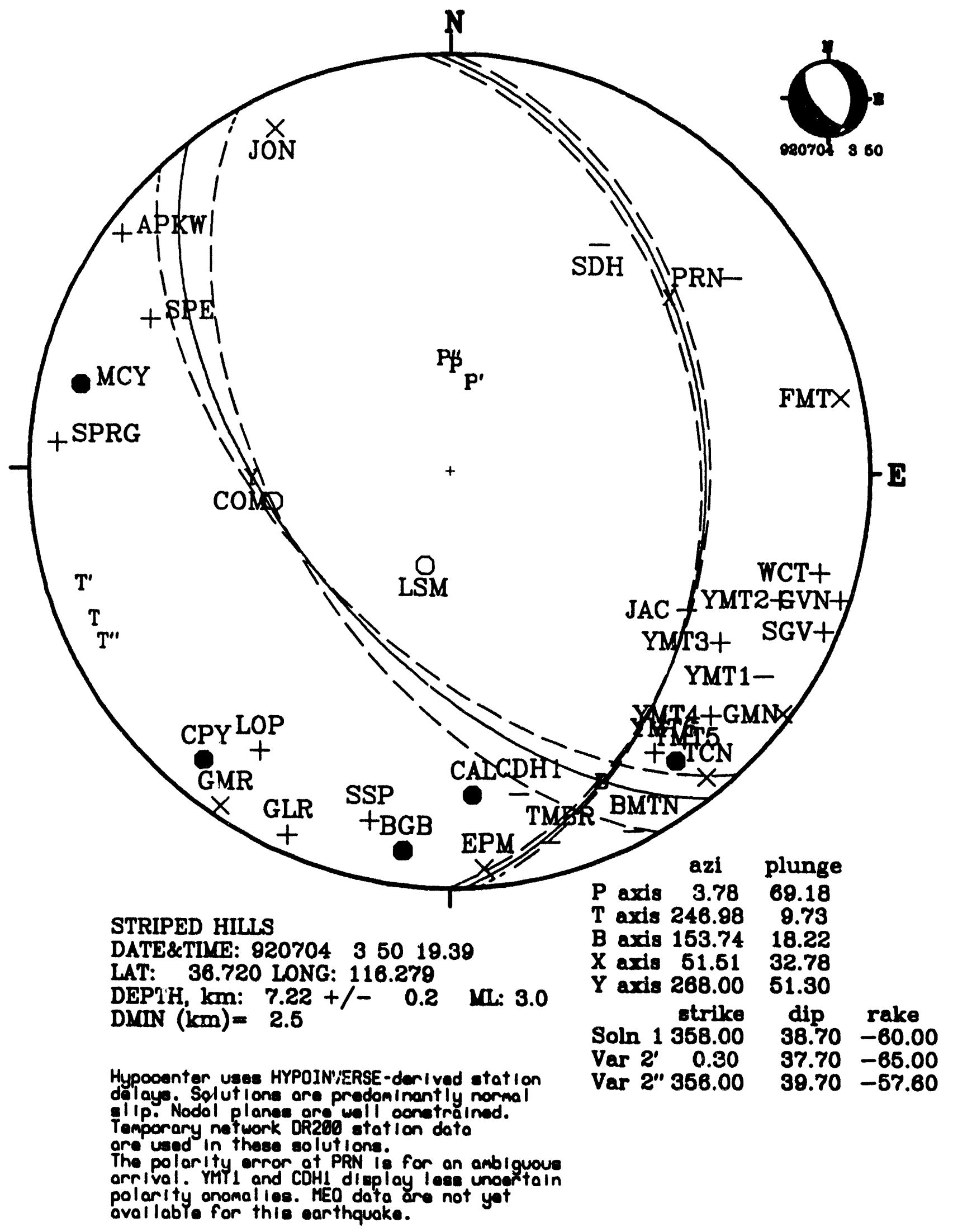




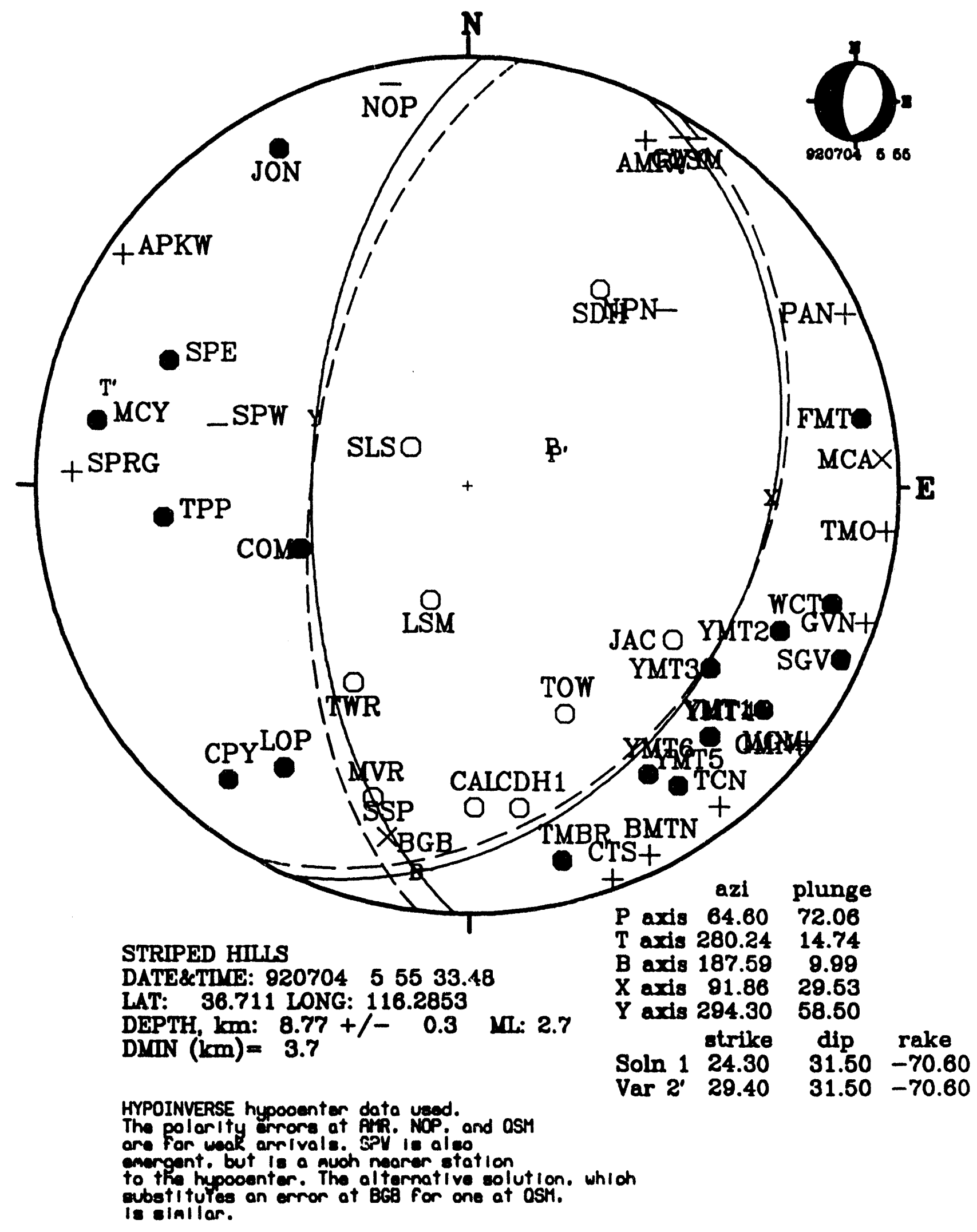




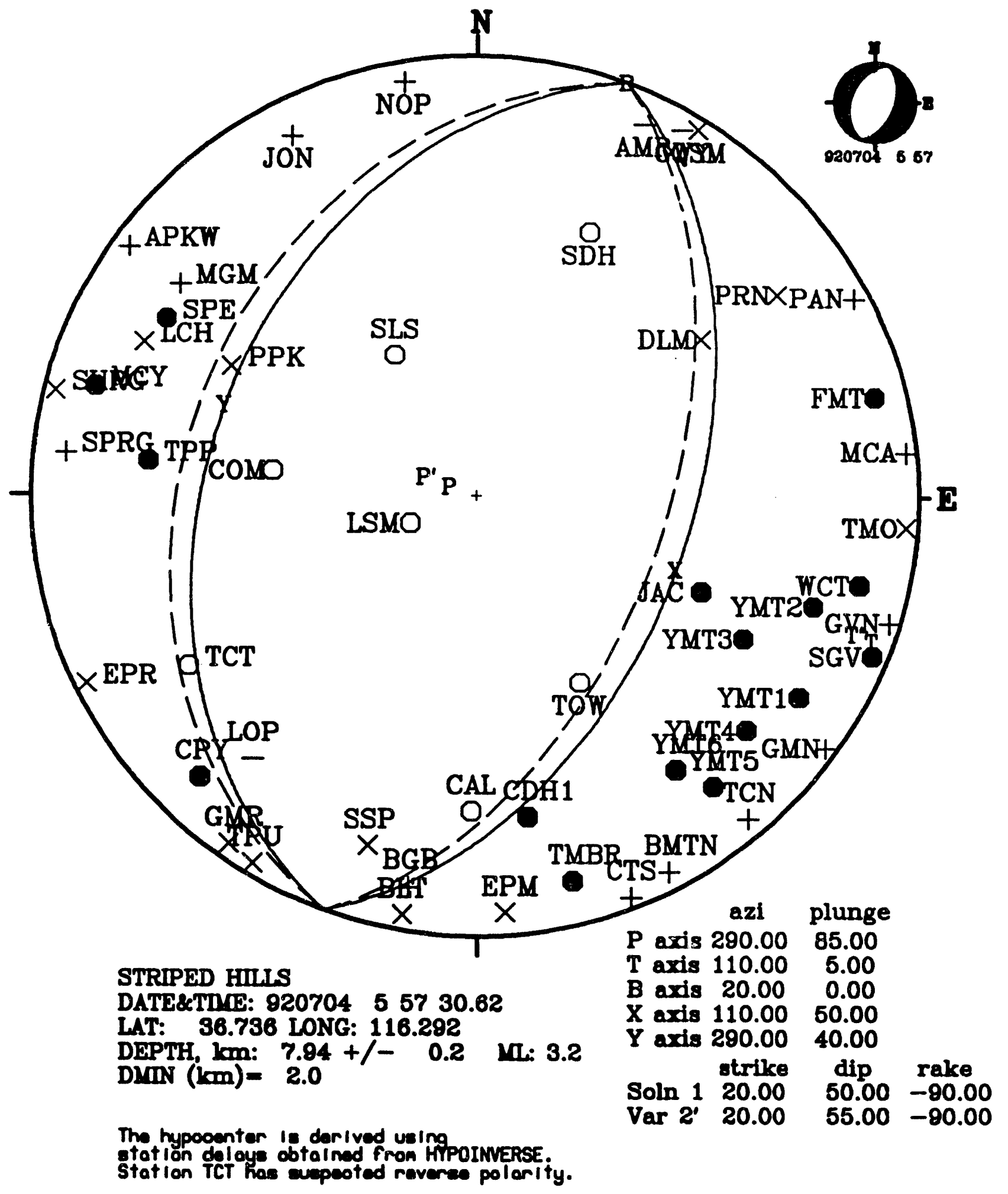




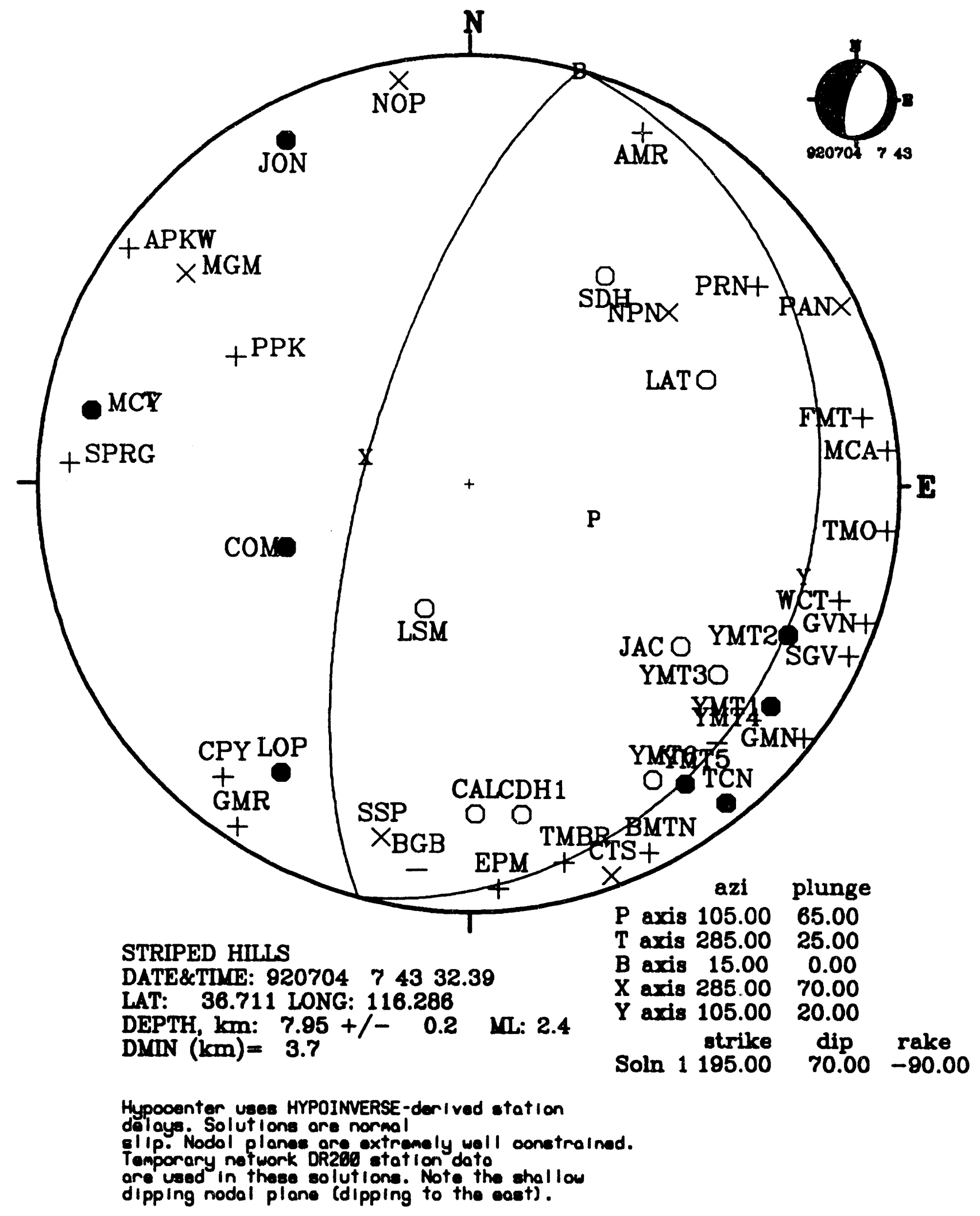




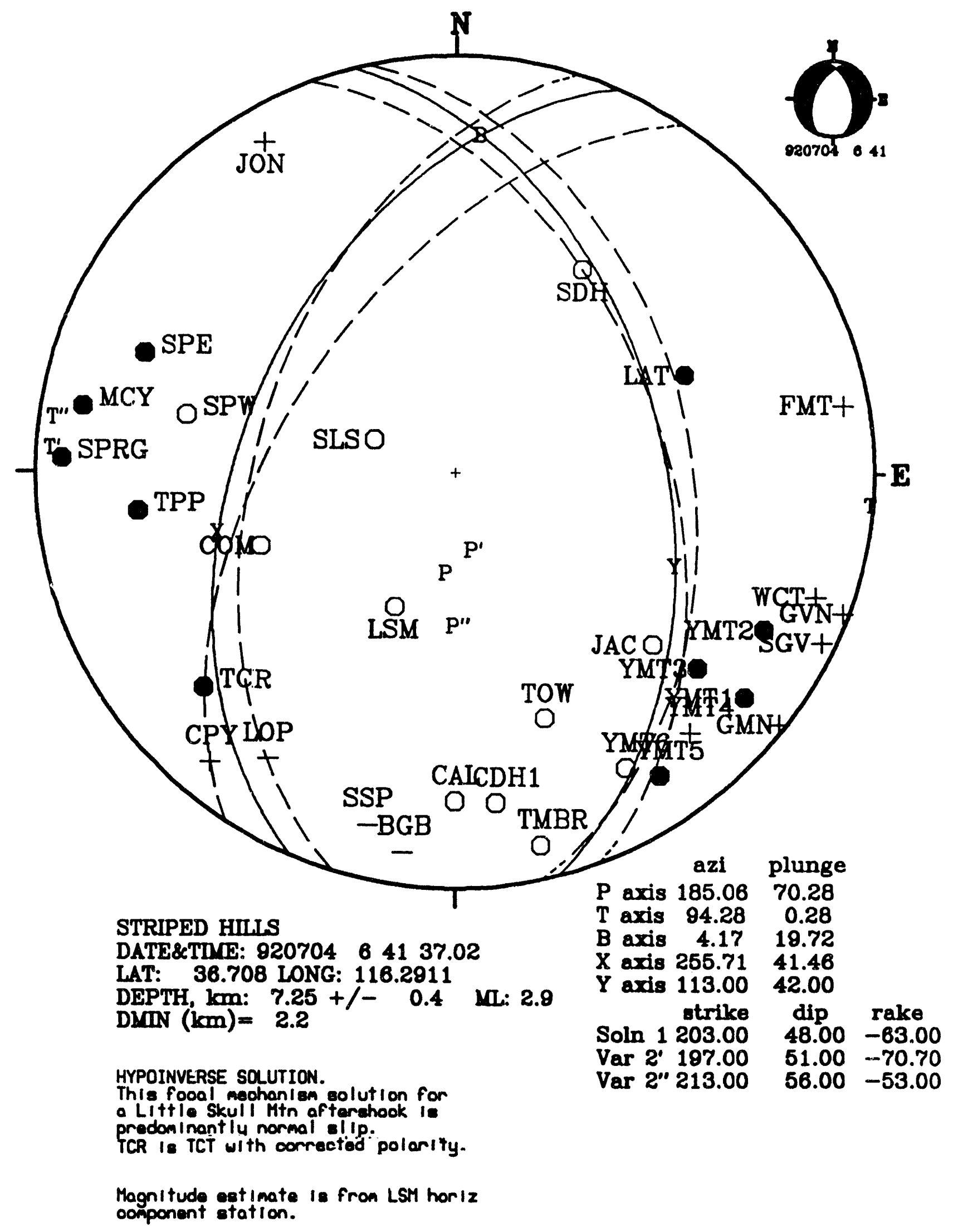




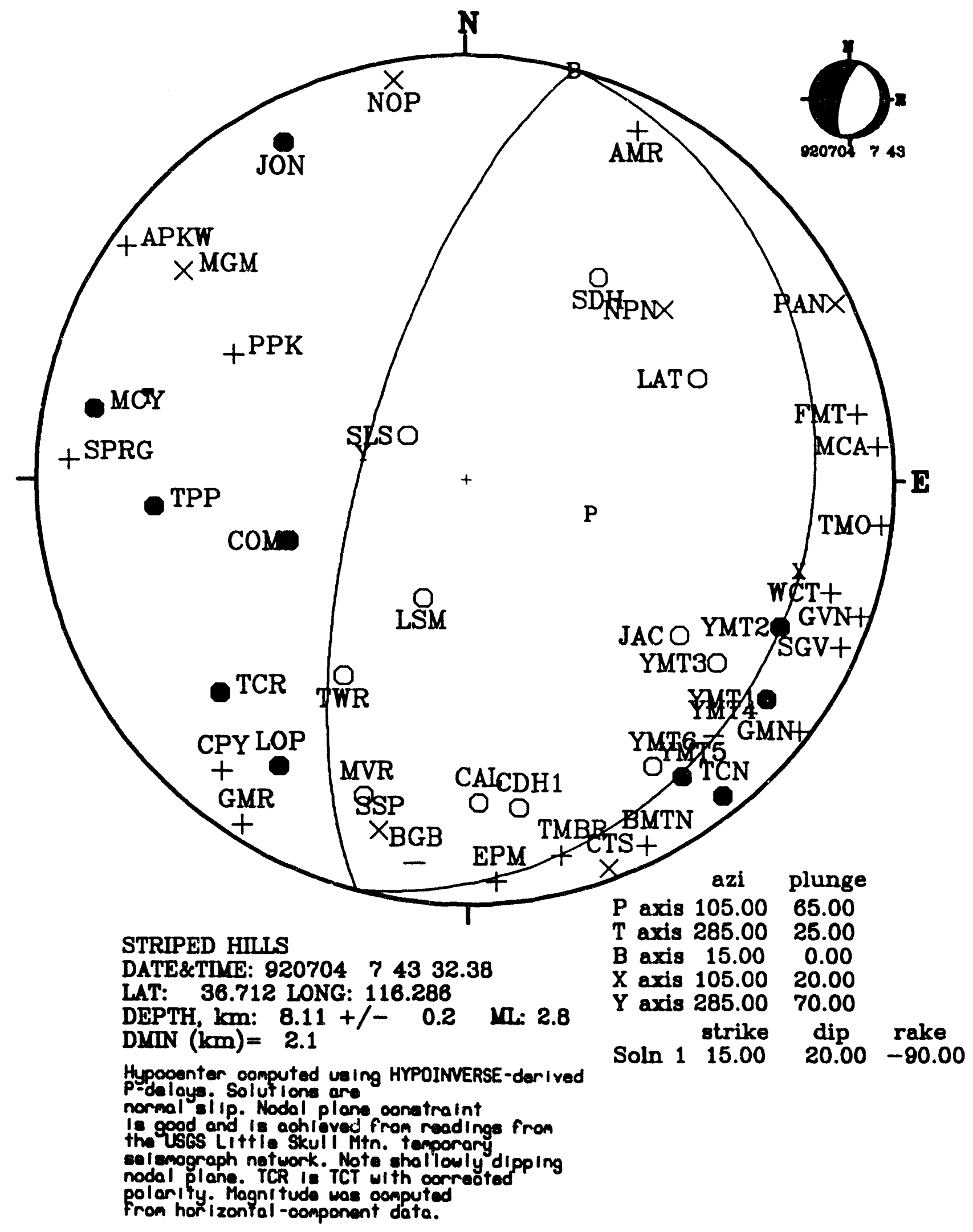




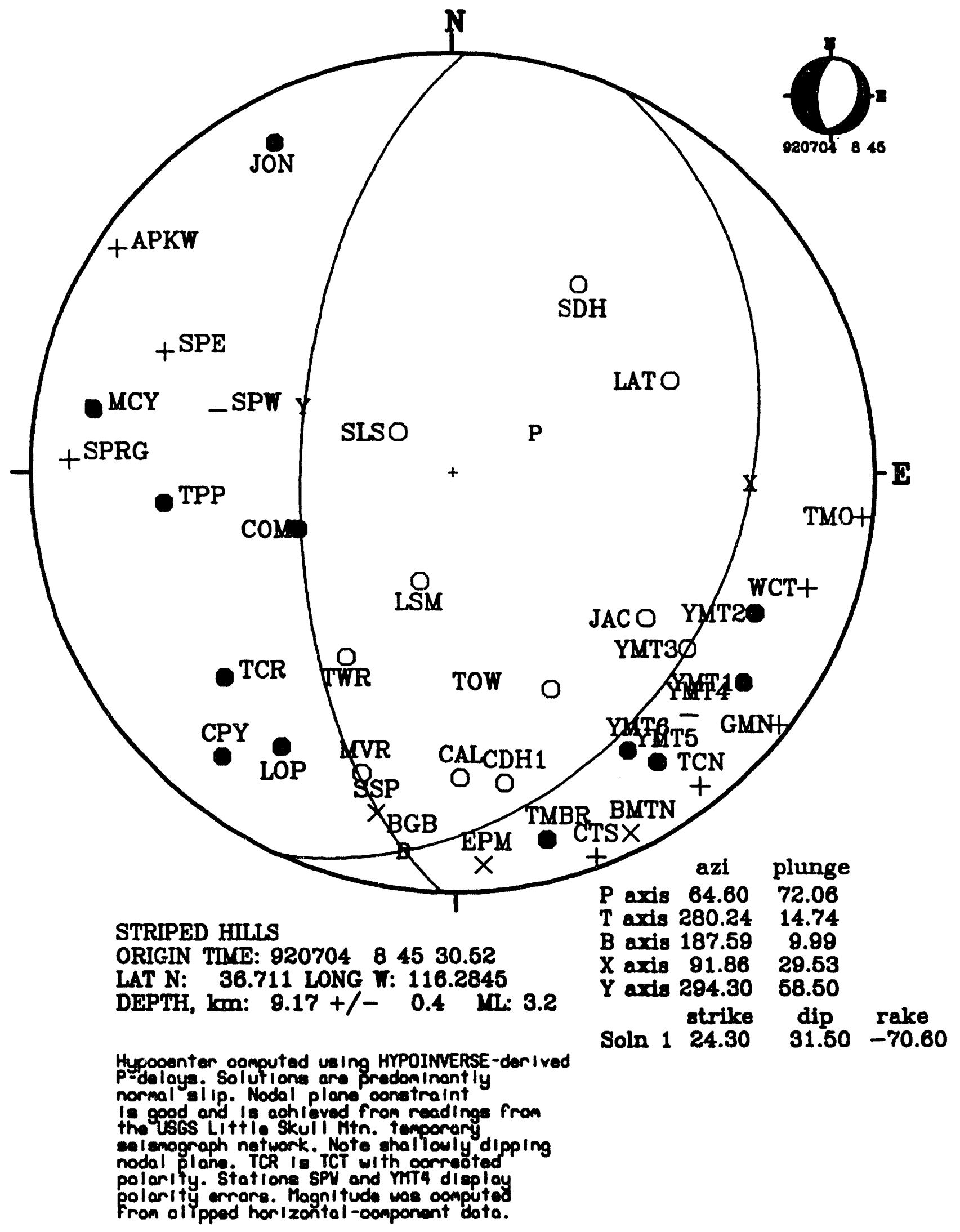




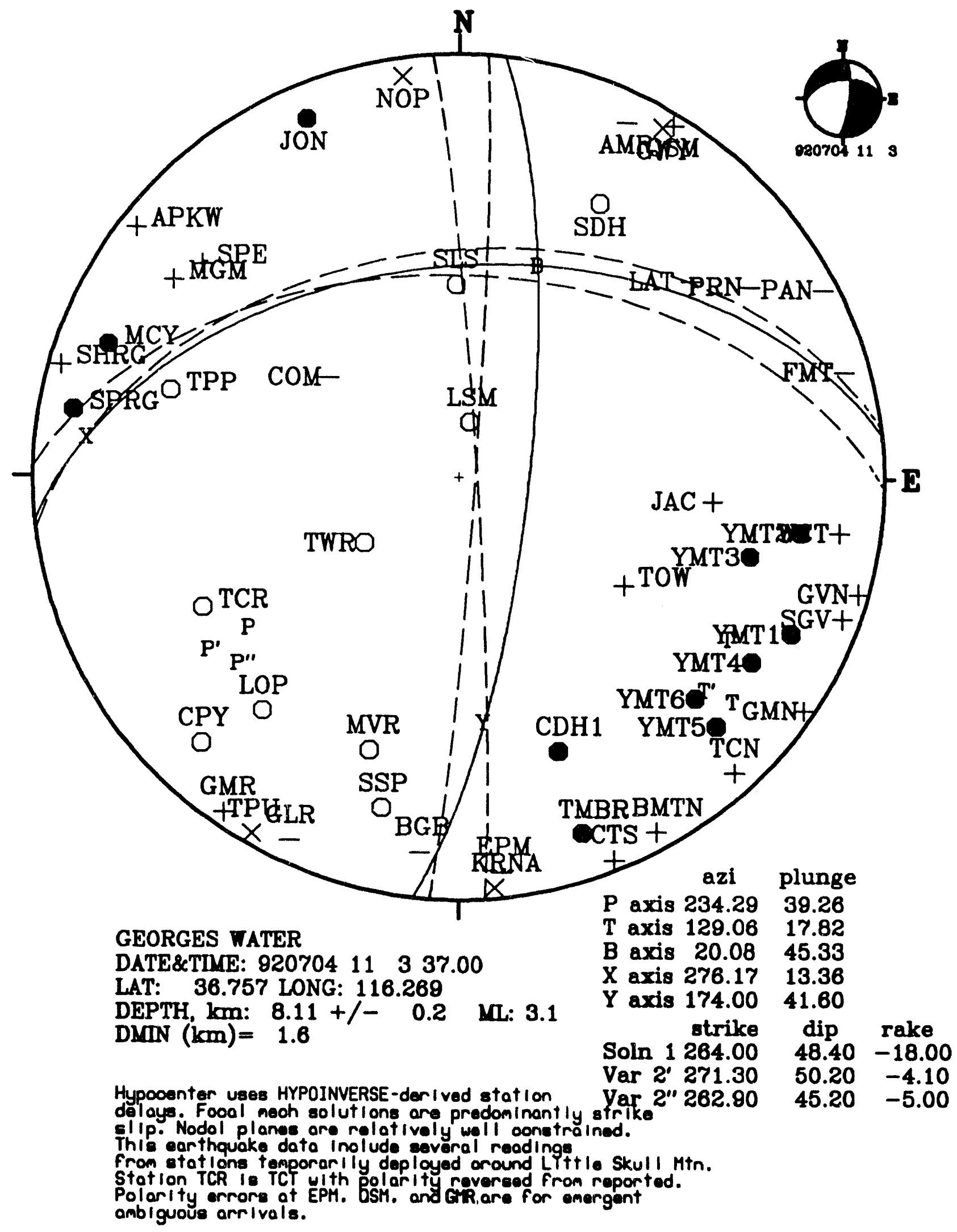

Magnitude estimate is rough. based on LSM hor iz-oomp. amplitude. 


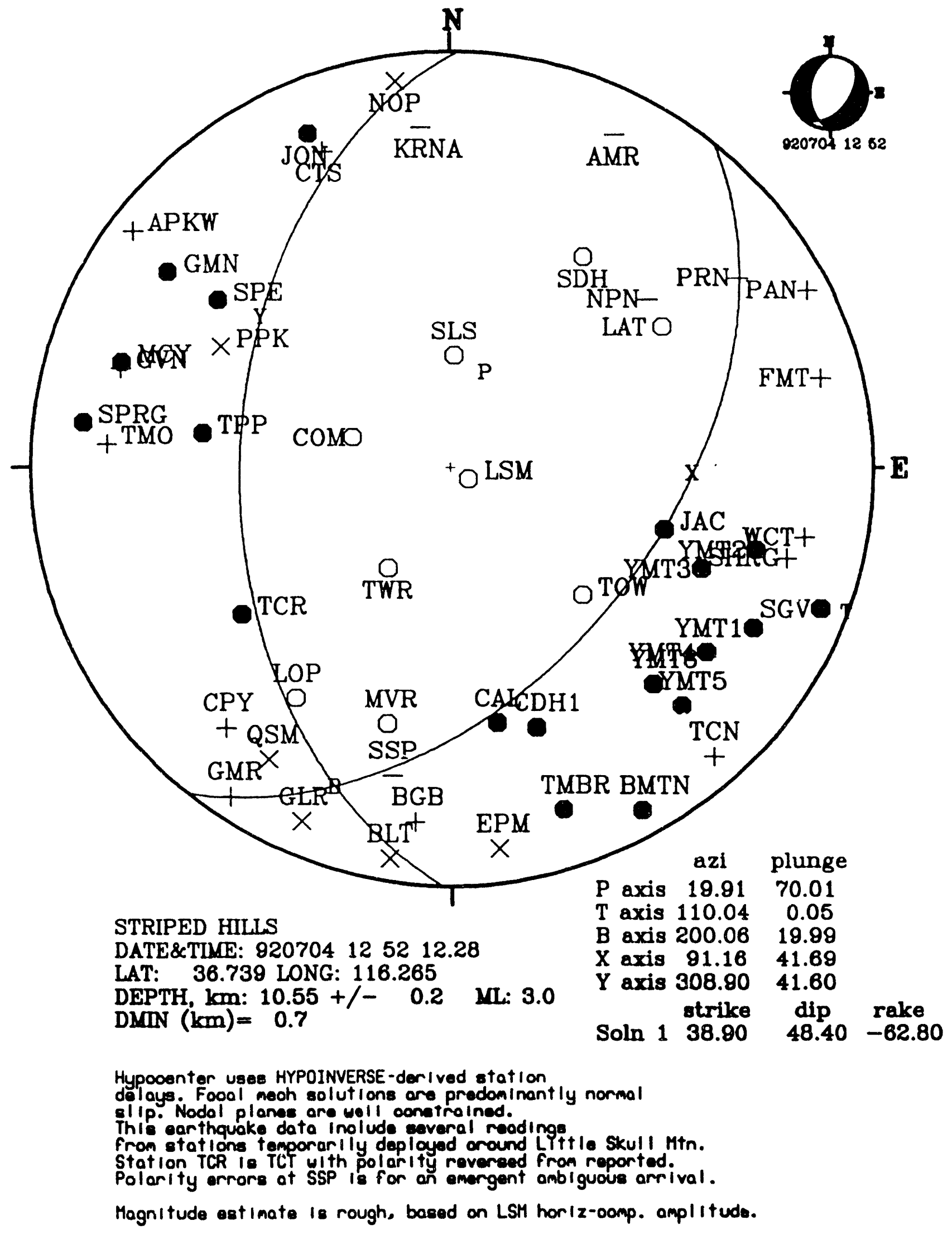




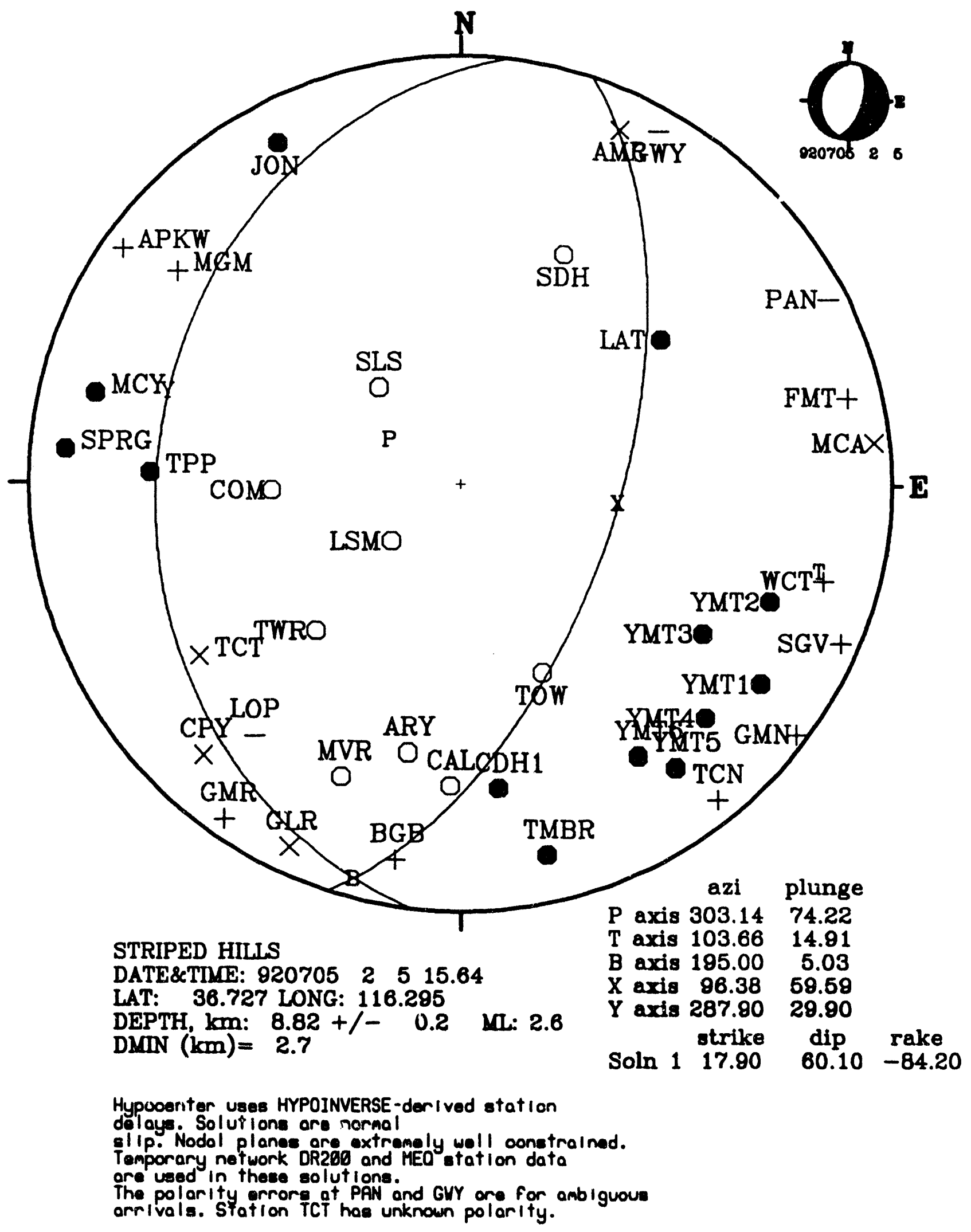




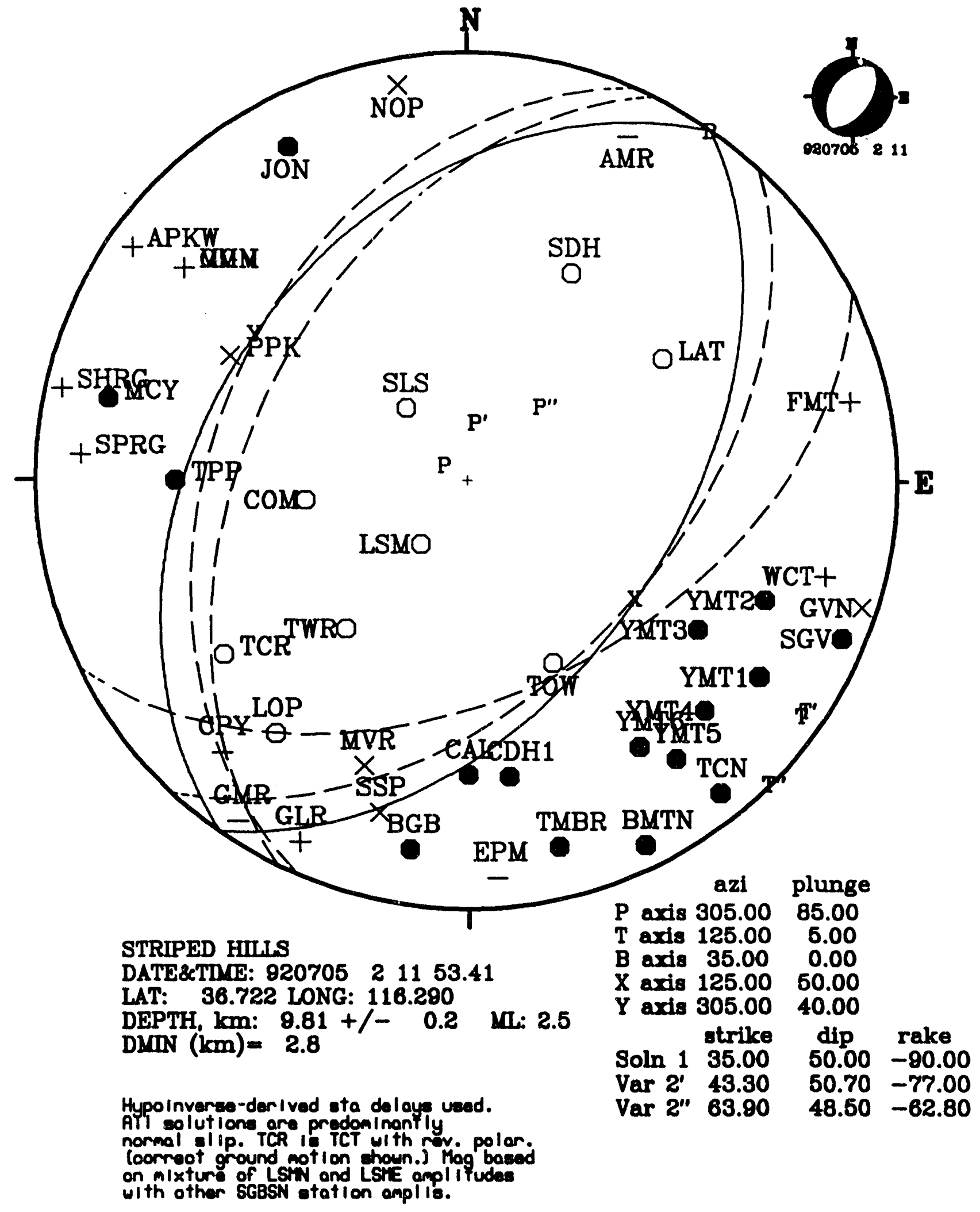




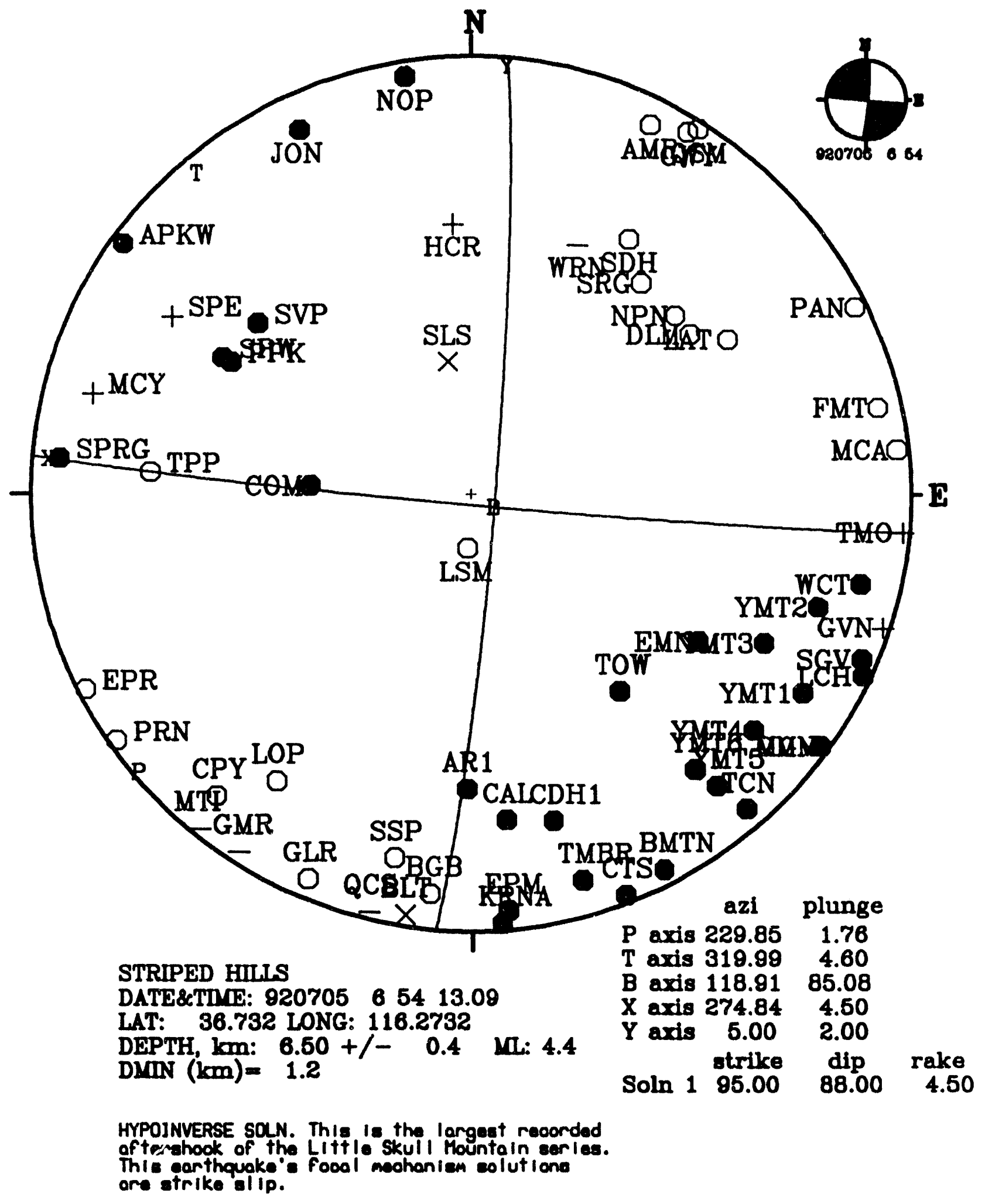

Magnitude eatimate is a lower bound. 


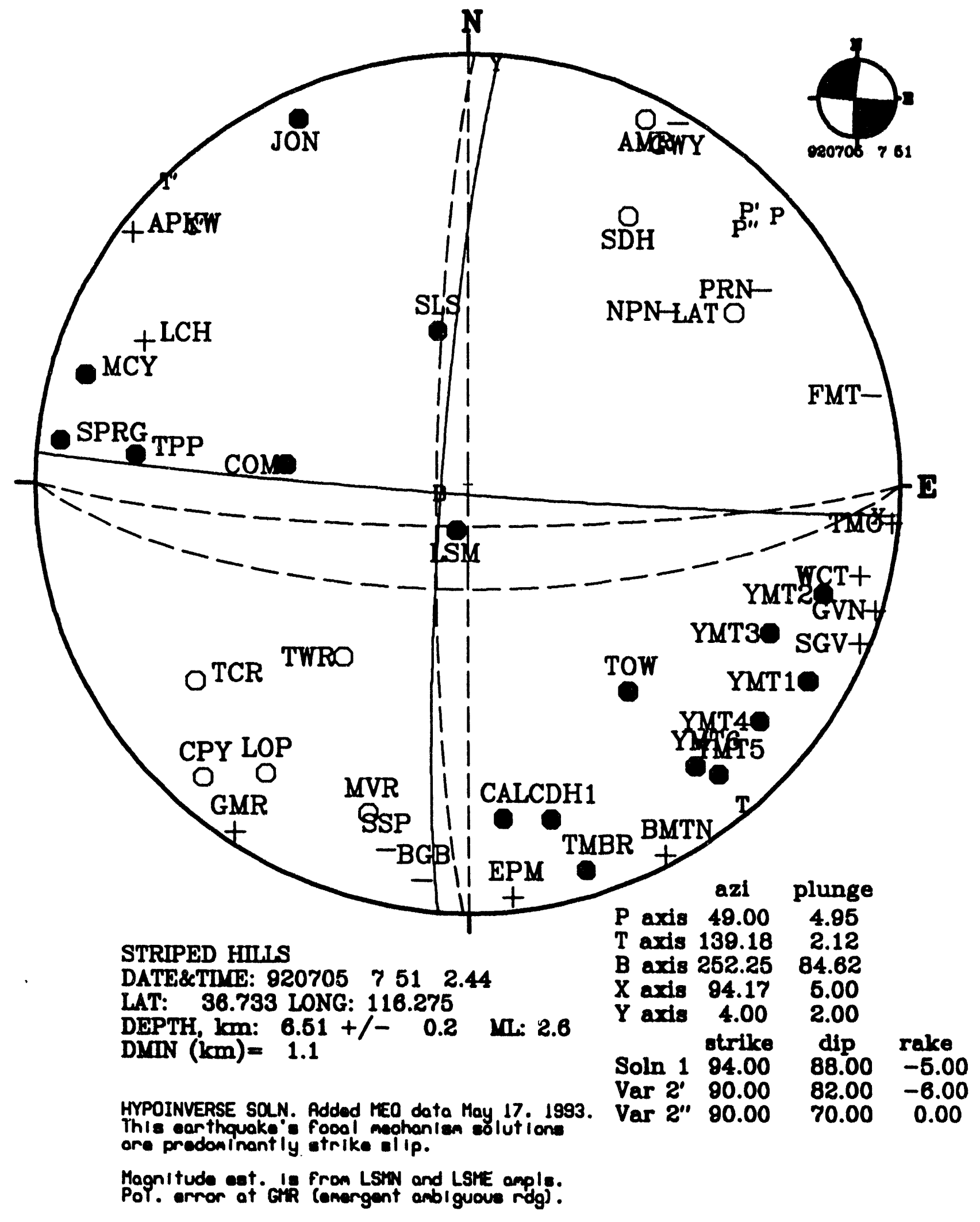




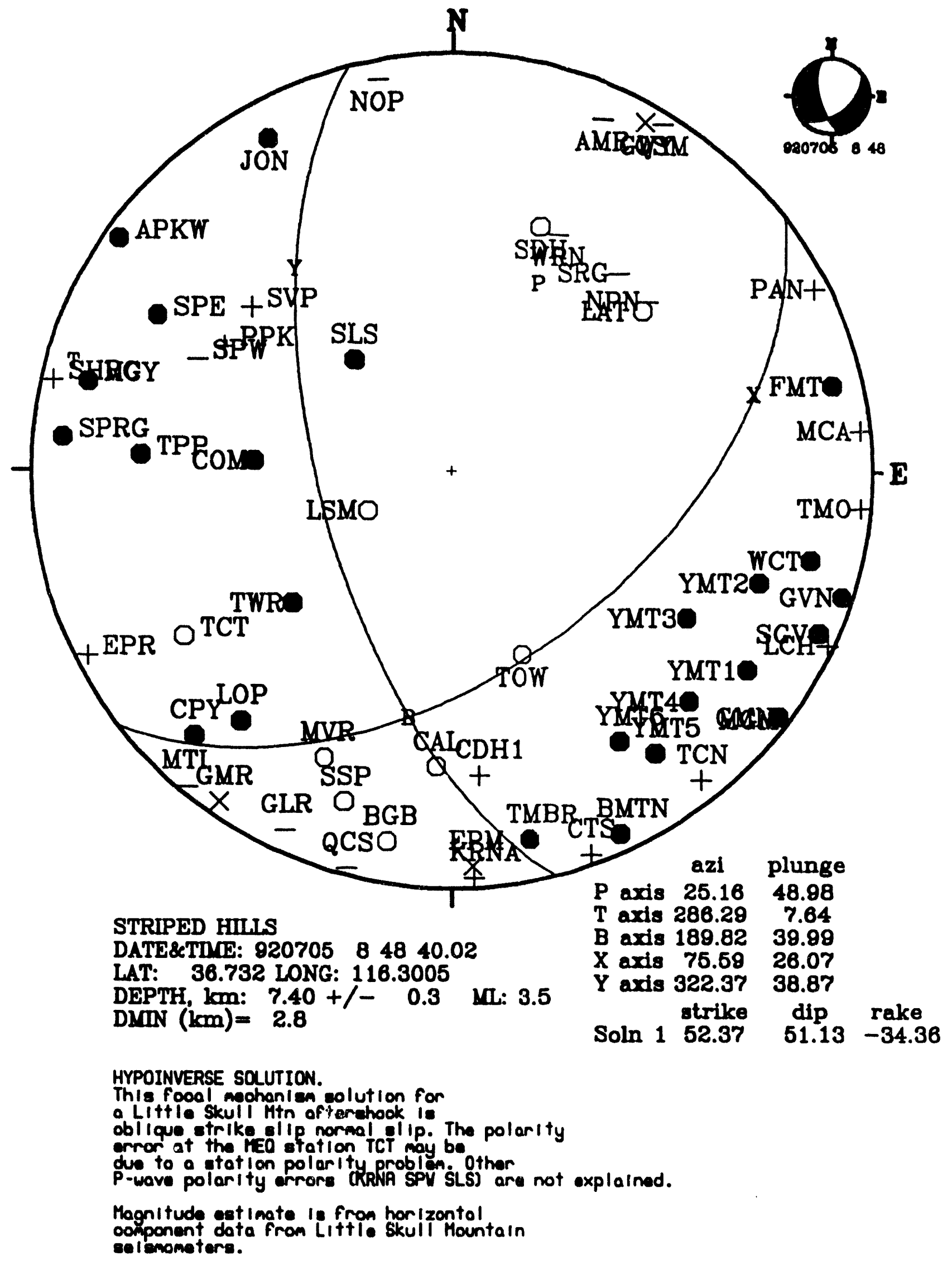




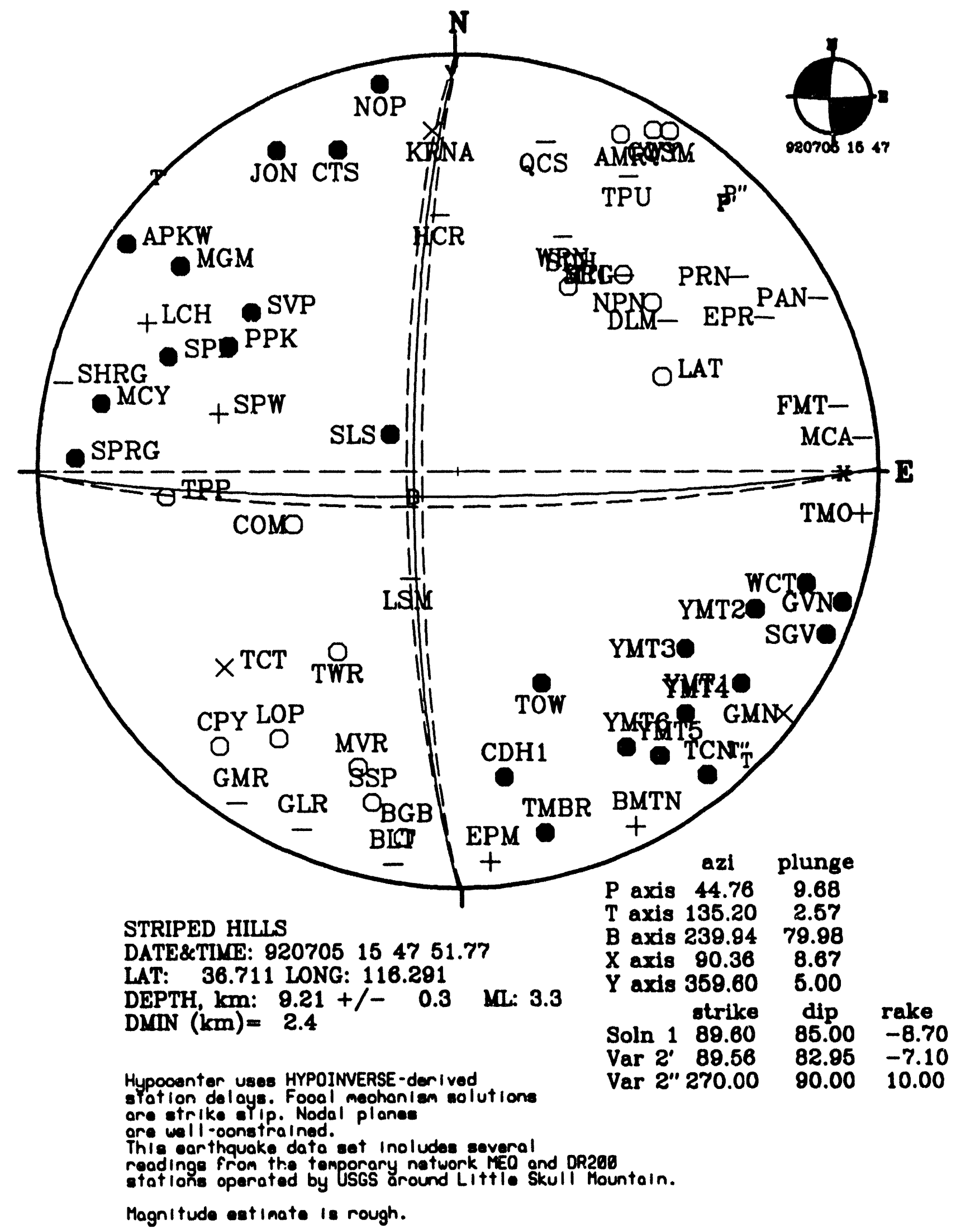




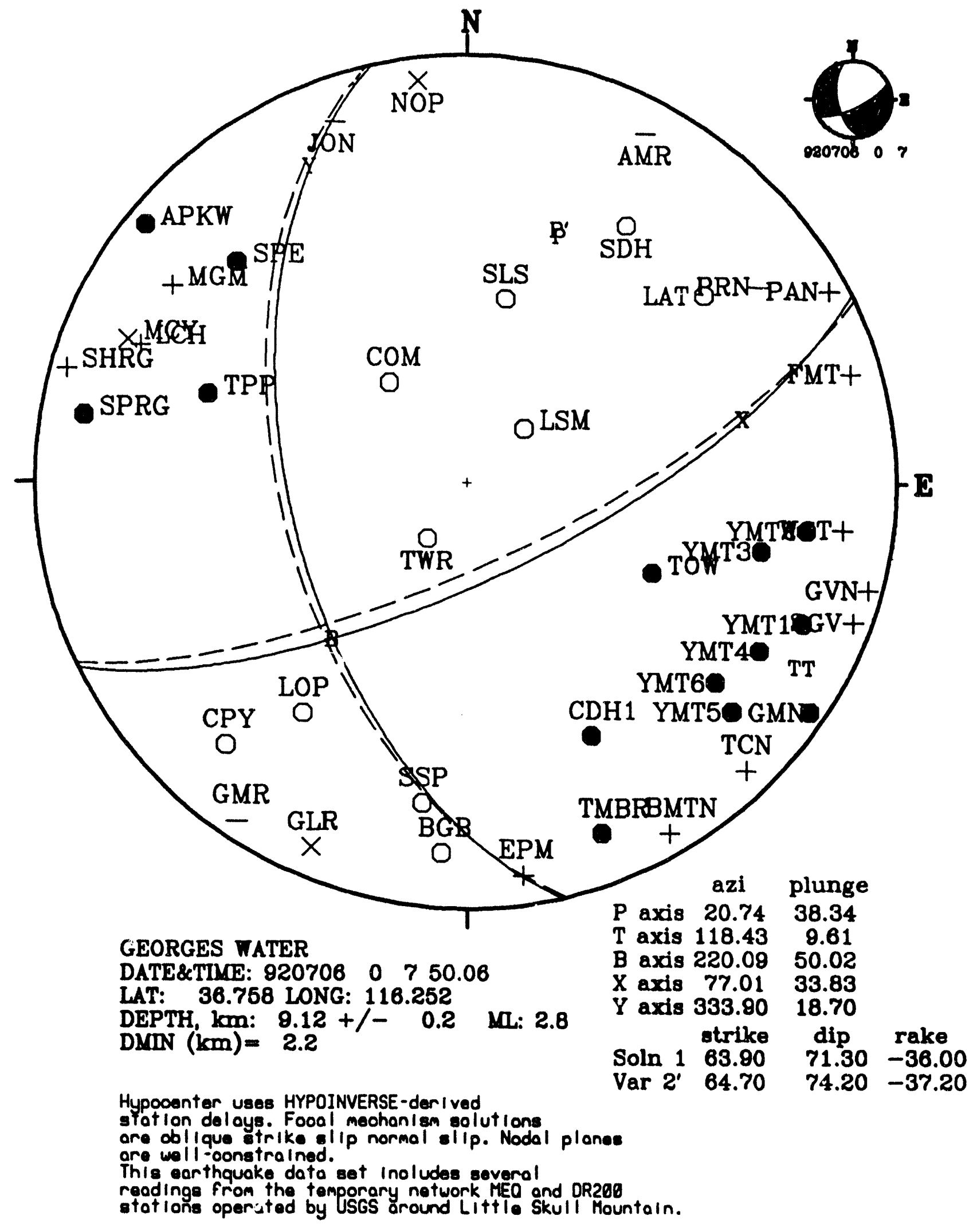




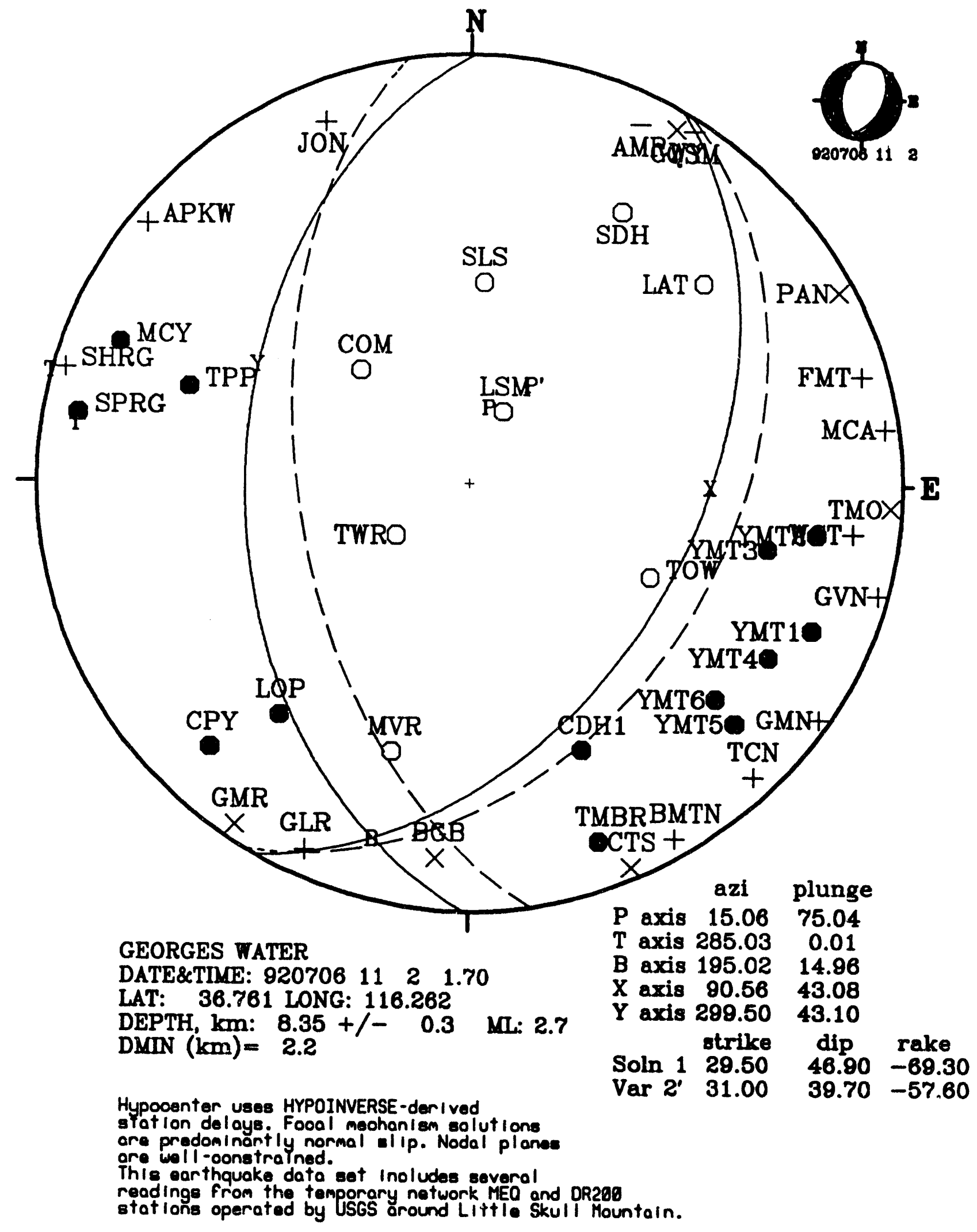




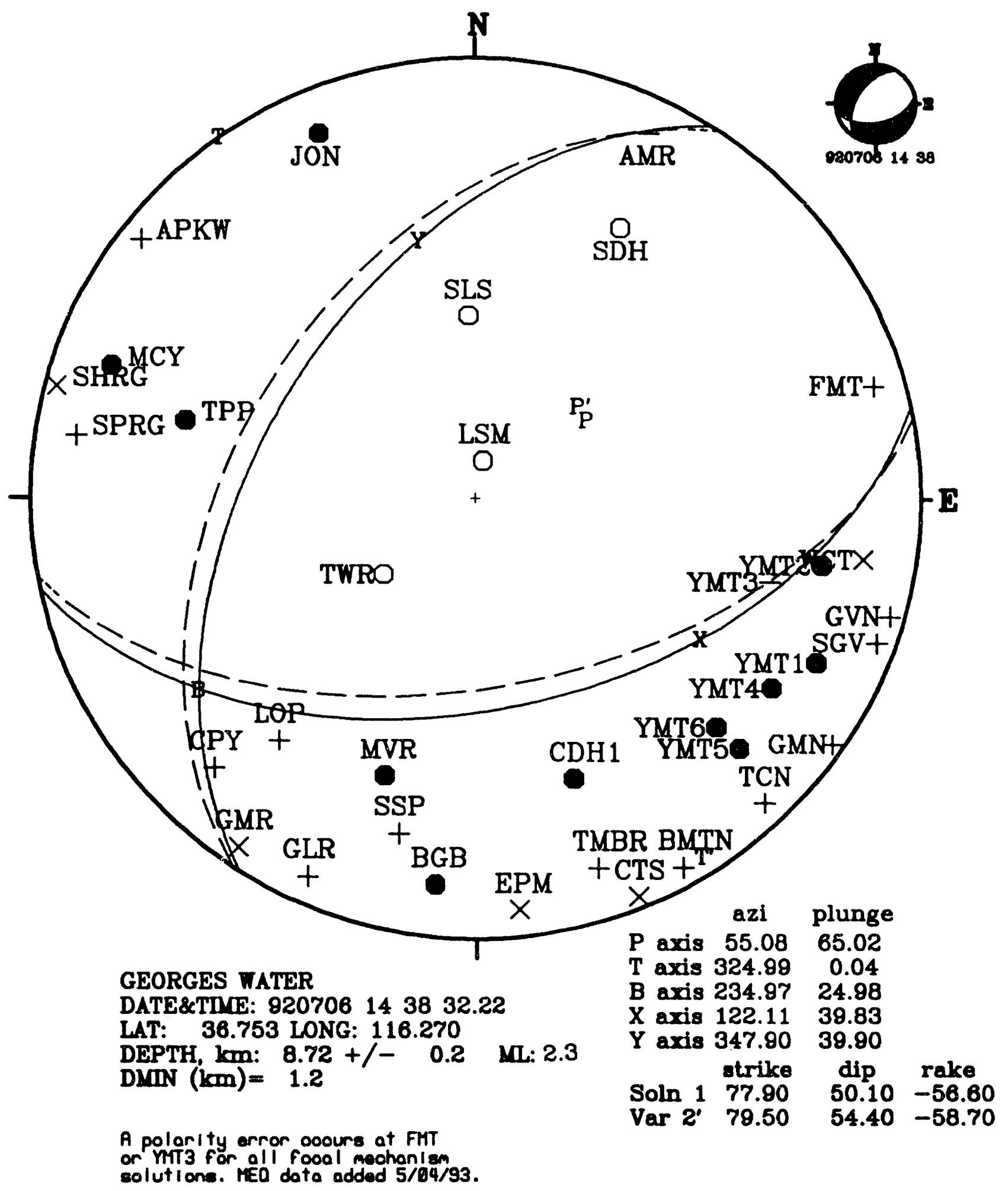




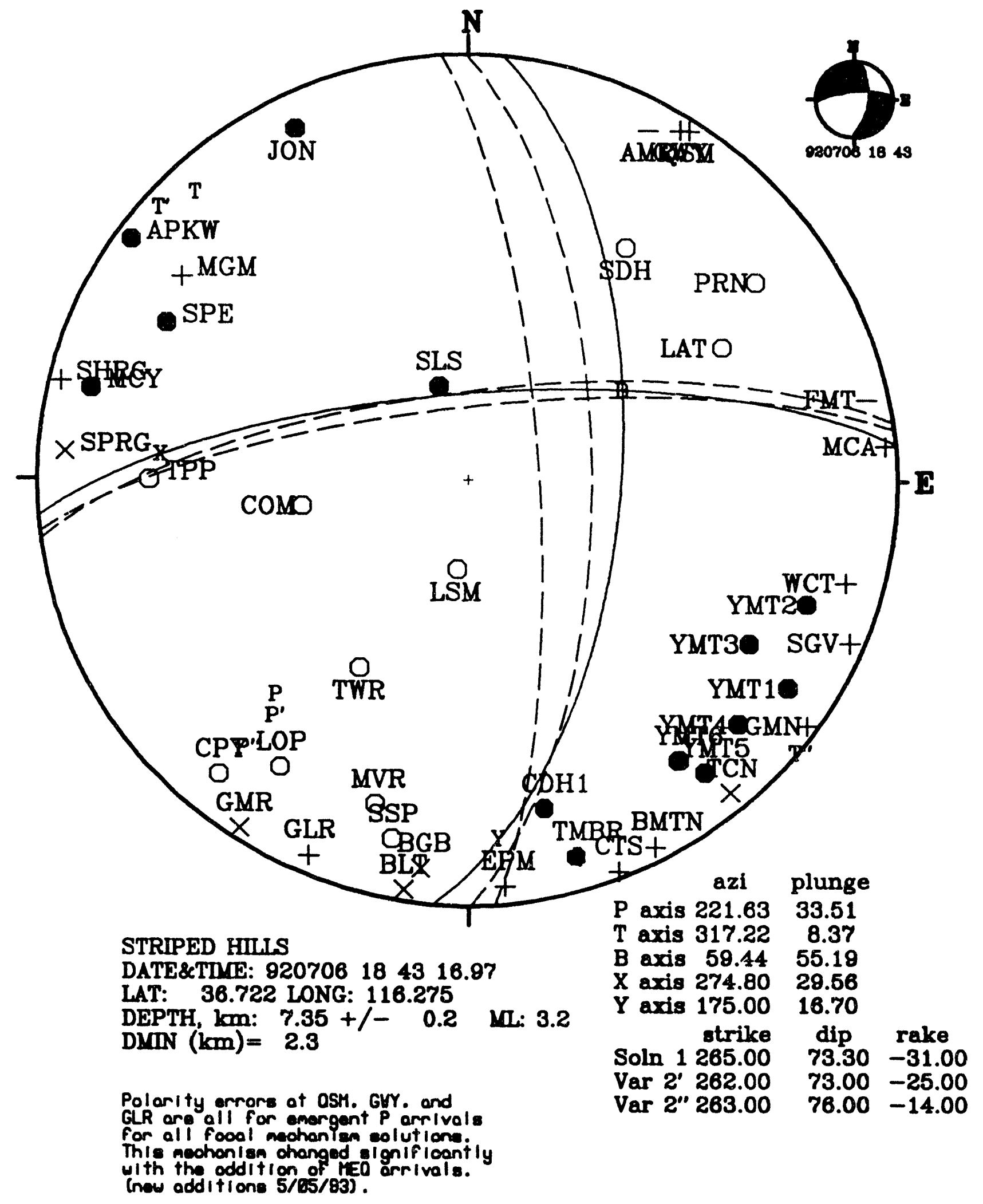




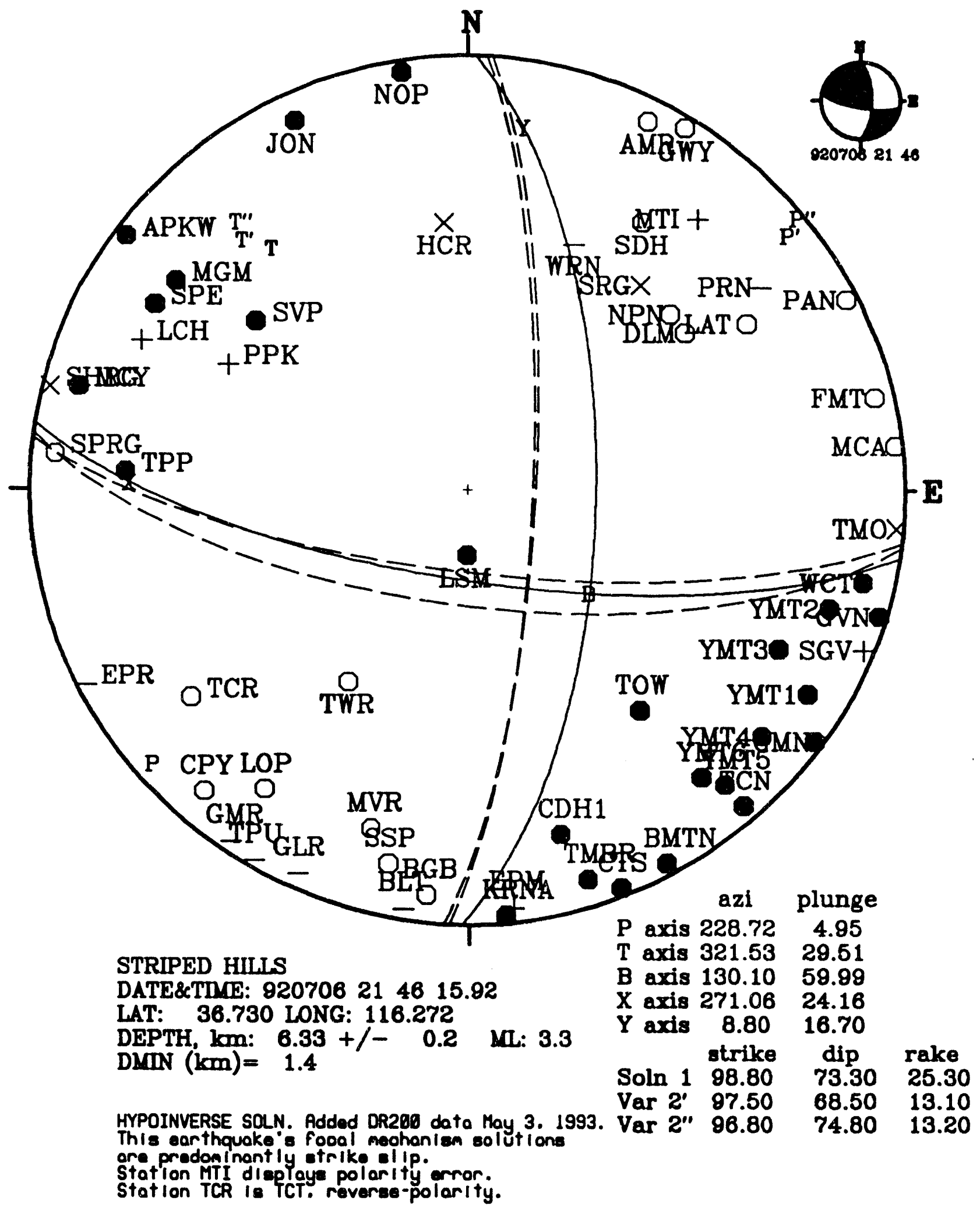




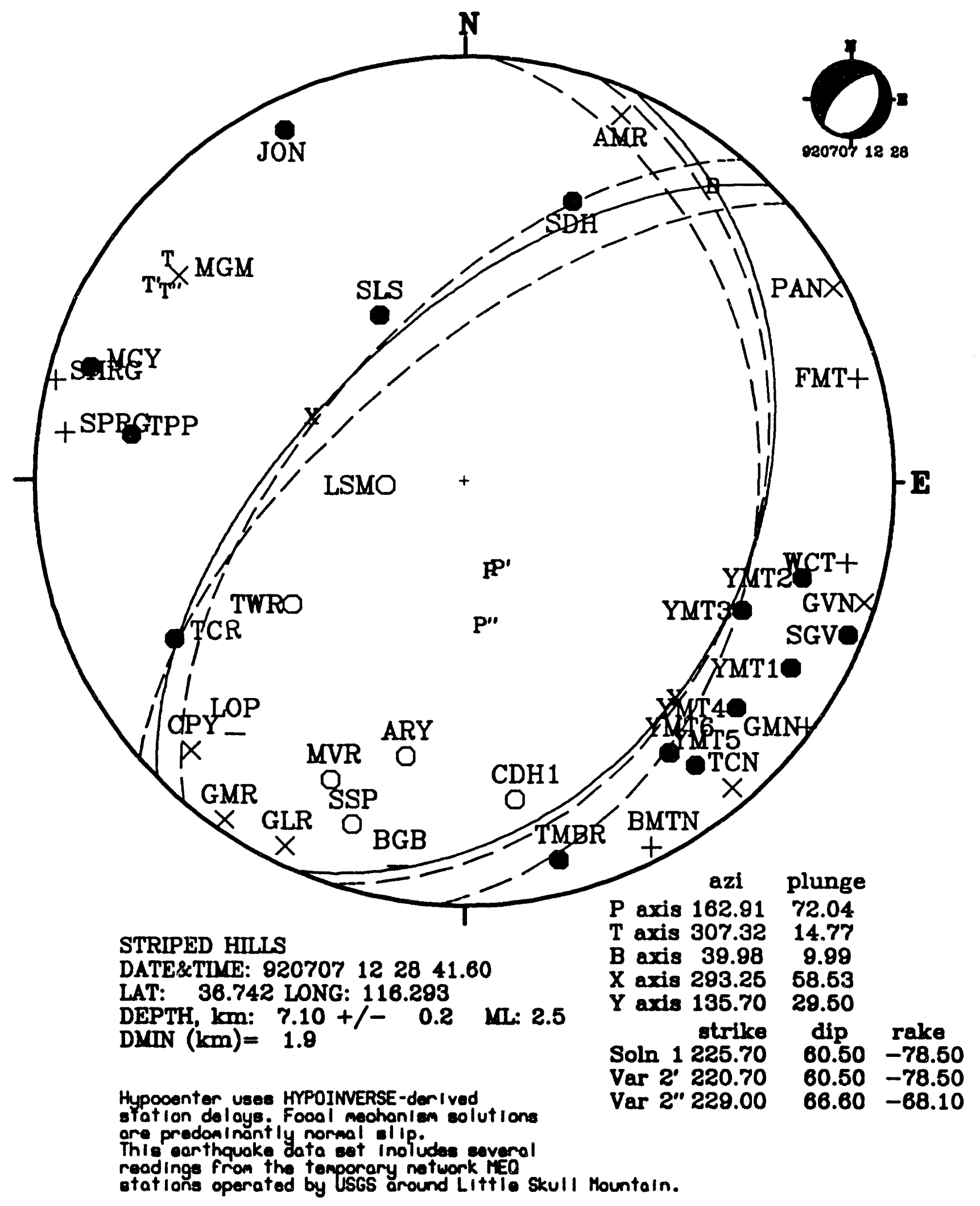




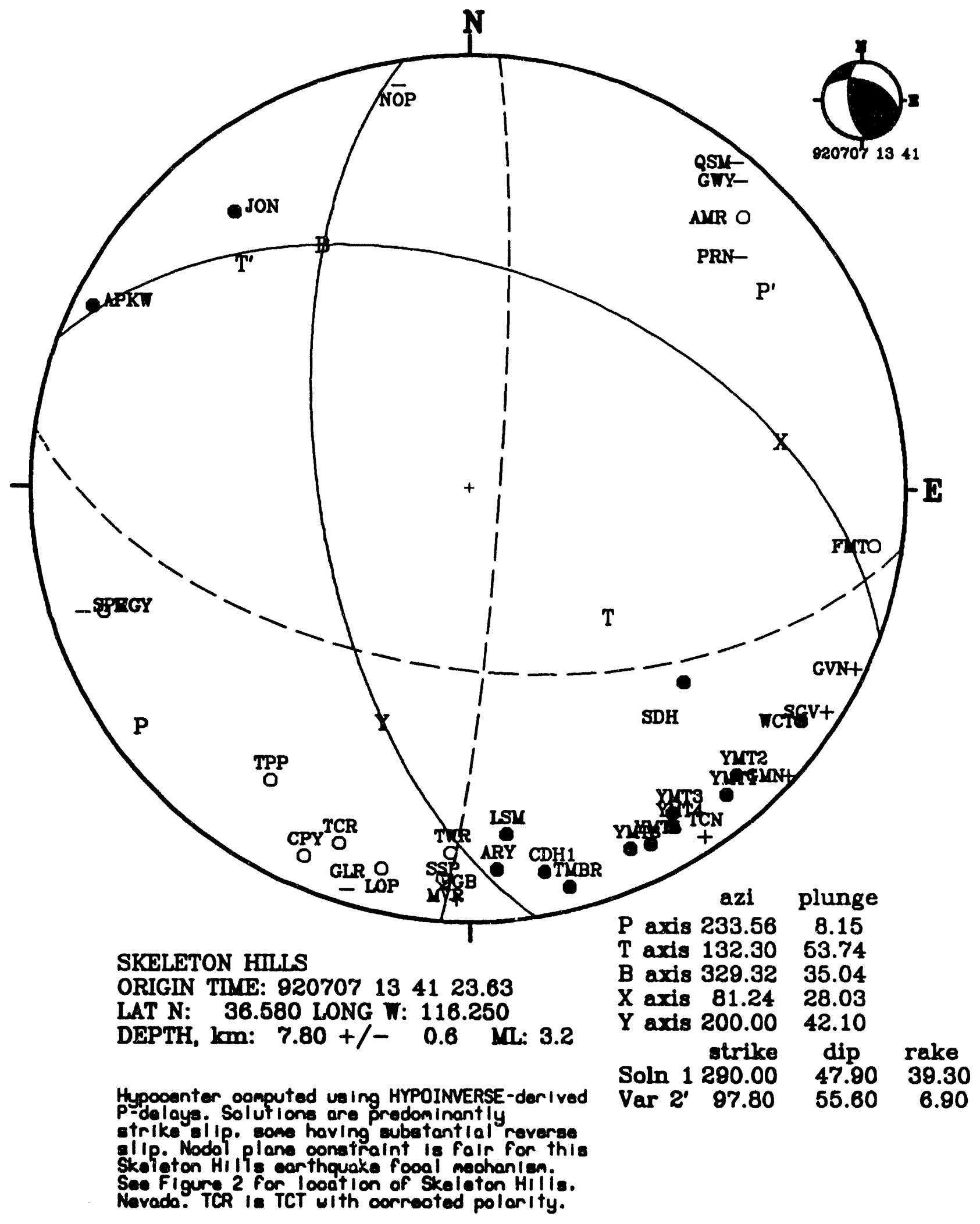




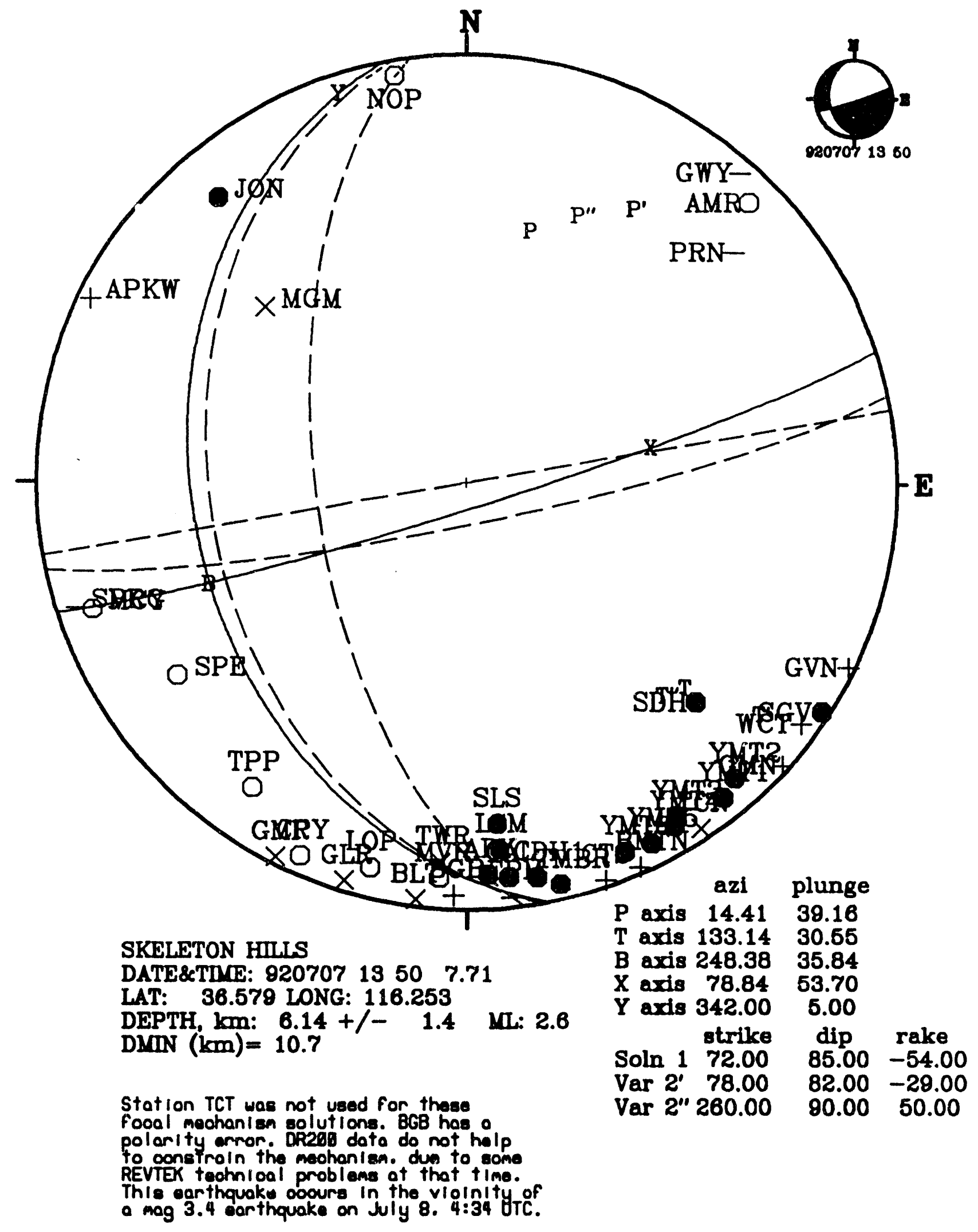




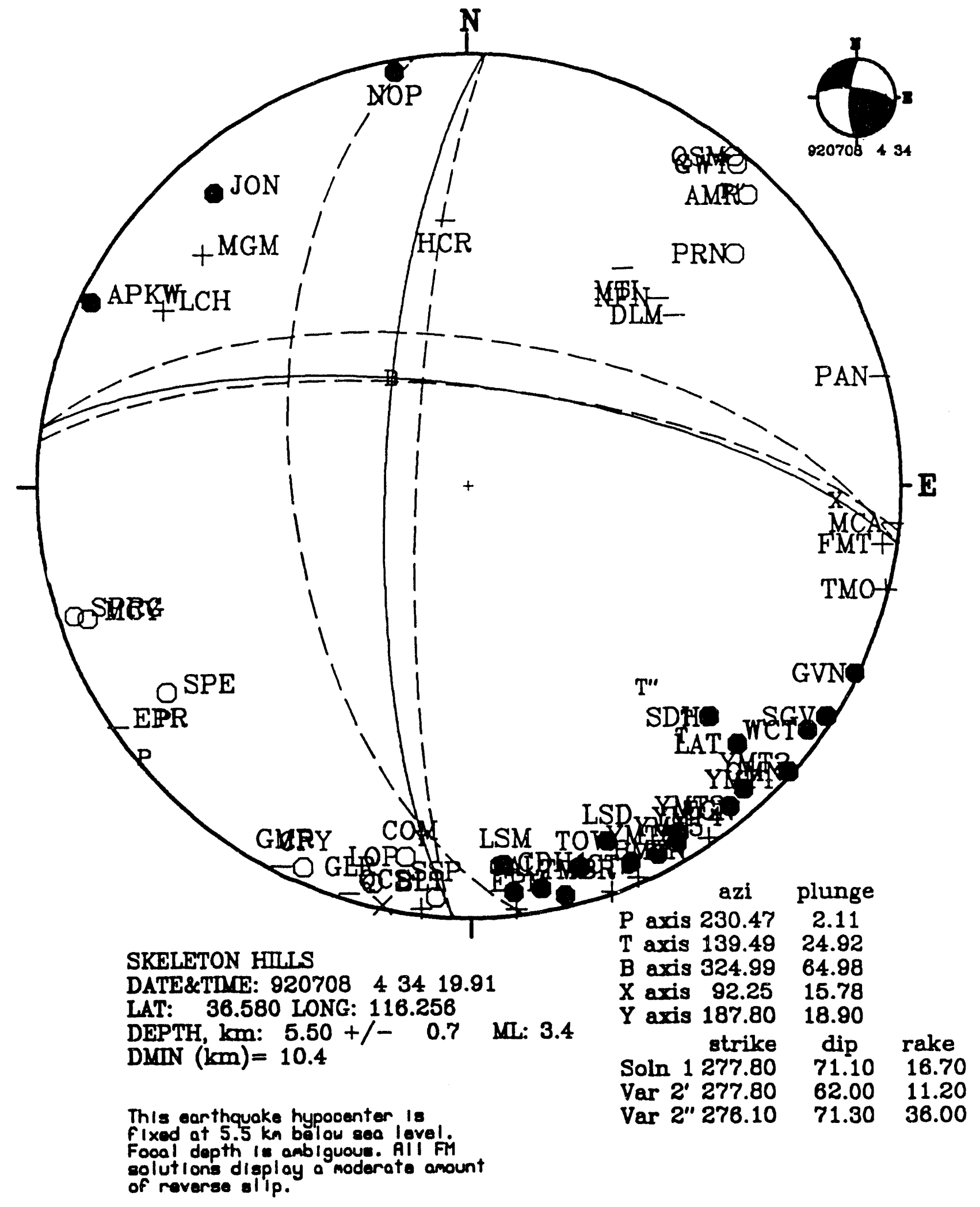




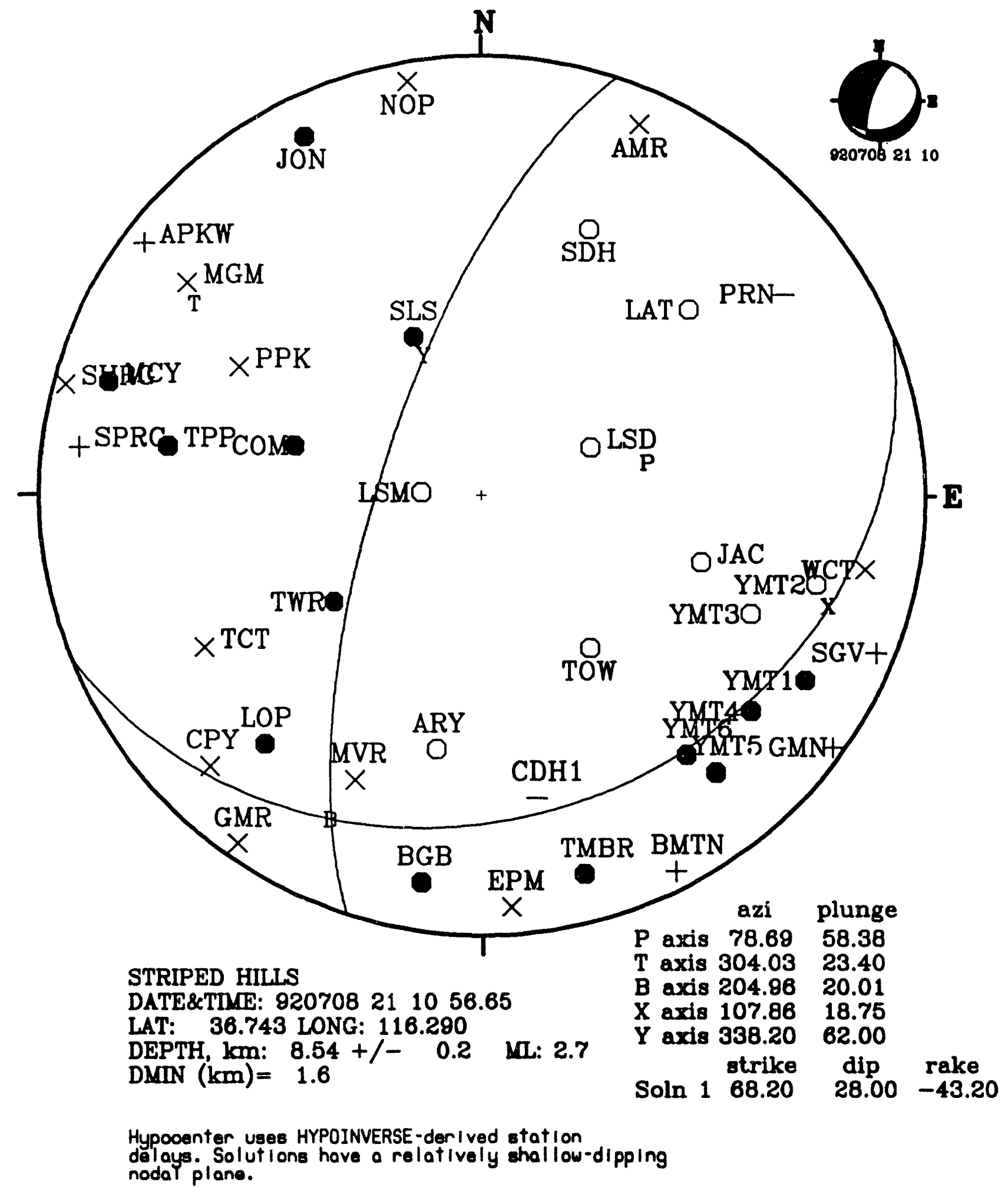




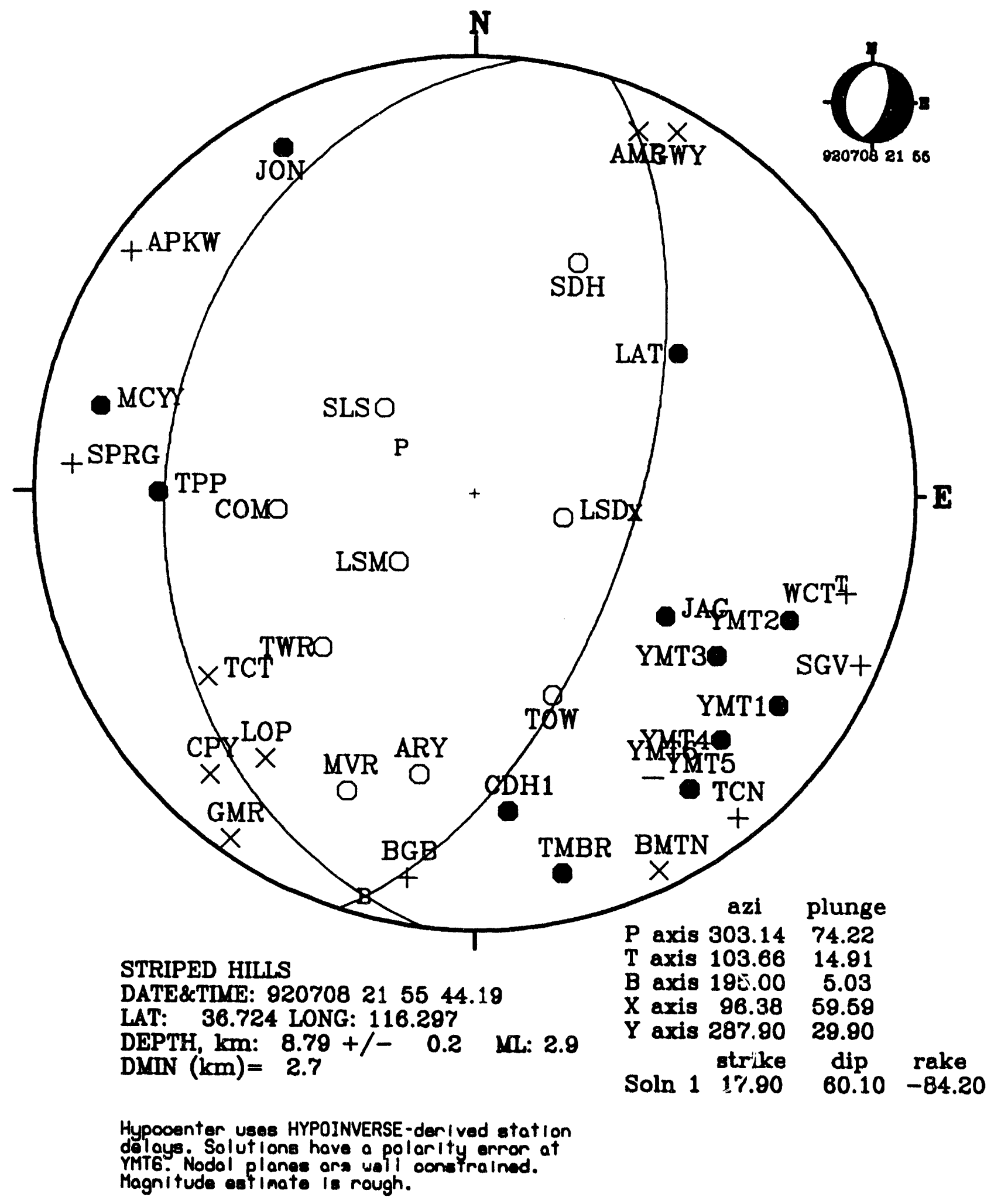




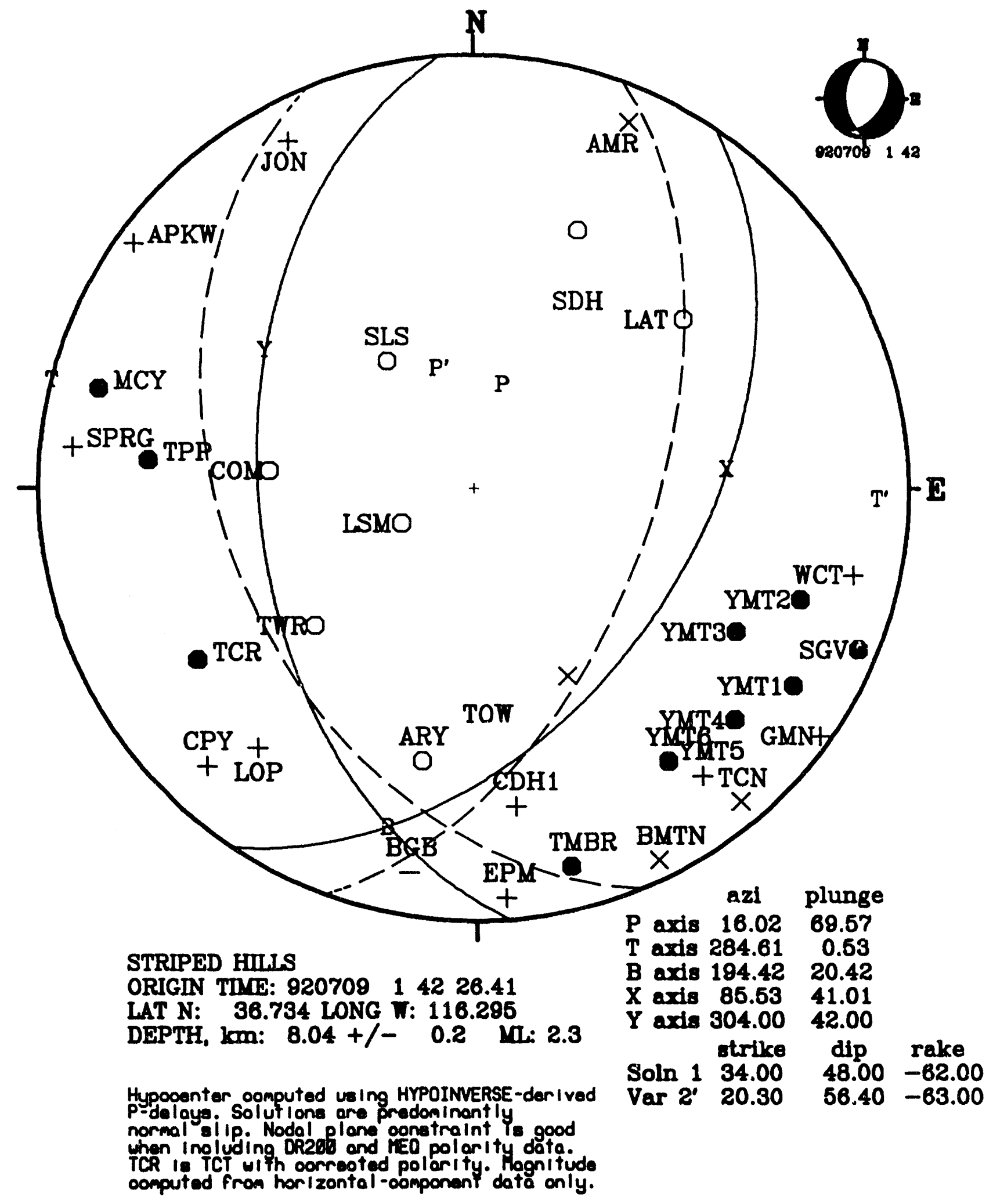




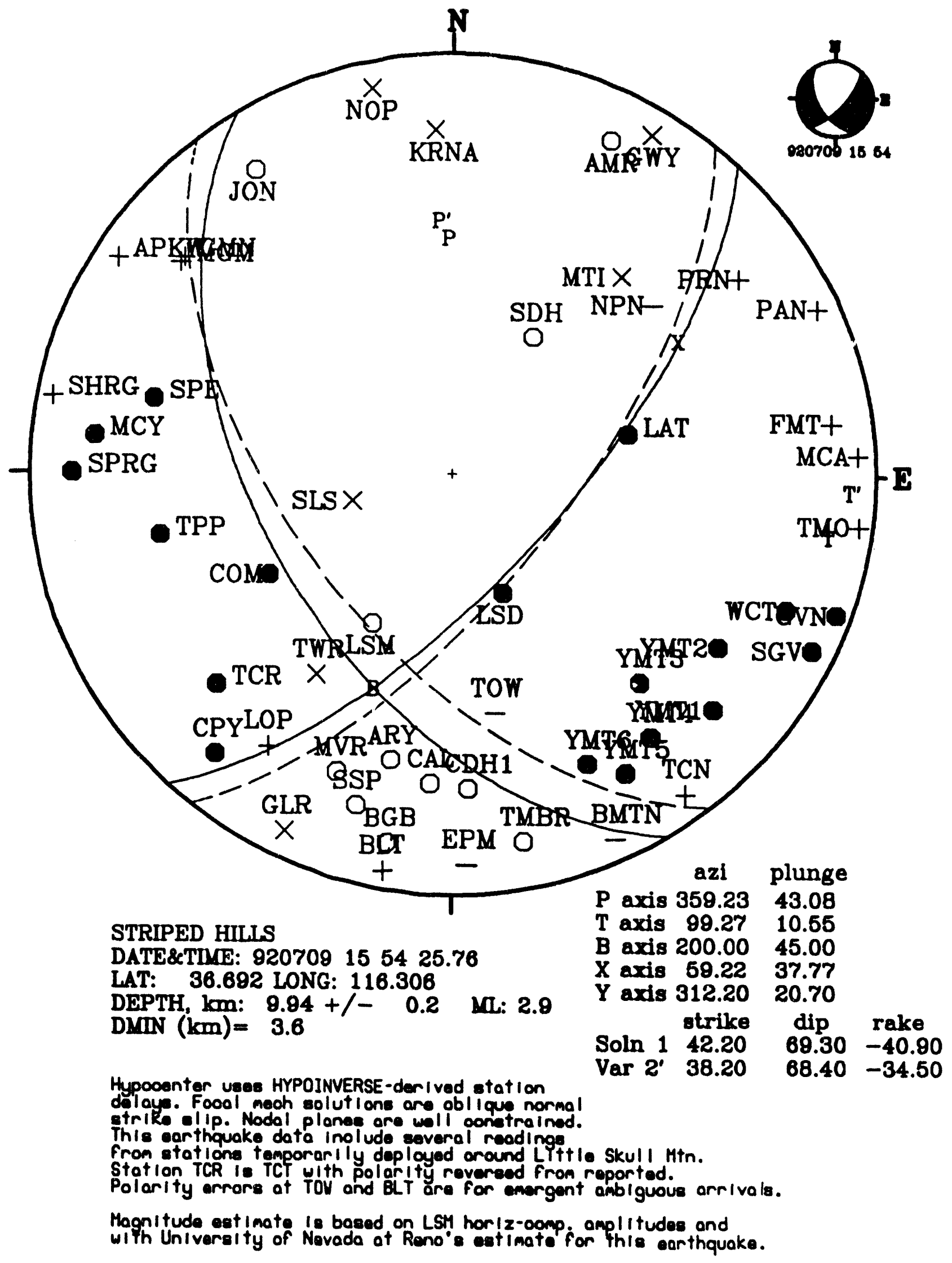




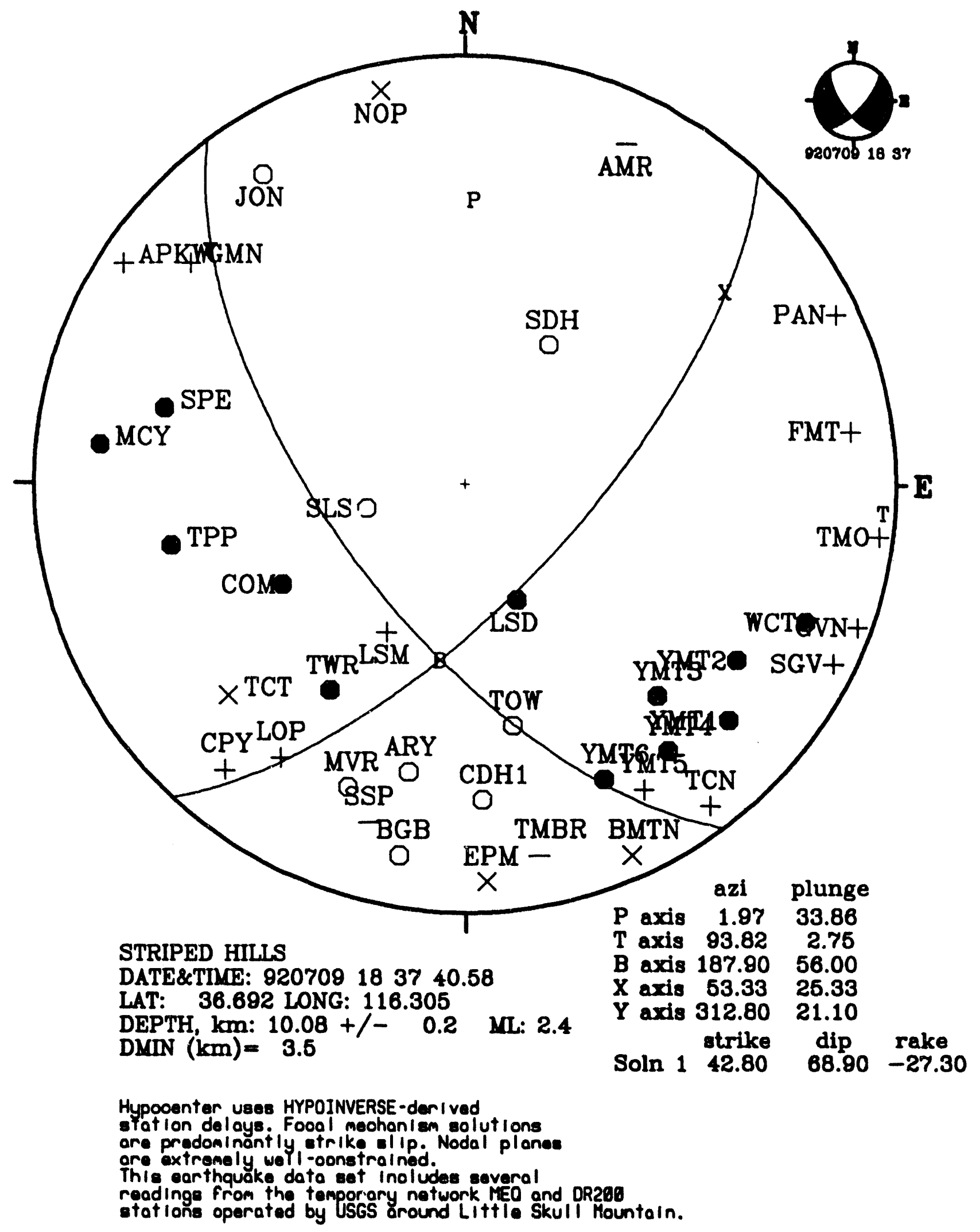




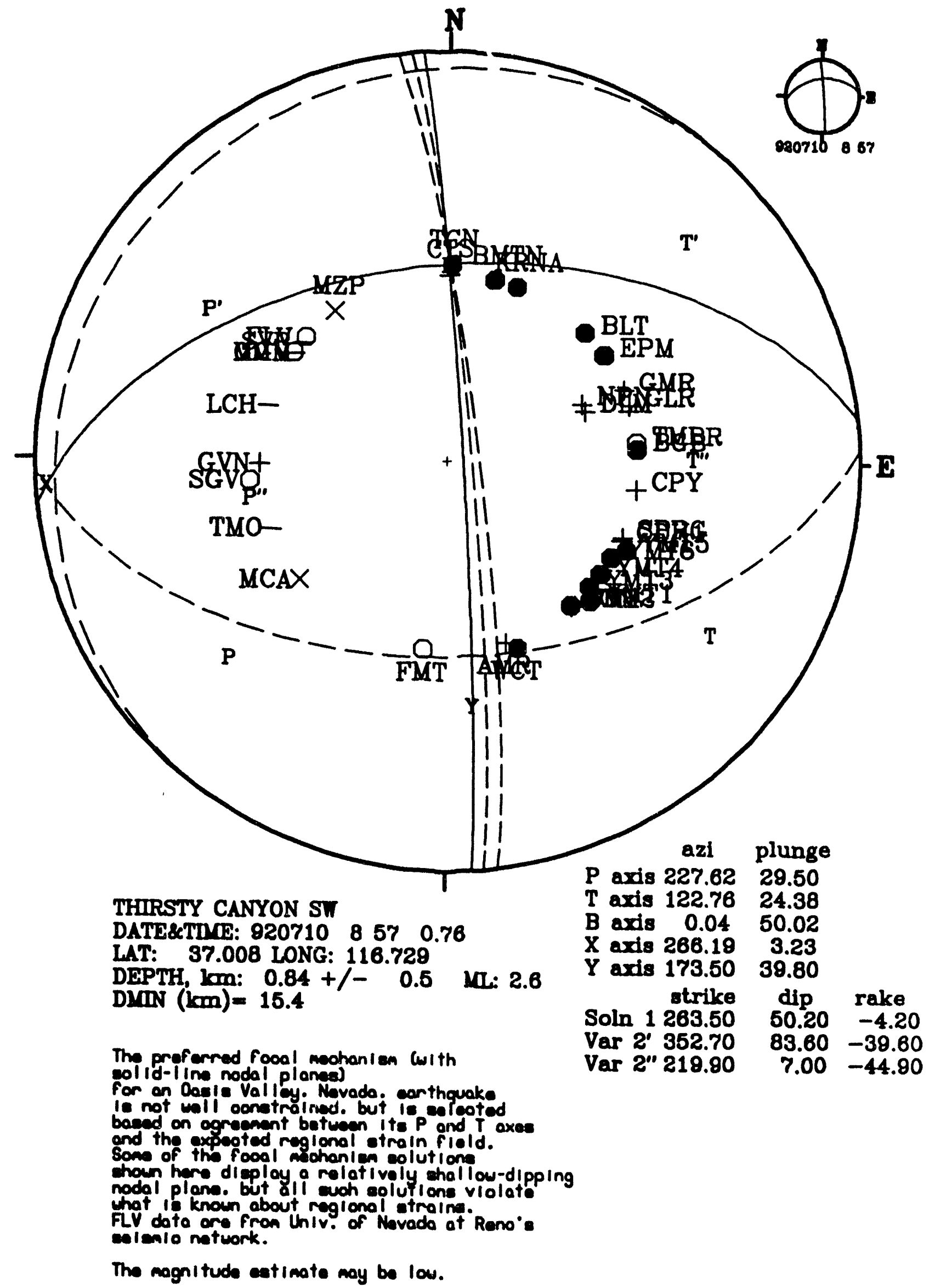



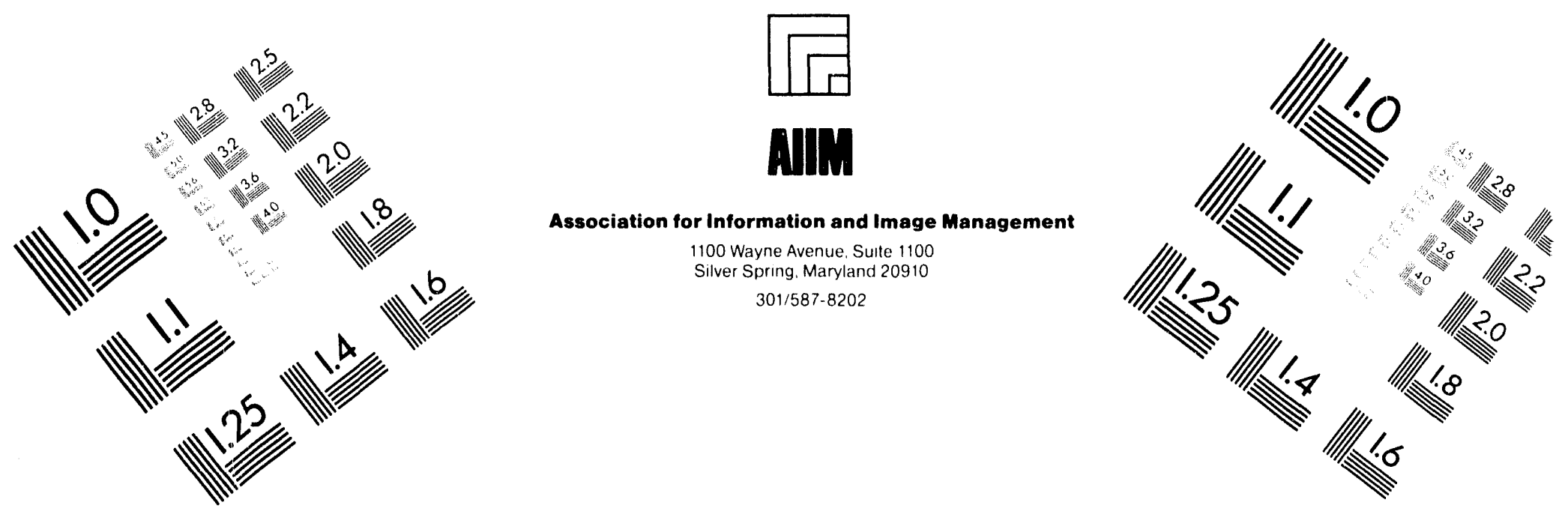

\section{Centimeter}

1
$\mid$
$\mid$ Inches
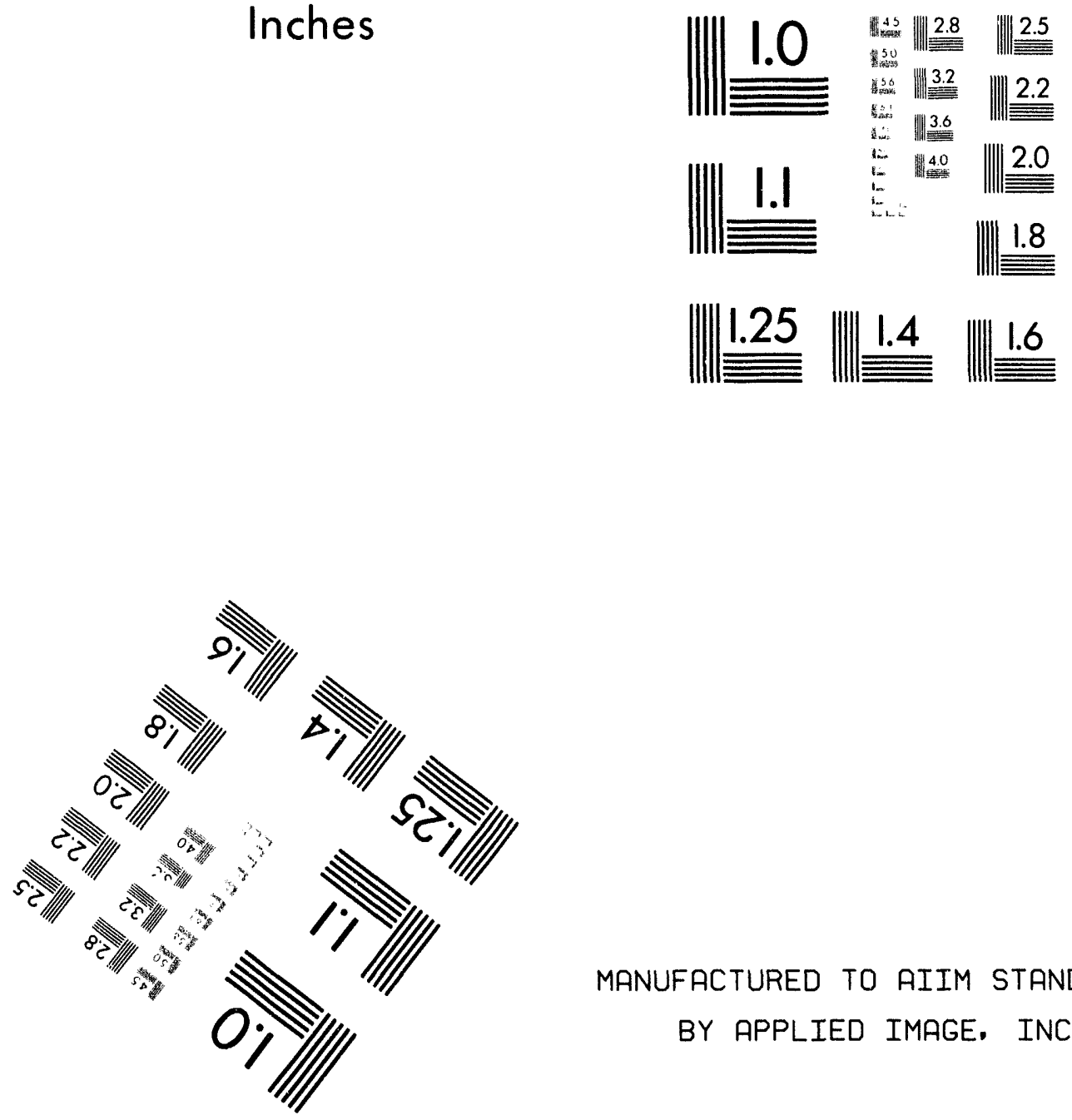

MANUFACTURED TO AIIM STANDARDS

BY APPLIED IMAGE, INC.

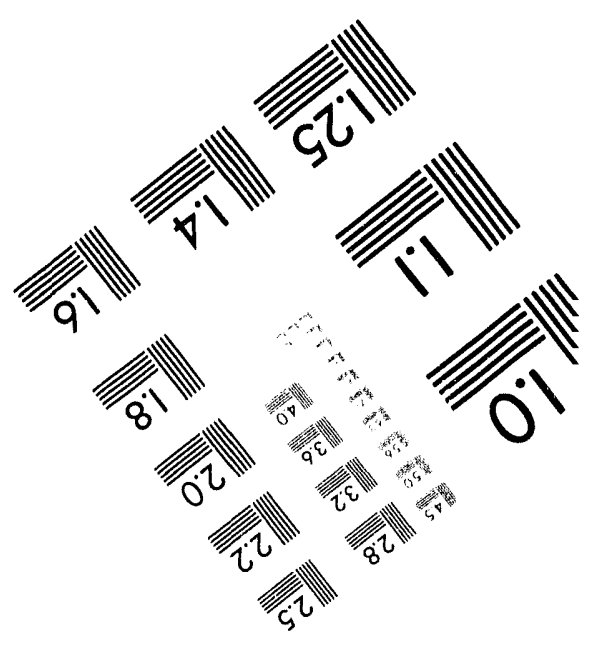



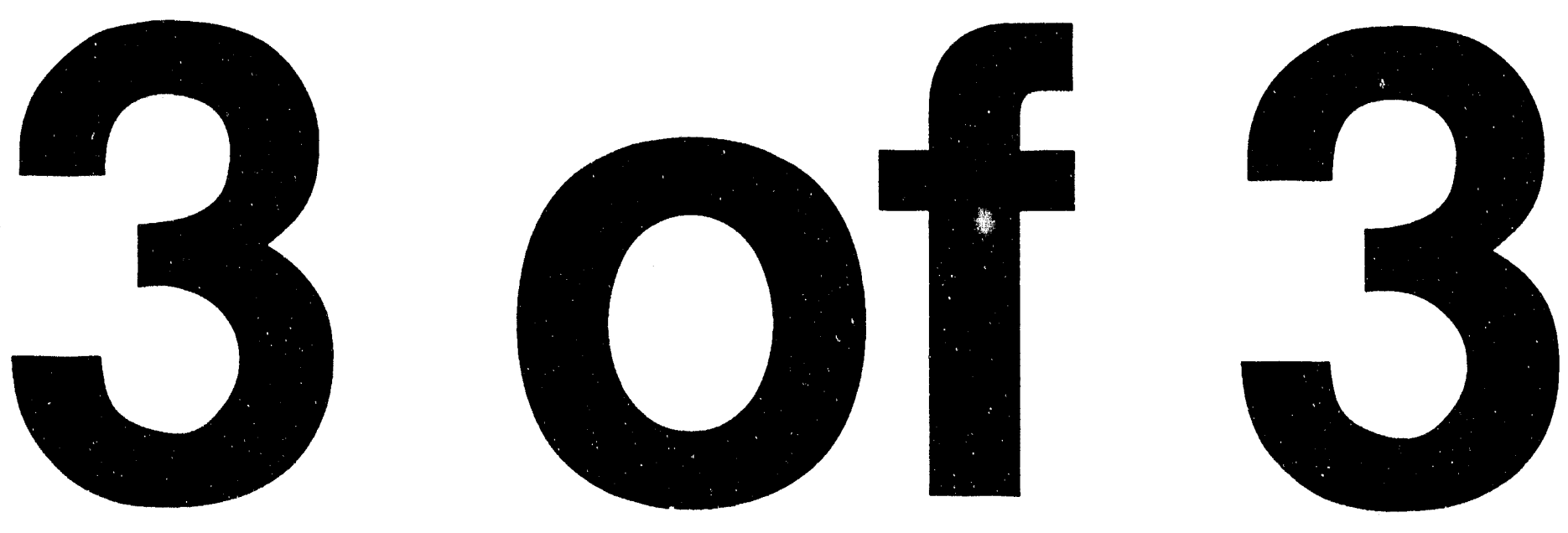


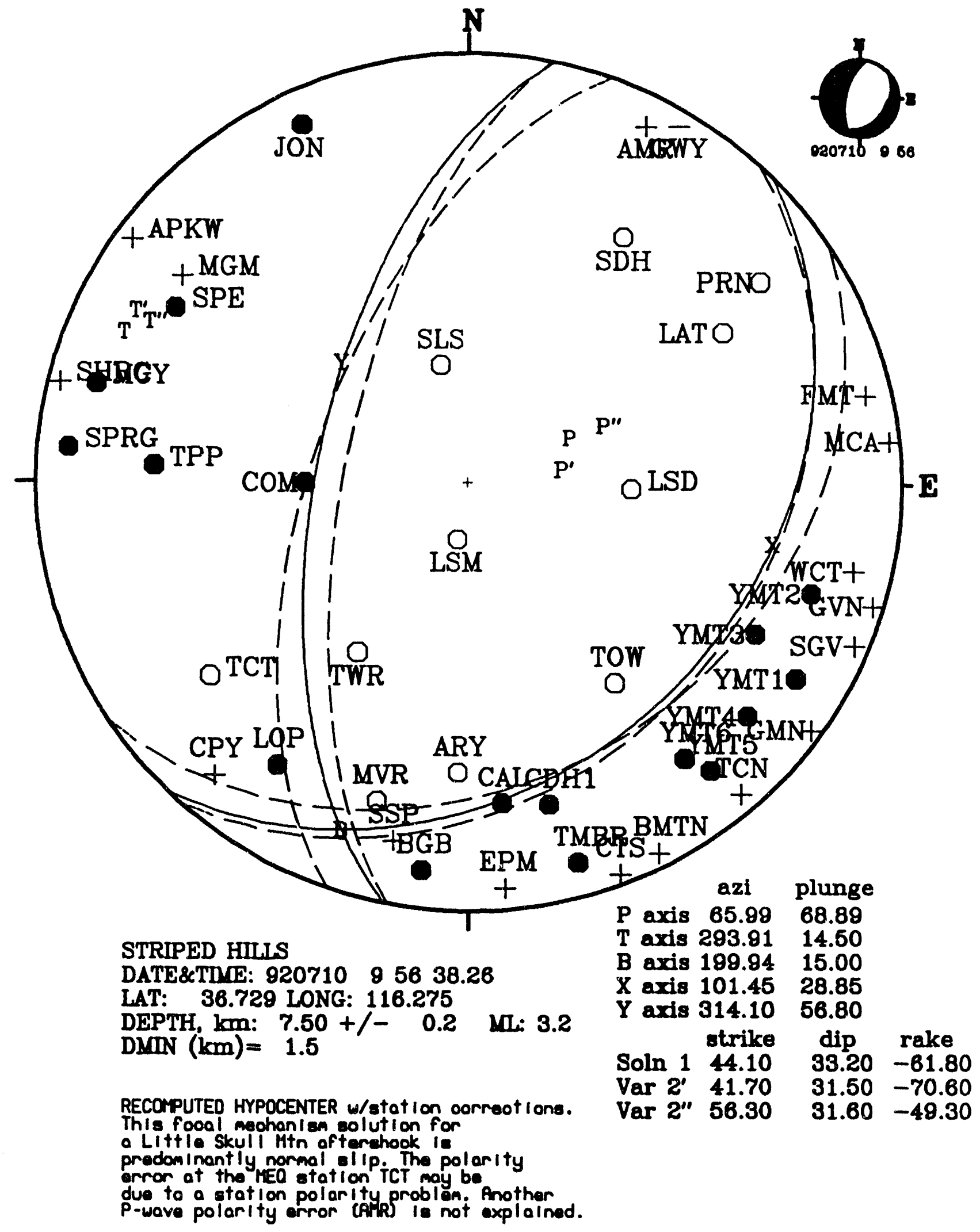

Magnitude eatimate Is from DR2ag intograted nel ismograns. 


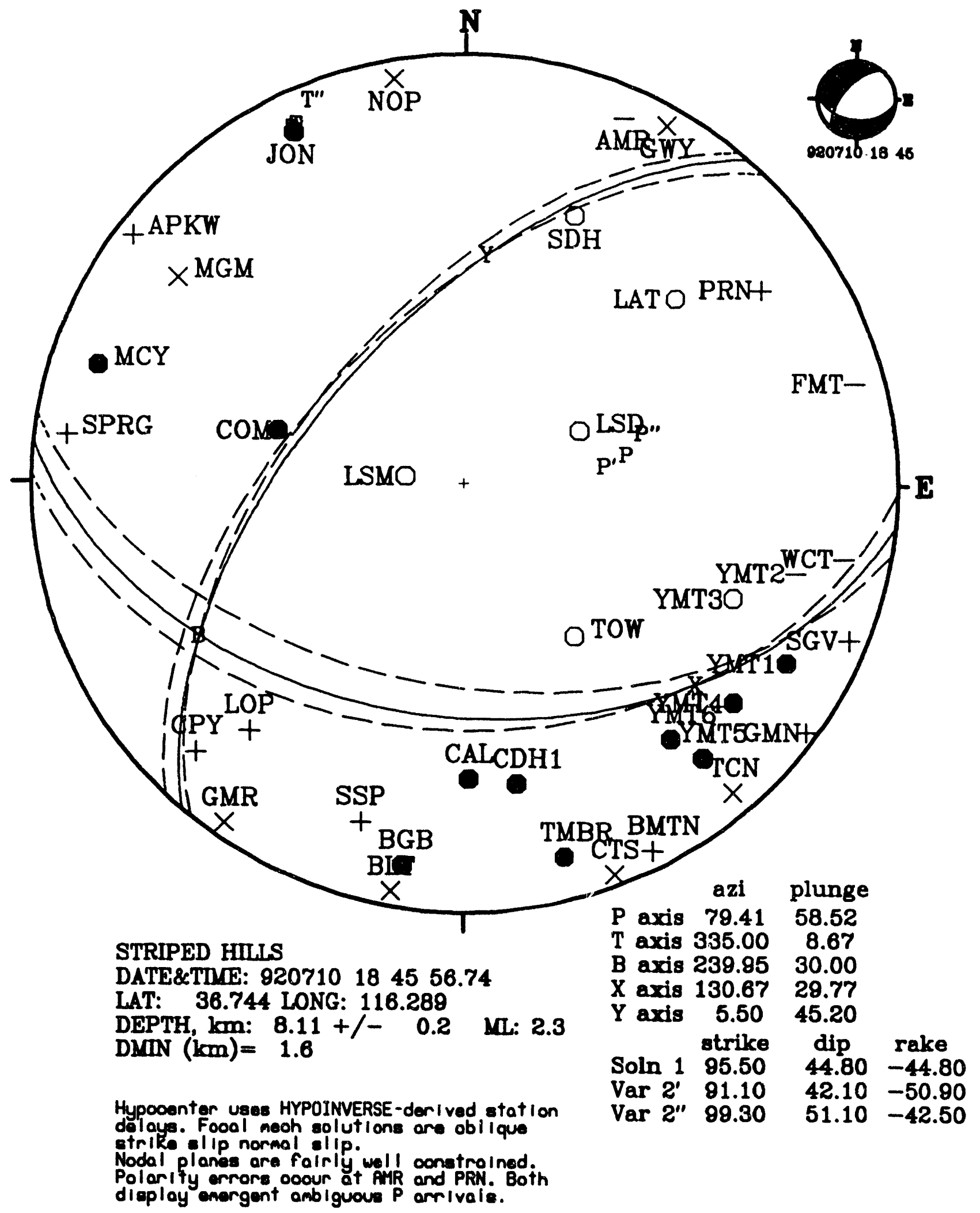




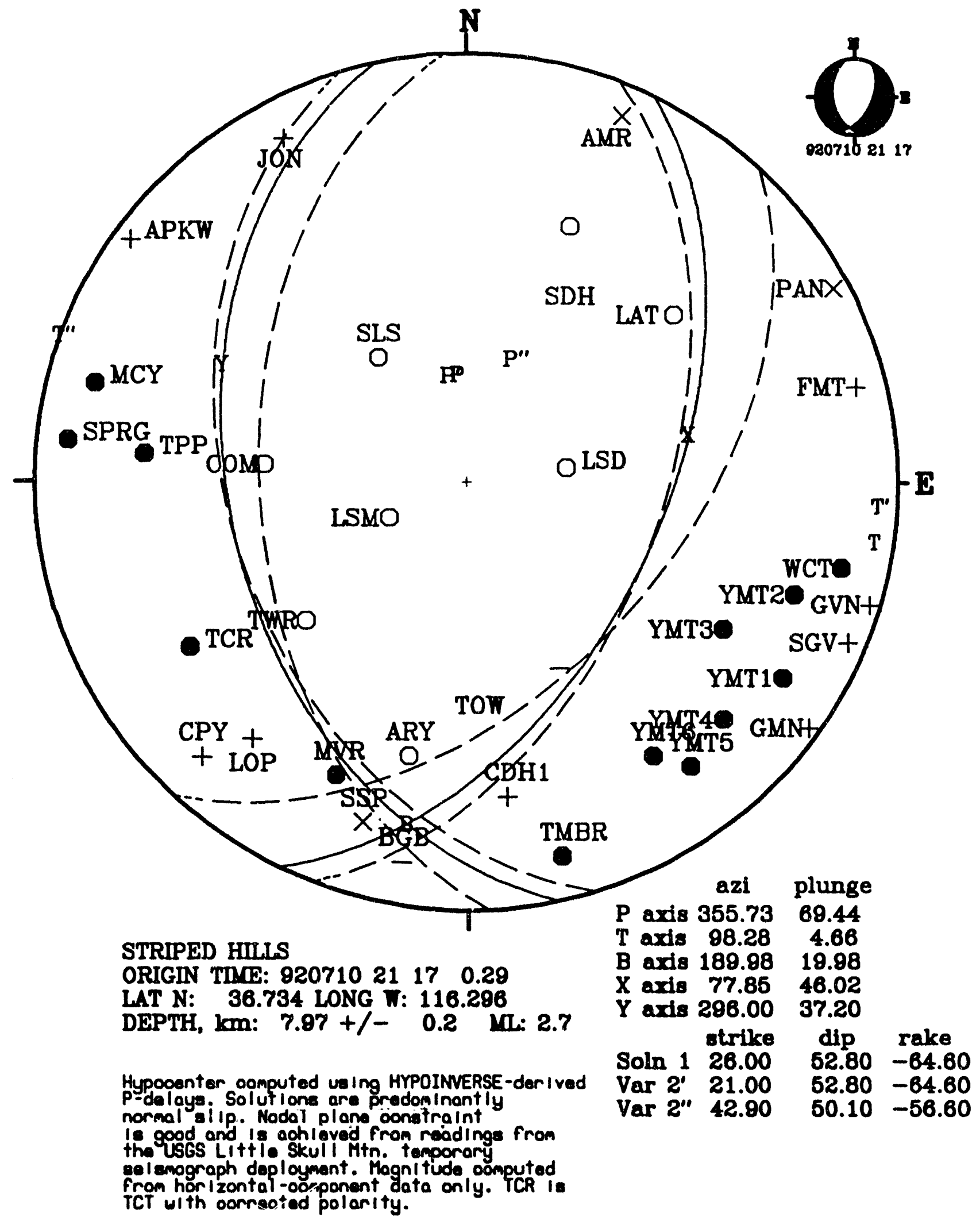




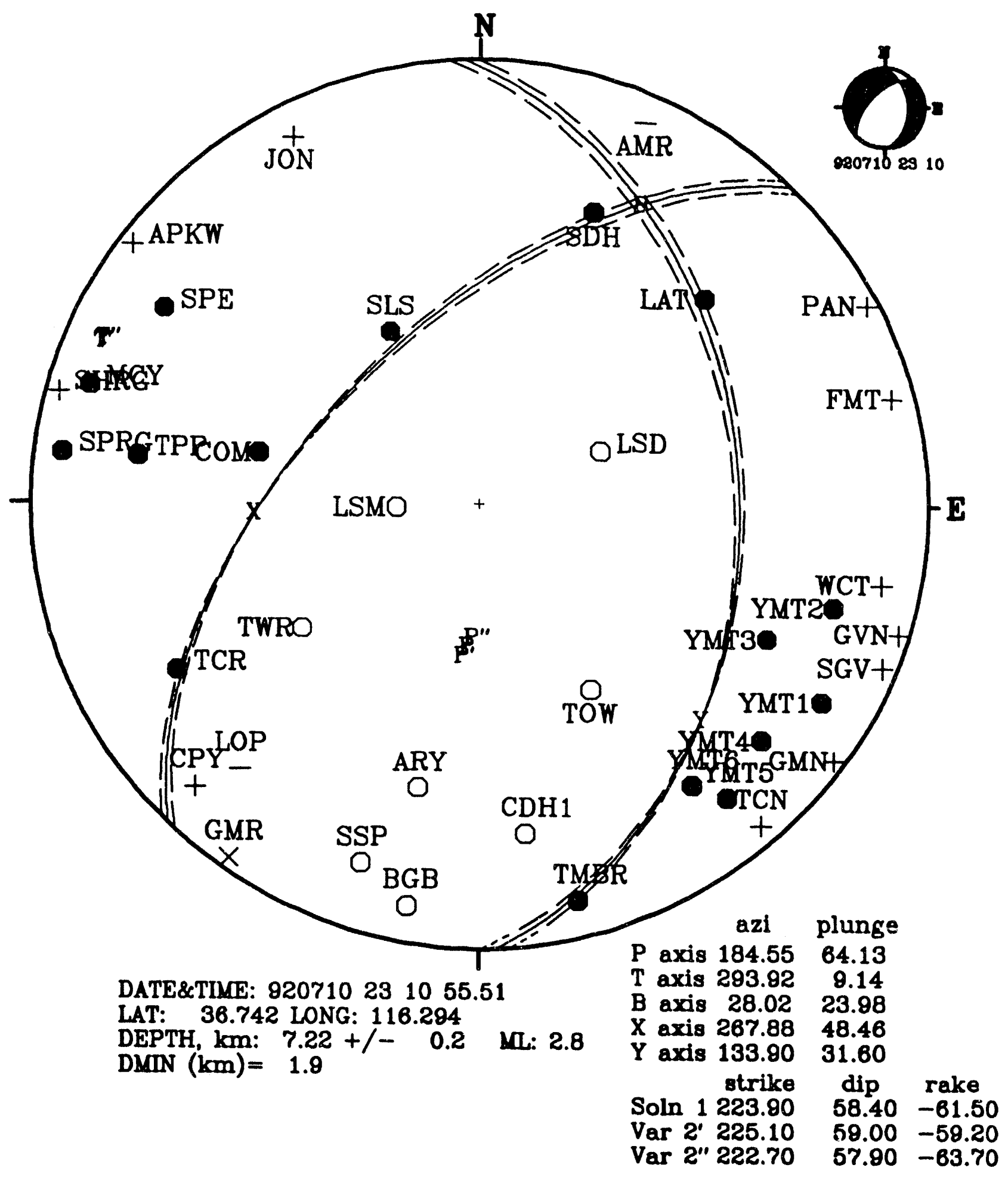




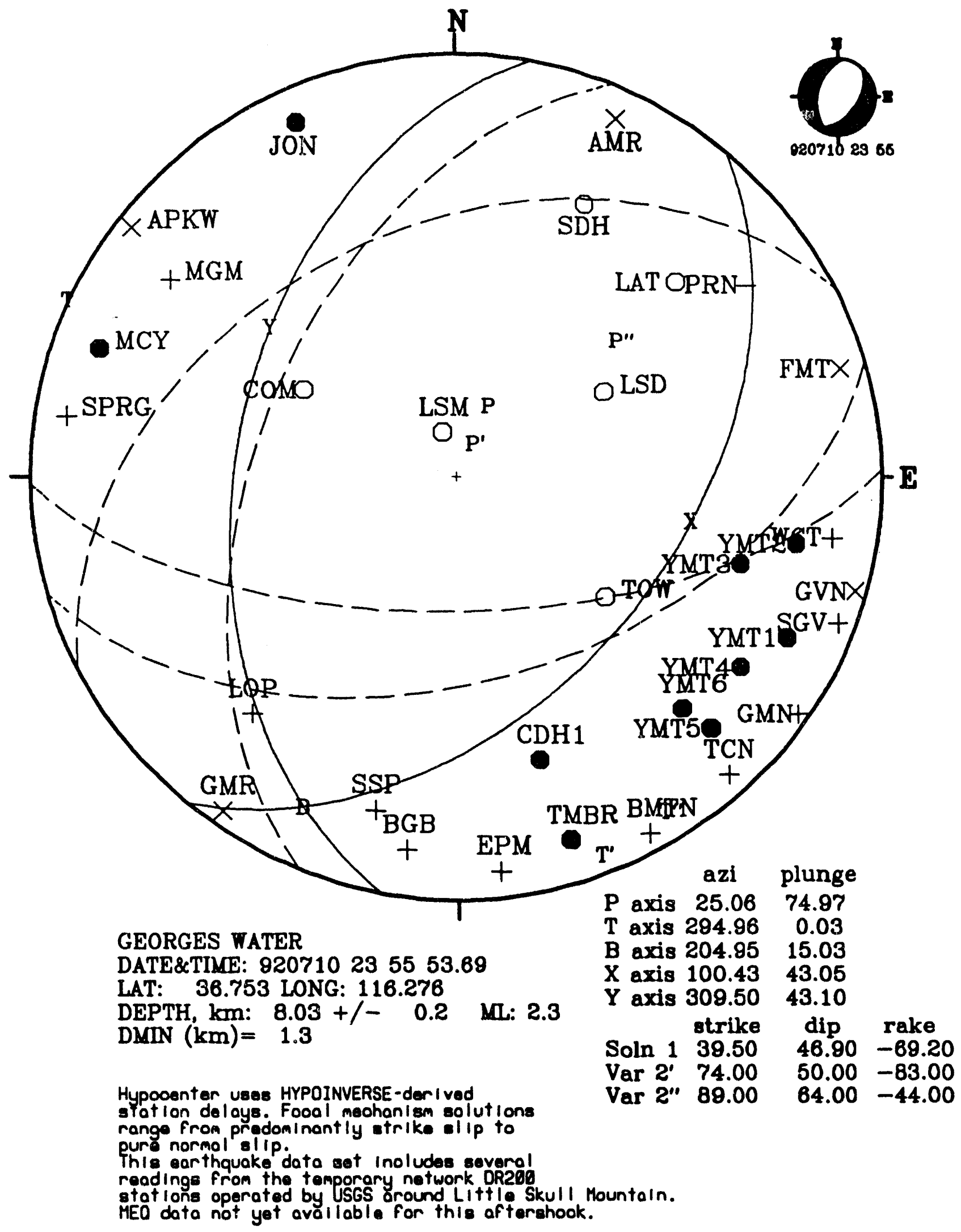




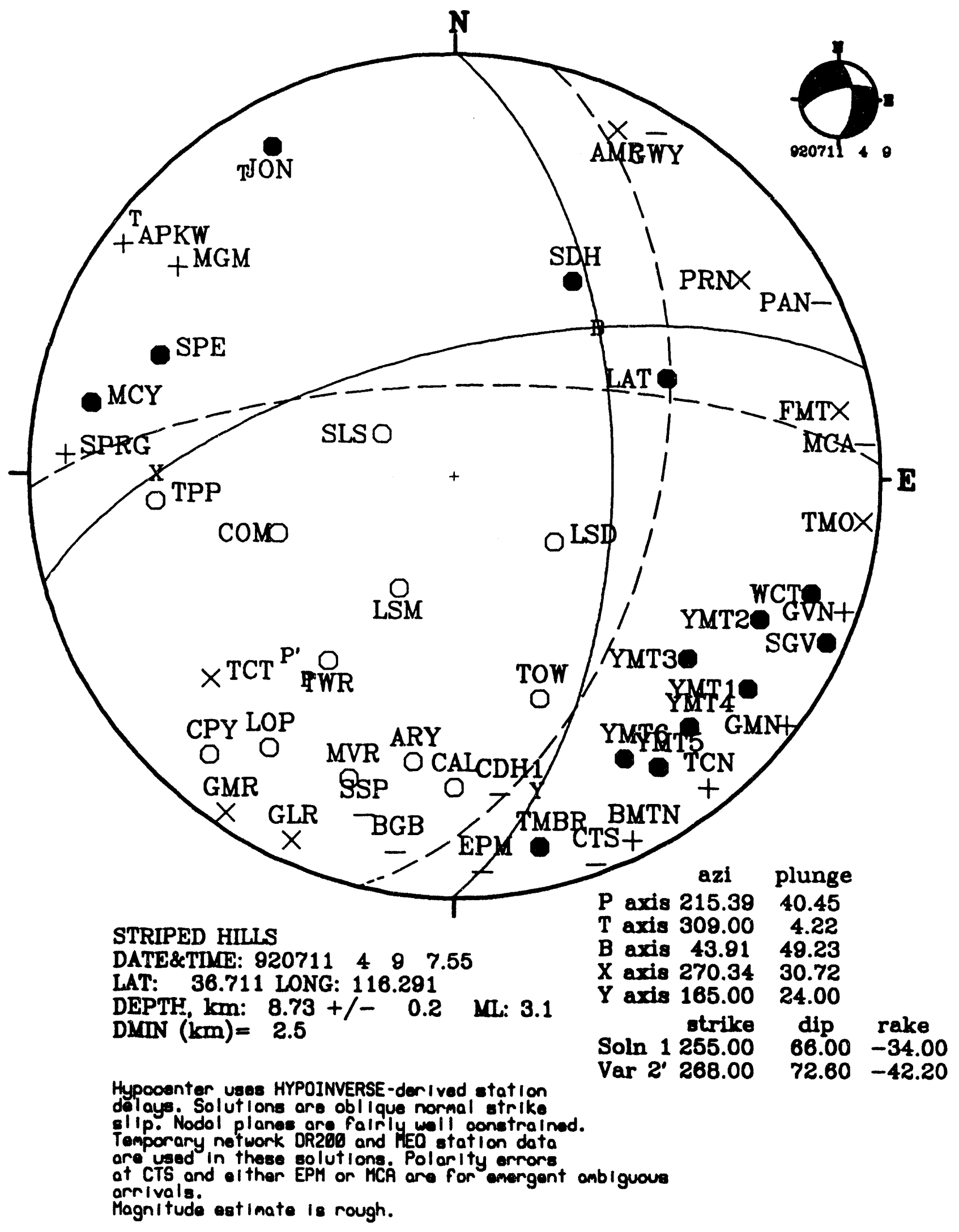




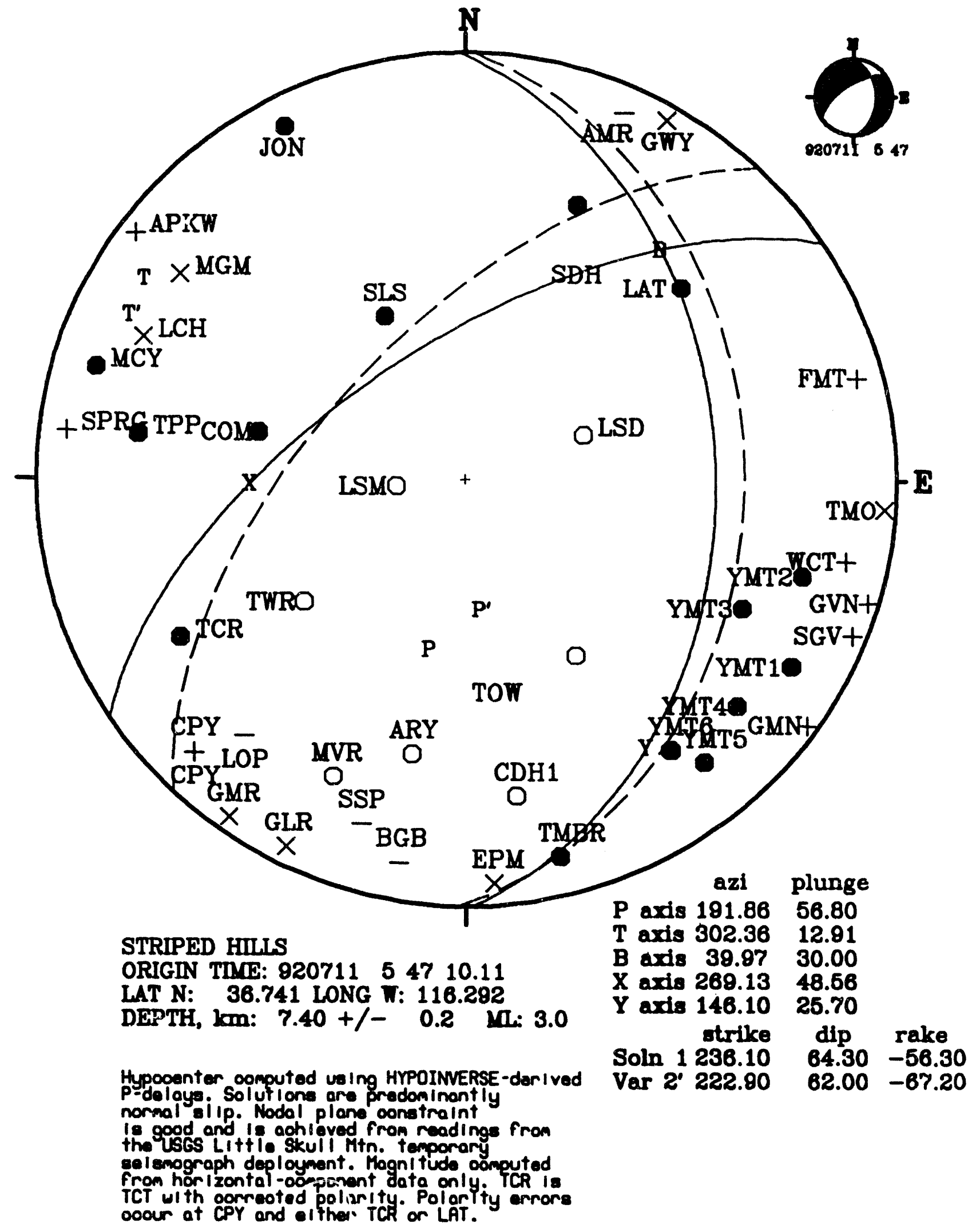




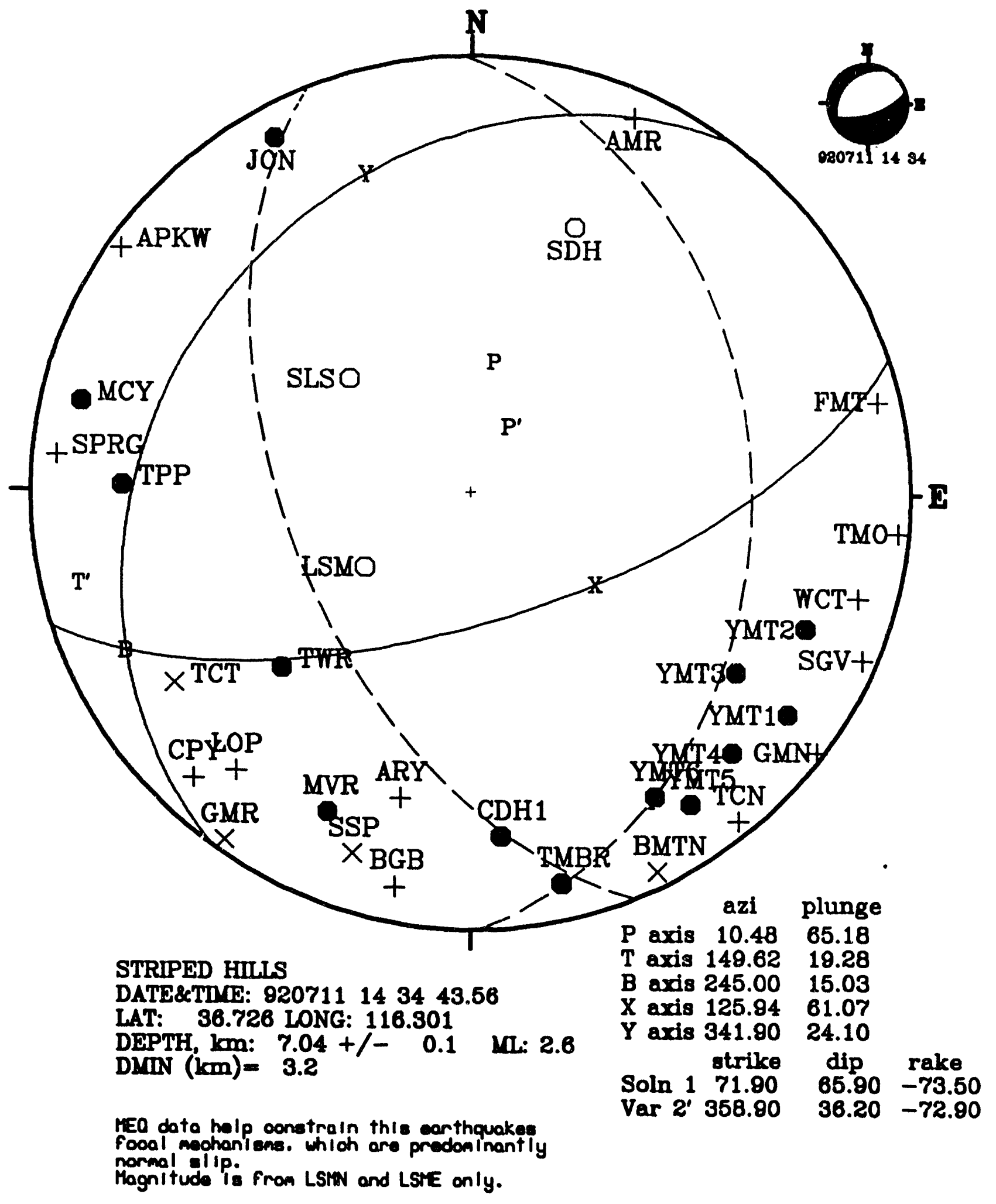




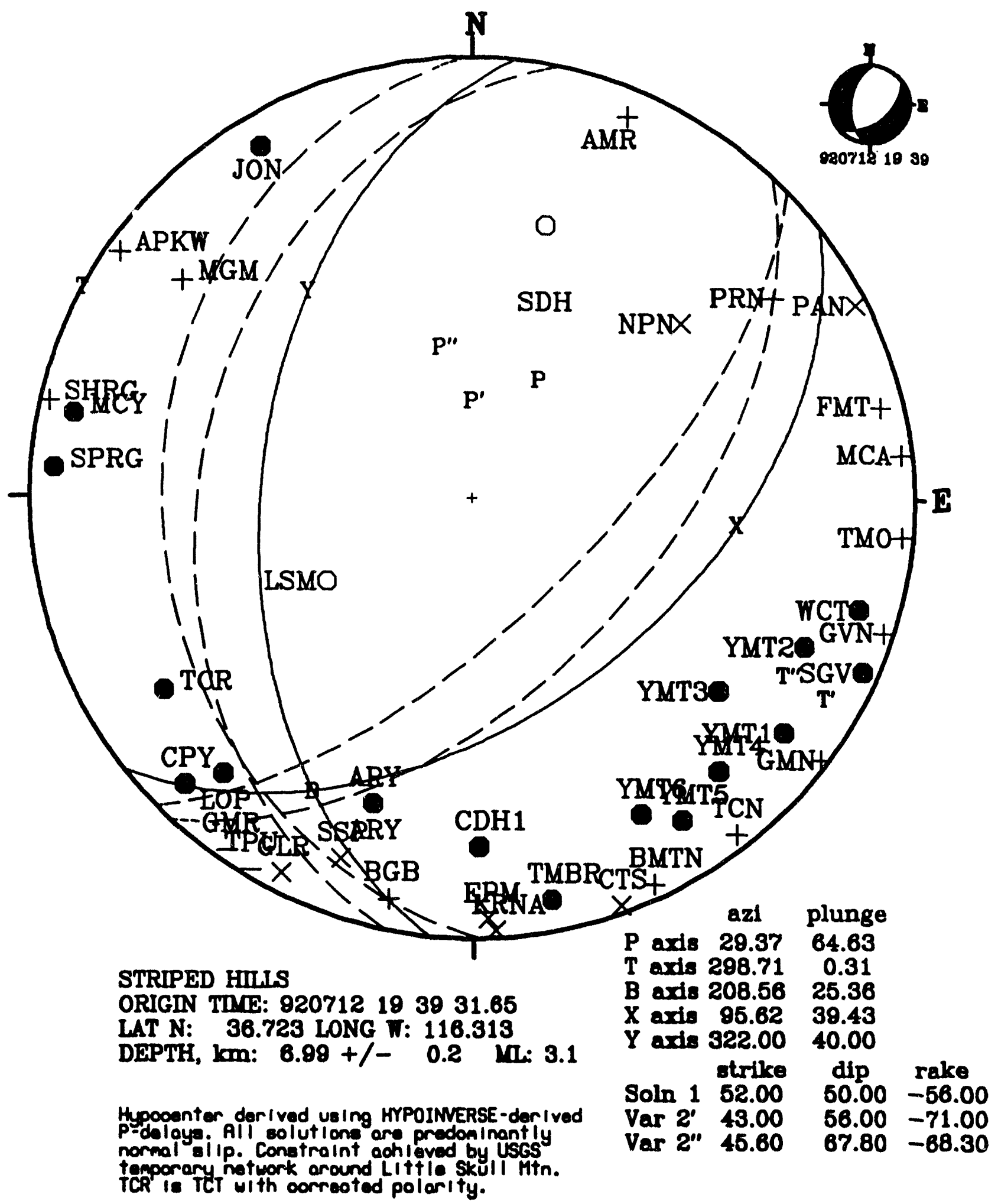




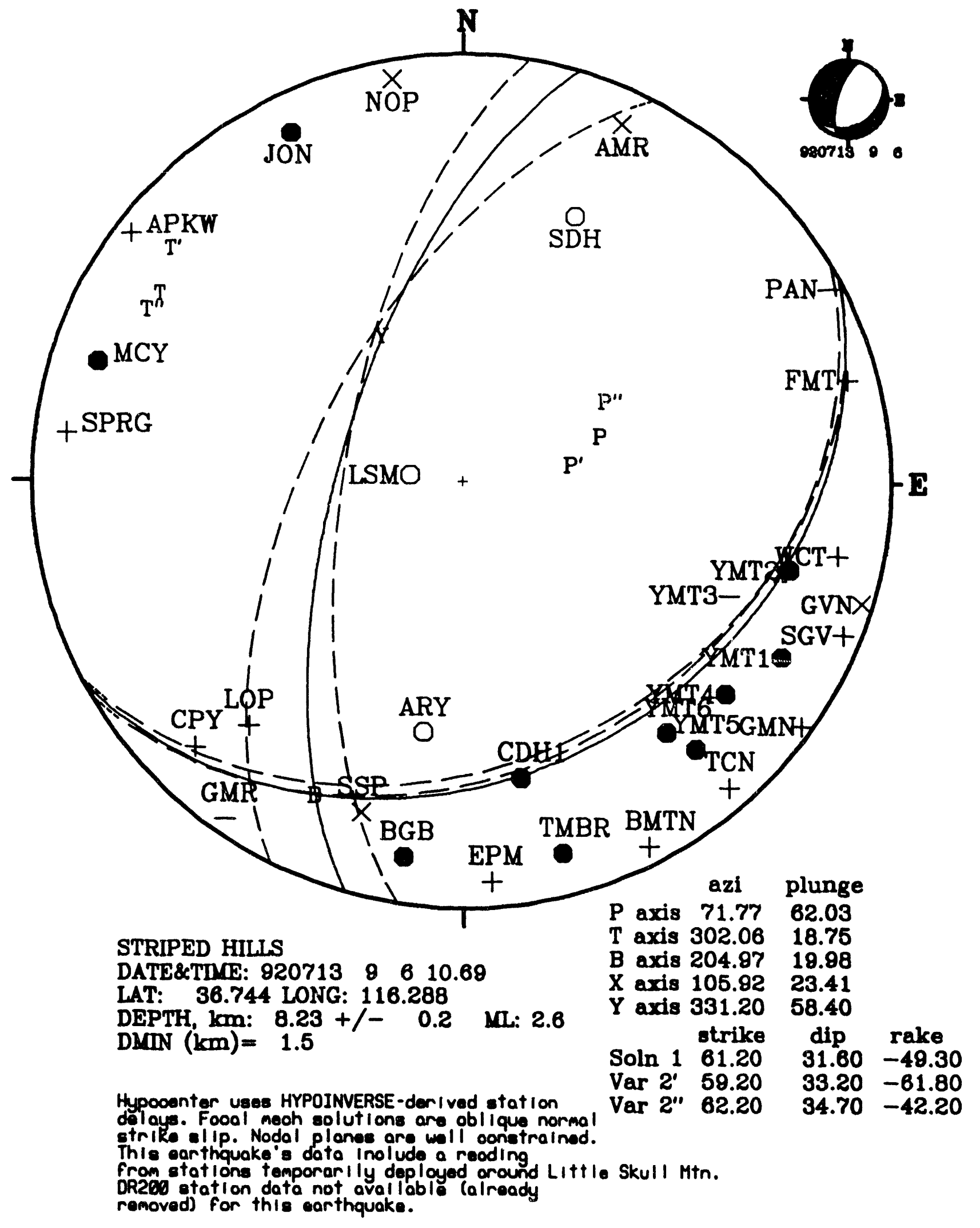




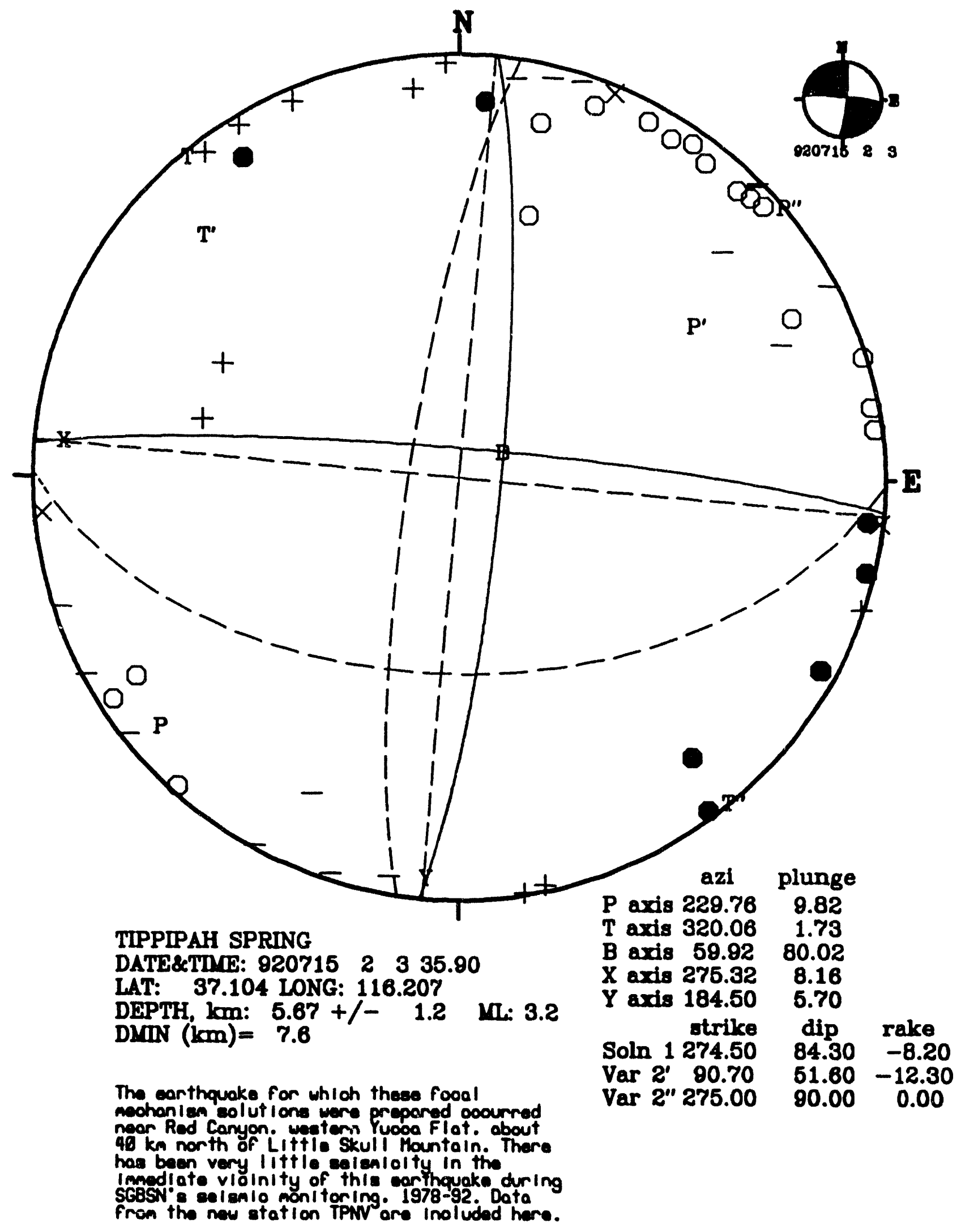




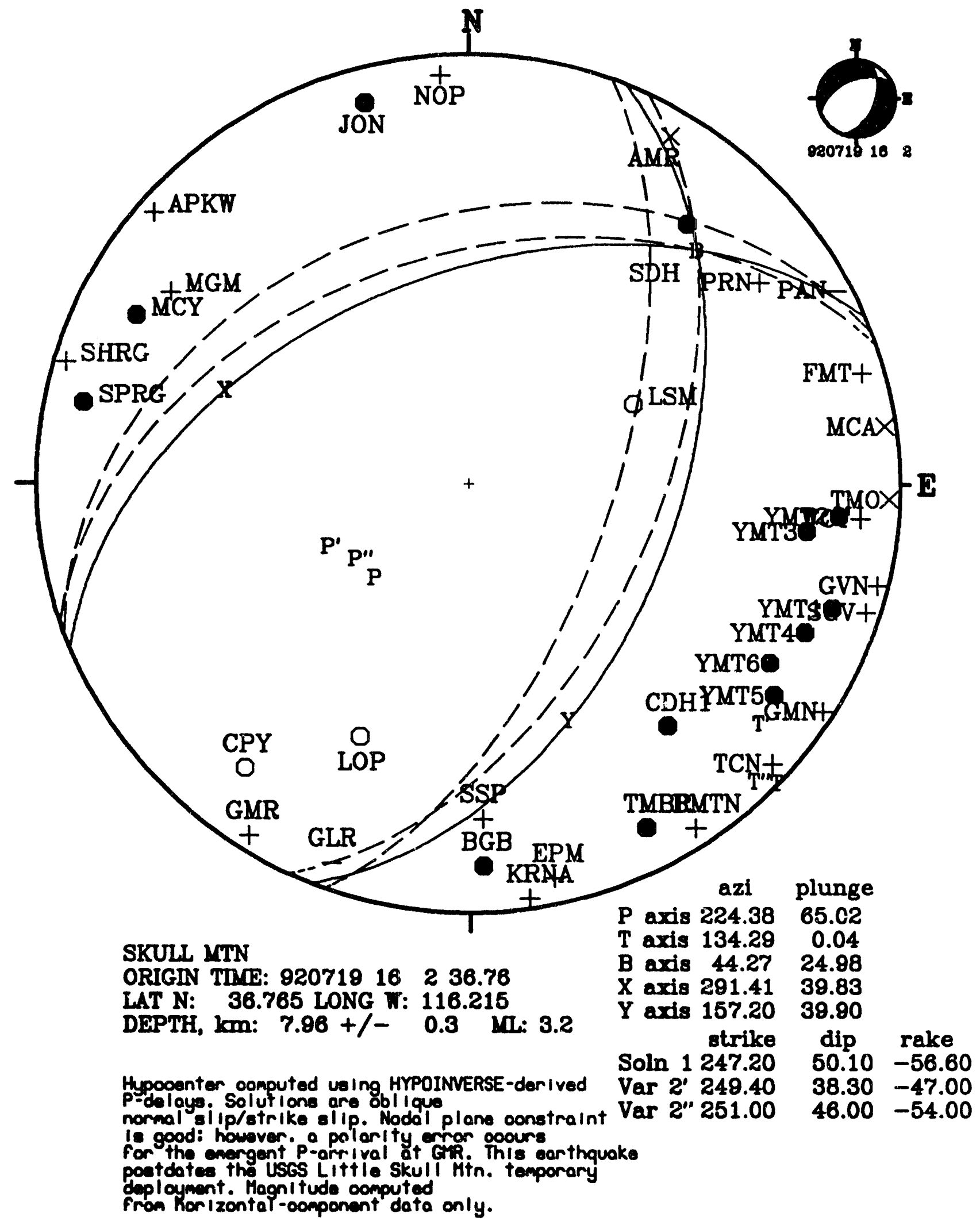




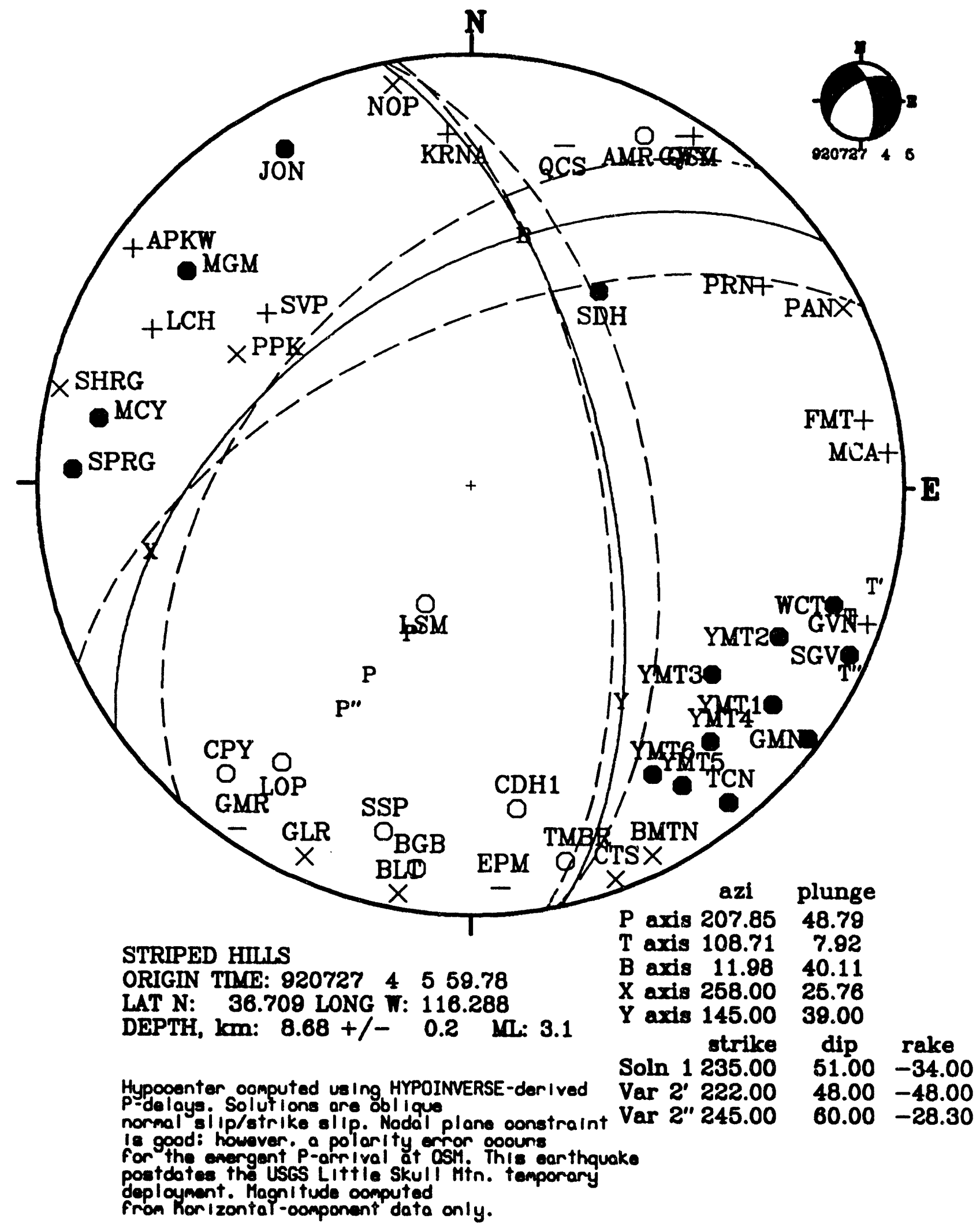




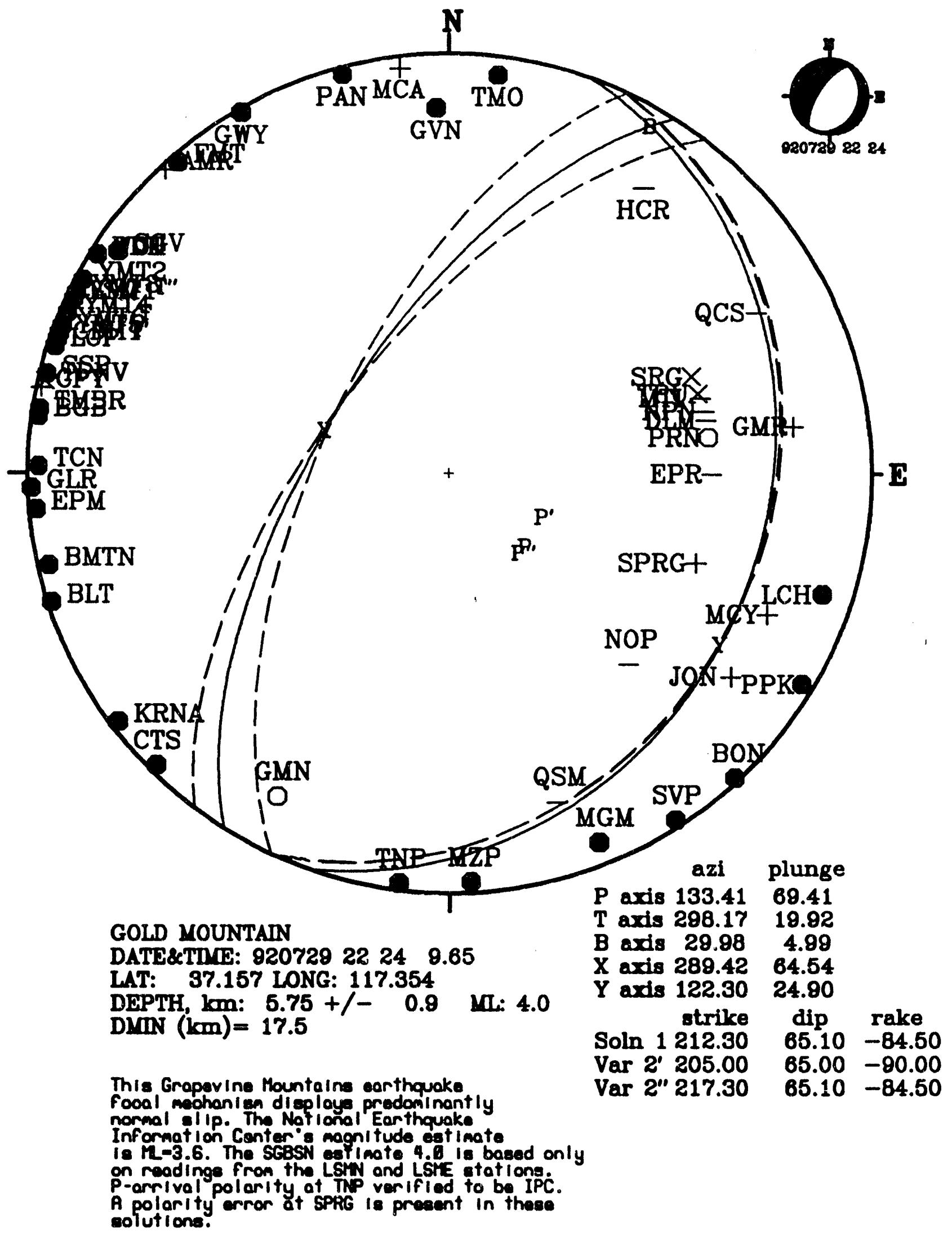




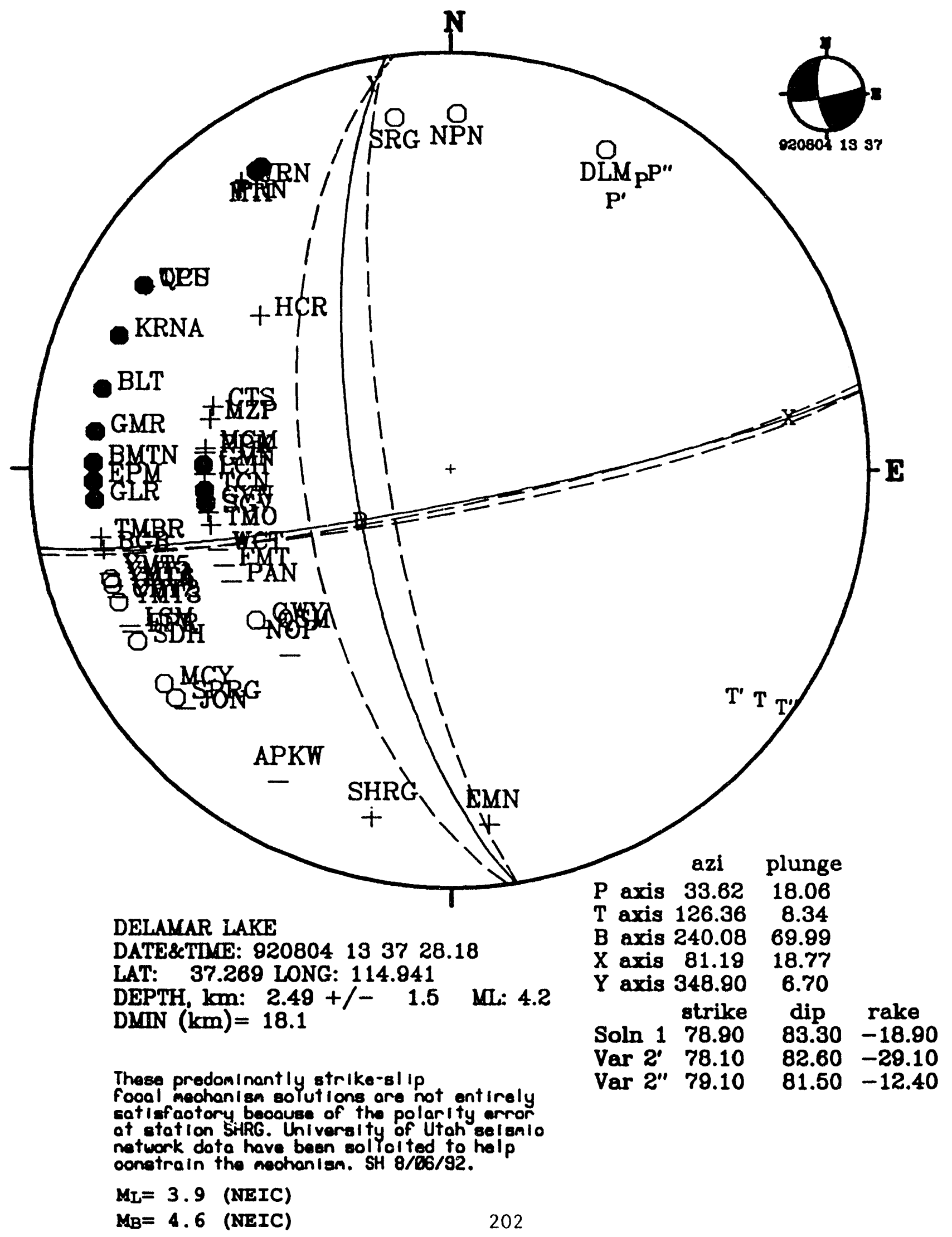




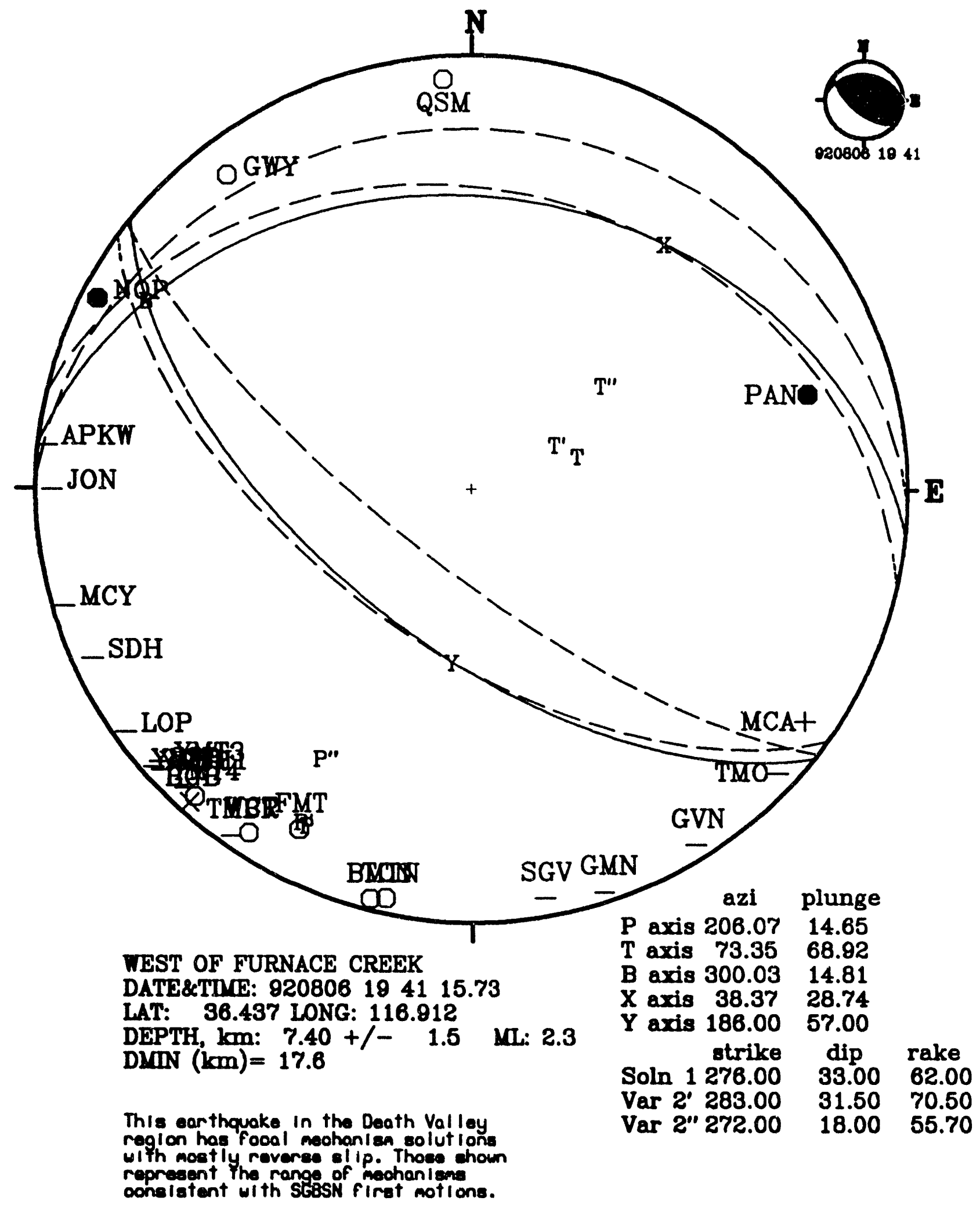




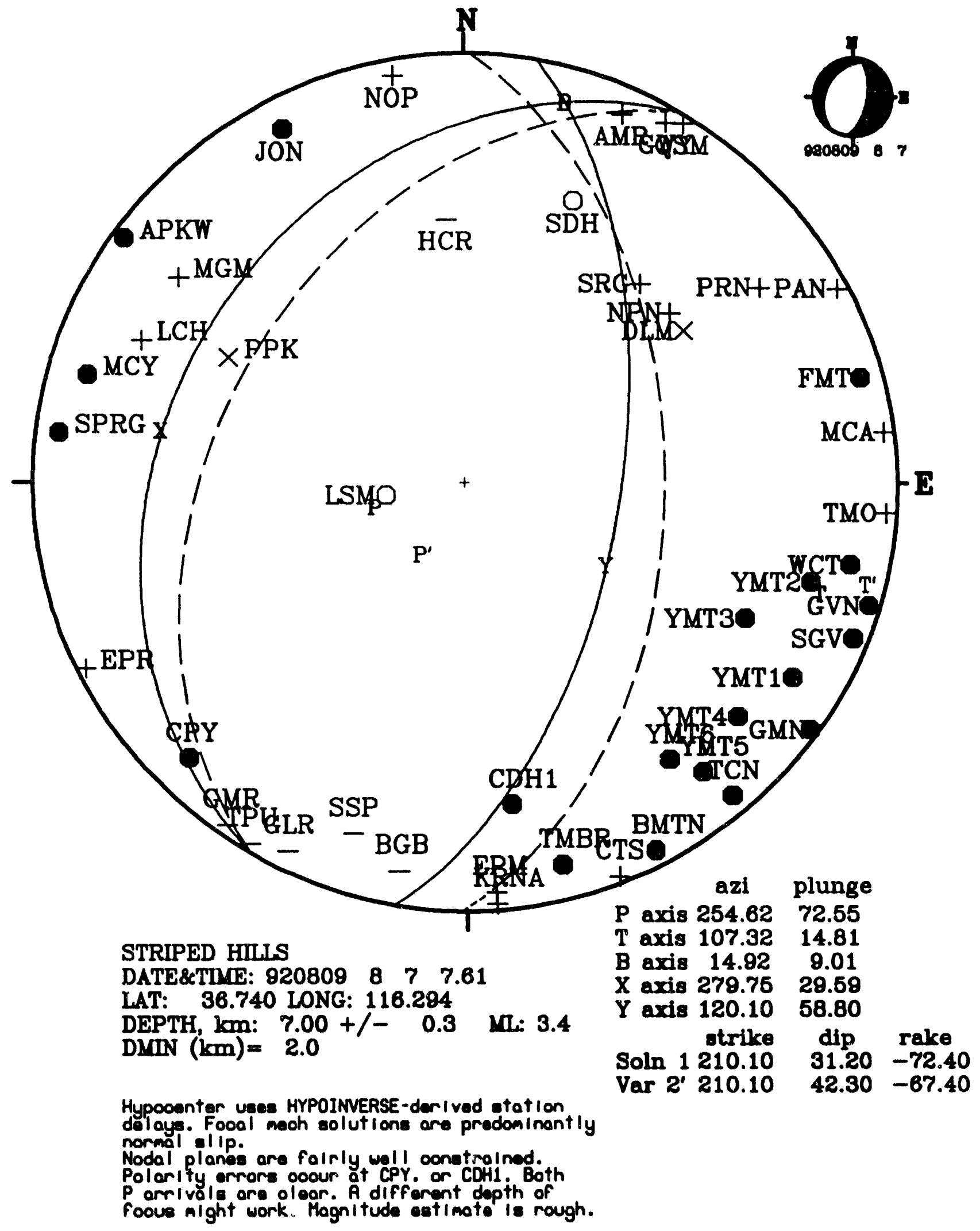




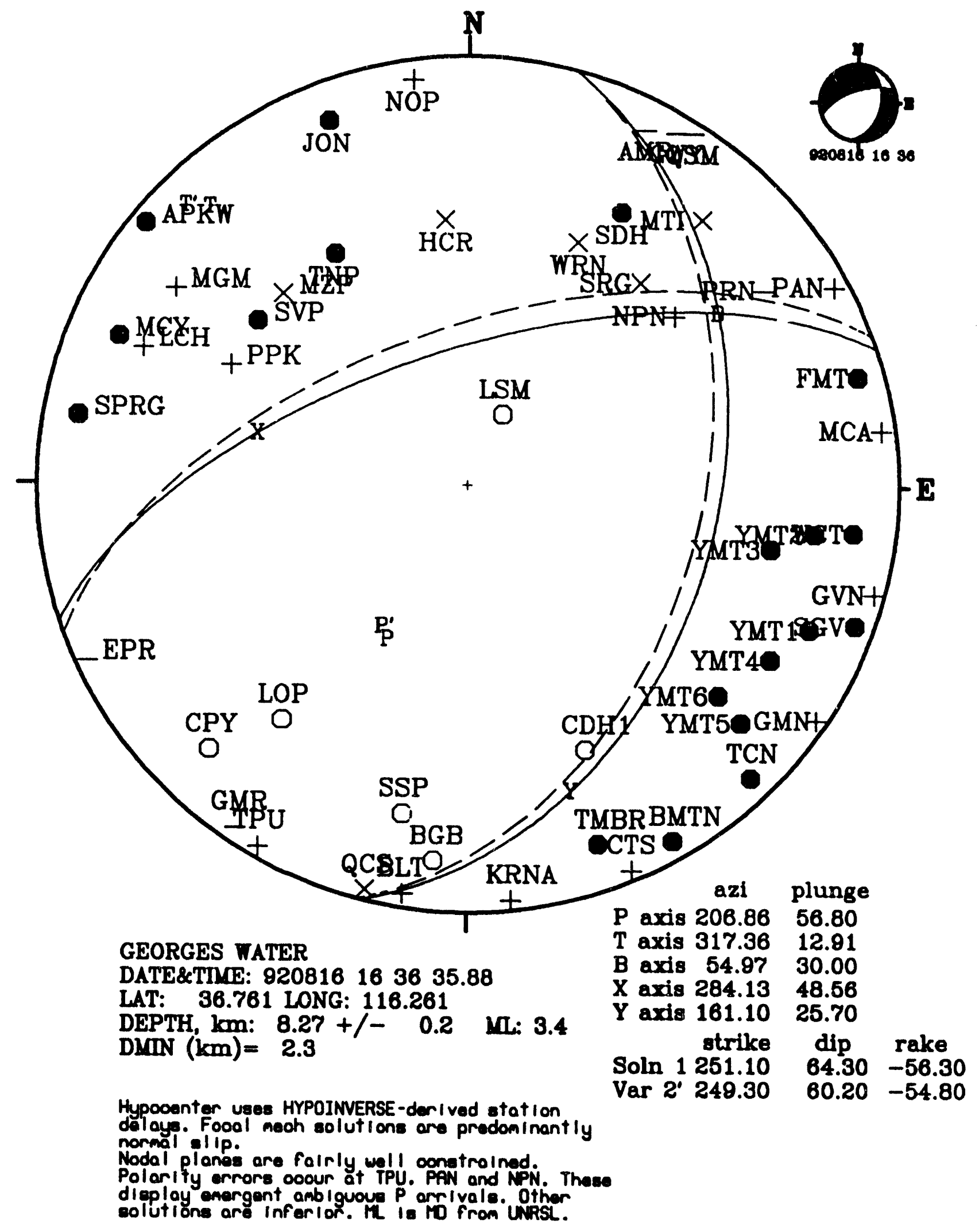




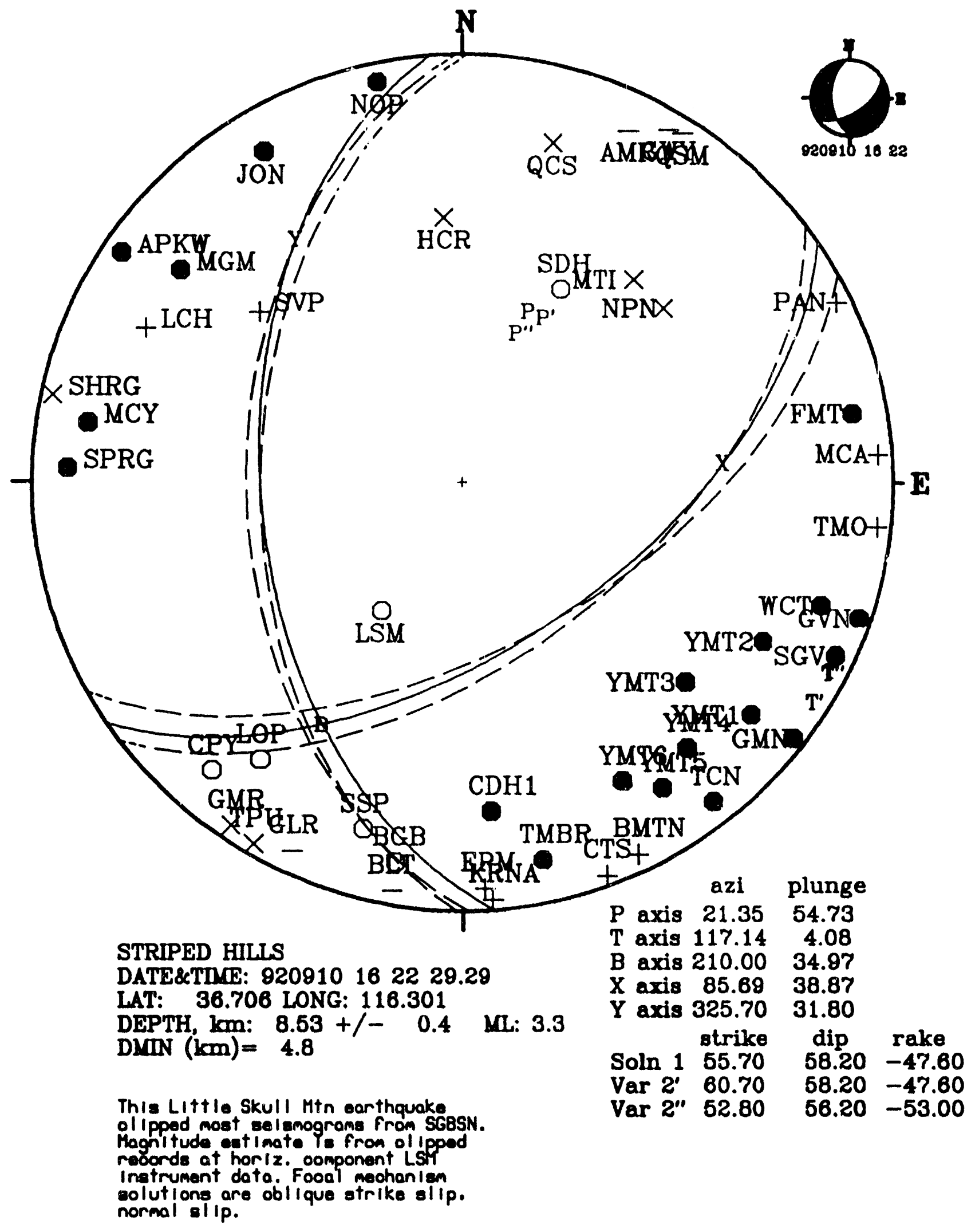




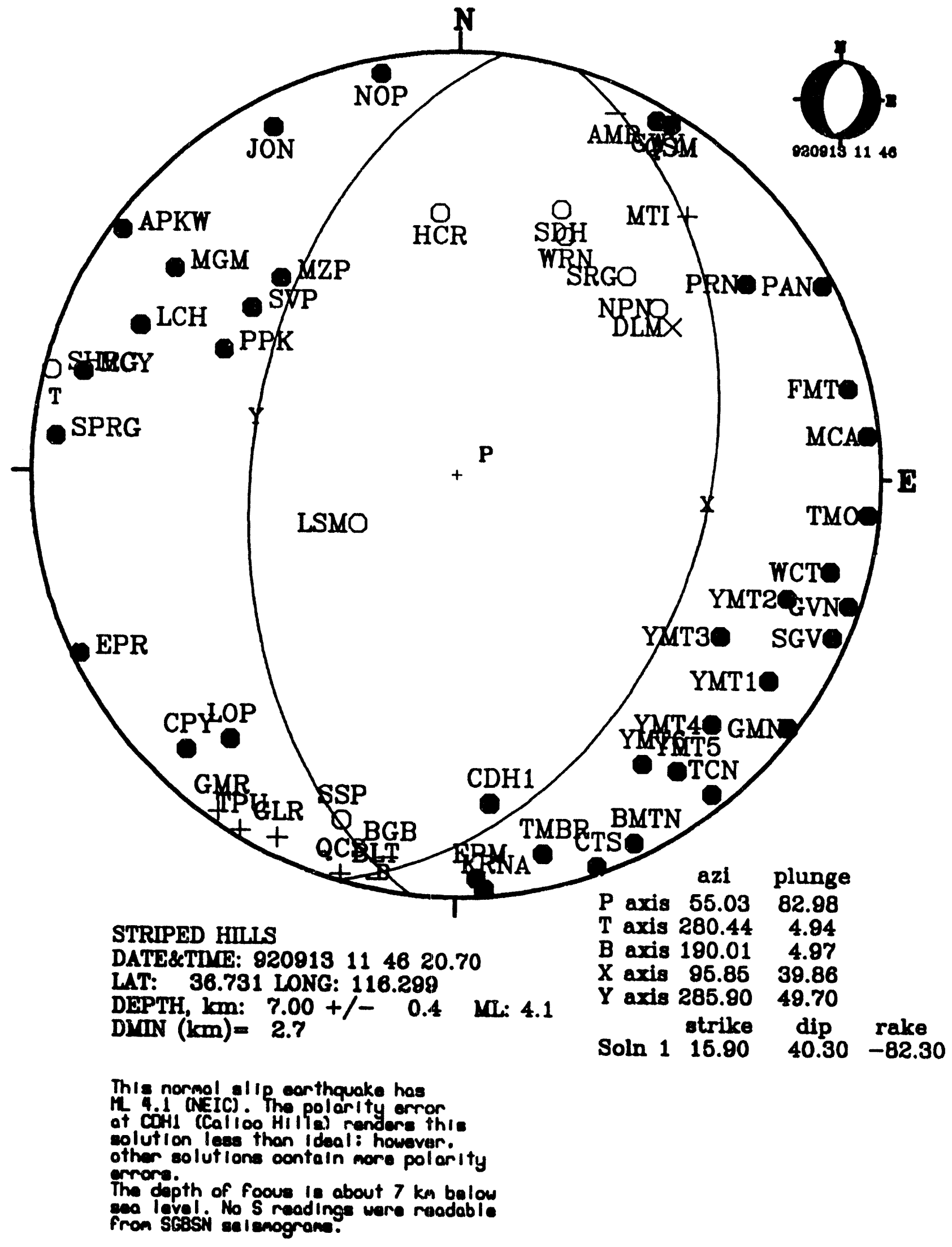




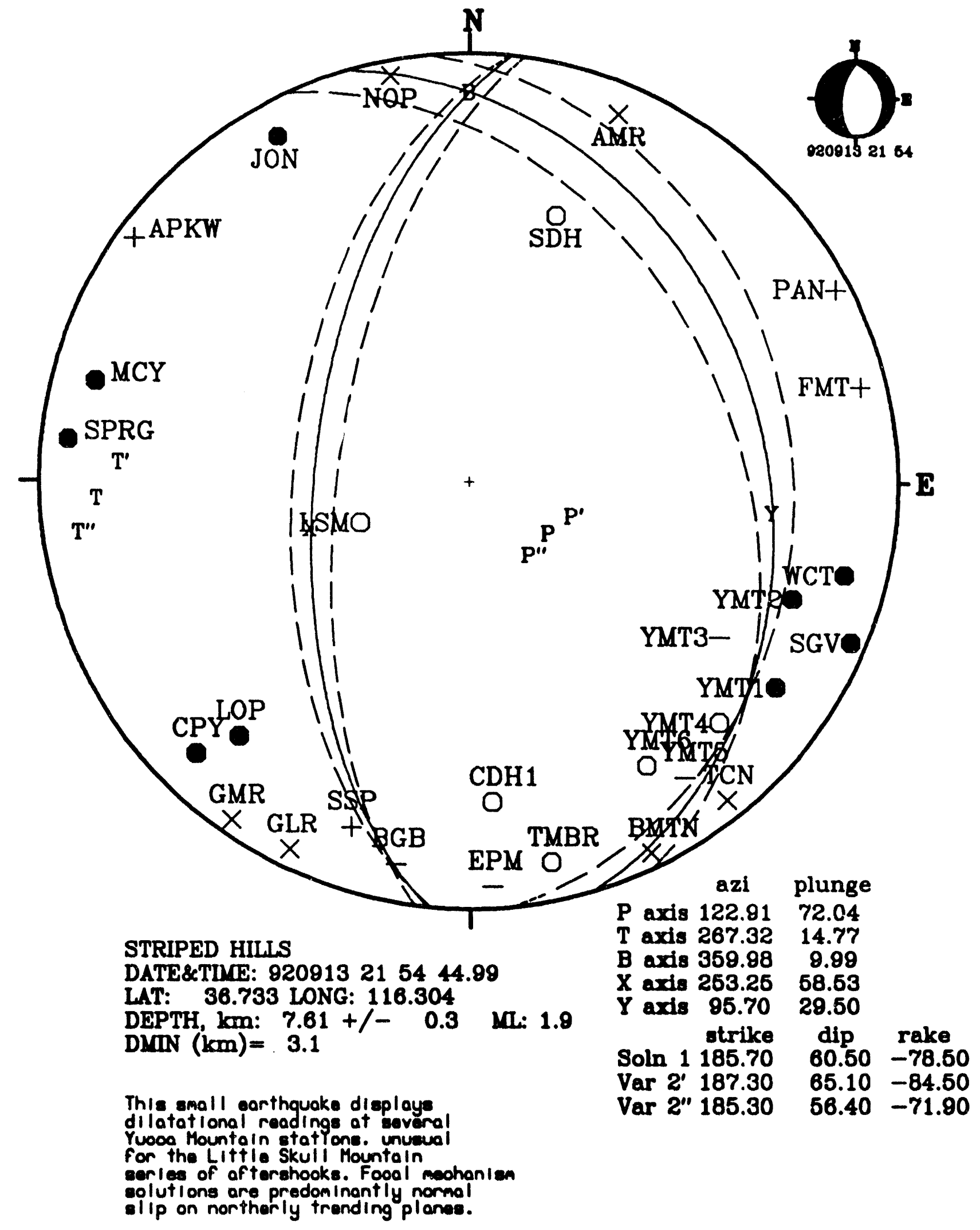




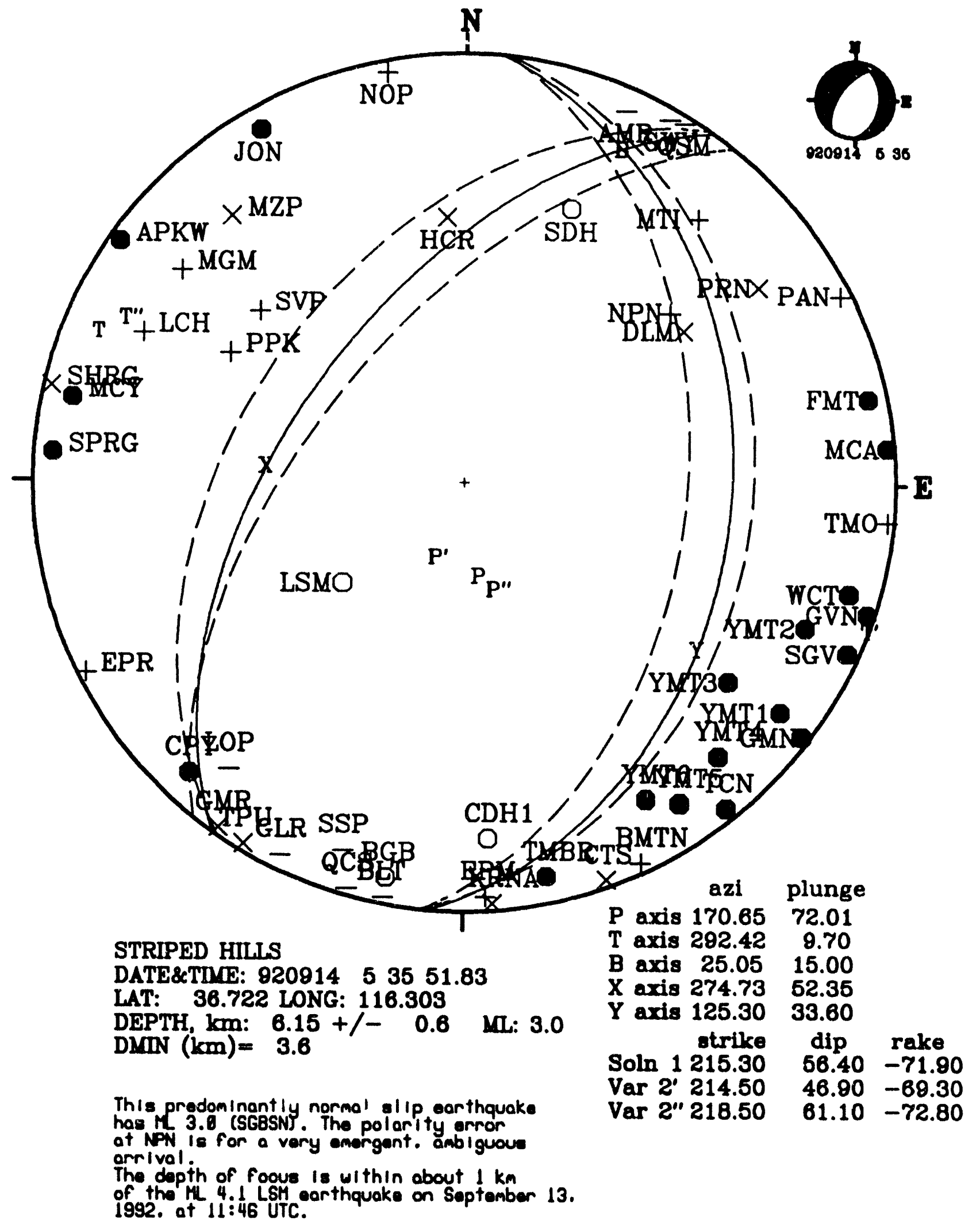




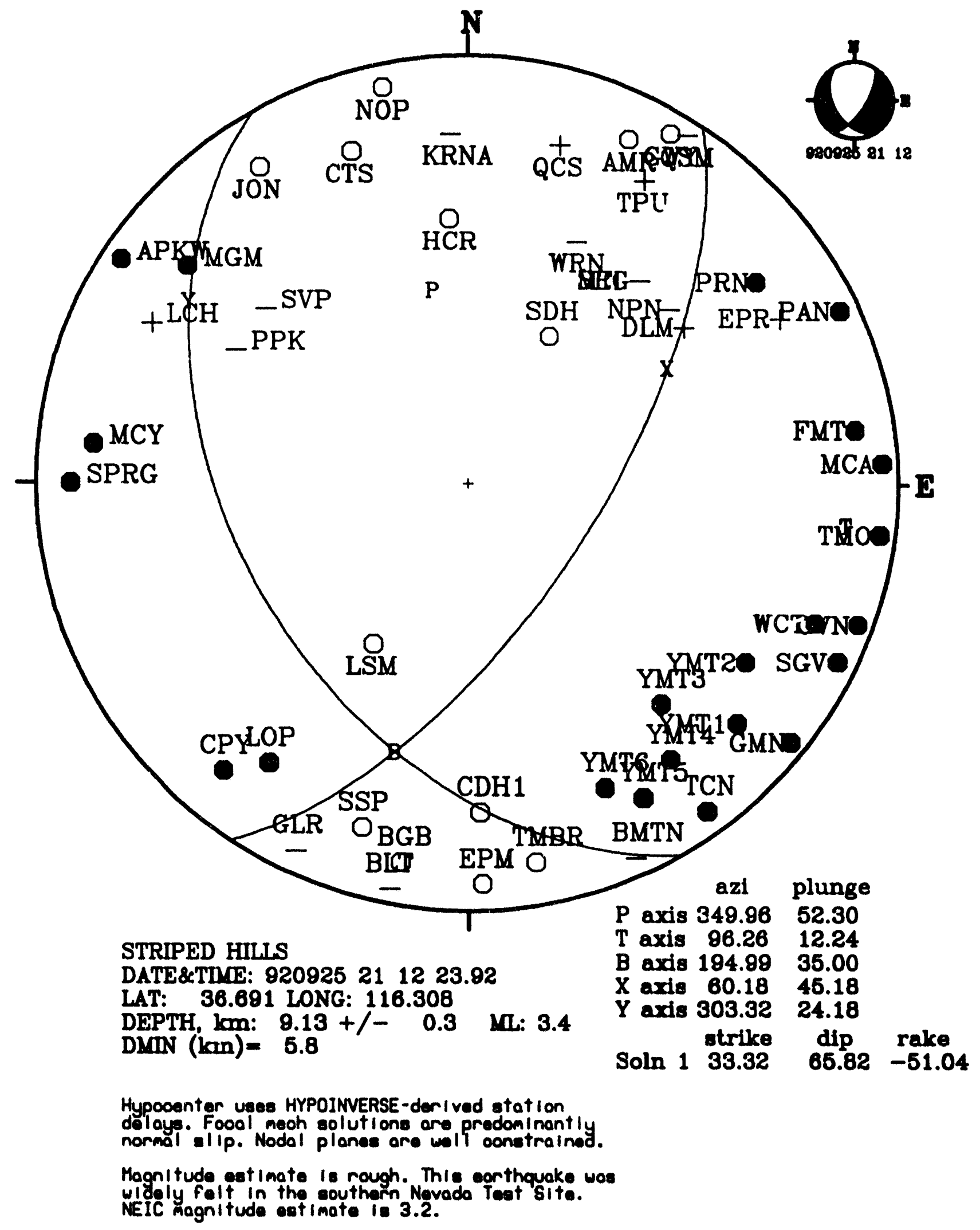




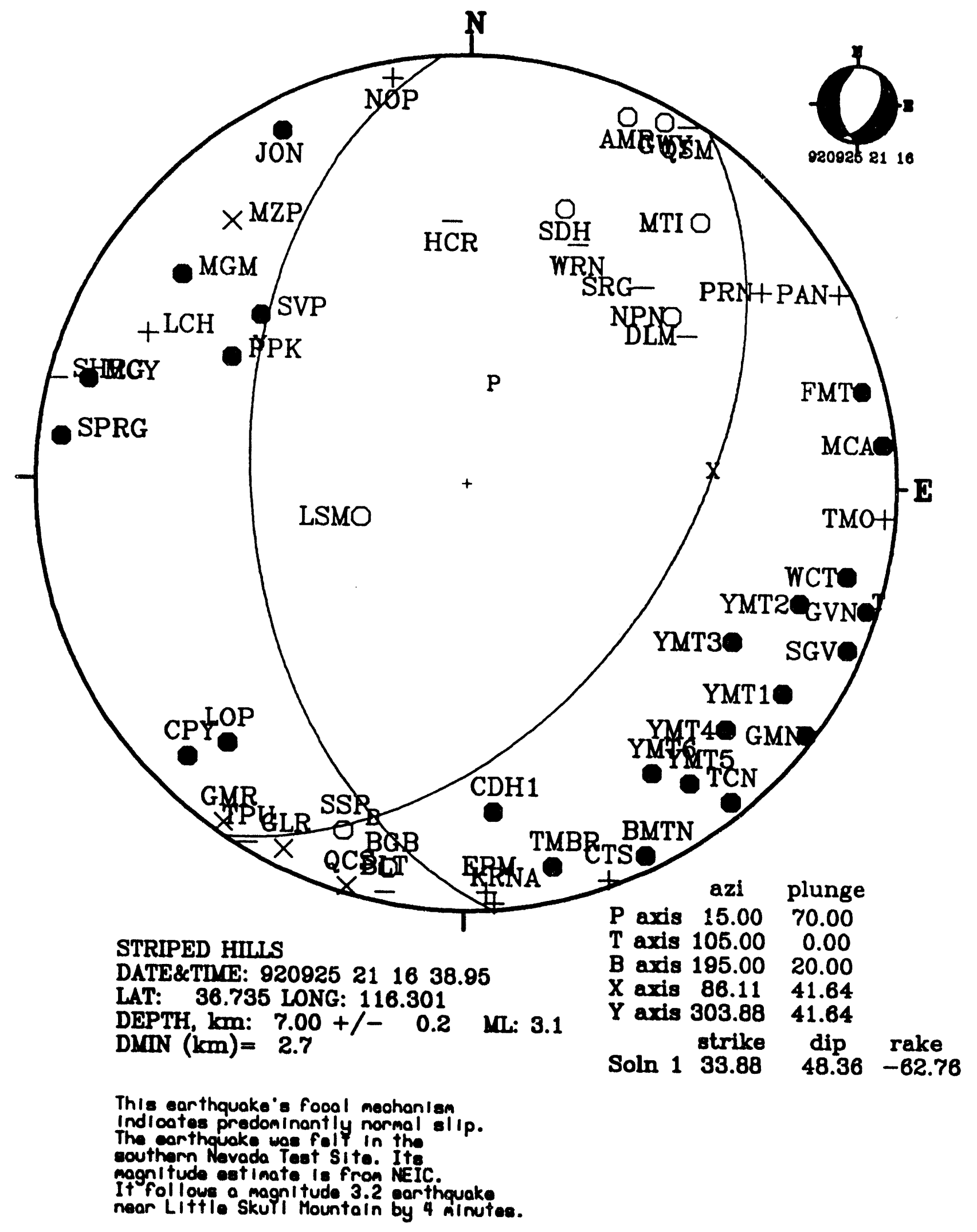




\section{Appendix D}

Table D1. SGBSN earthquake data-acquisition computer downtime log for 1992

\begin{tabular}{|c|c|c|c|c|c|}
\hline \multicolumn{2}{|c|}{ Down } & \multicolumn{2}{|c|}{ Up } & \multirow[b]{2}{*}{ Problem noted } & \multirow{2}{*}{$\begin{array}{c}\text { Techniclan/ } \\
\text { Analyst }\end{array}$} \\
\hline $\begin{array}{l}\text { Date } \\
\text { Mo/Da }\end{array}$ & $\operatorname{\prod ime}_{\text {Hr:Mn UTC }}$ & $\begin{array}{c}\text { Date } \\
\mathrm{Mo} / \mathrm{Da}\end{array}$ & $\begin{array}{c}\text { Tlme } \\
\text { Hr:Mn UTC }\end{array}$ & & \\
\hline $01 / 01$ & $00: 00$ & $01 / 06$ & $22: 30$ & Extensive hardware repairs & \\
\hline $01 / 07$ & 02:00 & $01 / 07$ & $18: 30$ & Hardware adjustments & SH \\
\hline $01 / 08$ & $19: 48$ & $01 / 08$ & $23: 52$ & Power problems, cables & SH \\
\hline $02 / 06$ & 08:07 & $02 / 06$ & $14: 49$ & Tapes filled & JD \\
\hline $02 / 11$ & $18: 29$ & $02 / 11$ & 19:58 & Operator error & JD \\
\hline $02 / 16$ & $16: 50$ & $02 / 16$ & $17: 10$ & Power outage & TB \\
\hline $02 / 16$ & $20: 00+$ & $02 / 17$ & $15: 41$ & Power outage & TB \\
\hline $02 / 25$ & $10: 46$ & $02 / 25$ & $14: 57$ & Tapes filled & JD \\
\hline $03 / 09$ & $00: 00+$ & 03/04 & $14: 59$ & Power outage, some uptime & TB \\
\hline $03 / 15$ & $14: 39$ & $03 / 15$ & 15:00 & Tape subsystem problems & TB \\
\hline $03 / 25$ & $17: 08$ & $03 / 25$ & $17: 22$ & Telos maintenance A/D\#2 & SH \\
\hline $03 / 27$ & $13: 10$ & $03 / 27$ & $14: 08$ & LPA subsystem + select err & JD \\
\hline $03 / 27$ & $20: 42$ & $03 / 27$ & $22: 28$ & LPA subsystem & JD \\
\hline $04 / 21$ & 23:04 & $04 / 22$ & $14: 00$ & LPA subsystem problems & $\mathrm{JD}$ \\
\hline $04 / 23$ & 05:30 & $04 / 23$ & $13: 59$ & LPA-select error & JD \\
\hline $04 / 24$ & $14: 18$ & $04 / 24$ & $14: 30$ & Tapes full select error & JD \\
\hline $04 / 29$ & $20: 24$ & $04 / 29$ & $20: 35$ & DISK backup BRU64K & JD \\
\hline $05 / 01$ & $12: 39$ & $05 / 01$ & $14: 35$ & Tapes filled & SH \\
\hline $05 / 14$ & $20: 35$ & $05 / 14$ & $21: 07$ & LPA error-system reboot & JD \\
\hline $05 / 27$ & $02: 23$ & $05 / 27$ & $15: 02$ & LPA error select ERR & JD \\
\hline $05 / 30$ & $01: 40$ & $05 / 30$ & $18: 25$ & LPA error select ERR & JD \\
\hline $06 / 28$ & $13: 40$ & $06 / 28$ & $16: 15$ & Tapes filled & JD \\
\hline $06 / 28$ & $16: 52$ & $06 / 28$ & $17: 14$ & Tapes filled & JD \\
\hline $06 / 29$ & $02: 17$ & $06 / 29$ & $02: 31$ & Tapes filled & SH \\
\hline $06 / 29$ & $05: 55$ & $06 / 29$ & $12: 04$ & Tapes filled & SH \\
\hline $06 / 29$ & $23: 42$ & $06 / 30$ & $00: 22$ & Tapes filled & SH \\
\hline $06 / 30$ & $10: 08$ & $06 / 30$ & $11: 29$ & Tapes filled & SH \\
\hline $06 / 30$ & $12: 35$ & $06 / 30$ & $12: 55$ & Tape drive MT0: error & SH \\
\hline $06 / 30$ & $14: 20$ & $06 / 30$ & $14: 42$ & Tape drive MT0: error & JD \\
\hline $06 / 30$ & $19: 33$ & $06 / 30$ & $19: 43$ & MT0: tape ran off end & JD \\
\hline $06 / 30$ & $21: 40$ & $06 / 30$ & $21: 49$ & MT0: tape ran off end & JD \\
\hline $07 / 01$ & $04: 05$ & $07 / 01$ & $13: 00$ & Tapes filled & JD \\
\hline 07/01 & $16: 03$ & $07 / 01$ & 16:08 & MT0: tape ran off end & JD \\
\hline $07 / 02$ & $12: 47$ & $07 / 02$ & $12: 56$ & Tapes filled & JD \\
\hline $07 / 05$ & $16: 00$ & $07 / 05$ & $16: 15$ & Tapes filled & TB \\
\hline $07 / 06$ & $16: 16$ & $07 / 06$ & $17: 02$ & Bldg. power failure & SH \\
\hline 07/06 & $22: 16$ & $07 / 06$ & $22: 30$ & Tape drive overwrites & JD \\
\hline $07 / 06$ & $23: 15$ & $07 / 07$ & $02: 02$ & Tape $2 \mathrm{C}$ was overwritten & SII \\
\hline 07/09 & $12: 13$ & $07 / 09$ & $14: 08$ & Tapes filled & JD \\
\hline $07 / 14$ & $16: 44$ & $07 / 14$ & $17: 20$ & Tape drive hard error & SH \\
\hline $07 / 16$ & $22: 00$ & $07 / 16$ & $22: 03$ & Power outage & JD \\
\hline $07 / 18$ & 19:00 & $07 / 19$ & 07:00? & $\begin{array}{l}\text { Write to tape err: } 40 \mathrm{C} \\
\text { Data may be part salvageable }\end{array}$ & SH \\
\hline
\end{tabular}


Table D1. SGBSN earthquake data-acquisition computer downtime log for 1992 --Continued

\begin{tabular}{|c|c|c|c|c|c|}
\hline \multicolumn{2}{|c|}{ Down } & \multicolumn{2}{|c|}{ Up } & \multirow[b]{2}{*}{ Problem noted } & \multirow{2}{*}{$\begin{array}{c}\text { Techniclan/ } \\
\text { Analyst }\end{array}$} \\
\hline $\begin{array}{c}\text { Date } \\
\mathrm{Mo} / \mathrm{Da}\end{array}$ & $\begin{array}{c}\text { nme } \\
\text { Hr:Mn UTC }\end{array}$ & $\begin{array}{c}\text { Date } \\
\mathrm{Mo} / \mathrm{Da}\end{array}$ & $\begin{array}{c}\text { Tlme } \\
\text { Hr:Mn UTC }\end{array}$ & & \\
\hline $07 / 20$ & $04: 08$ & $07 / 20$ & $12: 12$ & Tape drive MM1: hard err & JD \\
\hline $07 / 25$ & $15: 14$ & $07 / 25$ & 18:01 & Tapes filled & JD \\
\hline $07 / 28$ & 19:16 & $07 / 28$ & $19: 22$ & Scheduled power outage & JD \\
\hline $07 / 31$ & 18:57 & $07 ß 1$ & $19: 31$ & Scheduled power outage & JD \\
\hline $07 / 31$ & 21:07 & $07 / 31$ & $21: 12$ & Power outage & JD \\
\hline $07 / 31$ & $21: 20$ & $07 / 31$ & $22: 50$ & Power outage & JD \\
\hline $08 / 01$ & $12: 56$ & $08 / 01$ & $14: 42$ & Power outage & JD \\
\hline $08 / 01$ & $17: 00+$ & $08 / 01$ & $18: 31$ & Power outage & JD \\
\hline $08 / 03$ & 05:22 & $08 / 03$ & $13: 10$ & Power-outage related & $J D$ \\
\hline $08 / 04$ & 06:08 & $08 / 04$ & $12: 32$ & Watchdog ("auto") asleep & JD \\
\hline $08 / 06$ & $05: 26$ & $08 / 06$ & $14: 10$ & Tapes filled & JD \\
\hline $08 / 08$ & $09: 00+$ & 08/08 & $18: 00$ & Tape controller problem & SH \\
\hline $08 / 12$ & 03:40 & $08 / 12$ & $14: 15$ & Tape controller problem & SH \\
\hline $08 / 13$ & $17: 21$ & $08 / 14$ & 01:30 & Tape controller problem & SH \\
\hline $08 / 19$ & 19:04 & $08 / 19$ & 19:47 & Vacuum prob tape drive & JD \\
\hline $08 / 30$ & $17: 00+$ & $08 / 30$ & $17: 38$ & Telos PM-drive repair & JD \\
\hline $09 / 13$ & $12: 44$ & $09 / 13$ & $18: 46$ & Select error & TB \\
\hline $09 / 23$ & $15: 00$ & $09 / 23$ & $15: 22$ & Select error tapes full & SH \\
\hline $09 / 30$ & $15: 30$ & $12 / 31$ & $11: 59.9$ & SGBSN offline at Golden & SH \\
\hline
\end{tabular}

For the period 01/01/1992 through 05/31/1992, develocorder films have been read and hypocenters determined for local earthquakes. For later downtime, films have been scanned briefly, but no arrival time data have been recorded. 

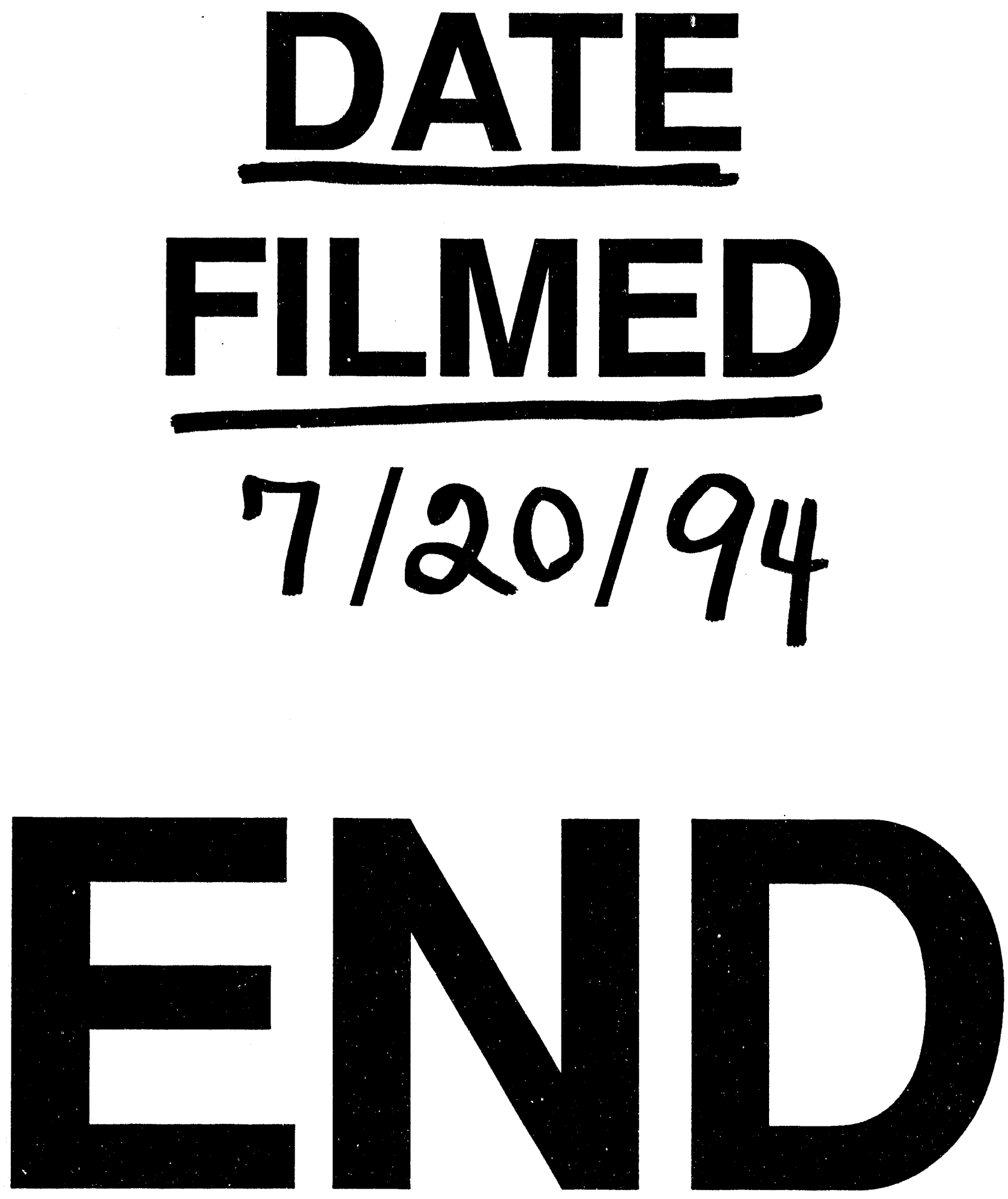


\section{1}

